\title{
EXPLORING THE POTENTIAL OF URBAN LEFTOVER SPACES
}

BY

JASIM AZHAR

\author{
A thesis submitted to \\ Victoria University of Wellington \\ in fulfillment of the requirements for the degree of
}

Doctor of Philosophy

Victoria University of Wellington

2019 


\section{Published Work}

Some of the text has been cited from my published work during the $\mathrm{PhD}$, where I am the first author, and I have taken verbal approval from my co-authors to include the text in this thesis. The references for these papers are:

Azhar, J; Vale, B and Gjerde, M. (2018). Reimagining Urban Leftover Spaces, Journal of Architecture, SASBE $6^{\text {th }}$ International Conference Proceedings (7-9 Dec), Sydney, Australia.

Azhar, J; Vale, B and Gjerde, M. (2018). Urban Leftover Spaces: Transformation from Within', Journal of Architecture, DAKAAM Conference Proceedings (12-14 April), Dubrovnik, Croatia. (ISBN: 978-605-9207-99-7)

Azhar, J and Gjerde, M. (2016). "Rethinking the role of Urban In-between Spaces, Journal of Architectural Science Review, ASA Conference Proceedings (7-9 Dec), Taylor \& Francis, Adelaide. (ISBN: 978-3-033-04654-2) 


\section{Abstract}

A city's spatial environment emerges from the ongoing negotiation between the constructed environment, urban processes, and bodily experience. Many spaces do not represent a static notion but are continually challenged and reconstituted, including spaces that appear to be 'leftover'. The ability to recognise leftover spaces in the urban context is an integral part of the urban redevelopment process, where structured and layered approaches become useful in understanding how to transform these spaces into places. Consequently, leftover spaces in the urban fabric can be seen both as having potential and as threatening. Researchers have pointed out the issues, conditions, and importance of the positive utilisation of leftover spaces. These spaces can be designed, transformed, and integrated into the main urban fabric to achieve environmental and social gains. Creative and flexible design should lead to psychologically healthy places by improving the image of a city from within. However, there is insufficient information available on how to go about designing such spaces.

The revitalisation and aesthetic quality of leftover spaces could expand the dynamism of a city through strategic design interventions. This study explores how the visual perception of leftover spaces in Wellington City that influences both personal experiences and their potential usage could be enhanced. The research aims to investigate the potential of different types of urban leftover spaces, which could be used in a more effective way than they are present. The mixed methodology undertaken in this study seeks to inform planning initiatives by knowing what people feel about leftover spaces and their aspects that need improvement. This research, therefore, examines how such leftover spaces are defined and can be redesigned to become part of a built environment. The research thus consists of three studies starting with an initial visual preference study to understand human perceptions that could lead to better design solutions. The second study explored the differences in design preferences among participants coming from different fields of study, forming the main visual preference study. Visual preferences can guide behaviour and the emotional responses of different users in the redesign of such spaces and their essential attributes. Lastly, focus group discussions were held with built and non-built environment participants. To sum up, the results revealed that providing more vegetation is a critical design attribute for such spaces. The study contradicts theories 
that hold there are differences in the ways built and non-built environment experts perceive the environment.

Keywords: Urban Leftover Spaces, Environmental Perception and Aesthetics, Visual Preference Study 


\section{Acknowledgements}

I want to express my heartfelt gratitude to some of the kindest people around me, those who continuously supported me in composing my research.

Firstly, I would like to thank my supervisors Brenda Vale and Morten Gjerde sincerely. Their motivation and immense knowledge have helped me in all areas of my research. Without their guidance, I would not have been able to conduct this thesis to the best of my abilities.

Besides my encouraging mentors, I want to thank Lisa Woods for always providing me with useful feedback and correcting the general mistakes in my statistics. I would also like to thank the financial, academic and technical support staff at the Victoria University of Wellington. They all have been very insightful and comforting throughout my writing journey. I would also like to show my appreciation for my research fellows with whom I have learned many new things, explored new places, and shared memorable moments.

My warmest thanks and humility go to God Almighty, for providing me with an opportunity to take my career to a higher level. Moreover, to my parents, brothers, sisters and my wife, who have all always stood by my side with their precious support.

Finally, I would like to thank my beloved parents, parents-in-law and wife in particular. My father and mother have always guided me with their pearls of wisdom, both academically and about life in general. A special thanks goes to my beloved wife, who always stood patiently by during the times of stress and anxiety while trying her best to make things easier for me. I appreciate you all for giving me faith and believing in me. 


\section{Table of Contents}

Published Work ....................................................................................... i

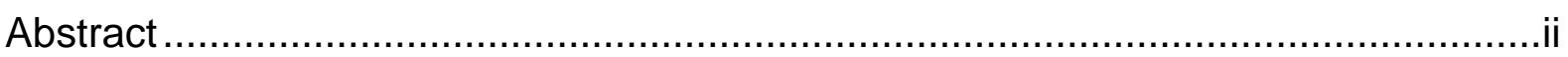

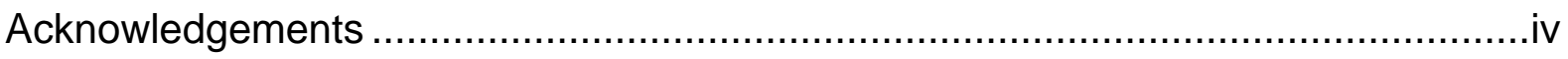

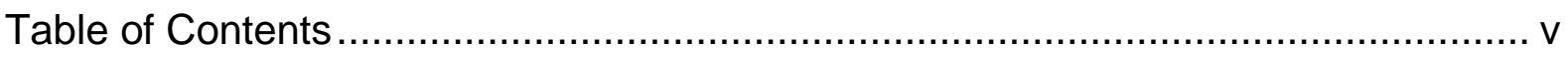

List of Figures....................................................................................

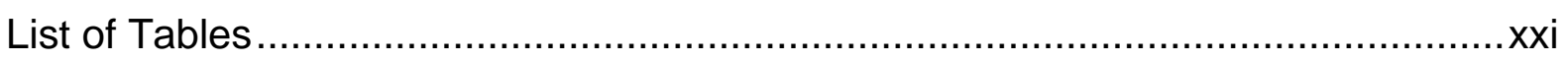

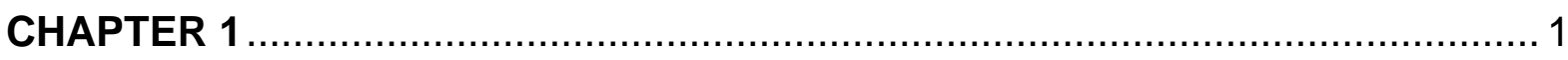

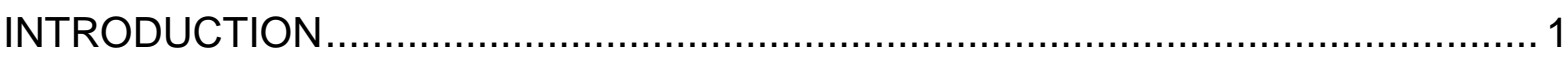

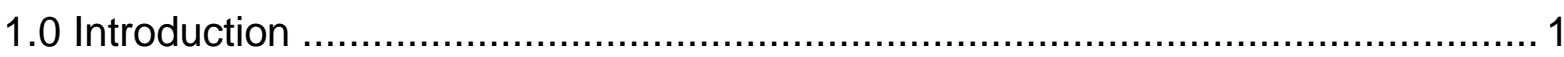

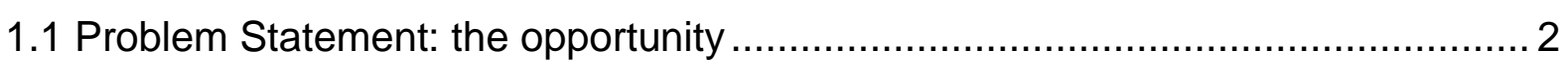

1.1.1 Significance of Leftover Spaces ........................................................ 4

1.1.2 Significance of Environmental Aesthetics ................................................ 5

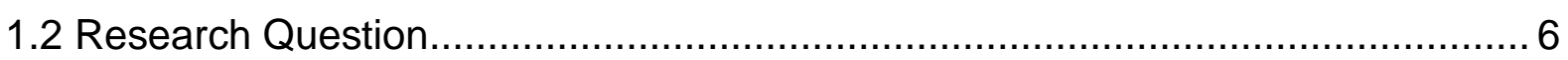

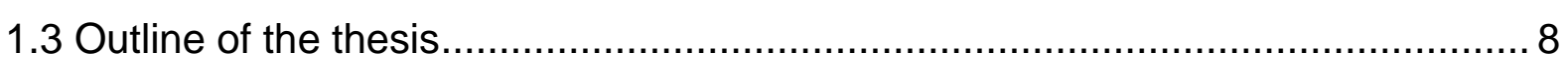

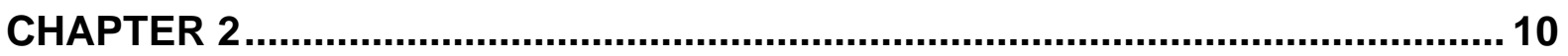

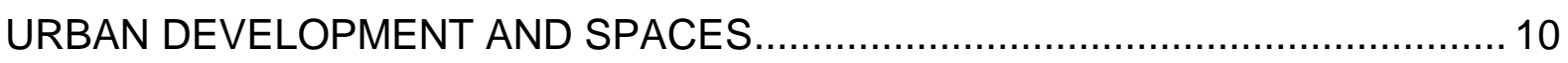

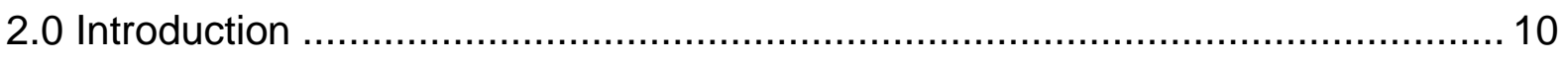

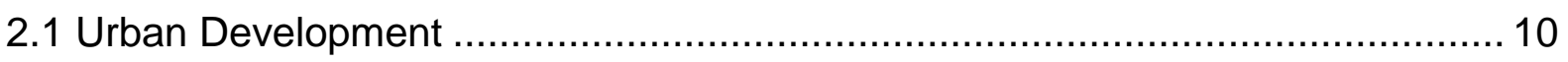

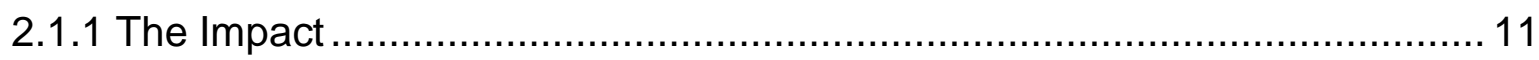

2.1.2 The Compact City Approach .......................................................... 13

2.2 Urban Spaces ............................................................................... 15

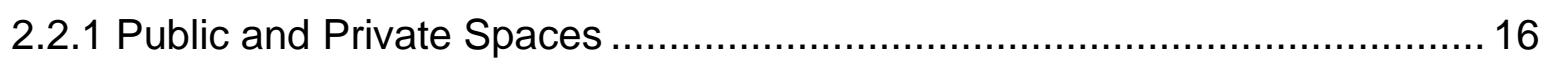

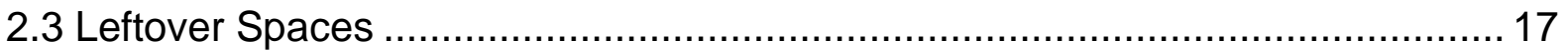

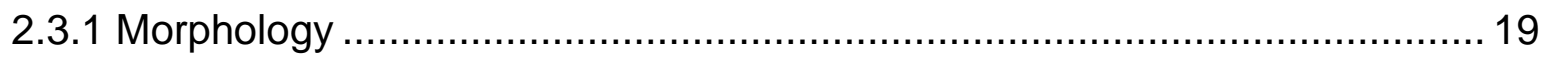

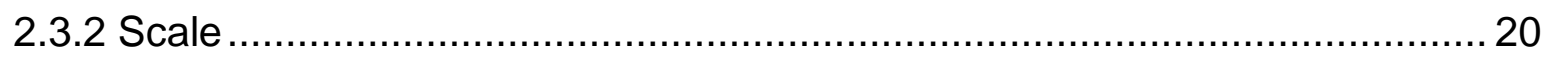

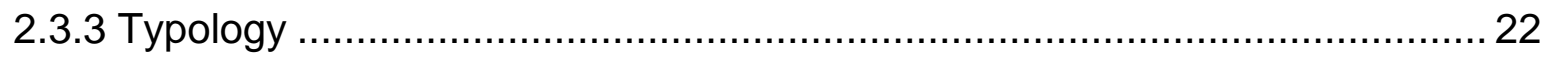

2.4 Leftover Spaces as 'Inbetween Spaces' ................................................. 26

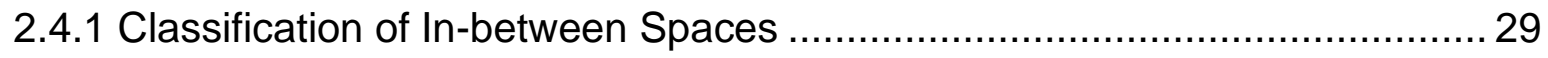

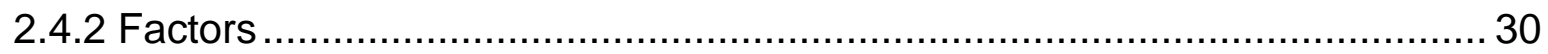

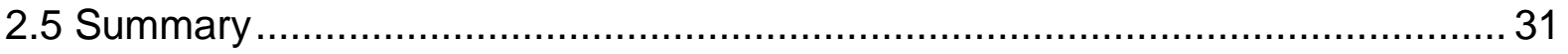

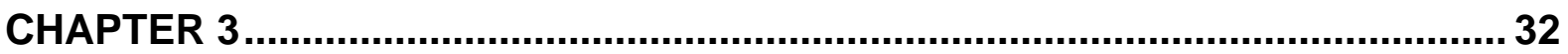

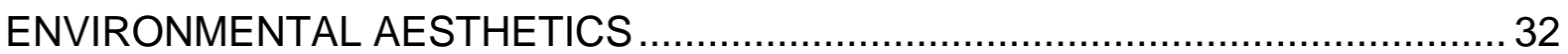


3.0 Introduction 32

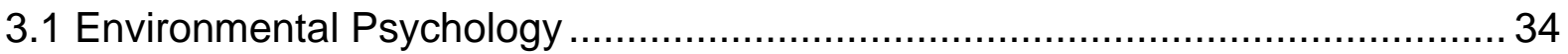

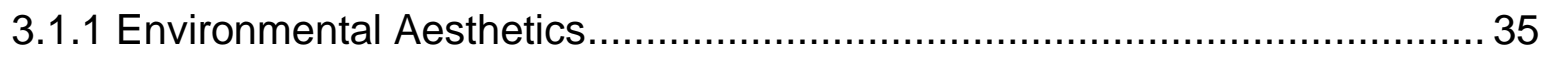

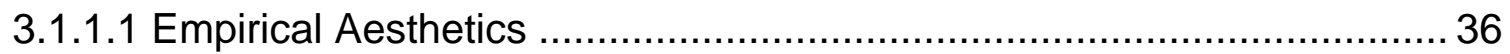

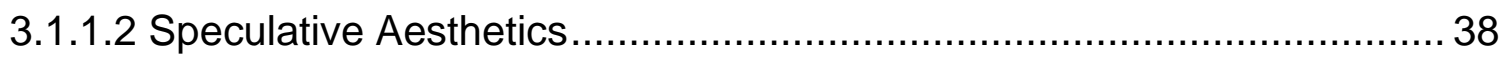

3.2 Sensory Stimulation and the Built Environment ............................................ 38

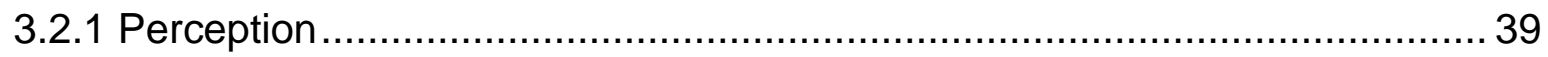

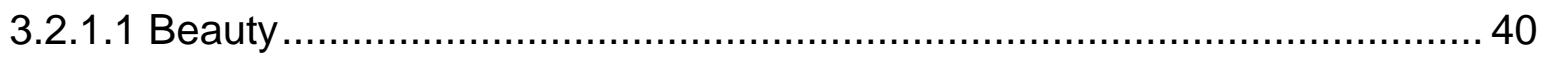

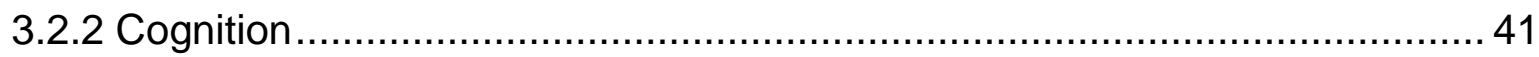

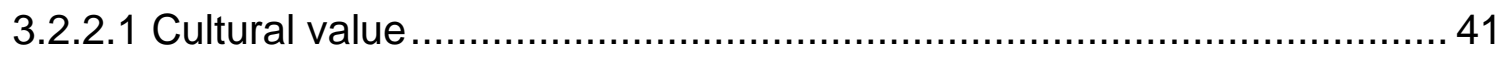

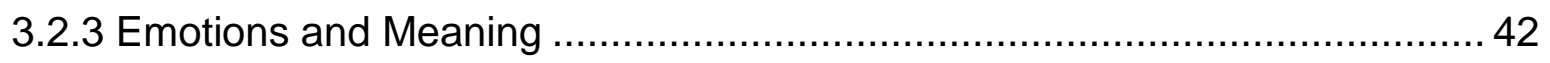

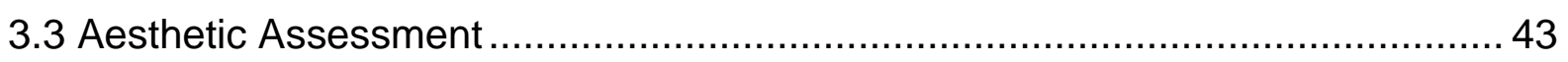

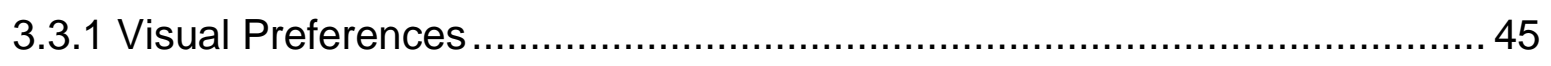

3.3.2 Difference of Opinions in Perceived Values ............................................. 46

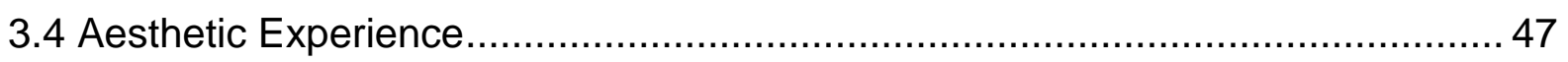

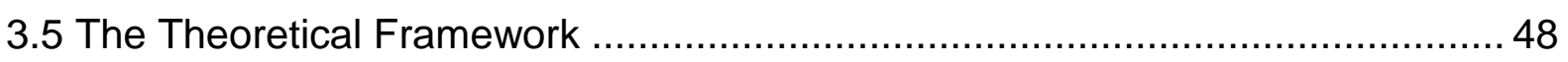

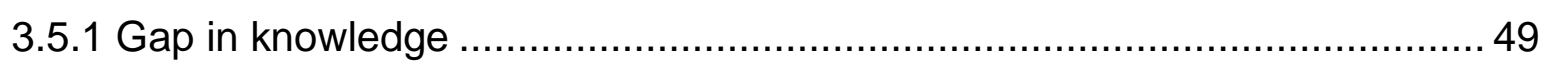

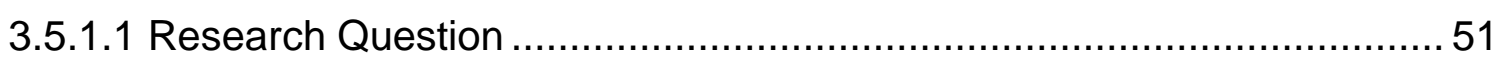

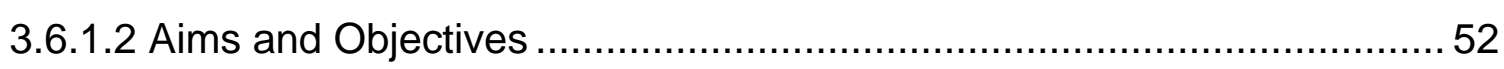

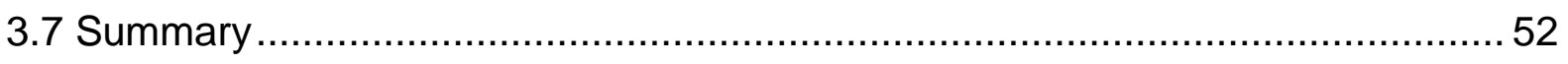

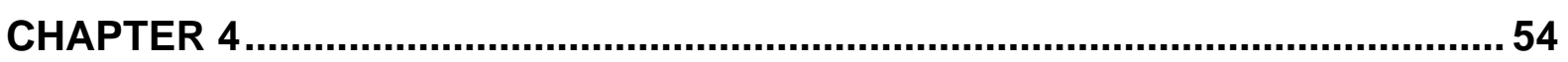

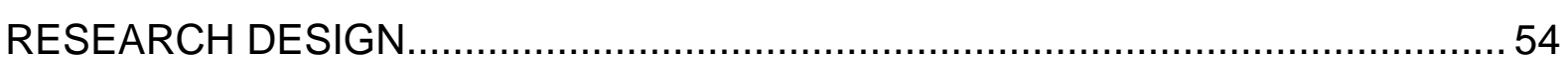

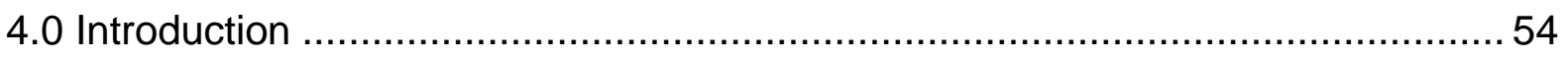

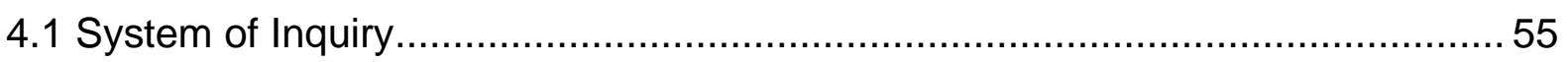

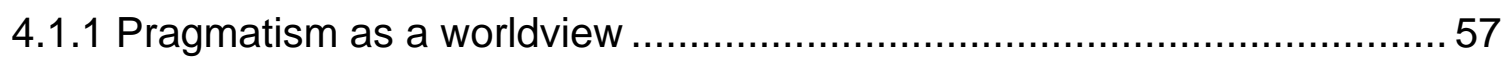

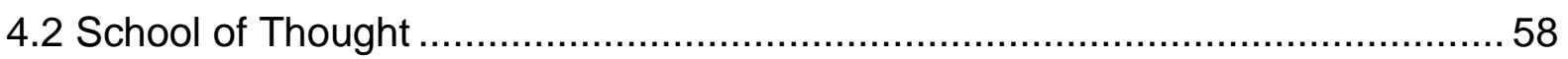

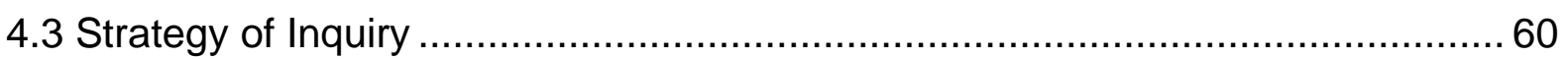

4.3.1 Concurrent Triangulation Method ......................................................... 61

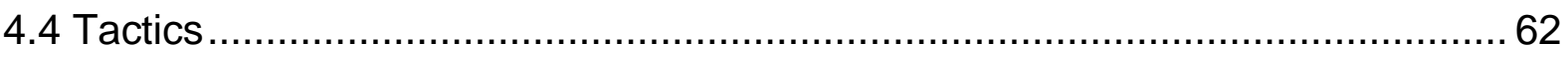

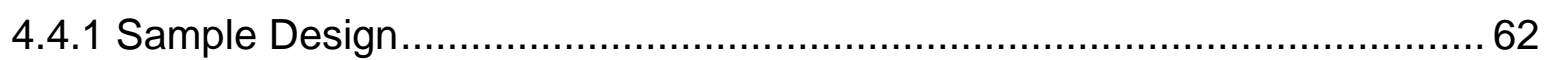

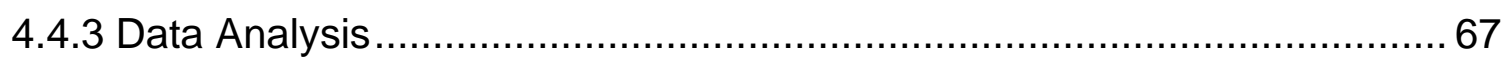

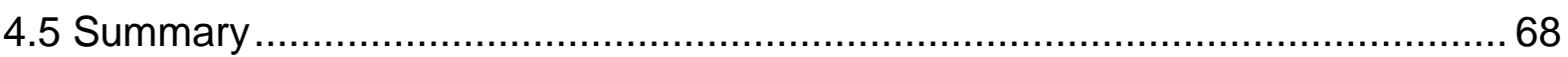

CHAPTER 5

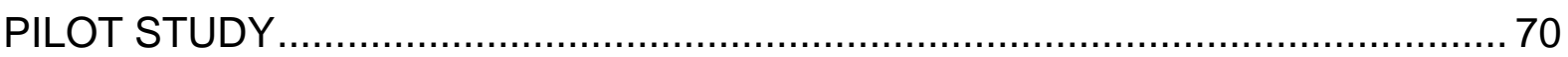

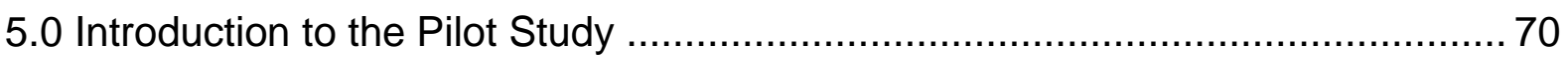




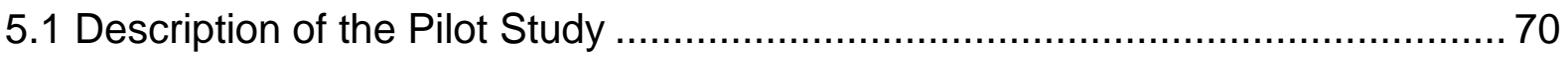

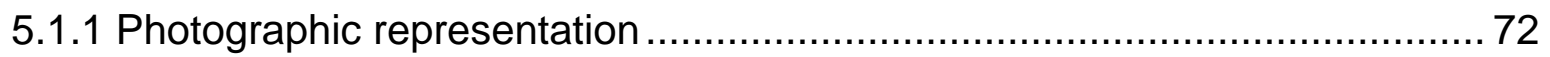

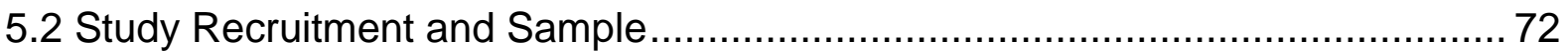

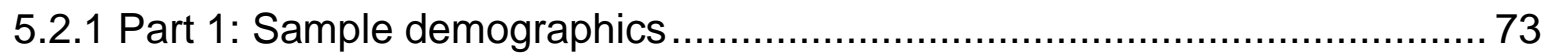

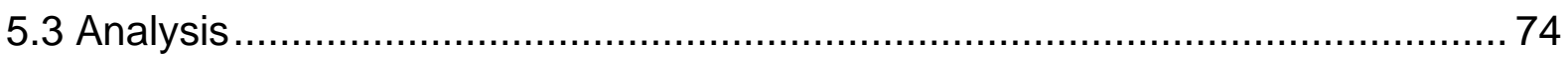

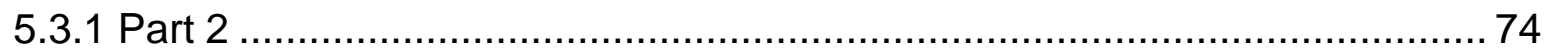

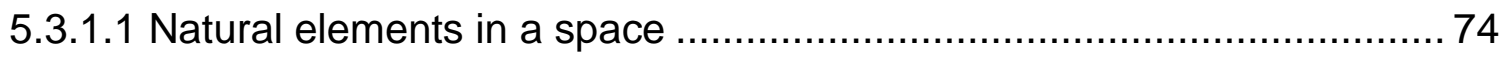

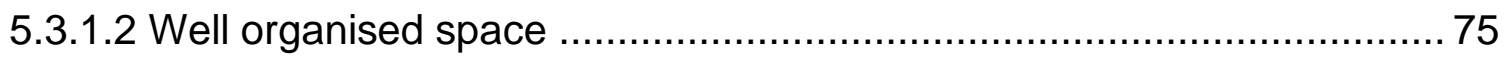

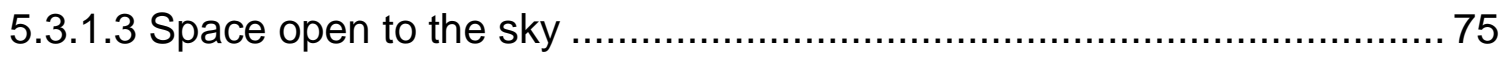

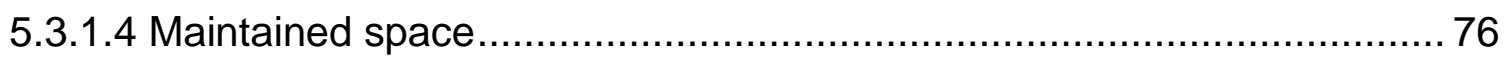

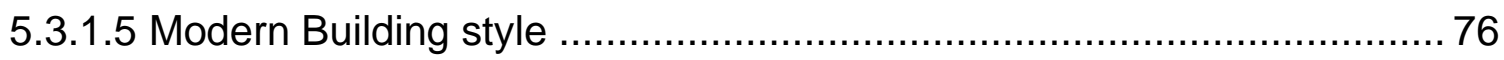

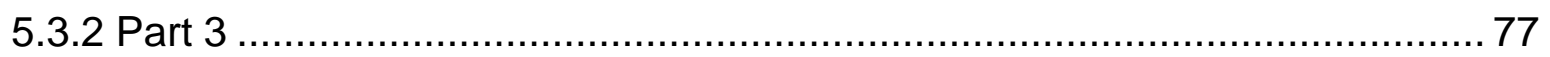

5.3.2.1 Space underneath a building …................................................ 78

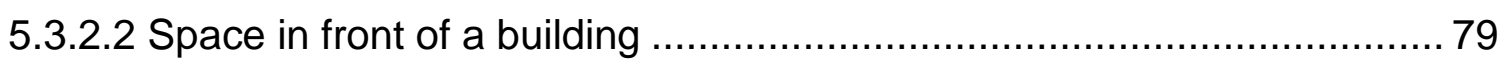

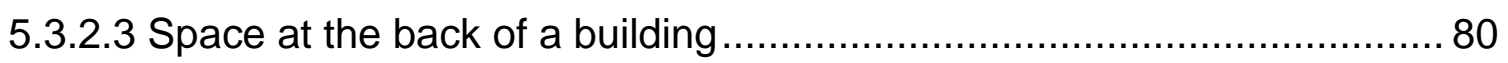

5.3.2.4 Space enclosed by buildings on three sides ........................................ 81

5.3.2.5 Space enclosed by buildings on two sides........................................... 82

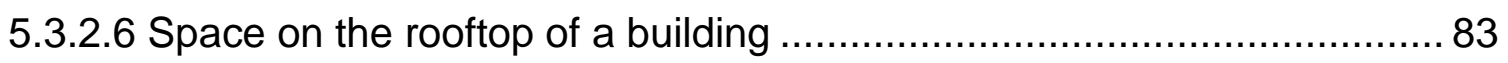

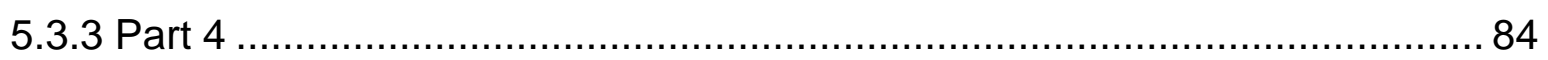

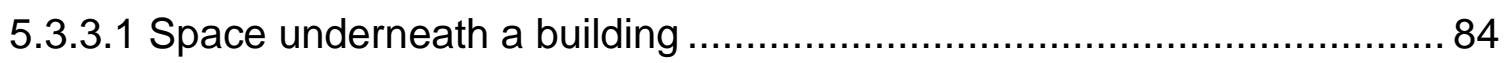

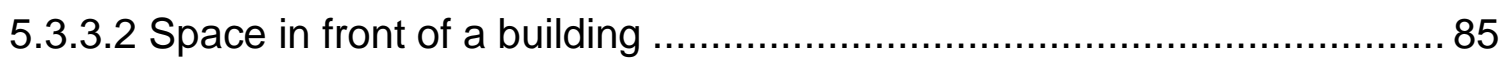

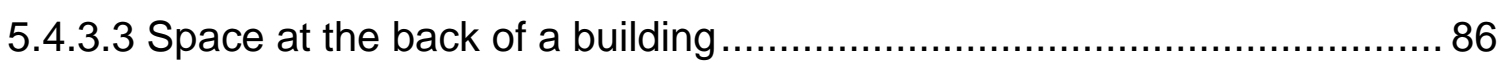

5.3.3.4 Space enclosed by buildings on three sides ....................................... 87

5.3.3.5 Space enclosed by buildings on two sides......................................... 88

5.3.3.6 Space on the rooftop of a building ………....................................... 90

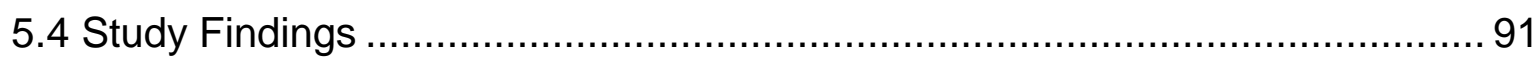

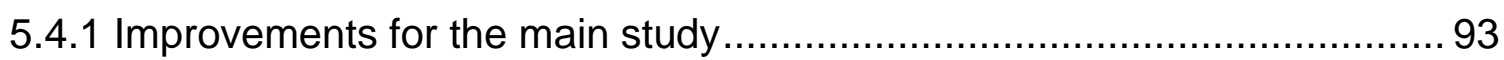

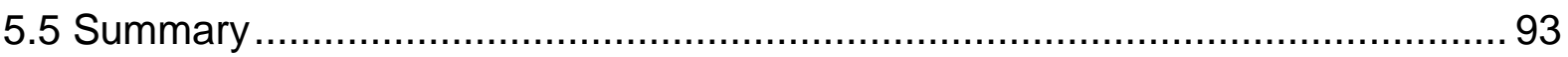

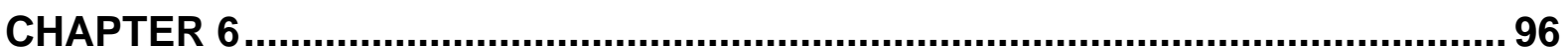

Study One: FIRST VISUAL PREFERENCE STUDY ............................................. 96

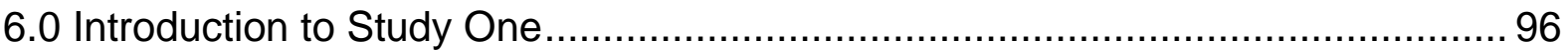

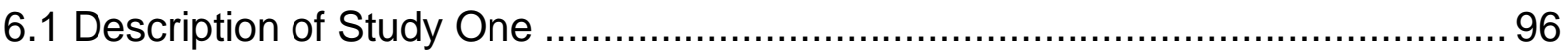

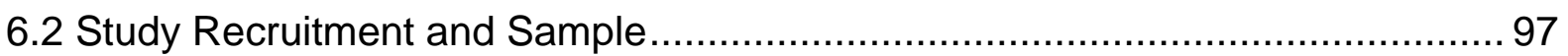

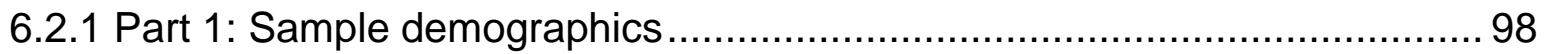

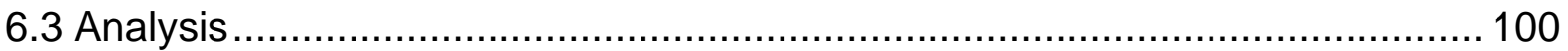




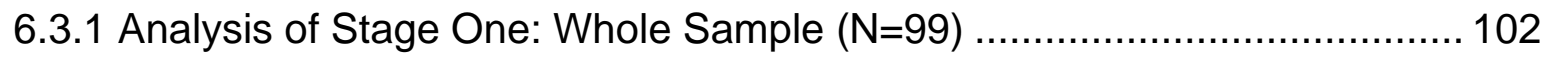

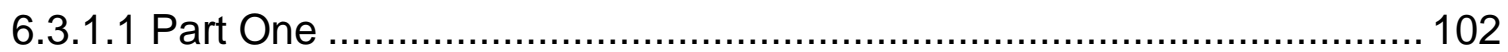

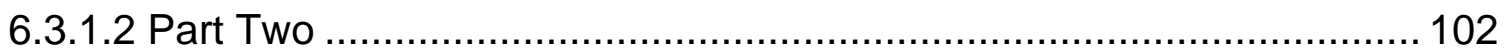

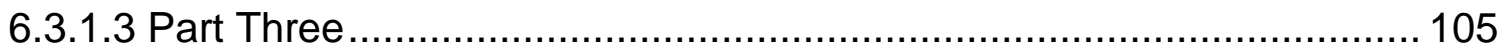

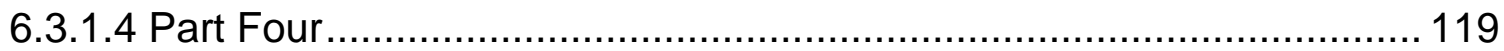

6.3.2 Analysis of Stage two: Gender Differences..................................... 125

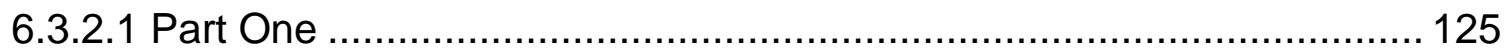

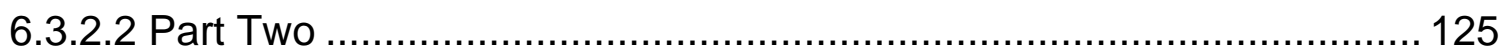

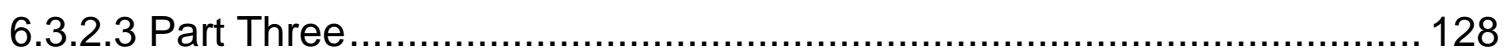

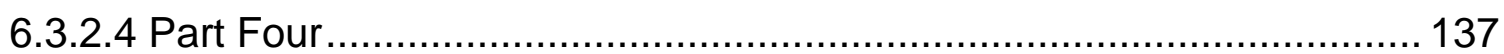

6.3.3 Analysis of Stage three: Built Environment (BE) and Non-Built Environment

(NBE) Participants ................................................................... 144

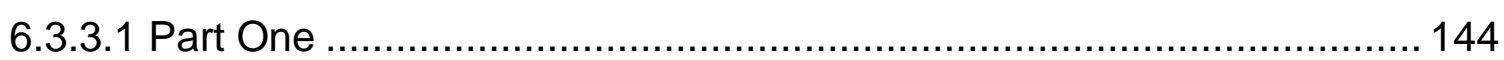

6.3.3.2 Part Two ............................................................................ 145

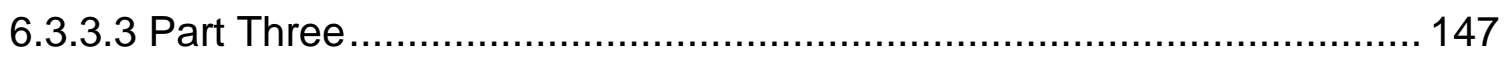

6.3.3.4 Part Four ............................................................................. 157

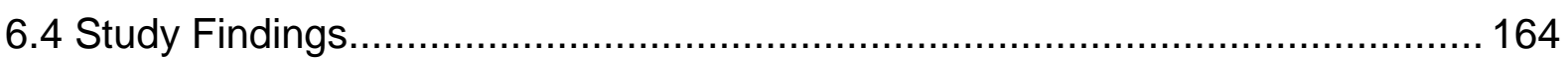

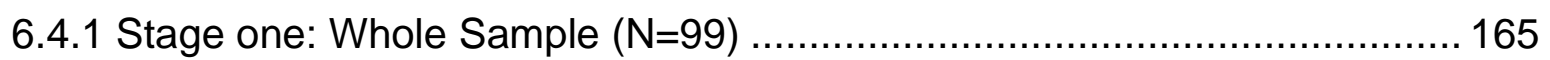

6.4.2 Stage two: Gender analysis ........................................................ 166

6.4.3 Stage three: Built Environment (BE) and Non-Built Environment (NBE)

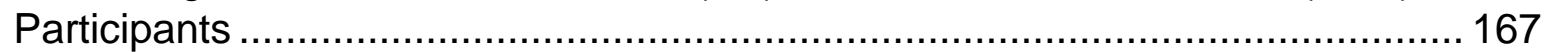

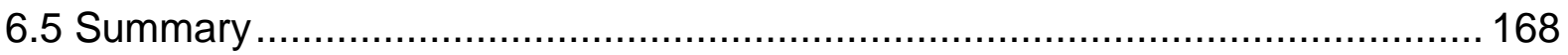

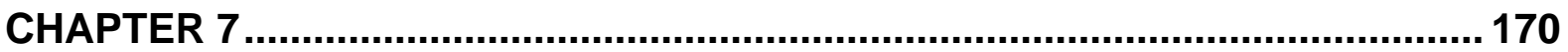

STUDY TWO: SECOND VISUAL PREFERENCE STUDY ............................. 170

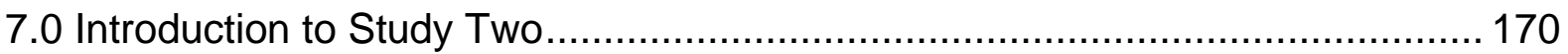

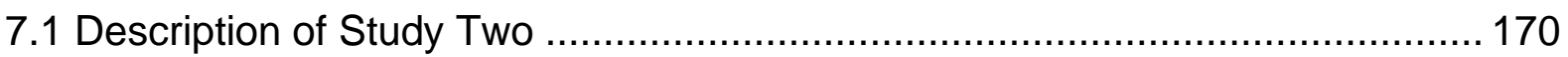

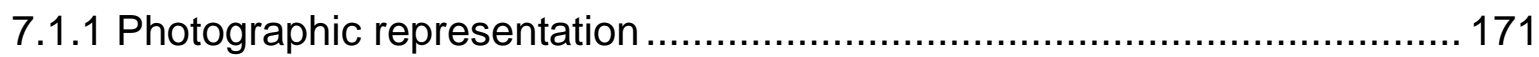

7.2 Study Recruitment and Sample ......................................................... 172

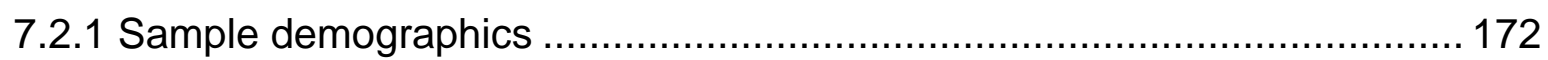

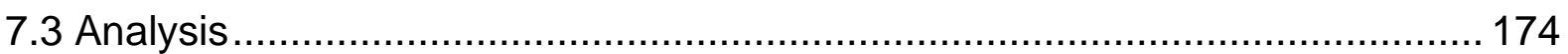

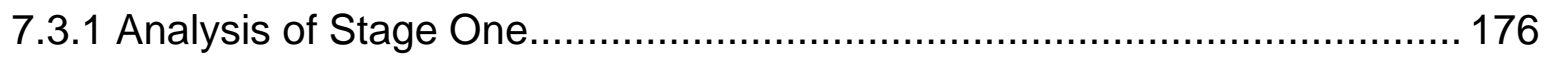

7.3.1.1 Space underneath a building .............................................. 177

7.3.1.2 Space in front of a building ................................................... 179

7.3.1.3 Space at the back of a building ............................................... 182

7.3.1.4 Space enclosed by buildings on three sides .................................. 185 
7.3.1.5 Space enclosed by buildings on two sides...................................... 188

7.3.1.6 Space on the rooftop of a building ................................................ 191

7.3.1.7 Participant selections for improving the leftover spaces .................... 194

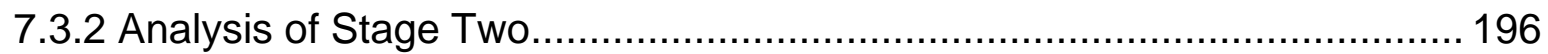

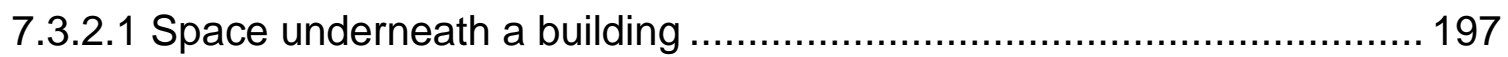

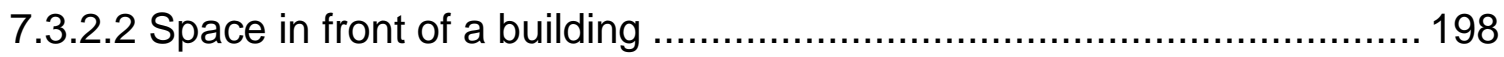

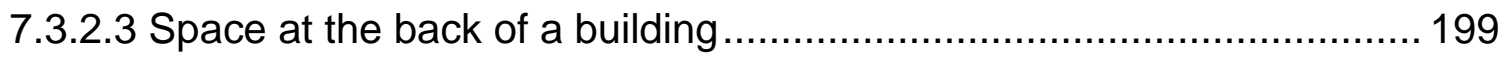

7.3.2.4 Space enclosed by buildings on three sides ..................................... 200

7.3.2.5 Space enclosed by buildings on two sides......................................... 201

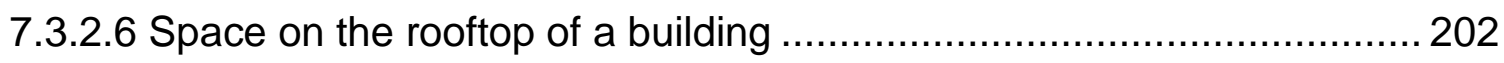

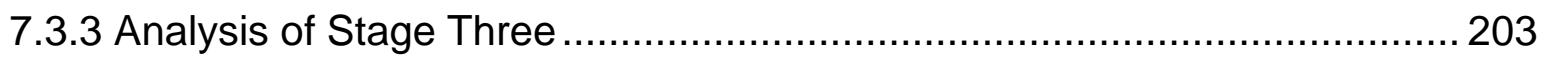

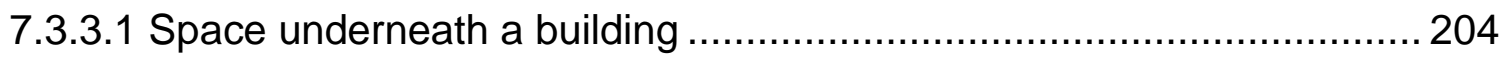

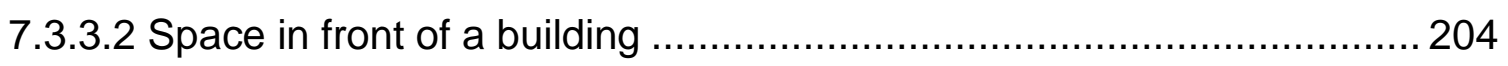

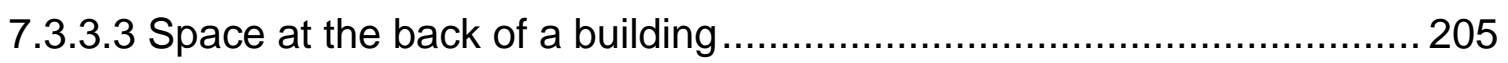

7.3.3.4 Space enclosed by buildings on three sides ..................................... 206

7.3.3.5 Space enclosed by buildings on two sides........................................ 207

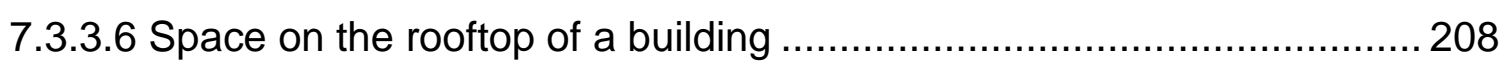

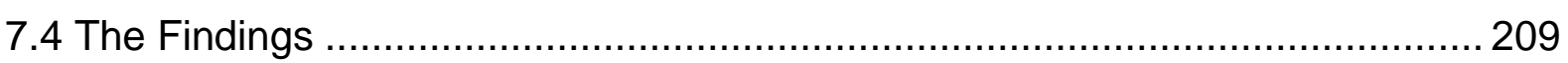

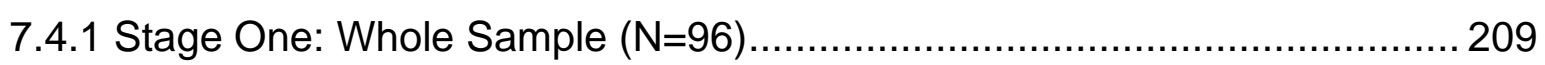

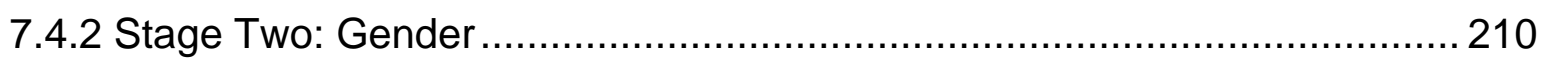

7.4.3 Stage Three: Built Environment (BE) and Non-Built Environment (NBE)

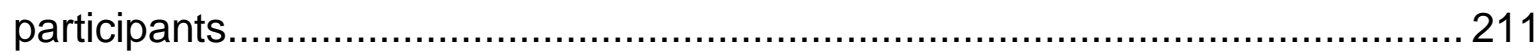

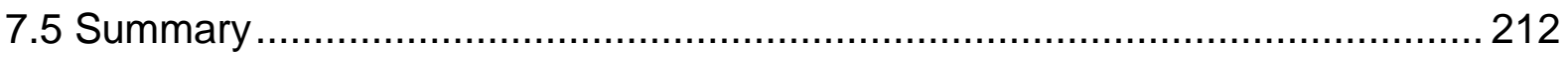

CHAPTER 8

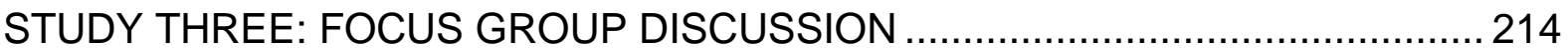

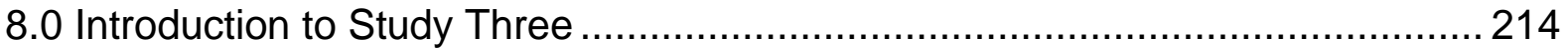

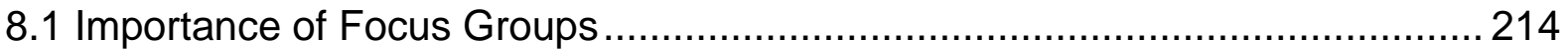

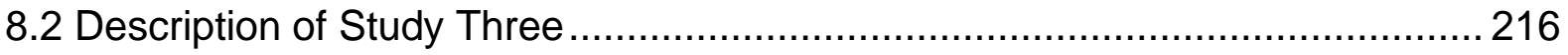

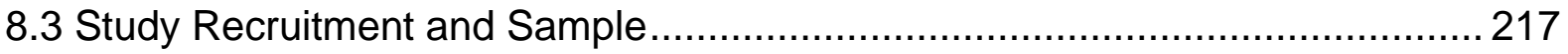

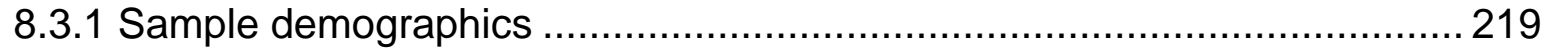

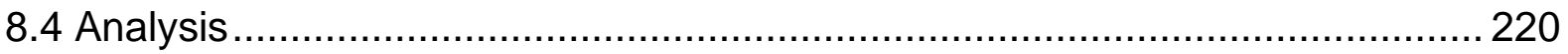

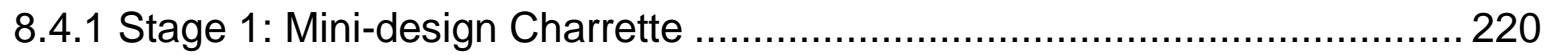

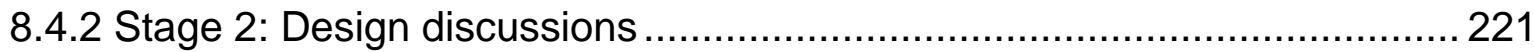

8.4.3 Built Environment (BE) and Non-Built Environment (NBE) Participants..... 222

8.4.3.1 Space underneath a building ….................................................. 223 


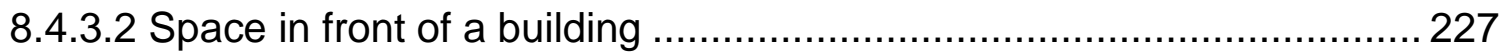

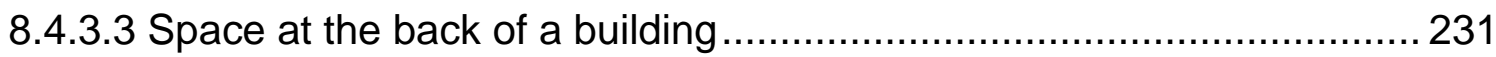

8.4.3.4 Space enclosed by buildings on three sides .................................... 235

8.4.3.5 Space enclosed by buildings on two sides..................................... 238

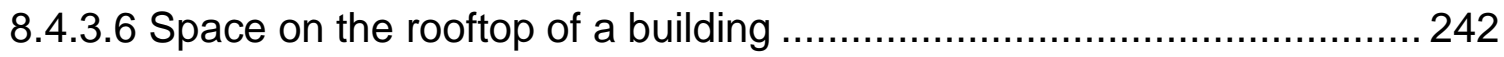

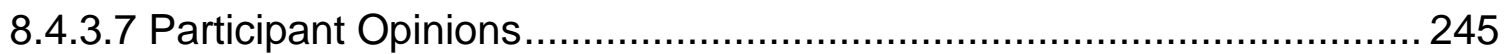

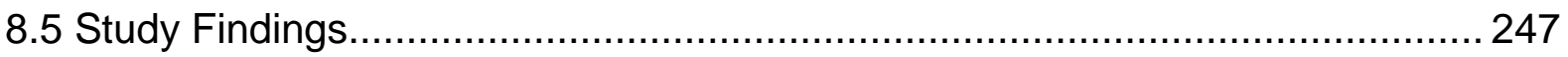

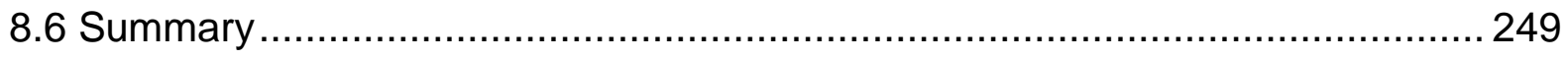

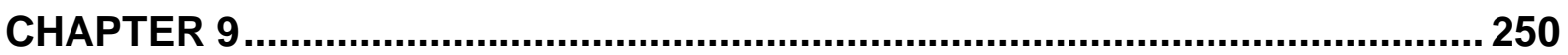

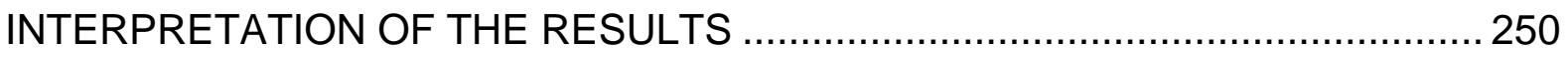

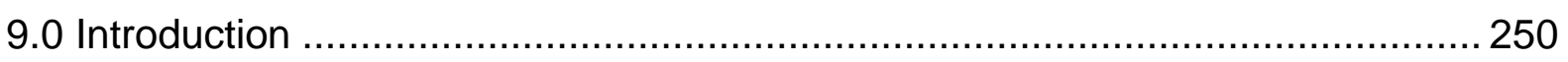

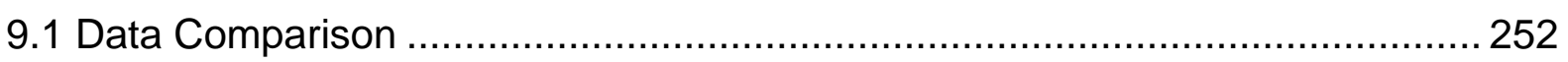

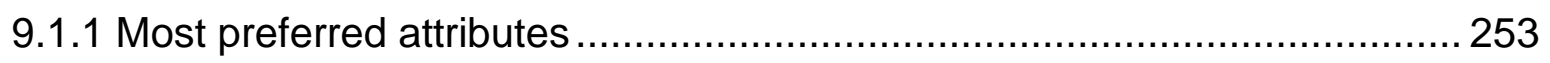

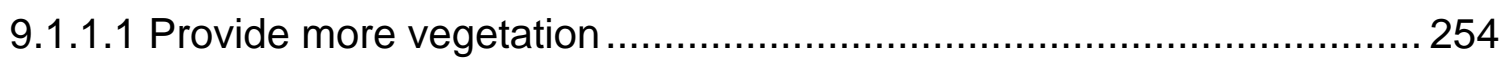

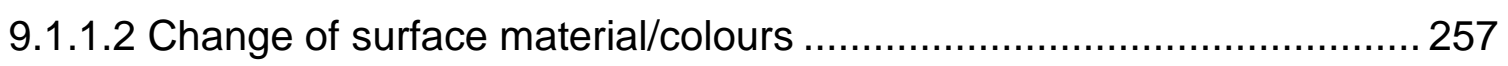

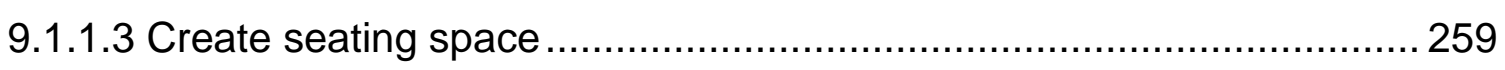

9.1.1.4 Improve maintenance and cleanliness............................................... 260

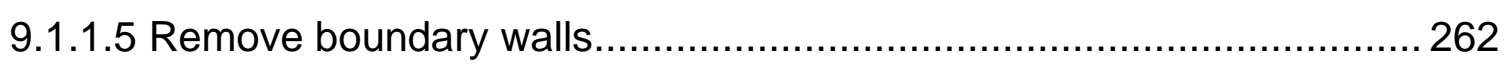

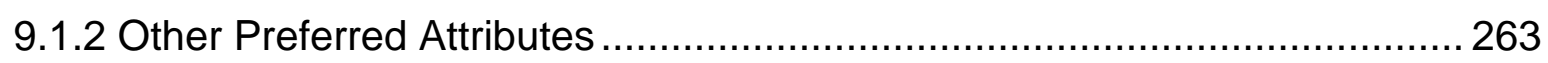

9.1.2.1 Create a clear (pedestrian) pathway .................................................... 264

9.1.2.2 Install wind turbines and solar panels ................................................ 266

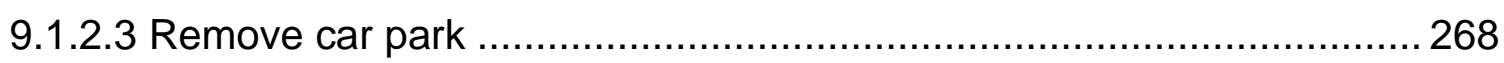

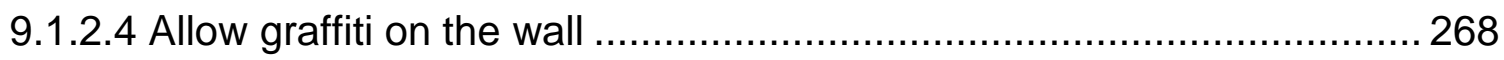

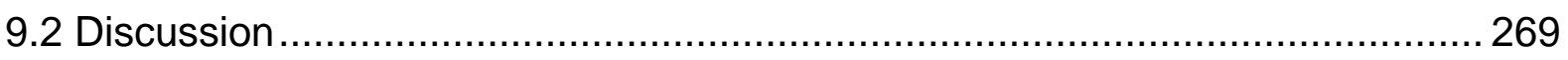

9.2.1 Similarities with Nasar's framework ………...................................... 278

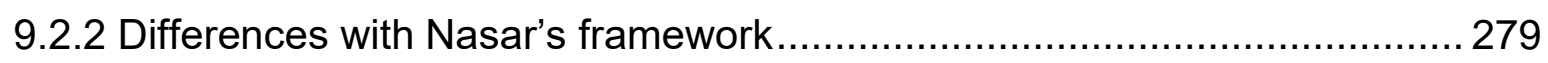

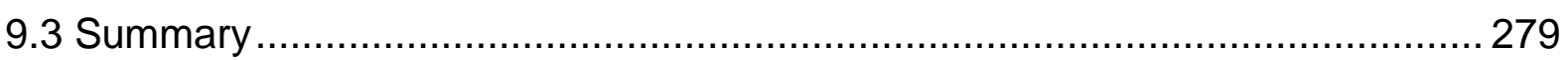

CHAPTER 10

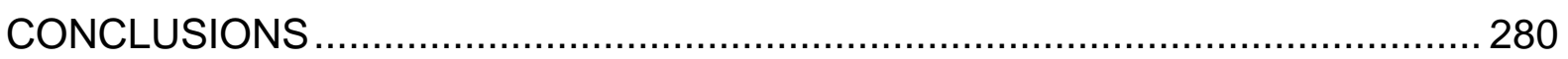

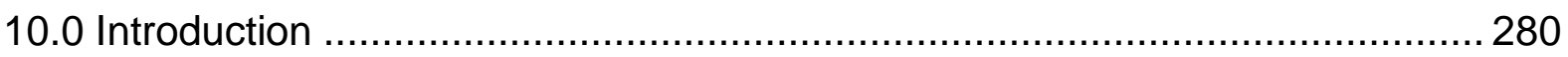

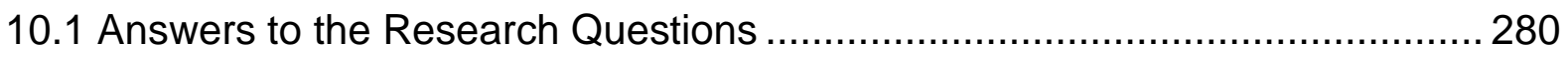

10.1.1 What are people's visual preferences for urban leftover spaces in

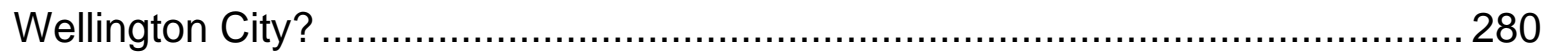

10.1.1.1 What attributes do people prefer for the design of urban leftover spaces? 
10.1.1.2 Are there any differences in design preferences between male and female participants?

10.1.1.3 Are there any differences in design preference between participants with a built environment background and those without?

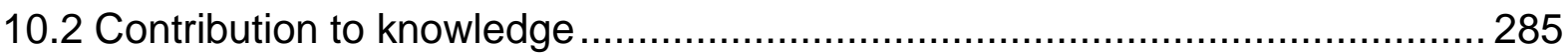

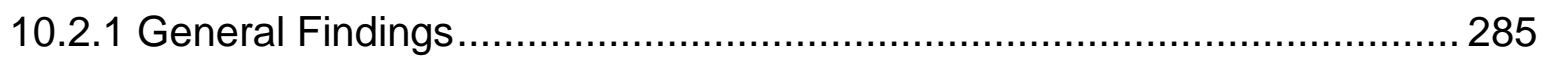

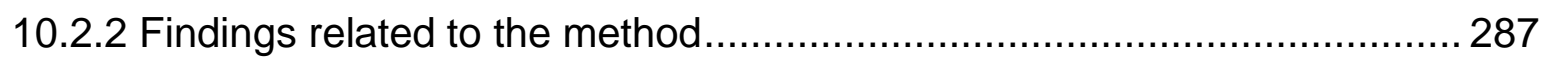

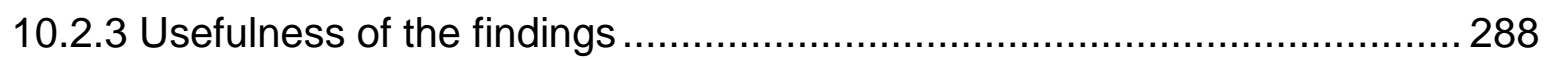

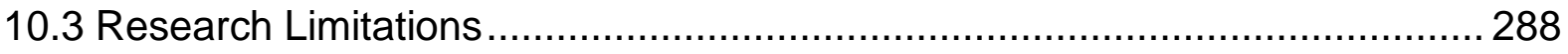

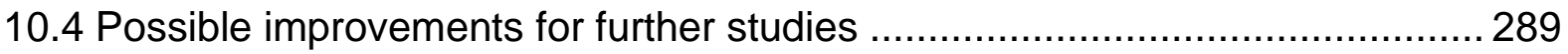

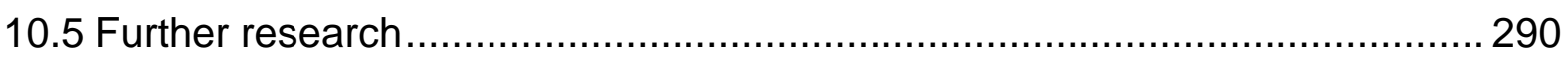

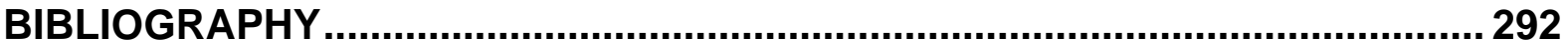

APPENDICES 


\section{List of Figures}

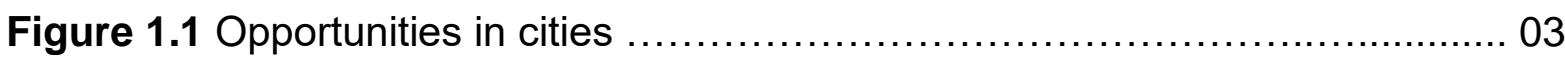

Figure 2.1 New Zealand primary energy supply................................ 12

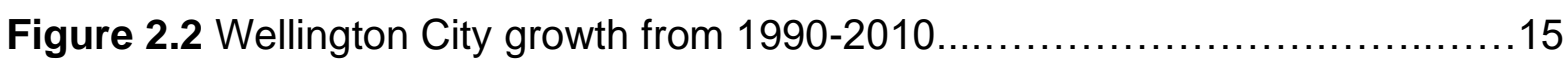

Figure 2.3 Space configuration proposed by Newman (1972) ...................... 17

Figure 2.4 Different scales of space in cities..................................... 21

Figure 2.5 Different unused and unmanaged spaces in Wellington City............. 28

Figure 2.6 Spatial classification for in-between spaces........................... 30

Figure 3.1 Diagram of analytical framework.......................................... 33

Figure 3.2 A theoretical framework for this research ............................... 43

Figure 3.3 Overview of this research............................................... 51

Figure 4.1 A nested framework for research design .............................. 54

Figure 4.2 Hierarchical order of a paradigm ......................................... 55

Figure 4.3 Fields examined in this research .................................... 58

Figure 4.4 Diagram of the concurrent triangulation strategy to be used in this study

Figure 5.1 Space with natural elements was preferred over space with artificial

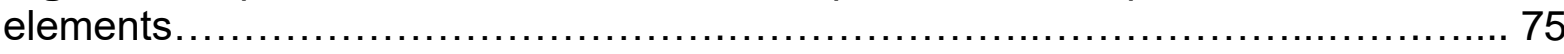

Figure 5.2 Well-organised space was preferred over unorganised

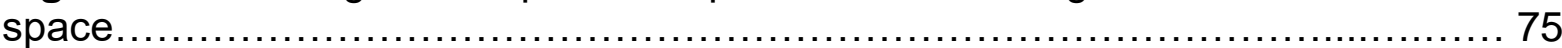

Figure 5.3 Space with openness was preferred over an enclosed

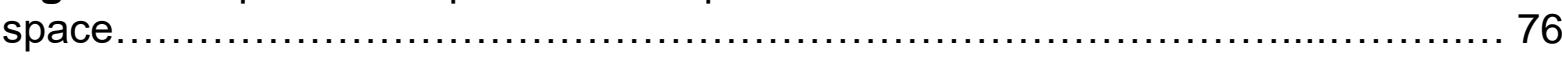

Figure 5.4 Well-maintained space was preferred over unmanaged space.

Figure 5.5 Modern building style was preferred over the old building style

Figure 5.6 Options shown to participants ….................................... 78

Figure 5.7 Participant preferences for the different options......................... 78

Figure 5.8 Options shown to participants …....................................... 79

Figure 5.9 Participant preferences for the different options......................... 79

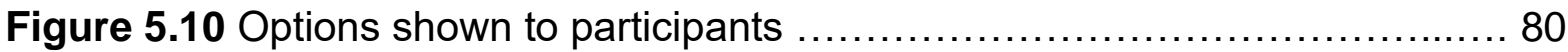

Figure 5.11 Participant preferences for the different options....................... 80 
Figure 5.12 Options shown to participants 81

Figure 5.13 Participant preferences for the different options........................ 81

Figure 5.14 Options shown to participants........................................ 82

Figure 5.15 Participant preferences for the different options...................... 82

Figure 5.16 Options shown to participants....................................... 83

Figure 5.17 Participant preferences for the different options........................ 83

Figure 5.18 Two options shown to participants..................................... 84

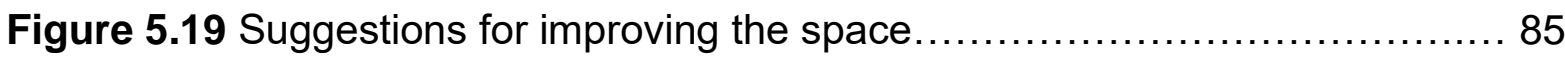

Figure 5.20 Two options shown to participants................................... 85

Figure 5.21 Suggestions for improving the space .............................. 86

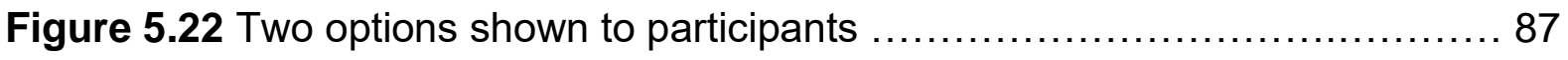

Figure 5.23 Suggestions for improving the space............................... 87

Figure 5.24 Two options shown to participants ................................ 88

Figure 5.25 Suggestions for improving the space............................... 88

Figure 5.26 Two options shown to participants .................................. 89

Figure 5.27 Suggestions for improving the space.................................. 89

Figure 5.28 Two options shown to participants ................................ 90

Figure 5.29 Suggestions for improving the space................................ 91

Figure $\mathbf{5 . 3 0}$ Overall modifications suggested by participants for the leftover

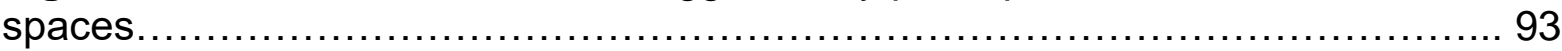

Figure 6.00 Three stages of Study One and the parts for each stage.

Figure 6.01 Space with natural elements was preferred over space with artificial

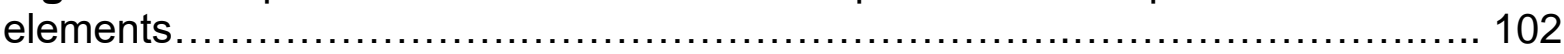

Figure 6.02 Well-organised space was preferred over unorganised space...........103

Figure 6.03 Space with openness was preferred over an enclosed space.

Figure 6.04 Well-maintained space was preferred over unmanaged space. 104

Figure 6.05 Modern building style was preferred over the old building style. 104

Figure 6.06 Preferences for spaces with different attributes........................104

Figure 6.07 Options shown to participants ......................................106

Figure 6.08 Participant preferences for the different options......................106 
Figure 6.09 Participant reasons behind the rating of images A-C ................. 107

Figure 6.10 Options shown to participants ...................................... 108

Figure 6.11 Participant preferences for the different options...................... 109

Figure 6.12 Participant reasons behind the rating of images A-C ................ 110

Figure 6.13 Options shown to participants ......................................111

Figure 6.14 Participant preferences for the different options..................... 111

Figure 6.15 Participant reasons behind the rating of images A-C .................. 112

Figure 6.16 Options shown to participants ...................................... 113

Figure 6.17 Participant preferences for the different options..................... 113

Figure 6.18 Participant reasons behind the rating of images A-C ................. 114

Figure 6.19 Options shown to participants .................................. 115

Figure 6.20 Participant preferences for the different options....................... 115

Figure 6.21 Participant reasons behind the rating of images A-C .................. 116

Figure 6.22 Options shown to participants ......................................... 117

Figure 6.23 Participant preferences for the different options..................... 118

Figure 6.24 Participant reasons behind the rating of images A-C ................. 119

Figure 6.25 Two options shown to participants................................ 120

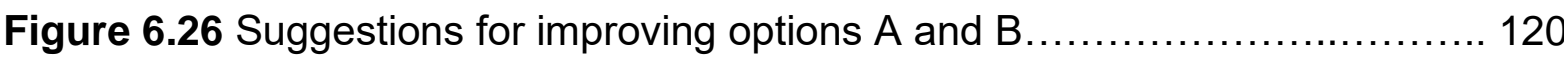

Figure 6.27 Two options shown to participants............................... 121

Figure 6.28 Suggestions for improving options A and B........................ 121

Figure 6.29 Two options shown to participants................................. 122

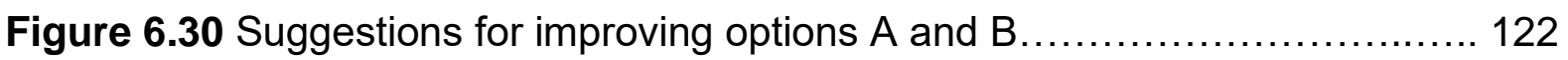

Figure 6.31 Two options shown to participants................................. 122

Figure 6.32 Suggestions for improving options A and B......................... 123

Figure 6.33 Two options shown to participants.................................. 123

Figure 6.34 Suggestions for improving options A and B........................ 124

Figure 6.35 Two options shown to participants................................... 124

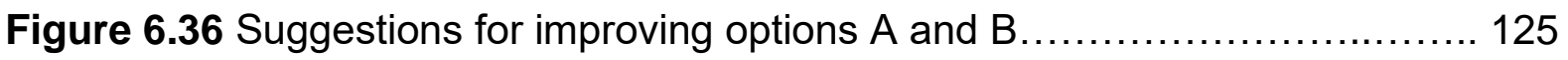

Figure 6.37 Space with natural elements was preferred over space with artificial

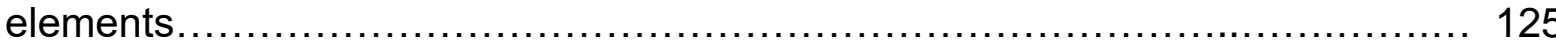

Figure 6.38 Well-organised space was preferred over unorganised space. 
Figure 6.39 Space with openness was preferred over an enclosed space.....

Figure 6.40 Well-maintained space was preferred over unmanaged space.

Figure 6.41 Modern building style was preferred over the old building style

Figure 6.42 Preferences for spaces with different attributes.......................128

Figure 6.43 Options shown to participants .....................................129

Figure 6.44 Gender preferences for the different options......................... 129

Figure 6.45 Options shown to participants ...................................... 130

Figure 6.46 Gender preferences for the different options...................... 131

Figure 6.47 Options shown to participants ..................................... 132

Figure 6.48 Gender preferences for the different options........................ 132

Figure 6.49 Options shown to participants ....................................... 133

Figure 6.50 Gender preferences for the different options........................ 134

Figure 6.51 Options shown to participants ........................................... 135

Figure 6.52 Gender preferences for the different options........................ 135

Figure 6.53 Options shown to participants ..................................... 136

Figure 6.54 Gender preferences for the different options...................... 137

Figure 6.55 Gender preferences for spaces to be improved ..................... 138

Figure 6.56 Gender preferences for improvement options........................ 138

Figure 6.57 Gender preferences for spaces to be improved....................... 139

Figure 6.58 Gender preferences for improvement options........................ 139

Figure 6.59 Gender preferences for spaces to be improved........................ 140

Figure 6.60 Gender preferences for improvement options........................ 140

Figure 6.61 Gender preferences for spaces to be improved....................... 141

Figure 6.62 Gender preferences for improvement options....................... 142

Figure 6.63 Gender preferences for spaces to be improved........................ 142

Figure 6.64 Gender preferences for improvement options........................ 143

Figure 6.65 Gender preferences for spaces to be improved....................... 144

Figure 6.66 Gender preferences for improvement options........................ 144

Figure 6.67Space with natural elements was preferred over space with artificial elements. 
Figure 6.68 Well-organised space was preferred over unorganised space.

Figure 6.69 Space with openness was preferred over an enclosed space. 146

Figure 6.70 Well-maintained space was preferred over unmanaged space.

Figure 6.71 Modern building style was preferred over the old building style. 146

Figure 6.72 Preferences for spaces with different attributes 147

Figure 6.73 Options shown to participants. 148

Figure 6.74 Preferences for different options as rated by BE and NBE participants 149

Figure 6.75 Options shown to participants 150

Figure 6.76 Preferences for different options as rated by BE and NBE participants 150

Figure 6.77 Options shown to participants. 151

Figure 6.78 Preferences for different options as rated by BE and NBE participants 152

Figure 6.79 Options shown to participants.

Figure 6.80 Preferences for different options as rated by BE and NBE participants 153

Figure 6.81 Options shown to participants 154

Figure 6.82 Preferences for different options as rated by BE and NBE participants 155

Figure 6.83 Options shown to participants 156

Figure 6.84 Preferences for different options as rated by BE and NBE participants 156

Figure 6.85 Preference for options $A$ and $B$ for BE and NBE participants

Figure 6.86 Preferences for improvement as rated by BE and NBE participants 158

Figure 6.87 Preference for options A and B for BE and NBE participants 159

Figure 6.88 Preferences for improvement as rated by BE and NBE participants

Figure 6.89 Preference for options A and B for BE and NBE participants 
Figure 6.90 Preferences for improvement as rated by BE and NBE participants.

Figure 6.91 Preference for options A and B for BE and NBE participants

Figure 6.92 Preferences for improvement as rated by BE and NBE participants.

Figure 6.93 Preference for options A and B for BE and NBE participants

Figure 6.94 Preferences for improvement as rated by BE and NBE participants

Figure 6.95 Preference for options $A$ and $B$ for $B E$ and NBE participants

Figure 6.96 Preferences for improvement as rated by BE and NBE participants 164

Figure 6.97 Overall modifications suggested by participants for the leftover spaces. 166

Figure 6.98 Overall modifications suggested by male and female participants

Figure 6.99 Overall modifications suggested by BE and NBE participants 168

Figure 7.01 5-point Likert scale converted to 3-point Likert scale.

Figure 7.02 Three stages conducted in Study Two with the three parts in each stage. 176

Figure 7.03 Options presented to participants in Study

Two.

Figure 7.04 Preferences for the space underneath a building

Figure 7.05 Attitudes of respondents to the three options of the space underneath a building

Figure 7.06 Options presented to participants in Study Two. 180

Figure 7.07 Preferences for the space in front of a building. 181

Figure 7.08 Attitudes of respondents to the three redesigns of the space in front of a building 182

Figure 7.09 Options presented to participants in Study Two 183

Figure 7.10 Preferences for the space at the back of a building.... 184 
Figure 7.11 Attitudes of respondents to the three options of the space at the back of

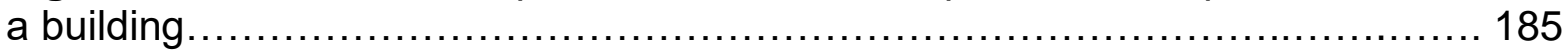

Figure 7.12 Options presented to participants in Study Two...................... 186

Figure 7.13 Preferences for the space enclosed by buildings on three sides...... 187

Figure 7.14 Attitudes to the three options for a space enclosed by buildings on three sides............................................................................... 188

Figure 7.15 Options presented to participants in Study Two ........................ 189

Figure 7.16 Preferences for the space enclosed by buildings on two sides........ 190

Figure 7.17 Attitudes to the three options for a space enclosed by buildings on two sides.

Figure 7.18 Options presented to participants in Study Two...................... 192

Figure 7.19 Preferences for the space on the rooftop of a building................. 193

Figure 7.20 Attitudes of respondents to the three options of the space on the rooftop

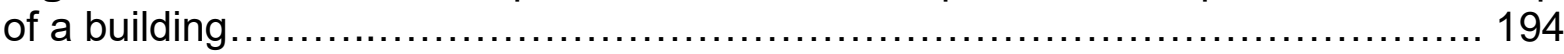

Figure 7.21 Four options shown to participants in part 3 of Study Two............. 195

Figure 7.22 Preferred functions for four different leftover spaces................... 196

Figure 7.23 Gender preferences for space underneath a building ................ 198

Figure 7.24 Gender preferences for the space in front of a building................ 199

Figure 7.25 Gender preferences for space at the back of a building............... 200

Figure 7.26 Gender preferences for space enclosed by buildings on three

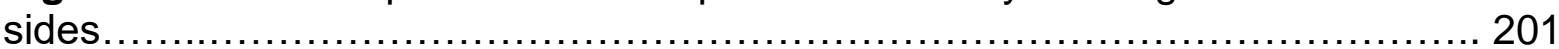

Figure 7.27 Gender preferences for space enclosed by buildings on two

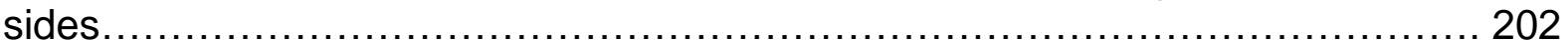

Figure 7.28 Gender preferences for space on the rooftop of a

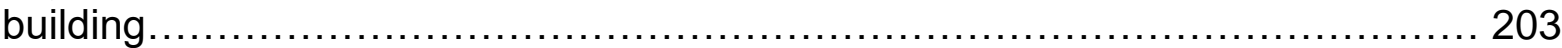

Figure 7.29 BE and NBE preferences for space underneath a building 204

Figure 7.30 BE and NBE participant preferences for space in front of a building 205

Figure 7.31 BE and NBE preferences for space at the back of a building. 206

Figure 7.32 BE and NBE preferences for space enclosed by buildings on three sides. 207

Figure 7.33 BE and NBE preferences for space enclosed by buildings on two sides 208

Figure 7.34 BE and NBE preferences for space on the rooftop of a building 209 
Figure 7.35 Preferences for redesigned leftover spaces for all 96 respondents... 210

Figure 7.36 Preferences by Gender group ..........................................

Figure 7.37 Preferences of BE and NBE participants...........................212

Figure 8.1 Procedures taken in Study Three.......................................

Figure 8.2 Images sketched and doodled by focus group participants..............221

Figure 8.3 Image shown to focus group participants ...............................224

Figure 8.4 Sketches drawn by focus group participants............................ 224

Figure 8.5 Design preferences of focus group participants.........................225

Figure 8.6 Preferences of NBE and BE participants for the space underneath a

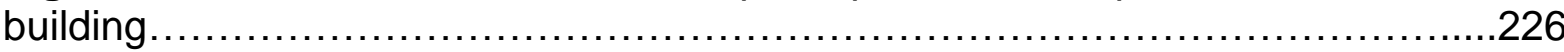

Figure 8.7 Image shown to focus group participants.............................228

Figure 8.8 Sketches drawn by focus group participants............................228

Figure 8.9 Design preferences of focus group participants........................229

Figure 8.10 Preference of NBE and BE participants for the space in front of a

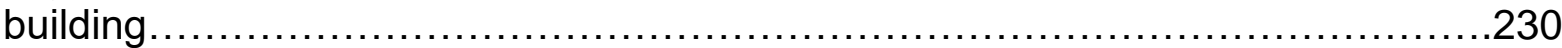

Figure 8.11 Image shown to focus group participants.............................232

Figure 8.12 Sketches drawn by focus group participants.........................232

Figure 8.13 Design preferences of focus group participants......................233

Figure 8.14 Preferences of NBE and BE participants for space at the back of a

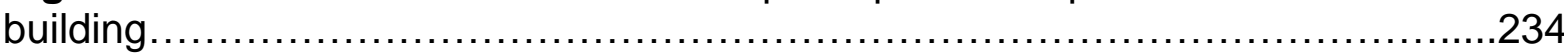

Figure 8.15 Image shown to focus group participants............................236

Figure 8.16 Sketches drawn by focus group participants.........................236

Figure 8.17 Design preferences of focus group participants.......................237

Figure 8.18 Preferences of NBE and BE participants for the space enclosed by

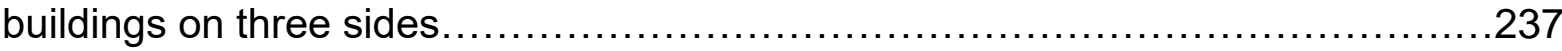

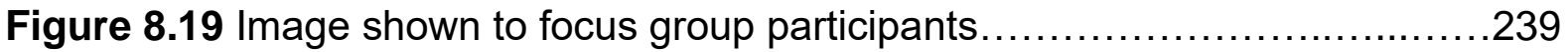

Figure 8.20 Sketches drawn by focus group participants..........................239

Figure 8.21 Design preferences of focus group participants.......................240

Figure 8.22 Preferences of NBE and BE participants for the space enclosed by buildings on two sides............................................................

Figure 8.23 Image shown to focus group participants............................242

Figure 8.24 Sketches drawn by focus group participants..........................243

Figure 8.25 Design preferences of focus group participants.......................244 
Figure 8.26 Preferences of NBE and BE participants for space on the rooftop of a

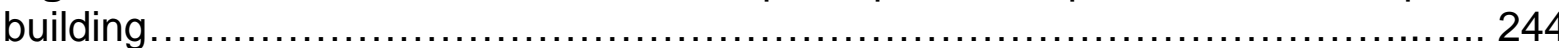

Figure 8.27 Overall preferences of all participants...............................248

Figure 9.1 Built environment participant sketches with an emphasis on providing

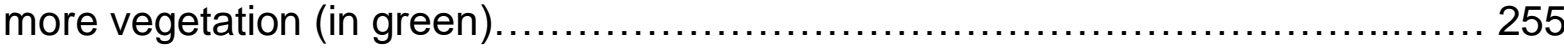




\section{List of Tables}

Table 2.1 Thoughts and terms used by researchers to describe leftover

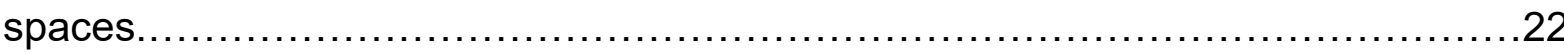

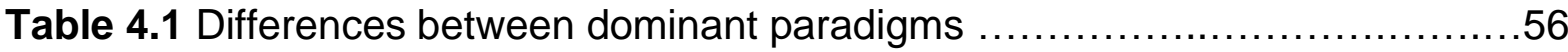

Table 4.2 The concurrent triangulation method used in this research.............. 61

Table 4.3 Procedures employed to achieve the research goals...................65

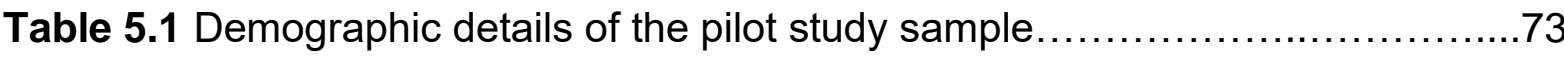

Table 5.2 Preferences for spaces with different attributes $\ldots \ldots \ldots \ldots \ldots \ldots \ldots \ldots \ldots 77$

Table 6.1 Demographic details of the study sample........................ 100

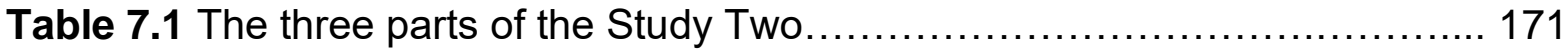

Table 7.2 Demographic details of the Study Two sample..................... 174

Table 8.1 Demographic details of the Study Three sample $\ldots \ldots \ldots \ldots \ldots \ldots \ldots \ldots \ldots \ldots \ldots \ldots \ldots \ldots$

Table 9.1 Most preferred design attributes of different leftover spaces............ 254

Table 9.2 Participant groups by age from Studies One and Two have a similar

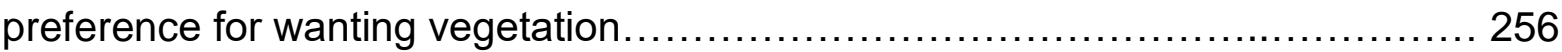

Table 9.3 Participant groups by age from Studies One and Two and preferences for a change in surface materials/ colours ............................................ . 58

Table 9.4 Participant groups from Studies One and Two and preferences for creating

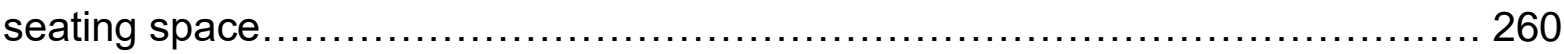

Table 9.5 Participant groups from Studies One and Two and preference for improving maintenance and cleanliness.

Table 9.6 Participant groups from Studies One and Two and preference for removing boundary walls

Table 9.7 Other liked design attributes. 264

Table 9.8 Participant groups from Studies One and Two and preference for creating a clear pathway. 266

Table 9.9 Participant groups from Studies One and Two and preferences for installing wind turbines and solar panels 


\section{CHAPTER 1}

\section{INTRODUCTION}

Humanity will be living on credit (from $8^{\text {th }}$ August 2016) as in eight months it has consumed the natural capital which our planet can renew in a year, which is the fastest of all time (Canfin, 2016).

\subsection{Introduction}

Urbanised areas cover up to $6 \%$ of the Earth's surface (Meyer \& Turner, 1992) and yet they have large 'ecological footprints', which collectively lead to significant environmental impacts (Rees \& Wackernagel, 1994). The ecological footprint of a city extends over an area many times larger than its physical size. The many complex problems that cities in the world are facing come from human population growth. By 2050, another 2.5 billion people will live in cities, and urban dwellers will comprise more than $70 \%$ of the total human population (United Nations, 2014). Wellington, the capital city of New Zealand, expects a significant population growth of 200,000 to 250,000 inhabitants from 2015-2040 (Department of Interior Affairs, 2015). This rapid population growth is a problem numerous cities face worldwide. New Zealand's population increased by $1.9 \%$ in 2015 (Statistics New Zealand, 2015), outpacing Australia, which had a 1.4\% increase in the same year (Australian Bureau of Statistics, 2016). Of the New Zealand population, $87 \%$ resides in 138 recognised urban centres ranging in size from around 1,000 people to more than 1,000,000 (Department of Interior Affairs, 2015). Globally the shift from rural to city living has increased the demand for resources, including water, food, and energy for urban populations (Satterthwaite et al., 2010). This growth will lead cities taking up a higher proportion of the available land area (Johnson, 2001).

Different studies (Czech et al., 2000; Duany et al., 2000 and Johnson, 2001) have detailed out adverse environmental impacts associated with urban sprawl like loss of productive land, and increases in air pollution, poverty, traffic congestion, and resource scarcity, and spiralling infrastructure costs. The availability of resources, the flows of energy, food, and water, together with air quality and global warming, are all becoming critical for human life. The growth and quality of future urbanisation will, therefore, have a considerable impact on international resource availability and sustainability, affecting 
the quality of life for many people. If current practices remain unchanged, the impacts that urban settlements have on the planet will increase significantly while affecting human well-being.

With each passing year, it becomes obvious that cities should be able to grow sustainably from within in order to diminish impacts on natural ecosystems and to avoid unnecessary appropriation of precious agricultural land. According to Shochat et al. (2006), the urban environment is a potent force that alters the behaviours, physiologies, and morphologies of city-dwelling organisms. In order to cope with urban environmental crises, a paradigm shift is essential, and this needs to include recognising leftover urban leftover spaces as having the potential to be designed using sustainable strategies.

This thesis explores the potential of urban leftover spaces within the fabric of Wellington City to be used effectively and redesigned aesthetically based on people's preferences. The opportunity of developing neglected spaces could contribute to the social and ecological health of the city.

\subsection{Problem Statement: the opportunity}

As populations grow in large cities, this, in turn, creates demand for further expansion and development that causes cities to rely on surrounding hinterlands for food and other services (Folke et al., 1997). A shift in boundaries between urban and rural is created, and these boundaries also become blurred. However, over the past century, this urban hinterland area has extended to become global. Through specialisation of markets and transport technologies, cities are becoming less and less able to provide for the wants and desires of their residents. For cities to respond effectively to global climate change, both mitigation and adaptation strategies are required, which will affect economic markets. Cities need to become contributors to the promotion of global sustainability, rather than impediments to proceeding towards it. Future cities will also have to deal with limited urban spaces and resources, undertake the conservation of sense of place (Scannell \& Gifford, 2010), and consistently enhance the current urban environment. 
Cities have the potential to be organised without excessive reliance on the surrounding countryside (Siemens, n.d.). With people and resources located close to one another in cities, it is possible to save energy and resources through effective planning (Viradiya, 2014). Cities may also benefit the economy by bringing people together in one area where ideas for future development can be initiated, which in turn can lead to a higher drain on local resources. Jacobs (1961) suggested that urban centres generate problems but can also solve these by creating jobs and enhancing the quality of life. There has been a growing interest for the past twenty years in how the form of cities - their densities, size, shapes, forms and layouts - can contribute to their sustainability (Williams, n.d.). One opportunity is to make use of areas that are currently not being used effectively. An example of these is the spaces' "leftover" by patterns of uncoordinated development. These leftover spaces are unplanned, unmanaged and are not considered purposeful. However, these spaces could contribute to sustainable urban solutions (figure 1.1) and have the potential for diversification, revitalisation, and densification within an area of the urban fabric.

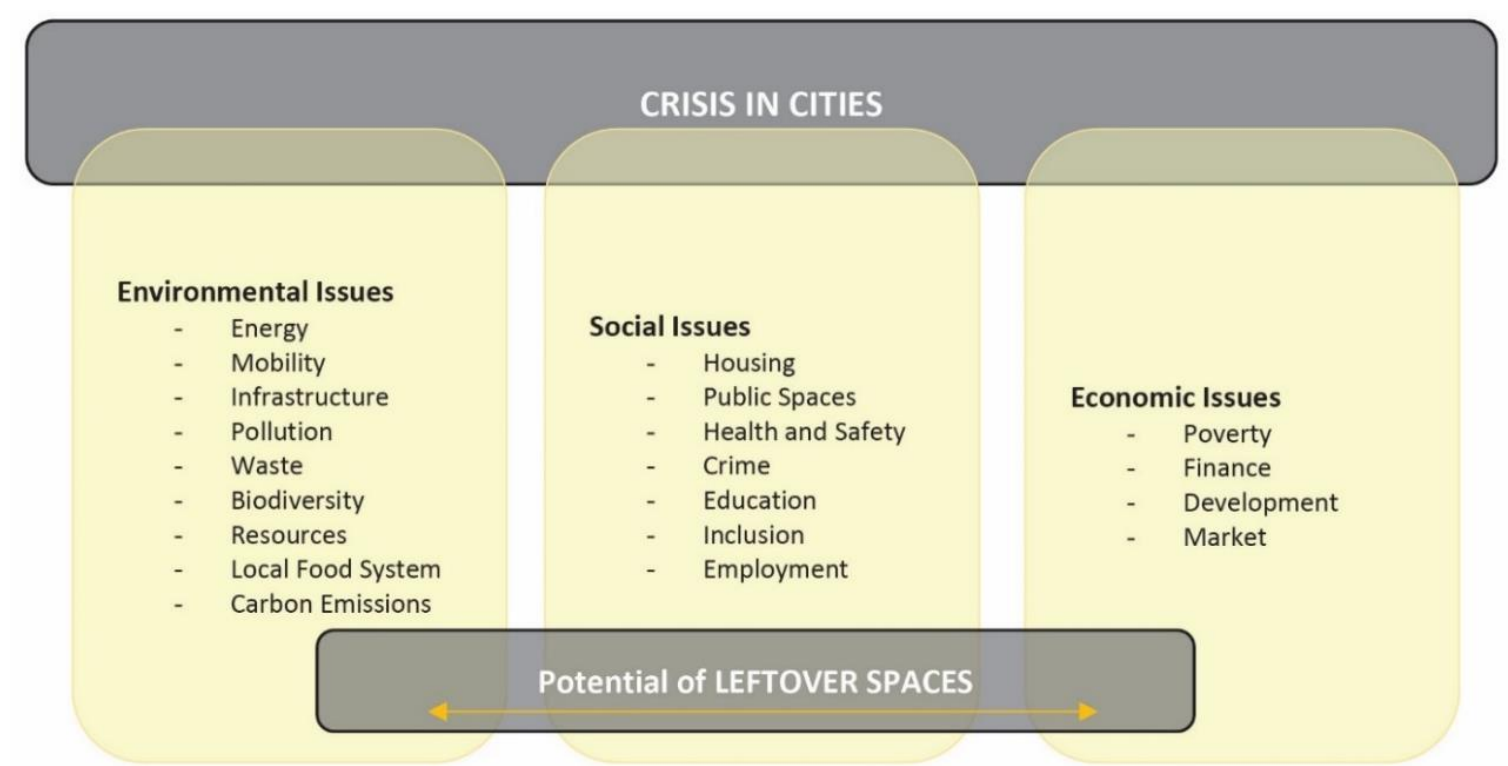

Figure 1.1 Opportunities in cities

Source Author

The film-maker Wim Wenders (1988) observed that the most beautiful parts of the city are precisely those areas where nobody has ever done anything. The work of artist Rachel Whiteread encourages the viewer to observe the space between objects through their shapes and forms as depicted in her negatively cast sculptures that evoke a sense of a forgotten spirit. Trancik (1986) claimed that the built environment 
needs to be treated as a whole rather than as an isolated object. Moreover, spaces that do not serve any purpose are lost occur due to their lack of aesthetic quality within the urban setting. Since urbanisation has adverse environmental effects, it is vital to find new approaches to realising future cities. Therefore, the role of sustainability should be emphasised within cities through using leftover spaces, not only to mitigate adverse climate effects but also to provide amenities in higher density settlements that can connect people to natural cycles.

\subsubsection{Significance of Leftover Spaces}

Urban growth varies from area to area, making it almost impossible to follow only one development model (Turok \& McGranahan, 2013). To that end, any development will depend on the current infrastructure, traditional and cultural desires, topography, financial resources, and the institutional scope for planning and political stability for growth management. The redevelopment process, including space assessment, has layers of integrating designs with structures and these range from reading space to interpreting it while generating meanings through diverse activities. As cities continue to expand out across productive arable land, it is crucial to investigate the potential value and usage of the unused land or spaces that are currently forgotten in developed cities.

The New York State Department (2009) defined such leftover spaces as opportunities waiting to happen, although they represent natural social, economic, spatial, environmental and temporal tensions mainly because of their lack of functionality, typology, visual attractiveness and their attributed name (Sousa, 2009). These spaces present a threat to environmental health, potential housing, neighbourhood development, economic opportunities, and sometimes public safety within cities (Wilkinson, 2011). Different forms of leftover space can be found in cities, and each has the potential to become a steppingstone for nature to flourish. By and large, leftover spaces are deprived of human activity, but they could be used, either permanently or temporarily for future urban regeneration toward more sustainable urban living.

Capturing and engaging with the qualities of the intermediate, often invisible phenomena of the city suggests the need for an alternative approach to utilising 
leftover spaces efficiently and productively. Muller \& Busmann (2002) asserted that leftover spaces are a site for activities and represent a space of social compensation, where the excess pressure of a society can be discharged. However, urban leftover spaces could invite many possibilities for the integration of new techniques from integrating natural attributes to tactical solutions for the built environment. Local inhabitants could use them as a shortcut, they could be used for meetings or greeting people, painting could be allowed on the surrounding walls, or vegetables could be grown (Sousa, 2009). At present, the need to establish an urban identity in many cities that suffer from sameness through globalisation, coincides with the need to restore an ecological sense of nature in cities. Since surplus land is an asset in growing cities, these leftover sites have great potential for generating economic revenue by reconsidering the diverse range of urban activities they might support, and which might be flexible in time and space.

\subsubsection{Significance of Environmental Aesthetics}

A thing of beauty is a joy forever (Ruskin, 1857)

Krupat (1985) claimed that a city is a geographical environment with physical parameters (objective) that have a direct impact on the behaviour of people (subjective). People often describe a city in terms of their association with it rather than through its objective qualities. Rapoport (1993) argued that the ambience of space is felt by human beings, and this is attached to their feelings. The quality of a space is linked with the emotional response to it through aspects like scale, form, colour, light, and texture, which affect the behaviour of people both mentally and physically (Holgate, 1992). Moreover, feelings associated with being lost, claustrophobia, and a sense of terror represent a built environment that affects mental health (Evans, 2003).

Urban aesthetics not only deal with urban beauty but also comprise perceptual experiences through different senses, which leads an individual to associate meanings with the environment (Berleant, 1998). According to Philipp et al. (1999), a feeling of satisfaction, pleasure and relaxation is enhanced through an environment that has a high aesthetic quality, whereas beauty is a visual stimulus that initiates a pleasant experience and response (Grinde \& Patil, 2009). 
Clavera (2017) argued that the aesthetic quality of materials, and appliances or tools which can be touched, visually seen and experienced, all shape human behaviour daily. The human senses play a critical part in understanding the relationship of a building with its environment. The aesthetic quality of a built environment incorporates intangible and perceivable attributes that depend on the association between spatial and design configurations. The studies by Sternberg (1991) and Nasar (1994) argued that the aesthetic judgement of a built context depends on both formal aspects, including proportion, rhythm, scale, shape, colour, and illumination, and symbolic aspects, including personal experience and idiosyncrasies, as determined by culture. Culture has a substantial impact on how an environment is perceived and interpreted (Berleant, 1998).

Moreover, Amin (2002) claimed that the spaces that are visually appealing invite more people and are where people make choices over sitting, walking, or standing. Scholars like Gobster \& Chenoweth (1989) and Herzog (1989) claimed that the perceptionbased assessment of an environment attains a high degree of reliability. Saito (2008) emphasised that the aesthetics of the environment should not be neglected when it comes to improving quality of life and human wellbeing, which satisfies psychological needs. Nasar (1998) asserted that the things that are perceived as good or bad by an individual because of their appearance could affect and even harm a community. Thus, the aspects of aesthetics and perceptions make it more vital for spaces to be designed with appealing attributes which are preferred by the community. This research focuses on exploring design solutions for developing urban leftover spaces based on people's preferences as to what they want to see or experience and find aesthetically pleasing.

\subsection{Research Question}

According to Blanc (2013), rapid urbanisation has substantially transformed the human association with the natural and built environment. The urban experience, visions and narratives shared within a community are associated with aesthetic engagement with the built environment. Every person interprets and perceives an environment differently and reacts accordingly. Aesthetic engagement gives the urban environment its full meaning, which is about understanding the natural, emotional and physical 
aspects through visual information. Nasar (1998) argued that 'visual attributes' and 'human evaluative responses' are key variables for assessing a built environment. The appearance of the built environment affects human well-being, can influence the usability of space, and even reduce property values if not appropriately managed. Handszuh (1991) emphasised that environmental values are correlated with physical health and economic stability. Therefore, this research focuses on highlighting the importance of using neglected leftover spaces that are an essential part of the built environment, and that could be designed based on people's perceptions of them. This research also identifies a gap in knowledge related to finding the attributes people feel are aesthetically pleasing and practical for designing leftover spaces.

Leftover spaces represent an opportunity in the context of Wellington City, where such spaces could be designed using people's preferences. To explore the design potential of leftover spaces, this research has posed the primary question

\section{What are people's visual preferences for urban leftover spaces in Wellington City?}

Three sub-questions related to the main question are formed (see section 3.6.1.1). These are:

i. What attributes do people prefer for the design of urban leftover spaces?

ii. Are there any differences in design preferences between male and female participants?

iii. Are there any differences in design preference between participants with a built environment background and those without?

This research investigates the attributes that are liked by people in order to use these to inform the design decisions for transforming urban leftover spaces in the future. This research uses photographs of real spaces and photomontages to help generate feedback from the public in order to answer the research questions. Furthermore, Section 3.6.1 explains the gap in knowledge related to the research question. 


\subsection{Outline of the thesis}

This thesis is comprised of 10 Chapters starting with Chapter 1, which details the background to this research, explains the research problem, and highlights the research question. The key literature, which supports the research, is discussed in Chapters 2 and 3 . Chapter 2 reviews the background, potential, and challenges in the field of urban leftover spaces. Chapter 3 discusses the importance of environmental aesthetics and how people perceive and evaluate the built context. Chapter 3 thus creates a theoretical framework for collecting data for this research and also discusses the gap in knowledge. Chapter 4 explains the research paradigm and the strategy and tactics involved in generating the data. It also explains the rationale for conducting the three main studies. Two studies are based on photographs, and one study is based on photomontages, which represent the design schemes. Chapter 5 discusses the result of a pilot study that was initiated to suggest improvements in the initial visual preference study. Chapters 6 to 8 highlight the findings of the three studies. Chapters 6 and 7 detail the result of the first two studies, which were done online, and where participants gave their opinions about leftover spaces and the attributes that they liked or disliked. Chapter 8 compares the results of two focus group discussions. Chapter 9 elaborates and interprets the findings of the three studies related to what attributes people prefer for designing leftover spaces in Wellington City. Chapter 10 concludes the thesis, answers the research questions, highlights the research limitations, and outlines the research contribution. 


\section{CHAPTER 2}

\section{URBAN DEVELOPMENT AND SPACES}

\subsection{Introduction}

Chapter 1 discussed the significance and opportunities related to designing leftover spaces (see section 1.2.1) and environmental aesthetics (see section 1.2.2). Chapter 2 begins by explaining the importance of leftover spaces in urban development and forms the context of this study. This Chapter examines the literature related to leftover urban spaces and highlights some of the ways by which these spaces are created and named, and the factors involved that are essential in understanding the relationship of such spaces in an urban context and exploring their potential purposefulness in the City.

\subsection{Urban Development}

The concept of urban defines the characteristics of a city (Snieska \& Zykiene, 2015) which relates to spatial built structures and spaces to support the quality of life through subjective and objective aspects (McCrea et al., 2006). The growth of cities is a constant threat (see section 2.1.1) to the environment and happens due to the massive population shift from rural to urban centres, and which fuels their transformation. This causes cities to expand through accommodating and initiating different strategies related to urban design and planning. Urban design is associated with urban planning, but there is a slight difference as urban design emphasises designing physical places in more detail and creating a relationship between people and the built environment, while urban planning focuses on land-use, and the structural and operational plans for organising a city and meeting the demands of people (Shareen \& Voghera, 2016). The expansion of cities can happen in vacant or less populated areas, or through renovating decayed areas (Brooks, 2017). Urbanisation thus has diverse facets that are associated with the transformation of geographical, societal, economic, and physical aspects. The process of development is expensive and time-consuming as it requires funding, joint efforts, and policymaking. 


\subsubsection{The Impact}

Urban areas consume $75 \%$ of the world's natural resources, and $80 \%$ of the global energy supply (UNEP, n.d.). The repercussion of urban development is that it isolates, fragments and degrades natural habitats and it modifies hydrological systems (Arnold \& Gibbons, 1996) while disrupting energy flows and nutrient cycles (McDonnell \& Pickett, 1990). In terms of the problems that come with urbanisation, the Clinton Foundation (2008) suggests that urban areas contribute approximately $75 \%$ of all heat-trapping greenhouse gas (GHG) emissions to the atmosphere, while only comprising $25 \%$ of the land mass. Primary greenhouse gas sources in cities are the burning of fossil fuels for heating and cooling or mechanical processes, and transportation. GHG are expected to grow by $52 \%$ by 2050 (Millennium Project, 2008). The clearing of land for cities and the demands for goods or resources by urban residents are the major drivers of environmental change in land use and carbon sinks (Grimmond, 2007).

According to UN-Habitat (2016), the energy supplied globally in 2012 was mainly generated through the use of fossil fuels (oil, coal, and gas) forming $81.3 \%$ total, almost $9.7 \%$ was based on nuclear power, and just $9 \%$ was renewable energy made up of hydro, wind, biomass, and solar. Carbon based energy production has a high ecological footprint, not only due to rising GHG emissions and pollution caused by burning fuels but also through extraction and production techniques (UN-habitat, 2016). In 2012, New Zealand's GHG emissions per person were the fifth highest among 40 developed countries, at 17.2 tonnes $\mathrm{CO}_{2}$ per person (MFE, 2015). New Zealand is doing quite well by international standards by generating nearly $67 \%$ of its electricity from renewable sources (Cook, 2010) and $40 \%$ of its primary energy (Figure 2.1). According to the New Zealand Ministry of Economic Development (2007), renewable electricity generation is to be increased to $90 \%$ of total by 2025 , but currently, fossil fuels continue to satisfy a large proportion of the country's increasing appetite for energy. 
Chapter 2 - URBAN DEVELOPMENT AND SPACES

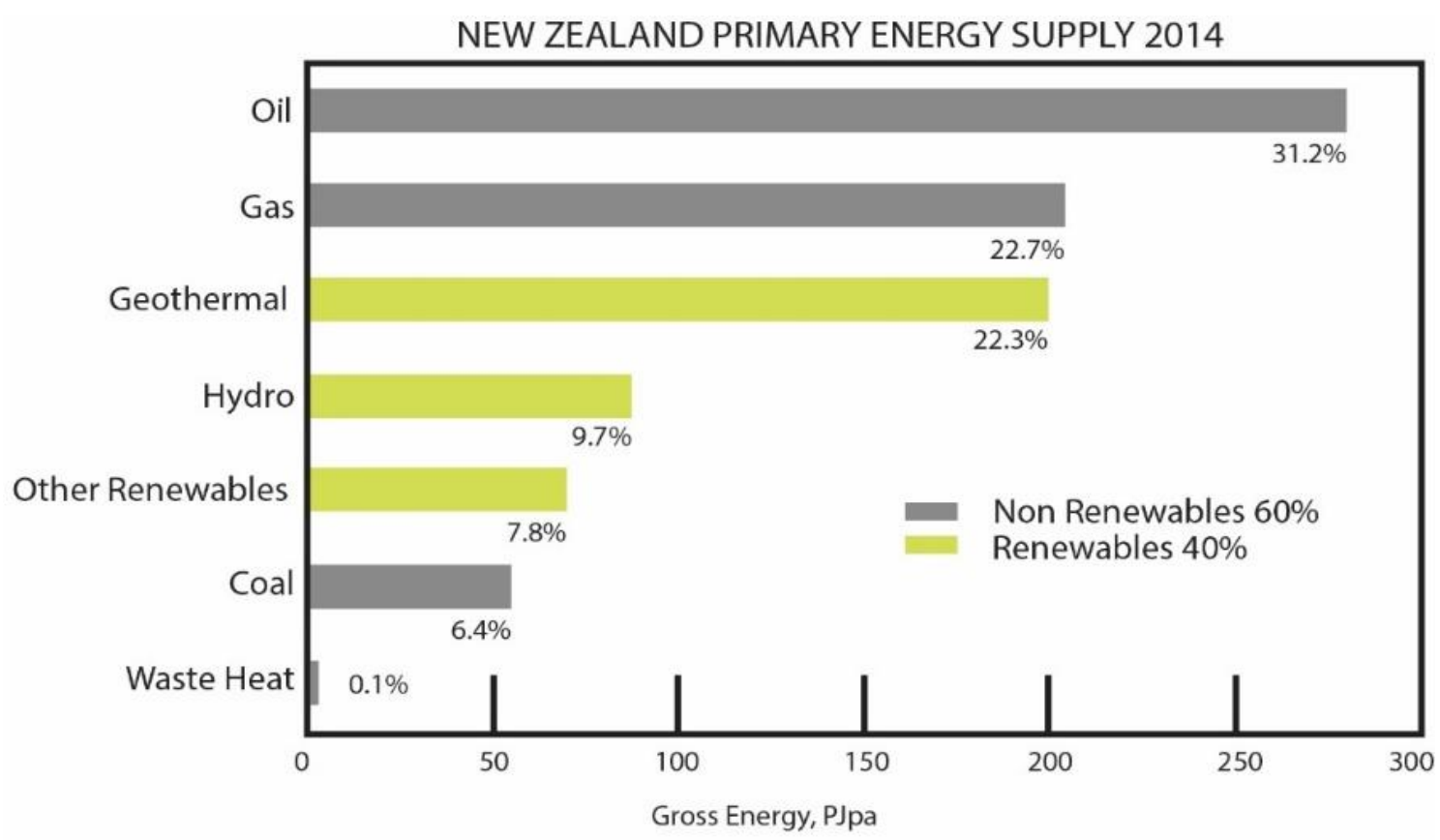

Figure 2.1 New Zealand primary energy supply, 2014

Source MBIE Energy New Zealand, 2014

On a large scale, the city as a whole also modifies the regional climate conditions that result in differences between the city and its surrounding rural area causing low wind speeds, less cloud cover, more solar irradiation, a difference in air temperature, and less precipitation. On a smaller scale, the geometry, spacing, and orientation of buildings and outdoor spaces strongly influence the microclimate in the city. Urban areas have higher surface and air temperatures than their rural surroundings, especially at night (Oke, 1997). On average, urban temperatures may be $1-3^{\circ} \mathrm{C}$ warmer, but air temperatures can be more than $10^{\circ} \mathrm{C}$ warmer than surrounding rural environments (Oke, 1981). The Urban Heat Island effect (UHI) is a local phenomenon with a negligible effect on the global climate (Trenberth et al., 2007). The UHI may create photochemical smog and affect local air-circulation patterns in cities. However, green spaces, which tend to lower the local UHI, directly influence the health of people and the presence of native species. However, urban development reduces species richness for most biotic communities (McKinney, 2002) and causes the extinction of many native species (Vale \& Vale, 1976). 


\subsubsection{The Compact City Approach}

"Urban development without landscape consumption" (von Grot et al., 1988) and the discussion of sustainability and its link to the demand to constrain land take (Rose, 1989) are permanent challenges with differing priorities. To curb the need for further expansion, urban planners have been exploring ways of making better use of existing areas. One response to this challenge is intensification, which is implemented through the redevelopment of existing sites in more intensive ways. As Jenks et al. (1996) and Williams et al. (2000) have argued, compact cities are efficient for promoting more sustainable modes of transport and reducing dependence on the car. Also making cities more compact through high density with mixed-use can save people time as they can live near to their workplace.

According to Elkin et al. (1991), compactness encourages social interaction, can enhance social cohesion or cultural development, and is more cost-effective per capita. Given that a primary goal of the compact city model is to reduce the impact of urban development upon the countryside, most future urban growth will need to occur within existing city boundaries (Williams et al., 1999). Besides this there is the compact city paradox: smaller distances may cut down mobility problems but concurrently produces more air and noise pollution in the urban centres. The primary focus of compactness is to utilise space to its full potential before taking greenfield sites (Lock, 1995).

Scoffham \& Vale (1996) argue that it is highly relevant to question what the compact city is; whether buildings should be brought closer together; whether the number of people living in buildings should be increased; whether it is dwelling density or activity density that needs to be compacted; and what role a mix of urban uses has in the compact city debate. However, no densification schemes have considered the role of food supply and where the land to grow food is located and how sewage nutrients are returned to the soil (Vale \& Vale, 2010). Balancing the compact city approach with the transformation of infrastructure and adaptation to eco-friendly systems are understood to be crucial (Bolton \& Foxon, 2015) for developing sustainable, resource efficient cities. The potential of leftover spaces in compact cities has generally been overlooked. Such vacant land could be an asset in strengthening the ability of cities to respond to climate change and the need to move towards sustainable urban living. 


\subsubsection{Wellington City}

Wellington City is growing, with diverse topography and a population of 203,800 . People aged $15-65$ compromise $73 \%$ of the population and the population is projected to increase by $19 \%$ within 25 years (WCP, 2015). According to Wellington City Council (2014), residential zones make up about $75 \%$ of Wellington City's urban area. In 2014 , the primary source of carbon emissions in the City was from transport $(60 \%)$ with $29 \%$ from stationary energy (AECOM, 2016), the latter being emissions from fuel consumption for electricity generation, fuels consumed in the manufacturing, construction and commercial sectors, and other sources like domestic heating (EPA, 2010).

Wellington City has a long history of development, once being covered in dense forest, and is also vulnerable to flooding and earthquakes. The suburbs of Thorndon and Te Aro were the first areas to be developed from the time of European colonisation. In the late 1850s, New Zealand company surveyors attempted to apply the grid town plan designed in Britain to the hilly terrain, and the land was sold to immigrants in one-acre blocks (Menzies, n.d.). The advantages of a grid pattern were to impose regularity over the landscape, create flexibility for sub-division and ensure air circulation in the streets. However, the pattern could not be overlaid onto the hilly sites because of their steepness and the consequent construction of expensive streets using cut and fill (Schrader, 2010). The essential functions were located at the centre of the grid city, and new blocks were added to expand it, a process that is still happening. This is where the opportunity to utilise leftover spaces can be realised. Over time, the idea of the grid plan was compromised by landowners who built streets and lanes not on the grid. The city has an elevated outer green belt, helping to reinforce compactness, and also form a cornerstone for ecological recovery (Foster, 2015). Figure 2.2 shows the growth pattern of the Wellington City shaped by this ecological belt (shown in grey) and coastline to the east. The urban growth plan of the city has been developed to find ways to make the city more compact, resilient, and liveable for people. This represents an opportunity to identify and use spaces that have lost their identity and function over the years. 


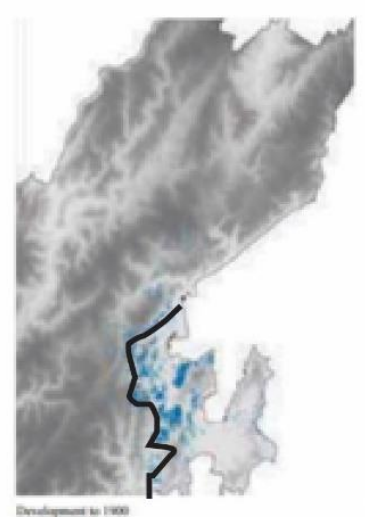

1900

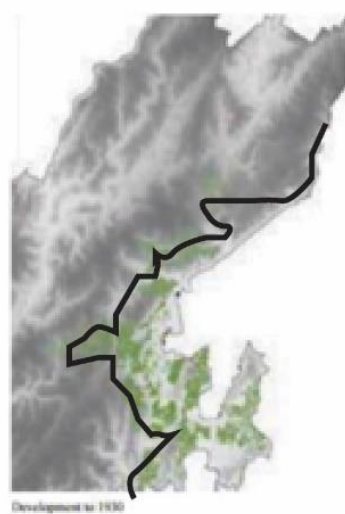

1930

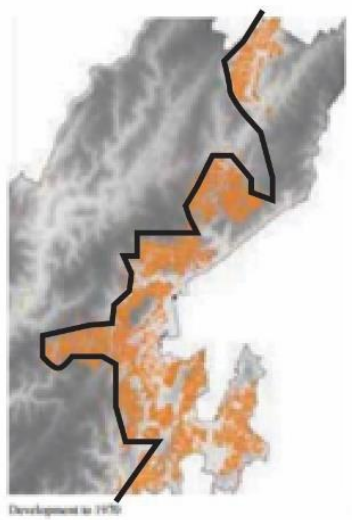

1970

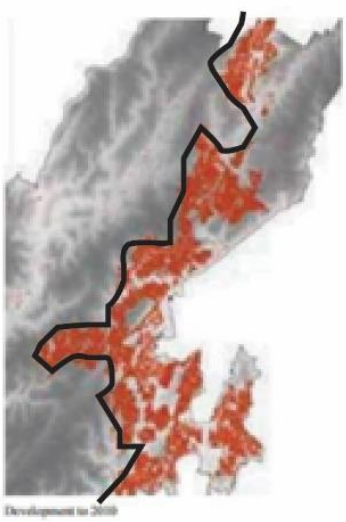

2010

Figure 2.2 Wellington City Growth from 1990 -2010

Source Wellington Urban Growth Plan, 2014

\subsection{Urban Spaces}

Urban spaces have a part in the conflicts, challenges, and transactions within a society, which shape the environment for people. Social and cultural practices or codes play a significant role in defining or transforming urban spaces. The term 'space' has developed from a geometric and mathematical understanding of the word, into interpretations of social space (Lefebvre, 1991). Lynch (1960) proposed that implementing urban scale plans could improve the physicality of spaces. Alexander (1997) suggested that there are two different kinds of outdoor space: negative space and positive space. He regarded outdoor space as unfavourable when such space is shapeless and without enclosure, and claimed an outdoor space is positive when it has a definite shape and is enclosed by its surroundings. Positive outdoor spaces can be identified through the character of the surrounding facades, which can also contribute to visual appropriateness and richness. The existence of negative and positive spaces comes from the geometry of the enclosure in terms of the physical form and physical size of the space in between buildings (Alexander, 1997). The difference between thinking in terms of 'space' and thinking in terms of 'place' has deep philosophical roots (Casey 1997). According to Hajer and Reijndorp (2001), the quality of a place will not be measured in terms of space and accessibility but will increasingly become a question of how it influences the ambience and quality of the surroundings, and how people use it. Furthermore, Buchanan (1988) commented that 
urban design is fundamentally about creating places that are not just a specific space, but that make possible all the activities and events within them. Jacobs (1965) claimed that urban diversity could be accomplished by intertwining functional spaces or zones. In order to transform urban vacant or unused spaces, these will need a diversity of activities and cognitive meanings (Montgomery, 2008).

\subsubsection{Public and Private Spaces}

A public space is a place that is used by and accessible to the public, and where they enjoy meeting and greeting other people (Jackson, 1984), whereas private space implements rules for using a particular place by controlling the accessibility of individuals or groups of people (Smith \& Low, 2006) and social activity occurs without state control. According to Lefebvre (1991) and Dovey \& Polakit (2007), division of private and public space is in a constant state of negotiation between society and state, where written or unwritten regulations control the usage of spaces. This separation of public and private space is caused socially and has a historical background (Weintraub \& Kumar, 1997). Senneth (2003) mentioned that the differences in the class system of a society are the cause of segregation of spaces. Madanipour (2003) argued that private spaces have specific boundaries which interact and mediate with shapeless public spaces. However, liveliness can only be enhanced through the active usage of streets and the interaction of people with public and private spaces (Panerai et al., 2004).

Furthermore, Newman (1972) created a hierarchical system for defining unambiguous spaces in which semi- or pseudo-private space is connected to a semi-public condition and is in a state of transformation, lying between the private and public realms with their different ownership rights (see figure 2.3). It is also suggested that naming such spaces in the urban fabric gives them a feeling of inclusion, and this can play a positive role in reducing the crime rate and providing a sense of security among people. According to Baum et al. (1978), semi-private space is private space, which is regulated by a group of people or the public and is located at a border, whereas, Mitchell \& Staeheli (2006) asserted that semi-public spaces are officially owned by the public or state but are controlled or regulated by private interest. Various names in the literature for such spaces, like interactional territory (Lyman \& Scott, 1967), secondary 
territories (Altman, 1975), or defensible spaces (Newman 1972), which are neither part of public realm nor private, have created a complication in understanding them. However, this research uses the term leftover to define spaces that are in a state of transition in order to probe their potential within Wellington City.

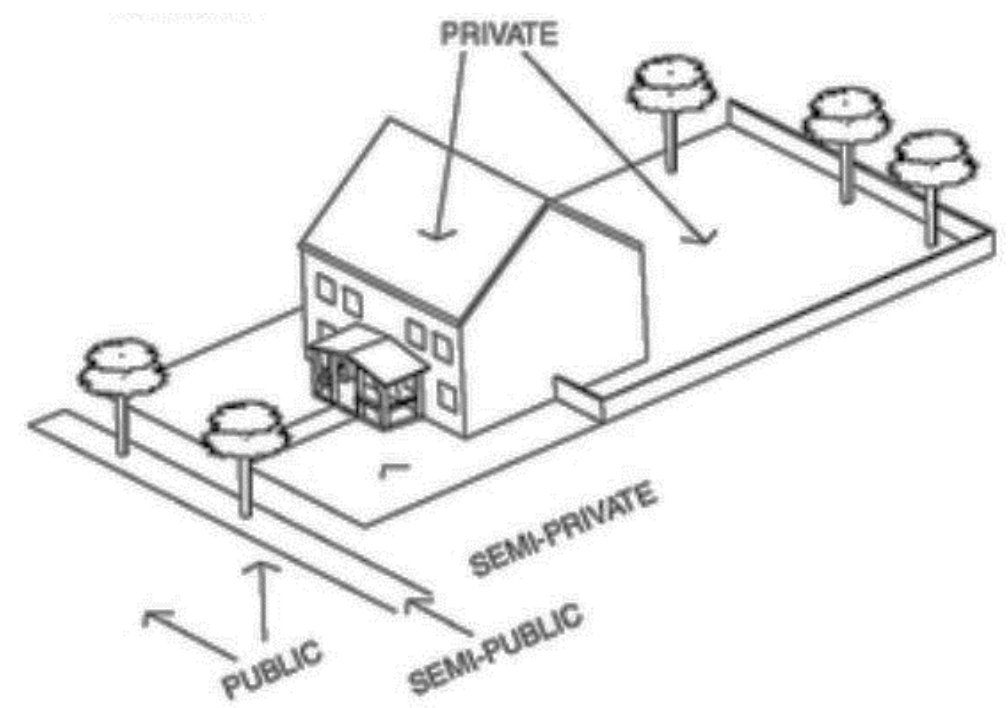

Figure 2.3 Space configuration proposed by Newman (1972)

Source: Pradinie et al. (2015)

\subsection{Leftover Spaces}

In everything, whatever it may be, uniformity is undesirable. Leaving something incomplete makes it interesting, and gives one the feeling that there is room for growth and improvement (Yoshida Kenko, 1998).

Analytical research into leftover spaces was started approximately 30 years ago by Roger Trancik (1986). He examined the notion of leftover spaces, referring to them in the title of his book as "lost spaces" as they seemed to have no significant positive impact on the surroundings. He claimed these spaces were ill-defined, without measurable boundaries and failed to connect elements coherently. Since the 1990s, as land values have soared in city centres, scattered unused parcels of land varying in size and shape located in valuable inner-city land have increased. Greenberg et al. (1990) defined these abandoned lands as Temporarily Obsolete Abandoned Derelict Sites (TOADS). 
Within growing cities, the qualities of leftover spaces are often unique to each spatial context, and they are frequently disregarded and lost through "unrelenting development occupying these spaces within the urban form of late capitalism" (Armstrong, 2006). According to Sousa (2009), for more than two centuries, the traditional idea of a city has moved beyond its walls in dispersion to indefinite limits, while at the same time transforming cities into objects of economic and political speculation (Portas et al., 2003). Urban leftover spaces are a fundamental part of an urban system that can occur next to a planned development or along and under highways and railways, often stumbled upon unnoticed, either publicly owned or no man's space, or being land set aside for development or greening and not developed. These spaces of uncertainty (Muller and Busmann, 2002) are considered to be underutilised, unnoticed or meaningless by a large segment of the community (Akkerman and Cornfeld, 2009). Lacking officially assigned uses, leftover spaces being mostly abandoned spaces, lie outside the rush and flow as well as the control regulations of a city (Qamaruz-Zaman et al., 2012). Thus, in the name of progress, they are commonly considered devoid of function as a result of an imposed social, political and economical frame, and are frequently portrayed as a "tabula rasa" (Doron, 2008).

Leftover spaces are generally vacant, unkempt, unused with no defined function, and between stages of formal development, sometimes indefinitely waiting for future use. Cresswell (1996) argued that leftover spaces are consequently perceived negatively. According to the National Vacant Properties Campaign (n.d.), leftover spaces pose a threat to public safety or exhibit traits of neglect from the property owner and can range from abandoned to unused vacant lots that attract trash and debris. Hudson \& Shaw (2011) noted that planning guidance and policy for appropriate implementation is critical for urban leftover spaces, which are overlooked and yet depicted as unfavourable within the realms of planning, architecture, design, and urban theory. Because of this, they often fade away with time or slip out of the main urban actor's notice and are left with no significant purpose.

These leftover spaces vary in size and shape and could be dangerous without any proper strategic intervention. Lack of proper designation of function has led them to be used as a space for rubbish-disposal or short-cut walkway to a back lane. These spaces contain a range of identities that needs to be addressed so they can form a 
background to city life. Sola-Morales (1995), an urban planner, suggested that empty leftover spaces are fundamental to the evocative potential of the city and are hidden places, where the absence of use can create a sense of freedom and expectancy. Leftover spaces in a city are accustomed to being empty but contain a range of possible uses that could benefit people by providing ways to engage, connect, and contribute to knowledge sharing within the community. To understand the potential of leftover urban spaces, different characteristics related to the formation, scale and types of such spaces are discussed in the following section.

\subsubsection{Morphology}

Urban morphology is the study of the plan and buildings of a city as seen through its concept, development and function (Chen, 2014). As the world entered the postindustrial age, a decline in manufacturing industry, suburbanisation, and changing urban policy and planning systems have resulted in producing diverse empty spaces (Cybriwsky, 1999; Pagano \& Bowman, 2000). Over time, there have been different reasons for the creation of these urban leftover spaces, including war, disaster, new means of technology, migration of people, poor land management, disinvestment, a new mode of transportation, and social conflicts. However, Aruninta (2004) pointed out that a radical restructuring of the global economy in recent decades has created several leftover spaces. She has furthermore explained the reasons for this, citing inefficient decision making, poor land management, and poor coordination. According to Jalaian (2015), the mode for travelling before the Industrial Revolution was on foot, and in traditional cities, this created a sense of continuity and stability within the physical urban environment. In the $18^{\text {th }}$ century, the use of vehicles led to the creation of a dedicated space for cars with separate sidewalks and pedestrian space. Crisman (2009) argued that the concept of roads and mobility centred approaches created discontinuities in the physical and social fabric of the city. Advances in technology during the $19^{\text {th }}$ and early $20^{\text {th }}$ century changed the development pattern within cities leading to private owners being in the majority (Jalaian, 2015).

Nipesh (2012) classified leftover spaces into three main categories: planning, functional neglect, and geographical. Planning leftovers are the creation of planning practices that leave gaps within the urban fabric, scattering such gaps throughout cities (Edensor, 2005). Land-use policies and zoning help create defined boundaries and lines within the landscape. They also form a separation between uses and divide 
the public and private realms (Trancik, 1986). Functional void spaces have lost positive usage within the city and have become defunct as a result. Reversing this process has a significant legal aspect associated with it, as a reallocation of functions has to go through a bureaucratic process. Geographical voids are spaces that are the result of geographical features, such as rivers and hills. There are also many extreme situations that result in the creation of spatial voids, such as natural disasters or conflict, and a decreasing population leading to urban decay (Gallagher, 2010). However, Sola-Morales (1995) suggested that empty leftover spaces are fundamental to the evocative potential of the city and are latent places, where the absence of use can create a sense of freedom and expectancy.

\subsubsection{Scale}

Tsai (2005) argued that the form of the city has a spatial order and structure to it, where human activities happen over time, and that these shape the built environment at different scales. Modernists and postmodernists emphasised the different scales of spaces as being large or small, respectively. The modernist approach focused on incorporating the public into abstract designs on the grand scale, while postmodernism laid stress on designing small scale places with specific functions. When referring to the design of cities, consideration of both the macro and micro scale is essential. Both scales address different issues related to settlements as a whole or the design of some parts of a city but complement each other in the process of urban design (Madanipour, 1996). Sharifi (2019) claimed that the macro scale is concerned with recognising the development type, for example, whether it is compact or dispersed, the overall urban structure, and arrangement of shapes and connectivity. The macro scale deals with the broader challenges and problems related to the function and physical urban form of spaces.

The microscale in urban studies aims at defining the inter-relationship of buildings or private spaces and adjacent street segments (Van Nes \& Lopez, 2010). Microscales are diverse in terms of their functionality, positioning, sizes, shapes, and magnitude. At the microscale, leftover spaces become more easily recognised because the microscale focuses on much smaller but detailed aspects of designs related to architecture, buildings, and public spaces within a city. According to Blair et al. (2014), microscale spaces have problems like vandalism, littering, and poor management, which creates insecurity and social disorder in the city. The poor quality of microscale 
environments is also associated with raised stress levels and negatively affects the cognitive function (Cassarino \& Setti, 2015). However, even at the microscale, the expression of otherness and wilderness can be perceived in patches of moss in the shadow of neighbouring buildings. Microscale spaces evolve with time, as bottom-up, small scale initiatives can reinforce or support the development at a macro scale. The shift in scale makes it necessary to comprehend the potential of urban leftover spaces at the microscale.

Different scales of urban leftover space range from the scale of the building, plot, and block, even up to the neighbourhood scale (figure 2.4). A plot is a basic unit of urban space, and this unit can be dealt with efficiently when landowners want to develop or modify property, which is why the plot is regarded as a basic unit of development. Marcus (2010) argued that the plot scale also affects socioeconomic processes and creates urban diversity in a city. The block is a larger unit which gathers plots together. Lastly, a community, the largest unit, comprises lots, blocks, streets, and sometimes large empty spaces between apartment complexes or low-rise housing (Lee et al., 2015). The otherness in vague spaces lies in the way people seek to see them. The culmination of blocks and lots forms the community/neighbourhood scale, which together forms cities, and thus can be related to the macro scale. However, the two aspects of lot and block divisions can be related to the microscale. Furthermore, a gap in the urban fabric often corresponds to a single plot gap, something commonly found in residential neighbourhoods, whereas consecutive lots that are empty are formed of two or more plots combined. Empty blocks offer the potential for large scale redevelopment, and lastly, corridors are the connecting spaces in the urban and suburban fabric.

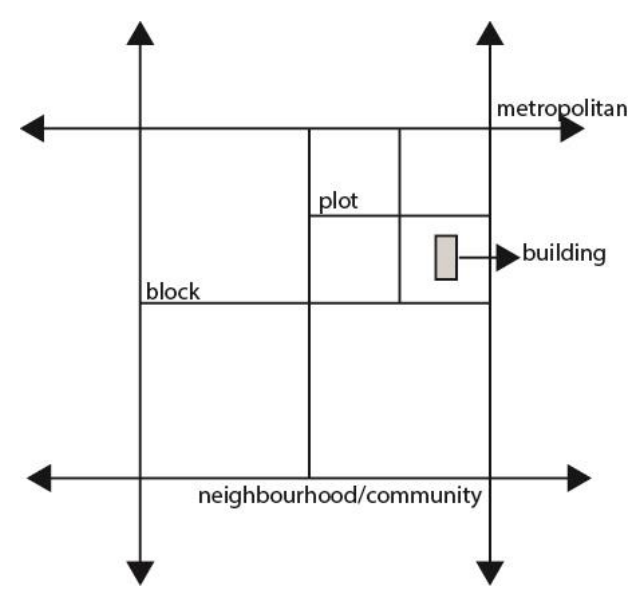

Figure 2.4 Different scales of space in cities Source Author 


\subsubsection{Typology}

Typologies of urban space can provide a way of analysing existing, and designing new, urban forms. Over time, the subject of urban leftover spaces has produced interpretations related to taxonomy (Auge, 1995). Researchers have explored the topic using different interpretations and terms such as loose, liminal, lost, vacant, transitional, indeterminate, neglected, and derelict. In most cases, by adding slight semantic differences, these definitions have only contributed to increased confusion about the subject and have focused on one type of space rather than their broader connections (De-Girolamo, 2013). Leftover spaces have primarily been defined according to their aesthetic merits and other qualitative aspects. Trancik (1986) and Sola-Morales (1995) have also based their evaluations on cause and effect criteria, overlooking the potential opportunities for regeneration such spaces offer. In urban design, there has been little analysis of this aspect. Temporariness and impermanence are rarely viewed as attributes of growth and regeneration (De-Girolamo, 2013). Each leftover space is an anomaly located in a place and time but could be managed appropriately if not colonised (Doron, 2006). Leftover spaces have been given different names throughout history, but their scale, spatial quality, and usability remain the real parameters with which to describe them (table 2.1).

\begin{tabular}{|c|c|c|c|}
\hline \multicolumn{4}{|c|}{ URBAN LEFTOVER SPACES } \\
\hline Year & Author & $\begin{array}{l}\text { Space } \\
\text { Name }\end{array}$ & Description \\
\hline 1971 & Focault & $\begin{array}{l}\text { Heterotopia } \\
\text { and Dual } \\
\text { Space }\end{array}$ & $\begin{array}{l}\text { Spaces that have a hierarchy of layers and } \\
\text { personal associations to describe an } \\
\text { environment. The concept of a mirror } \\
\text { describes as a metaphor for the duality, } \\
\text { which shows contradiction and } \\
\text { reaffirmation of utopian projects. These } \\
\text { space deviate for what society considers } \\
\text { as normal (Focault, 1971). }\end{array}$ \\
\hline 1984 & Suttles & $\begin{array}{l}\text { Residual } \\
\text { space }\end{array}$ & $\begin{array}{l}\text { Residual spaces are often publicly owned } \\
\text { and of low value, as they have little } \\
\text { prospect for commercial or residential } \\
\text { development (Suttles, 1984). Typically } \\
\text { considered eyesores or waste zones or }\end{array}$ \\
\hline
\end{tabular}




\begin{tabular}{|c|c|c|c|c|}
\hline $\begin{array}{l}\text { M } \\
\text { A }\end{array}$ & & & & $\begin{array}{l}\text { unfinished buildings that lie at the } \\
\text { periphery of city structures. Whereas } \\
\text { Wiskstrom (2004) argued that four } \\
\text { categories are interzones, fringes, } \\
\text { infrastructural border zones, and } \\
\text { expansion areas. }\end{array}$ \\
\hline $\begin{array}{l}\text { C } \\
\text { R } \\
\text { O }\end{array}$ & 1990 & $\begin{array}{l}\text { Greenberg, } \\
\text { Popper \& } \\
\text { West }\end{array}$ & $\begin{array}{l}\text { Temporarily } \\
\text { Obsolete } \\
\text { Abandoned } \\
\text { Derelict Sites } \\
\text { (TOADS) }\end{array}$ & $\begin{array}{l}\text { Vacant properties within neighbourhoods, } \\
\text { commercial, and industrial buildings and } \\
\text { vacant lots that pose a threat to public } \\
\text { safety or whose owners or managers have } \\
\text { purposively neglected the fundamental } \\
\text { duties of property ownership and are } \\
\text { discarded (Greenberg et al., 1990). }\end{array}$ \\
\hline $\begin{array}{l}\text { C } \\
\text { A } \\
\text { L } \\
\text { E }\end{array}$ & 1992 & $\begin{array}{l}\text { US } \\
\text { Environmenta } \\
\text { I Protection } \\
\text { Agency }\end{array}$ & $\begin{array}{l}\text { Brownfield } \\
\text { land }\end{array}$ & $\begin{array}{l}\text { Such spaces or land that were used for } \\
\text { industrial or commercial activity but now } \\
\text { are polluted or contaminated with } \\
\text { hazardous waste (USEPA, } 1992 \text { in Franz } \\
\text { et al., 2006). }\end{array}$ \\
\hline & 1996 & Sola-Morales & $\begin{array}{l}\text { Terrain } \\
\text { Vague }\end{array}$ & $\begin{array}{l}\text { Relating to marginal, semi-abandoned } \\
\text { space in or along the edge of the city, and } \\
\text { landscapes that exist outside the city's } \\
\text { active circuits and productive structures } \\
\text { (Sola-Morales, 1996). }\end{array}$ \\
\hline & 2000 & $\begin{array}{l}\text { Pagano \& } \\
\text { Bowman }\end{array}$ & Vacant Land & $\begin{array}{l}\text { Can either be publicly owned or privately } \\
\text { held and may lie vacant due to various } \\
\text { factors like contamination and } \\
\text { deindustrialisation (Pagano \& Bowman, } \\
\text { 2000). }\end{array}$ \\
\hline & 2002 & Nielsen & $\begin{array}{l}\text { Superfluous } \\
\text { Landscape }\end{array}$ & $\begin{array}{l}\text { Spaces that creates a horrible image of the } \\
\text { urban field and haunts planners or public. } \\
\text { The undeveloped parts of the city are } \\
\text { understood as a part of an ongoing } \\
\text { process of excretion and re-appropriation } \\
\text { (Nielsen, 2002). }\end{array}$ \\
\hline & 2002 & $\begin{array}{l}\text { Cupers \& } \\
\text { Miessen }\end{array}$ & $\begin{array}{l}\text { Uncertainty } \\
\text { and Margins }\end{array}$ & $\begin{array}{l}\text { A city with inner peripheries, sudden } \\
\text { changes, breaks, voids and } \\
\text { inconsistencies, whereas, the margin is }\end{array}$ \\
\hline
\end{tabular}




\begin{tabular}{|c|c|c|c|c|}
\hline & & & & $\begin{array}{l}\text { the place where architecture reaches the } \\
\text { border of intentional intervention. It is the } \\
\text { very space in which the designer loses his } \\
\text { power and is confronted with the } \\
\text { impossibility of designing an environment } \\
\text { on both scales, small or large (Cupers \& } \\
\text { Miessen, 2002). }\end{array}$ \\
\hline & 2005 & $\begin{array}{l}\text { Graff \& } \\
\text { Bomans }\end{array}$ & Pause land & $\begin{array}{l}\text { Deserted commercial, industrial and } \\
\text { housing properties and unbuilt sites that } \\
\text { become toxic waste dumps, temporary } \\
\text { housing for the homeless and crack } \\
\text { houses for drugs (Graff \& Bomans, } 2005 \\
\text { in Greenburg et., 1992). }\end{array}$ \\
\hline & 1969 & Sommer & $\begin{array}{l}\text { Personal } \\
\text { Space \& } \\
\text { territory }\end{array}$ & $\begin{array}{l}\text { Personal space is carried around and is } \\
\text { portable while the territory is relatively } \\
\text { stationary and marked for exclusive us, } \\
\text { whether temporary or permanent } \\
\text { (Sommer, 1969). }\end{array}$ \\
\hline M & 1967 & Turner & $\begin{array}{l}\text { Liminal } \\
\text { Space }\end{array}$ & $\begin{array}{l}\text { Threshold spaces are seen neither here } \\
\text { nor there; they are betwixt and between } \\
\text { the positions assigned and arrayed by law, } \\
\text { custom, convention, and ceremony } \\
\text { (Turner, 1967). }\end{array}$ \\
\hline $\begin{array}{l}\text { C } \\
\text { R } \\
\text { O }\end{array}$ & 1971 & Northam & $\begin{array}{l}\text { Vacant } \\
\text { Parcel }\end{array}$ & $\begin{array}{l}\text { Five conditions: Land small in size, often } \\
\text { irregular in shape; parcels with physical } \\
\text { limitations, such as steep slope or flood } \\
\text { hazard; corporate reserve parcels held for } \\
\text { future expansion or relocation; parcels } \\
\text { held for speculation; institutional reserve } \\
\text { parcels set aside by public or quasi-public } \\
\text { entities for future development (Northam, } \\
\text { 1971). }\end{array}$ \\
\hline $\begin{array}{l}L \\
E\end{array}$ & 1988 & $\begin{array}{l}\text { Deleuze \& } \\
\text { Guattari }\end{array}$ & $\begin{array}{l}\text { Striated } \\
\text { space \& } \\
\text { Smooth } \\
\text { Space }\end{array}$ & $\begin{array}{l}\text { Striated spaces is a referred to a space } \\
\text { that is geometrically homogeneous, and } \\
\text { standardised. Smooth space } \\
\text { accommodates social life experiences } \\
\text { historically. }\end{array}$ \\
\hline
\end{tabular}




\begin{tabular}{|c|c|c|c|}
\hline 1989 & Burroughs & Interzone & $\begin{array}{l}\text { Spaces that becomes the point of } \\
\text { maximum visibility, or a coagulum, and } \\
\text { that become a concentration of } \\
\text { experiences, as in-front of buildings, and } \\
\text { that are related to time and movement } \\
\text { (Burroughs, 1989). }\end{array}$ \\
\hline 1991 & Lefebvre & Third Space & $\begin{array}{l}\text { The first space perspective focused on the } \\
\text { real material world and sociality (the } \\
\text { second space perspective interprets the } \\
\text { imagined representations of the world) } \\
\text { through the insertion of a third space: that } \\
\text { of spatiality. Thereby a trialectic is created } \\
\text { with the third space being a space of } \\
\text { extraordinary openness, a place of critical } \\
\text { exchange (Lefebvre, 1991). }\end{array}$ \\
\hline 1995 & Auge & Non-Space & $\begin{array}{l}\text { Spaces of institutions formed about certain } \\
\text { ends like transport, transit, commerce, } \\
\text { leisure. These spaces are never totally } \\
\text { completed, and such non-spaces are the } \\
\text { real measure of our time (Auge, 1995). }\end{array}$ \\
\hline 1996 & $\begin{array}{l}\text { Loukaitou- } \\
\text { Sideris }\end{array}$ & $\begin{array}{l}\text { Cracks in the } \\
\text { City }\end{array}$ & $\begin{array}{l}\text { Cracks are the in-between spaces- } \\
\text { residual, underutilised and often } \\
\text { deteriorating-that frequently divide } \\
\text { physical and social worlds (Loukaitou- } \\
\text { Sideris, 1996). }\end{array}$ \\
\hline 1996 & Campari & $\begin{array}{l}\text { Uncertain } \\
\text { Space }\end{array}$ & $\begin{array}{l}\text { Uncertainty in the boundaries of urban } \\
\text { space, e.g., administrative boundaries and } \\
\text { boundaries of urban artefacts (Campari, } \\
\text { 1996). }\end{array}$ \\
\hline 1999 & Delgado & $\begin{array}{l}\text { Interstitial } \\
\text { space }\end{array}$ & $\begin{array}{l}\text { Transitional spaces that may well be } \\
\text { situated in the city centres, to be crossed } \\
\text { and circulated through, as opposed to } \\
\text { fixed places (Delgado, } 1999 \text { in } \\
\text { Huffschmid, 2012). }\end{array}$ \\
\hline 2000 & Doron & Dead Space & $\begin{array}{l}\text { Spaces that cannot be zoned or delimited } \\
\text { and presents suspension, solitude, the }\end{array}$ \\
\hline
\end{tabular}




\begin{tabular}{|l|l|l|l|}
\hline 2003 & La Varra & Post-it City & $\begin{array}{l}\text { silence within the bustling city (Doron, } \\
\text { 2000). }\end{array}$ \\
\hline 2005 & $\begin{array}{l}\text { Post-it city is a functional urban space } \\
\text { where citizens develop specific non- } \\
\text { conventional ways of relating (La Varra, } \\
\text { Corjin }\end{array}$ & $\begin{array}{l}\text { Indeterminate } \\
\text { Space }\end{array}$ & $\begin{array}{l}\text { New, transitional re-appropriations, which } \\
\text { are assumed by civil or informal official } \\
\text { agents outside the institutionalised realm } \\
\text { of politics, urban design and planning } \\
\text { (Groth \& Corjin, 2005). }\end{array}$ \\
\hline 2007 & $\begin{array}{l}\text { Worpole \& } \\
\text { Knox }\end{array}$ & Slack Space & $\begin{array}{l}\text { Places where the participants do not want } \\
\text { to be seen or heard by others. These } \\
\text { spaces are dependent on the level of } \\
\text { tolerance from society (Worpole \& Knox, } \\
\text { 2007). }\end{array}$ \\
\hline 2007 & $\begin{array}{l}\text { Franck \& } \\
\text { Stevens }\end{array}$ & $\begin{array}{l}\text { Loose Space, } \\
\text { Tight space }\end{array}$ & $\begin{array}{l}\text { A dynamic space that allows people to } \\
\text { carry out their desired action while } \\
\text { recognising the presence and rights of } \\
\text { others whereas, tight space is static in } \\
\text { action as dependent on action or } \\
\text { regulation (Franck \& Steven, 2007). }\end{array}$ \\
\hline 2010 & $\begin{array}{l}\text { Jarnang } \\
\text { Interspaces which have lost their identity, } \\
\text { official usage and encroached by a new } \\
\text { group of people for diverse use (Jarnang, } \\
\text { 2010). }\end{array}$ \\
\hline Space
\end{tabular}

Table 2.1 Thoughts and terms used by researchers to describe leftover spaces

\subsection{Leftover Spaces as 'In-between Spaces'}

Leftover space is a shapeless, empty, isolated space deprived of any spatial relationship with its context and one that is unable to create a transitional condition. In contrast, in-between spaces are in a state of transition. Such spaces form gaps in the figure-ground are mainly generated by existing boundaries of buildings at the micro-scale. Leftover spaces exist as a residue (Alexander et al., 1977), whereas in- 
between spaces act as a third space (Lefebvre, 1991) with no identity as an interspace (Jarnang, 2010). In the built environment, in-between spaces are confined by the boundaries of surrounding buildings and infrastructure, which form the shape and configuration that could allow a specific function to inhabit such a space. In-between spaces lie within the functional setting of a place and could connect the neighbouring building to an environment and create interconnections within the fabric of the city. Being in-between becomes a spatial category by highlighting the value and variety of between-ness from a spatial and transient perspective.

Urban in-between spaces can be seen as ephemeral objects, sites that are not only space but also a possible future (Rahmann \& Jonas, 2014). Theorists Lefebvre (1991) and Soja (2000) suggest the existence of an in-between reality, a third instance or another reality that both reconstitutes and expands upon the original opposition. Hajer \& Reijndorp (2001) first used the term in-between spaces and claimed that these urban spaces could be used to bring together "disparate activities and character in a manner that creates valuable exchanges and connections" (Carmona, 2010). Naming this spatial category in-between is mainly due to the need to underline its main characteristic of between-ness, both from a spatial and a temporal point of view. The term can also refer to spaces of absence, voids, and the gaps between the forms of objects (Piccinno and Lega, n.d.). These are the edges, and leftover spaces on a micro scale, which are ever-present in urban and architectural design and also tend to collide or unintentionally create unfortunate divisions. These spaces intervene between adjacent objects, which are ultimately problematic for both the physical and social fabric. Instead of looking only at the built elements of the urban environment, more weight should be given to leftover in-between spaces at the micro level.

The concept of in-between also suggests juxtaposition, overlapping, and concurrence, particularly between things, for example, the amorphous and abstract space between public and private spaces is, in fact, a real space that is both public-and-private, commonly prefixed with the term semi-, leading to an inter-situation or middle location (Luz, 2001). Spatially, the in-between implies a central location between two events or distinct spaces, for instance: between here and there, this and that, or inside and outside. While in-between spaces are often conceived of merely as a connection between two extreme ends, the logic of duality or a binary system can also be blurred to recognise the physical presence of this connector. These events consist of large- 
and small-scale places, public or private and built or unbuilt, which are in some inbetween phase, creating a pause in functionality (Smet, 2008). These spaces represent sites for spontaneous activities to unfold and are places for activities and experiments evocative of a future beyond the restrictive capitalist present (Hudson and Shaw, 2012). Such spaces are mostly found in between two buildings, in front of, and at the sides or the rear of buildings, but rooftops can also be leftover spaces. These spaces intervene between adjacent objects and often become problematic for the physical and social fabric of the city. There is a need to search for transformational opportunities. Figure 2.5 suggests a few of the underutilised spaces that exist in the urban fabric and that be neglected. The photographs represent some examples of spaces between buildings, at the back and underneath a building. These leftover spaces exist in-between building lots and blocks, on rooftops, underneath a building, and in front of and to the rear of a building.
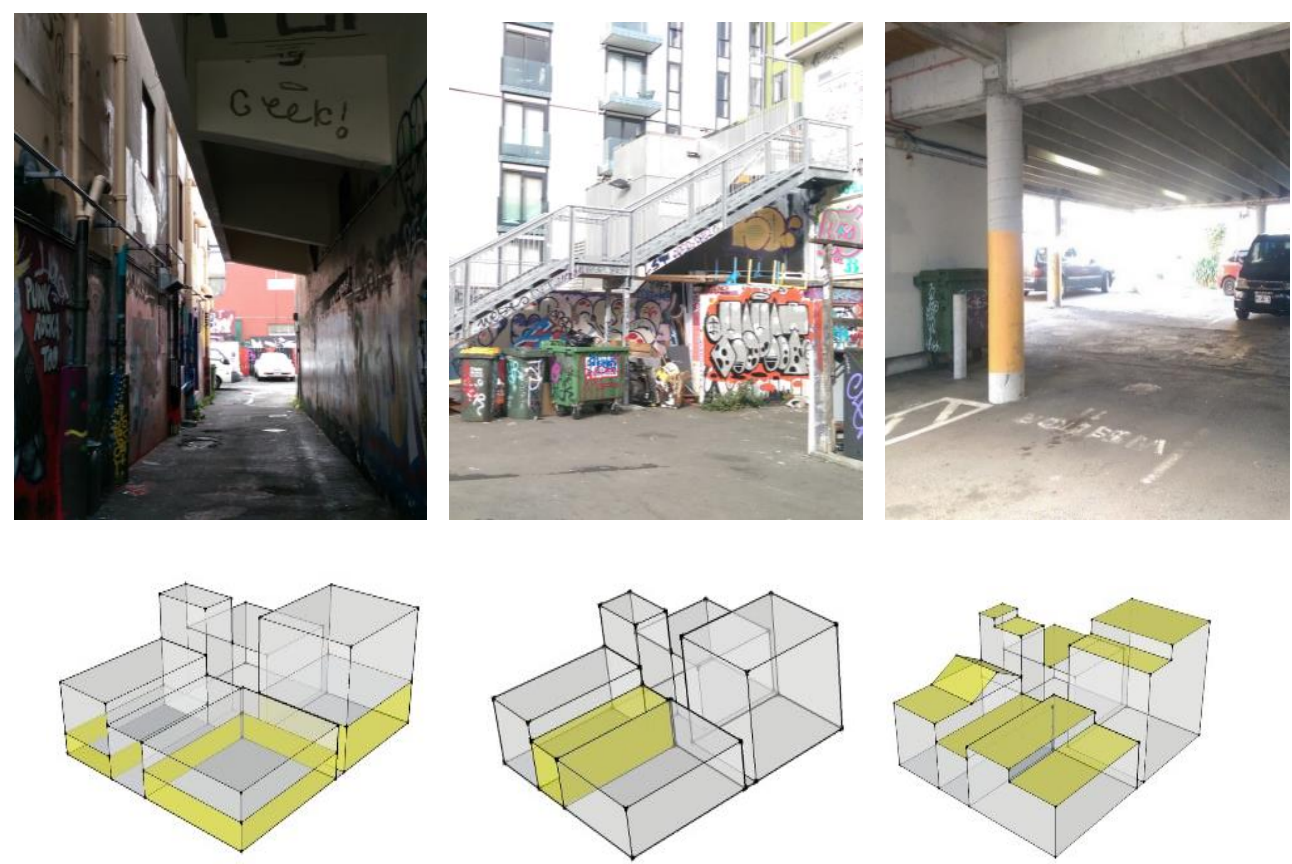

Space Underneath

Space In-Between

Space on Roof top

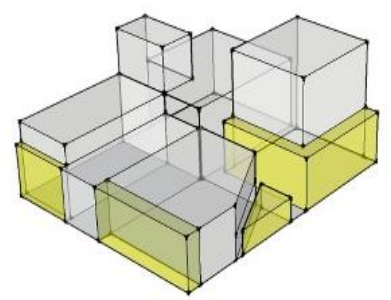

Space Around

Figure 2.5 Different unused and unmanaged spaces in Wellington City

Source Author 


\subsubsection{Classification of In-between Spaces}

By way of field observations, these spaces have been identified as being in-between buildings or structures, and at their edges and within them. These spaces extend across the boundaries of open/closed, interior/exterior, private/public and generally have no official usage, often taking the form of underutilised and informal parking areas (Doron 2006). In-between becomes a spatial category by highlighting the value and variety of between-ness from a spatial and transient perspective. There are distinctive terminologies that are associated with in-between spaces, differentiated through their use, scale and size.

According to Sola-Morales (1995), urban in-between spaces are identified through their sense of absence and emptiness, characteristics that could also apply to leftover spaces. In order to understand leftover spaces, they invite spatial classification. The typology of urban leftover spaces is mainly derived from the literature by classifying different types of spaces at a macro and micro scale. Urban leftover spaces can be divided into two qualitative categories at the macro scale of continuous and discontinuous spaces. The continuous spaces are those in transition where dimensions of time, space, and other circumstances are relevant. Discontinuous space is temporal, having various physical circumstances and which do not relate well to the setting. These spatial types are cracks, openings or gaps since qualities of leftover spaces are often disregarded in the unrelenting development of urban form. In the existing fabric of cities, many unplanned leftover spaces, which arise during development, are not considered purposeful and are known as Spaces left Over After Planning or SLOAP. For this research, a thorough in the field observation was carried out to draw up a list of spaces that accord with the qualitative definition of leftover (inbetweenness) space and that do not support any useful function within the built environment. The investigation found that urban leftover over spaces fall into six major categories (see figure 2.6) at the micro-scale, i.e. enclosed by buildings on two and three sides, underneath a building, the rooftop, and in front and at the back of a building. 


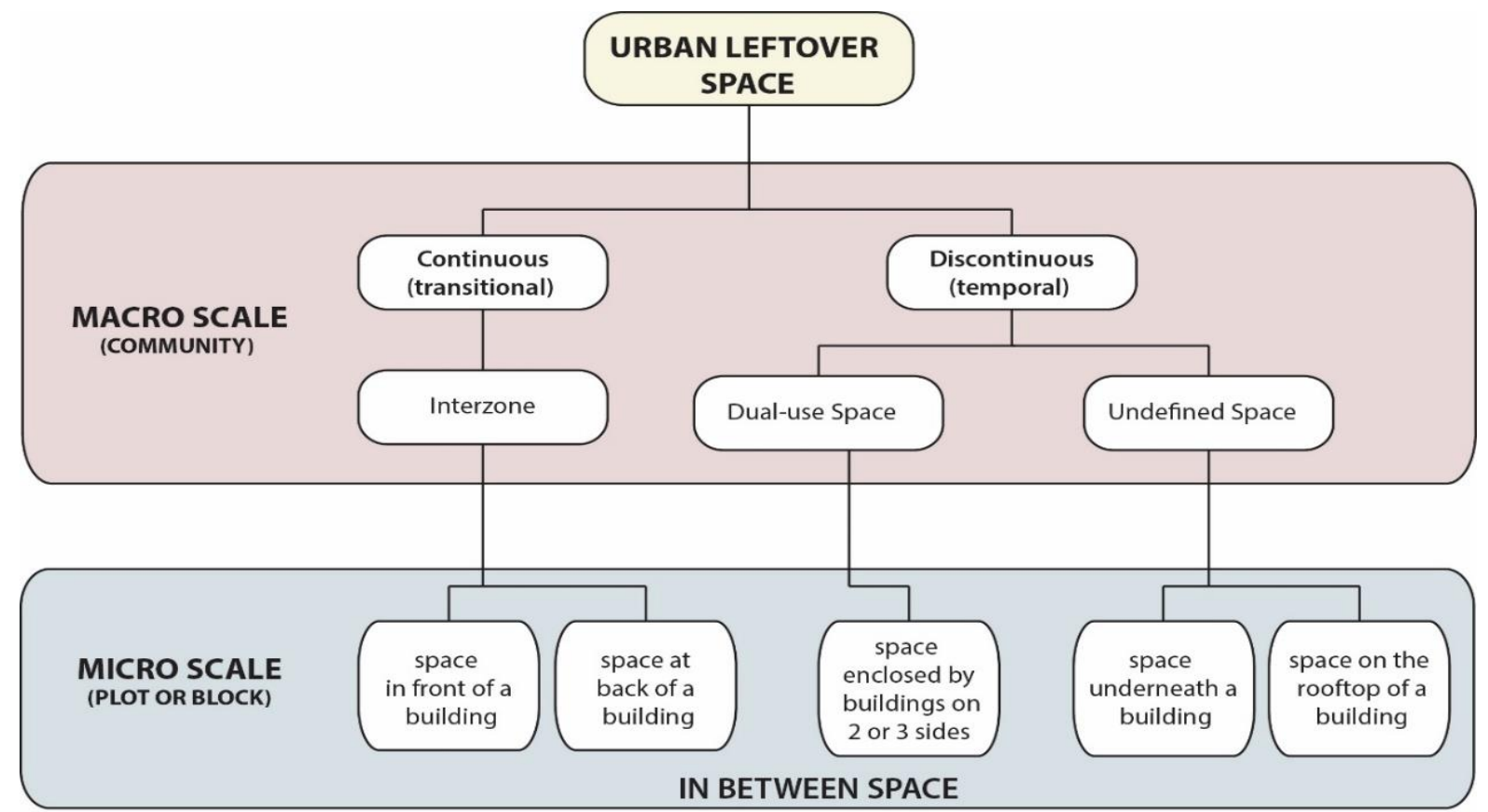

Figure 2.6 Spatial classification for inbetween spaces

Source Author

\subsubsection{Factors}

Multiple criteria for defining urban in-between spaces can be considered, such as formation background, scale and shape, solar orientation and climate, context, current activity, management, accessibility, and ownership. Newman (1972) suggested the feeling of ownership, which often leads to personalisation (Bently et al., 1987), creates psychological feelings about the management and utilisation of space. The space in between buildings could sometimes be considered dull due to its low level of natural illumination. The level of brightness has been one of the factors that contribute to the liveliness and deadness of a space (Abbaszadeh, 2006). At night, unlit space might risk the safety of end users. Also, non-lit spaces are beyond visual surveillance, examples being areas at the rear of buildings. According to Bentley et al. (1987), if a comparison were made between an isolated and an exposed area, the degree of surveillance was much higher for the more visually accessible area. Therefore, it becomes an advantage to allow pedestrians and users to pass by the spaces in between buildings. Some spaces can be hazardous if located adjacent to high-risk zones such as open space around a power sub-station, an oxidation pond, an accident-prone area, or a construction site. To avoid public access, these spaces are buffered and posted with safety signage, making the public conscious of such spaces in the city (Samadi \& Hasbullah, 2008). The characteristics of spaces in between 
buildings are highly influenced by the architecture style of the surrounding building facades. For example, the space in between a row of historical buildings can affect the spirit of the surrounding spaces due to the image of heritage (Samadi \& Hasbullah, 2008). The enhancement of small urban spaces in between buildings should be treated as a revitalisation of the concept of a city outdoor living room. Lefebvre (1991) encourages the inhabitants of urban societies to fight for the restoration of the places in their cities to become spaces for multiplicity, meetings, games, and festivity. His work celebrates the urban grid and the streets, squares, and parks of the traditional city (Shukla, 2013).

\subsection{Summary}

Although an increase in density with high-quality development can lead to more vibrant places and social spaces, problems like congestion, over crowdedness, and pollution, can have detrimental effects on the quality of life (Williams et al., 2000). In that regard, urban spaces could be designed to instigate change through their aesthetic merits and other qualities. The role of sustainability can be supported within the cities by using inbetween spaces to not only mitigate the negative effect of climate but also to promote the well-being of the inhabitants. This Chapter highlighted the morphological aspects, physical dimensions and classifications of leftover spaces. Such spaces also represent design opportunities through their active usage, which can create a sense of identity by enhancing natural and social interaction (see section 9.3). However, a gap in knowledge emerged as the public have not been consulted about the appearance of such spaces. According to Beierle (2002), public views are critical in urban design as they can improve decision-making, thus ensuring public benefits and democratic rights. Chapter 3 discusses the literature of environmental aesthetics related to understanding the perceptions and preferences of people, which could, in turn, inform design decisions for creating aesthetically pleasing spaces for the public. 


\section{CHAPTER 3}

\section{ENVIRONMENTAL AESTHETICS}

\subsection{Introduction}

Chapter 2 reviewed the literature on urban development and the relevance of leftover spaces to the city. Leftover spaces could enhance the image of a city by creating aesthetically pleasing spaces for people that would improve their well-being. However, little attention has been paid to the physical renewal of urban leftover spaces, and no information is available about what design attributes people would prefer most. Nasar (1998) asserted that the physical form of a city is ever evolving through the actions of private and public entities. This affects the aesthetic appearance of the city, which can be evaluated through the preferences of the people who experience it. Urban development processes can be linked to urban aesthetics as a way of improving the quality of the designed environment for people (Ojo \& Kayode, 2006). Thus, Chapter 3 starts by examining the literature related to environmental psychology and focuses on the subfield of environmental aesthetics. This Chapter highlights the factors involved in perceiving, assessing, and experiencing a space in order to understand how human beings engage with and gauge the built environment. Nevarez (2007) asserted that the use of aesthetic guidelines could improve the appearance of contemporary spaces. These can also define the function and help to create order while preventing crime through proposing aesthetically pleasing spaces for the public. This Chapter identifies and develops the gap in knowledge that emerged from Chapters 2 and 3 , and which forms the theoretical framework of this research.

This research adopts the model of environmental aesthetics from Nasar (1998), Gjerde (2015) and Redies (2015). All approaches acknowledged the aesthetic responses, both formal and symbolic, that are perceived through the visual form and includes aesthetic parameters. This Chapter highlighted four critical stages of environment aesthetics by which an environment is experienced (see figure 3.1). The first stage involves the interaction of a user with the built environment (see section 3.2) that creates a stimulus. The second stage is concerned with the external information of the context that is visually perceived and processed (see section 3.3). The 
information about the built environment is then divided into the three parameters of perception of beauty (the affective state), cognition (cultural experience), and associations or meanings that arouse feelings. These three parameters are both interrelated and work independently (Gjerde, 2015; Redies, 2015). The sensory and perceptual nervous system plays a vital role in carrying the information of a context and its form, which is then encoded by the human brain. Redies (2015) argued that an aesthetic cognition process involves a specific context and cultural content by which to acquire knowledge, while perception involves a visual stimulation coming from an environment. Meanings are associated with spaces or objects that create emotions based on appearance. The third stage creates the information from stage 2 and makes an individual judge the aesthetic quality of an object as to whether they like or dislike it(see section 3.3), and stage 4 creates an aesthetic experience through an internal neural mechanism (see section 3.4).

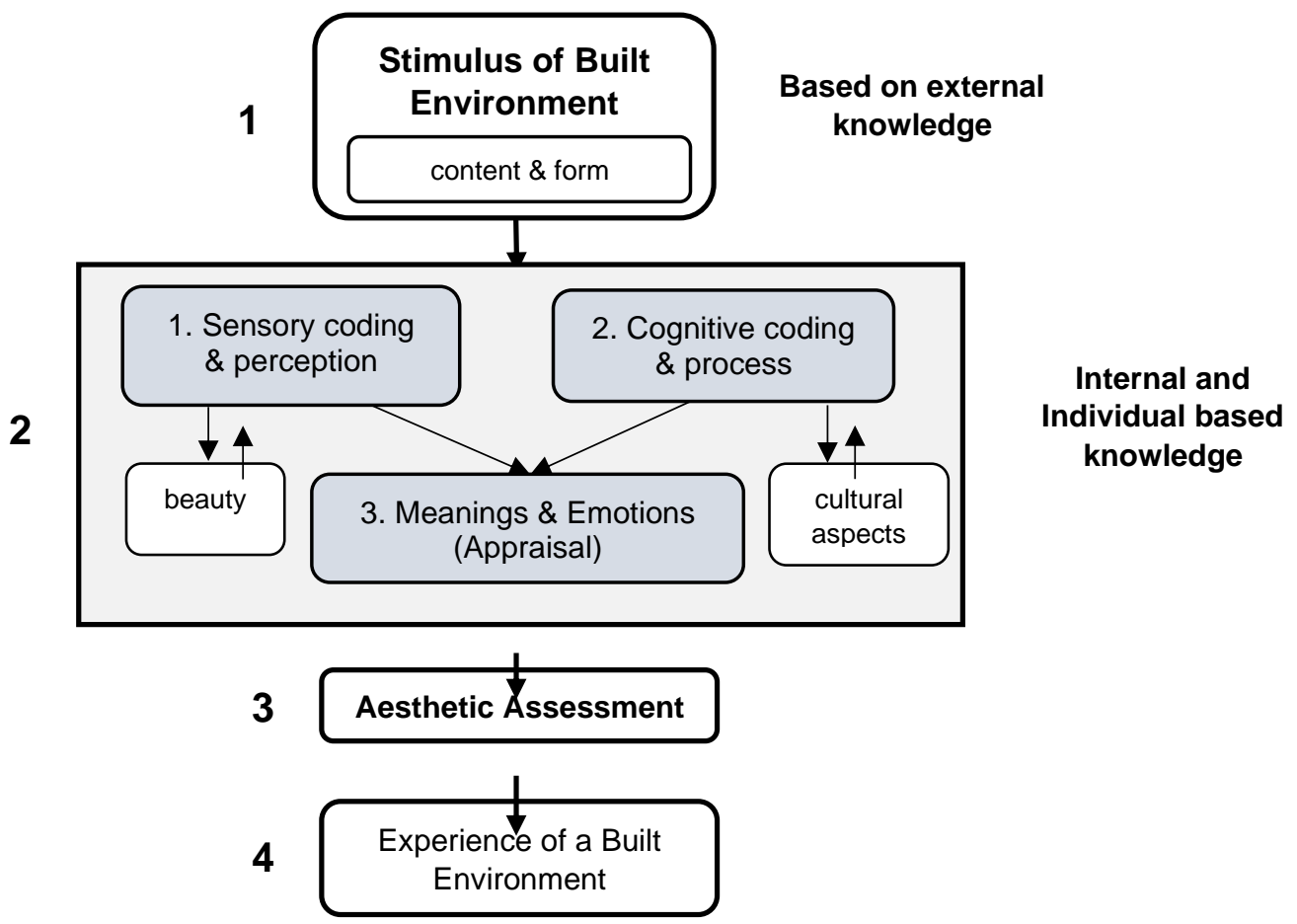

Figure 3.1 Diagram of analytical framework Adapted from Nasar (1998); Gjerde (2015) and Redies (2015) 


\subsection{Environmental Psychology}

We design things and things design us (Lerup, 1977).

The field of aesthetic is generally elaborated under two broad domains of philosophy and psychology. Philosophy aims at investigating the knowledge behind a creative idea and its concern (Scruton, 1979). While psychology plays a vital role in investigating the science of interaction between humans and their environment (Keniger et al., 2013). The psychology of an environment focuses on human thoughts, people's attitudes, behavioural relationship with their surroundings and its causes. The field of Environmental Psychology (EP) evolved in the middle of the nineteenth century, but it was not until the mid-twentieth century that the importance of understanding the human-made and natural environment interaction became vital. EP focuses on upgrading the importance of the natural setting while it also encourages constructing liveable built environments by designing buildings centred on human needs and desires. EP seeks to find a human-nature relationship and how people experience or behave in an environment (Craik, 1973). EP is a subtopic of ecological psychology (Breker, 1968) and incorporates knowledge from behavioural sciences (Lang, 1988) that study human actions and behaviours. Cassidy (1997) argued that EP is a reciprocal cause-effect transaction between an environment and human beings. Currently, most people's environment includes both man-made and natural settings. Barker (1968) and Greenway (2010) argued that it is crucial to understand the relationship between the natural world and human beings, as this relationship is reciprocal. Ottosson \& Grahn (2005) found that a natural setting affects human health, and reduces stress (Wells \& Evans, 2003) and levels of aggression (Kuo \& Sullivan, 2001), while the quality of an environment affects people's behaviour and the usability of space (Gehl, 2010).

Environmental psychology and human behaviour are a personal thing, which includes an element of vulnerability since no-one can predict the outcome as to how a person behaves and adapts to a condition with an environment (Zubin \& Spring, 1977). Similarly, Pomeranz (1980) argued that behaviour is a dependent variable and the environment is independent, while the interaction of internal personnel and external environmental variables is critical to understanding a person's behaviour and psychological functioning. Gifford (2002) asserted that the behaviour of an individual is flexible and can be influenced by the circumstances of an environment. However, 
the biggest challenge for EP is to promote environmental and social sustainability that could be achieved by enhancing, preserving, or upgrading the quality of natural conditions for human beings in the constructed setting. According to Orr (2002), psychology has much to offer to the building of a sustainable future by giving importance to human needs and the fulfilment of human desires.

Saunders (2003) divided the EP into two subfields of eco-psychology and conservation psychology. Eco-psychology studies the relationship of human beings with the built environment while conservation psychology looks at the association of human beings with the natural environment. Eco-psychology deals with urban transformation and influences specific activities which are unique to the citizens involved. According to Gieseking (2014), experience and perception are the most commonly used keywords in an understanding of EP. Experience relates to the transaction between intuition and already assessed knowledge (see section 3.5). It differs from person to person and produces social contrasts, whereas perception is about identifying and interpreting knowledge through the use of different senses (see section 3.2.1). According to Moos \& Insel (1974), there are six ways by which EP can be understood. These influential aspects are based on ecology, context, structural organisation, individual characteristics, psycho-social relationship to climatic attributes, and lastly making reinforcements to or changes within a particular object or space. According to Nasar (1998), knowledge of EP and aesthetics could help professionals to enrich their understanding of an environment by answering questions and finding solutions or new strategies in the field of environmental design research.

\subsubsection{Environmental Aesthetics}

The term aesthetics is derived from the Greek word 'Aisthetikos', which refers to the understanding of sensations and concepts related to the assessment of an object (Blackburn, 1994). In the late eighteenth century, Kant proposed the concept of aesthetics by linking personal taste and judgements. He argued that judgement involves the two aspects of personal taste related to subjectivity (the creation of emotions, happiness or sadness) and objectivity (the object that is perceived) and he described design feelings related to the physical environment (Kant, 2000). According to Zangwill (2003), the field of aesthetics is concerned with the subjective quality of an 
object that is perceived through senses to induce emotions. Moreover, Madanipour (1996) argued that the built environment uses both subjective and objective attributes to enhance the design of spaces for people.

Aesthetics also refers to the perception of beauty and the arousal this creates (Lang, 1988). However, there is a difference between the beauty of an object and aesthetics. The beauty of a space or object is associated with meanings and induces emotions of pleasure within an individual. Aesthetics is concerned with perceived factual information and involves a logical thought process to understand the beauty of an object (Habibi, 2016). Environmental Aesthetics (EA) is about rethinking the association between the design of the built environment and nature (Svabo \& Ekelund, 2015). The field of EA focuses on developing concepts to improve the aesthetic appearances of designed and natural environments. According to Saito (2008), EA investigates and defines the aesthetic dimensions of daily life, which in turn are related to ambience and atmosphere. Ambience describes the experience and acknowledgement of an attribute, whereas atmosphere refers to a particular setting, situation or event. Manning (1991) argued that EA refers to the process of examining the aesthetic attributes that still have to be added to an environment. The domain of aesthetics in the field of arts and the built environment can be further divided into the two major categories of empirical and speculative aesthetics (Lang 1987).

\subsubsection{Empirical Aesthetics}

Lang (1987) argued that empirical or experimental aesthetics is about formulating the process of cognition, perception and one's sentiment. The scientific approach of measuring people's perceptions and their behaviour in society was initiated by Gustav Fechner in 1876. The primary objective of empirical aesthetics was to establish a standardised approach that could evaluate the visual stimuli of an environment (Cuthbert 2006) and justify the aesthetic appreciation people have for a particular object. This domain of knowledge flourished in the middle of the $20^{\text {th }}$ century. Researchers Berlyne (1974), Arnheim, (1974), Cupchik, (1986), Kaplan (1988), Martindale, (1990), and Nasar (1998) have confirmed the significance of evaluating people's perceptions, and validated the theories that relate to analysing the process involved in measuring the perception of people. Different methods and techniques 
have been applied by researchers to reveal the cause and effect relationship between an individual's experience and a specific object. Berlyne (1974) claimed that collative qualities like innovation, complexity, and being startling affect the aesthetic experience related to arousal and interest.

Furthermore, he argued that hedonic values have a relationship with an individual's feelings that can be either negative or positive. Lang (2003) claimed that empirical aesthetics is about measuring the hedonic reactions of people within an environment that can be scientifically evaluated. Lynch (1960) identified five attributes of the built environment such as edges, districts, nodes, landmarks and paths, which could be recognised to evaluate the aesthetic quality, aesthetic meanings and organisation of urban form. Kapan \& Kaplan (1982) developed a preference predictor model to investigate the built environment and emphasised that people should explore and investigate their immediate environment. This can be done by using the four informational variables of coherence, complexity, legibility and mystery. Furthermore, empirical aesthetics include formal and symbolic aspects.

\subsection{Formal Aesthetics}

The aesthetic appreciation of a built or natural environment can be related to formal and symbolic aesthetic variables, which affect the usability of spaces. Formal aesthetics include attributes such as enclosure (openness, spaciousness, density, shape, proportion, rhythm, scale, mystery, illumination, shadowing), complexity (diversity, visual richness, ornamentation, information rate, colour, illumination), and order (clarity, hierarchy, legibility spatial relations, incongruity, ambiguity, surprise, novelty), which together can be used to understand the overall form (Wohlwill, 1976; Herzog et. al, 1976). However, often, human responses are neglected (Groat \& Despres, 1990). Nasar (1994) and Gjerde (2010) argued that formal characteristics such as order and pleasant views influence aesthetic judgments.

\subsection{Symbolic Aesthetics}

Wilson \& Baldassare (1996) described the built environment as the relationship of people's needs to their surroundings but stated that it also has to provide symbolic and functional aspects. Symbolic aesthetics are not solely defined by physical attributes but are judged by their representations and include denotative, connotative, and abstract meanings (Nasar, 1994). Recognising a style as a denotative and 
emotional attachment to an object is connotative, as this depends on the cognitive process at a personal level. Lastly, the abstract provides an in-depth view of values associated with an object (Rapoport, 1990). Appleyard (1976) confirmed that inhabitants perceive the built environment in an evaluative way. Consequently, this research focuses on investigating connotative attributes to understand the meanings that influence decision making and affect human behaviour within a built environment.

\subsubsection{Speculative Aesthetics}

The speculative aesthetics explains the values, concerns, terminologies and attributes linked to art and beauty (Berlyne, 1974). Lang (2003) asserted that the speculative aesthetics philosophy investigates individual's perception that is related to understanding the beauty that gives emotional pleasure. Nasar (1998) argued that the theory of speculative aesthetics is related to historiography, which provides evidence for making decisions by analysing certain patterns. The concept of speculative aesthetics creates an argument in support of a particular statement or piece of work. However, Rappaport (1990) asserted that the arguments should be scientifically based than art based 'metaphors' since it provides reliability, validity and helps to predict the future better. Gjerde (2015) claimed that historically specific proportions and their arrangements of shapes created aesthetically beautiful buildings and such precise measurements are still in use (Ching, 2007). Vitruvius, a Roman architect, mentioned that three aspects could create a better quality in architecture design of building, which is related to 'firmitas' (choice of material and construction quality), utilities (functionality and the usability of the building) and most importantly 'venustas' (beauty achieved through symmetry or order) Kraft (1994).

\subsection{Sensory Stimulation and the Built Environment}

Human behavioural change depends on the perceived environment and whether it is liked or disliked. This has been an area of manifestation in the history of aesthetic studies. According to Bell (1999), perception is regarded as a way of gaining and comprehending information through different senses. Though some senses are more significant than others, like touch, vision, and to some extent, smell, all senses are integrated and are never utilised in isolation. All the information from human senses is 
interpreted in the brain to give a full understanding and experience of the environment (Bell, 1999; Bundy et al., 2002). Holbrook (1980) argued that the aesthetics of an environment are discovered through perceiving an object by using the five senses of sight, smell, touch, hearing, and taste, which directly affect the emotions and behaviour of an individual. The stimuli of an environment are perceived through senses and structured in an individual's mind to develop an overall experience. Nasar (1994) found that positive stimuli create a higher aesthetic experience, but pleasure starts to diminish after achieving a certain level.

Lynch (1960) argued that city design is a piece of art for diverse people with different functions and associations attached to it. Furthermore, the image of a city is created through senses that are interconnected to many other attributes regarding the usage of space. People connect a mental picture of space with their experiences and past associations, and this informs them when they take action. Gjerde (2010) confirmed that the human aesthetic experience of a built environment is stimulated by visual perception, as people have preferences for attributes that affect the usability of a space. Brebner (1998) argued that human thoughts, emotions, and feelings are influenced but also affected both physically and emotionally by what surrounds them. Therefore, it is essential to merge human sensations to achieve a high-quality built environment (Bentley et al., 1985).

\subsubsection{Perception}

Rapoport (1999) argued that there is an unclear distinction between cognition and perception, but both are essential elements for an urban context. The direct sensory experience of an environment or a stimulus is known as perception (Krupat, 1985). Through perception, a person collects or gains knowledge about the environment. Aesthetic perception focuses on finding the components or attributes that evoke aesthetic reactions (Bar \& Neta, 2006). The structure of human perception is based on language. Language-based categories determine the differences through a selection process and connections through a construction process that allow people to perceive, evaluate, and interpret their surroundings. Appearance, feelings or emotions, and impressions may also affect judgments, so preference is an accumulation of knowledge regarding how individuals feel about their environment. Rapoport (1982) claimed that the interdependence of a person on his/her environment 
is most essentially linked to sensual experiences and perception, while Gibson (1977) elaborated the idea of perception more deeply. He claimed that human perception was not just an attachment to the environment, but also accounted for the potential outcomes of that environment to the benefit of the person.

Kaplan (1985) reported that human beings not only respond to things but to the setting that surrounds them within a space, making people act and respond differently depending on their prior knowledge and context. Taylor et al. (2008) differentiated the two facets of perception: the dimension of sensory passiveness or the idea of having any sensual experience, and the physical response that involves the action of a body. However, Seamon (2010) contradicted this idea by emphasising that both aspects were intertwined with each other. He said that in a day-to-day routine, both bodily actions and sensory responses are working continuously. Thus, actions are coupled outcomes instead of separate responses, and action should be viewed as an integrated response.

\subsubsection{Beauty}

Although different civilisations have had different aesthetics, their apprehension of beauty has been somewhat the same. People react varyingly to different environments around them, depending on their past occurrences and experiences, their closeness to all the views, and their expectations and the duration of exposure. The idea of beauty or beautifying by the processing of human cognition and perception is aesthetics (McWhinnie, 1968). Beauty is an inbuilt part of a visual stimulus (Redies, 2015), whereas in linguistics, beauty is a visual attraction that is associated with capacity, efficacy, and ability (Mansouri, 2005). Lang (1987) claimed that personal ideas or concepts of beauty generate analytical aesthetics. According to Dijkstra et al. (2017), beauty is perceived directly by the brain, and the visual information is encoded by neural function. The neuronal functions send the sensory coded information through the retina to the brain and involve a complex internal mechanism of the body. Brizee (2003) and Chatterjee (2011) argued that human beings distinguish and recognise different things or appreciate beauty by utilising visual cues, such as familiar shapes and colours, which involves the cognitive and affective processes. 


\subsubsection{Cognition}

The characteristics of a city speak to people (Kurupat, 1985) and provide cues for how to behave in an environment. The attributes of space have intended meanings which depend on situations and cultural values. Cognition is a process by which the information collected is structured or organised and sorted into meaningful groups. Rapoport (1990) claimed that a pragmatic approach could be used to identify the impact of nonverbal environmental signs, which are decoded by the users, leading to recognition of spaces and the intentions behind them. According to Tversky et al. (2006), the visual aspects of an environment exist in the human cognition and can be as significant as real expressions. Nasar (1998) stressed that the evaluative response to an environment is generated through perception and cognition. The evaluative response concerns the ongoing interaction of human beings and the environment and can lead to a negative or positive feeling about the surroundings. Lynch (1960) used the approach of mental or cognitive maps by linking the psychological information of inhabitants regarding urban form and its transformation. Siegel \& White (1975) pointed out that identifying landmarks is the first step in developing a cognitive mental map, and the route network is then given landmarks to create a wayfinding network in cities.

\subsubsection{Cultural value}

Culture and the social context have a major role in defining and organising the processes of a person's mind (Burnston, 2017). The cognitive process is developed through an individual's experience while living, and this combines the physical and experiential dimensions (Varela et al., 1991). Cultural practices are transformed over time and differ from culture to culture. For example, the style of artwork varies and is influenced by the cultural setting. Triandis (1989) argued that culture makes people act differently while shaping an individual through the perception of self and other individuals in a context. According to Redies (2015), cultural stimuli and factors like innovation, imagination, uncertainty, individual desire, historical background, and financial concerns affect the interpretation of content. Thus, the culture of an environment establishes a person's behaviour, expression and aesthetic preferences. However, Fiske \& Taylor (1991) claimed that social cognition is linked with culture, and this defines how an individual reacts to a certain environment, takes decisions, makes another individual aware about a particular situation, and understands how another individual feels or processes information. Thus, in this research, there is a 
need to identify the differences in aesthetic opinions of groups of people with innate qualities and abilities.

\subsubsection{Emotions and Meaning}

Korpela et al. (2001) found that human beings use spaces in cities that can give them positive emotions and feelings. Such spaces are easily recalled by memory, and they generally generate strong feelings and look aesthetically pleasing (Rapoport, 1970). Every person feels an environment differently through arousing the emotions of the user, and an environment generates meanings through its usage, or the associations assigned to it (Nasar, 1998) and the feelings that are aroused on an individual basis (Stamp III, 2000). Associations can induce negative or positive feelings for a space or an object. Positive feelings might come from a public space or building with ceremonial and spiritual functions, whereas a concentration camp has negative associations, no matter how beautiful as a space or object it is (Gjerde, 2010). Aesthetic meanings are thus associated with a person's emotion for a particular incident or event. Lynch (1960) also argued that identifying places with names creates an associational meaning in the human mind, which is vital in structuring the built form and creating an image map of specific spaces. However, the meanings associated with spaces are constantly evolving and change with time (Lang, 1988). Thus, it becomes vital for design professionals to pay attention to and determine the attributes that have associative values in a particular society as these affect experience.

Rapoport (1982) used the term affordances to describe the function and usage an object can support. Osgood et al. (1957) used a linguistic analytical approach. They created a bipolar grouping to test the efficacy of affective domains. As a result, photomontage became a way of creating altered images by coupling or omitting elements to form a well-composed picture of future reality (Waldheim, 2006). All emotional responses depend on sensory exposure and include the basic emotions (Ekman, 1992) like fear, anger, sadness, enjoyment, or sympathy (Ledous, 1996; Freedberg \& Gallease, 2007). However, Zeki (1999) found in his research that responses are generated through the use of different cognitive processes and he used the term neuroaesthetics to describe environmental and aesthetic experiences based on biological evidence. This appreciation of an environment creates emotions which are connected to a person's cognitive system. Norman (2004) found that emotion 
involves the three levels of subconscious perception, behaviour, and reflection. Gjerde (2015) concluded that built form has five characteristics which invoke emotions and have meaning related to the usability of space, these being scale, style of architecture, heritage, maintenance, and the materials of construction.

\subsection{Aesthetic Assessment}

People react varyingly to different environments around them, depending on past happenings and experiences, their closeness to all the views, and their expectations and the duration of exposure. Aesthetic assessment is about judging feelings based on the feeling of pleasure associated with an object. The feeling of a person is a subjective element and the most vital parameter for aesthetic judgement (Stamp III, 2000). Ulrich (1983) stated that the aesthetic response is about the individual preference for something that provides a feeling of happiness or sadness and works through cognitive activity by visual confrontation. The aesthetic quality of any built or natural environment is a measure of a viewer's visual perception and responsiveness to that area (Cerosaletti \& Loui, 2009). McWhinnie (1968) used aesthetics as a benchmark to explain the responsiveness of people towards a visual stimulus. Whether the stimulus is beautiful or not, it creates an analogy of aesthetics through human cognition. Also, if a particular visual appearance is more beautiful or pleasing, the preference is automatically diverted to it. Beauty rating is a result of this hypothesis. Hagerhall et al. (2008) argued that visual impacts could be explained through various elements and not just as a single factor. These include visual character and quality, including form, line, colour, and texture, visual exposure, the viewer's idealised mental image, and the number of viewers who are expected to see the project. Rapoport (1977) claimed that judgments or intuitions are a reflection of people's feelings for the environment around them. However, this claim was contradicted by Kaplan \& Kaplan (1982), who insisted that human perception could only be interpreted through preferences.

According to Garcia-Domenech (2015), a space with aesthetic quality could encourage people to use it but there should be a desirable balance between perceptions and practicality. Nasar (1998) assists this idea by stating that appearances and meanings are not separate from usability but are essential to it. The way in which people perceive the environment can impact their enjoyment, behaviour, 
and emotions regarding a place, which can contribute to their sense of place. The visual impact of spaces can also leave strong marks on the memory and influence a person's experience significantly because people react to what appears before them. Nasar (1998) also found that environmental spatial attributes produce strong responses, and these attributes are closely tied to people in terms of the context and places which offer different kinds of emotional appraisal.

The perception of an object's appearance creates psychological arousal, which compromises cognitive judgement and affective appraisal (Stamps, 2000) and leads to behavioural changes (Bitner, 1992). The cognitive assessment follows from an initial judgement, whereas the aesthetic judgement can be interpreted as affective (Osborne, 1979). According to Lazarus et al. (1980), an affective quality is a critical tool for examining the interaction of an individual with their context. The aesthetic stimuli invoke an affective reaction that is the result of cognitive processes. Radford (2007) argued that there is a need to investigate affective reactions since much focus has been given to cognitive assessments. Thumfart et al. (2011) proposed a mathematical concept by creating a link between aesthetic properties and aesthetic emotions with which to understand complexity. This approach represents a hierarchical system of aesthetic experience in which the visual sense stimulates an individual. Furthermore, this model divides the features into the three categories of affective appraisal, judgment, and emotional layer by which individuals experience a space using their internal sensorimotor mechanisms.

Many scholars (Hershberger et al., 1974; Kaplan \& Kaplan, 1983; Groat, 1988; Sanoff, 1991) have asserted that people react to visual attributes, and excitement is created when the space that surrounds a person can have meanings associated with it. Berlyne (1974) also proposed the concept of hedonic value that measures the stimulus function of an individual's arousal, whether this is positive or negative. Later, Nasar (1998) refined the hedonic values of arousal and pleasantness by associating meanings with these. Wohlwill (1976) asserted the significance of the visual aspect and its effect on human psychology to invoke emotions. Much literature (Lakoff \& Johnson, 1980; Ryan et al., 2010; Ishizu \& Zeki, 2013; Kirsch et al., 2016) has focused on understanding aesthetic perception, judgment and preferences linked to a person's sensory, cognitive, and motor capabilities. This also involves the whole neural system of a human being to create an aesthetic experience. 


\subsubsection{Visual Preferences}

Understanding preferences is a vital process used to analyse how people judge an environment, including how they characterise and project it. This judgment can be different from person to person based on individual preferences. Habe (1989) confirmed that visual elements in a building are essential in creating a spatial preference. His study found evidence that photographs, responsiveness, and multidimensional scaling were essential in deriving the dimensions of perception. Nasar (1998) asserted that visual quality effects the experience and feelings of a space. Thus, Nasar (1994) claimed that the visual study approach is a useful strategy for determining how people feel about the conditions of an environment. Researchers like Kaplan \& Kaplan (1989) and Sanoff (1991) earlier studied the reliability of the visual study approach for getting visual responses.

In visual preference studies, photos of an environment are used as substituent agents for the original (Arriaza et al., 2004). Nasar \& Stamps (2009) and Nasar (1994) suggested that showcasing photographs of a scenario or environment induce the same response in people as if the pictures were real. Hartig \& Staats (2006), Herzog (1989), and Kaplan (1973) all made use of photos or slides as a means of drawing out people's preferences. In 1970, Tony Nelessen (1979) ratified this way of conducting a survey and referred to as an Image-Based Survey/Visual Preference Study (VPS), as a means of engaging with non-technical people. He held a test for VPS in 1979, in which he used a photo-based process as a way of discerning the impressions of residents about different spaces in a city. They were shown fast-paced sequential photos, which they then rated. The idea of this survey was to facilitate public participation in placemaking. The rating system makes it easy to interpret the mean values or central tendencies when assessing most to least preferred images. The survey used a descriptive vocabulary for identification and relation of spatial elements to understand the form, function, and consequent appearance of a given space. It thus seems photographs can be used with confidence in preference judgments and perceptual studies as surrogates for actual spaces (Kaplan, 1985). For all these reasons, this research used photographs of different leftover spaces to probe people preferences regarding them (see Chapters 5-8). 


\subsubsection{Difference of Opinions in Perceived Values}

Maron \& Spreckelmeyer (1982) asserted that consensus judgement about the quality of architecture could be achieved if the preference values of users group are given significance and consideration by designers. According to Groat (1982), architects give more importance to the organisation of the site and its massing than the public. A study by Nasar \& Devlin (1989) showed that architects preferred 'high style' buildings, whereas participants with no knowledge of design preferred popular style buildings and concluded that an architect's perception of style is different from that of the public. Nasar (1998) also asserted that design professionals do not share the same design values as the public. Similarly, Gifford et al. (2002) found that architects perceive buildings to have more defined meanings than non-architects who view buildings in simple terms such as being complex or strong. Akalin \& Yildirim (2009) found that users living in a building with a curved façade had layout and orientation difficulties, while the designers had wanted a distinguishable style for the building. Montana et al. (2013) found differences between architects and laypersons in the selection of a residential property. They found that architects evaluated the design related attributes of light, external façade, and creativity highly, while other people focused on the functional aspects of good layout design and living in a home-made for family living.

Janssens (2001) found that architects have a higher level of coherence or consistency for liking colour patterns than other participants, who chose a variety of colours schemes. Similarly, a study by Gjerde (2010) found that the views and aesthetic judgment of lay participants with no professional knowledge of the built environment were similar to those of architects and planners, although professional participants were more confident and explicit when expressing their concerns. Brown \& Gifford (2001) concluded that the conceptual properties (formal or physical) of a building should be examined to discover differences in preference between architects and the public. The literature indicates contrasting positions for preferences between built environment participants and others, which opens the need to further examine people's aesthetic feelings. Consequently, this research evaluates the difference in preferences between built environment participants and others as these could play a significant role in designing the built environment of the future and in developing design guides. 


\subsection{Aesthetic Experience}

The aesthetic experience refers to the psychological action that involves a concern for a particular object (Cupchik \& Winston, 1996). According to Ellison\& Woodward (2010), aesthetic experience is about the object-subject association in which the object creates a feeling in the subject's memory through a dominating quality in the mind. Aesthetic responses to spaces can produce pleasurable emotions through neurophysiological activity inspired by a visual experience (Ulrich, 1983). Kaplan \& Kaplan (1989) found that the two distinct sorts of perceptual categories derived from preference ratings are content-based and spatial configurations. These classifications appear to demonstrate that individuals perceive situations and create patterns of like and dislike. Rapoport (1982) suggested that individuals respond to and assess the environment in more associational or meaningful terms. Visual preferences can guide behaviour and the emotional responses of users for the redesign of leftover spaces, which could contain both physical and social attributes. The likelihood of stimulating the interest of users should increase if the attributes of an environment are made visible. According to Evan \& Garling (1991), the meanings related to a city can be assessed by identifying the most liked attributes or features. This can help in understanding what people feel and know about their city. Through research the most preferred visual attributes in a built environment are related to providing a water element or fountain (Wolley, 2003), sculptures (Mehta, 2009), vegetation (Nasar, 1998; Herzog et al., 2003), sense of enclosure (Stamps 2005), and the height width relationship (Cullen 2007). Nasar (1998) created a framework for evaluating the responses of people to an environment. He emphasised that the content meaning of a form is essential in evaluating an environment, and this is linked with the mental capability of recognising a subject, drawing inferences, and then evaluating it.

It emerged from the literature review that there is an urgent need for probing the preferences of people towards a built environment. However, different methods and characteristics for judging the aesthetic responses have been used, and few efforts have been in assessing the aesthetic reaction to the parts of the built environment that are not well used, such as leftover spaces. Thus, this research focuses on evaluating people's responses to the physical attributes of different urban leftover spaces by using both quantitative and qualitative methods. This research also highlights the significance of the visual but tangible environmental attributes that affect people's 
aesthetic experiences. Six different types of urban leftover space (see section 2.4.1) from Wellington City are selected and represented through two-dimensional photographs to stimulate aesthetic responses. The studies by Nasar (1998) and Stamp (2000) found that the preferences produced using photographs are correlated to the built environment and can be used to evaluate responses.

\subsection{The Theoretical Framework}

Leftover spaces are spaces that are unused, underused or currently used but could be in a better usable condition. The primary focus of this research is about finding the role of private and public leftover spaces that are between buildings (enclosed on two or more sides), and their adjacent facades and rooftops. Through the literature, it became evident that there is a need to examine people's aesthetic responses to different leftover spaces both in their current and redesigned conditions. This research aims at developing leftover spaces based on people's preferences, so the work could be used as a reference by design professionals to enhance design qualities and promote sustainable solutions within the city. The literature on urban leftover spaces, environmental psychology, and aesthetics formed the basis for a theoretical framework to give a structure to this research (figure 3.2). The diagram shows the essential elements that are crucial to understanding the built environment and its impact on individual perception when it comes to the design of leftover spaces.

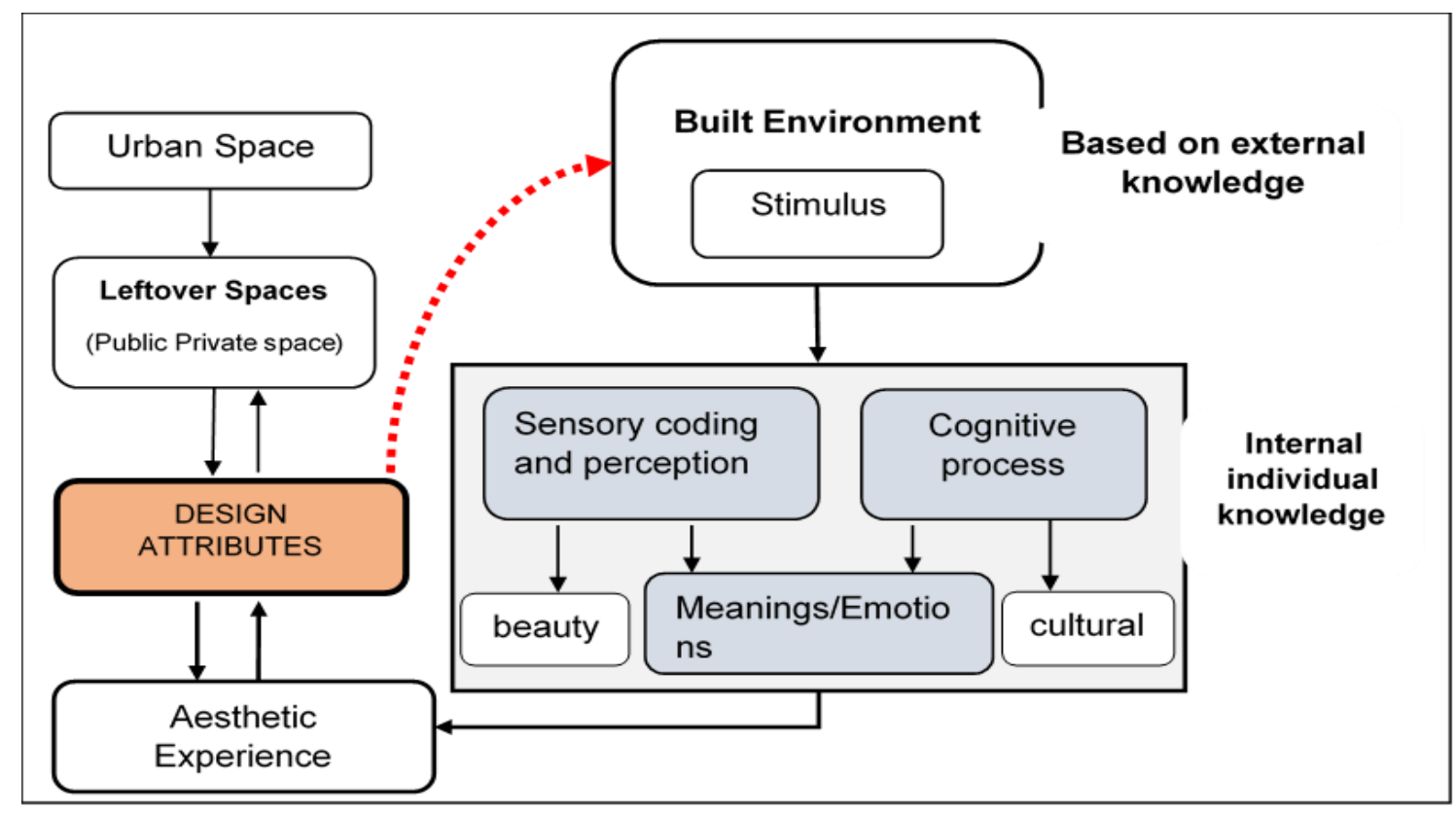

Figure 3.2 A theoretical framework for this research Adapted from Nasar (1998); Gjerde (2015); Redies (2015) 
The left side of the diagram represents the position of urban development trying to meet the demands of rapid urbanisation in which leftover spaces if designed, could play a crucial part in making cities more sustainable. Gehl (2010) argued that cities are built for human beings and shape them. The aesthetic experience that gives a higher level of pleasure (Kubovy, 1999) provokes and encourages an individual to use spaces. Thus, it is necessary to design aesthetically pleasing environments for people that could enhance the appearance of the built environment and improve social wellbeing.

The right side of the diagram shows the built environment affecting human perception based on the setting. Gifford (2007) argued that people are aroused by natural aspects, and the interpretation of stimuli varies from person to person. Wohlwill (1966) asserted that physical setting is fundamental to creating stimulation and is a source of sensory knowledge. Moreover, Nasar (1998) claimed that the processes in an environment related to the perception of beauty and cognition affect the experience of an individual. The emotions or meanings associated with feelings are connected to cognition (Robinson, 2005) that leads to the evaluation of problems, objectives, and values (Roseman \& Smith, 2001). Thus, experiences are associated with the stimuli of an environment, and these lead to how much a person feels aroused (Berlyne, 1967). Scherer (2001) posited that emotions are inclusive or based on the personal and that feelings can be evaluated through the subjective appraisal approach. Human perception, cognition and meanings associated with each person's feelings become an essential factor in experiencing a space positively or negatively. The dotted line in the diagram suggests a gap in knowledge when it comes to developing urban leftover spaces that could be aesthetically designed based on people's preferences. There is also an inconsistency in knowledge in the liking for particular design attributes between people with built and non-built environment backgrounds. This knowledge is vital for guiding design driven by the users rather than making design speculations that might not satisfy the expectations of the public.

\subsubsection{Gap in knowledge}

This study seeks to understand the potential of different urban leftover spaces to be used more effectively than they appear to be at present based on people's 
preferences. It will contribute to the knowledge domain of environmental aesthetics, which means investigating design attributes that are liked by the public. This will also contribute to making the built environment look aesthetically pleasing. Leftover spaces within the built context of Wellington City should be seen as positive spaces that can enhance social and environmental sustainability. The developed world cities are taking sustainable initiatives to improve the built environment and mitigate adverse carbon effects, and the use of leftover spaces could provide further opportunities in supporting these endeavours. At the same time, taking these opportunities should improve the aesthetic quality of the city.

Chapter 2 discussed the issues, conditions and importance of the positive utilisation of leftover spaces. However, there is insufficient information available for how to go about using these. Also, the semantic confusion of different names has caused problems in understanding the issue (see section 2.3.1.3). The literature employs different names for leftover spaces, often with varying scales, but no authors have dealt with or tested possible solutions for future regeneration from within. As some of the names suggest, these spaces seem vague and unloved. The vital issue of time and temporality is entirely excluded from the official definitions of leftover spaces.

This research focuses on the potential design solutions for different types of leftover space, which has not been fully explored for expanding cities. Little is known about how leftover spaces could be designed using people's preferences, and knowledge of this could also benefit design professionals. A broad spectrum of information about leftover spaces (see section 2.3) and environmental aesthetics (see section 3.3) has been analysed to form the basis of this research. A crucial aspect is how leftover spaces could be used and designed to make them a support system for the built fabric (see section 9.3). As a result, the research explores design attributes for different leftover spaces for improving the aesthetic quality of Wellington City. The leftover spaces can be seen both as having potential and as threatening. However, the revitalisation of micro spaces could expand the dynamism of a city. The challenging goal of urban design is to consider leftover spaces in terms of redefining the relationship between closed and open spaces through design interventions intended to raise aesthetic quality (figure 3.3). These leftover spaces could be developed as an active part of cities to help the citizens maintain social interaction and introduce biodiversity. 


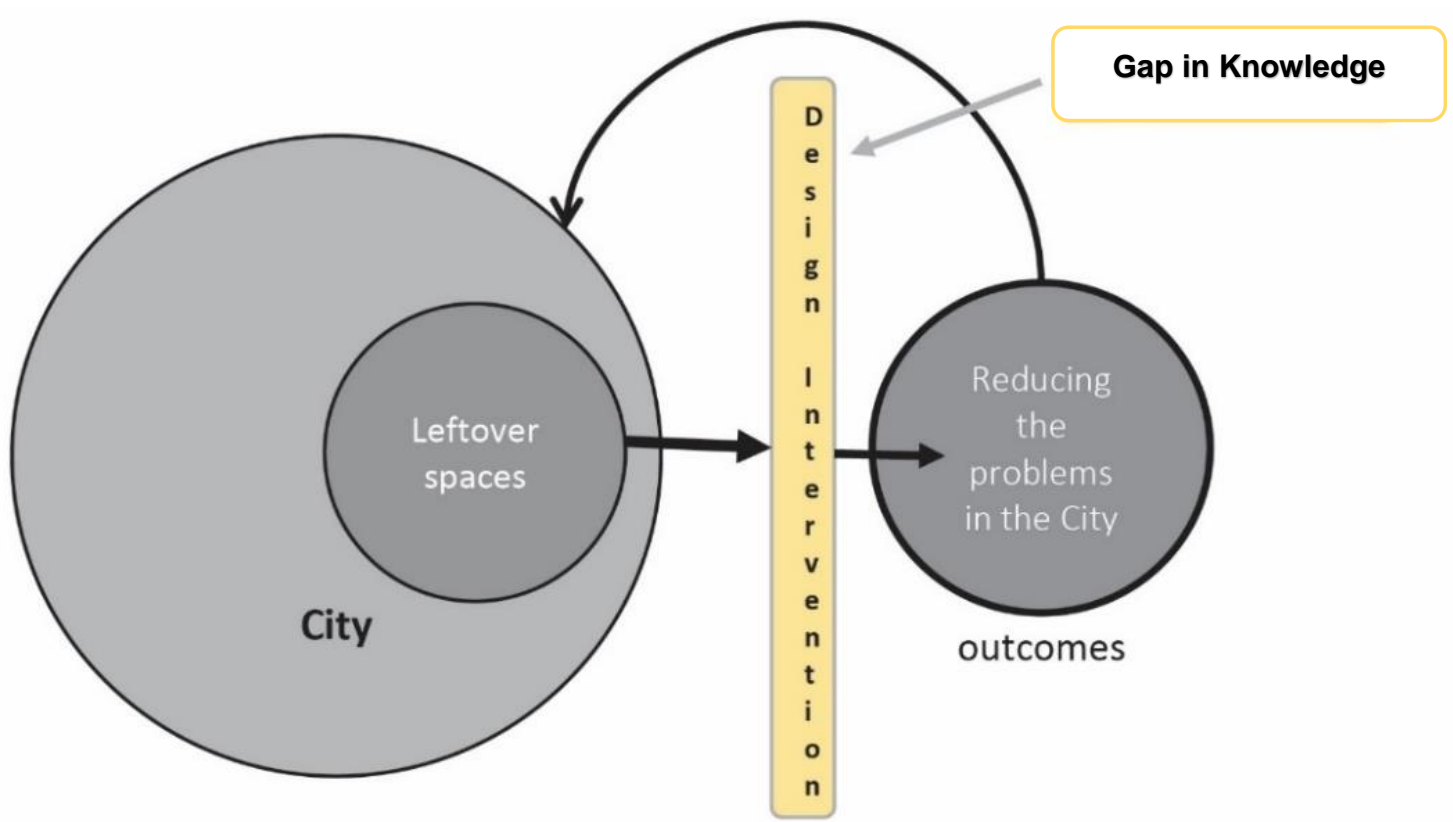

Figure 3.3 Overview of this research

Source Author

\subsubsection{Research Question}

The research focuses on the contribution of leftover spaces within the fabric of Wellington City that can be designed to make the city look aesthetically more appealing and contribute to sustainable initiatives. Different types of leftover space have the potential of becoming a steppingstone for nature in the urban fabric (European Commission, 2012). Realising the value of local leftover spaces in Wellington City could be necessary before applying the concept of sustainability across the whole city. The motivation of this research is to understand how leftover spaces are perceived by the public and examine the affective responses of designs related to environmental and social interaction. This research is triggered by one primary question:

What are people's visual preferences for urban leftover spaces in Wellington City?

Widgery (1982) argued that the aesthetic quality of a place affects community satisfaction and social belonging. Aesthetics play a critical role in satisfying a community and contribute to economic conditions (Belot et al. 2007). Moreover, Green (1999) found that the perception of the community is associated with the features of a town's character and natural aspects are positively correlated with the image of a place. This research has identified a gap in knowledge for designing leftover spaces 
with attributes that are perceived as appealing by the public in Wellington. Also, the literature (see section 3.3.2) was found ambiguous as to whether design professionals value design similarly to the general public. Therefore, the initial question also leads to three sub-questions:

i. What attributes do people prefer for the design of urban leftover spaces?

ii. Are there any differences in design preferences between male and female participants?

iii. Are there any differences in design preference between participants with a built environment background and those without?

\subsubsection{Aims and Objectives}

This study examines the potential of public and privately-owned urban leftover spaces for being used positively with pleasing aesthetics and in ways that can help meet future needs. Previous studies have focused on public spaces and neglected the potential of private spaces, which make up a significant proportion of a city, to be used constructively. The spatial conditions are those between buildings and those where the original purpose of the space has or will become obsolete. In order to answer the questions, this research has the following main aim:

\section{To identify the preferred design attributes for six different types of leftover space in Wellington City.}

The focus of this research is to design an aesthetically pleasing built environment using people's preferences that could become part of the development process in Wellington. The process of finding answers to the main research question produces the following research objectives:

i. Identifying the significance of leftover spaces in the city and identifying their types.

ii. Discovering the importance of aesthetic perception in the built environment.

\subsection{Summary}

A theoretical framework for investigating the preferences and perceptions of people for different leftover spaces before and after the design was introduced in this Chapter. The importance of leftover spaces in the city's fabric was discussed in Chapter 2, and 
Chapter 3 reviewed issues around environmental aesthetics for designing spaces. Together these created the theoretical framework of this research. It should be noted that no knowledge exists in the literature about designing leftover spaces based on people preferences (see figure 3.9). This Chapter has highlighted the role of aesthetics in evaluating an environment and its effects on human psychology. Groat (1988) found that features affect the judgement of contextual fit, whereas perception is a crucial element for measuring preferences (Kaplan \& Kaplan, 1989), while the appearance of a space and meanings associated with it are fundamental to any function (Nasar, 1994). This research deals with the subjective evaluation of feelings about leftover spaces to understand preferences in the built environment. To this end, this Chapter presented the main research question supported by two sub-questions, together with the research aims and objective. In Chapter 4, the research methodology with which to answer the research questions is developed. 


\section{CHAPTER 4}

\section{RESEARCH DESIGN}

\subsection{Introduction}

Having outlined the theories related to urban leftover space and environmental perception in Chapters 2 and 3, this Chapter explains the background to the design of the research undertaken to answer the research question and fulfil the goals and objectives, which were set out in section 3.7. The research design is a strategic framework, which acts as a connection between the research questions and achieving the research goals through specific procedures (Durrheim, 2004). Researchers like Collis \& Hussey (2003), Groat \& Wang (2013), and Creswell (2009) claimed a theoretical underpinning is essential to conducting systematic research. It is thus helpful to situate the research in its broader context, before setting up the procedures for collecting and analysing the data.

Groat \& Wang (2013) described four frameworks, which are nested together in a system of research (figure 4.1) and based on this, this Chapter explains each phase of the design of the research in detail. The first section explores the system of inquiry in which the research is situated. The second highlights the school of thought, followed by the specific strategy for acquiring data through the implementation of tactics, i.e. the collection and analysis of data and the interpretation of the results (Creswell, 2009).

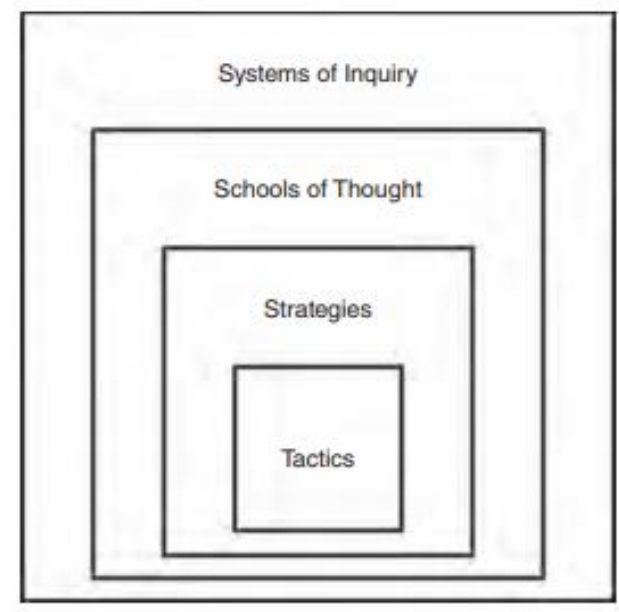

Figure 4.1 A nested framework for research design Source Groat \& Wang, 2013 


\subsection{System of Inquiry}

The foremost framework in the design of this research is the system of inquiry. This is also known as the paradigm or worldview. It involves the assumptions used for understanding the real world and its existence (Groat \& Wang, 2013). According to Fossey et al. (2002), a worldview is a cluster of opinions and involves a systematic process for developing new knowledge. Mills et al. (2006) asserted to achieve robustness in research design, the researcher needs to select a worldview that is parallel to his/her beliefs about the physical world. However, there are also opposing worldviews with different beliefs about reality, and these can also help the researcher to achieve an outcome from the research topic (Tashakkori \& Teddlie 1998).

When comparing or selecting a worldview, there are three crucial questions (figure 4.2):

1. What is actual or real (ontology)?

2. How can something be learned (epistemology)?

3. Which approaches should be used to plan the research (methodology)?

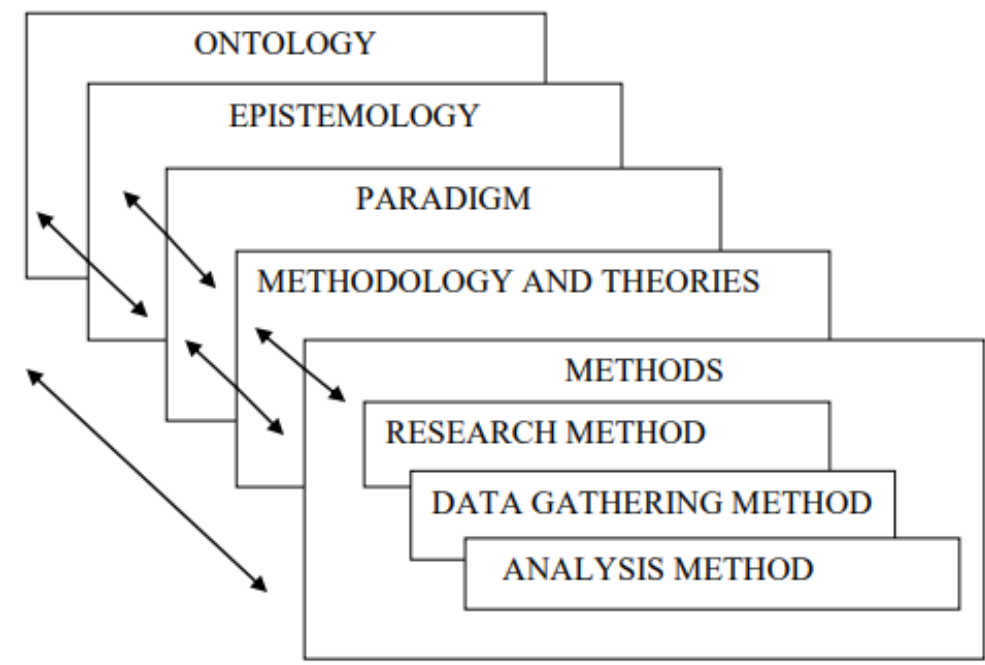

Figure 4.2 Hierarchical order of a paradigm Adapted from Kyro, 2003

Ontology is described by Crotty (2003) as an inquiry into the physical world. Questions such as 'what is the nature of reality?' or 'what is there that can be known?' can be answered by ontological presumptions (Guba \& Lincoln, 1989). Kyro (2003) claimed that ontology is the most basic, but also most significant, level of understanding reality, followed by epistemology, which is derived from the 
ontology. Ontology is also concerned with unique techniques for acquiring information, which is a methodology. The present research sits within the approach known as pragmatism, which uses an ontology regarding social life issues and realities. It asserts that there is a single reality (positivist), and all individuals have their unique interpretation and understanding of reality (interpretive). The study adopts both objectivist and subjectivist ontological perspectives. Table 4.1 presents different paradigms and critical differences in them.

\begin{tabular}{|c|c|c|c|}
\hline Description & Positivism & Interpretivism & Pragmatism \\
\hline $\begin{array}{l}\text { Ontology } \\
\text { What is real? }\end{array}$ & $\begin{array}{l}\text { Objectivist, } \\
\text { reality is one } \\
\text { (what an individual } \\
\text { sees) }\end{array}$ & $\begin{array}{l}\text { Subjectivist, } \\
\text { socially constructed } \\
\text { realities (differs } \\
\text { between individuals) }\end{array}$ & $\begin{array}{l}\text { Seeks to minimise } \\
\text { questions about } \\
\text { reality (by } \\
\text { concentrating on } \\
\text { practical actions) }\end{array}$ \\
\hline $\begin{array}{l}\text { Epistemology } \\
\text { What is true? }\end{array}$ & $\begin{array}{l}\text { The only knowledge } \\
\text { is scientific } \\
\text { knowledge, which is } \\
\text { the truth; reality is } \\
\text { apprehensible }\end{array}$ & $\begin{array}{l}\text { Findings approximate } \\
\text { to the truth, the } \\
\text { reality is never fully } \\
\text { apprehended }\end{array}$ & $\begin{array}{l}\text { Deem as } \\
\text { appropriate for the } \\
\text { research, both } \\
\text { objective and } \\
\text { subjective }\end{array}$ \\
\hline Methodology & Quantitative & Qualitative & Mixed methods \\
\hline $\begin{array}{l}\text { Data } \\
\text { collection }\end{array}$ & $\begin{array}{l}\text { Experiments, quasi- } \\
\text { experiments, tests, } \\
\text { scales }\end{array}$ & $\begin{array}{l}\text { Interviews, } \\
\text { observations, visual } \\
\text { data, document } \\
\text { reviews }\end{array}$ & $\begin{array}{l}\text { May include tools } \\
\text { from positivist and } \\
\text { interpretive } \\
\text { paradigms }\end{array}$ \\
\hline
\end{tabular}

Table 4.1 Differences between dominant paradigms Adapted from Guba \& Lincoln, 1994; Crotty, 2003

Epistemology investigates the existence of physical things in terms of how and what they are (Crotty, 2003). According to Maynard (1994), epistemology is about the philosophical understanding of various types of knowledge, while assuring the knowledge is adequate and authentic. The epistemological stance in this research is pragmatic (section 4.1.1) and involves two steps. 
1. Objective method (quantitative data collection)

2. Subjective method (qualitative data collection).

The former calls for no interaction with research subjects, whereas the latter is defined by the fact that involves interacting with subjects to collect data (Teddlie \& Tashakkori, 2009). The pragmatic approach enables a researcher to be flexible enough to adopt the most practical approach to investigating the research question.

The third stage is the plan of action, also referred to as the methodology. The methodology is the process of selecting and utilising the specific techniques to accomplish the goals of a study (Crotty, 2003). It aims to explain, interpret, and justify the selection of a specific method for conducting a study (Wellington, 2000). This study uses a mixed methods approach by conducting both quantitative and qualitative sub-studies within a pragmatic paradigm (Howe, 1988; Maxcy, 2003; Johnson \& Gray, 2010). While objective (quantitative) and subjective (qualitative) approaches link theory to data using deductive and inductive methods respectively, the pragmatic approach (Modell, 2009) relies on abduction in order to reveal and strategise the best possible way to explain and understand the outcomes.

\subsubsection{Pragmatism as a worldview}

A pragmatic approach essentially means a realistic approach. This leads to a research design that is potentially more realistic, and that could, therefore, have more relevant results, thus making the study findings more authentic (Kloppenberg, 1996). According to Dewey (1958) \& Pohlstrom (1998) the effectiveness of an inquiry is associated with its practical outcomes, or in other words, what works. Rossman \& Wilson (1985) claimed that a researcher needs to identify the nature of the research in order to put more focus on the study problem and what is needed to solve it rather than emphasising possible research methods. The integration of knowledge and action is known as pragmatism (Goldkuh, 2002). Goldkuh (2012) claims that pragmatism is concerned with having practical outcomes and the consequences of these. An example of such an ontology is related to how humans are in a constant state of action and change in this world. Blumer (1969) argued that the basis of human society lies in action and constant change. An activity causes individual to react in a certain way, 
which in turn transforms the physical setting. The pragmatic worldview is drawn out from present actions and situations and not from the past conditions of being (Creswell, 2009). Morgan (2007) added that pragmatism can act a passageway for motivating researchers who make use of a variety of methods in paradigms to point out meanings. It includes an understanding of shared actions and the behaviours of individuals. Pragmatism, as a worldview is adopted for social and administration practice-based research and focuses on the importance of working with mixed qualitative and quantitative approaches to answering the research questions (Armitage, 2007).

\subsection{School of Thought}

The second nested framework is the school of thought. According to Groat \& Wang (2013), commitment to a school of thought is necessary to determine what procedures are required for analysis and to investigate how the research questions are framed. This research sits within the field of the built environment and emphasises the significance of environmental psychology (see figure 4.3).

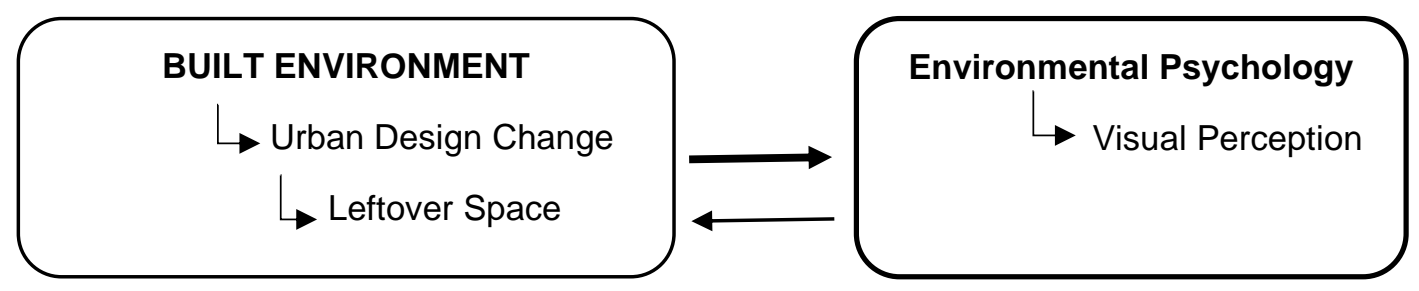

Figure 4.3 Fields examined in this research Source Author

Built Environment refers to the transformation of natural surroundings by the intentional application of human energy to serve human purposes and needs (Kropf, 2017). Scholars like Jacobs (1993), Allan \& Bryant (2011), and Carmona (2013) argued that the quality of physical space and its design, in terms of the configuration of buildings and open space to create an urban morphology, affects public life through the social functions it offers and the economic values it enshrines. Cowan et al. (2005) and Krier (2009) claimed that urban design is about enriching the experiences and values of individuals and groups, and the society wherein they reside. However, these needs and demands vary physiologically, socially, and psychologically (Bartuska \& McClure, 2007). The spaces that surround a person are critical since they can change 
the mood, behaviour, and affect a person's mental wellbeing (Bell et al., 1996; Evans et al., 2013).

The psychological field emphasises human perceptions, actions, and reactions to the physical setting (Gifford, 2008) In general psychology, the most crucial process is perception, which compromises the three processes of preference for something, association with something, and interpretation of these reactions. Thus, for this research the field of environmental psychology (see section 3.2) becomes significant that studies interaction of individual human beings or groups of people with their physical setting. It includes how individuals see a thing and alter their environment as a consequence, or even how human cognition, behaviours, and experiences are shaped by the physical setting (Bechtel \& Churchman 2002), which thus involves both natural and built environments. The sub field of an environmental aesthetics (see section 3.2.1) is linked to environmental psychology that focuses on understanding the human perception, cognitive representation of physical settings and emotions that are aroused, which can then be interpreted, described and assessed. The perception of an environment (see section 3.2.1) incorporates both natural and built environments, individuals, aesthetics, intuition, and cultural values. Rapoport (1970) reported that the meaning of a place is attached to individual feelings for it, whether these are strong or weak, and is also linked to the cognitive associations for the recalled, and hence image-able, parts of the urban fabric. Driver \& Spence (1998) stated that people perceive information related to the environment by using different senses, such as sight, smell, hearing, touch, and taste. This causes people to take actions influenced by their preferences as to how they experience the world and react to a particular situation, whether it is ugly or beautiful, simple or complex. Such actions are based on the process of perception. More than $80 \%$ of sensory data accessible to a human being comes through vision (Wood \& Troutbeck 1992; Porteous 1996). The visual perception of the built environment is seen as the most critical information for architects (Crosby, 1997). All such information is engaged through using vision, which is coordinated and interpreted by the brain (Matlin \& Foley, 1992). 


\subsection{Strategy of Inquiry}

The third nested framework is the strategy of inquiry. The strategy is skilful management and planning to structure a research study (Groat \& Wang, 2013). The mixed methods approach is utilised for this study to identify the attributes affecting the perception of people when it comes to the design of leftover spaces. The idea of mixing unique approaches was developed by Campbell and Fisk in 1959, as they utilised multi-methods to inquire into the validity of psychological characteristics (Creswell, 2009). Different names have been used for the mixed methods approach, such as multi, hybrid, integrated, and combined (Creswell \& Clark, 2007). In this approach, the qualitative and quantitative information is assembled individually and examined separately, the data is then brought together in the final interpretation phase, known as the stage of meta-inferences or integrated mixed inferences (Tashakkori and Teddlie, 2003). Researchers like Schmidt (2005) and Patton, (1990) argued that the mixed methods approach resolves the issue of public participation and strengthens the overall design process by overlapping the shortcomings of quantitative methods (large sample size, trends, and generalizability) with those of qualitative methods (small sample, details, and particularities).

According to Van der Merwe (1996), a quantitative study is an approach aimed at investigating theories, determining facts, establishing connections between different factors, and concluding results. This method is derived from natural sciences and is aimed at ensuring objectivity, and is concerned with numbers, rationale, generalizability, and reliability (Weinreich, 2009). Qualitative research, however, focuses on the qualities of a situation, procedures, and implications that are not measured or analysed in terms of quantity (Denzin and Lincoln, 2005). Weinreich (2009) suggested the rationale for a subjective study is that it provides the researcher with a view of the target group, and with the views of individuals about a particular situation in which they live or with which they are somehow connected.

As explained in section 3.6 the research explores the design potential of leftover spaces by referring to people's perceptions of what makes environments aesthetically pleasing and usable. Thus, to understand the people preferences for current leftover spaces and their design expectations, this research uses a mixed methods strategy with the integration of quantitative data (preferences for design, correlations of most liked option with the affective appraisals), and qualitative data (focus group 
discussions) to gain a more holistic understanding of the potential uses of leftover spaces.

\subsubsection{Concurrent Triangulation Method}

The most familiar and acknowledged way of mixing methods is known as triangulation (Creswell et al., 2003). This approach aims to understand the research issues by getting diverse and complementary information on the research topic (Jick 1976; Morse, 1991). Triangulation is a one-phase approach in which the analyst executes separate data collection and analysis techniques for objective (quantitative) and subjective (qualitative) methods during the same timeframe and giving equal importance to both (table 4.2). This is known as concurrent triangulation (Creswell et al., 2003).

\begin{tabular}{|c|c|c|c|}
\hline Design Type & Variants & Timing & Mixing \\
\hline $\begin{array}{l}\text { Concurrent } \\
\text { Triangulation }\end{array}$ & $\begin{array}{l}\text { 1. Convergence } \\
\text { 2. Data } \\
\text { Transformation } \\
\text { 3. Validating } \\
\text { quantitative data } \\
\text { 4. Multilevel } \\
\text { (quantitative and } \\
\text { qualitative samples) }\end{array}$ & $\begin{array}{c}\text { Concurrent: } \\
\text { quantitative } \\
\text { and qualitative } \\
\text { data collected } \\
\text { in the same } \\
\text { period }\end{array}$ & $\begin{array}{l}\text { Data is } \\
\text { merged } \\
\text { during the } \\
\text { interpretation } \\
\text { or analysis }\end{array}$ \\
\hline
\end{tabular}

Table 4.2 The concurrent triangulation method used in this research

Source Creswell \& Clark, 2007

Morse \& Niehaus (2009) and Guest (2013) argued that the qualitative and quantitative approaches need to be mixed, connected, and integrated at one point of interface (figure 4.4). This is also done for cross-validation, affirmation, corroboration, and overcoming the shortcomings of one approach with the strength of the other (Greene et al., 1989; Morgan, 1998). 


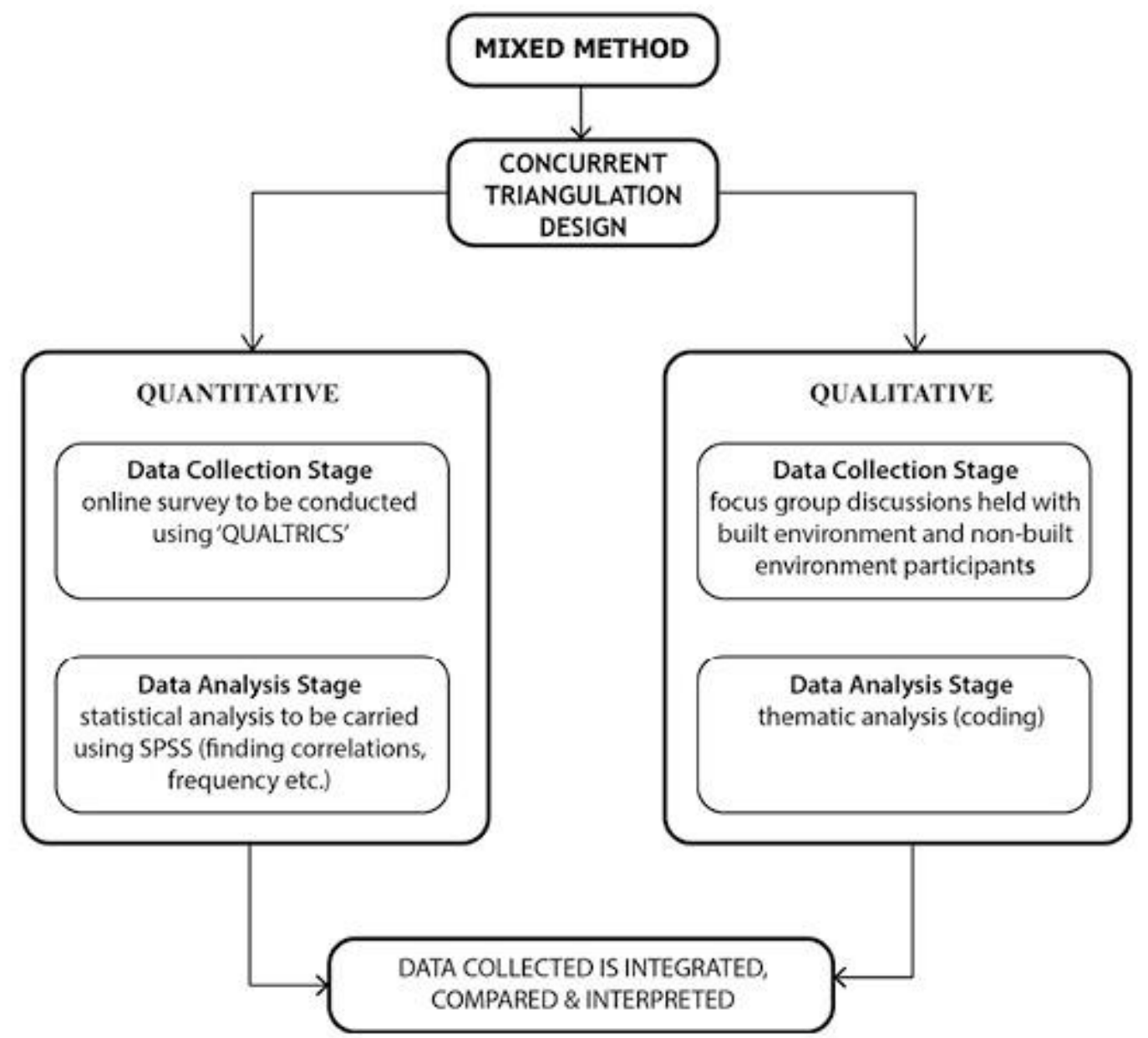

Figure 4.4 Diagram of the concurrent triangulation strategy to be used in this study Adapted from Morse \& Niehaus (2009) and Guest (2013)

\subsection{Tactics}

The final nested framework is the tactics related to the design of the research. To answer a research question or hypothesis, the researcher must utilise particular but detailed methods or procedures to investigate the data, which is also referred to as tactics (Crotty, 2003). According to Sargeant (2012), it is critical for the researcher to clarify the three methods to be used for determining the sample, the method of data collection, and for doing the analysis, as discussed below.

\subsubsection{Sample Design}

Leech et al. (2006) argued that the research findings are more valid and reliable if the sample size is large. However, according to Lenth (2001), the study should only 
involve enough recruitment to realise a successful outcome. Nayak (2010) suggested providing reasons for what is an adequate number of participants as this saves time, energy, consumes fewer resources, and ensures that participants are valued equally. Assistance was sought for estimating the necessary sample size for studies One and Two in this research from the free statistical software known as GPower (Buchner et al., 1997). According to Aberson (2010), the power analysis calculates the probability and effectiveness of the test for determining statistically significant outcomes for a study. Furthermore, it helps researchers to generate a relevant sample size, which is accurate, valid, and reliable. The test was performed in Gpower software with an effect size of 0.5 by using the two-tailed T-test to determine the mean differences in participant opinions. The test revealed that at least 64 participants were required for studies One and Two (128 participants in total). The effect size represents the strength and magnitude of the difference between groups (Durlak, 2009). Onwuegbuzie and Leech (2007) have classified 24 sampling techniques (5 random and 19 purposive sampling schemes), which can be used to select participants in either the quantitative or qualitative phases of a mixed methods research study. In this research, the probability sampling technique is used to select the sample randomly in which every participant has an equal probability of being selected from the population. Lastly, study Three consisted of focused discussions with two independent groups of people based on their field of study or expertise (see section 8.2).

\subsubsection{Data Collection}

This exploratory research utilised the cross-sectional design technique of a survey to collect the information at one time and make the data more generalizable and thus representative of the whole national population. The data were collected in four stages to achieve the goals (see section 3.7) of the study and different techniques for collecting the data were adopted. Table 4.3 provides a brief overview of all the stages undertaken in this study. 


\section{RESEARCH AIM}

To identify the preferred design attributes for six different types of leftover space in Wellington City.

\begin{tabular}{|c|c|c|}
\hline OBJECTIVE & METHODS & $\begin{array}{c}\text { RESOURCE } \\
\text { S }\end{array}$ \\
\hline $\begin{array}{l}\text { Development of a } \\
\text { theoretical framework by } \\
\text { taking account of: } \\
\text { i. The significance of } \\
\text { leftover spaces in the city } \\
\text { and identifying their types. } \\
\text { ii. The importance of } \\
\text { aesthetic perception in the } \\
\text { built environment. }\end{array}$ & Literature Review & \\
\hline $\begin{array}{l}\text { i. To understand the } \\
\text { perception of participants } \\
\text { regarding different leftover } \\
\text { spaces. } \\
\text { ii. Assist in developing a } \\
\text { precise understandable } \\
\text { survey instrument for the } \\
\text { main study. }\end{array}$ & $\begin{array}{l}\text { Pilot Study } \\
\text { The photographic survey collected } \\
\text { information from participants about } \\
\text { their preferences for leftover spaces } \\
\text { with different attributes (see Chapter } \\
\text { 5). }\end{array}$ & $\begin{array}{l}\text { Photographs } \\
\text { Field Survey } \\
\text { Qualtrics (to } \\
\text { conduct an } \\
\text { online } \\
\text { survey) } \\
\text { Statistical } \\
\text { Package for } \\
\text { the Social } \\
\text { Sciences } \\
\text { (SPSS) } \\
\text { version } 23\end{array}$ \\
\hline $\begin{array}{l}\text { i. To understand the } \\
\text { perception of participants } \\
\text { and their subgroups about } \\
\text { preferences for different } \\
\text { leftover spaces and their } \\
\text { attributes. }\end{array}$ & $\begin{array}{l}\text { Study One: First Visual Preference } \\
\text { Study (see Chapter 6) } \\
\text { i. The photographic survey collected } \\
\text { information from participants about } \\
\text { their preferences for different leftover } \\
\text { spaces and their attributes. The } \\
\text { participants also recommended }\end{array}$ & $\begin{array}{l}\text { Photographs } \\
\text { Field Survey }\end{array}$ \\
\hline
\end{tabular}




\begin{tabular}{|c|c|c|}
\hline $\begin{array}{l}\text { i. To understand the } \\
\text { preferences for spaces } \\
\text { (before and after their } \\
\text { redesign) and their } \\
\text { attributes. } \\
\text { ii. To identify the differences } \\
\text { in design opinions between } \\
\text { built environment experts } \\
\text { and others. }\end{array}$ & $\begin{array}{l}\text { changes for improving the visual } \\
\text { aesthetics and usability of the } \\
\text { spaces. } \\
\text { ii. Analysis of data about participant } \\
\text { preferences, and comparison by } \\
\text { gender, built environment } \\
\text { participants, and other factors. } \\
\text { Study Two: Second Preference } \\
\text { Study (see Chapter } 7 \text { ) } \\
\text { i. Collection of data about } \\
\text { preferences using photomontages } \\
\text { ii. Analysis of data from all } \\
\text { participants, and comparison by } \\
\text { gender, built environment experts, } \\
\text { and other factors. } \\
\text { iii. Correlation of the most preferred } \\
\text { designed option with its affective } \\
\text { appraisal. }\end{array}$ & $\begin{array}{l}\text { Photoshop } \\
\text { CS5 } \\
\text { Qualtrics (to } \\
\text { conduct an } \\
\text { online } \\
\text { survey) } \\
\text { SPSS version } \\
23\end{array}$ \\
\hline $\begin{array}{l}\text { To understand the } \\
\text { perception and design } \\
\text { preferences of built } \\
\text { environment participants } \\
\text { and others for different } \\
\text { leftover spaces. }\end{array}$ & $\begin{array}{l}\text { Study three: Focus Group } \\
\text { Discussions (see Chapter } 8 \text { ) } \\
\text { i. Data collected from discussions } \\
\text { between built environment experts } \\
\text { and others for preferences for the } \\
\text { design of leftover spaces. } \\
\text { ii. Thematic analysis to probe for } \\
\text { differences between the two groups. }\end{array}$ & $\begin{array}{l}\text { Voice } \\
\text { Recorder } \\
\text { Photographs } \\
\text { Microsoft } \\
\text { Word (for } \\
\text { transcription) } \\
\text { Meeting room }\end{array}$ \\
\hline
\end{tabular}

Table 4.3 Procedures employed to achieve the research goals

\subsubsection{Pilot Study}

Before conducting the primary studies, six different leftover spaces in Wellington City were identified and documented. The initial exploration involved ethnographic methods such as observation, walkthroughs, maps, photographs, and site visits to identify leftover spaces with different parameters such as current use, shape, context, 
ownership rights, accessibility, visibility, and barriers. The photographs of six different leftover spaces were taken in perspective view with the original setting and without any people in the scene. Participants would also be asked to suggest improvements to the spaces. This pilot study was a small-scale replica of study One, targeting students, both those with a built environment background and others (see section 5.2). This study was aimed at developing a clear and understandable survey instrument for the primary studies to follow. Gosling et al. (2004) found that conducting online research is more viable than paper-based surveys, easily accessible, and easy to conduct. As a result, the internet-based software Qualtrics was used for the pilot study, with the aim of using it for both Study One and Study Two if this was successful.

\subsubsection{Study One: First Visual Preference Study}

Study One was aimed at measuring people's general preferences for the characteristics of different urban leftover spaces. The results would, in turn, inform the development of Study two. Study One principally employed descriptive, quantitative methods to gain an understanding of people's preferences for urban leftover spaces, using photographs to stimulate the responses (see Chapter 6). Simple methods such as finding the mean, standard deviation, and percentages were deployed to probe the preferences of participants in this study. The study sought to collect data from a higher number of participants than in the pilot study to add to the robustness of findings. The survey was performed online using Qualtrics and analysis was done using SPSS software. The recruitment of participants was made by sending invitation emails and by setting up posters in cafés and the Wellington City library. On average, it took 21 minutes for participants to complete the survey. The survey link remained open for a month to gather as many responses as possible.

\subsubsection{Study Two: Second Visual Preference Study}

In the second study, a rendered image based questionnaire was given to a range of people using an online survey as before to discover what they liked and did not like about the different types of leftover space both before and after design interventions (see Chapter 7). Nasar (1998) has suggested that using pictures as the tool can elicit similar responses to showing people the actual circumstances. Study Two employed descriptive and inferential methods to calculate the mean preferences and correlation of affective appraisals for the most preferred redesigned option. The knowledge 
gathered from Study One led to different photomontages of leftover spaces with specific attributes to stimulate the responses.

Study Two also looked for differences and similarities in design preferences between participants with built environment expertise and those who lacked this. The recruitment technique was similar to that of Study One. It took participants on average 18 minutes to complete the Study Two survey. The survey link remained opened for a month to gather as many responses as possible.

\subsubsection{Study Three: Focus Group Discussions}

Study three comprised the focus group discussions with the built environment and non-built environment participants to understand their aesthetic preferences for various leftover spaces. The study was built upon the findings of Study One as participants were shown photographs of leftover spaces that were the outcome of Study One. Two separate focus group sessions were each divided into the two phases of a mini-design charrette and a discussion. The mini-design charrette is a collective design and planning workshop that aims to provide a solution for a particular issue (Lennertz \& Lutzenhiser, 2006). Participants were shown six different photographs of urban leftover spaces and were given the task of redesigning the spaces in order to improve their usability and aesthetics. The second phase discussed the design solutions made in the previous phase to understand the effectiveness and significance of design attributes for designing leftover spaces. The sessions were audio recorded and transcribed to look for the most recurring patterns and themes in the discussion. This qualitative data was used to assess the validity of the quantitative findings from Study Two (O'Cathain et al., 2008).

The findings from all studies are compared and interpreted (see Chapter 9 ) to enable a more detailed understanding of how people perceive leftover spaces in Wellington City.

\subsubsection{Data Analysis}

For this research, two instruments were used, namely questionnaires in Studies One and Two, and focus group discussions in Study Three. The questionnaires covered demographics (gender, the field of study, age, cultural ethnicity, and academic qualification); preferences for spaces before and after interventions, and suggestions 
for improvement. The data obtained from Studies One and Two were analysed and processed in SPSS version 23. The survey data was directly imported from Qualtrics into SPSS for analysis. The bar charts were made to represent the data using Microsoft Excel. However, Study Three employed transcribed data using Microsoft Word, and bubble diagrams were made in Adobe Illustrator. While Study One analysed the preferences for spaces and participant suggestions about the design attributes, Study Two analysed the preferences for redesigning the spaces, the correlation of semantic differentials with the preferred design, and T-tests to see the difference in opinions between participant groupings. Lastly, Study Three was the focus group discussions with the two independent groups of built-environment trained participants and of that that are not, to look for any differences in opinion of and design preferences for the various leftover spaces.

\subsection{Summary}

Chapter 4 has set out the research design and the methods to be used for collecting the data. The philosophical framework from which the research methodology is developed is discussed. This research is situated and aligned with the pragmatic approach that emphasizes taking concrete, practical actions guided by knowledge to understand and alter reality in an effective way. The research uses the mixed methods approach that involves both quantitative and qualitative data. The methodology involves three different studies and seeks people's perceptions of urban leftover spaces and their redesign using photographs and photomontages. Study One probes preferences for changing six different types of leftover space and takes suggestions about design attributes for their improvement. The information created from Study One feeds into Study Two, where the most significant suggestions are photomontaged to create three alternatives for each type of space. Study Two investigates people's aesthetic responses to different redesigned options. This study also provides insight into any changes in people's perceptions from Study One. The online software Qualtrics is used for conducting the survey. Study Three involves two focus group discussions with mini design charrettes. This study is conducted with built environment and non-built environment participants separately, to make people more familiar with the study and to have more open discussions. Chapter 5 concerns the pilot study that 
Chapter 4- RESEARCH DESIGN

was conducted to check the research instruments for conducting main studies (Study One and Study Two). 


\section{CHAPTER 5}

\section{PILOT STUDY}

\subsection{Introduction to the Pilot Study}

Chapter 5 highlights the importance of conducting a pilot study for testing the tools consisting of the set of photographs and related questions before the main preference studies are undertaken. According to LaGasse (2013), a pilot study is also recognised as a feasibility study, or a mini or trial version of a full study. The idea of this study was to pre-test the research instruments in the real world as what may differ from what has been envisaged, and this includes both questions and schedules (Van Teijlingen \& Hundley, 2002). A pilot study thus provides an insight into the research topic, questions, tools, and procedures, all to accomplish a comprehensive and successful study (Blaxter et al., 2001). Welman \& Kruger (1999) argued that a pilot study aims at averting wastage of time, energy, and money by detecting flaws in the process, identifying ambiguous questions, and noting any unsettling content for the respondents.

This pilot study sought to examine the potential of semi-public urban leftover spaces dispersed around Wellington City using a small group of participants and noting how they responded to the research topic. To help ensure any visual improvements to these spaces would enhance their appreciation and uses, it was first necessary to find out how the general public would react to the visual quality of the unused spaces. The expected outcomes of this study were to improve the research project and to minimise the risk of procedural failure in the main study.

\subsection{Description of the Pilot Study}

The pilot study was conducted online using the internet-based software Qualtrics, and approval by the Victoria University Human Ethic Committee (No 25265) was obtained beforehand. The study was divided into four parts. The first part sought demographic information by asking participants to provide details related to their age, gender, the field of education, and year of study. The remaining three parts had questions based 
on photographs of leftover spaces, and none of these photos contained people since this is problematic (Herzog, 1989). Furthermore, the photos were selected to present each type of leftover space in diffuse light so that sunlight would not have an impact on the choices of participants.

The second part consisted of photos based on Nasar's (1998) research that claimed the most likeable environmental features were natural rather than artificial elements, well maintained rather than poorly maintained space, open space rather than enclosed space, an ordered space rather than an unorganised space, and buildings with historical significance such as an old building façade rather than a modern one. According to Nasar (1998), the imageability of a built environment is connected to the viewer's perception as to how things are associated, evaluated, and then how the viewer reacts to these. In this section of the survey, Nasar's (1998) theory that there are preferences for these five aspects of the environment would be tested in the present study. The aim was to see whether individuals with different backgrounds or fields of study perceive and experience the elements of an environment in a similar way as suggested. Thus, participants were asked to choose the more pleasant space out of two photos and provide reasons for their selection.

In the third part, a psychometric technique of likability was deployed, which has also been used to measure traits such as perceptions, qualities, and outlooks (Shea \& Fortna, 2002). Participants were asked to rate different leftover spaces as to how much they liked each scene using a 7 -point Likert scale $(1=$ Strongly Unappealing to $7=$ Strongly Appealing). The scale was created by Rensis Likert in 1932 to measure and evaluate the strength of attitudes (Croasmun \& Ostrom, 2011). Foddy (1994) claimed that at least seven categories are required on a scale to assure the reliability and validity of the study. Four different pictures were shown for the same type of leftover space and participants had to write a short description of what caused them to give a particular score to each picture. Different photos were chosen with specific aspects found in leftover spaces such as vegetation, parking, seating, wall openings, graffiti, cleanliness, and having a clear thoroughfare.

The last part of this survey was more specific. Participants were asked to select one photo out of two settings for each type of leftover space. This procedure was carried out for all six different types of leftover space. The purpose of this section was to ask participants which picture they felt most needed overall improvement and transformation. This section included different suggestions for these changes, out of 
which participants had to select three or more options. Participants were allowed to suggest further options for change other than those listed. Later a content analysis examined the frequency of words and essential phrases used by the participants in this section. The repeated keywords were identified and categorised along with the complete list of attributes, including the suggestions made by participants. Finally, participants were asked to give their suggestions for improving the study.

\subsubsection{Photographic representation}

Kaplan (1974), Herzog (1989), and Hartig \& Staats (2006) all made use of photos or slides as a means of eliciting people's preferences. In this pilot study, participants were shown 46 photos of different urban leftover spaces. The study took an average of 26 minutes to complete with its 47 questions. All photos were selected to represent six different types of leftover space containing different attributes. The spaces were enclosed by buildings on two and three sides, underneath a building, a rooftop, and in front of and at the back of a building. At least two pictures of each space were included. Natural and built structures were evident in the photos, and the focus of the pictorial frame was on the space itself. Colour photos were used, and care was taken to try and ensure that lighting levels were the same across all images. The critical aspects that were tested in this survey included vegetation, cleanliness, the openness of the space, seating spaces, openings in a wall, having a clear thoroughfare, parking spaces, surface materials, graffiti, and maintenance or evidence of lack of maintenance. The survey used a descriptive vocabulary for identification of spatial elements in order to understand the form, function, and consequent appearance of the given space, on the understanding that photographs can be used with confidence in preference judgments and perceptual studies as surrogates for actual spaces (Kaplan, 1985).

\subsection{Study Recruitment and Sample}

The pilot survey was conducted from $10^{\text {th }}$ May 2017 until $21^{\text {st }}$ May 2017. The invitation emails were sent to students studying in the $3^{\text {rd }}$ year of the architecture and urban design course. Other email invitations were sent to engineering and science students at Victoria University. As a result, data were collected from 15 participants and imported for analysis in the SPSS software at the end of the survey period. Overall, 13 participants completed and two partially completed the survey. A draw was held on 
$25^{\text {th }}$ May 2017 , and one participant was contacted through email to receive an NZ $\$ 20$ voucher. At the end of the pilot survey, participants were asked to indicate their willingness to participate in the future main study (see Chapter 7.0) and 10 participants $(66 \%)$ volunteered for this.

\subsubsection{Part 1: Sample demographics}

Demographic information about the participants was collected to see the difference in preference opinions between built environment students and others (see table 5.1). Regarding gender, $53.3 \%$ of respondents were male and $46.6 \%$ female. As expected, a high $64 \%$ of respondents were young (aged 20 to 30 ), and $35.7 \%$ of participants were aged over 30. Participants with built environment knowledge formed $53.3 \%$ of the sample, with the remaining $46.6 \%$ being from a different field of study. For the year of study, $66.7 \%$ of students were in their $3^{\text {rd }}$ year, and $33.3 \%$ were in another year.

\begin{tabular}{|l|c|c|}
\hline Demographic Distribution & & \\
\hline Gender & Frequency & Percentage (\%) \\
Male & 08 & 53.3 \\
Female & 07 & 46.6 \\
\hline Age group & & \\
$20-35$ & 09 & 64.2 \\
Above 35 & 05 & 35.7 \\
\hline Undergraduate study level & & \\
\hline First Year Student & 02 & 13.3 \\
Second Year Student & 02 & 13.3 \\
Third Year Student & 10 & 66.7 \\
Fourth Year Student & 01 & 06.7 \\
\hline Educational Background & & \\
Architecture and Urban & 08 & 53.3 \\
Design & 07 & 46.6 \\
\hline Engineering and Sciences & & \\
\hline
\end{tabular}

Table 5.1 Demographic details of the pilot study sample 


\subsection{Analysis}

Frequency methods of analysis were used in SPSS to understand the relationships between the preferences of participants for space and its different attributes. The frequency statistics test determined the frequency and percentages for preferences among all respondents. The methods of finding the difference in opinions between the subgroups (gender and field of study) were not deployed in this pilot study but would be used in Study One (see section 6.4).

A frequency test was initiated for the second part of the pilot study, which measured the preferences for the spaces with different attributes based on Nasar's (1998) research. In the third part, the numerical values assigned to the 7-point Likert scale were used ranging from 'strongly unappealing $=1$ ' to 'strongly appealing $=7$ ', with other categories assigned a whole number within this range. Stevens (1951) claimed that statistical techniques are critical to measuring the strength of data. Jamieson (2004) argued that Likert type information is calculated with an ordinal level of estimation, which is ranked and arranged to know the difference between each item through an assigned value (Brown, 2011). The Likert scale measured the preferences for each space with different attributes. In the last part of the analysis, a preference for each leftover space was calculated using the frequency test.

The analysis for the pilot study was performed for the whole sample group of 15 respondents to identify the most appealing spaces and their attributes.

\subsubsection{Part 2}

Two different photos of spaces with different design attributes were shown out of which the participant had to choose the one they preferred. The themes for the photos were based on Nasar's (1998) theory, but these were not mentioned to the participants who were asked to give a reason for their choice. For each paired example discussed below, the most preferred space is marked with a red outline (figures 5.1-5.5).

\subsubsection{Natural elements in a space}

In the first pair of photos (figure 5.1), all 15 participants preferred the space with natural features. The reasons given for the choice were having a less unnatural landscape, having a more extensive view, having a green space with trees, and offering a peaceful sight. 

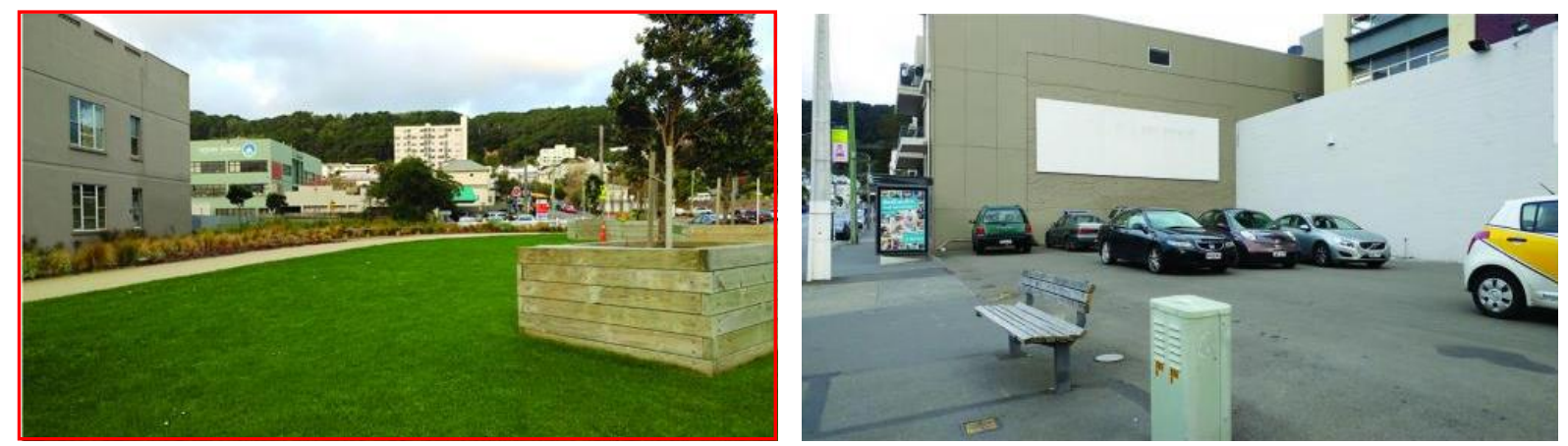

Figure 5.1 Space with natural elements was preferred over space with artificial elements

\subsubsection{Well organised space}

Most participants preferred the space that was organised (figure 5.2) with only three preferring the unorganised space. The reasons behind the preference related to the maintenance of the space and the light colour of the fences.
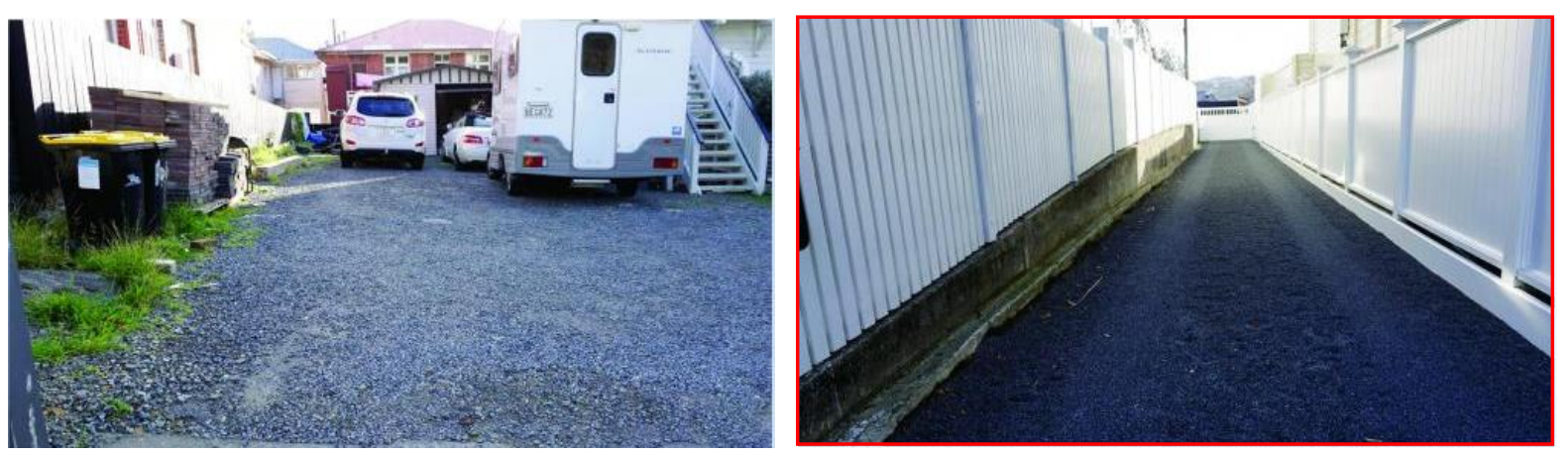

Figure 5.2 Well-organised space was preferred over unorganised space

\subsubsection{Space open to the sky}

The space open to the sky was preferred by 12 participants, with three respondents preferring the space that was enclosed and confined. The reasons for participants' preferences were the openness of the space and the fact that people would not feel trapped in it. 

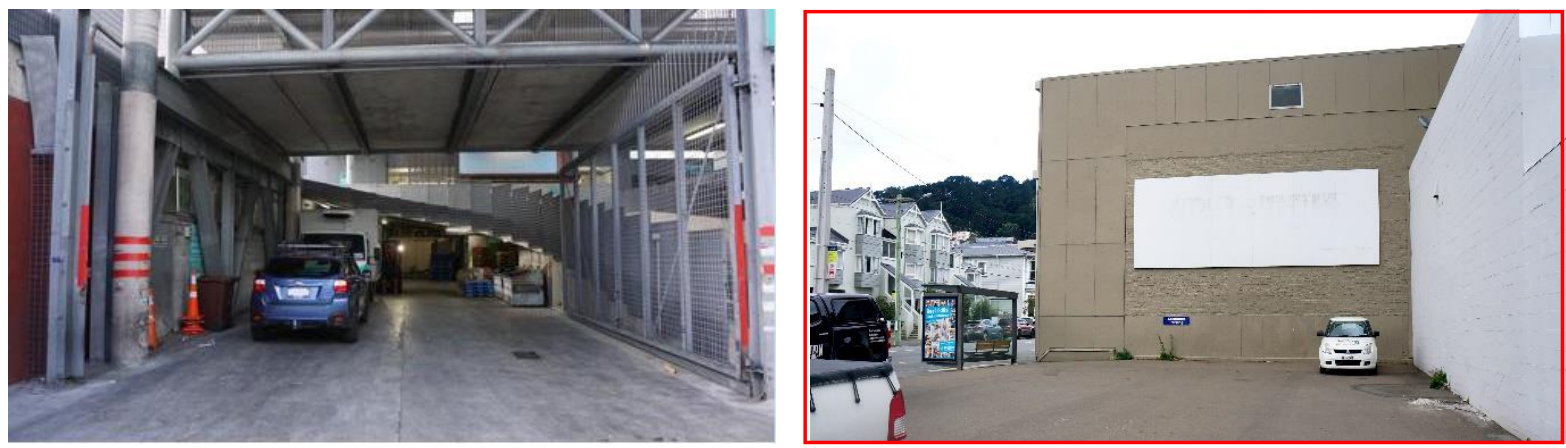

Figure 5.3 Space with openness was preferred over an enclosed space

\subsubsection{Maintained space}

Overall participants liked the space which was adequately managed (figure 5.4). Only 3 out of 15 participants preferred the space which was not well maintained. The reasons for the choice were related to cleanliness, the fact space appears to be private, and having a clear driveway.
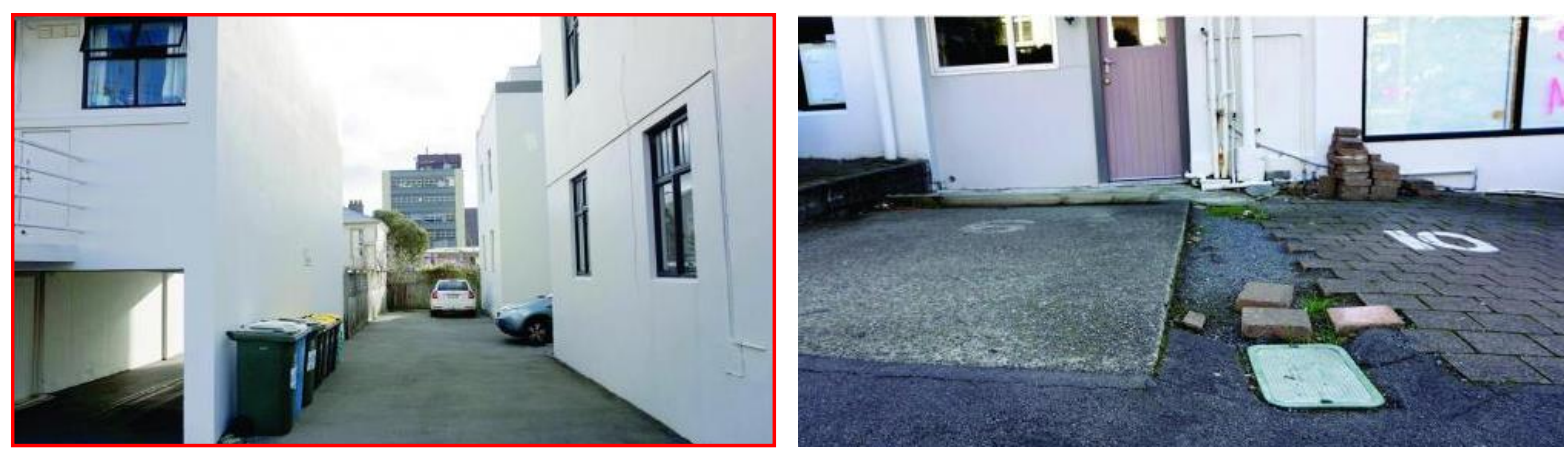

Figure 5.4 Well-maintained space was preferred over unmanaged space

\subsubsection{Modern Building style}

It became evident from the study that participants liked the modern style building (figure 5.1), with this being the choice of 14 participants. Only one participant liked the older style of building. The reasons behind the choices were having an aesthetically pleasing design, new construction materials, light colours, and cleanliness. 

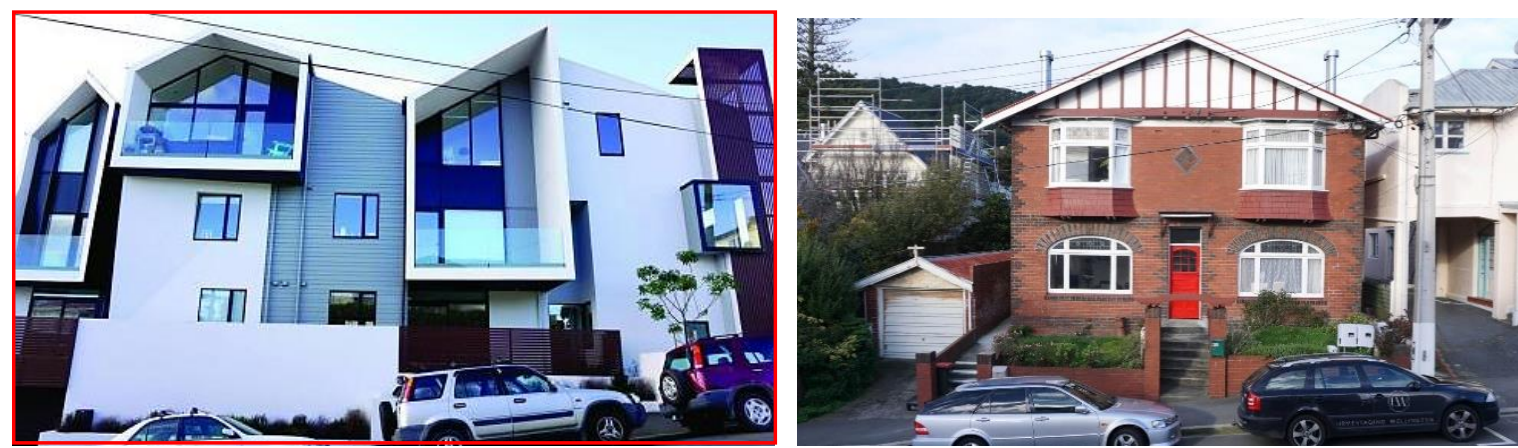

Figure 5.5 Modern building style was preferred over the old building style

The frequency of preference scores in part 2 of the pilot study is given in table 5.2

\begin{tabular}{|c|c|c|c|}
\hline Sequence & Theme & $\begin{array}{c}\text { Frequency } \\
\text { (N) }\end{array}$ & $\begin{array}{c}\text { Percentage } \\
(\%)\end{array}$ \\
\hline \multirow[t]{2}{*}{1} & Natural elements & 15 & 100 \\
\hline & $\begin{array}{l}\text { No natural } \\
\text { elements }\end{array}$ & 00 & 00 \\
\hline \multirow[t]{2}{*}{2} & Managed Space & 12 & 80 \\
\hline & $\begin{array}{c}\text { Unmanaged } \\
\text { Space }\end{array}$ & 03 & 20 \\
\hline \multirow[t]{2}{*}{3} & Open Space & 12 & 80 \\
\hline & Enclosed Space & 03 & 20 \\
\hline \multirow[t]{2}{*}{4} & Ordered & 12 & 80 \\
\hline & $\begin{array}{c}\text { Unorganised } \\
\text { Space }\end{array}$ & 03 & 20 \\
\hline \multirow[t]{2}{*}{5} & Modern Style & 14 & 93 \\
\hline & Older Style & 01 & 07 \\
\hline
\end{tabular}

Table 5.2 Preferences for spaces with different attributes

\subsubsection{Part 3}

The preferences for the most and least preferred photos were measured on the Likert scale (1-7) by using the descriptive frequency test in SPSS. This technique calculated the percentages of liking on the Likert scale for four different photos of each of the six types of leftover space. 


\subsubsection{Space underneath a building}

All the photos of space underneath a building have a way of entering it. All the participants preferred option $D$ that pictured a through the route with evidence that space was used by people in the café tables and chairs (figure 5.6). Option A was the second most liked space, which was adequately managed while option $C$ was the third most liked space being an organised parking space. The least preferred space was option B, which was a confined and gloomy space (figure 5.7).

A

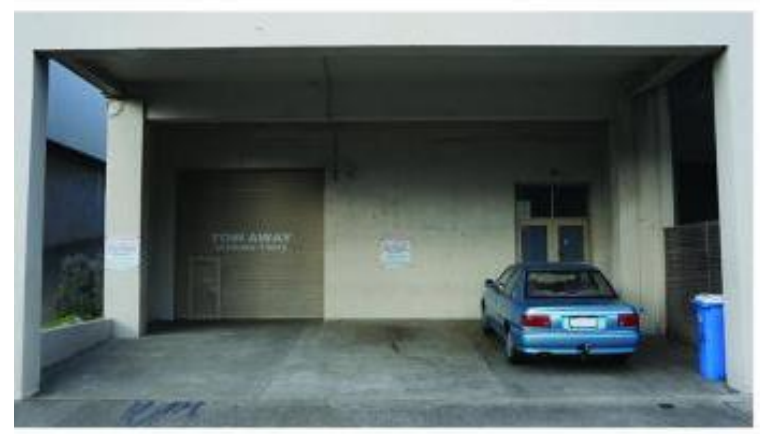

C

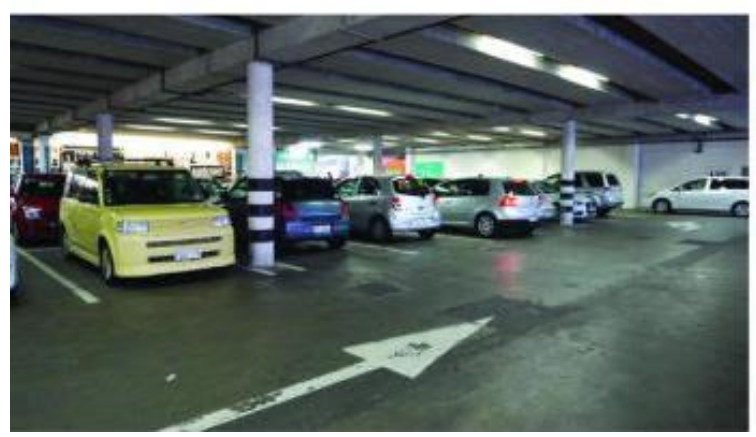

B

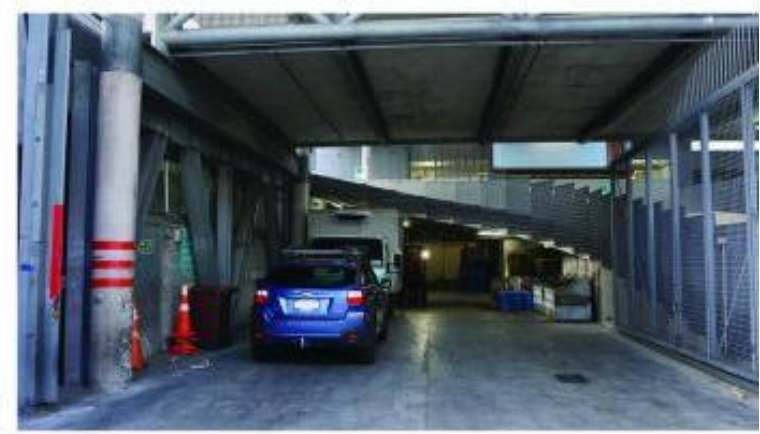

D

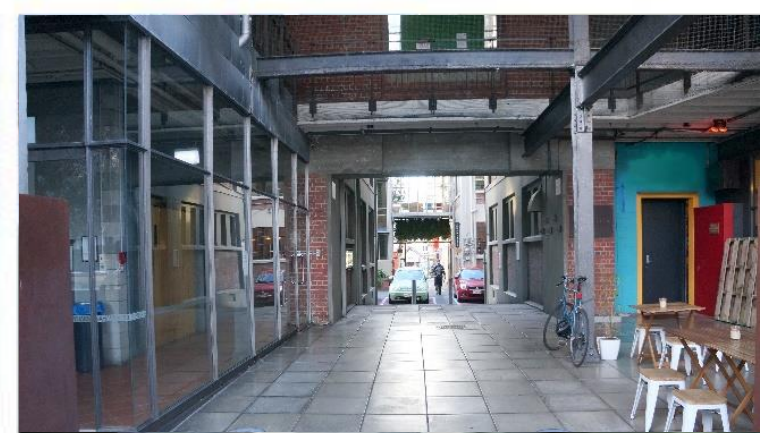

Figure 5.6 Options shown to participants

\section{SPACE UNDERNEATH A BUILDING}

Strongly Unappealing (1) Unappealing (2)

Neutral (4)

- Slightly Appealing (5)

Slightly Unappealing (3)

Appealing (6)

- Strongly Appealing (7)
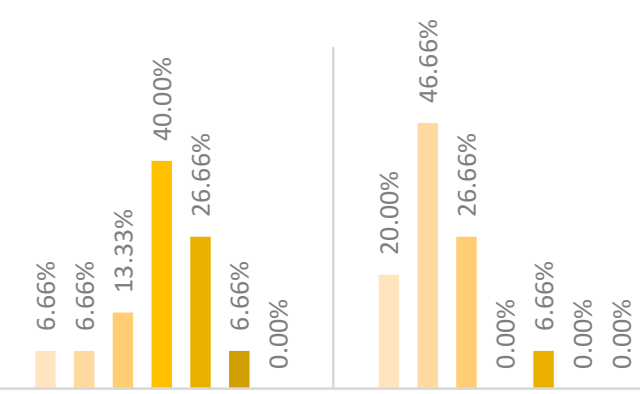

IMAGE B
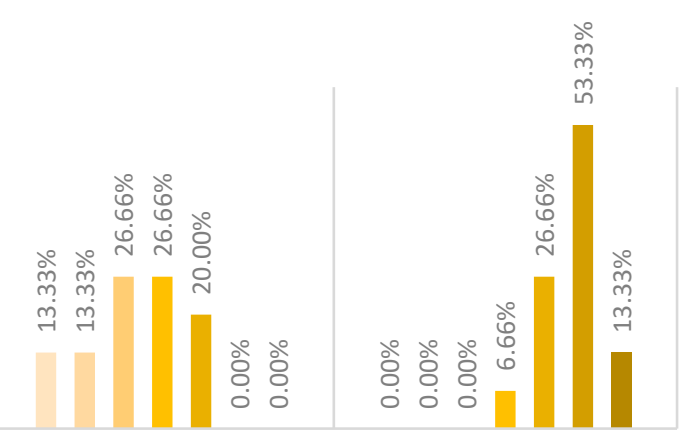

IMAGE C

IMAGE D

Figure 5.7 Participant preferences for the different options 


\subsubsection{Space in front of a building}

The respondents liked option $B$, which had natural vegetation in the space (figure 5.8). Option D was the second most liked space, being a public walkway. Options $A$ and $C$ were the least preferred. Option A was constructed with hard surface material, and the space in option $\mathrm{C}$ looked confined and gloomy (figure 5.9).

A

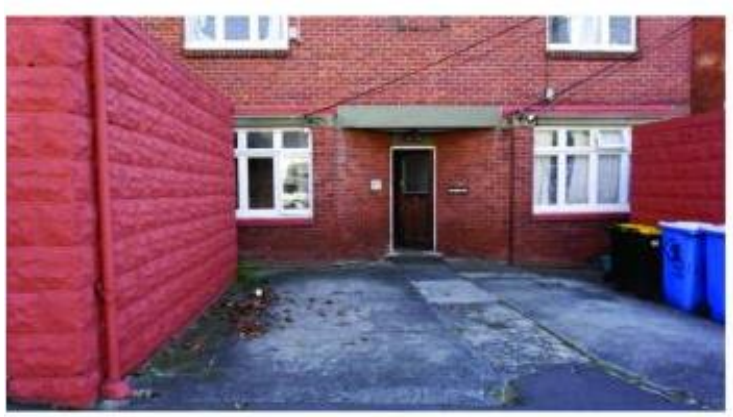

C

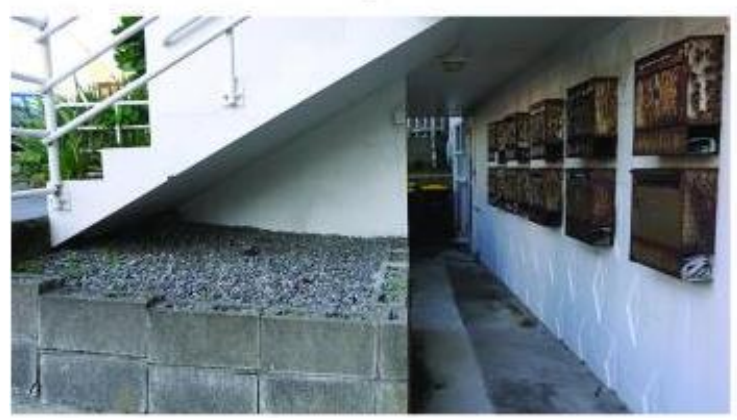

B

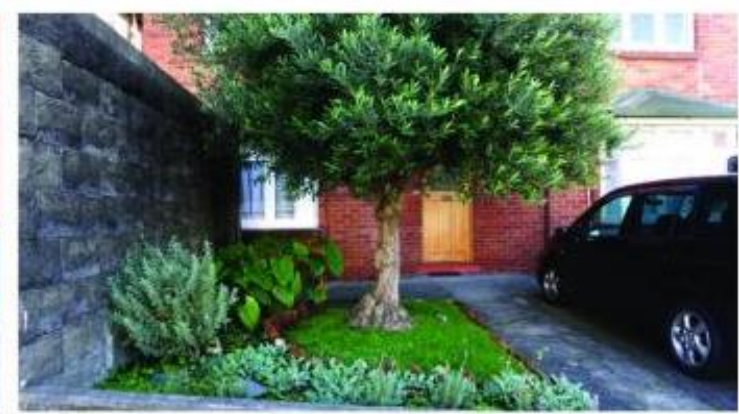

D

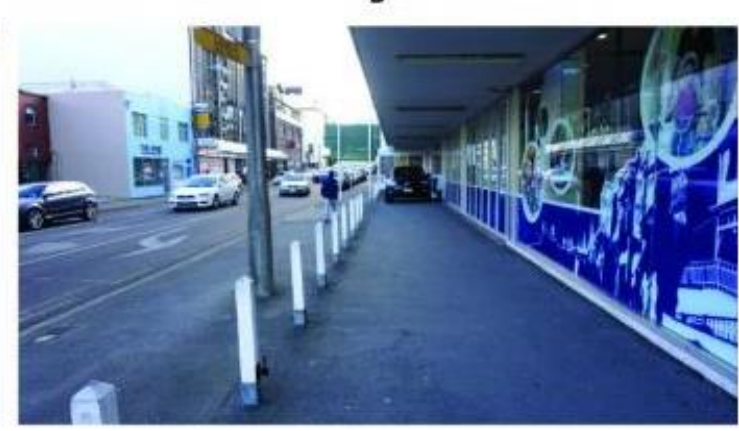

Figure 5.8 Options shown to participants

\section{SPACE IN FRONT OF A BUILDING}
Strongly Unappealing (1)
Unappealing (2)
Slightly Unappealing (3)
Neutral (4)
- Slightly Appealing (5)
Appealing (6)

- Strongly Appealing (7)

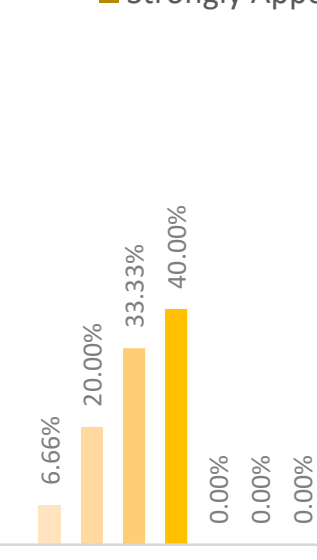

IMAGE A

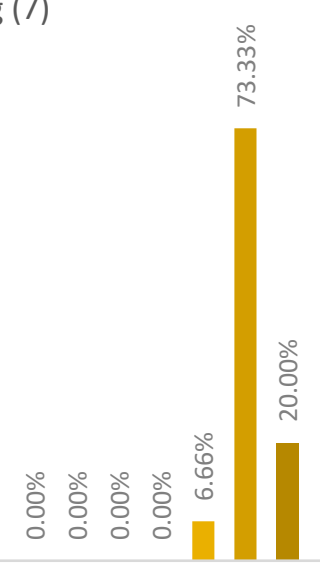

IMAGE B

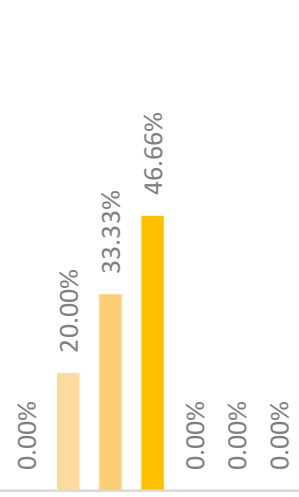

IMAGE C

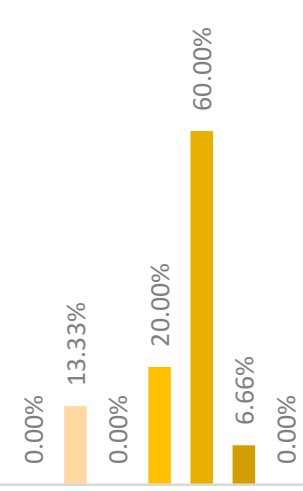

IMAGE D

Figure 5.9 Participant preferences for the different options 


\subsubsection{Space at the back of a building}

Option A was preferred by all participants, is open to the sky and adequately managed (figure 5.10). The option $D$ was the second most liked space, with its graffiti on the wall and public access. Options B and C were the least preferred spaces. Option B had access to the building on the side with storage space underneath a ramp, while option $\mathrm{C}$ was an organised parking space (figure 5.11).

A

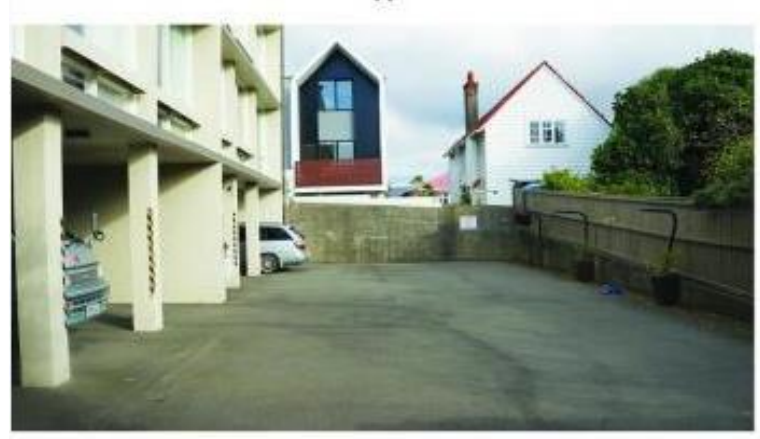

c

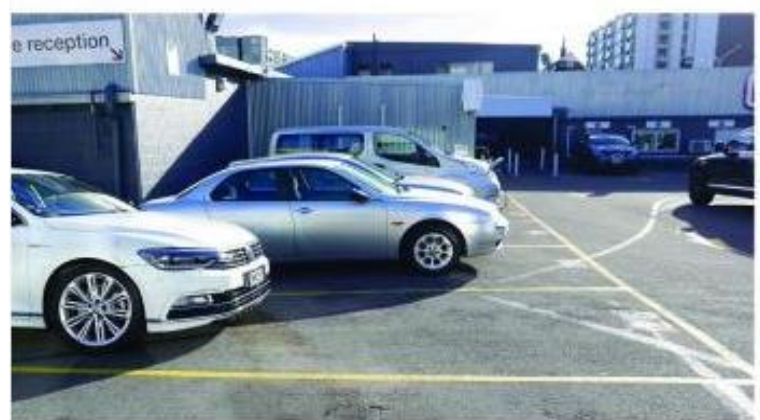

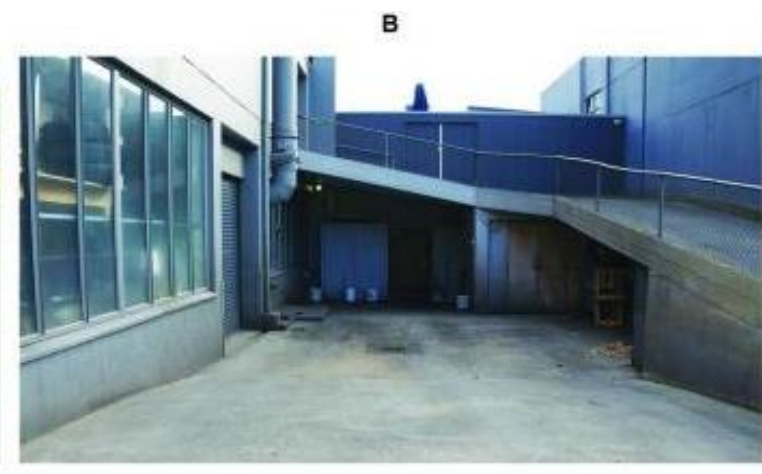

D

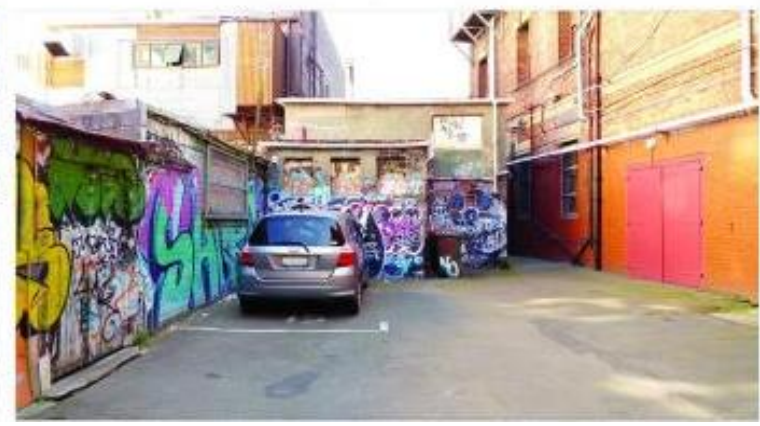

Figure 5.10 Options shown to participants

\section{SPACE AT THE BACK OF A BUILDING}

$\square$ Strongly Unappealing (1) $\square$ Unappealing (2)

- Neutral (4)

- Slightly Appealing (5)

- Strongly Appealing (7)

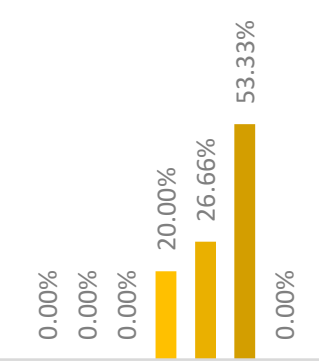

IMAGE A

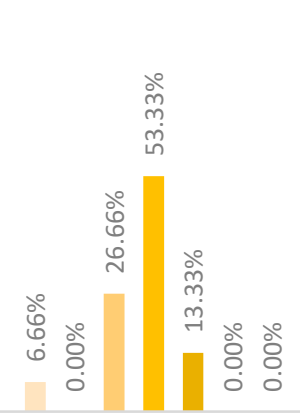

IMAGE B
Slightly Unappealing (3)

- Appealing (6)

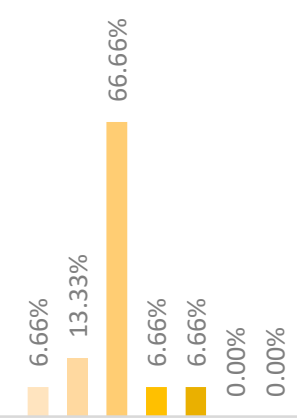

IMAGE C

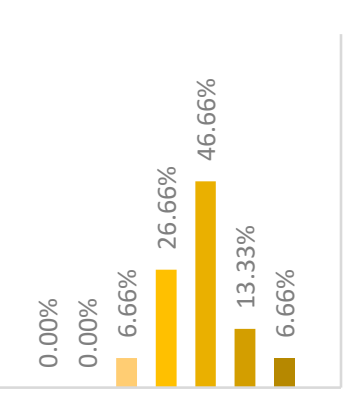

IMAGE D

Figure 5.11 Participant preferences for the different options 


\subsubsection{Space enclosed by buildings on three sides}

Option D was liked by all the participants with its vegetation and seating space (figure 5.12). Option $A$ was the second most liked space, being adequately maintained. Options B and $C$ were the least favoured spaces. The space in option B had garbage bins in it, while option $C$ was confined and poorly maintained (figure 5.13).

A

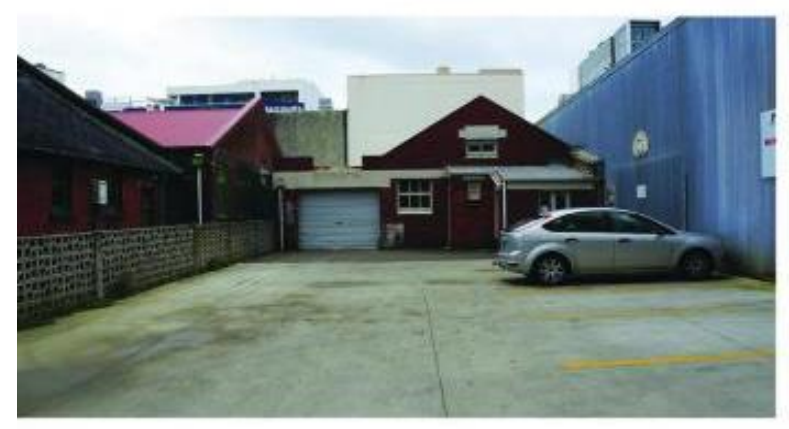

c

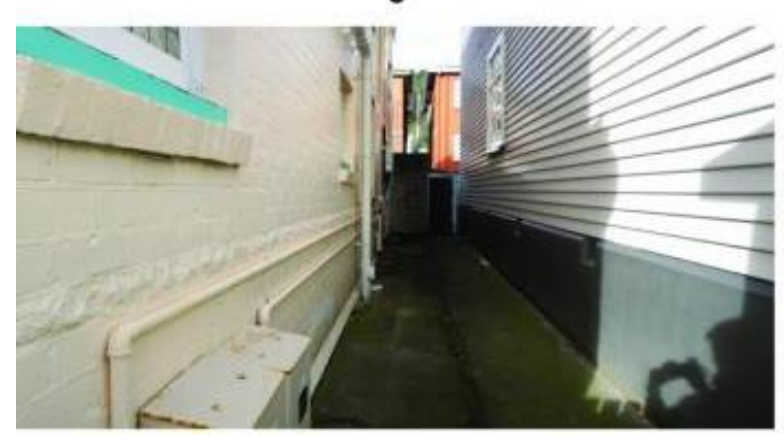

B

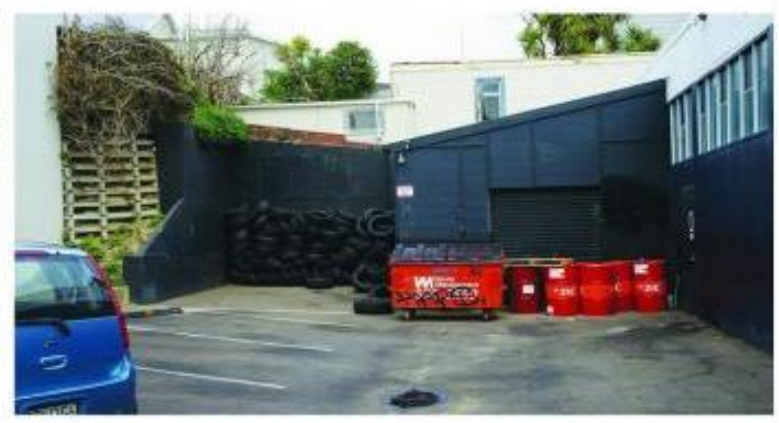

D

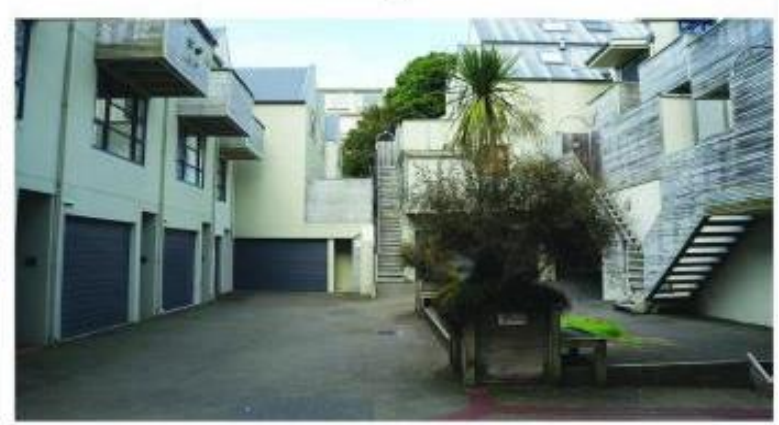

Figure 5.12 Options shown to participants

\section{SPACE ENCLOSED BY BUILDINGS ON THREE SIDES}

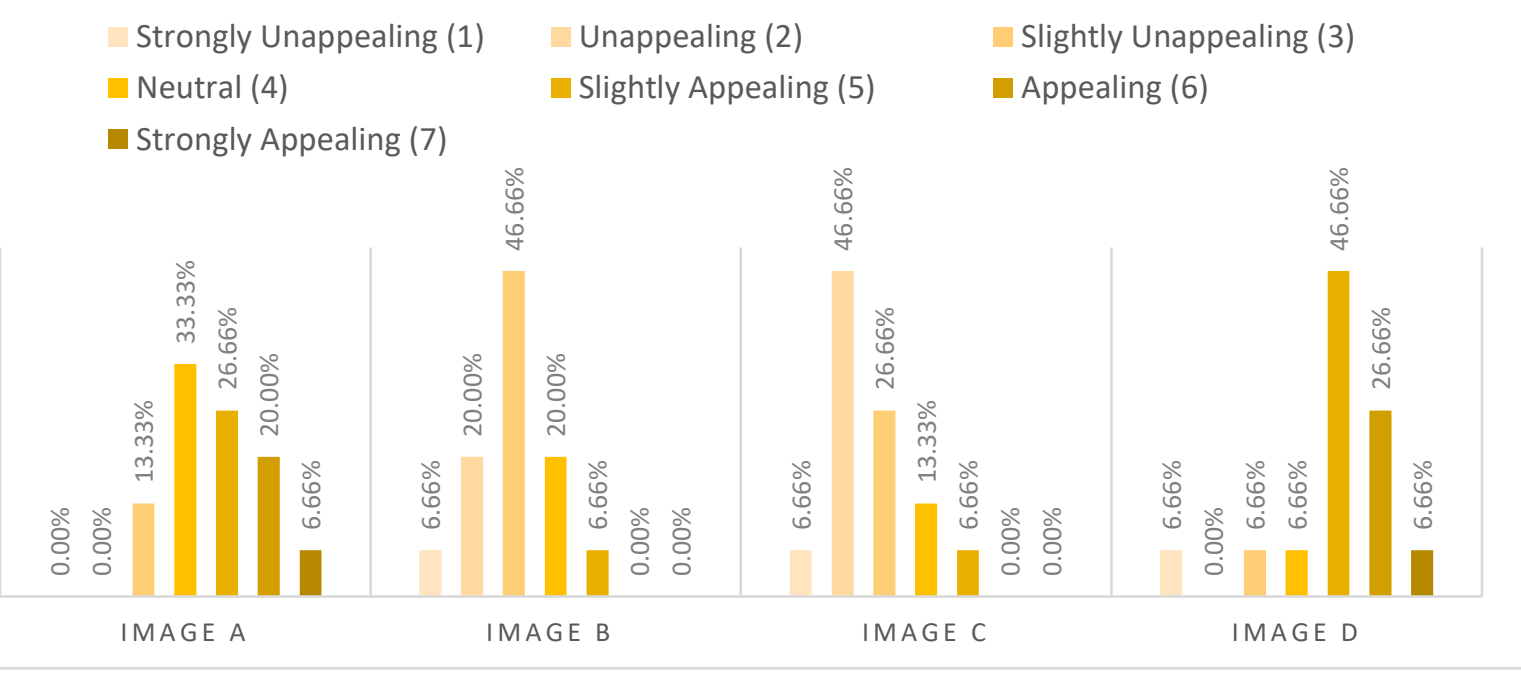

Figure 5.13 Participant preferences for the different options 


\subsubsection{Space enclosed by buildings on two sides}

All the participants preferred option $D$, which had a through route and was open to the sky (figure 5.14). Option $A$ was the second most liked space, being adequately maintained while option $B$ was the third most liked space with its organised parking space. The least preferred space was option $C$, which was confined and had blank walls on both sides (figure 5.15).

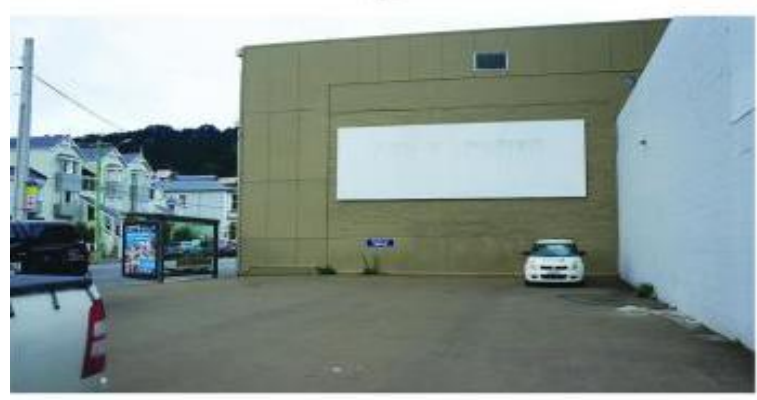

c

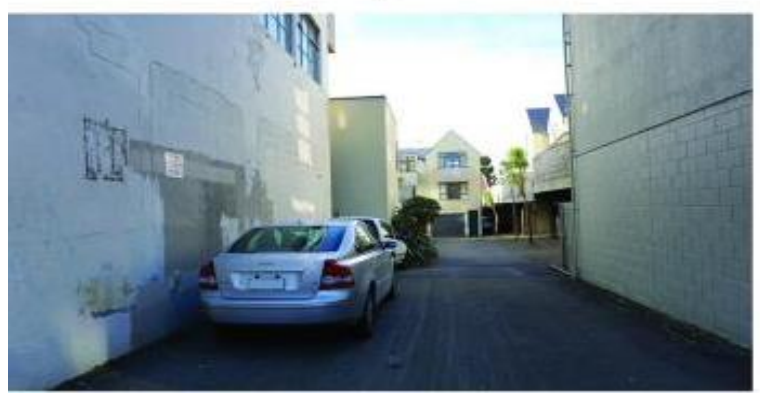

B

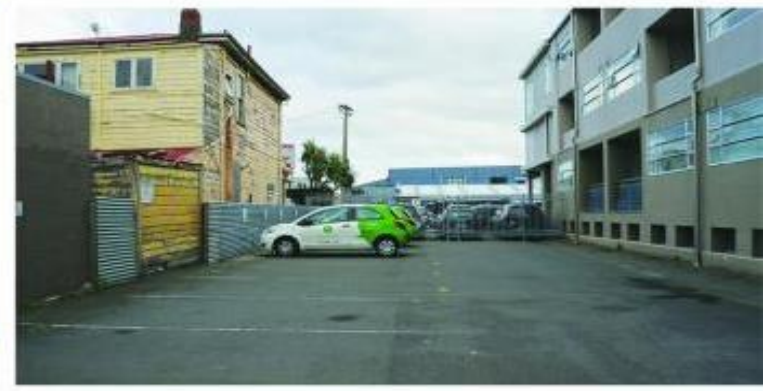

D

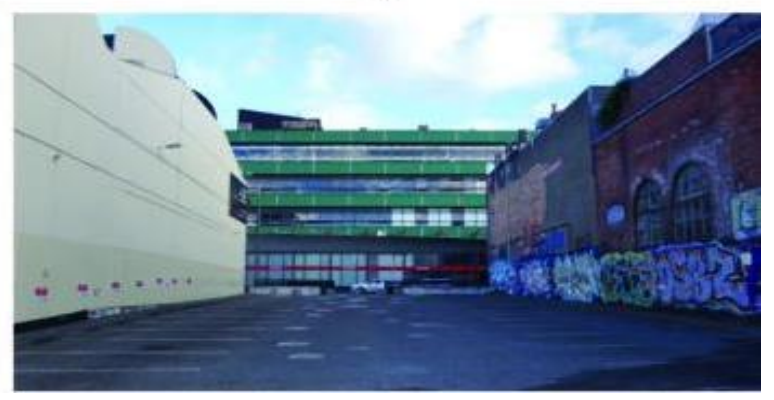

Figure 5.14 Options shown to participants

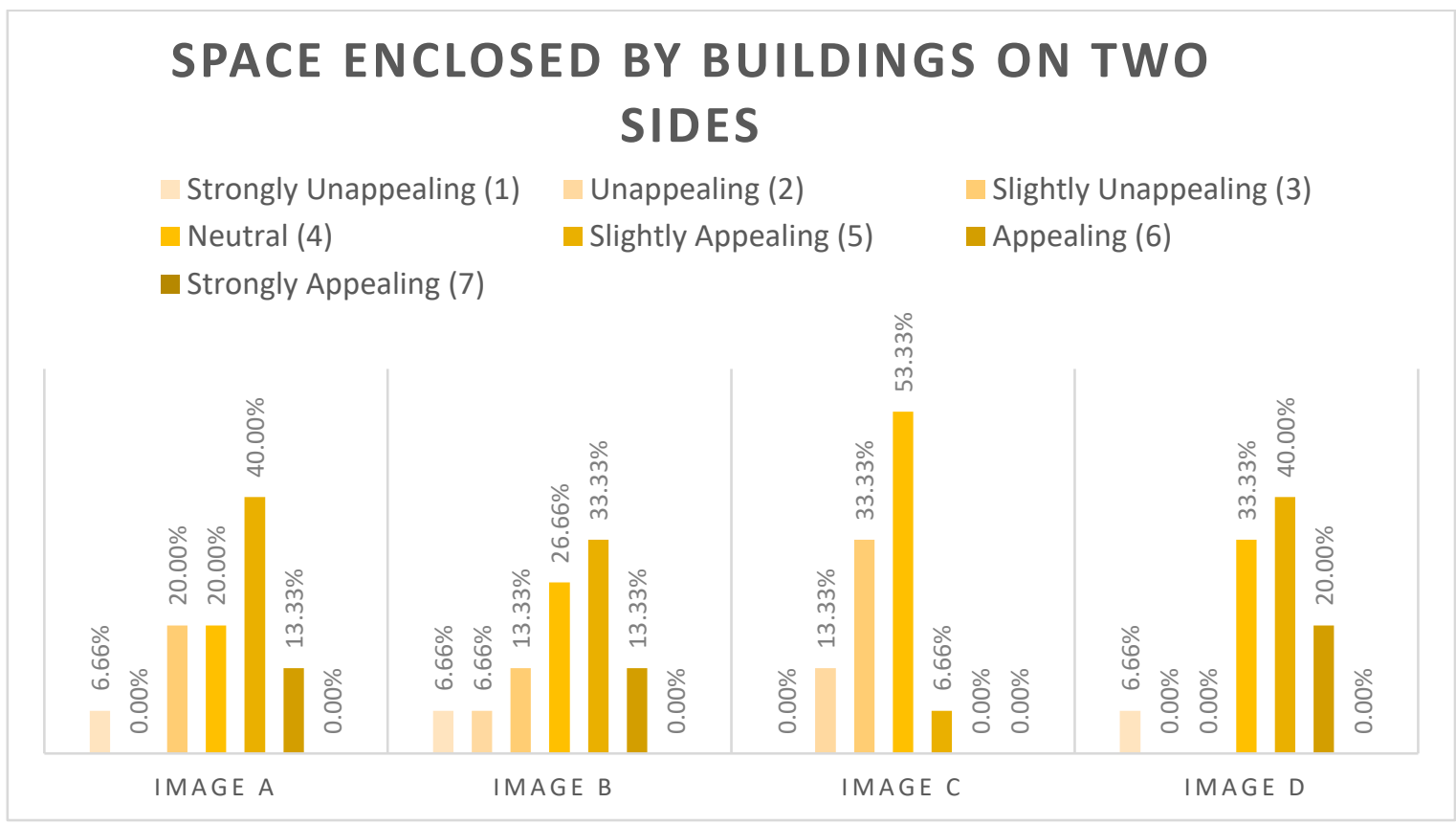

Figure 5.15 Participant preferences for the different options 


\subsubsection{Space on the rooftop of a building}

All the spaces on the rooftop of the building were open to the sky. Option D was preferred by all participants being an organised parking space (figure 5.16). Option $C$ was the second most liked space, with its clean sloping roof. Options $A$ and $B$ were the least preferred spaces. Option A had services units on the roof, whereas option $B$ was not maintained adequately (figure 5.17).

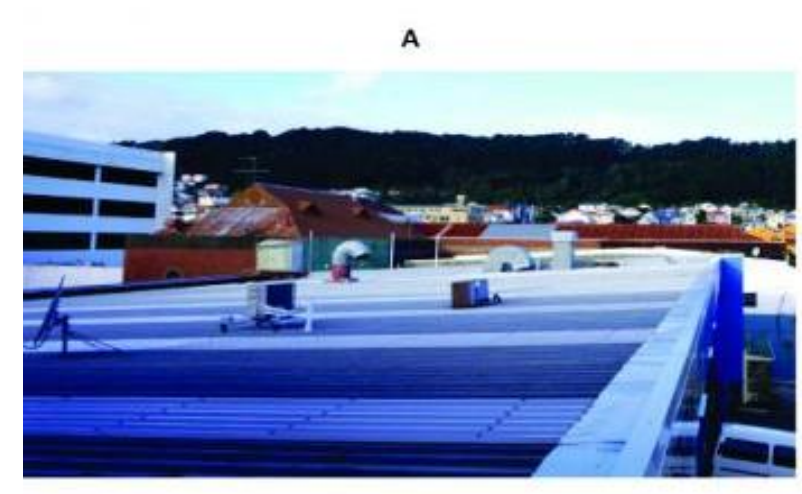

c
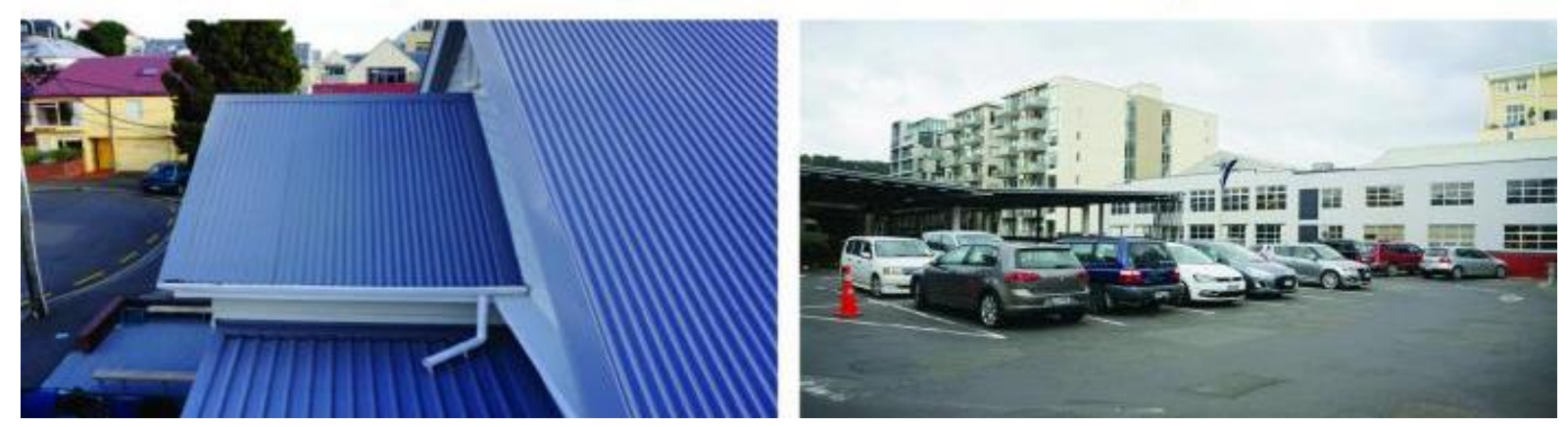

Figure 5.16 Options shown to participants

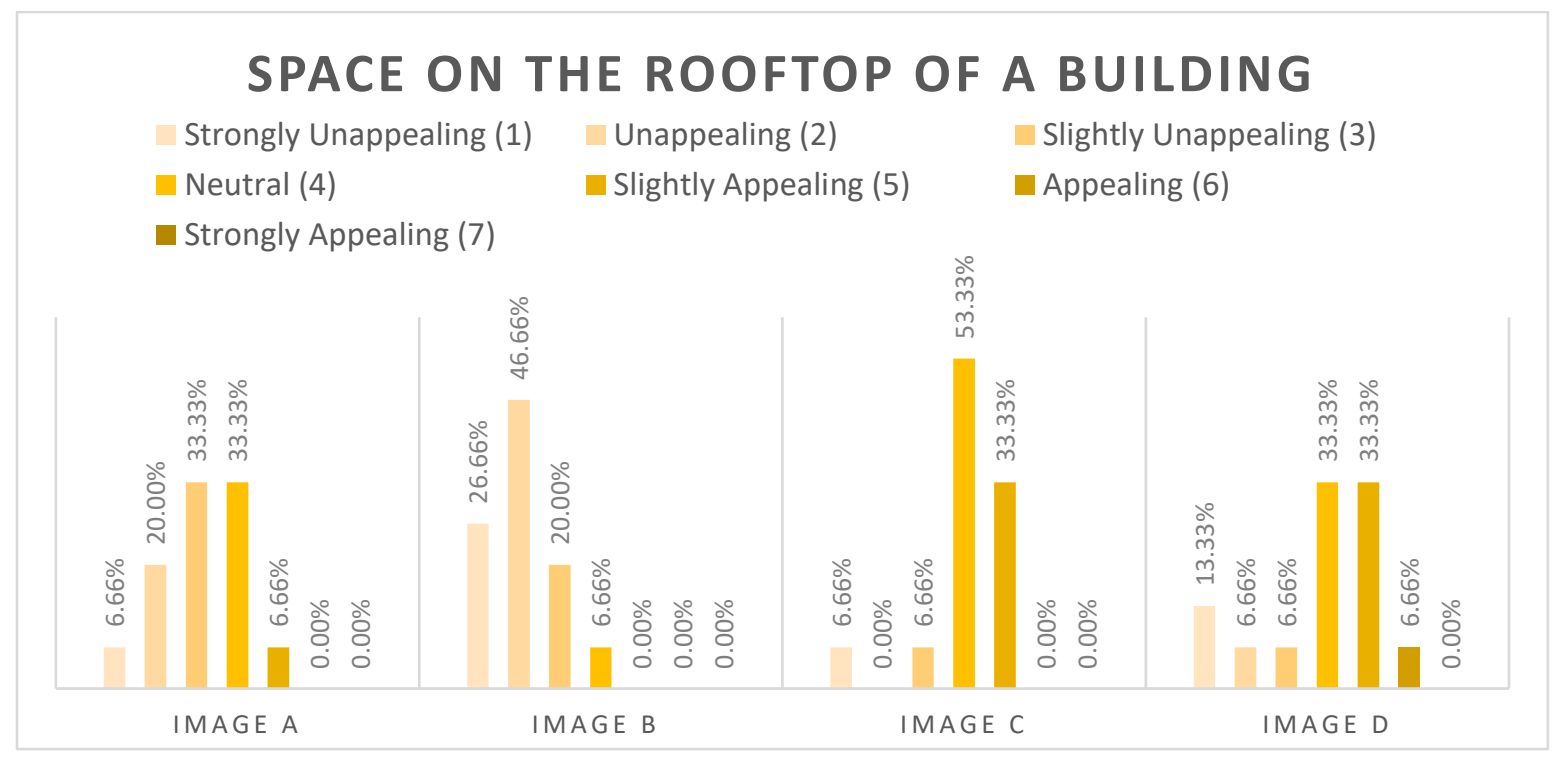

Figure 5.17 Participant preferences for the different options 


\subsubsection{Part 4}

Part 4 of this study showed pairs of photos to participants. All respondents were asked to select the one out of the two that they felt needed transformation. Participants were given a list of options for improving the aesthetics of the space by adding to and removing things from it.

\subsubsection{Space underneath a building}

Nearly three-quarters of participants preferred to see a change in option $A$. The popular suggestions for improving option $A$ were related to the removal of blank walls and the parking space. Participants also felt adding more vegetation, changing the surface material, and creating seating spaces could revamp the space. The suggestions for making refinements to option $B$ were related to the removal of the parking space and signage. Adding vegetation, creating openness, maintenance, and changing the surface materials were also thought to improve the aesthetics of the space.

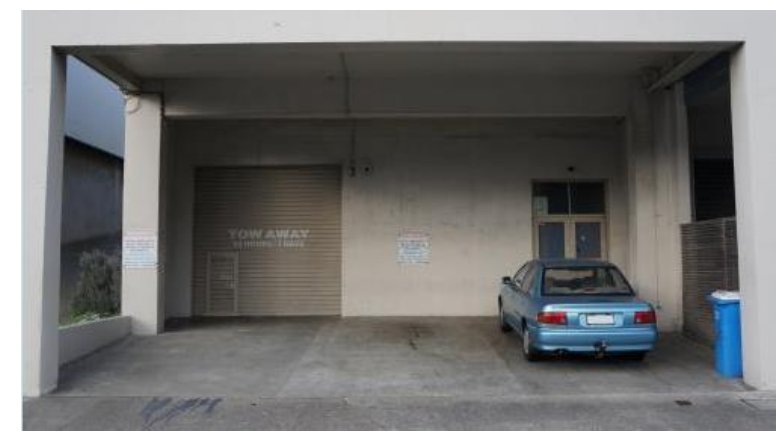

A $(73.33 \%)$

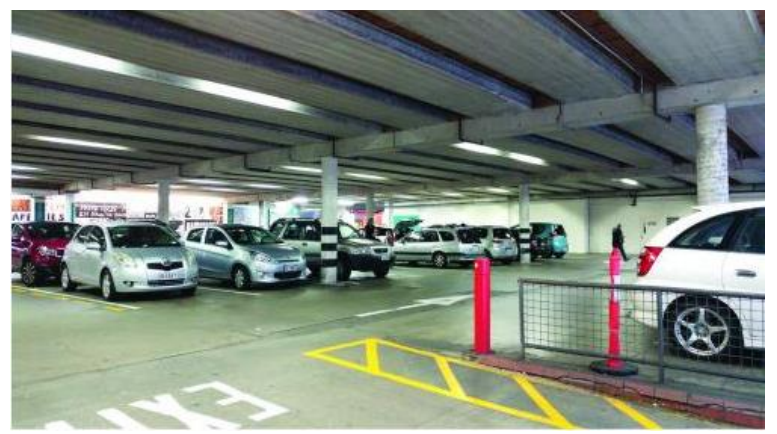

B $(26.66 \%)$

Figure 5.18 Two options shown to participants

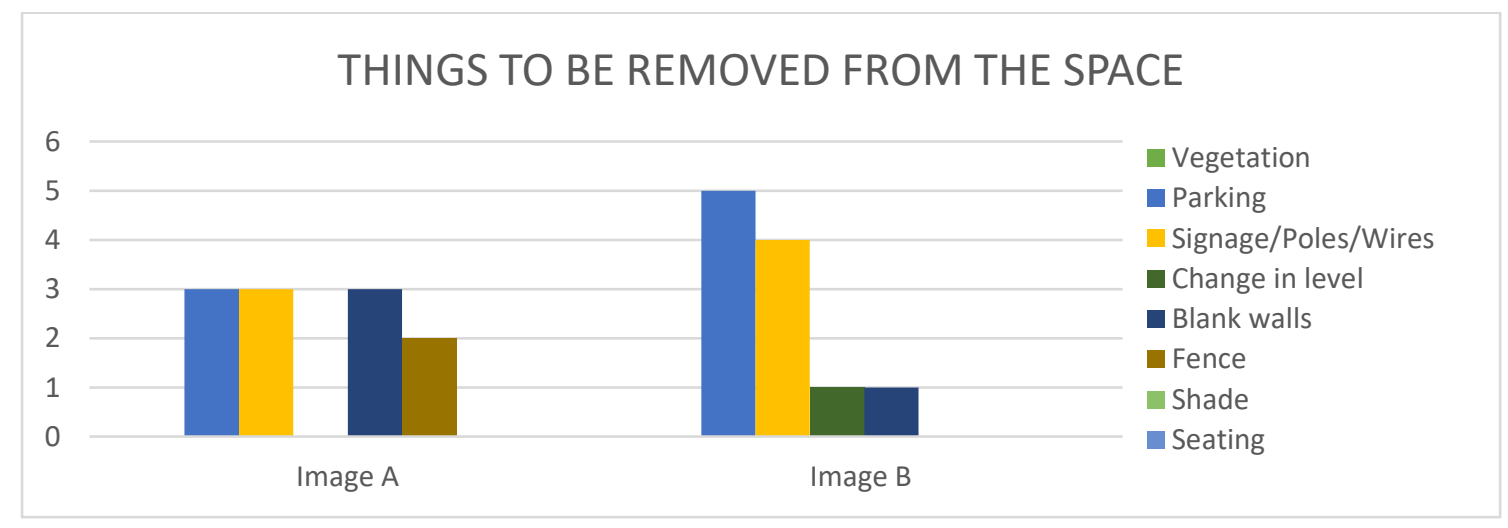




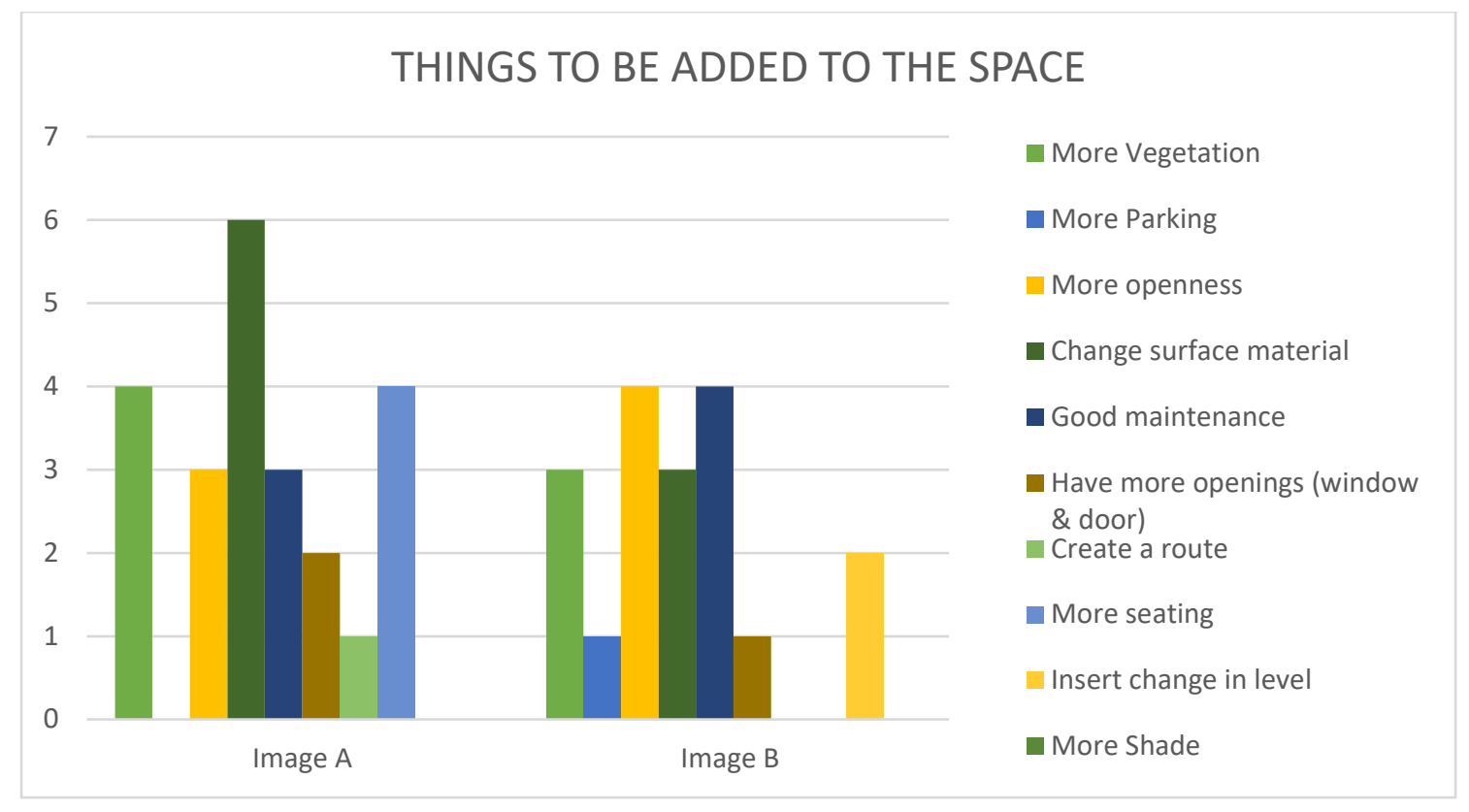

Figure 5.19 Suggestions for improving the space

\subsubsection{Space in front of a building}

Two-thirds of participants preferred to change option A. Upgrading the space in option A was related to the removal of the blank wall, fence, and parking space. Participants also thought the addition of more vegetation and improving the maintenance would improve the whole space. For option $B$, participants suggested removing the parking space and blank wall. Adding more vegetation and changing the surface materials was also suggested for improving aesthetics.

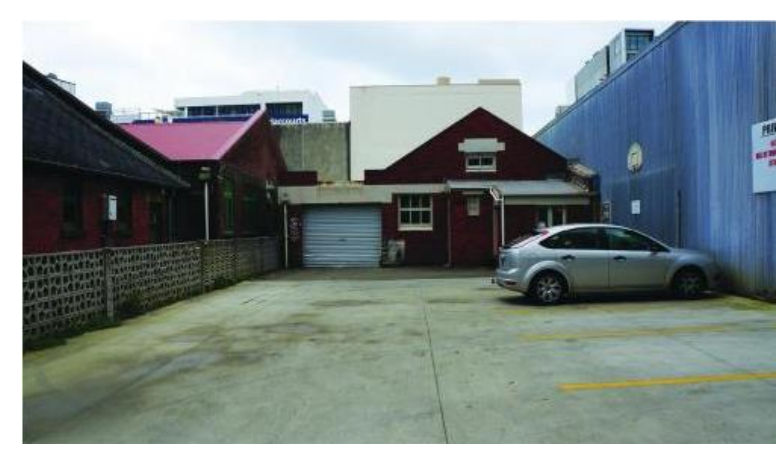

A $(66.66 \%)$

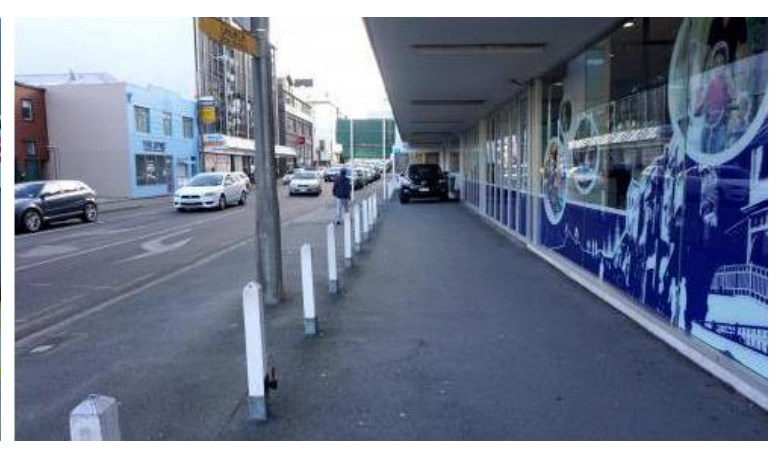

B (33.33\%)

Figure 5.20 Two options shown to participants 


\section{THINGS TO BE REMOVED FROM THE SPACE}
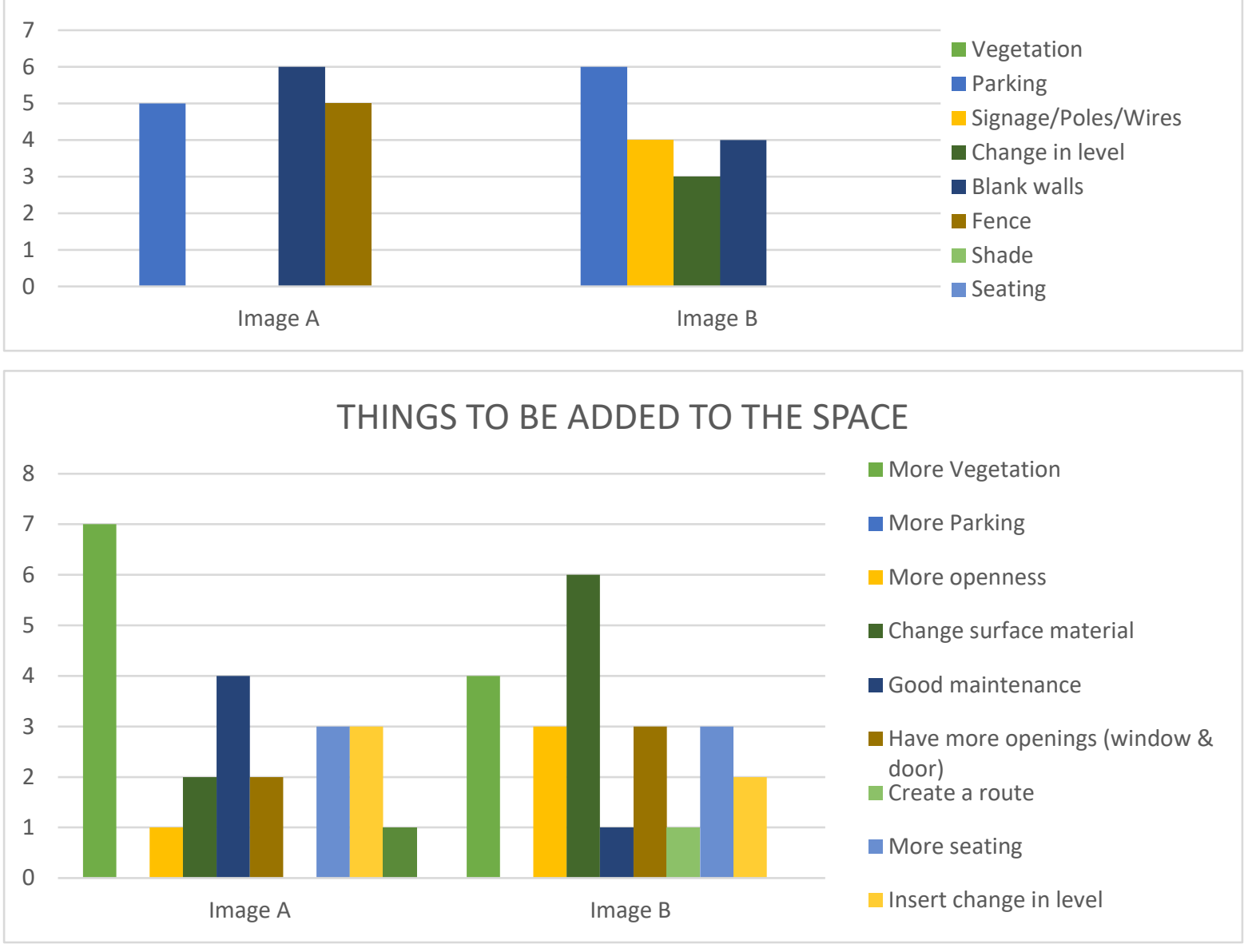

Figure 5.21 Suggestions for improving the space

\subsubsection{Space at the back of a building}

Nearly three-quarters of respondents wanted to change option B. The respondents proposed removing the change in level and blank wall from option $\mathrm{B}$. It was noted adding more vegetation, a change of surface materials and good maintenance would improve the aesthetics of the space. Participants also suggested removing car parking, blank walls and fence from image $A$ while adding more vegetation, different surface materials, good maintenance and more seating (figure 5.23).
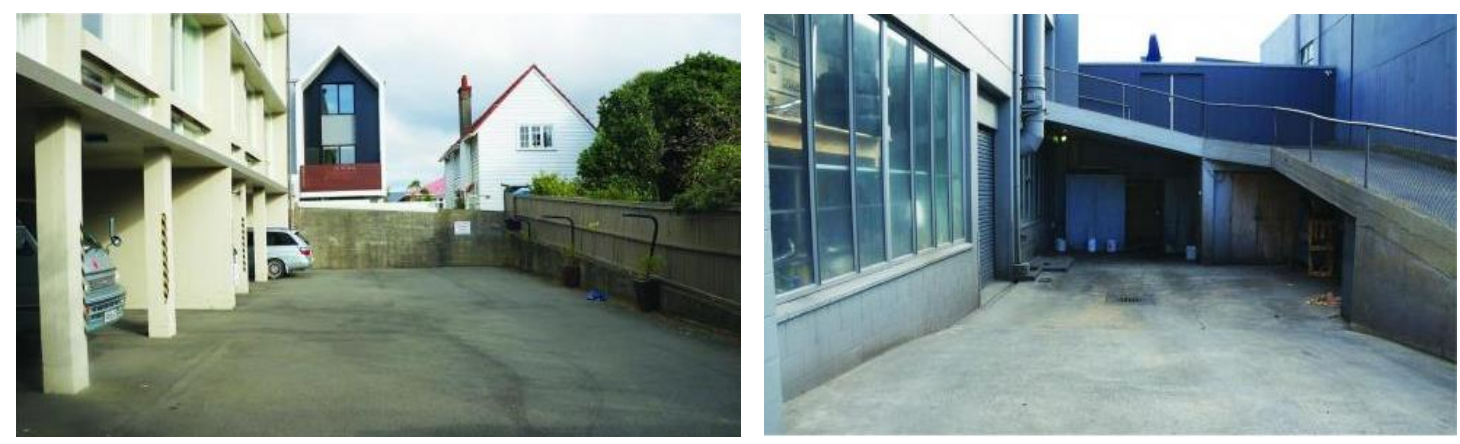

A $(26.66 \%)$
B $(73.33 \%)$

Figure 5.22 Two options shown to participants
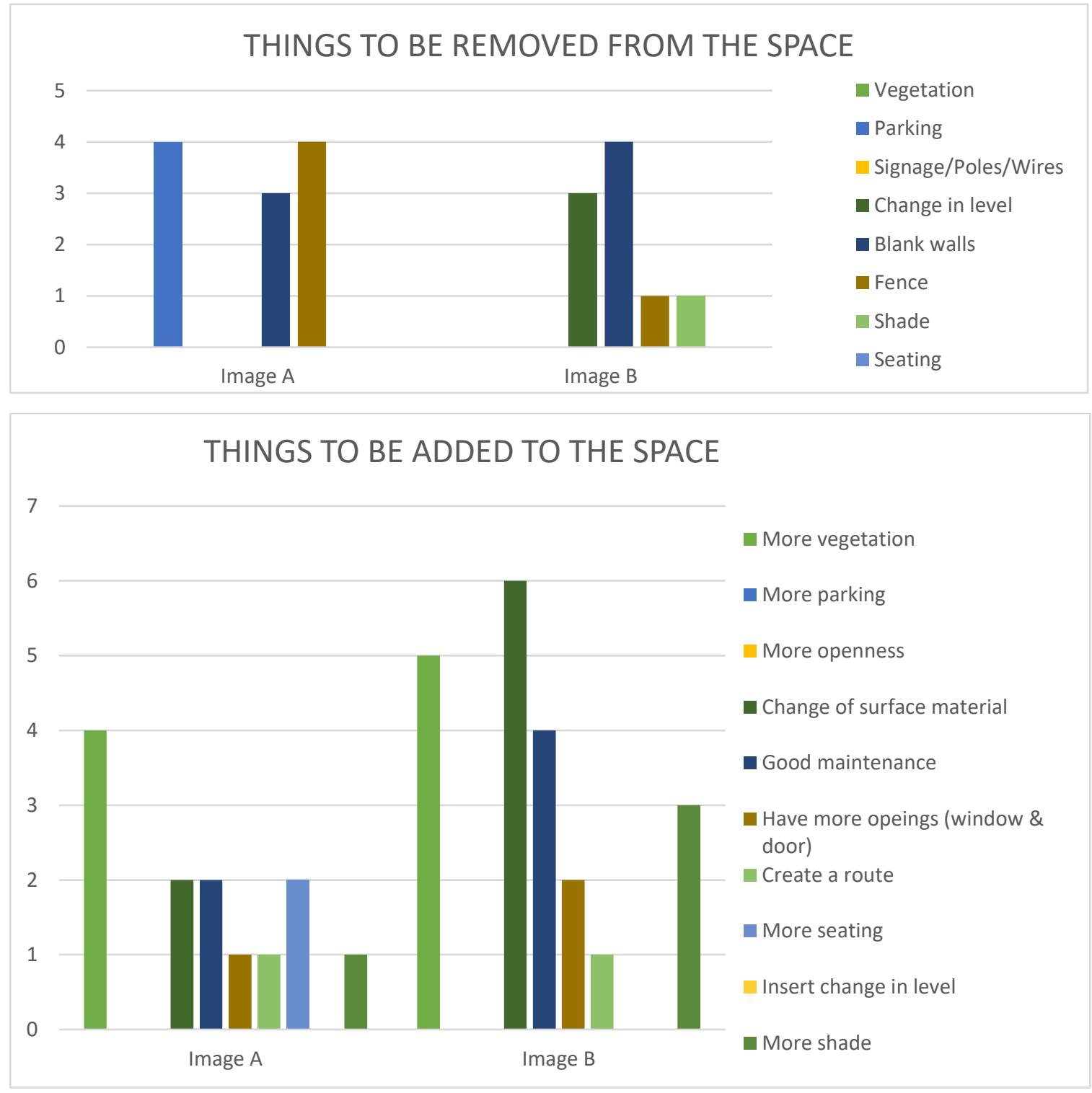

Figure 5.23 Suggestions for improving the space

\subsubsection{Space enclosed by buildings on three sides}

Two-thirds of respondents wanted to change option $A$. The popular suggestions for enhancing option $A$ related to the removal of the parking space and blank wall. Participants also thought the addition of more vegetation and creating seating could improve the space. The respondents proposed removing the pipes from option B while adding more vegetation and adequately maintaining the space was felt would improve its aesthetics. 


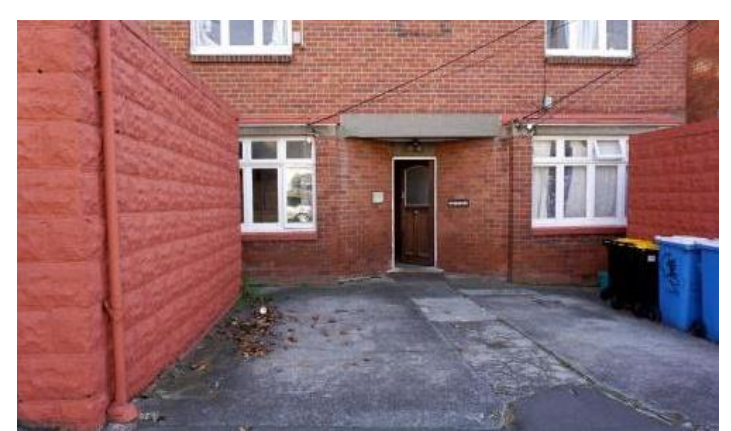

A $(66.66 \%)$

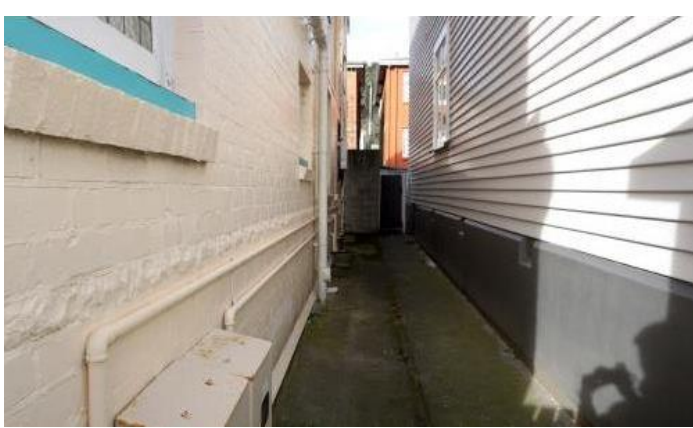

B (33.33\%)

Figure 5.24 Two options shown to participants
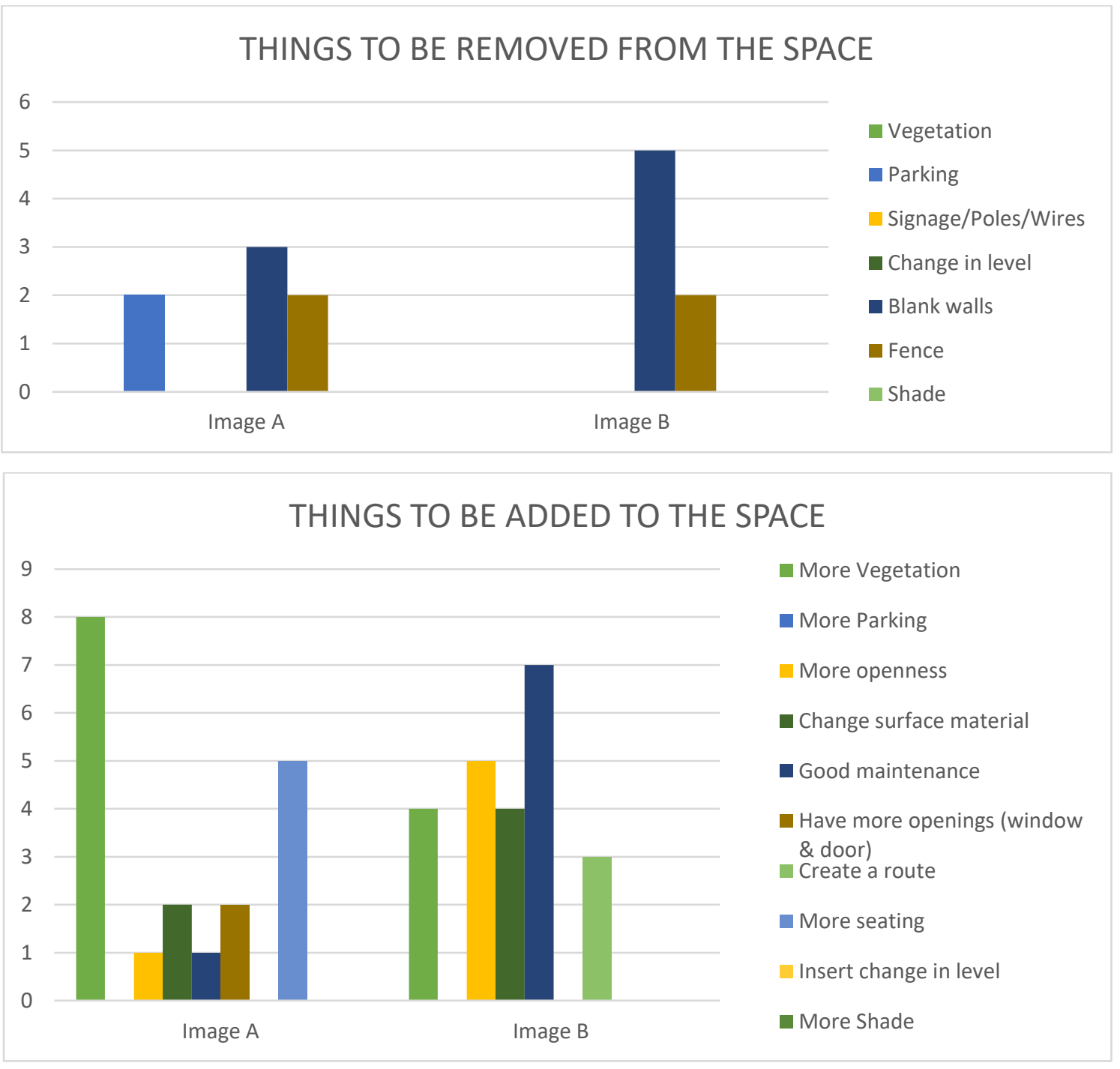

Figure 5.25 Suggestions for improving the space

\subsubsection{Space enclosed by buildings on two sides}

Just over half the participants wanted to see a change in option $A$. The most recurring suggestions for option $A$ related to the removal of blank walls, fence, and parking space. Participants also thought that adding more vegetation and creating a clear 
route and seating spaces could improve the space. The suggestions for making refinements to option $B$ related to the removal of the parking space, adding more vegetation, and forming openings in the building walls.

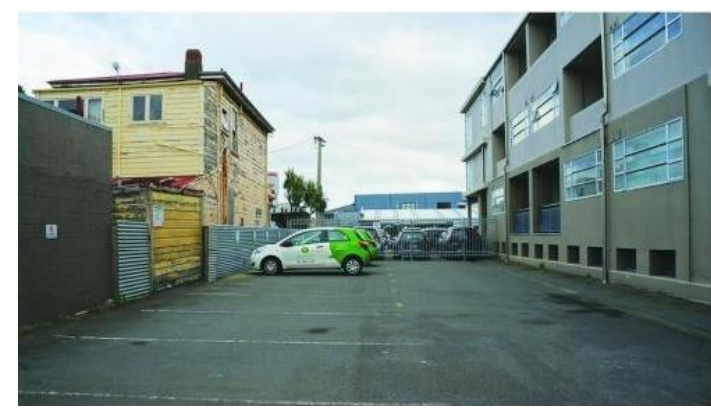

A $(53.33 \%)$

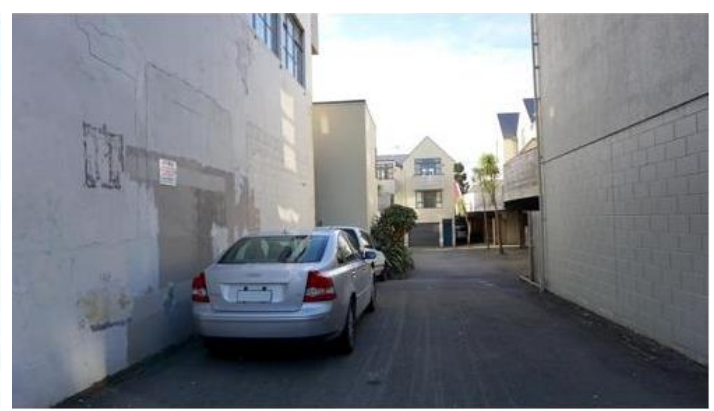

B $(46.66 \%)$

Figure 5.26 Two options shown to participants
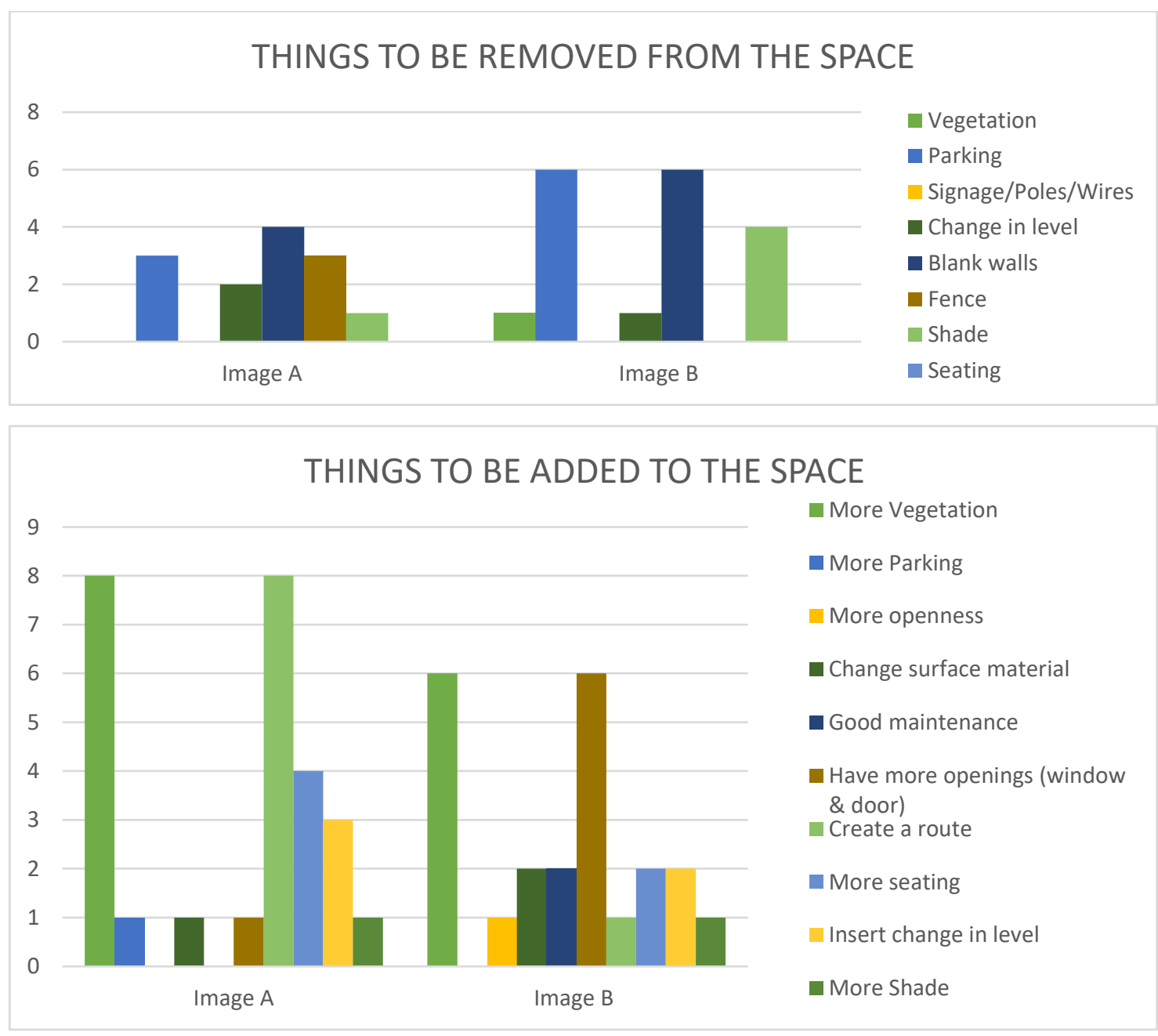

Figure 5.27 Suggestions for improving the space 


\subsubsection{Space on the rooftop of a building}

Most respondents $(80 \%)$ preferred to see a change in option $A$. The most common suggestions for improving option A related to the removal of the services units. The participants also suggested adding more vegetation and improving the maintenance of the space. For option B, the respondents proposed removing the fence/boundary wall and changing the surface material. They also felt that adding more vegetation, installing wind turbines and solar panels, and improving the upkeep would improve its aesthetics.

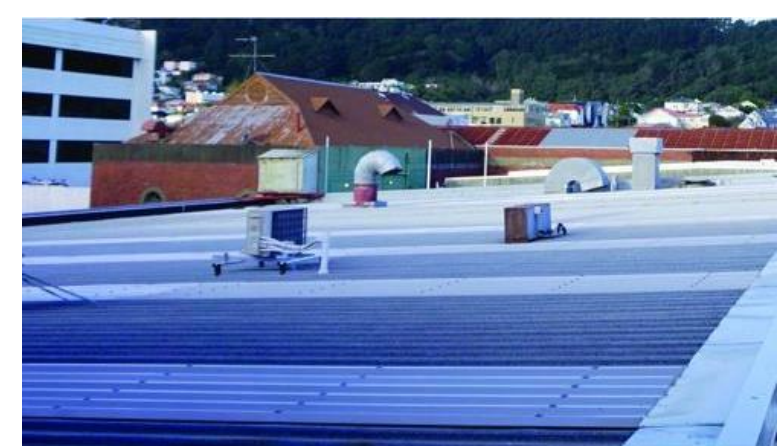

A $(80.00 \%)$

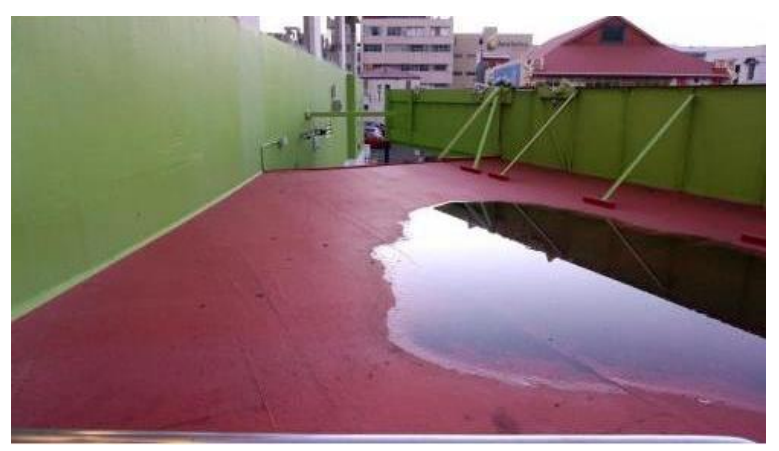

B $(20.00 \%)$

Figure 5.28 Two options shown to participants

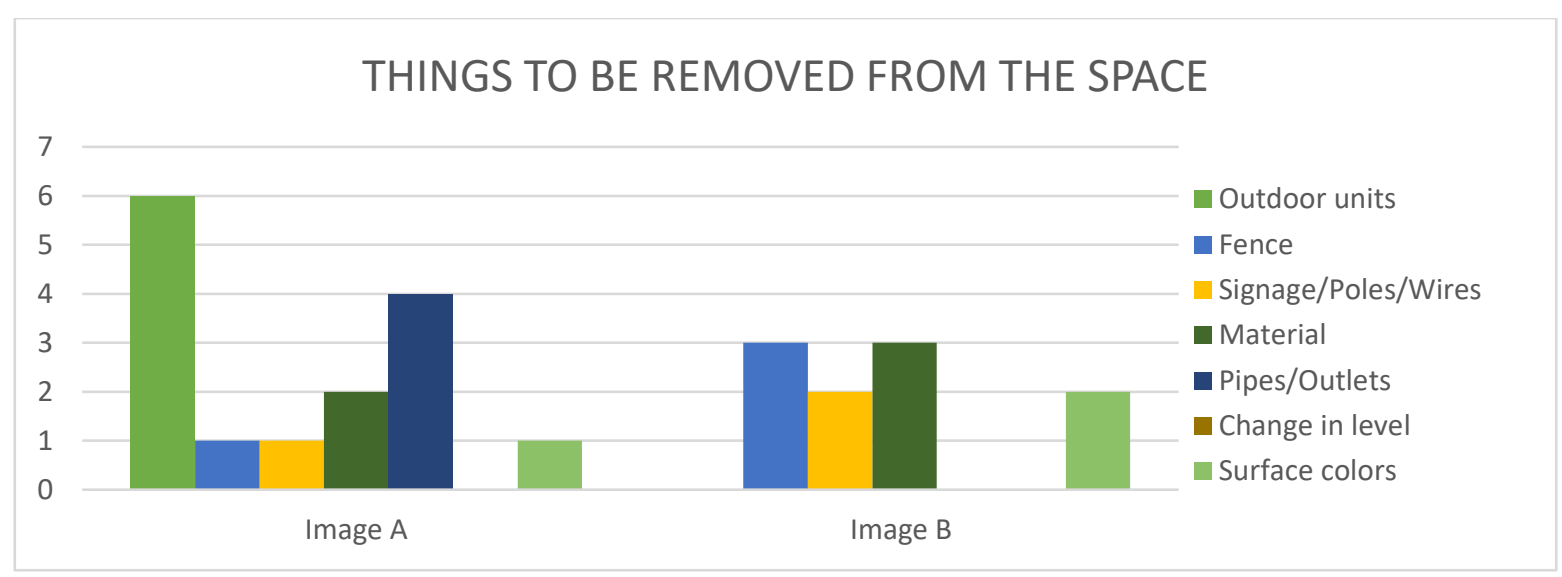




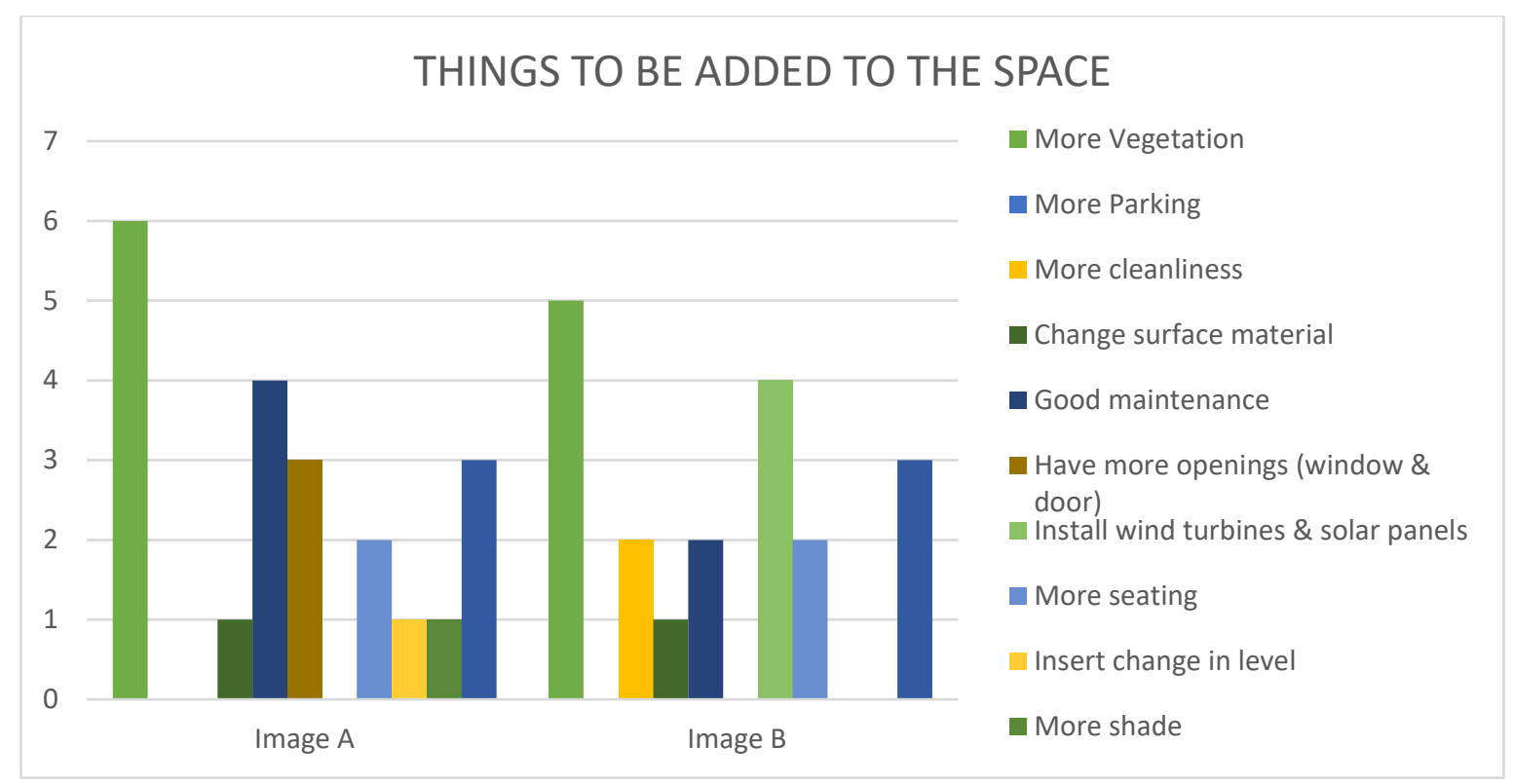

Figure 5.29 Suggestions for improving the space

\subsection{Study Findings}

This pilot study gave useful insights into what aspects could be improved in the primary survey. These included making survey questions shorter and having written phrases from which people could select, rather than making open ended suggestions. The pilot study also showed that people have diverse opinions about specific spaces, and there needs to be a careful categorisation of attributes when it comes to eliciting preferences. A larger sample would make it worth determining the relationship between different preferences and attributes. This will mean collecting the same demographic information that formed part one of the Pilot Study in Study One.

The second part of the study tested Nasar's (1998) theory for the aspects of naturalness, managed space, open space, and ordered space. These four aspects were found in the preferences of all participants. The one disagreement with Nasar was the preference for a historical style of building. The fact the photo only showed an older style of building rather than a historic building could have affected this result, as well as the young age of the pilot study sample, and the fact the sample was very small.

In the third part of this study, participants were asked to rate the photos on a Likert scale from 1 (strongly unappealing) to 7 (strongly appealing). For each space, there 
were four options to rate. The respondents preferred those images which had a clear route, vegetation, cleanliness, seating, and organised parking in the space. The most disliked photos were those that had garbage bins, disordered spaces implying poor management, those that had a parking space with no clear indication of usage, and spaces that were tightly confined. Different opinions were given regarding the reasons for these selections, but most prominent was the need to remove garbage bins, the need to add new colours, the need to have more light, and removal of the parking lots.

In the fourth part of this study, it became evident that people wanted to change those scenes that were in a state of chaos and those that were not being actively used and looked neglected. From the list of choices for improving the leftover spaces, the most prominent selections were related to adding more vegetation, changing the surface material, improving the maintenance, and providing more seating space. Respondents also wanted to remove the parking spaces, boundary walls or fences, blank walls, and signage or poles or wires from the space. Participants' overall preferences for improving the spaces are shown in figure 5.30, which represents the combined list of solutions from photos of all leftover spaces.

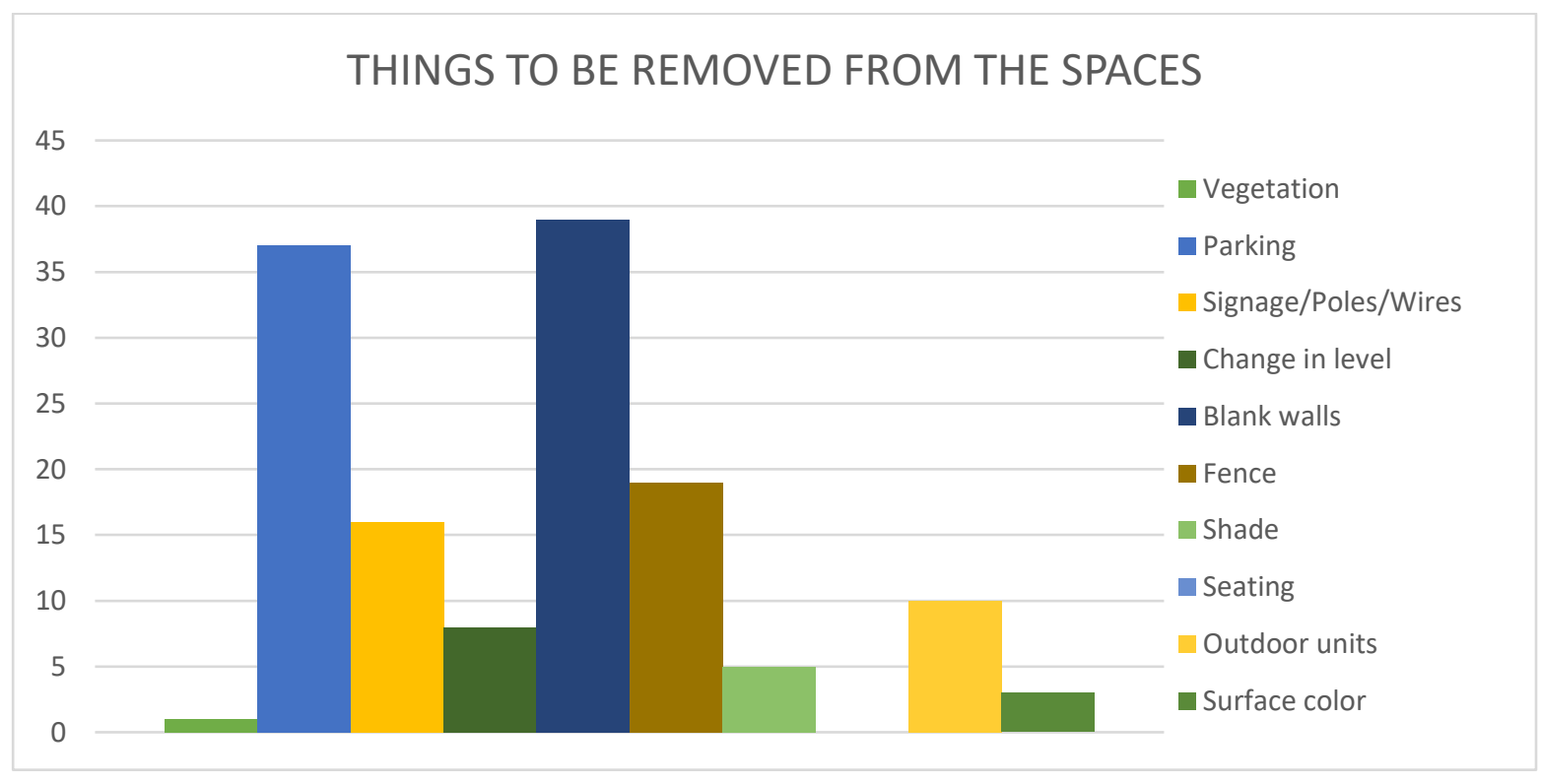




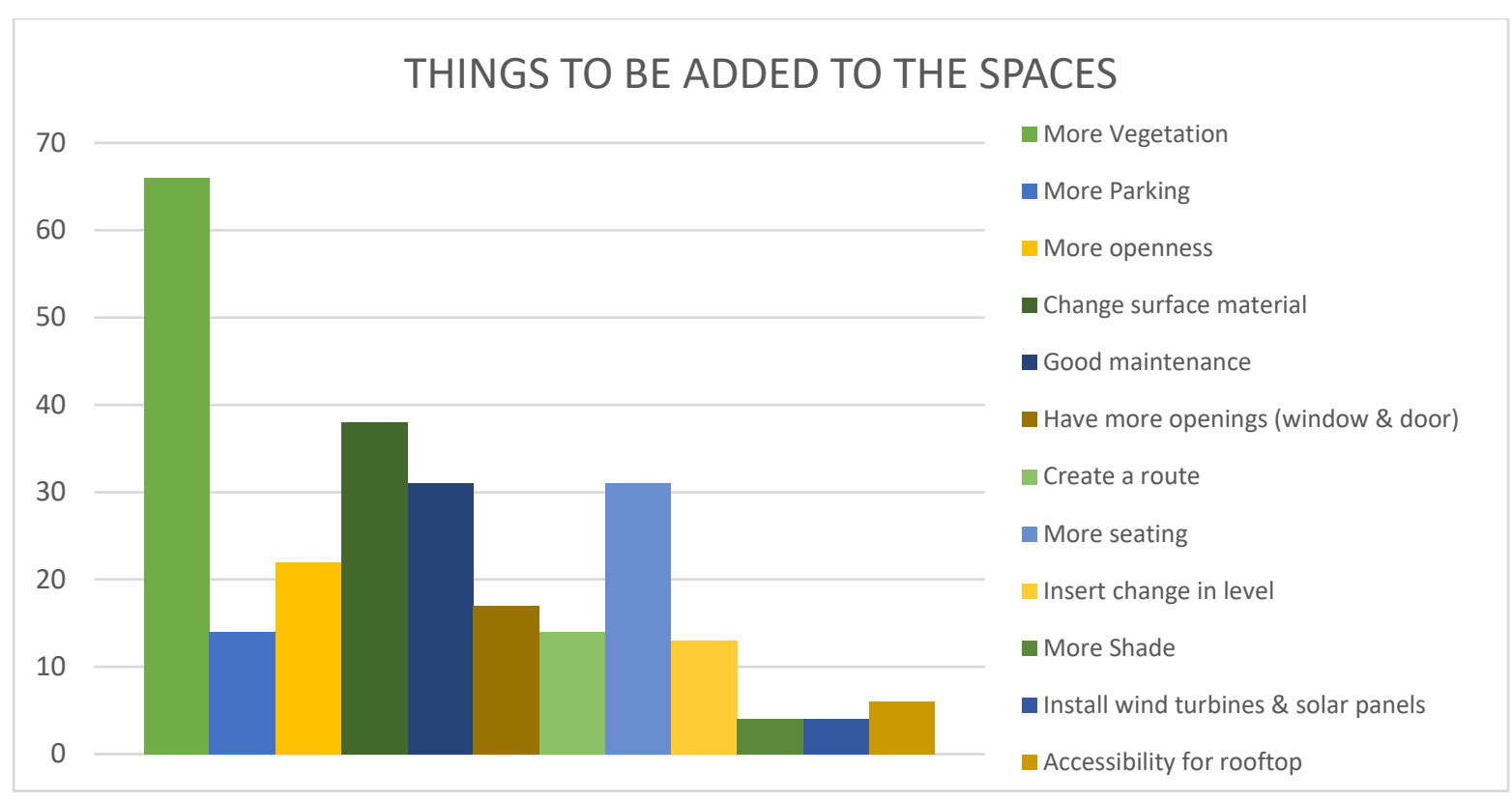

Figure 5.30 Overall modifications suggested by participants for the leftover spaces

\subsubsection{Improvements for the main study}

This pilot study led to the feeling that the main study needs to be more specific in letting participants know what they are viewing. The participants gave their suggestions for improvement at the end of the survey. These related to making the survey questions short of encouraging reliable answers, providing lists of suggestions for making improvement from which people could select options, making the images more prominent, and providing an easier scale than 7 points Likert scale for rating the photos (McKelvie, 1978). Additionally, the length of the survey could be decreased. The quality of the photos could be enhanced so that they appeared as similar as possible in terms of how the view was framed and the level of light. It was also thought helpful not to have the same photos in different sections of the questionnaire, which will make the result easier to be comprehended (Matell \& Jacoby, 1971).

\subsection{Summary}

This Chapter 5 tested the effectiveness of the research tools and techniques that would be used in Studies One and Two. The Chapter discussed the importance of photographic representations for investigating what people wanted to see in semipublic leftover spaces in Wellington (see section 5.1.1) and the procedures for recruiting the participants (see section 5.3). The study was divided into four parts with 
different questions related to demographics, preferences for five attributes from Nasar's theory (1998) about environmental perception, visual preferences for different types of space, and suggestions for improving the leftover spaces. Fifteen people participated in the survey. It became evident from the study that people had a preference for natural, maintained, and organised spaces. Participants disliked spaces that were confined, gloomy, poorly maintained and had blank walls. Participants wanted to see more vegetation, change in the surface materials, more seating, and good maintenance. The study gave useful insight for improving the primary studies. These were related to shortening the length of the survey by reducing a number of questions, providing a list of choices from which participants could select, and improving the quality of the images to emphasise the spaces with particular attributes. Thus, the pilot study gave useful insights into making improvements and resolving issues, which are reflected in the way Study One and Study Two were designed (see Chapter 6 and 7). 
Chapter 5 - PILOT STUDY

LEFT INTENTIONALLY BLANK 


\section{CHAPTER 6}

\section{Study One: FIRST VISUAL PREFERENCE STUDY}

\subsection{Introduction to Study One}

The outcomes of the pilot study (see section 5.5) led to revisions that were then incorporated into the first visual preference study (Study One). This study aimed to examine the potential improvements that could be made to semi-public leftover spaces dispersed around Wellington City by probing the preferences people had for different attributes. The participants were shown photographs of urban leftover spaces with no specific usage other than for casual parking and storage. The expectation was that changes would enhance the perceived value, appreciation, and uses of these spaces. This Chapter, therefore, sets out to discover how the study sample responded to the visual qualities of leftover spaces. The results of Study One feed into Study Two by gathering suggestions for how left-over spaces could be improved. Photomontaged representations of the changed spaces could then be investigated through Study Two (Chapter 7).

\subsection{Description of Study One}

This study was conducted online using the internet-based software Qualtrics, and ethics approval (25265) was obtained beforehand. The study is divided into four parts. The first part sought demographic information by asking participants to provide details about their age, gender, their field of education, and year of study. The remaining three parts all had questions based on photographs of leftover spaces (see section 6.3).

The second part consisted of photos based on Nasar's (1998) research that claimed the most liked environmental features were naturalness rather than artificial elements, a space kept in order rather than being disorganised, an open rather than an enclosed space, well maintained rather than poorly maintained space, and buildings with a historical rather than a modern appearance. Nasar's research was carried out for two cities in the United States, Knoxville and Chattanooga, both in Tennessee. In this section of Study One, Nasar's (1998) theory of the five preferred aspects of the environment formed the basis of the analysis to see if individuals with different backgrounds perceive and experience the elements of an environment similarly, as 
claimed by Nasar. Thus, participants were asked to choose one of two photos of a pair of spaces they perceived as the more pleasant and provide reasons for their selection.

In the third part of Study One, participants were asked two questions. The first asked respondents to rate how much they liked different leftover spaces using a 5-point Likert scale $(-2=$ Dislike to $+2=$ Like). Stem and Noazin (1985) concluded that a 5 -point scale had maximum reliability and validity for bipolar adjective scales. Three pictures were shown for the same type of leftover space and participants had to choose the descriptive option that best fitted why they gave a particular score to each picture. Different photos were chosen with specific aspects found in leftover spaces such as vegetation, parking, seating, wall openings, blank walls, organised parking spaces, dead ends, graffiti, cleanliness, and having a clear thoroughfare. The second question probed the reasons behind the scoring of each option. Participants were given a list of options from which they were asked to choose one reason behind their scoring.

The last part of Study One was more specific, as participants were asked to select one photo out of two settings for each type of leftover space. This procedure was carried out for all six typologies of leftover space. The purpose of this section was to ask participants which picture they felt most needed overall improvement and transformation (see section 6.1.1). This section included a list of different attributes for improvement, out of which participants had to select three or more options. This section aimed at extracting one image of each type of leftover space that could be photomontaged using the most preferred attributes for Study Two, the second visual preference study (see Chapter 7 ).

\subsection{Study Recruitment and Sample}

Study One was conducted from $12^{\text {th }}$ Sep 2017 until $15^{\text {th }}$ Oct 2017. The invitation emails were sent to staff and students in the Schools of Science, Architecture, Design, Health, Law, Engineering, and Education at Victoria University to participate. Other emails were sent to the administration of Victoria University societies, such as the Victoria Pakistan Association and Vic Muslims club, as well as to the New Zealand Institute of Architects, the University of the $3^{\text {rd }}$ Age Wellington, and Wellington City Council. Posters were set up in Raglan Roast Coffee, Wellington City Library, and Victoria University's School of Architecture. These efforts were made to generate responses from people with a broad range of demographic backgrounds. 
As a result, data were collected from 119 participants and imported for analysis in the Statistical Package for the Social Sciences (SPSS) software at the end of the survey period. Overall, 84 participants completed the survey, 15 respondents partially completed, and 21 failed to complete it. A draw was held on $25^{\text {th }}$ Oct 2017, and one participant was contacted through an email to receive an NZ\$20 voucher. At the end of this initial survey, respondents were asked to indicate their willingness to participate in Study Two (see section 7.3) and 54 participants (79\%) volunteered for this. To reduce biases, participants were asked to record their honest opinions and were reassured; there was no right or wrong answer.

\subsubsection{Part 1: Sample demographics}

Demographic information about the participants was collected to see the difference in preferences between the whole sample $(\mathrm{N}=99)$, and when broken down by gender, and built environment and non-built environment participants (table 6.1). Regarding gender, of those who answered this question ( $\mathrm{N}=99), 42 \%$ of respondents were male and $58 \%$ female. A high $78 \%$ of respondents were young (aged 18 to 35 ), with $22 \%$ of participants aged over 36. Participants with built environment knowledge formed $57 \%$ of the sample, with the remaining $43 \%$ being from a different field of study. Just under half $(46 \%)$ of participants had an NZ European background compared to $54 \%$ with different cultural ethnicity. When it came to formal education, $48 \%$ of respondents had a postgraduate qualification.

\begin{tabular}{|c|c|c|c|}
\hline \multicolumn{4}{|c|}{ Demographic Distribution ( $N=119)$} \\
\hline \multicolumn{4}{|c|}{ Frequency in numbers with Percentage } \\
\hline \multicolumn{4}{|l|}{ GENDER } \\
\hline Male & 42 & $35.0 \%$ & \\
\hline Female & 57 & $47.5 \%$ & \\
\hline Total & 99 & $100 \%$ & \\
\hline Prefer not to answer & 20 & & \\
\hline \multicolumn{4}{|l|}{ AGE } \\
\hline $18-25$ & 54 & $54.5 \%$ & $77.8 \%$ \\
\hline
\end{tabular}


Chapter 6 - STUDY ONE: FIRST VISUAL PREFERENCE STUDY

\begin{tabular}{|c|c|c|c|}
\hline $26-35$ & 23 & $23.3 \%$ & \multirow{3}{*}{$19.2 \%$} \\
\hline $36-45$ & 13 & $13.1 \%$ & \\
\hline $46-55$ & 06 & $06.1 \%$ & \\
\hline $56-65$ & 01 & $01.0 \%$ & \multirow[t]{2}{*}{$03.0 \%$} \\
\hline 65 Above & 02 & $02.0 \%$ & \\
\hline Total & 99 & \multicolumn{2}{|l|}{$100 \%$} \\
\hline Missing & 20 & & \\
\hline \multicolumn{4}{|l|}{ FIELD OF STUDY } \\
\hline Built Environment & 58 & $57.4 \%$ & $57 \%$ \\
\hline Health Science & 03 & $02.9 \%$ & \multirow{6}{*}{$43.0 \%$} \\
\hline Public Service & 11 & $10.8 \%$ & \\
\hline Education & 10 & $09.9 \%$ & \\
\hline Finance & 09 & $08.9 \%$ & \\
\hline Science & 07 & $06.9 \%$ & \\
\hline Other & 03 & $02.9 \%$ & \\
\hline Total & 101 & \multicolumn{2}{|l|}{$100 \%$} \\
\hline Missing & 18 & & \\
\hline \multicolumn{4}{|c|}{ ACADEMIC QUALIFICATION } \\
\hline Secondary School & 00 & $00.0 \%$ & \multirow{4}{*}{$52.0 \%$} \\
\hline High School / College & 02 & $02.0 \%$ & \\
\hline Undergraduate & 44 & $44.0 \%$ & \\
\hline Trade Certificate & 06 & $06.0 \%$ & \\
\hline Postgraduate & 41 & $41.0 \%$ & \multirow[t]{2}{*}{$48.0 \%$} \\
\hline Doctorate & 07 & $07.0 \%$ & \\
\hline Total & 100 & \multicolumn{2}{|l|}{$100 \%$} \\
\hline Missing & 19 & & \\
\hline \multicolumn{4}{|c|}{ CULTURAL ETHNICITY } \\
\hline Asian & 25 & $26.0 \%$ & \\
\hline European & 10 & $10.4 \%$ & \\
\hline
\end{tabular}




\begin{tabular}{|l|l|l|l|}
\hline African & 05 & $05.2 \%$ & \\
\hline Other & 13 & $13.5 \%$ & $54.0 \%$ \\
\hline Maori & 02 & $02.0 \%$ & \\
\hline Pacific Islander & 02 & $02.0 \%$ & \\
\hline NZ European (Pakeha) & 44 & $45.8 \%$ & $46.0 \%$ \\
\hline Total & 96 & $100 \%$ & \\
\hline Missing & 23 & & \\
\hline
\end{tabular}

Table 6.1 Demographic details of the study sample

\subsection{Analysis}

Different methods of analysis were used in SPSS to understand the critical relationships between the preferences of participants for a particular space with its different attributes. According to Boone \& Boone (2012), the ordinal data from a Likert scale can be analysed using descriptive statistics, such as the mode or median for central tendency, frequencies for variability, and the chi-square measure of association and Kendall Tau B, and Kendall Tau C to explain the relationships in the data. Tests such as percentage, mean, standard deviation, and cross tabulation were carried out to determine the preferences of the whole sample and its subgroups. Mean values represented the average for the most preferred space, while Standard Deviation (SD) determined the variation from the expected value.

In the second part of this study, the simple technique of calculating the percentage values was deployed to investigate the most liked options with their different attributes based on Nasar's (1998) research. All the photos of spaces with specific attributes were taken in Wellington City. Since this area of Wellington City (see section 2.4) does not have historic buildings as part of its leftover spaces, a photo of a building in the area in an older architectural style was used in place of one of a historic building. Nasar (1998) concluded that the definition of historical significance depends on the viewer's perception of historical content and the perception varies from built environment professionals to those whose expertise is in other fields, with historical buildings being more highly valued by built environment professionals.

In the third part, the numerical values assigned to the 5-point Likert scale were used to investigate the data, ranging from 'Dislike' $=-2$ and 'Like' $=+2$, with other categories assigned a whole number within this range. To understand the reasons behind the scoring the Likert scale was simplified (Benson, 1971), and a new 3 point Likert scale 
( $1(-1$ and -2$)$, = dislike, $2(0)$ neutral, and $3(+1$ and +2$)=$ like) was created by merging the relevant averages. The frequency of reasons for preferring different images was then calculated on this 3-point Likert scale. The Likert scale measured the preferences for each space with its different attributes. At the end of this part, participants were asked to select one reason from a list of options for their score.

In the fourth part of Study One, participants were asked to select one of two photos for each leftover space that they felt needed a change. Respondents were then asked to add or remove at least three things from the list of options for the selected photo. The selections of each leftover space with the suggestions for its improvement were determined by calculating percentages. The outcome of this fourth part was used for Study Two (see section 7.3) as the photomontages used were based on the particular attributes chosen by participants in Study One.

Before conducting the analysis, a general test determined the difference in opinion of participants by age group, i.e. 18-35 and 36 above, and also by cultural ethnicity, i.e. NZ European and other ethnicities. The results demonstrated no significant difference in preferences for the leftover spaces. The analysis for this was carried out in three stages with different groups (figure 6.0).

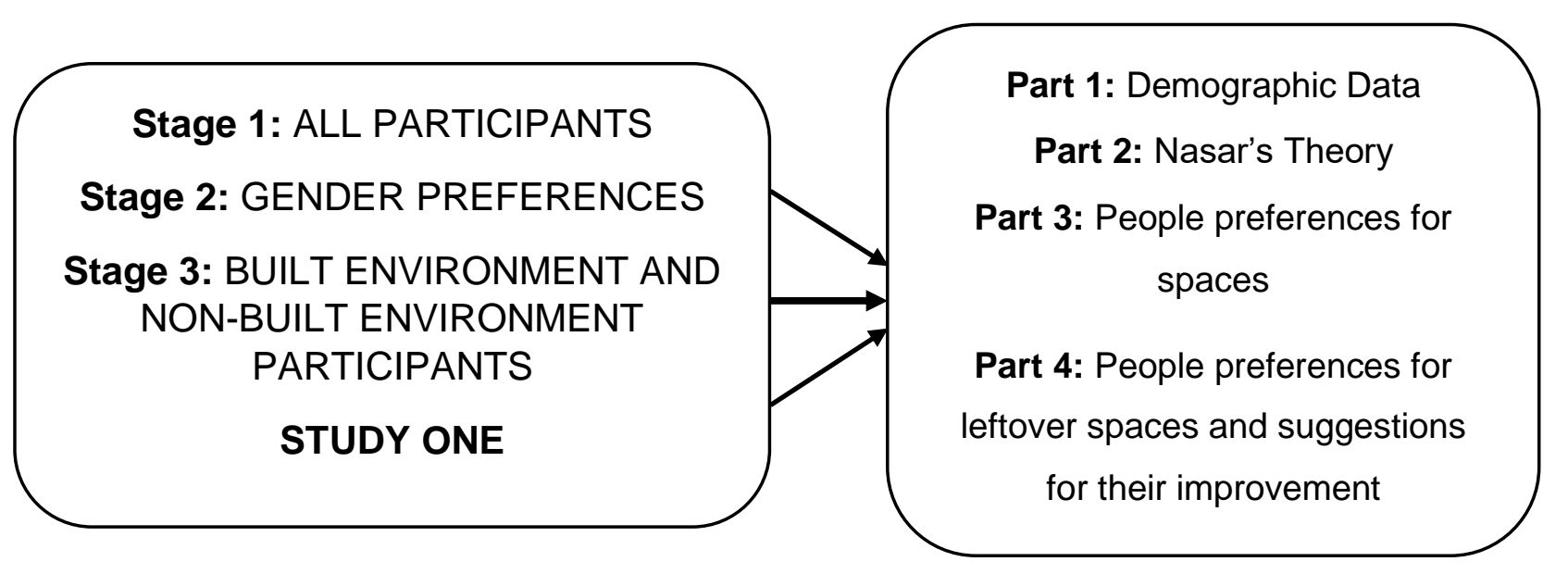

Figure 6.0 Three stages of Study One and the parts for each stage Source Author

Analysis of stage one was performed for the whole sample of 99 respondents to identify the most appealing space for all participants. The analysis of stage two was carried out for the subgroups of 42 males (42.4\%) and 57 females (57.6\%) to look for differences of opinion by gender. Lastly, the analysis of stage three was conducted with the subgroups of $61(64.2 \%)$ built environment participants and 38 (38.4\%) 
participants from other fields of study, to see again whether there were differences in the preferences expressed.

\subsubsection{Analysis of Stage One: Whole Sample $(\mathrm{N}=99)$}

In this stage, the analysis was conducted with all 99 participants.

\subsubsection{Part One}

Part one of the survey asked questions related to demographic characteristics that included gender, age, cultural ethnicity, education level, and field of study. This has been reported in 6.2.1

\subsubsection{Part Two}

Two photographs of leftover spaces with different aspects were shown to the respondents, out of which each respondent had to choose the one they liked better. Just as in the pilot study (see section 5.4), the themes for the photos were based on Nasar's (1998) theory. These themes were not mentioned to the participants who were asked to give a reason for their choice. For each paired example discussed below, the most liked space is outlined in red (figures 6.1-6.6).

\subsection{Natural elements in a space}

In the first pair, participants liked the space with vegetation (figure 6.1), as $96 \%$ preferred the space with its natural features, and only $4 \%$ preferred the space with artificial human-made elements in it. The reasons given for the choice were related to the presence of nature, being appealing to the eye, the openness of the space, and planting within the space.
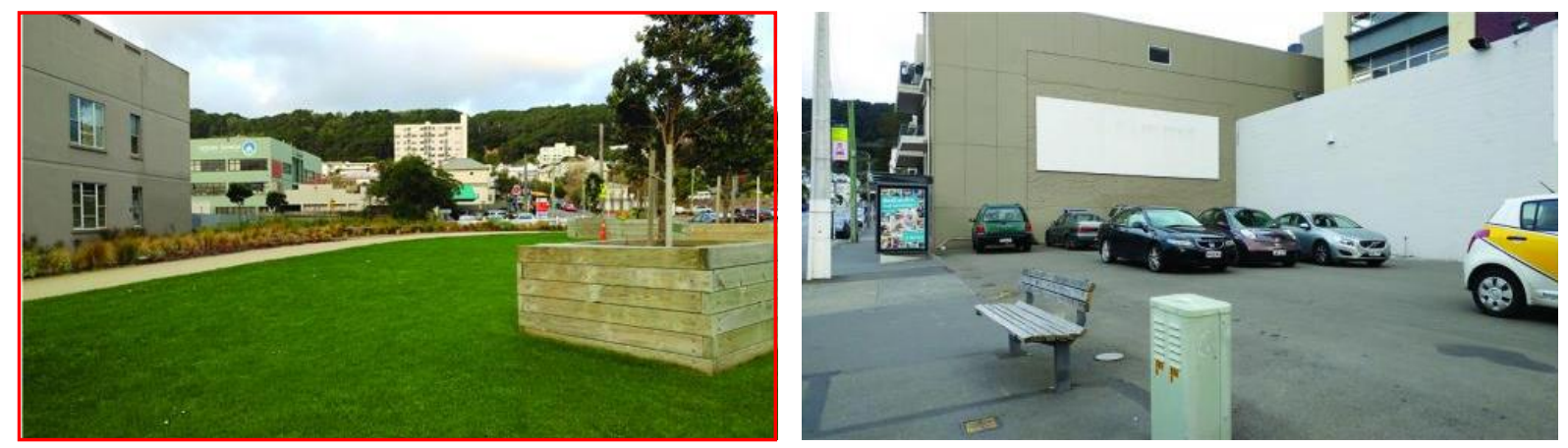

Figure 6.1 Space with natural elements was preferred over space with artificial elements 


\subsection{Organised space}

Overall, $88 \%$ of the participants liked the space that was well organised (figure 6.2) with only $12 \%$ preferring the disorganised space. The reasons for the choice were related to cleanliness and the fact that space has an entrance and exit and a sense of direction.
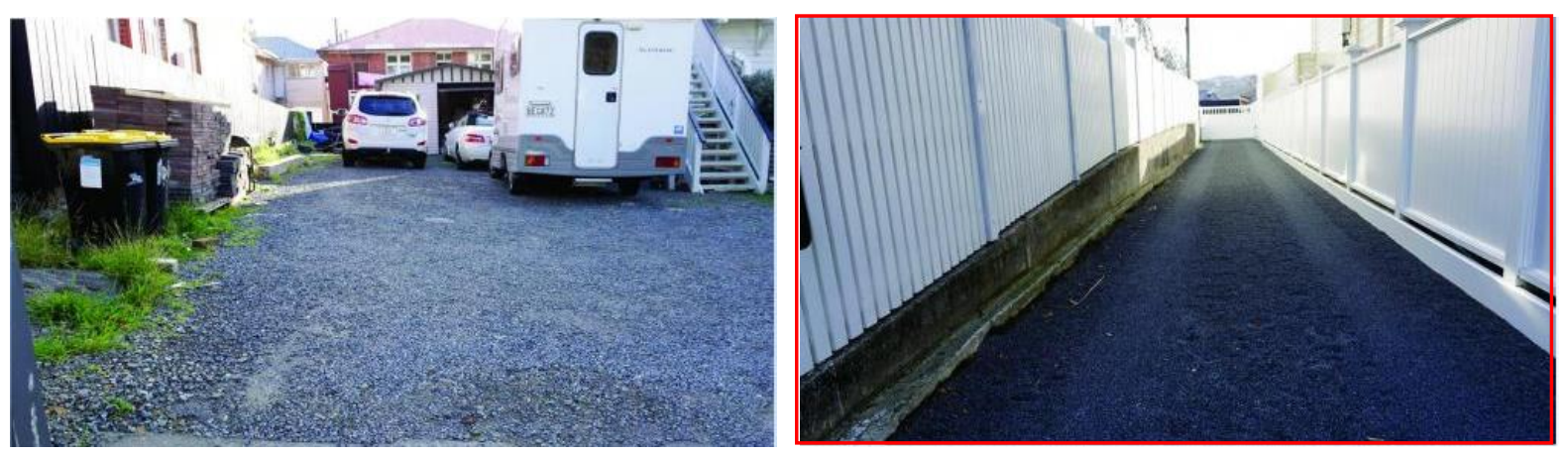

Figure 6.2 Well-organised space was preferred over the disorganised space

\subsection{Space open to the sky}

The space open to the sky was preferred by $64 \%$ participants, with $36 \%$ preferring the space that was more enclosed and confined. The reasons for participant preferences were related to openness that would attract sunlight and give warmth to space.
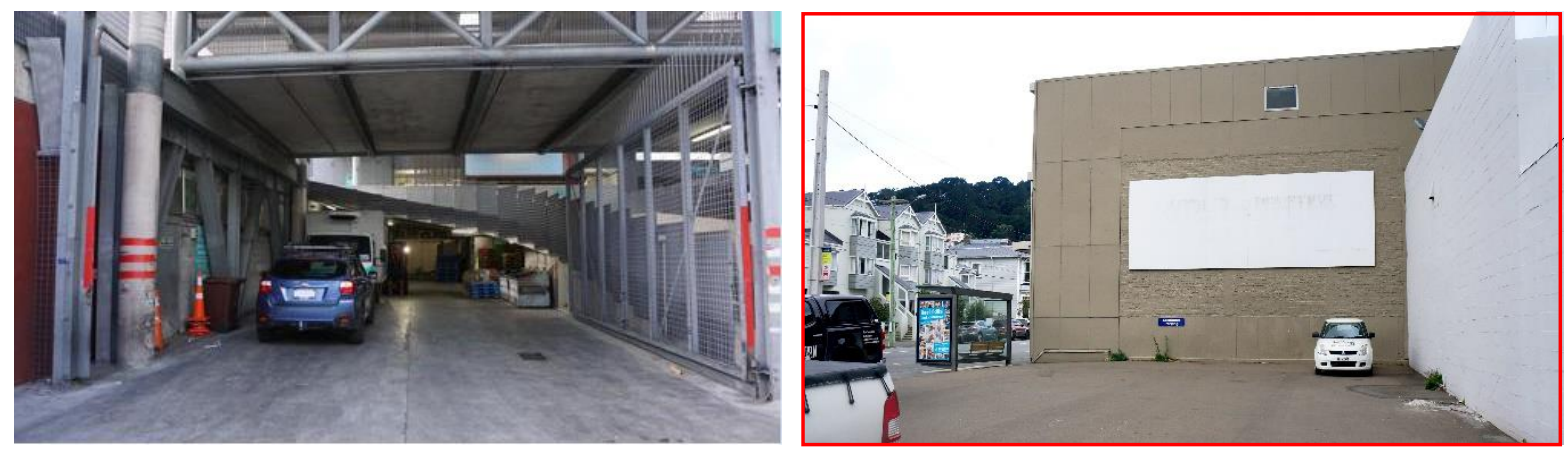

Figure 6.3 Space with openness was preferred over the enclosed space

\subsection{Well maintained space}

Some $79 \%$ of participants preferred the space that was adequately maintained (figure 6.4 ) with only $21 \%$ of participants preferring the less well-kept space. The reasons for the preferences related to cleanliness, openness, and the bright colour of the buildings. 

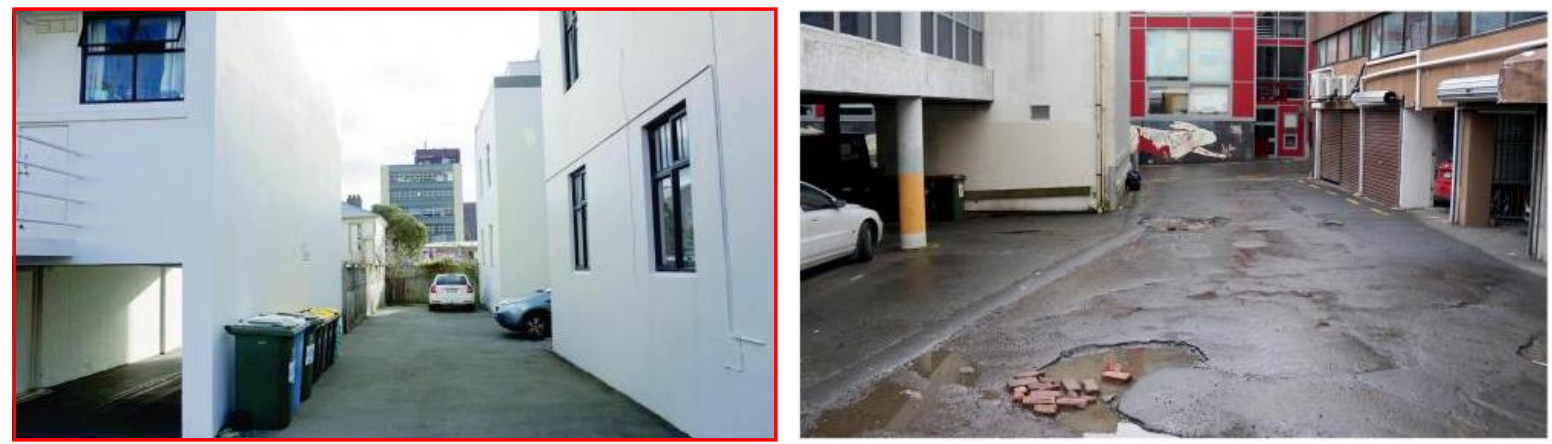

Figure 6.4 Well-maintained space was preferred over the poorly managed space

\subsection{Modern building style}

It became evident from the study that participants liked the modern style of building (figure 6.5), with this being the choice of $68 \%$ participants. Only $32 \%$ of participants liked the older style of building. The reasons behind the choice were the geometric shapes, the new construction materials, cleanliness, and the use of bright colours.
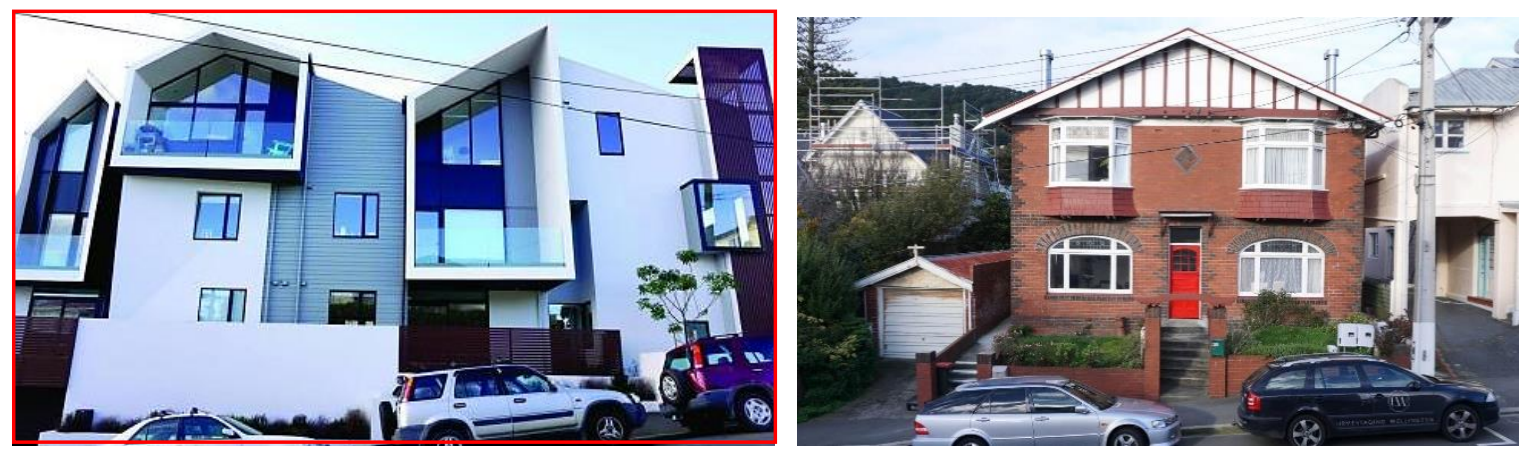

Figure 6.5 Modern building style was preferred over the older style of building The frequency of preference scores in part 2 of Study One is given in figure 6.6.

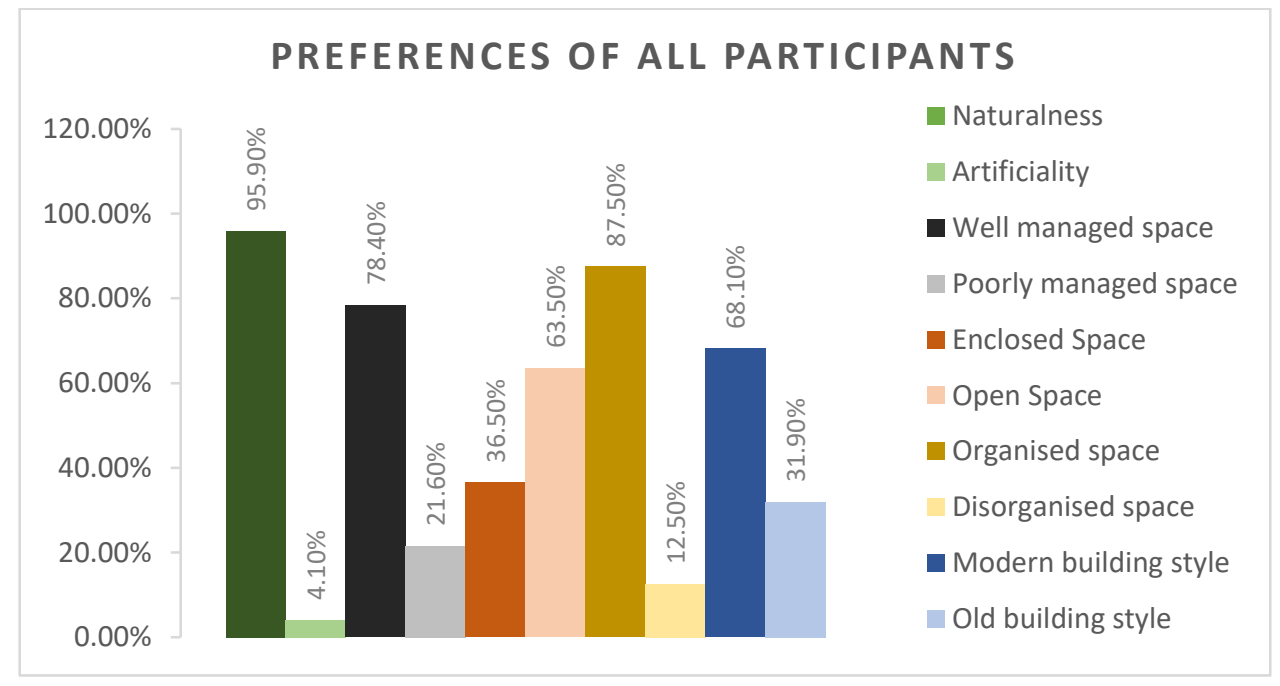

Figure 6.6 Preferences for spaces with different attributes 


\subsubsection{Part Three}

The preferences for the most and least preferred photos were measured on the Likert scale (1-5) by using the descriptive frequency test in SPSS. This technique calculated the percentages of likability on the Likert scale for the three different photos of each type of leftover space. At the end of this part, participants gave their reasons for preferring each space from a list of options.

\subsection{Space underneath a building}

Out of 99 participants, 84 rated the space underneath a building on the Likert scale (1-5). The overwhelming preference was for option $C$ that pictured a through route underneath the building that could be used by the public (figure 6.7). Option $C$ had the highest mean of 4.60 with a Standard Deviation (SD) of 0.54 , and $80 \%$ of participants liked or somewhat liked it (figure 6.8). The main reasons behind rating option $C$ highly (figure 6.9) were related to its different use of materials/colours $(85 \%)$, visual permeability ( $85 \%)$, good lighting $(68 \%)$, maintenance of space $(56 \%)$, and change in level (40\%). Option A was the second most liked with $45 \%$ participants either liking or somewhat liking it. Option A had a mean score of 2.77 and an SD of 1.20. The reasons for preferring option A were related to the cleanliness of the space $(70 \%)$, the sense of openness (37\%), and shade for the car park (36\%). Lastly, option B was the least favoured, but still with $27 \%$ of participants liking or somewhat liking it. This option received a mean score of 2.75, with an SD of 1.21 (appendix 6.1). The space in option B was liked because it was clean (56\%) and had an organised car park (38\%). 
Chapter 6 - STUDY ONE: FIRST VISUAL PREFERENCE STUDY

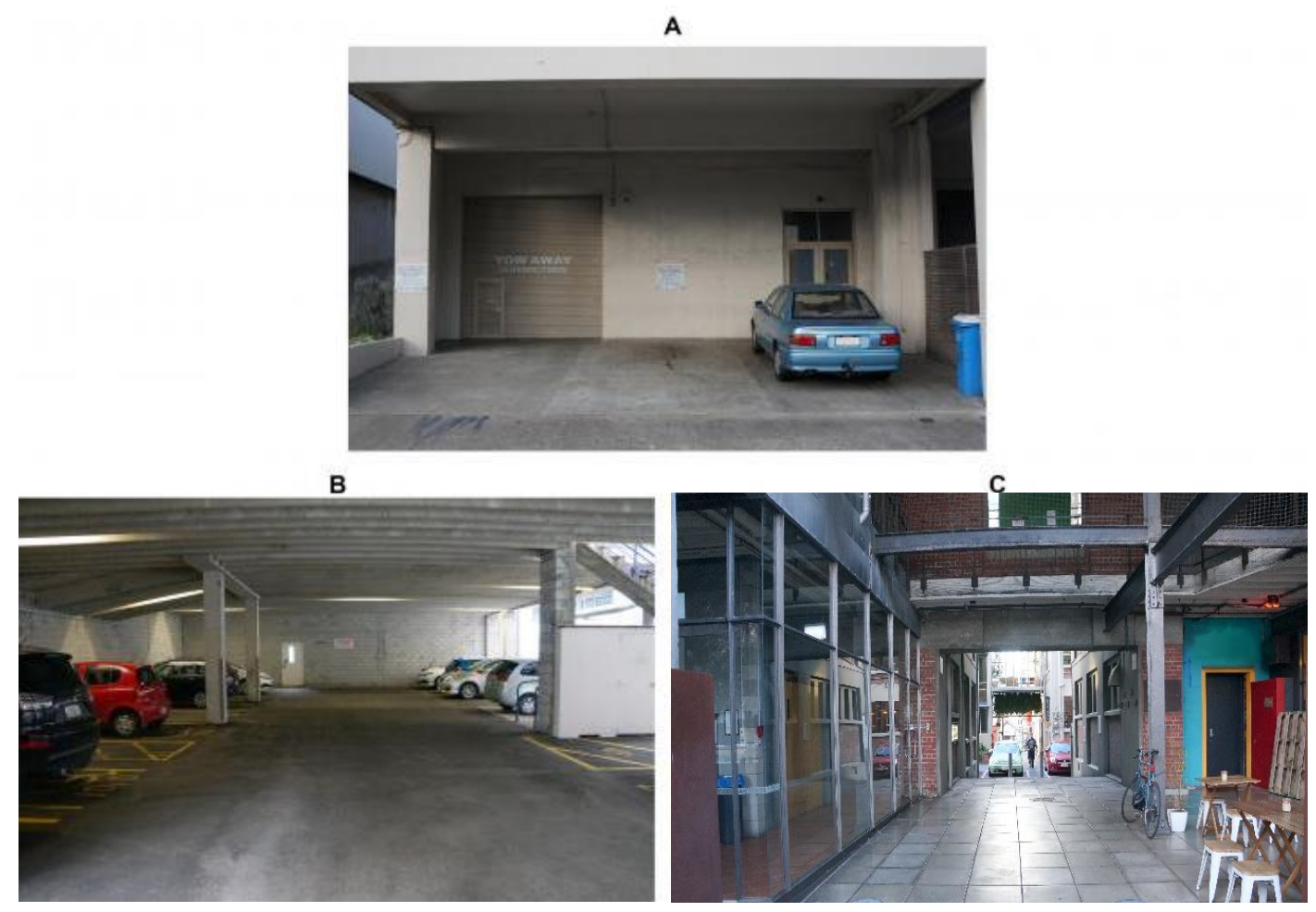

Figure 6.7 Options shown to participants

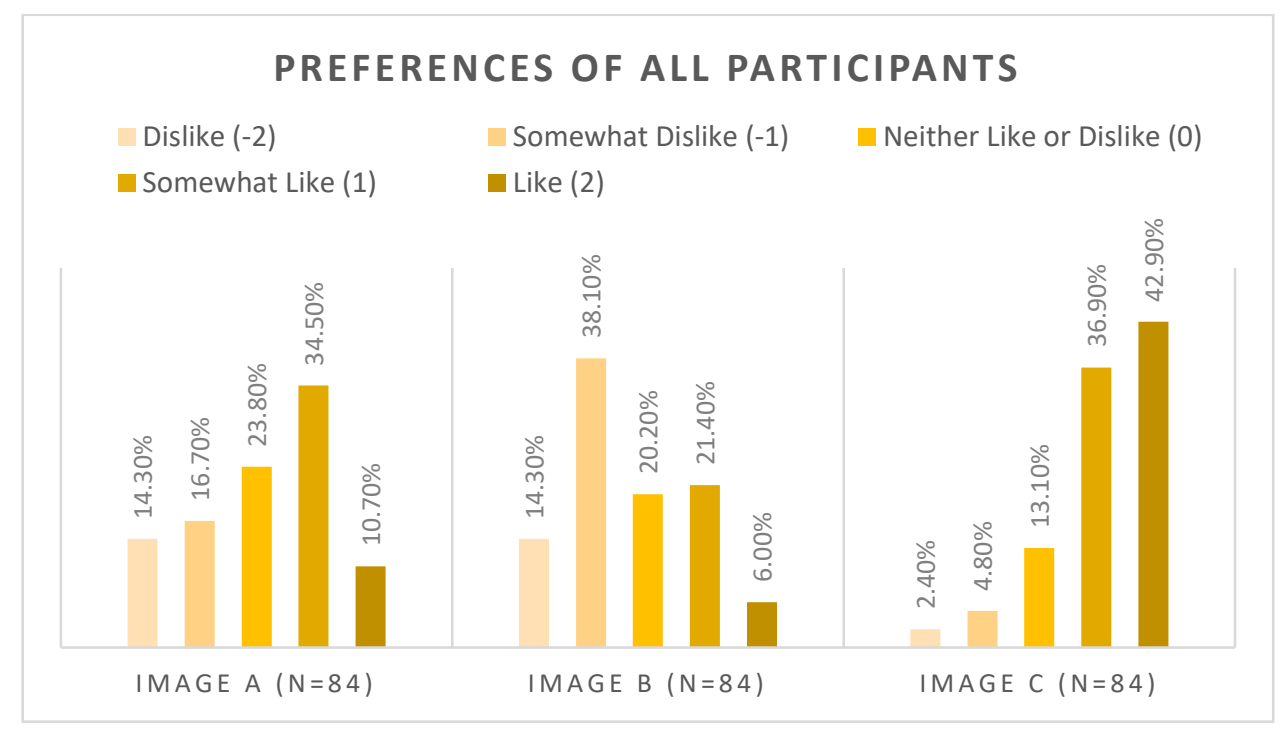

Figure 6.8 Participant preferences for the different options 

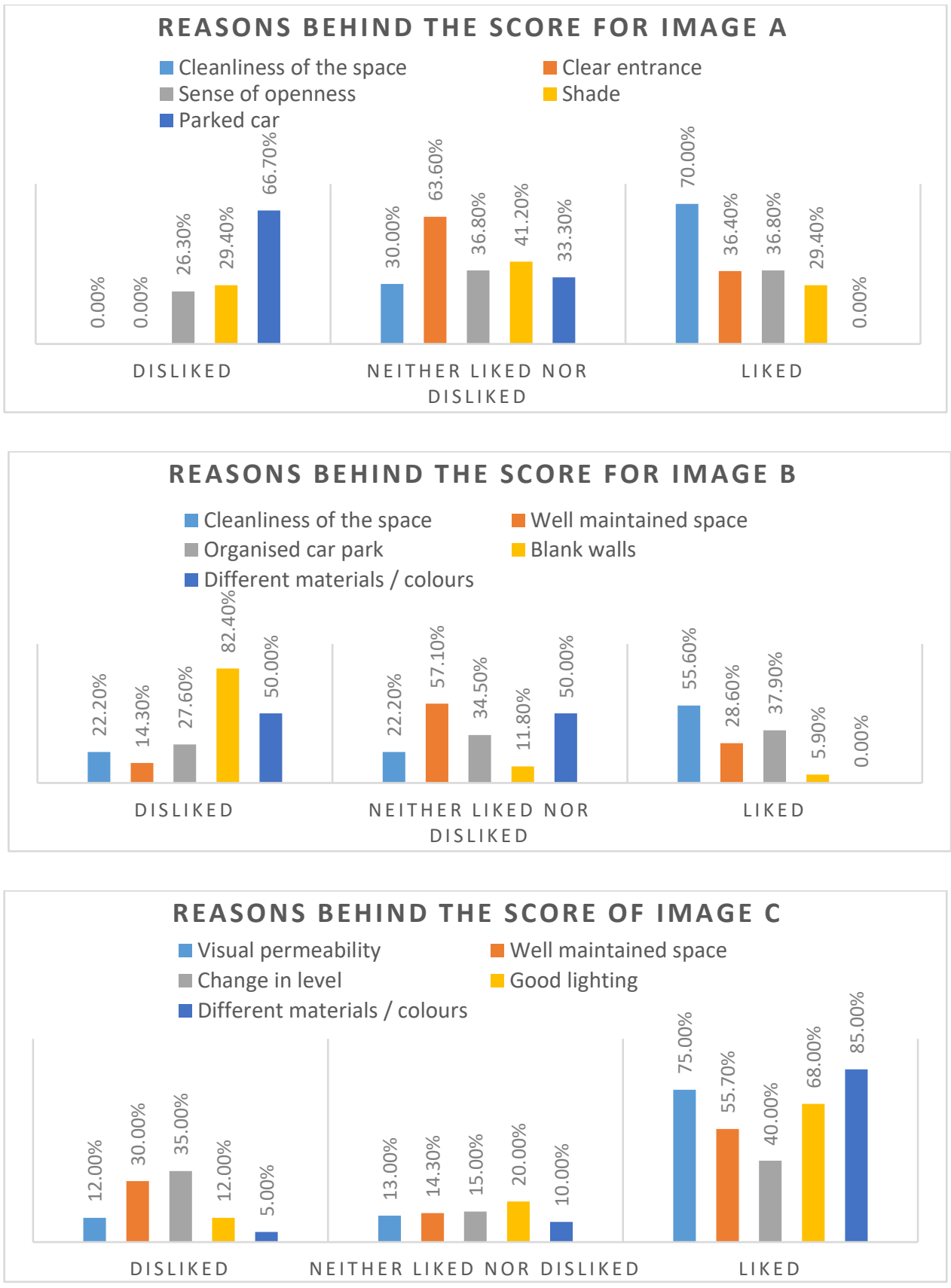

Figure 6.9 Participant reasons behind the rating of images A-C

\subsection{Space in front of a building}

95 rated the space in front of a building on the Likert scale (1-5). All participants preferred option A, which had natural vegetation in the space (figure 6.10). Option A scored the highest mean of 4.48 with a Standard Deviation (SD) of 0.75 , and $93 \%$ of people liked or somewhat liked it. The main reasons behind rating option A highly (figure 6.12) related to the cleanliness of the space $(78 \%)$, planting $(58 \%)$, clear 
entrance $(50 \%)$, use of different materials/colours (50\%), and organised car parking (40\%). Option C was the second most preferred, with $49 \%$ of participants liking or somewhat liking it (figure 6.11). Option $\mathrm{C}$ had a mean score of 3.28 and an SD of 1.09. The space in option $C$ was liked because it was visually permeable $(74 \%)$, had a clear thoroughfare $(63 \%)$, and a sense of openness $(46 \%)$. Lastly, option B was the least liked, with only $17 \%$ of participants liking or somewhat liking it. This option had a mean score of 2.49 and an SD of 1.21. The reasons for liking option B were related to use of different materials/colours (54\%) and having a clear thoroughfare (37\%).

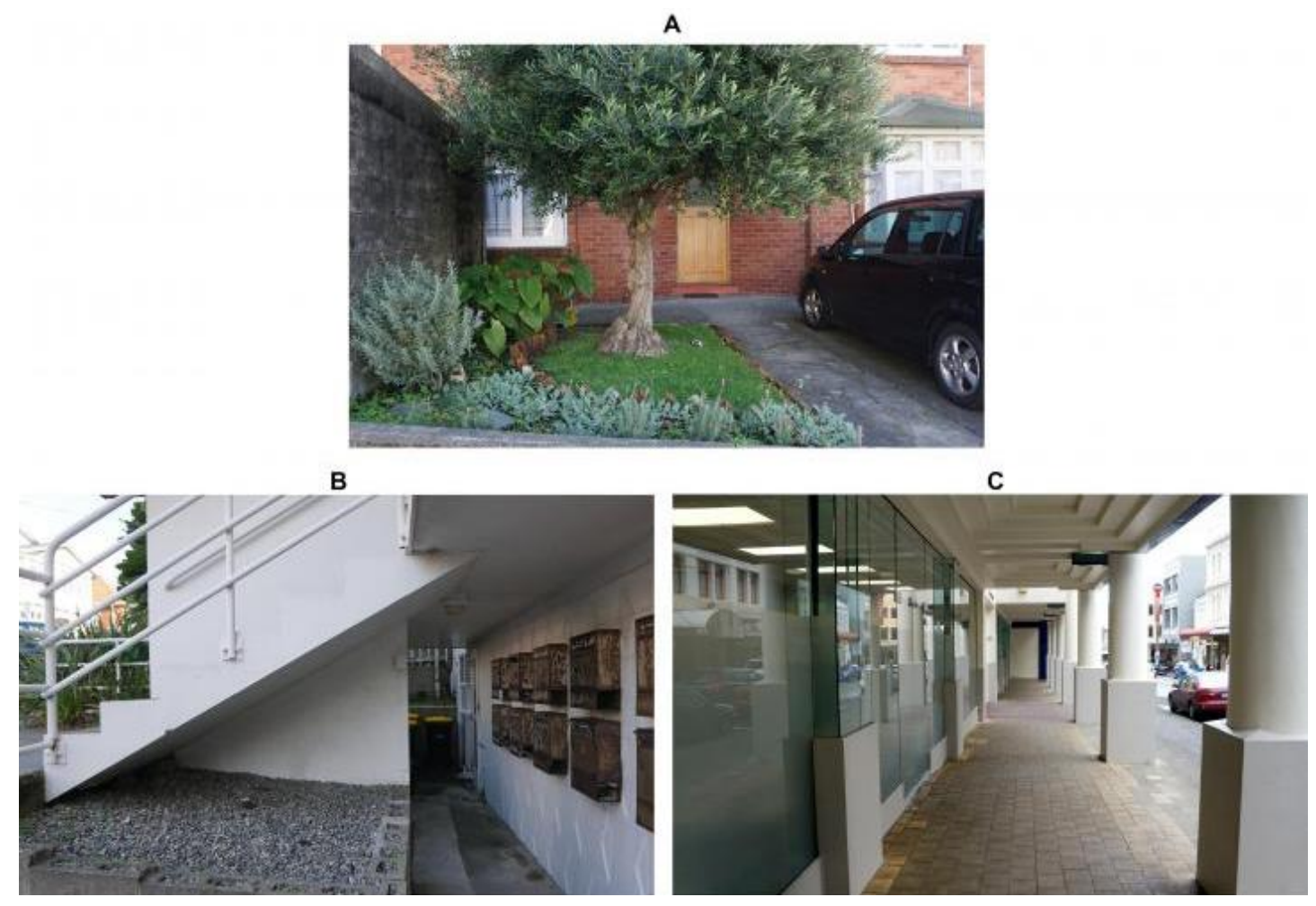

Figure 6.10 Options shown to participants 


\section{PREFERENCES OF ALL PARTICIPANTS}

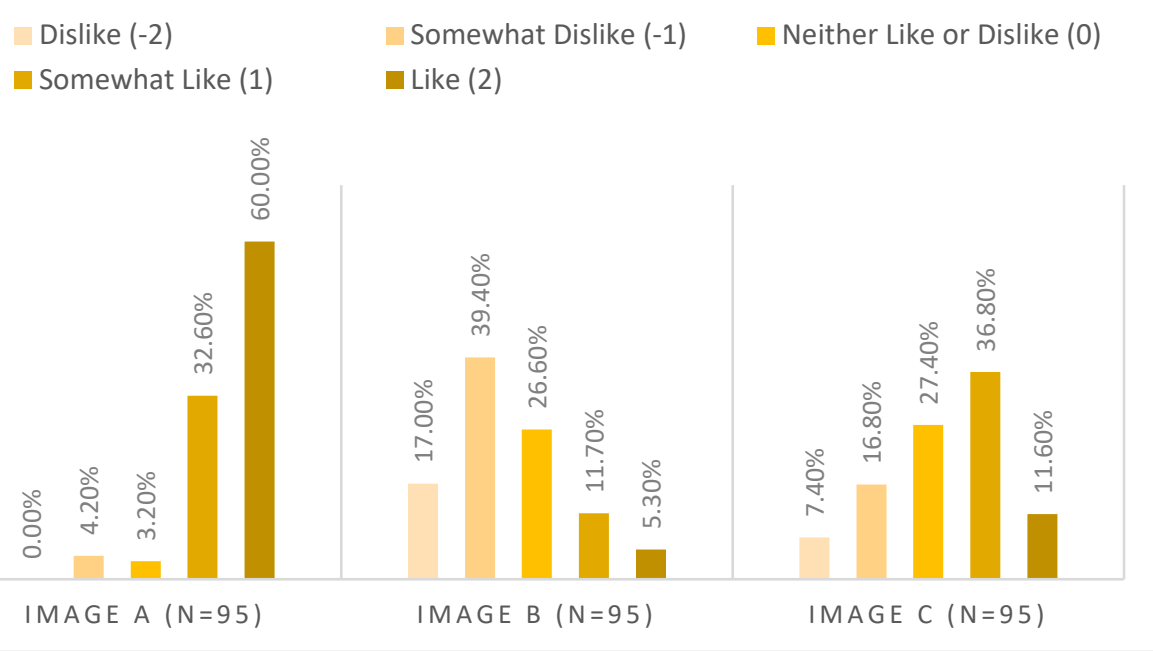

Figure 6.11 Participant preferences for the different options
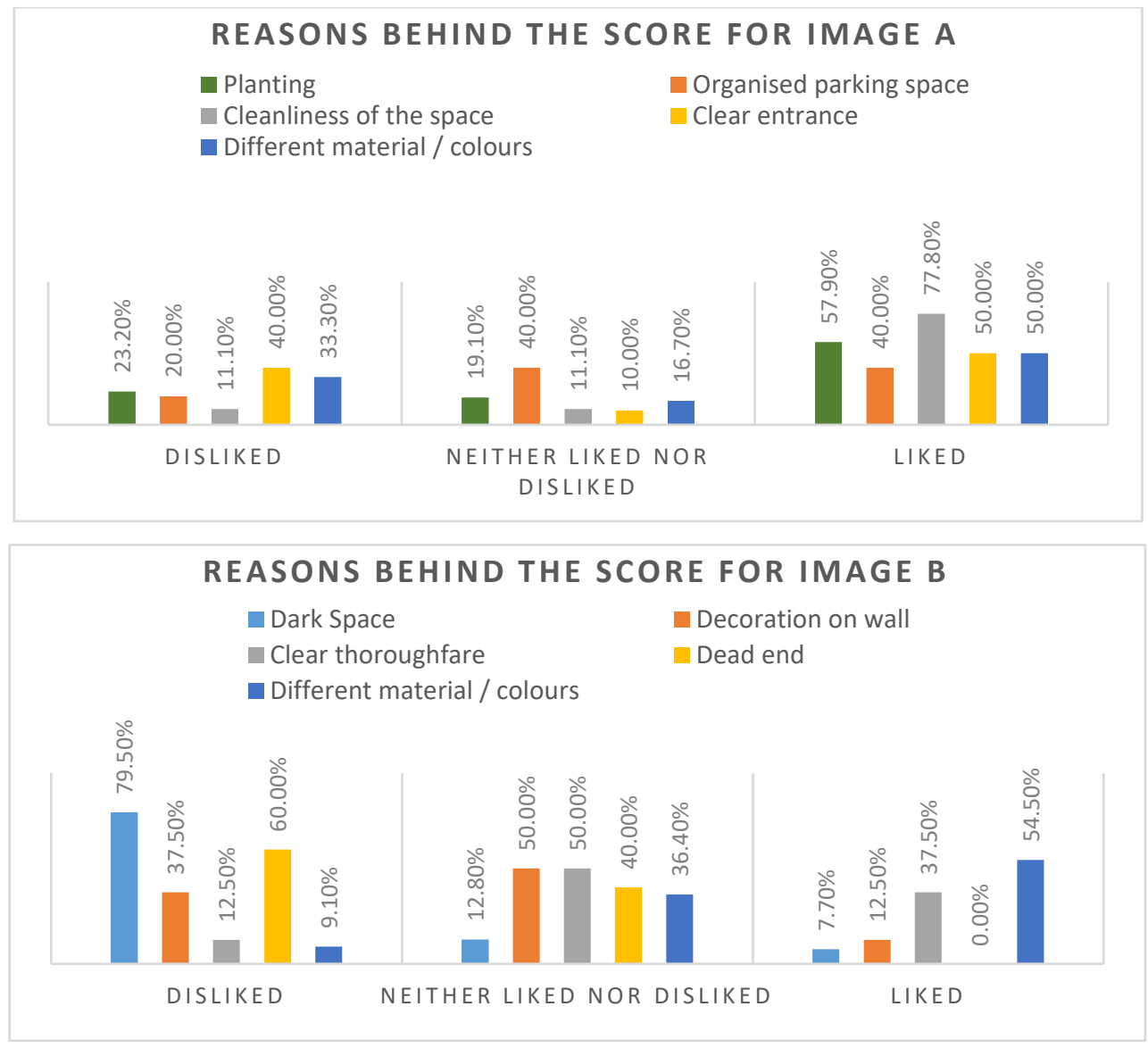


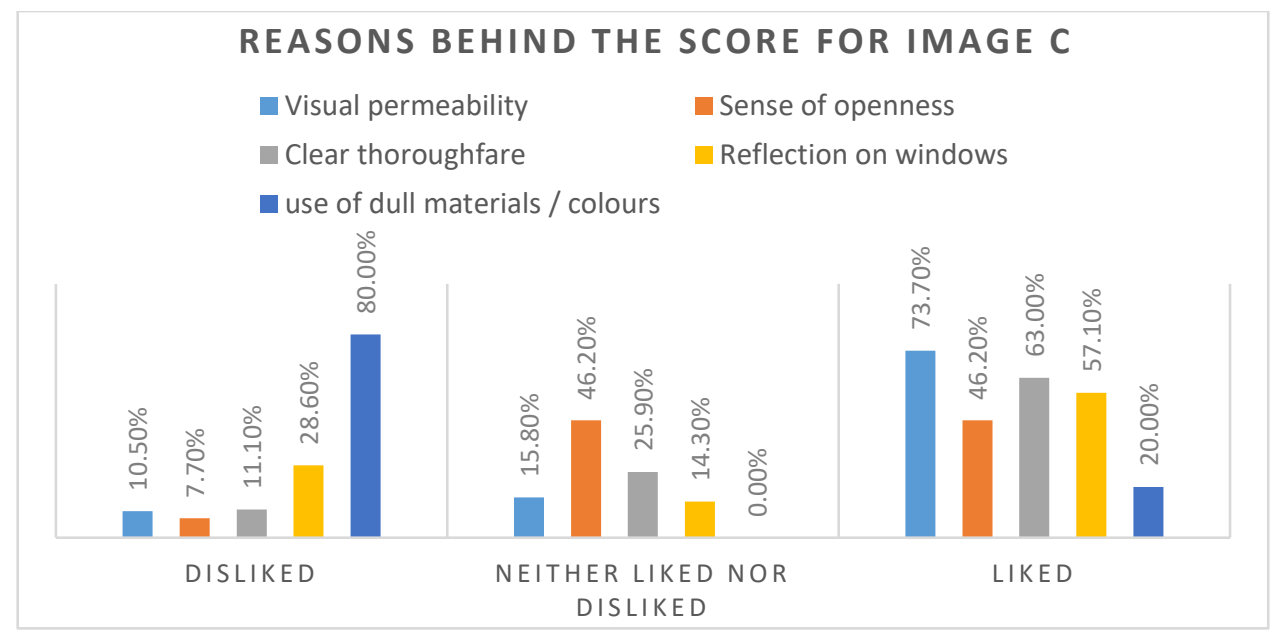

Figure 6.12 Participant reasons behind the rating of images A-C

\subsection{Space at back of a building}

91 rated the space at the back of a building on the Likert scale (1-5). Option B (figure 6.13), which scored the highest mean of 3.32 with a Standard Deviation (SD) of 1.01 was liked or somewhat liked by $54 \%$ of participants. The reasons behind rating option $B$ (figure 6.15) were related to its different surface materials (93\%) and graffiti on the wall $(57 \%)$. Option A was the second most preferred, with $44 \%$ of participants liking or somewhat liking it (figure 6.14). Option A had a mean score of 3.19 and an SD of 1.14. This option was liked because it had a clear entrance to the building (61\%), openness to the sky (47\%), and storage space under the ramp (43\%). Lastly, option C was the least liked, with only $37 \%$ of participants liking or somewhat liking it. This option received a mean score of 3.00 with an SD of 1.21 . The reasons for liking option $C$ were related to its good organisation (86\%) and planting in the space (62\%). 


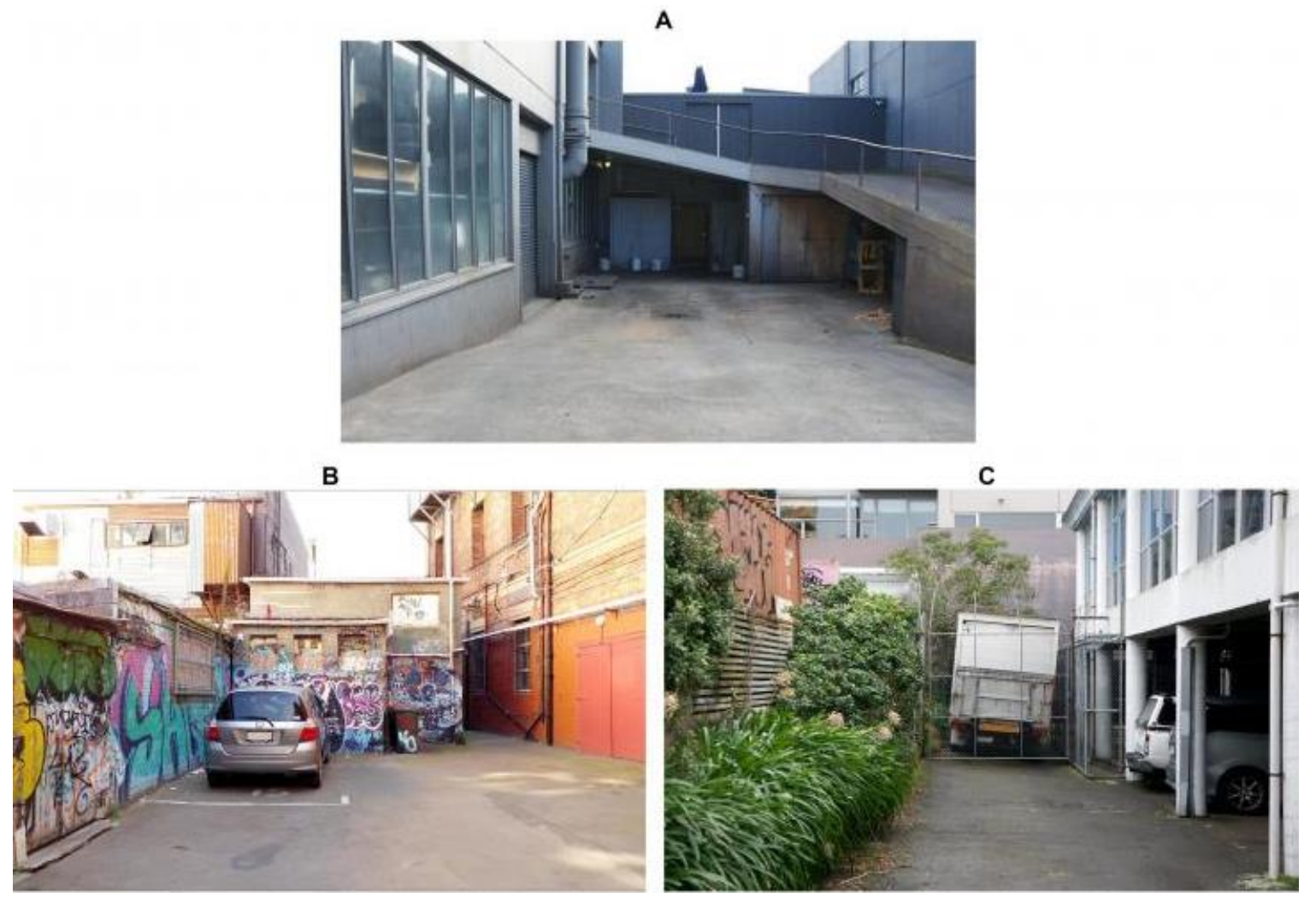

Figure 6.13 Options shown to participants

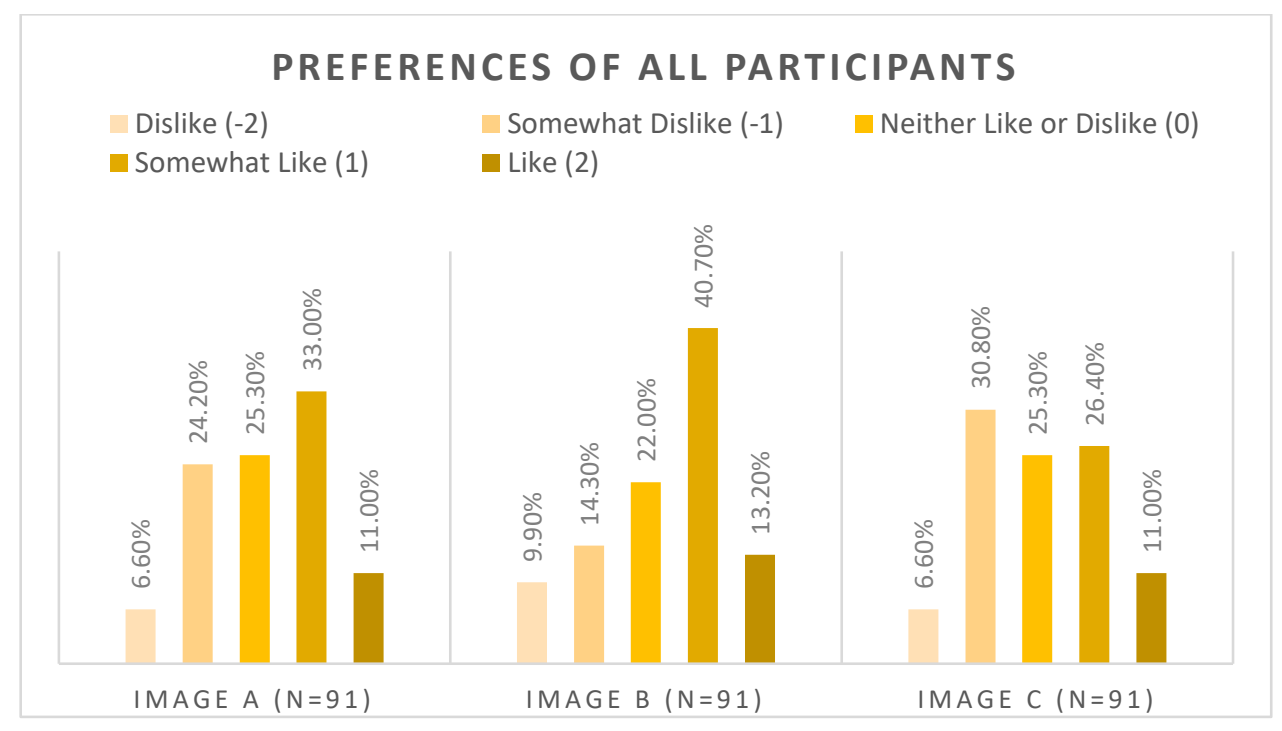

Figure 6.14 Participant preferences for the different options 

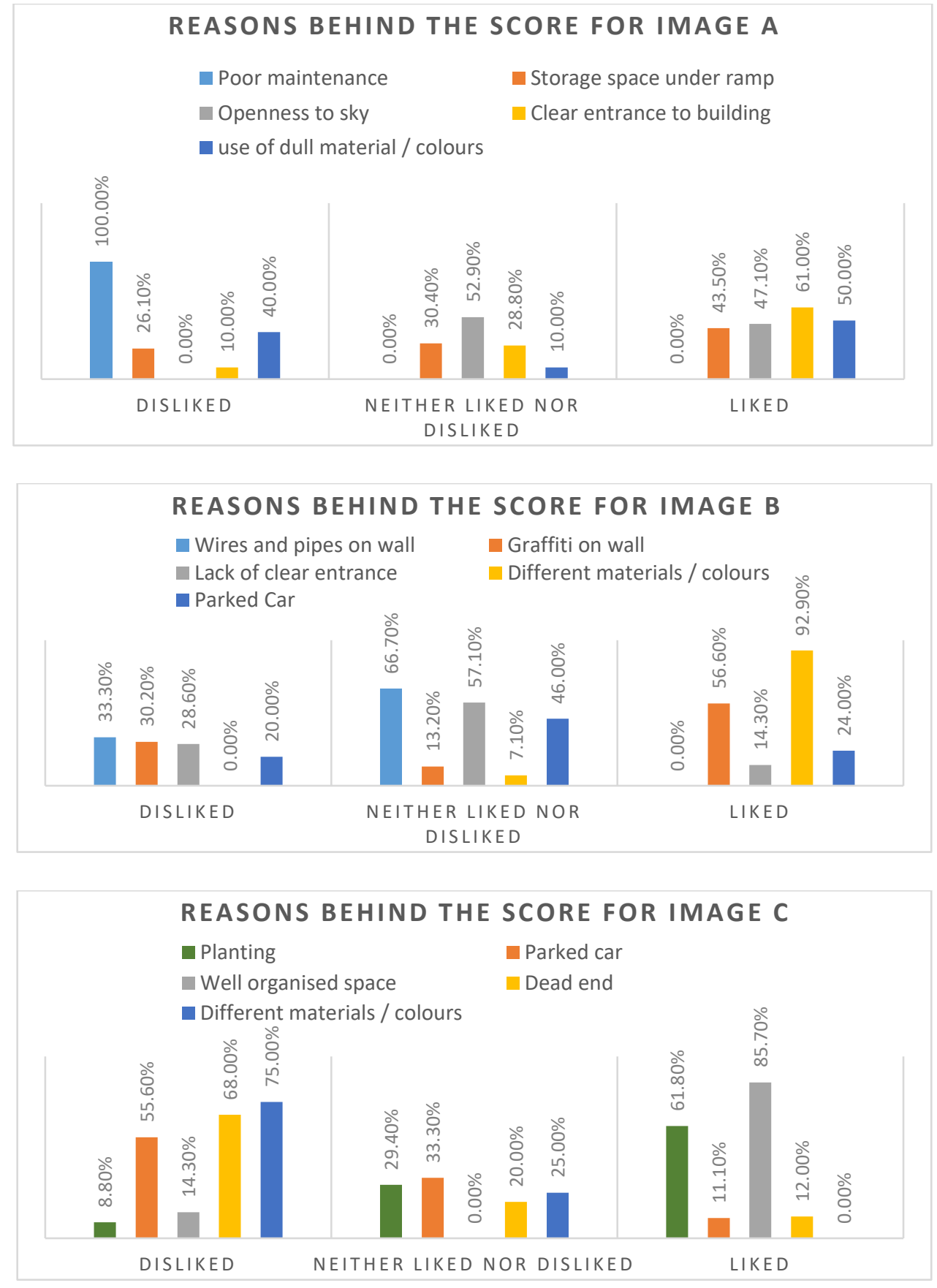

Figure 6.15 Participant reasons behind the rating of images A-C

\subsection{Space enclosed by buildings on three sides}

Out of 99 participants, 84 rated the space enclosed by buildings on three sides. Image C (figure 6.16) scored the highest mean of 4.13 with a Standard Deviation (SD) of 0.97 , with $80 \%$ of people liking or somewhat liking it. The main reasons behind rating option C highly (figure 6.18) were related to its openness to the sky $(90 \%)$, change in level $(88 \%)$, planting in the space $(81 \%)$, and the similar style of construction $(70 \%)$. Option A was the second most preferred, with $45 \%$ of participants liking or somewhat 
liking this space (figure 6.17). Option A had a mean score of 3.11 and an SD of 1.18. The reasons for liking the option A was related to openness to the sky $(79 \%)$, planting $(67 \%)$, and the use of different materials/colours (33\%). Option B was the least liked, with only $27 \%$ of participants liking or somewhat liking it. This option received a mean score of 2.67 with an SD of 1.27. The reasons for liking option B were related to the openings in the wall $(57 \%)$, and the space is well maintained $(50 \%)$.

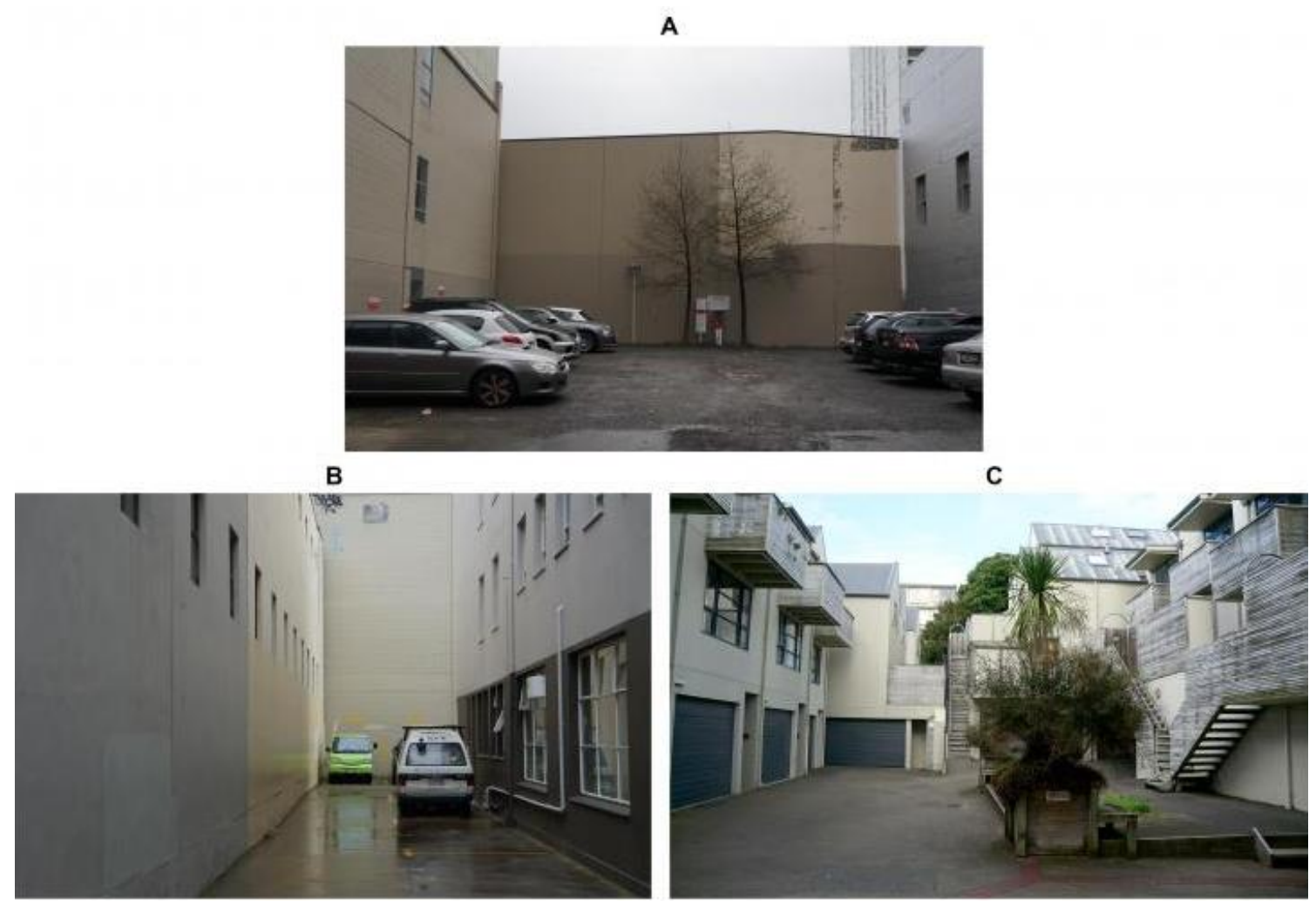

Figure 6.16 Options shown to participants

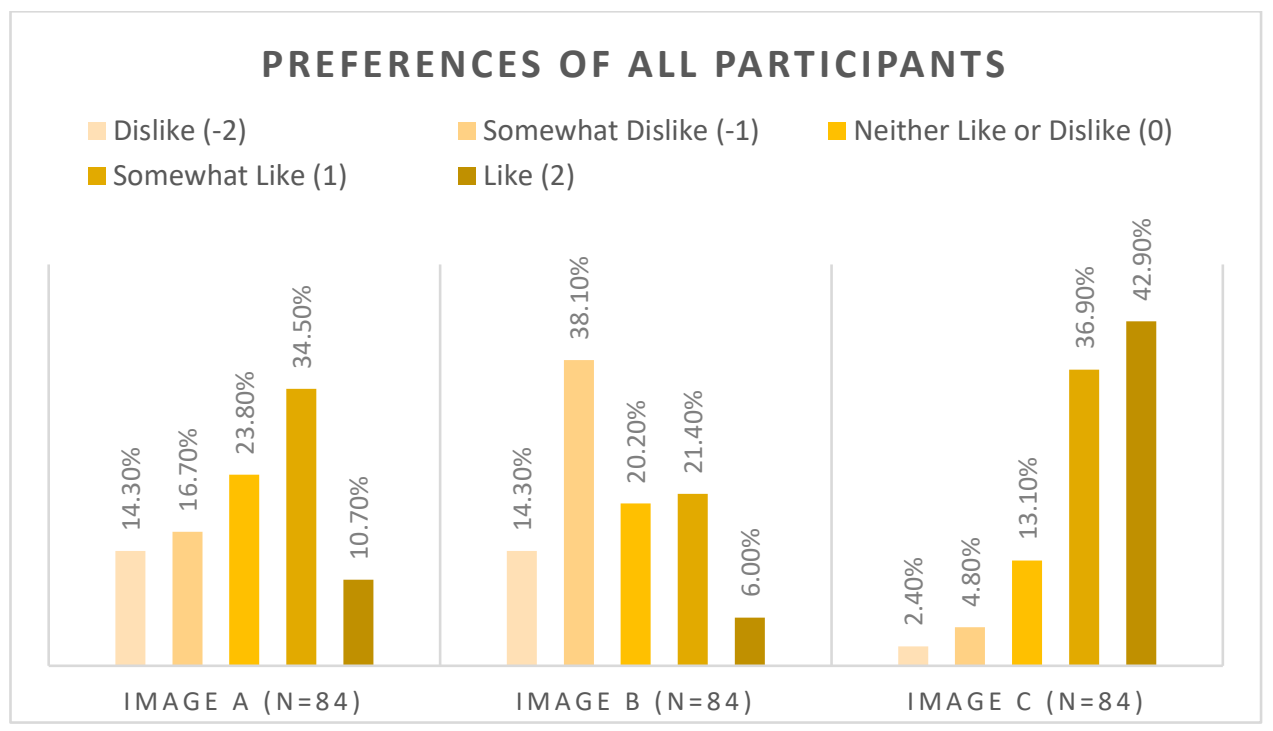

Figure 6.17 Participant preferences for the different options 

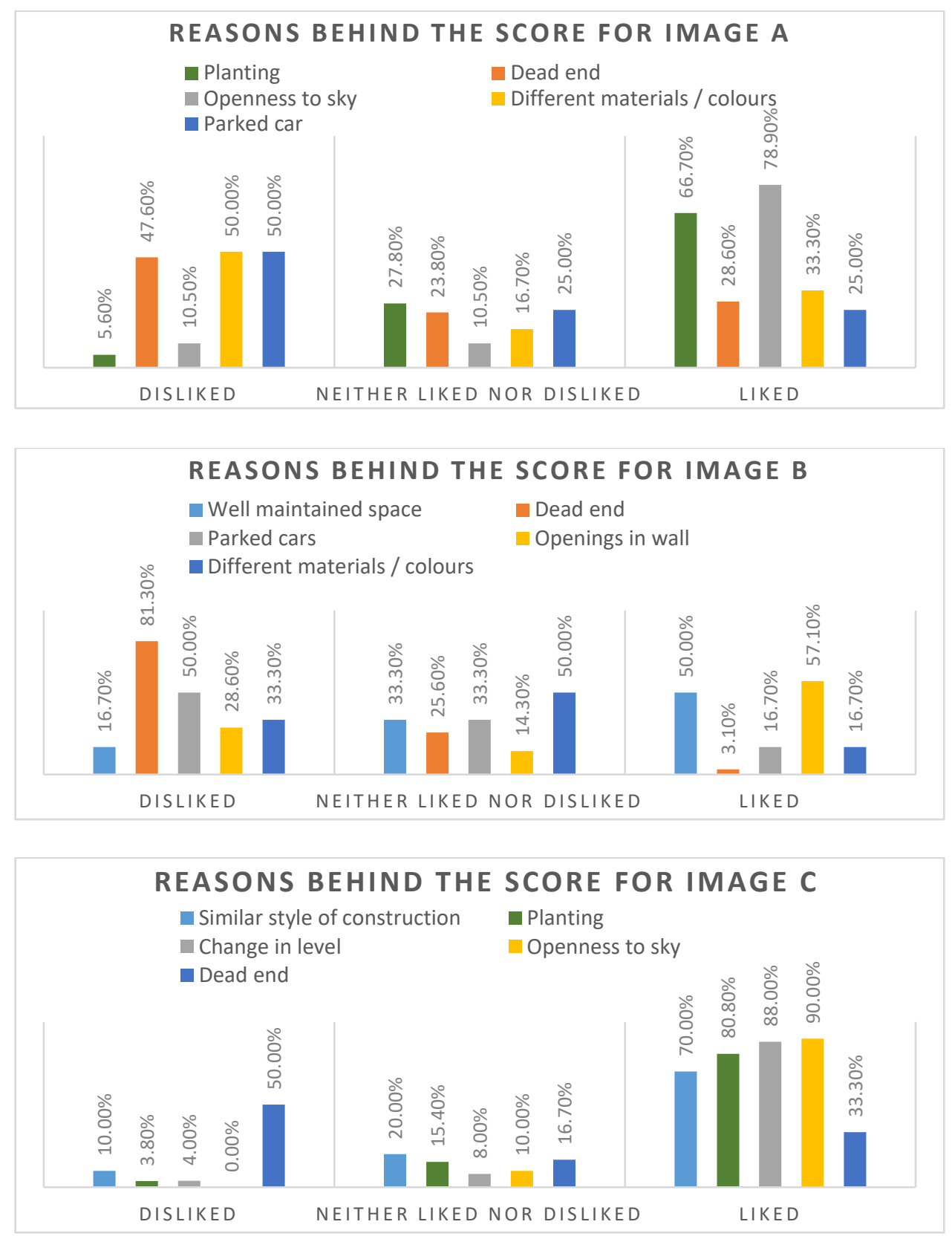

Figure 6.18 Participant reasons behind the rating of images A-C

\subsection{Space enclosed by buildings on two sides}

Out of 99 participants, 87 rated the space enclosed by buildings on two sides on the Likert scale (1-5). Option C (figure 6.19) scored the highest mean of 3.12 with a Standard Deviation (SD) of 1.13 . Overall $42 \%$ of people liked or somewhat liked option $\mathrm{C}$ and the main reasons for this (figure 6.21) were related to its openness to the sky $(56 \%)$, good maintenance of the space $(50 \%)$, the variety of facades $(43 \%)$, graffiti on the wall (39\%), and use of different materials/colours (33\%). Option B was the second 
most preferred, with $33 \%$ of participants liking or somewhat liking it (figure 6.20). Option B had a mean score of 2.95 and an SD of 1.19. The two main reasons for liking option B were related to planting (100\%) and having a clear thoroughfare $(51 \%)$. Lastly, option A was the least liked, with only $23 \%$ of participants liking or somewhat liking it. This option had a mean score of 2.70 and an SD of 1.24. The reasons for liking image $A$ were related to the variety of facades (43\%), and openness to the sky $(40 \%)$.

A

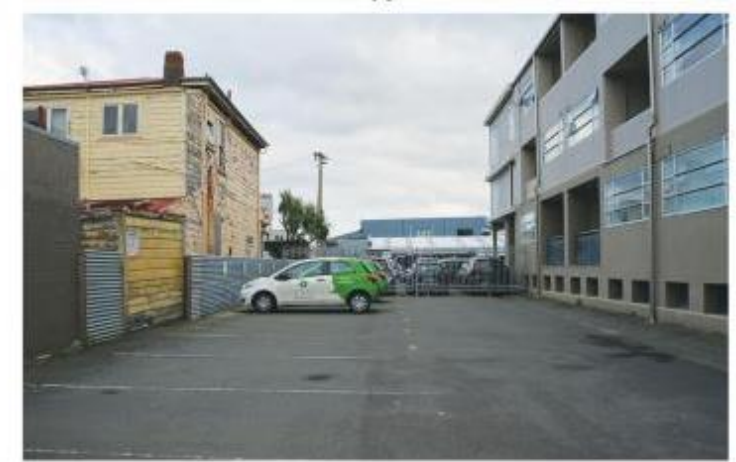

B

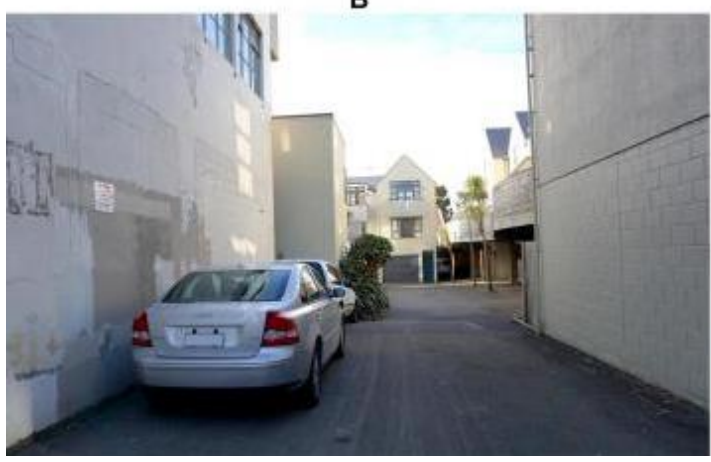

c

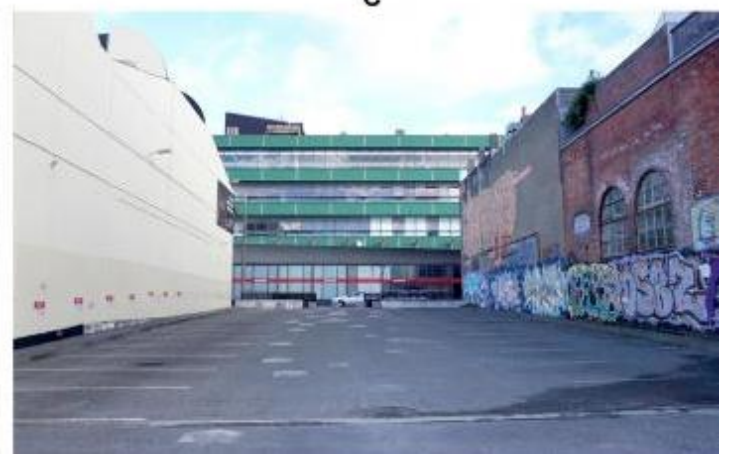

Figure 6.19 Options shown to participants

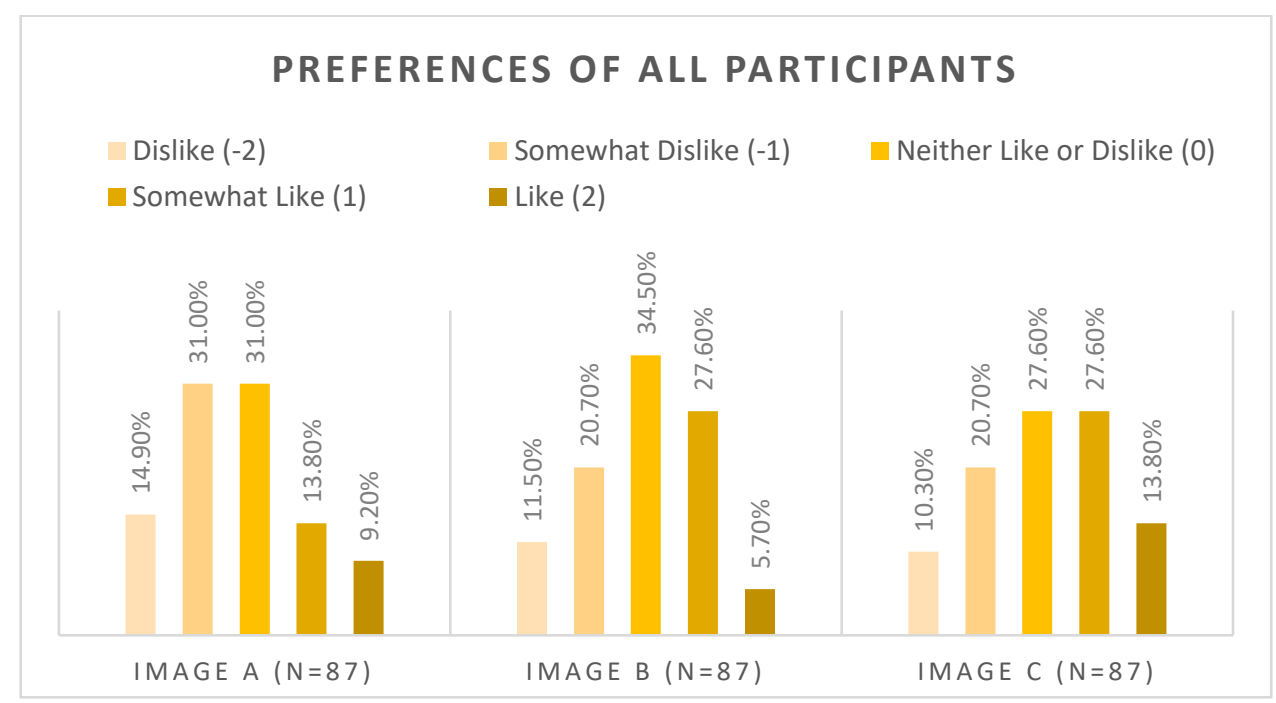

Figure 6.20 Participant preferences for the different options 

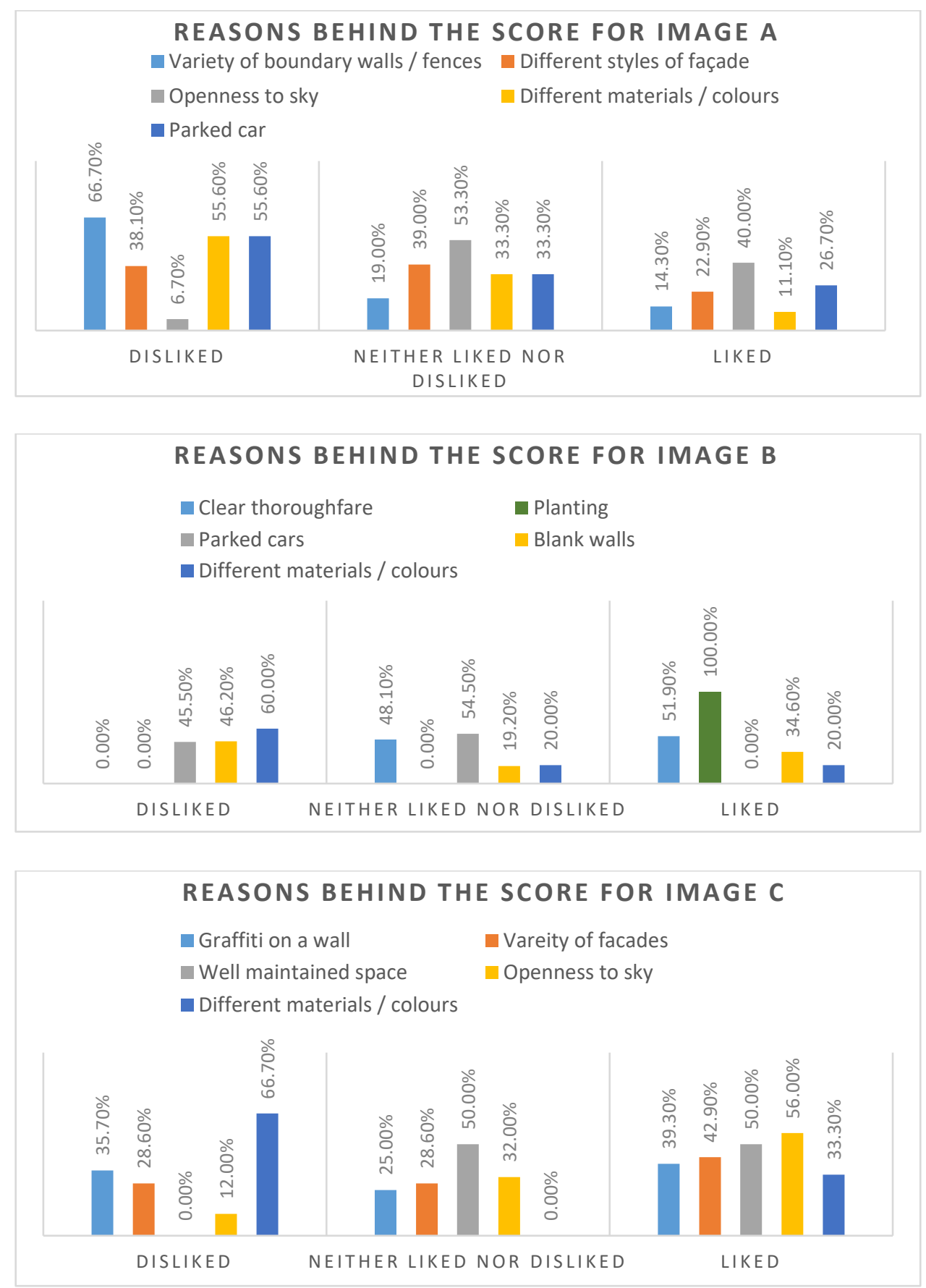

Figure 6.21 Participant reasons behind the rating of images A-C

\subsection{Space on the rooftop of a building}

Out of 99 participants, 84 rated the space on the rooftop of a building on the Likert scale (1-5). Option $C$ (figure 6.22) had the highest mean of 3.15 with a Standard Deviation (SD) of 1.11 , and with $37 \%$ of people liking or somewhat liking it. The main 
reasons behind the rating of option $C$ (figure 6.24) were its proper maintenance (57\%), openness to the sky $(57 \%)$, use of different materials/colours (46\%) and organised parking space $(31 \%)$. Option A was the second most preferred, with $35 \%$ participants liking or somewhat liking this space (figure 6.23). It received a mean score of 2.88 with an SD of 1.19. The reasons for liking image $A$ were its different levels (67\%), proper maintenance $(64 \%)$, and the use of different material/colours (43\%). Lastly, option B was least liked with only $18 \%$ of participants liking or somewhat liking it. This option had a mean score of 2.21 with an SD of 1.24. The reasons for liking image B were related to openness to the sky (33\%), and the use of different materials/colours $(30 \%)$.

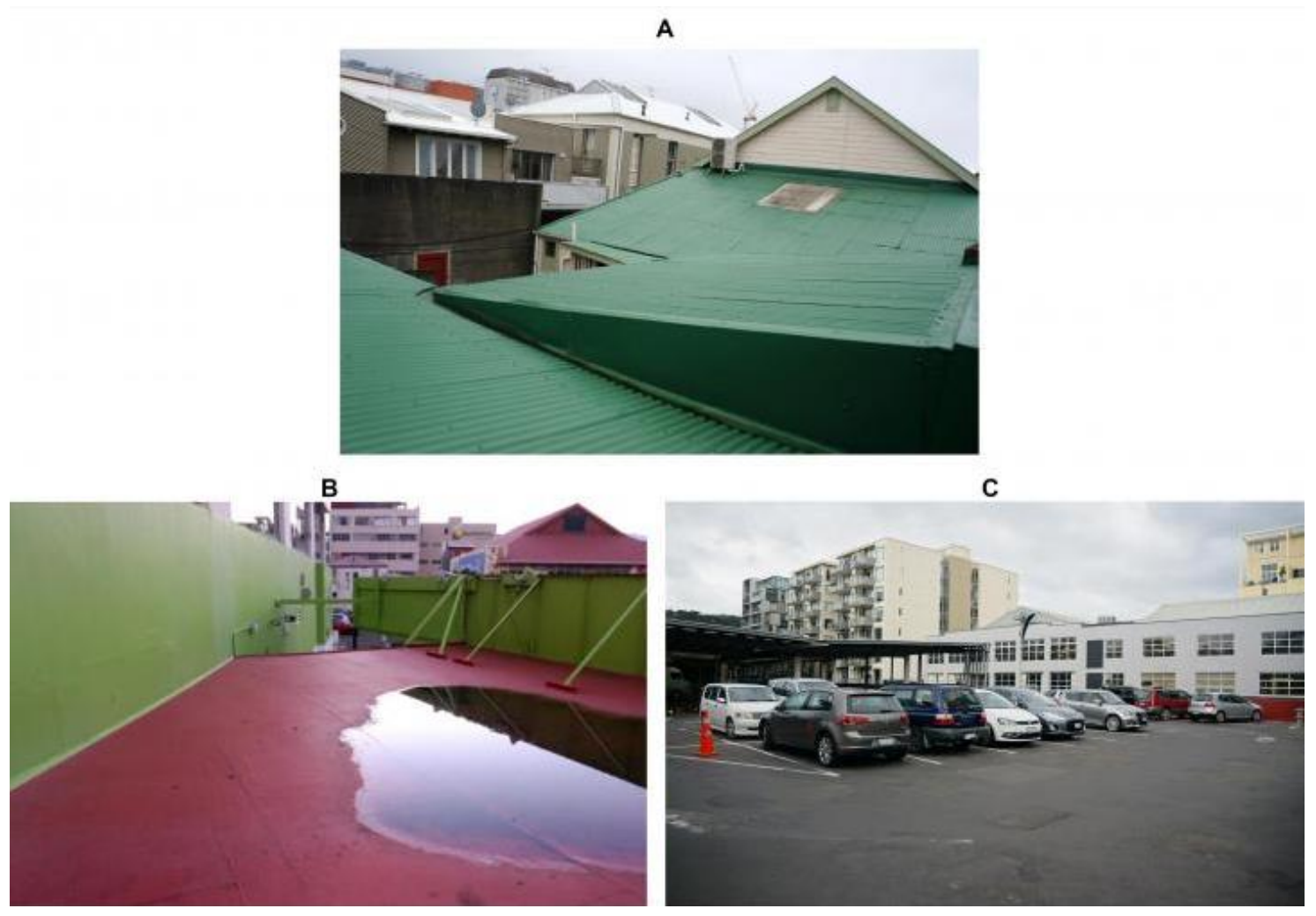

Figure 6.22 Options shown to participants 


\section{PREFERENCES OF ALL PARTICIPANTS}

$\square$ Dislike (-2) $\square$ Somewhat Dislike (-1) $\square$ Neither Like or Dislike (0)

- Somewhat Like (1) $\quad$ Like (2)

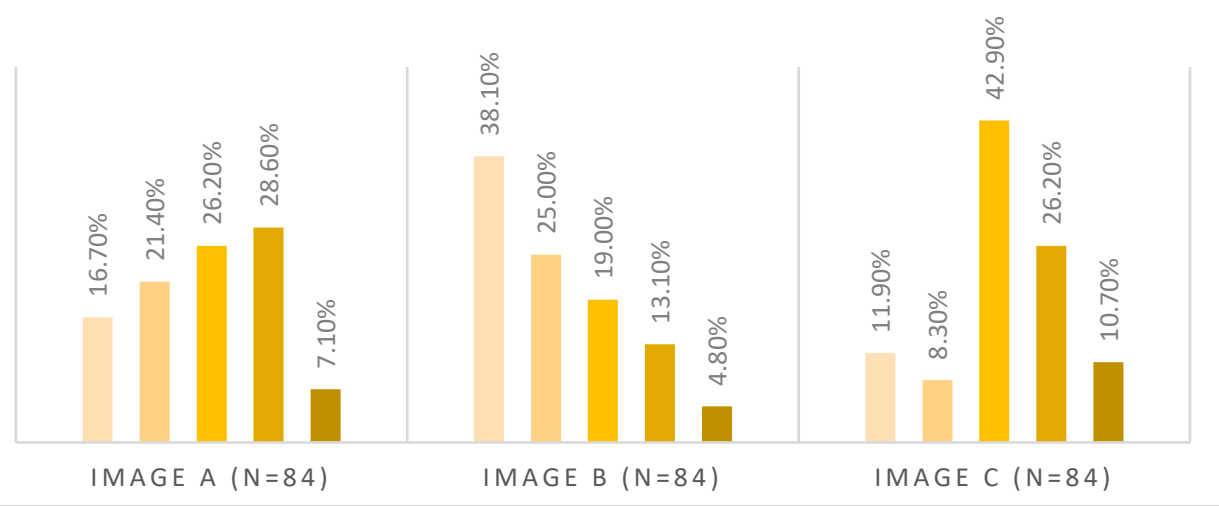

Figure 6.23 Participant preferences for the different options
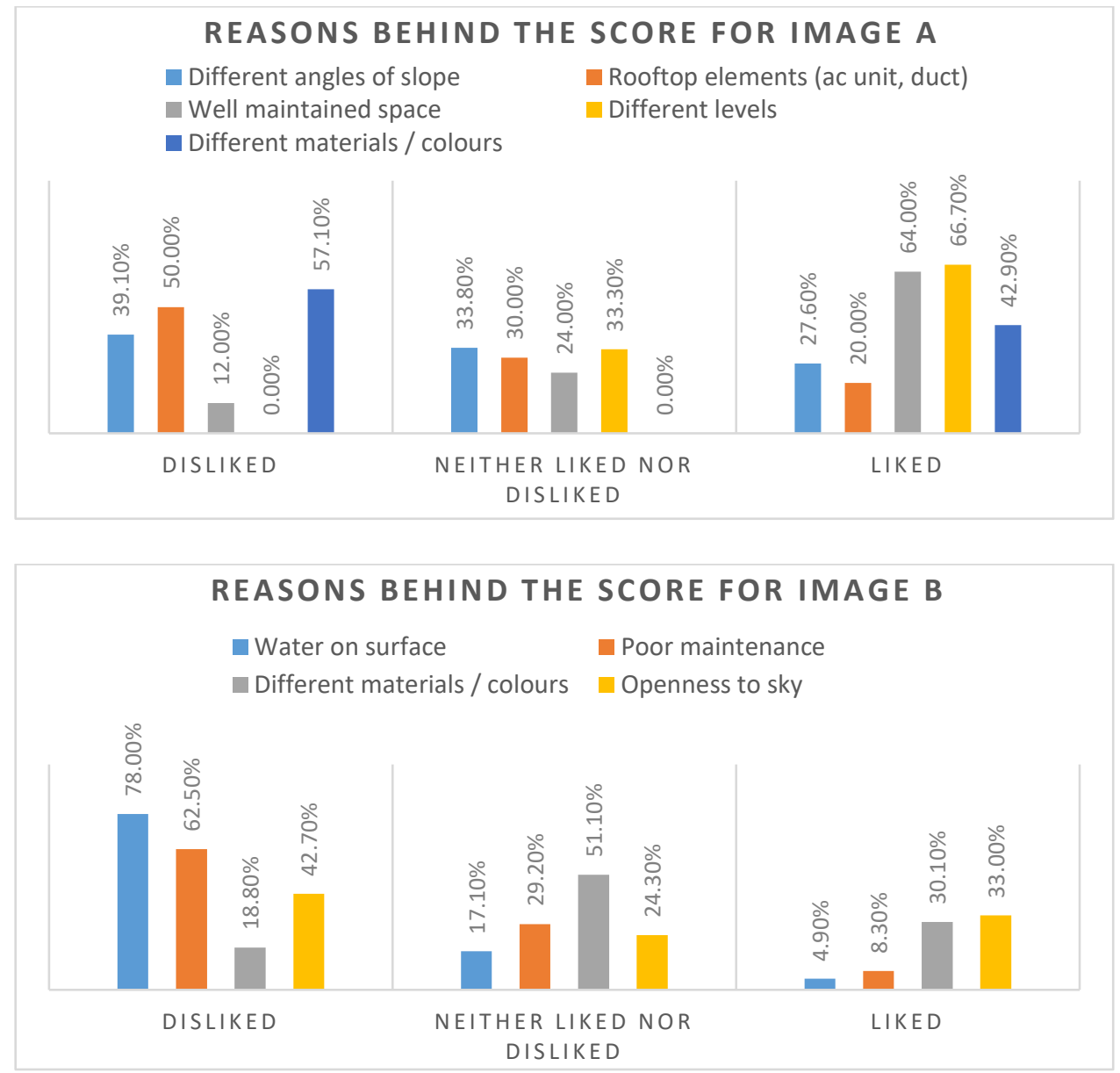


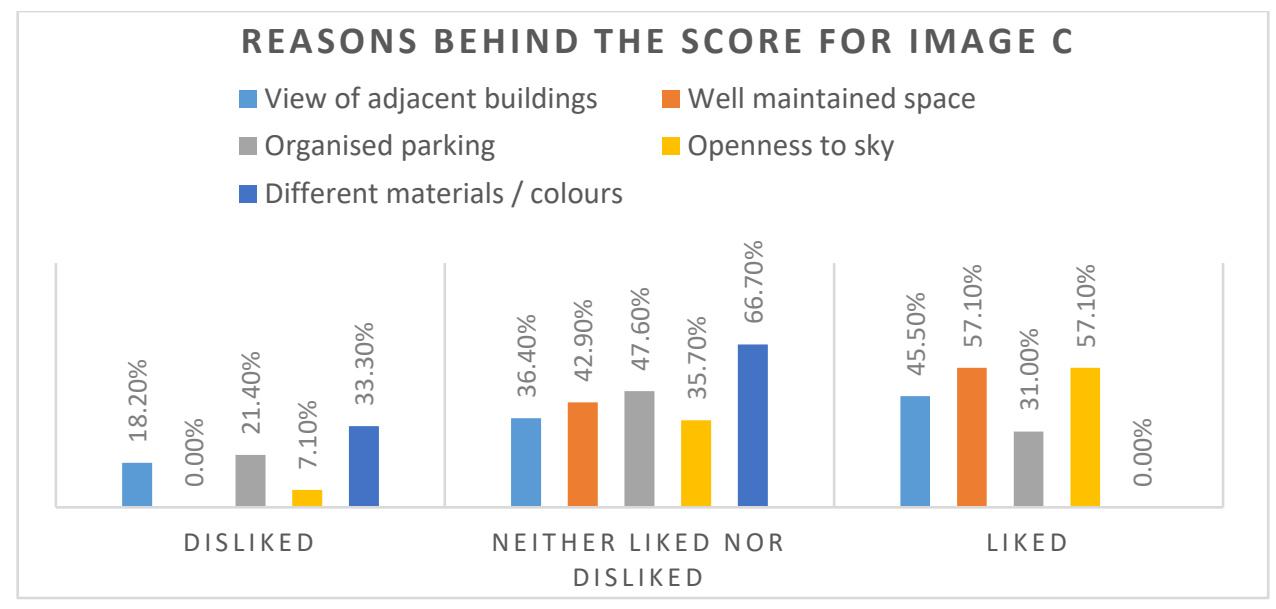

Figure 6.24 Participant reasons behind the rating of images A-C

\subsubsection{Part Four}

In part 4 of Study One participants were shown pairs of photos and were asked to select one of the two that needed transformation. Participants were given a list of options for improving the aesthetics of the space by adding to and removing things from it. Different suggestions were listed for each image out of which participants were asked to choose at least three options for making improvements in the space. The most preferred photo and its suggestion for improvement later formed the basis for the photomontages in Study Two visual preference study (see Chapter 7). However, the percentages of preferences are only calculated for participants who opted to see in option A or Option B.

\subsection{Space underneath a building}

Option A was selected for improvement by $80 \%$ of respondents. Popular suggestions for remodelling option A were related to creating a clear pathway (29\%), improving the lighting (29\%), improving the maintaining and cleanliness (28\%), providing more vegetation (28\%), and changing the surface materials (27\%) (figure 6.26). Common suggestions for improving option B were improving the lighting (10\%), creating a clear pathway $(9 \%)$ and improving the maintenance and cleanliness of the space $(9 \%)$. 


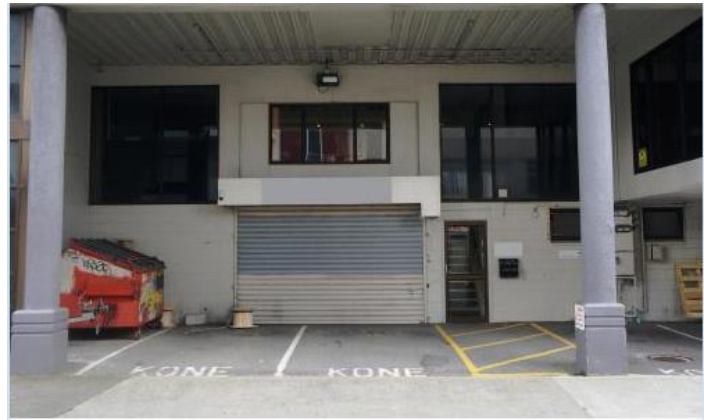

A $(79.50 \%)$

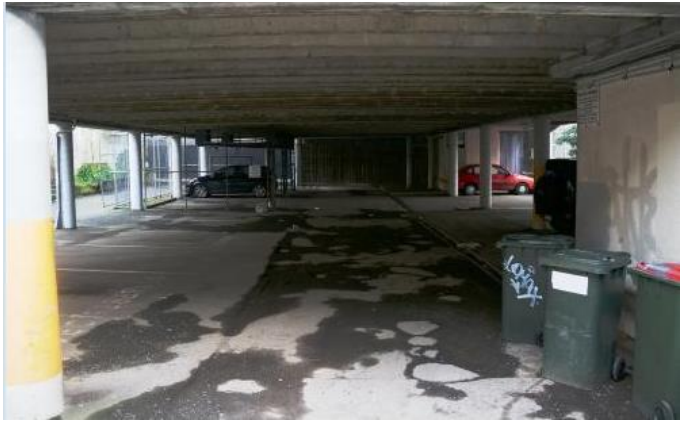

B $(20.50 \%)$

Figure 6.25 Two options shown to participants
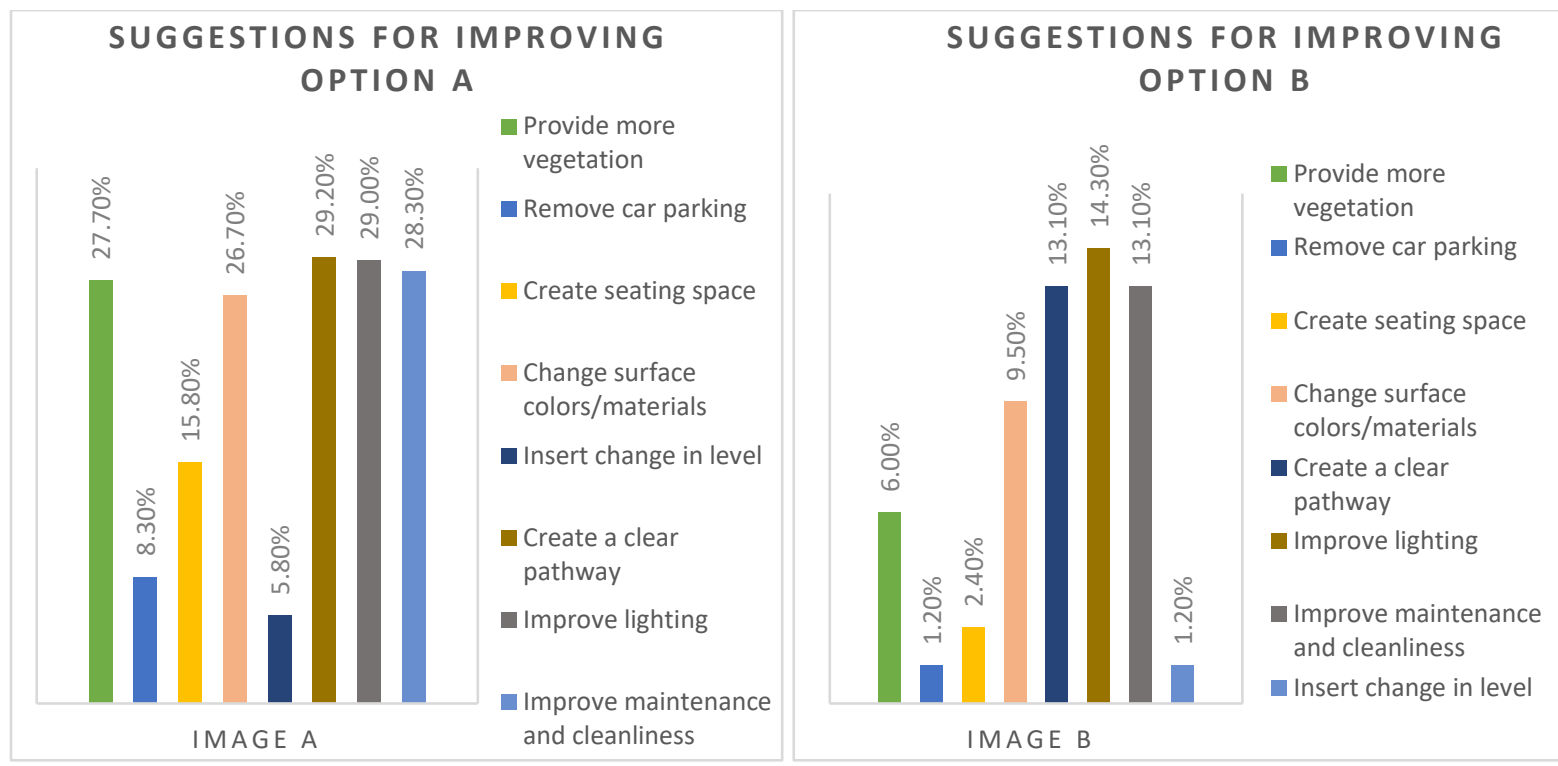

Figure 6.26 Suggestions for improving options $A$ and $B$

\subsection{Space in front of a building}

Overall, $70 \%$ of respondents wanted to change option B and 30\% Option A. Upgrading the space in option $B$ was related to the removal of the boundary wall (32\%), providing more vegetation $(30 \%)$, and creating a clear pathway to enter the building (18\%) (figure 6.28). For option A, participants suggested providing more vegetation (17\%), creating a clear pathway to enter the building (12\%), and removal of the boundary walls $(10 \%)$. 


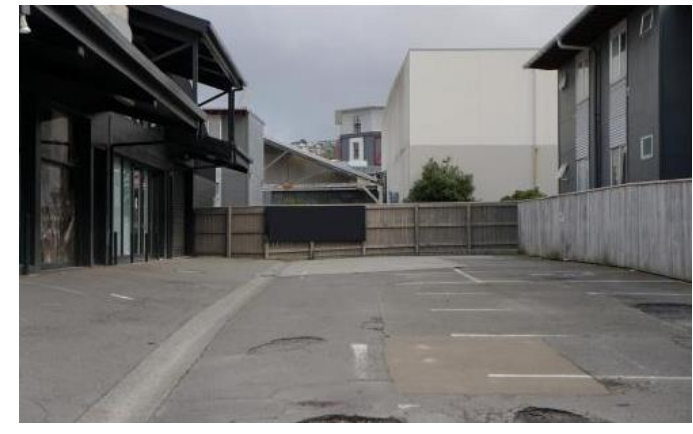

A $(29.80 \%)$

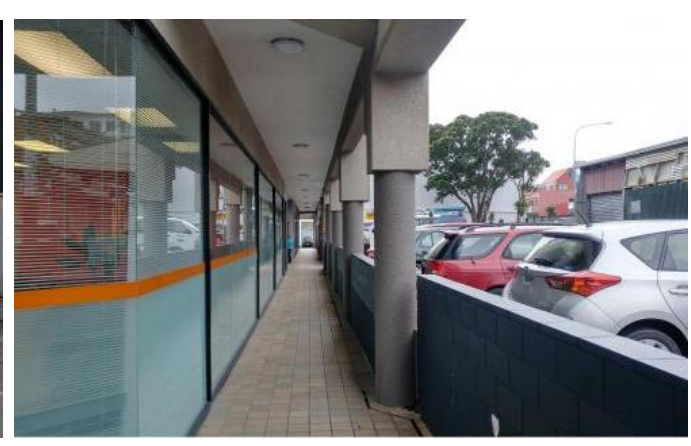

B $(70.20 \%)$

Figure 6.27 Two options shown to participants
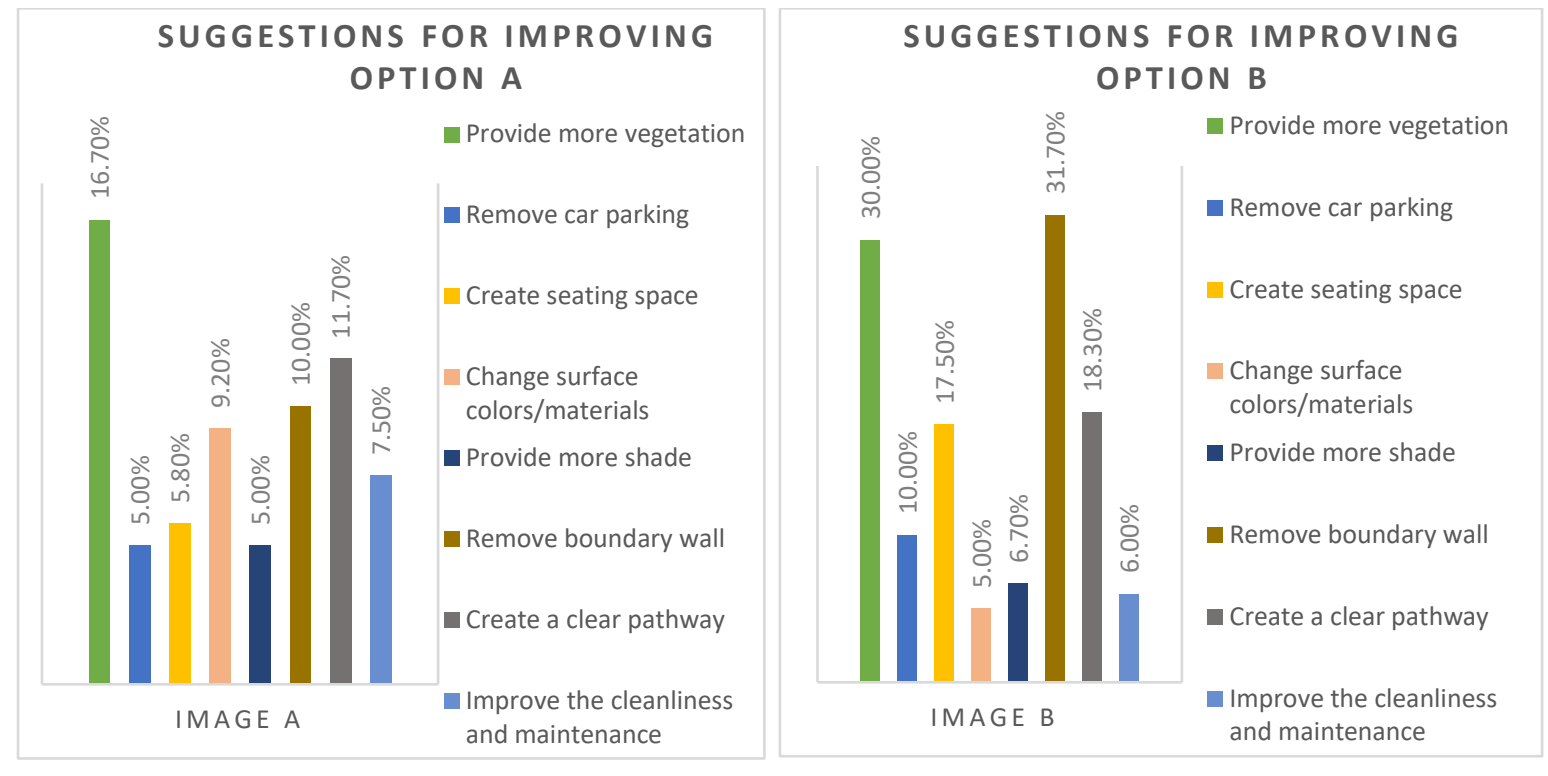

Figure 6.28 Suggestions for improving options A and B

\subsection{Space at the back of a building}

Some $62 \%$ of respondents wanted to change option B while $38 \%$ preferred to change option $A$. The most recurring suggestions for improving option $B$ were related to providing more vegetation (32\%), creating a clear pathway (23\%), and changing the surface materials (22\%) (figure 6.30). Respondents proposed providing more vegetation (19\%), improving the cleanliness and maintenance (15\%), and changing the surface materials (13\%) for option $A$.
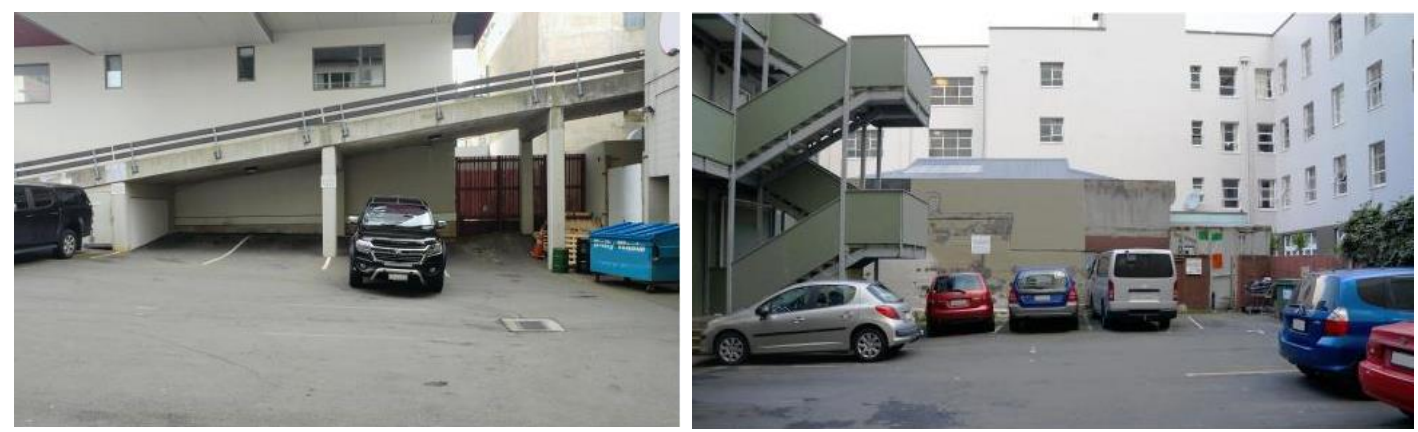

A $(38.60 \%)$
B $(61.40 \%)$

Figure 6.29 Two options shown to participants

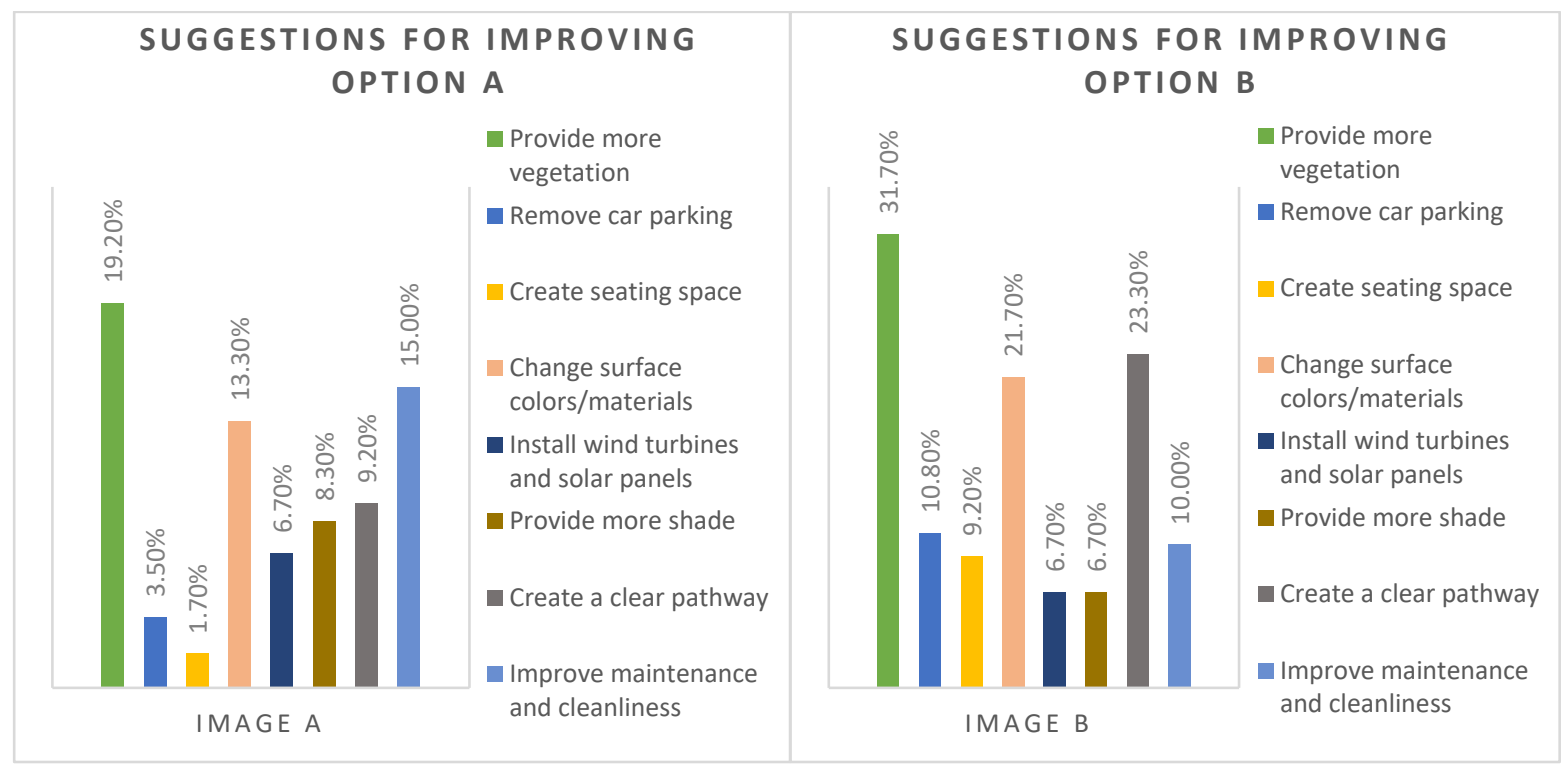

Figure 6.30 Suggestions for improving options A and B

\subsection{Space enclosed by buildings on three sides}

Some $60 \%$ of respondents wanted to change option B with $40 \%$ opting to improve option A. Popular suggestions for enhancing option B related to providing more vegetation $(29 \%)$, changing the surface materials $(21 \%)$, and creating a clear pathway (20\%) (figure 6.32). The respondents proposed providing more vegetation (21\%), creating a clear pathway (14\%), and allowing graffiti on the blank wall $(10 \%)$ as improvements for option $A$.

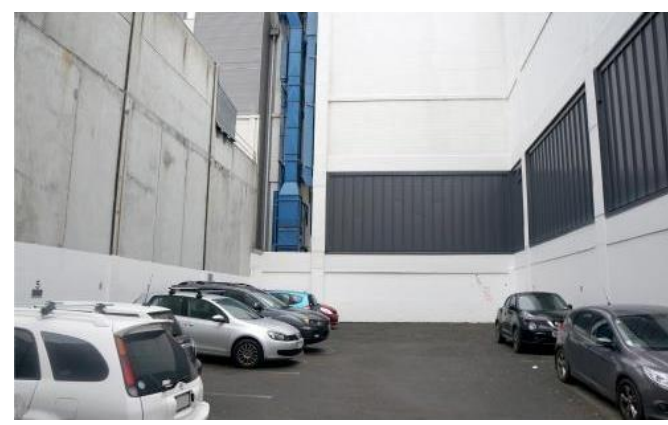

A $(39.80 \%)$

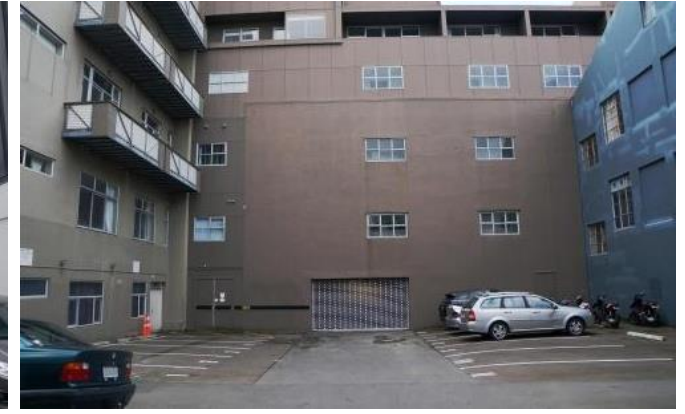

B $(60.20 \%)$

Figure 6.31 Two options shown to participants 


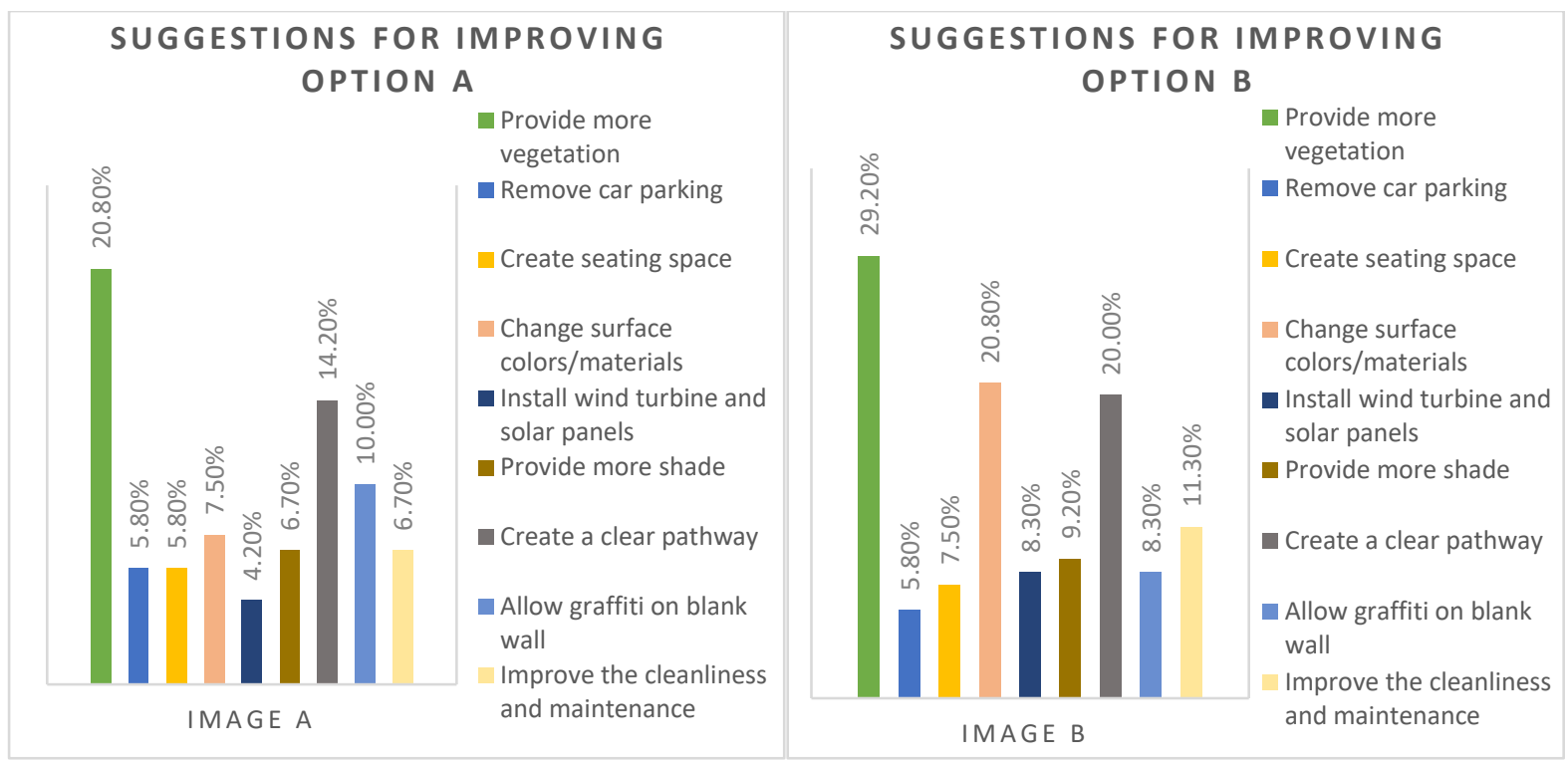

Figure 6.32 Suggestions for improving options A and B

\subsection{Space enclosed by buildings on two sides}

Of the respondents, $82 \%$ wanted to change option $A$ and $18 \%$ option $B$. The most recurring suggestions for option A related to providing more vegetation (36\%), creating a clear pathway (34\%), and changing the surface materials (20\%) (figure 6.34). The suggestions for improving option B were related to creating a clear pathway $(09 \%)$ and providing more vegetation (08\%).

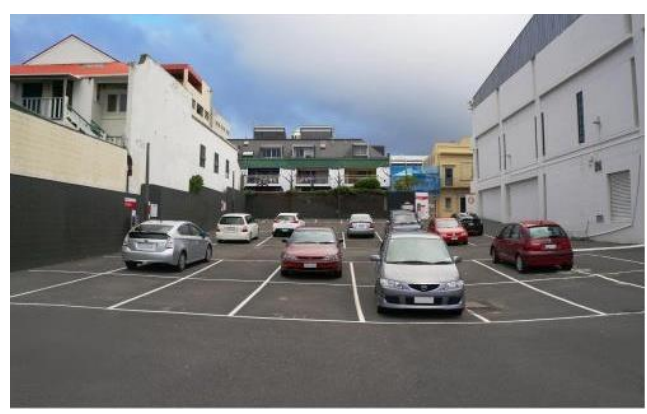

A $(81.90 \%)$

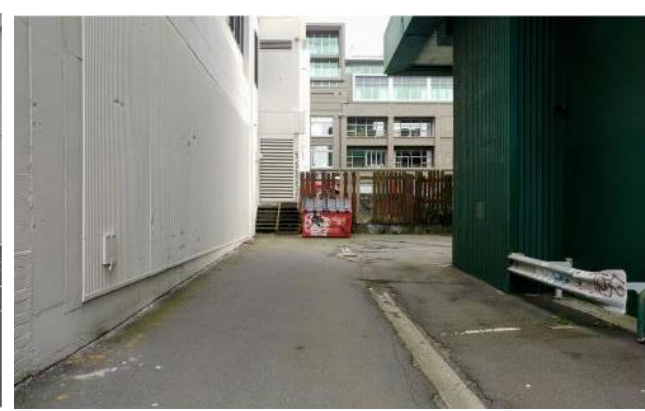

B $(18.10 \%)$

Figure 6.33 Two options shown to participants 

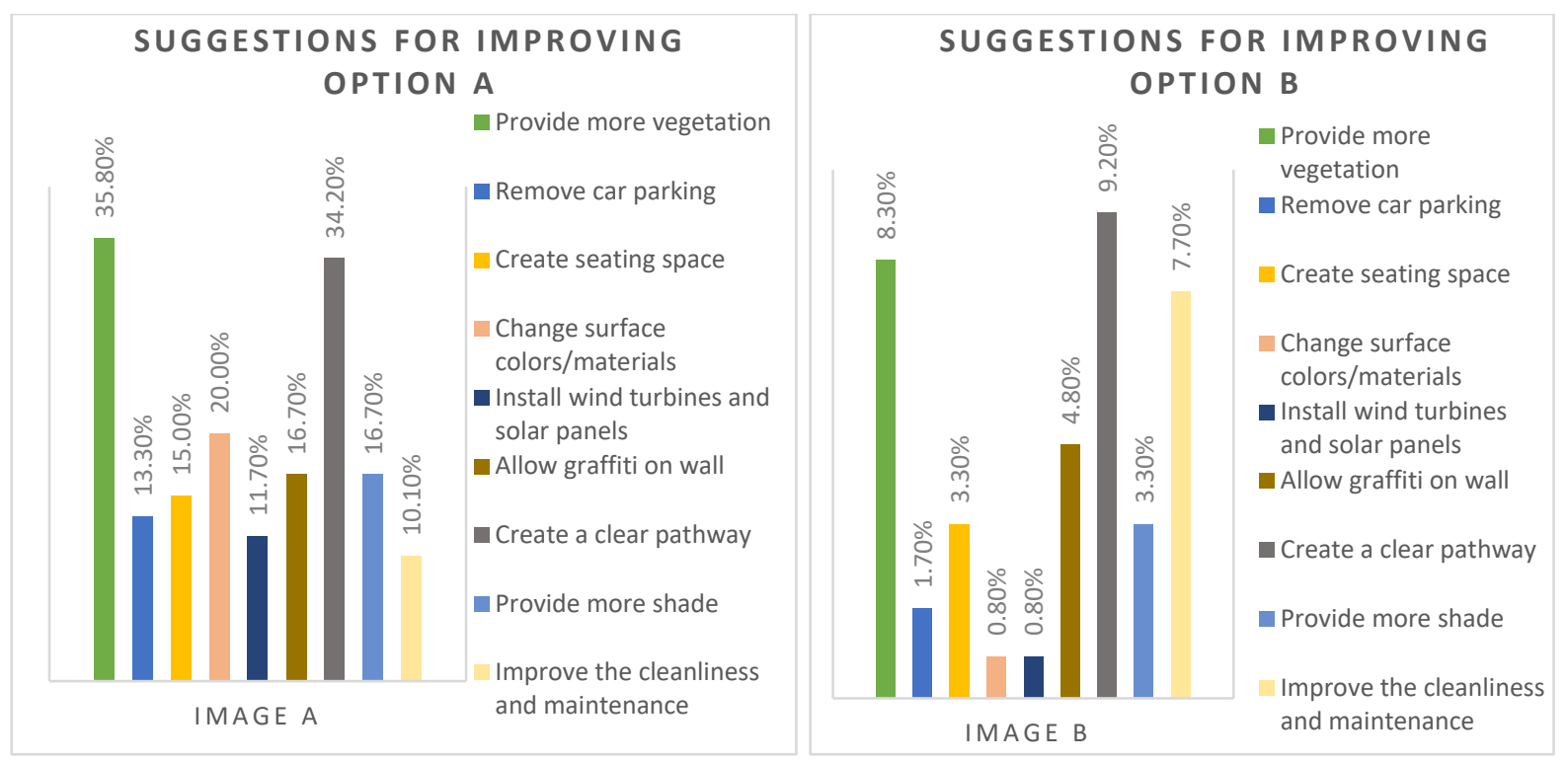

Figure 6.34 Suggestions for improving options A and B

\subsection{Space on the rooftop of a building}

Overall $54 \%$ of respondents wanted to change option B and $46 \%$ option A. The most common suggestions for improving option $B$ related to providing more vegetation $(29 \%)$, creating a seating space $(20 \%)$, and installing wind turbines and solar panels (17\%) (figure 6.36). For option $A$, the respondents proposed the same suggestions of installing wind turbines and solar panels (21\%), adding more vegetation (17\%), and creating a seating space (13\%).

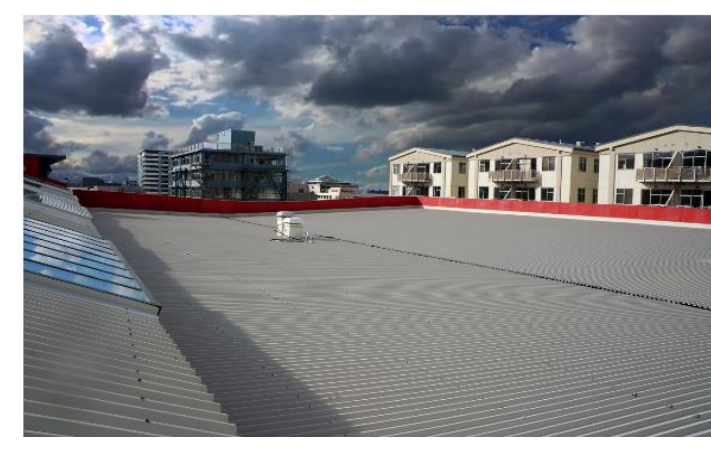

A $(46.30 \%)$

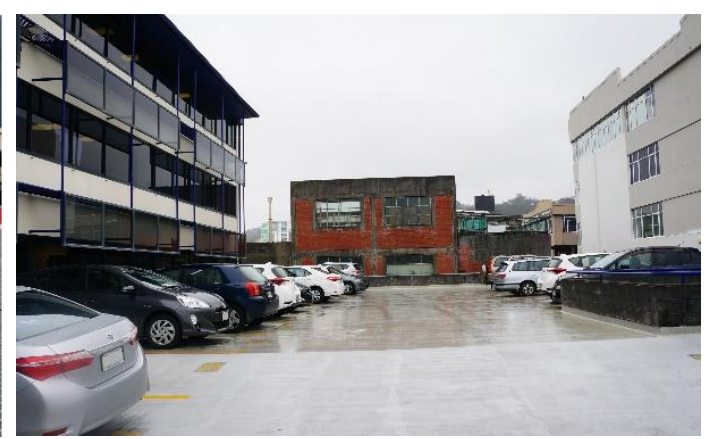

B $(53.70 \%)$

Figure 6.35 Two options shown to participants 
Chapter 6 - STUDY ONE: FIRST VISUAL PREFERENCE STUDY
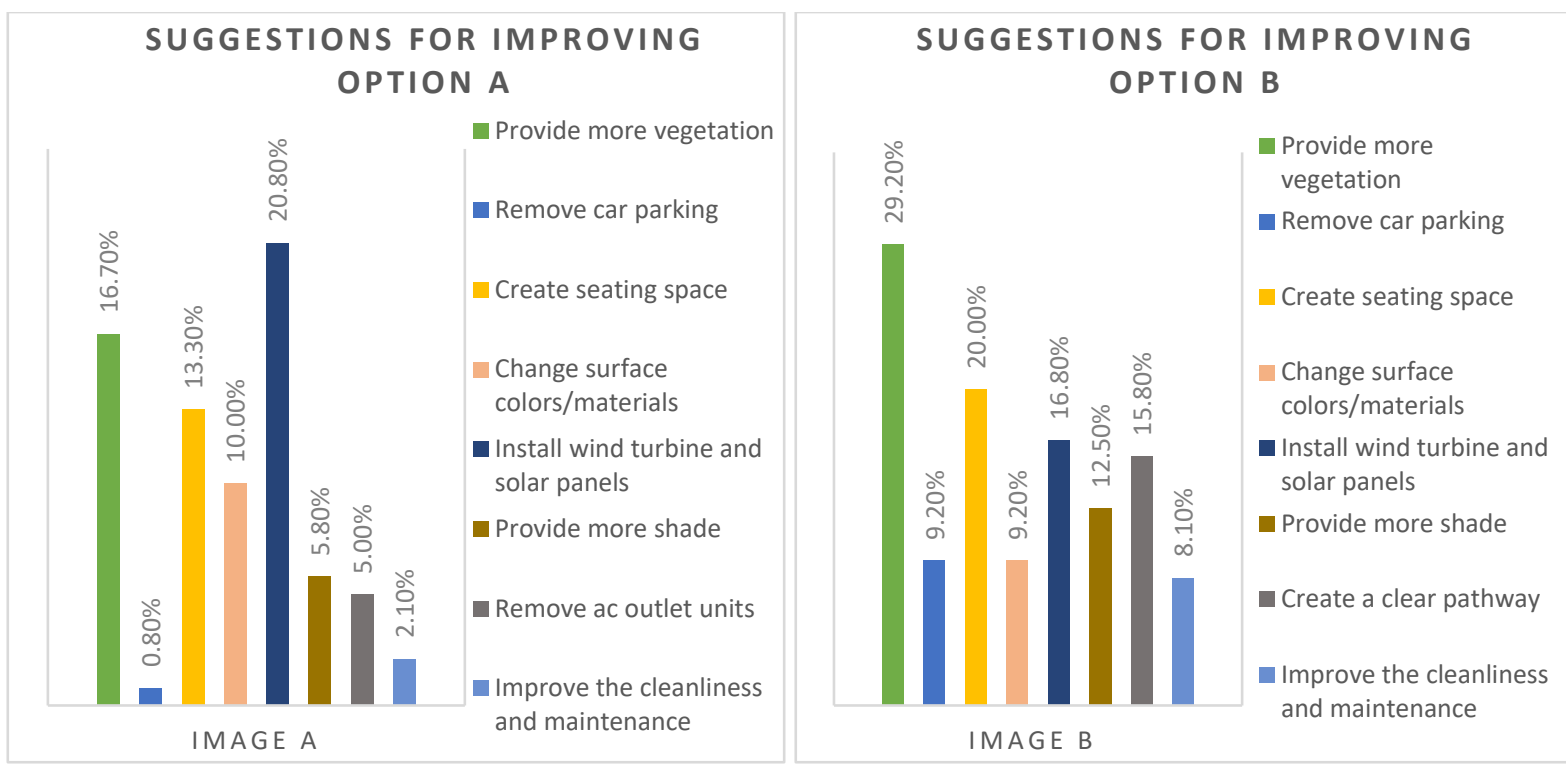

Figure 6.36 Suggestions for improving options A and B

\subsubsection{Analysis of Stage two: Gender Differences}

In the second stage, the analysis was carried out for the subgroups of 42 males (42.4\%) and 57 females (57.6\%) out of 99 participants.

\subsubsection{Part One}

Part one has already been reported (see section 6.3.1).

\subsubsection{Part Two}

\subsection{Natural elements in a space}

In the first pair, $95 \%$ of males and $96 \%$ of females favoured the space with natural features (figure 6.37 ), with the remaining $5 \%$ of males and $4 \%$ of females preferring the space with artificial elements in it.
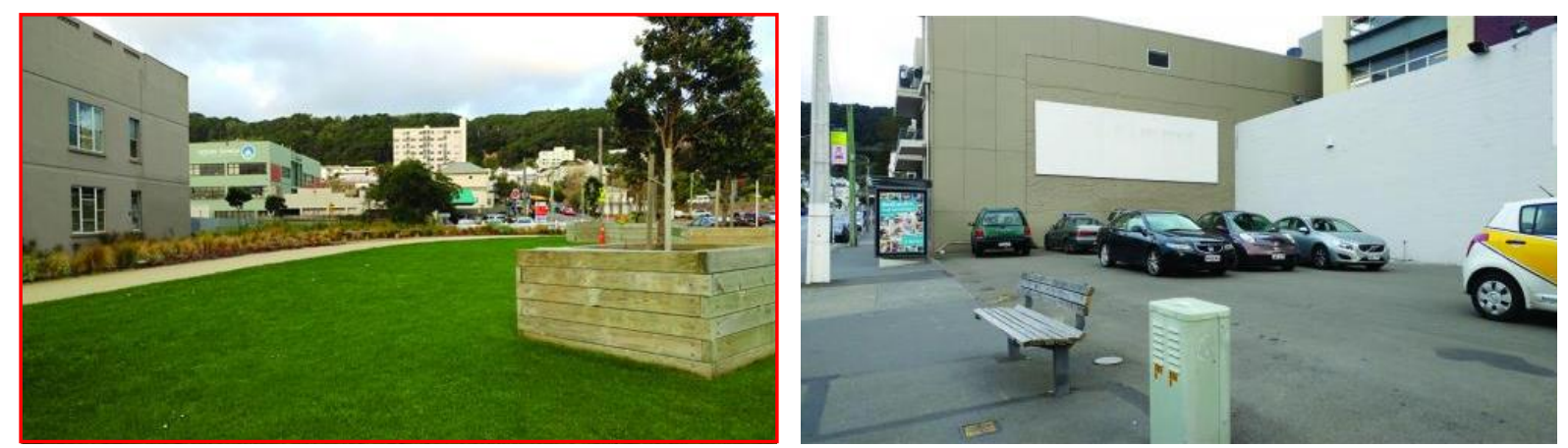

Figure 6.37 Space with natural elements was preferred over space with artificial elements 


\subsection{Organised space}

In the second pair, $86 \%$ of males and $89 \%$ of females preferred the organised space with $14 \%$ and $11 \%$ of males and females respectively preferring the less organised space (figure 6.38).
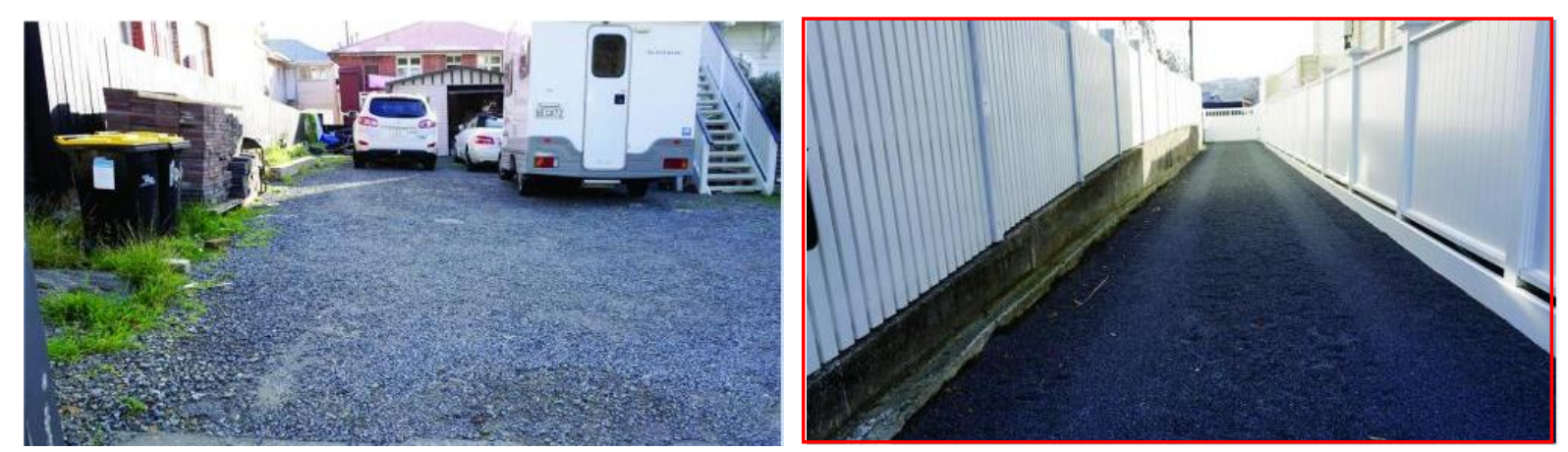

Figure 6.38 Well-organised space was preferred over disorganised space

\subsection{Open space}

In the third pair, although males showed little difference in preference with $51 \%$ preferring the space open to the sky and $49 \%$ opting for the more enclosed space, females showed a definite preference for the space open to the sky (72\% compared to $28 \%$ ) (figure 6.39 ).
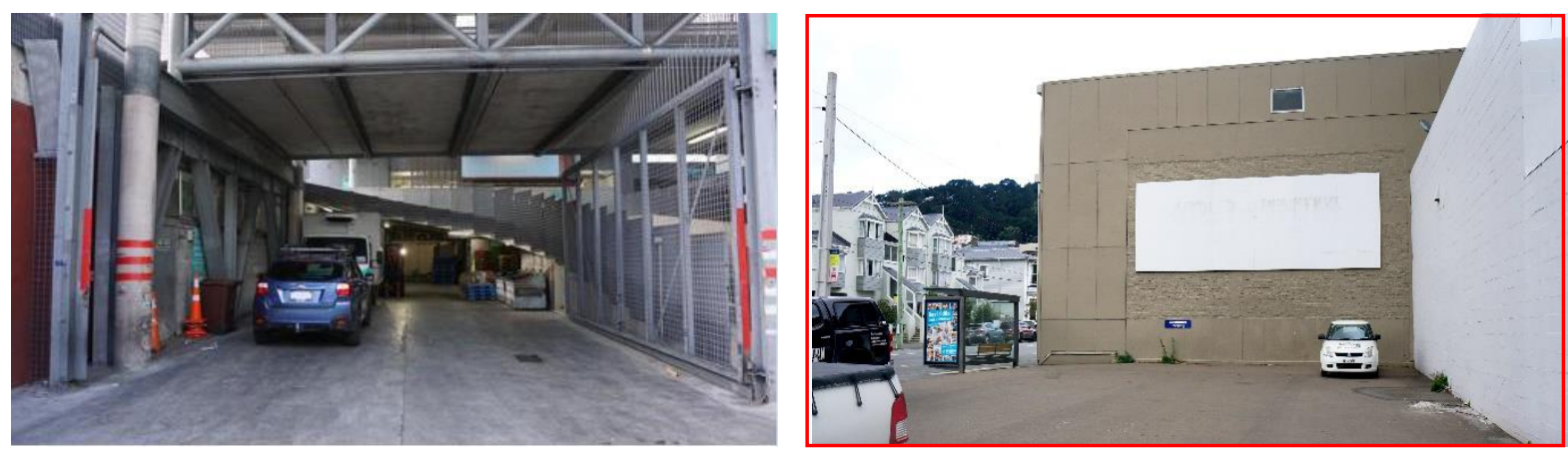

Figure 6.39 Space open to the sky was preferred over an enclosed space

\subsection{Well maintained space}

In the fourth pair, $74 \%$ of males and $82 \%$ of females preferred the well-maintained space, with $26 \%$ of males and $18 \%$ of females opting for the more unkempt space (figure 6.40). 

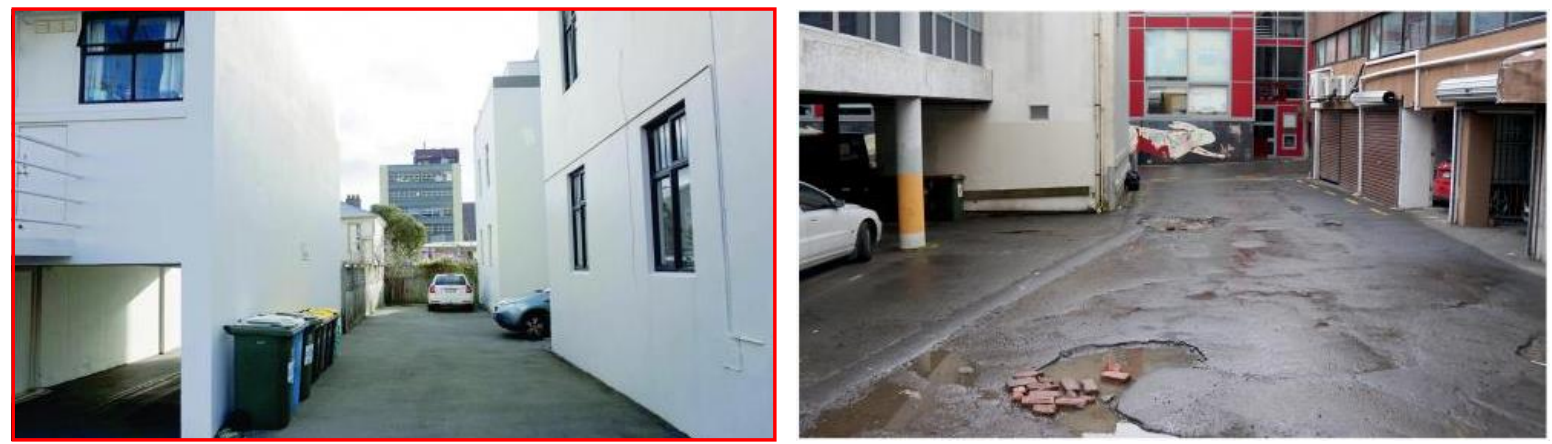

Figure 6.40 The well-maintained space was preferred over the less well-maintained space

\subsection{Modern Building style}

In the fifth pair, $73 \%$ of males and $65 \%$ of females preferred the image with the modern style building in it (figure 6.41). More females than males preferred the older style of building ( $35 \%$ compared to $27 \%$ ).
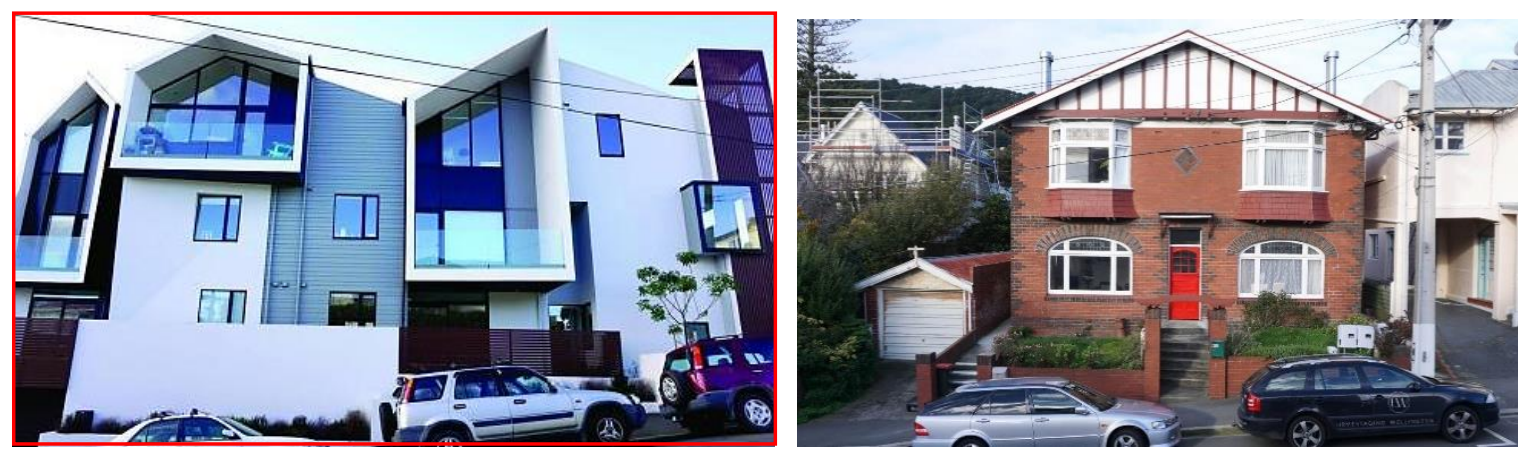

Figure 6.41 Modern building style was preferred over the older style of building The gender preferences for different spaces are presented in figure 6.42 


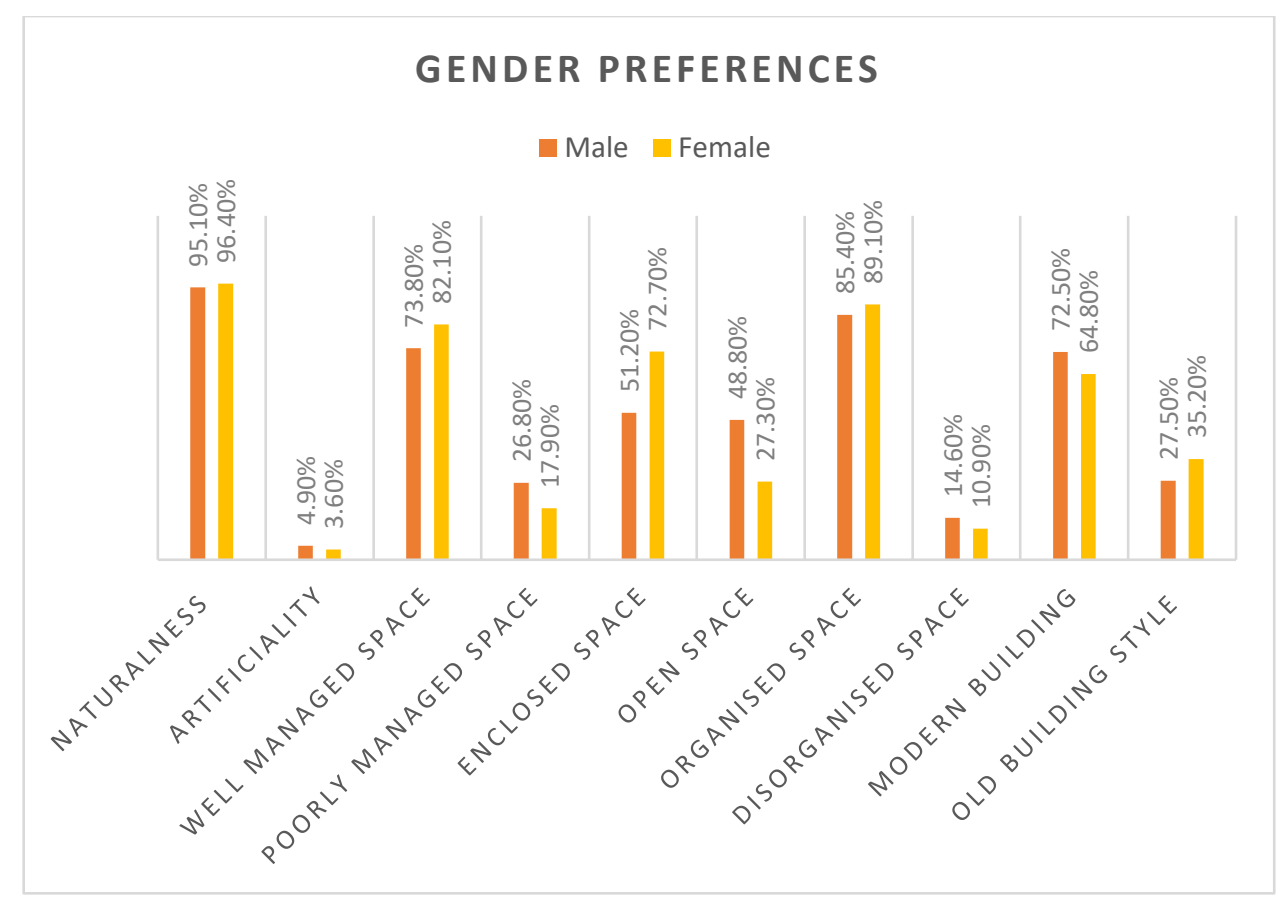

Figure 6.42 Preferences for spaces with different attributes

\subsubsection{Part Three}

\subsection{Space underneath a building}

For the space underneath a building, 39 males and 45 females responded to the question. Option C (see figure 6.43) was the most preferred by both genders, with $100 \%$ of males and $96 \%$ of females choosing it. This option had the highest mean score of 4.62 with a Standard deviation (SD) of 0.49 for males, and a mean score of 4.58 with an SD of 0.58 for females. The second highest preference was for option A, which was liked by $26 \%$ of males and $31 \%$ of females. This option had a mean score of 2.87 with an SD of 1.17 for males and a mean score of 2.85 with an SD of 1.19 for females. Option B was the least preferred, with only $32 \%$ of males, and $20 \%$ of females choosing it (figure 6.44). Though more males liked this option than $A$, the mean score was less than for option A. Image B had a mean score of 2.85 with an SD of 1.22 for males and a mean score of 2.67 with an SD of 1.26 for females. 
A
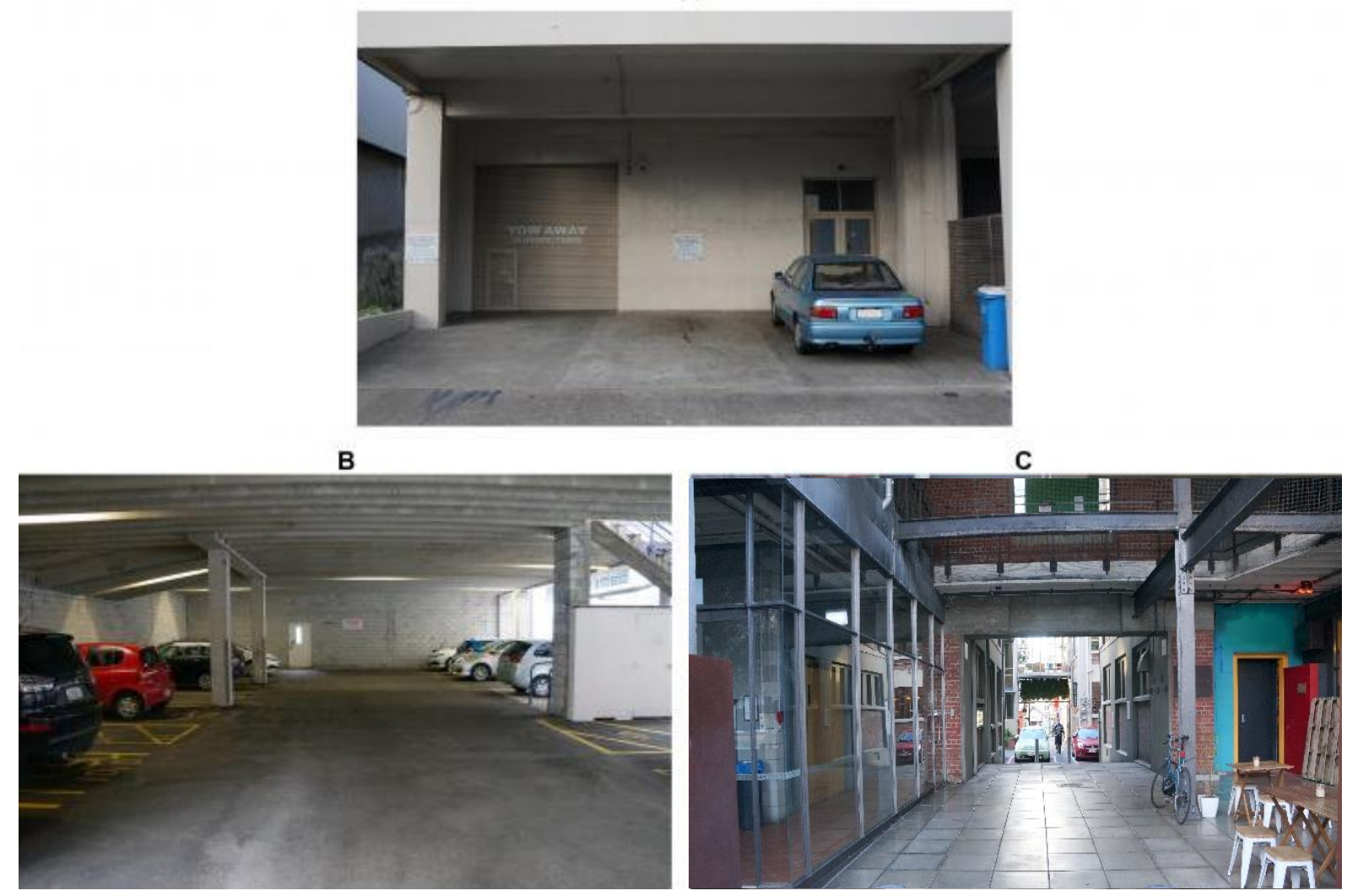

Figure 6.43 Options shown to participants

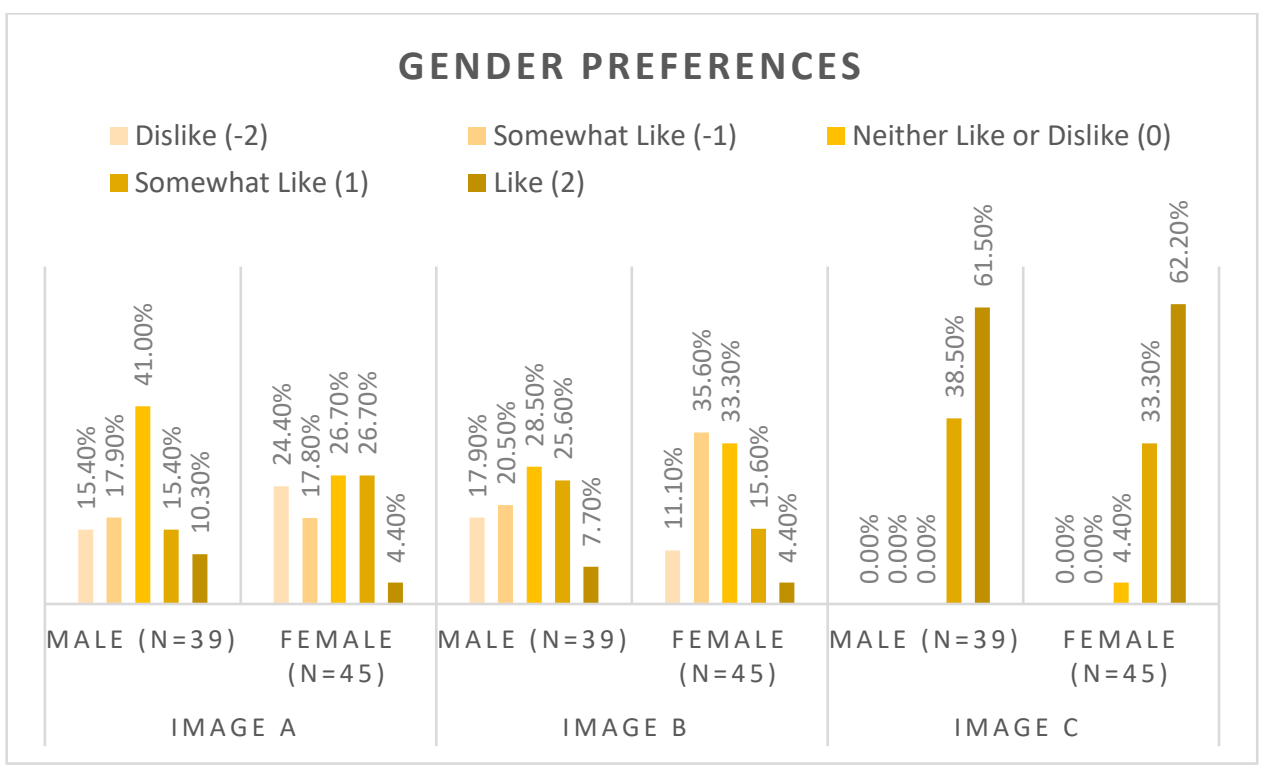

Figure 6.44 Gender preferences for the different options

\subsection{Space in front of a building}

For the space in front of a building, 41 males and 53 females responded to the question. Option A (see figure 6.45) was the most liked by both genders, with $100 \%$ 
of males and $96 \%$ of females liking it. This option had the highest mean score of 4.52 with a Standard deviation (SD) of 0.70 for males and a mean score of 4.45 with an SD of 0.79 for females. The second preference was for option C, which was liked by $41 \%$ of males and $55 \%$ of females. This option received a mean score of 3.19 with an SD of 1.23 for males and a mean score of 3.36 with an SD of 1.00 for females. Option B was liked by $12 \%$ of males, and $21 \%$ of females (figure 6.46 ), with a mean score of 2.44 with an SD of 1.22 for males and a mean score of 2.53 with an SD of 1.20 for females.

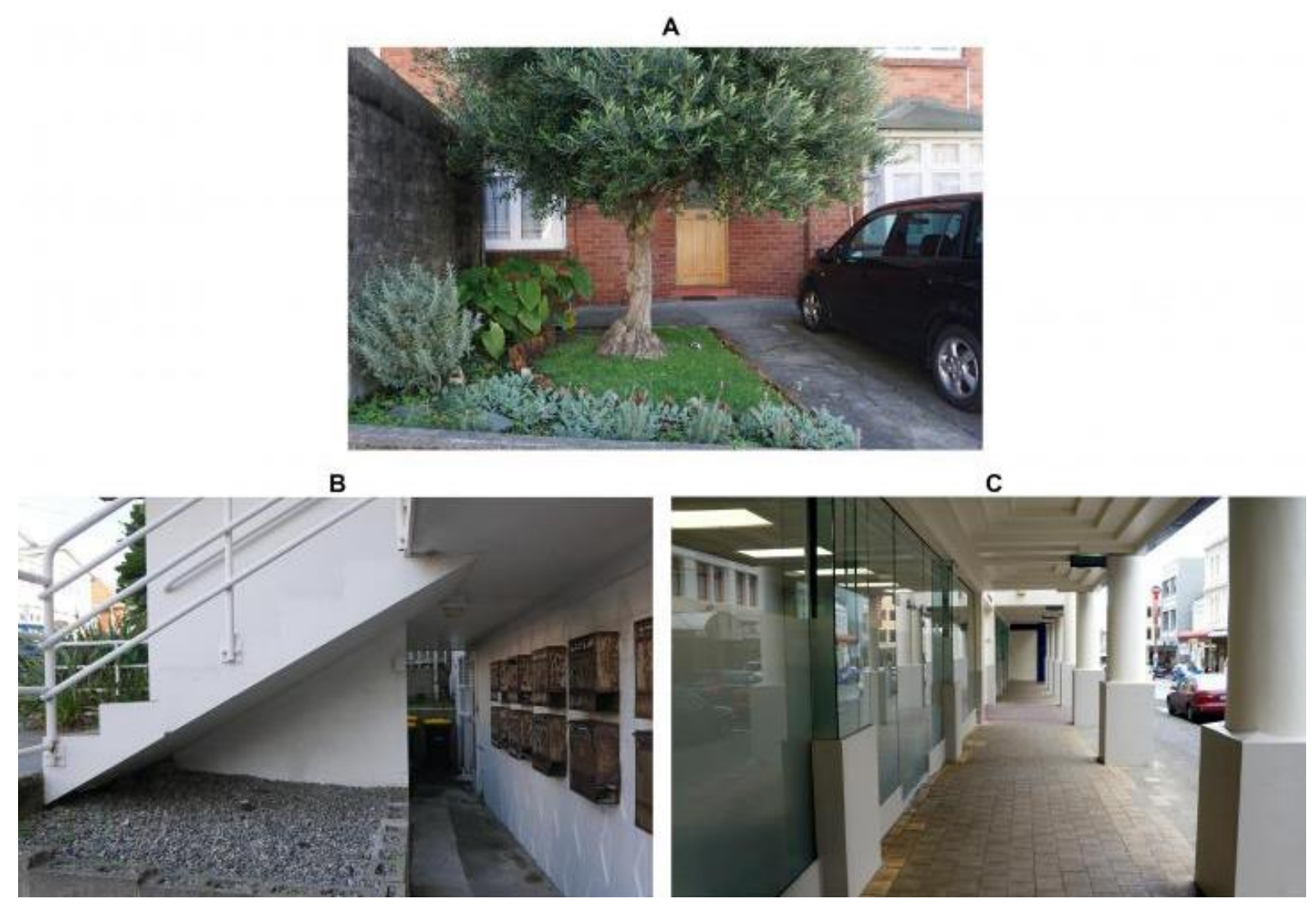

Figure 6.45 Options shown to participants 


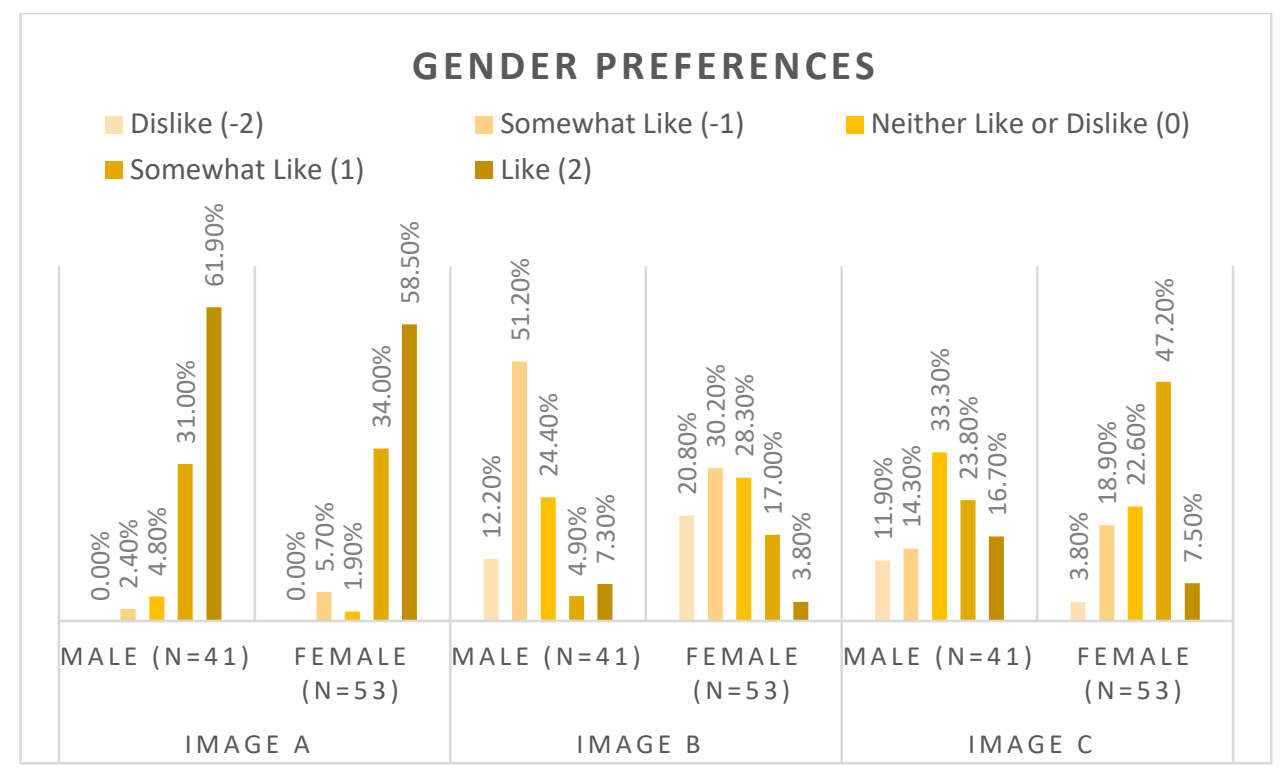

Figure 6.46 Gender preferences for the different options

\subsection{Space at the back of a building}

For the space at the back of a building, 40 males and 51 females answered the question. Option B (figure 6.47) was the most liked by both genders, with $50 \%$ of males and $57 \%$ of females liking it. This option had the highest mean score of 3.25 with a Standard deviation (SD) of 1.21 for males and a mean score of 3.39 with an SD of 1.15 for females. Option A was liked by $40 \%$ males and $47 \%$ females. This option had a mean score of 3.18 with an SD of 1.19 for males and a mean score of 3.20 with an SD of 1.18 for females. Option $C$ was only liked by $32 \%$ of males, but $41 \%$ of females liked the space (figure 6.48). Image $C$ had a mean score of 2.98 with an SD of 1.22 for males and a mean score of 3.10 with an SD of 1.20 for females. 

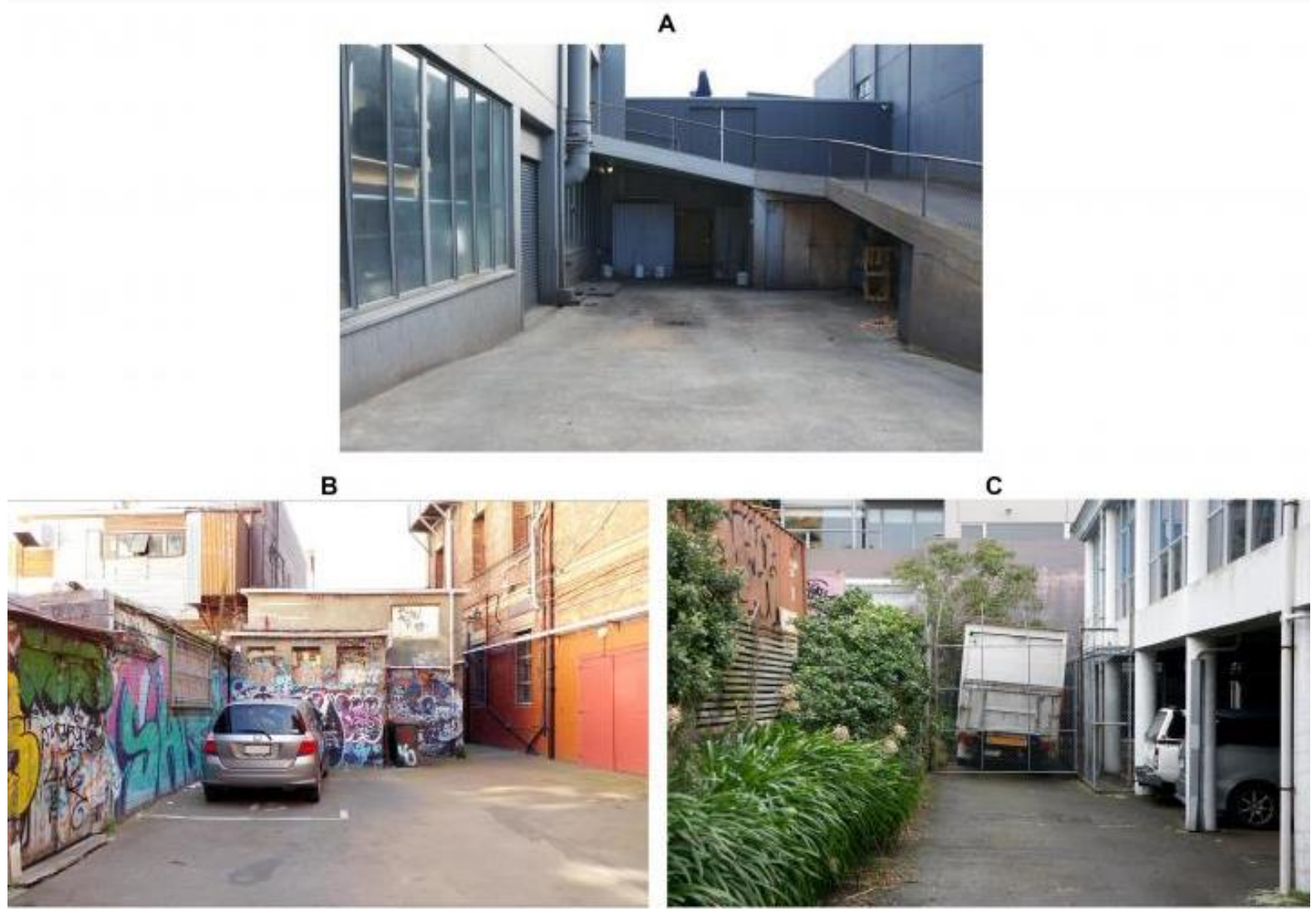

Figure 6.47 Options shown to participants

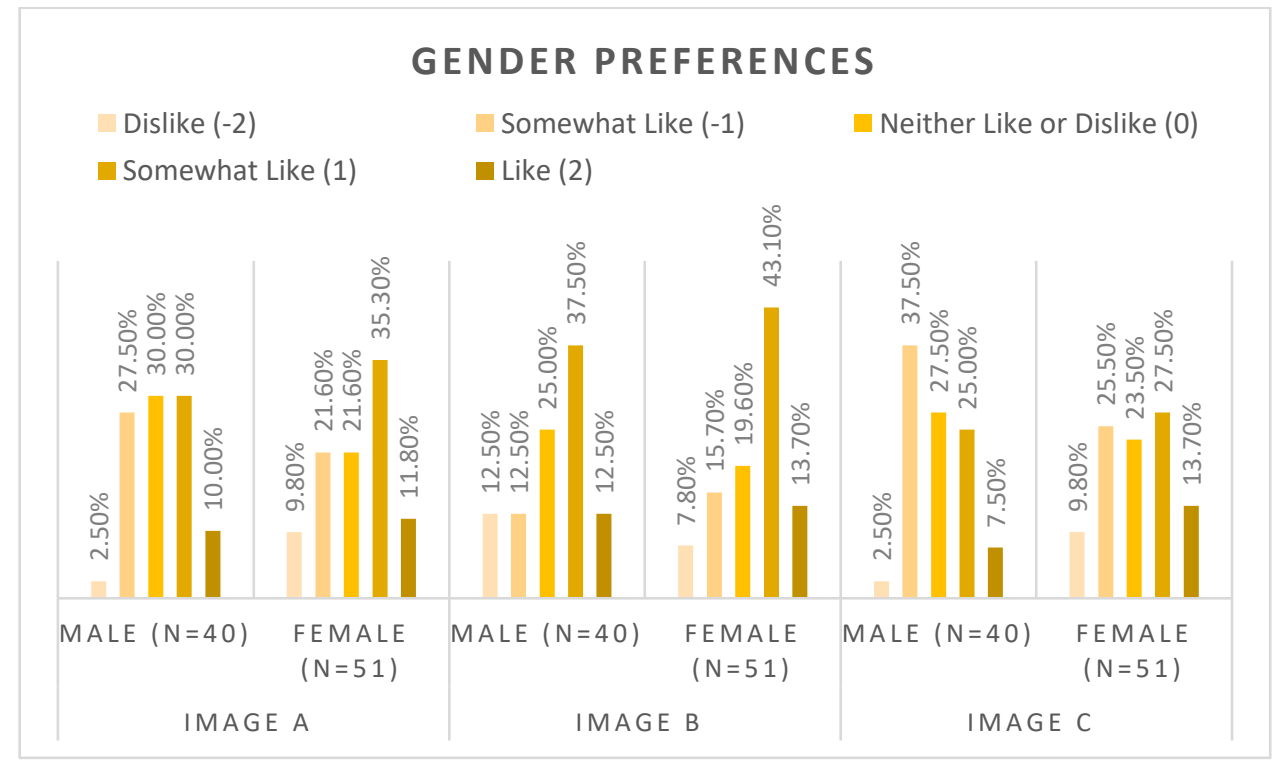

Figure 6.48 Gender preferences for the different options

\subsection{Space enclosed by buildings on three sides}

For the space enclosed by buildings on three sides, 39 males and 45 females answered the question. Option $C$ (figure 6.49) was the most liked by both genders, 
with $77 \%$ of males and $83 \%$ of females liking or somewhat liking it. This option also had the highest mean score of 4.00 with a Standard deviation (SD) of 1.10 for males and a mean score of 4.20 with an SD of 0.85 for females. The second most liked space was option A, which was liked by $46 \%$ of males and $44 \%$ of females. This option received a mean score of 3.08 with an SD of 1.25 for males and a mean score of 3.13 with an SD of 1.23 for females. Option B was least liked, with $26 \%$ of males and $29 \%$ of females liking or somewhat liking the space (figure 6.50). Image B had a mean score of 2.51 with an SD of 1.23 for males and a mean score of 2.80 with an SD of 1.15 for females.

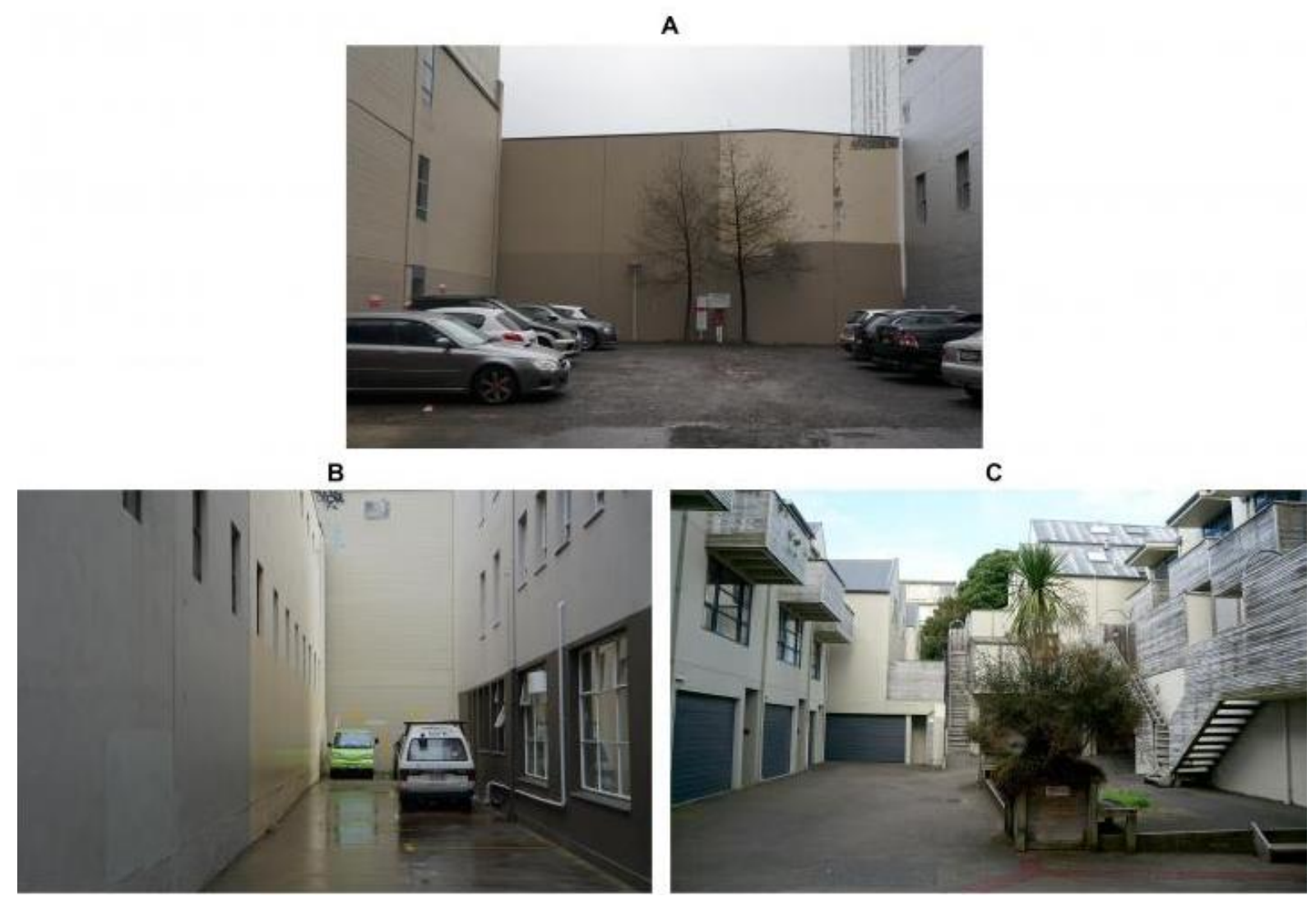

Figure 6.49 Options shown to participants 


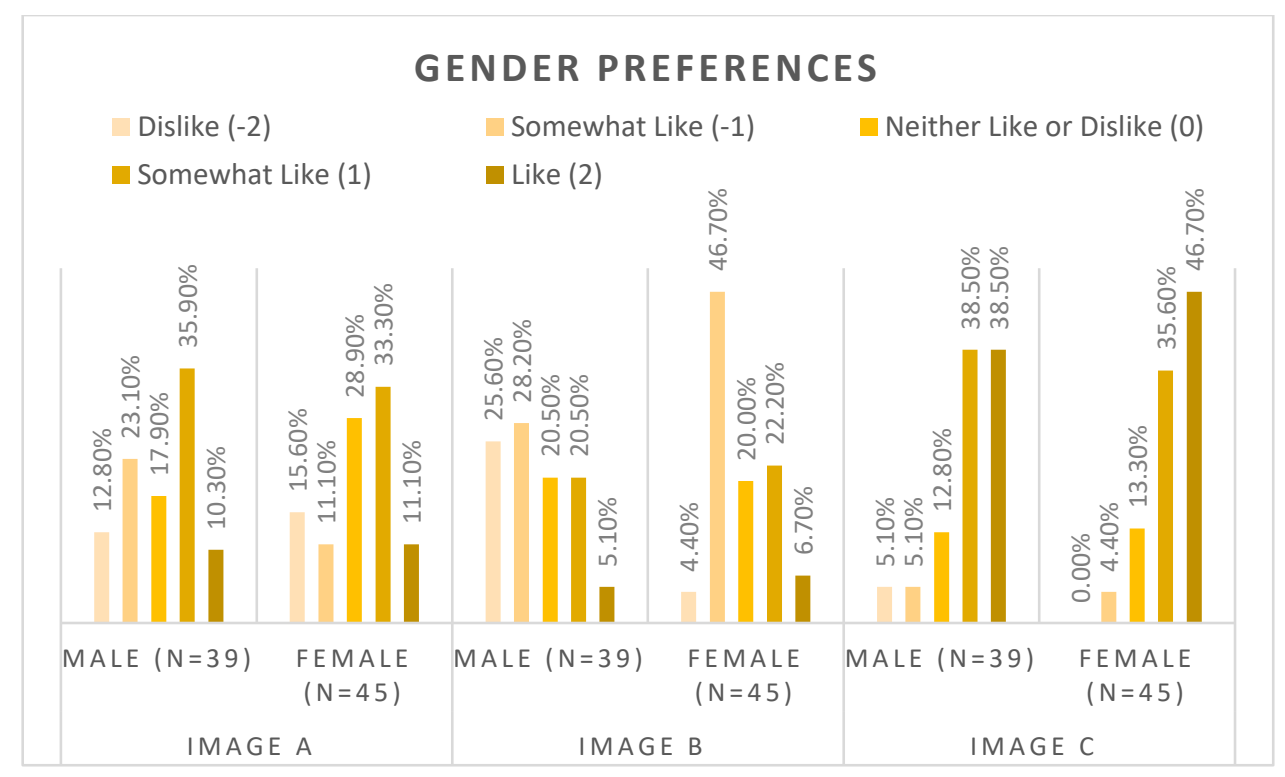

Figure 6.50 Gender preferences for the different options

\subsection{Space enclosed by buildings on two sides}

The question relating to the space enclosed by the buildings on two sides was answered by 39 males and 48 females. Option C (figure 6.51) was the most liked by both genders, with $44 \%$ of males and $40 \%$ of females liking or somewhat liking it. This option also had the highest mean score of 3.00 with a Standard Deviation (SD) of 1.20 for males and a mean score of 3.25 with an SD of 1.12 for females. The second most liked space was option B, which was liked/somewhat liked by $34 \%$ of males and $33 \%$ of females. This option received a mean score of 2.87 with an SD of 1.17 for males and a mean score of 3.02 with an SD of 1.12 for females. Option A was least liked with $26 \%$ males and $21 \%$ of females feeling positive towards space (figure 6.52 ). Image $A$ had a mean score of 2.77 with an SD of 1.22 for males and a mean score of 2.67 with an SD of 1.23 for females. 
A

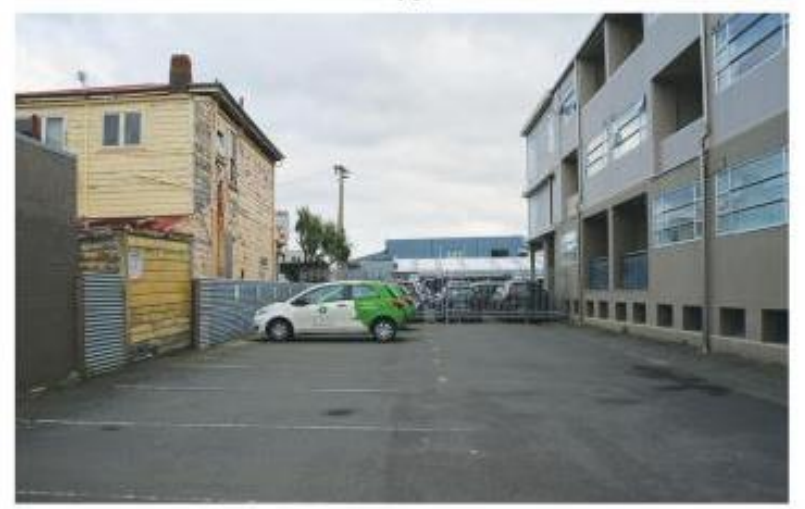

B
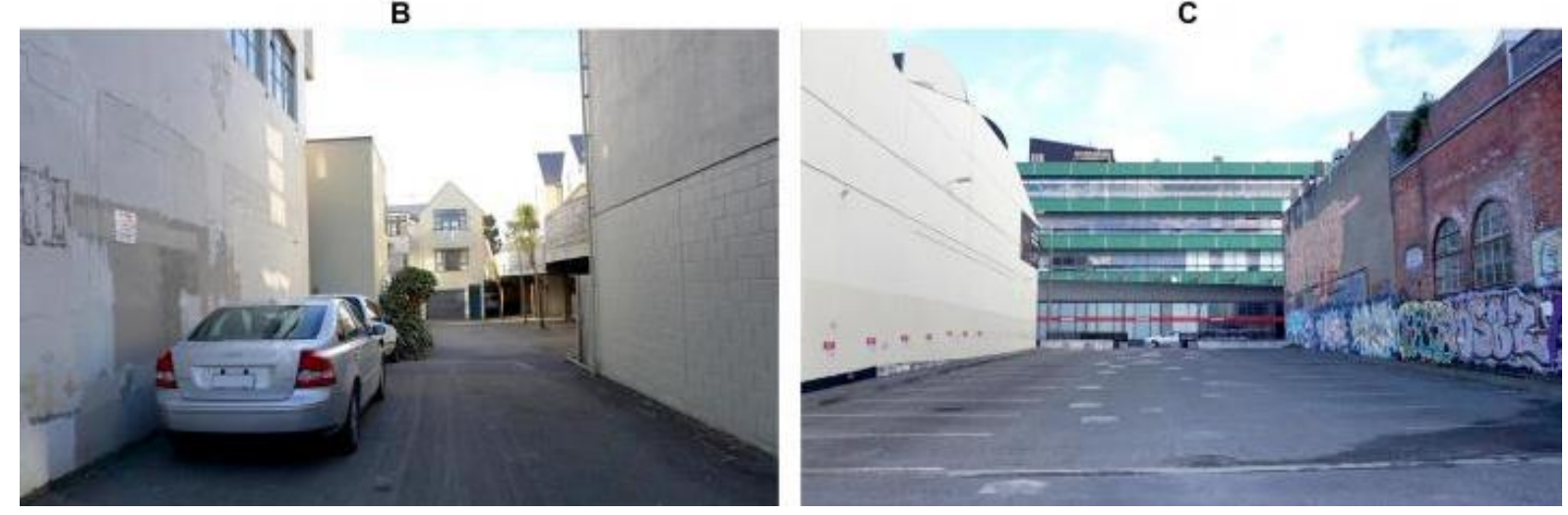

Figure 6.51 Options shown to participants

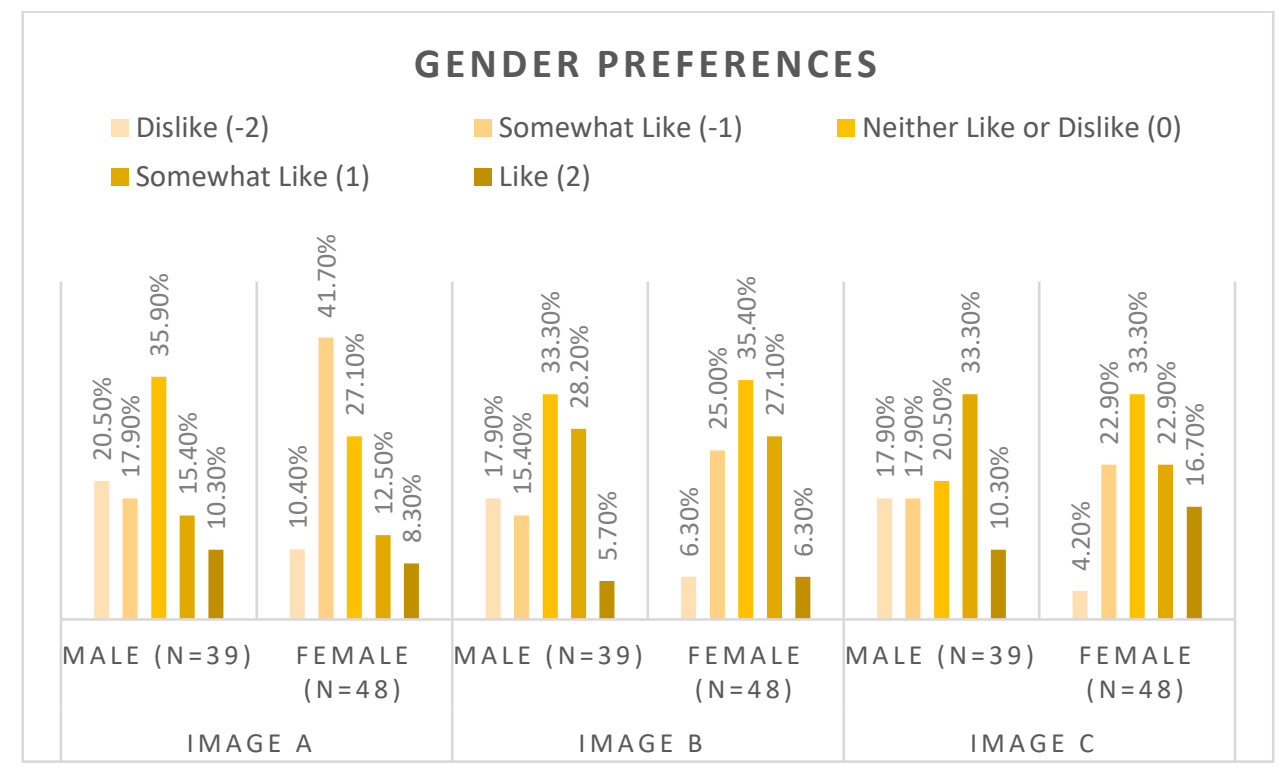

Figure 6.52 Gender preferences for the different options

\subsection{Space on the rooftop of a building}

The same 39 males and 45 females responded to this question. Option C (figure 6.53) was the most liked by both genders, with $41 \%$ of males and $33 \%$ of females liking/somewhat liking it. This option also received the highest mean score of 3.18 with 
a Standard Deviation (SD) of 1.09 for males and a mean score of 3.13 with an SD of 1.14 for females. The second most liked space was option A, as $31 \%$ of males and $40 \%$ of females felt positive towards it. This option had a mean score of 2.72 with an SD of 1.23 for males and a mean score of 3.02 with an SD of 1.17 for females. Option A was liked more by females than option $C$ but had a lower mean score. Lastly, just $18 \%$ males and $18 \%$ of females Liked option $B$ (figure 6.54 ), which had a mean score of 2.26 with an SD of 1.28 for males and a mean score of 2.18 with an SD of 1.30 for females.

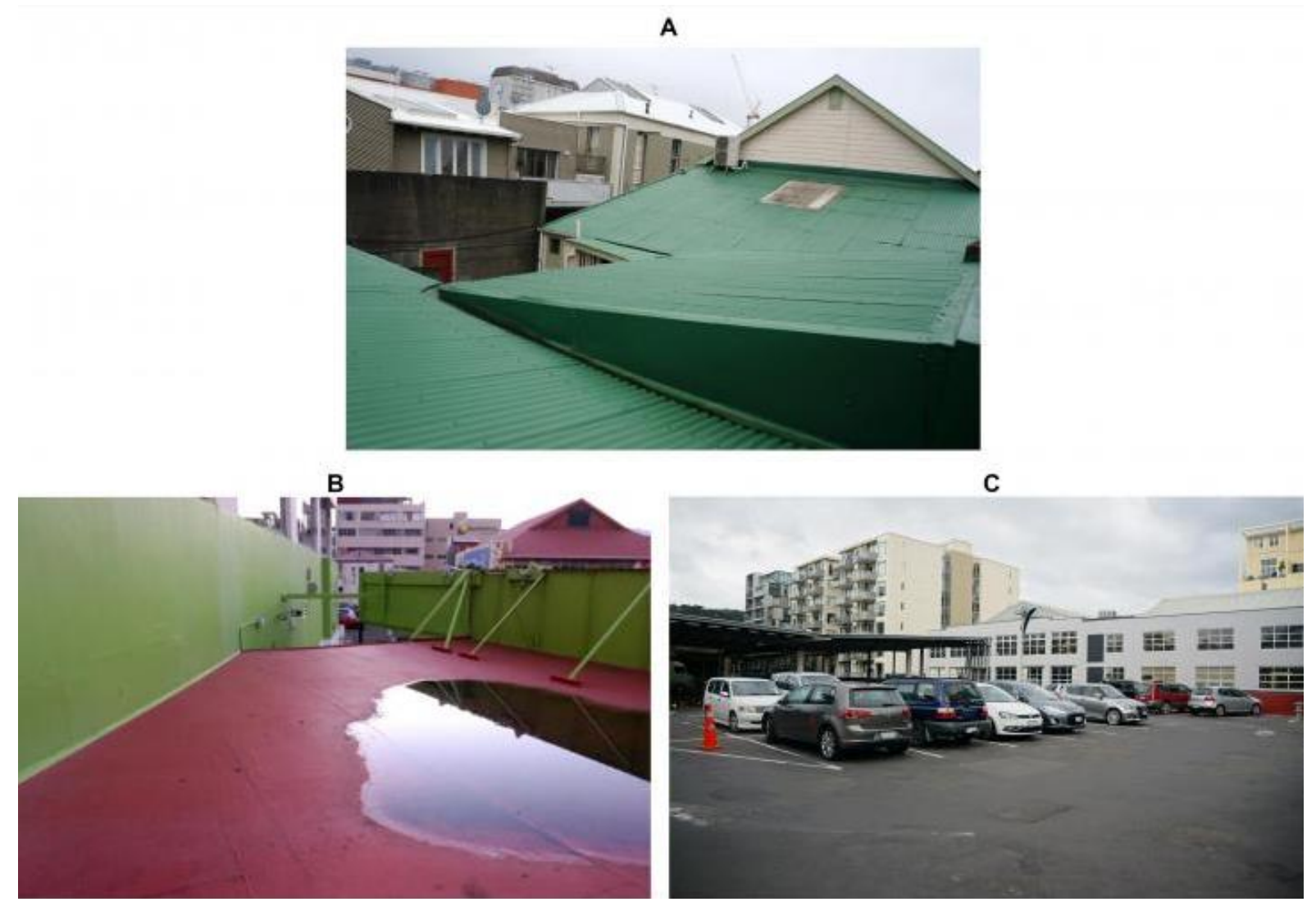

Figure 6.53 Options shown to participants 


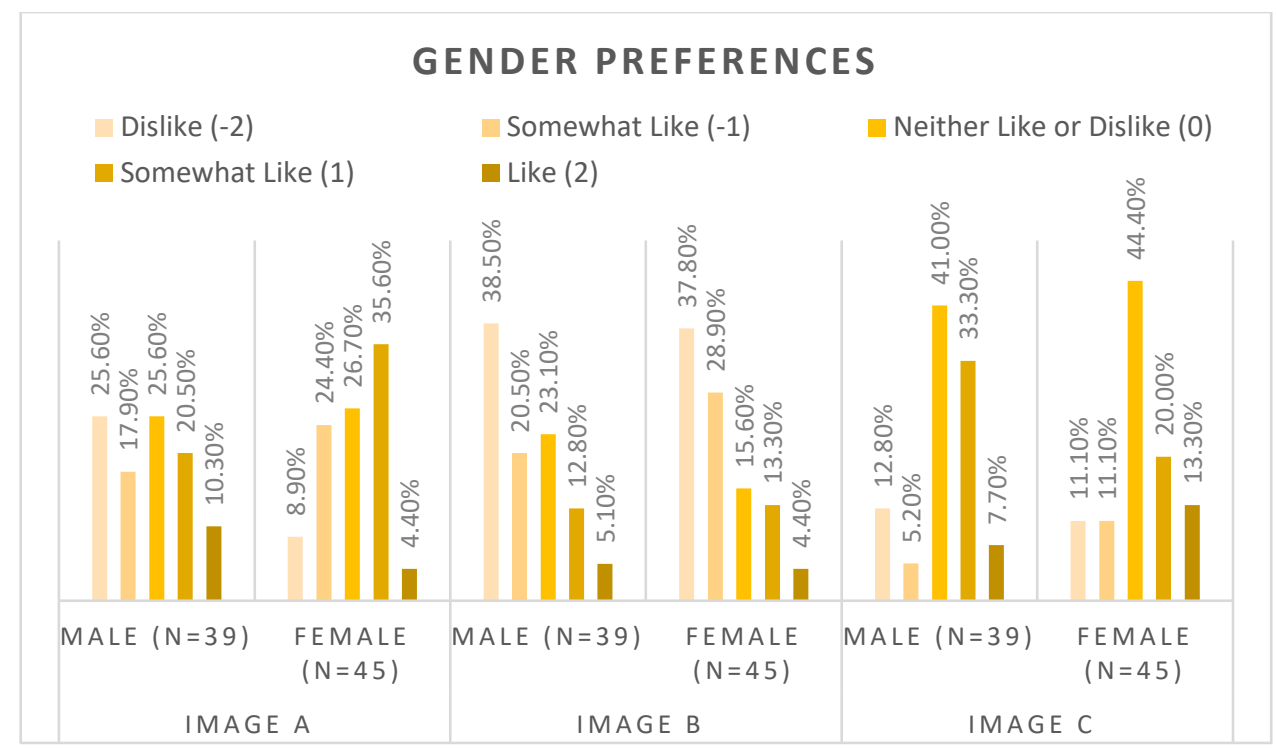

Figure 6.54 Gender preferences for the different options

\subsubsection{Part Four}

In part 4 of Study One pairs of photographs were shown to participants, who were asked to select one out of the two that needed transformation. Participants were given a list of options for improving the aesthetics of the space. The most liked images and the suggestions for improving them are set out below.

\subsection{Space underneath the building}

For the space underneath the building, 41 males and 53 females responded to the question with $79 \%$ of males and $80 \%$ of females wanting to see a change to option $\mathrm{A}$ (figure 6.55). The popular suggestions from males for improving option $A$ were improving the lighting (43\%), improving maintenance and cleanliness (40\%), creating a clear pathway to the building (36\%), and providing more vegetation (31\%). Females suggested providing more vegetation (37\%), changing the surface material $(37 \%)$, creating a clear pathway (35\%), and improving maintenance and cleanliness (29\%) (figure 6.56). 


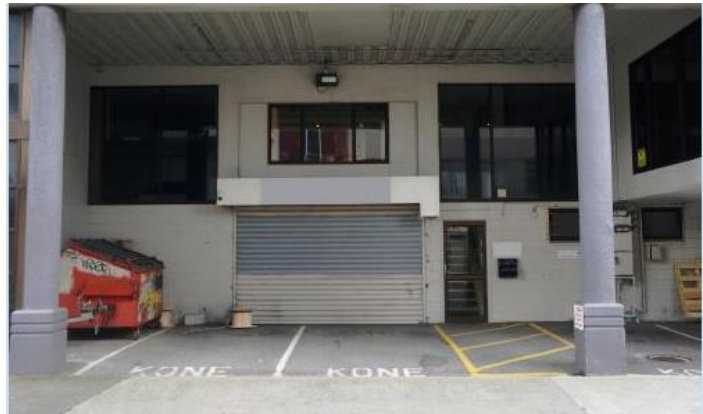

A

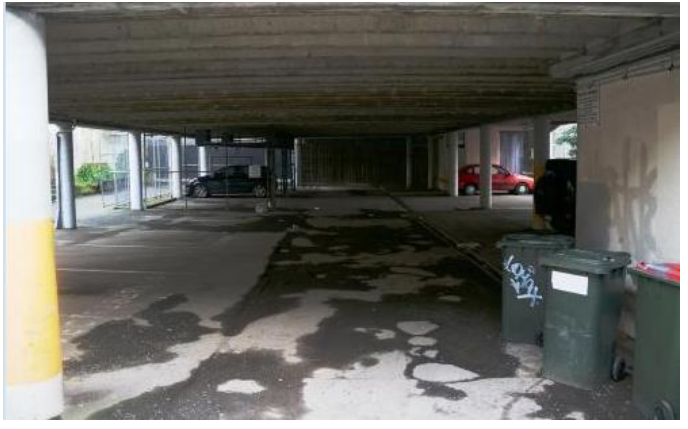

B

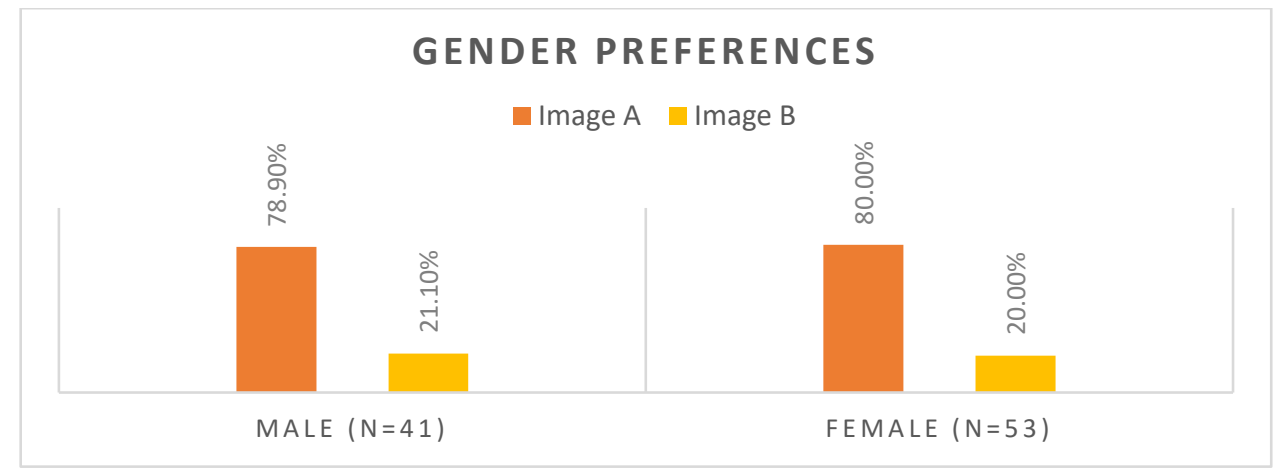

Figure 6.55 Gender preferences for spaces to be improved

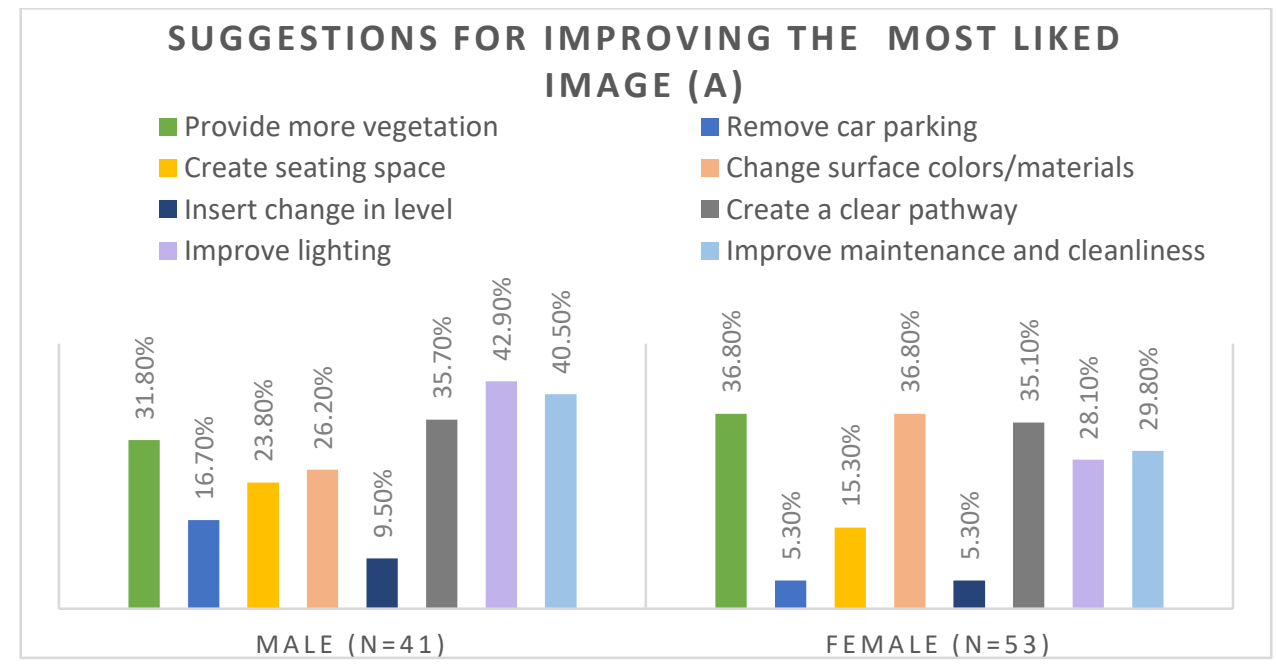

Figure 6.56 Gender preferences for improvement options

\subsection{Space in front of a building}

For the space in front of a building, 39 males and 48 females responded to the question. Option B was the one most people wanted to change $(62 \%$ of males and $78 \%$ of females). The most common suggestions from males for transforming option B concerned removal of the boundary walls $(31 \%)$, providing more vegetation $(26 \%)$, and creating more seating space (19\%). Females suggested providing more 
vegetation (44\%), removal of boundary walls (44\%), and creating a clear pathway to enter the building $(26 \%)$.

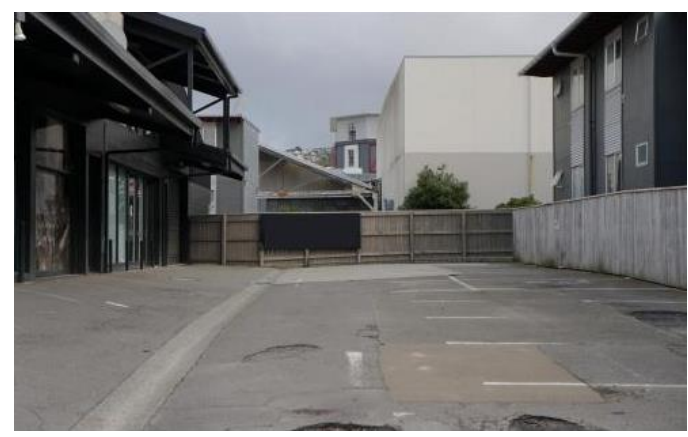

A

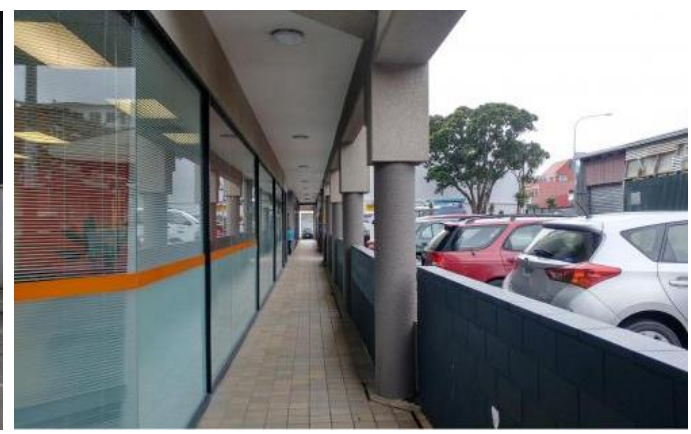

B

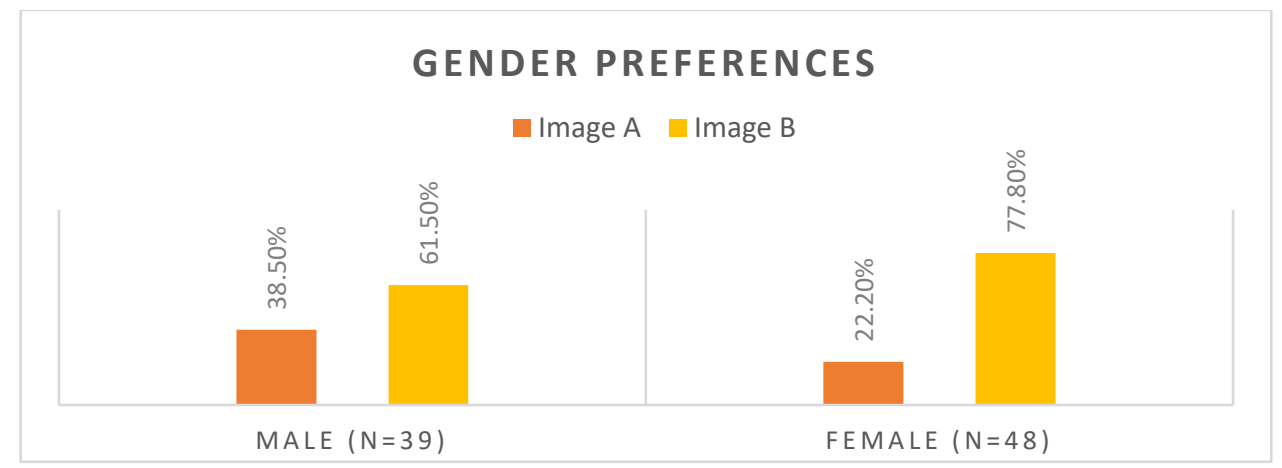

Figure 6.57 Gender preferences for spaces to be improved

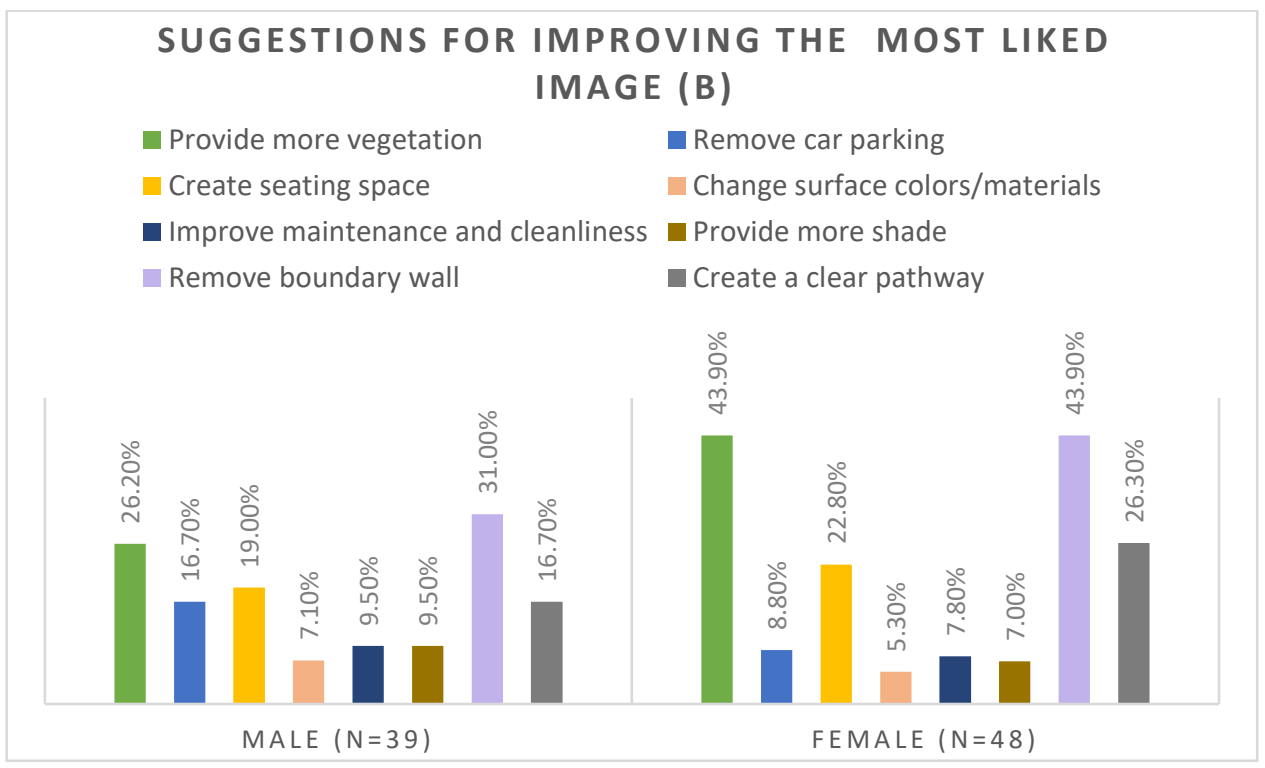

Figure 6.58 Gender preferences for improvement options

\subsection{Space at the back of a building}

For the space at the back of a building, 39 males and 51 females responded to the question, with $58 \%$ of males and $64 \%$ of females wanting to see a change in option $B$ 
(figure 6.59). The most common suggestions from males for upgrading option B concerned providing more vegetation (40\%), changing the surface material (33\%), and creating a clear pathway (24\%). These were similar to suggestions from females who suggested providing more vegetation (37\%), creating a clear pathway (32\%), and changing surface materials (21\%) (figure 6.60).

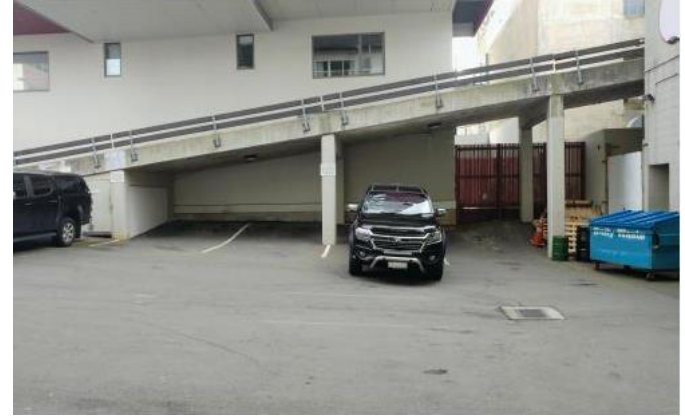

A

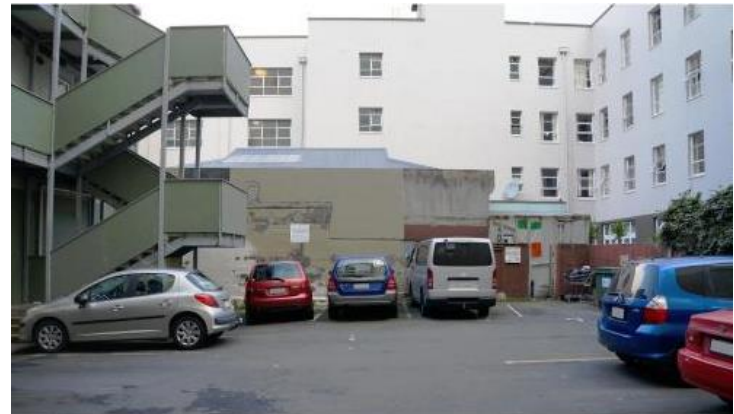

B

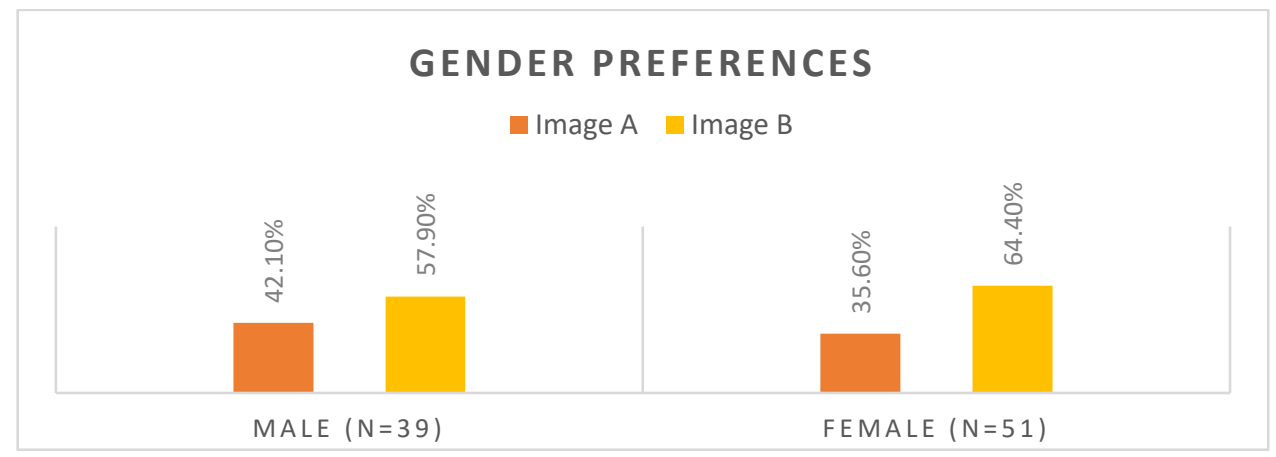

Figure 6.59 Gender preferences for spaces to be improved

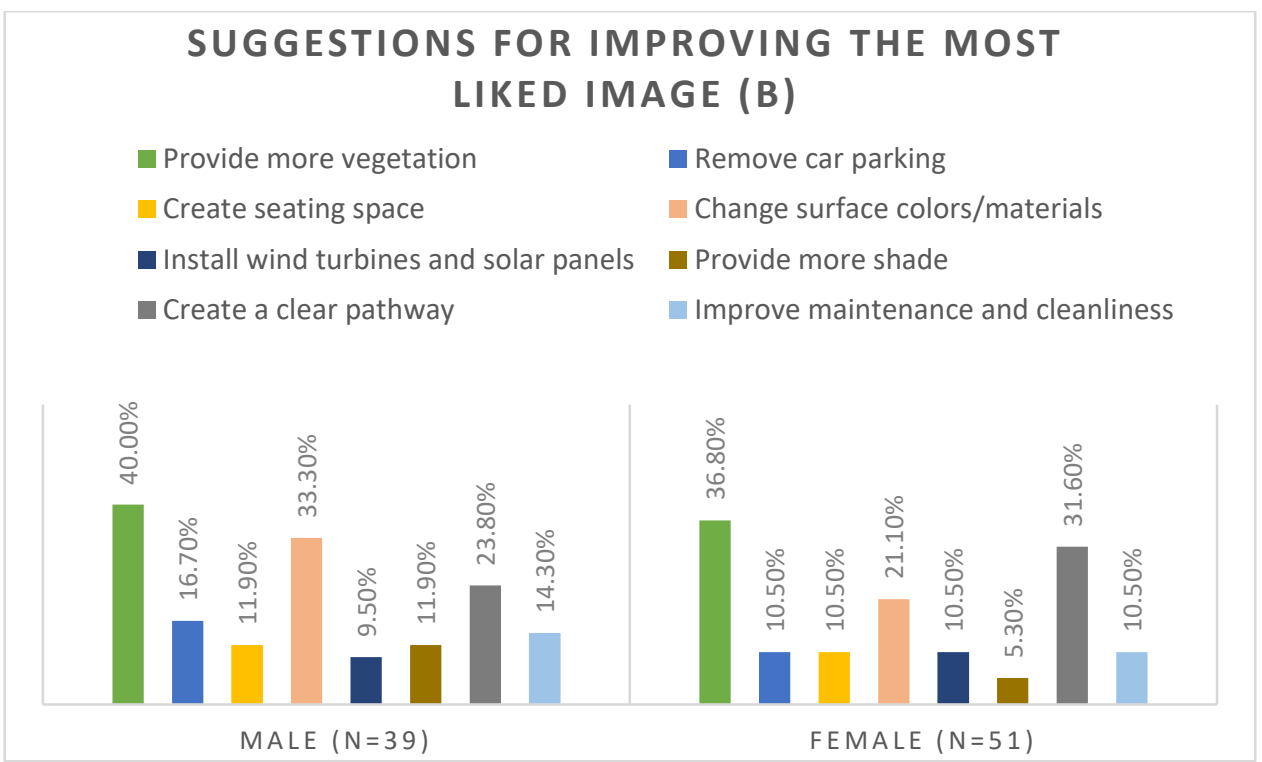

Figure 6.60 Gender preferences for improvement options 


\subsection{Space enclosed by buildings on three sides}

Overall, 39 males and 45 females responded to the question, with $76 \%$ of males and $53 \%$ of females wanting to change option B (figure 6.61 ). The most common suggestions among males for transforming option $B$ were providing more vegetation $(48 \%)$, changing the surface material $(36 \%)$, and creating a clear pathway $(31 \%)$. Female suggestions were somewhat different, by providing more vegetation (32\%), creating a clear pathway $(28 \%)$, and improving the maintenance and cleanliness (20\%) (figure 6.62).

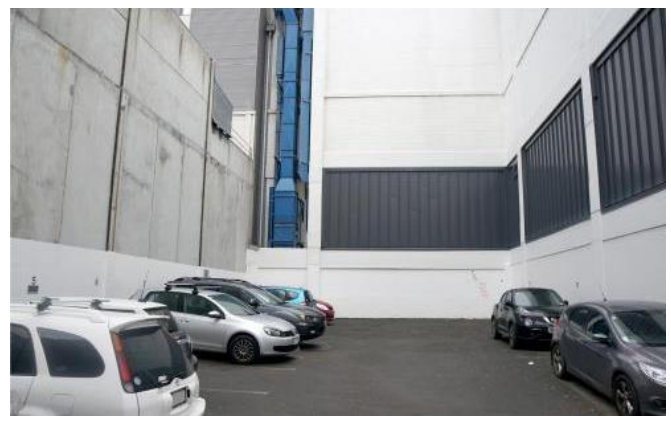

A

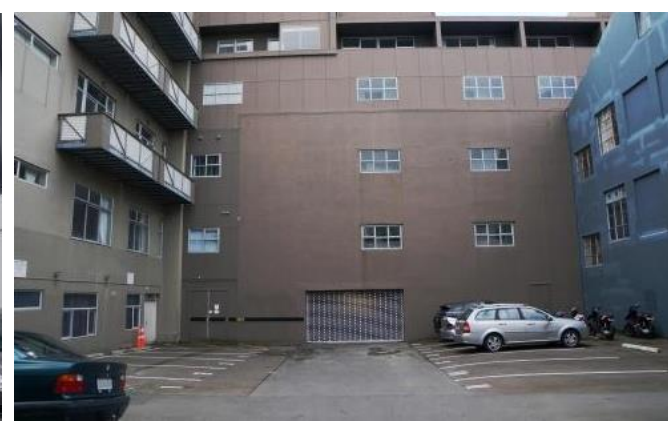

B

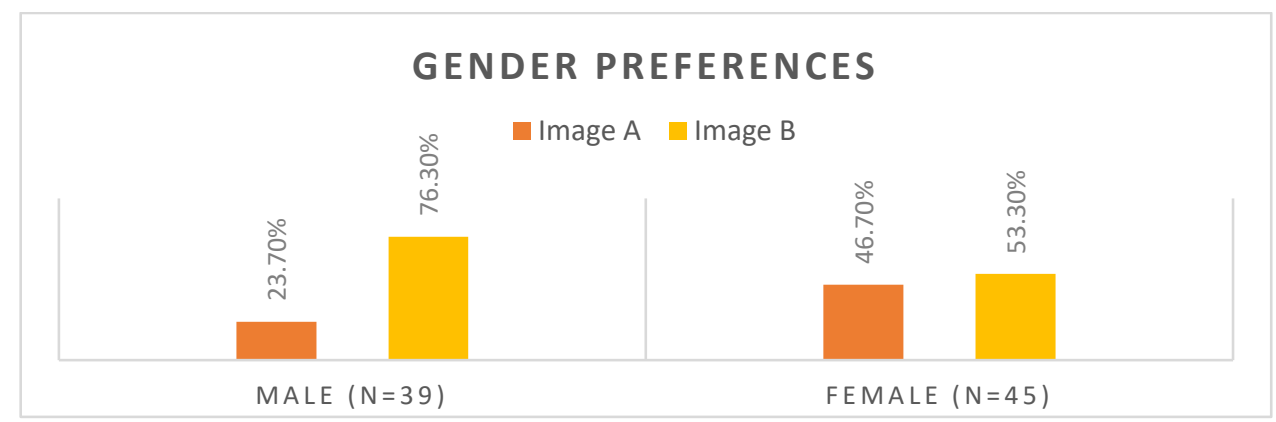

Figure 6.61 Gender preferences for spaces to be improved

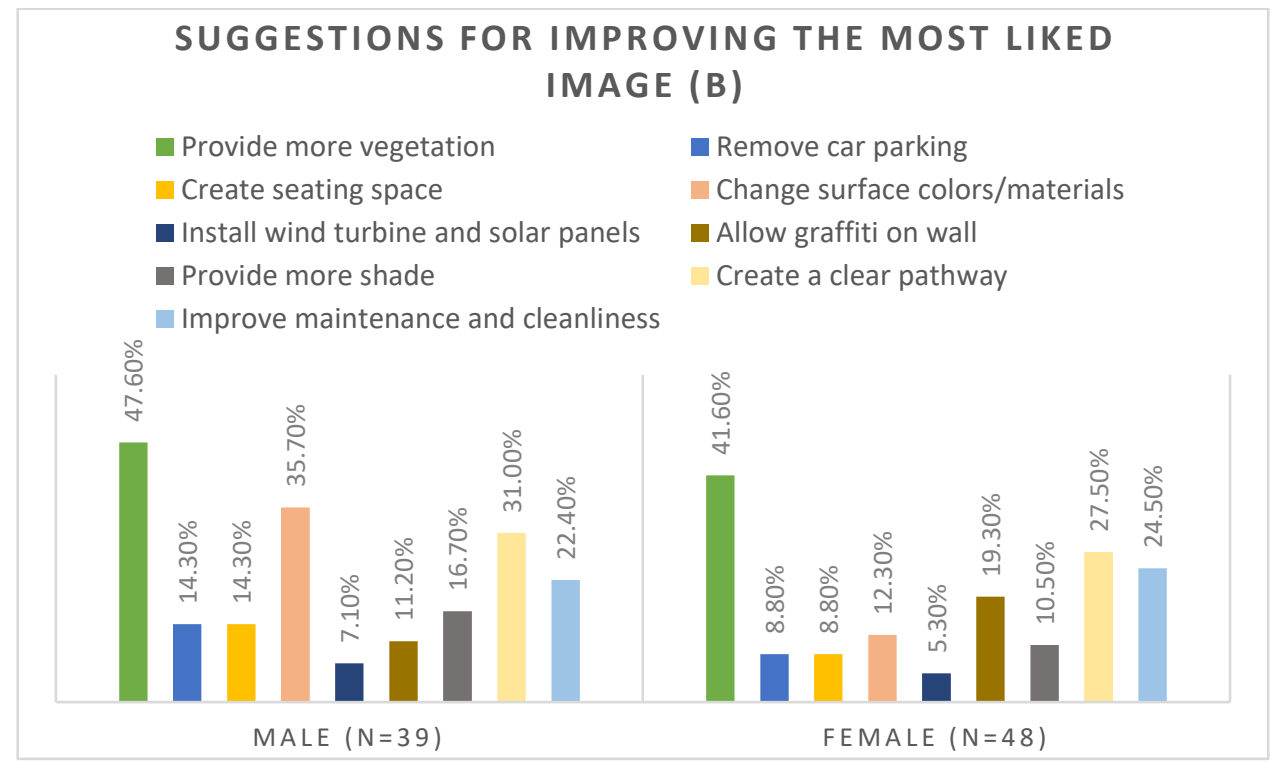


Figure 6.62 Gender preferences for improvement options

\subsection{Space enclosed by buildings on two sides}

For the space enclosed by buildings on two sides, 39 males and 45 females answered the question, leading to a high $84 \%$ of males and $80 \%$ of females wanting to see a change in option $A$ (figure 6.63). The most frequent suggestions among males for improving option A were providing more vegetation (46\%), creating a clear pathway $(45 \%)$, and changing the surface material $(31 \%)$. Female suggestions initially followed the same pattern with providing more vegetation $(42 \%)$, creating a clear pathway (39\%), and then changed with allowing graffiti on the wall (21\%) (figure 6.64).

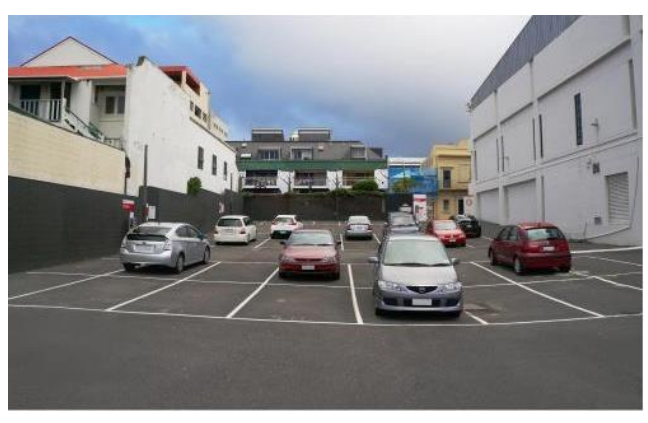

A

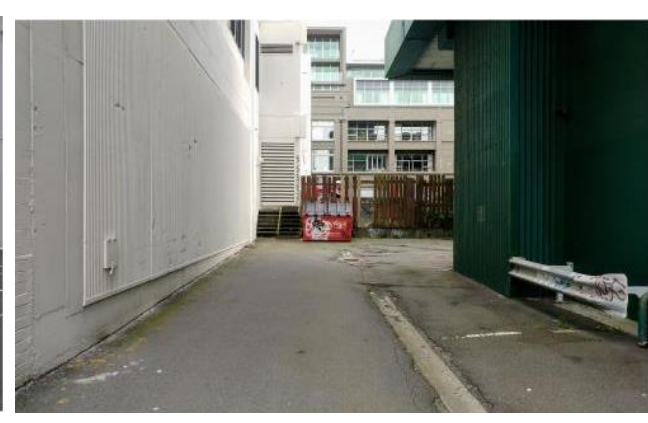

B

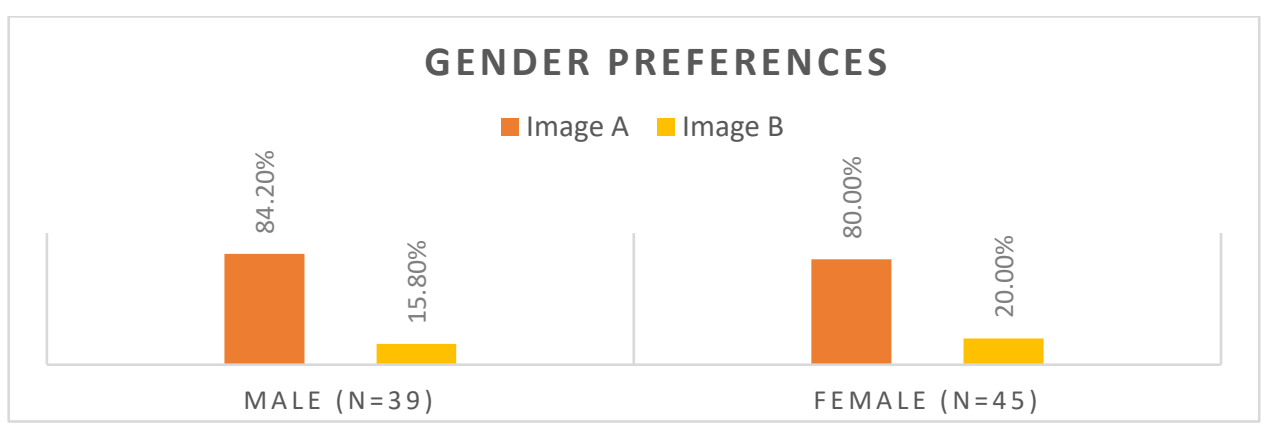

Figure 6.63 Gender preferences for spaces to be improved 


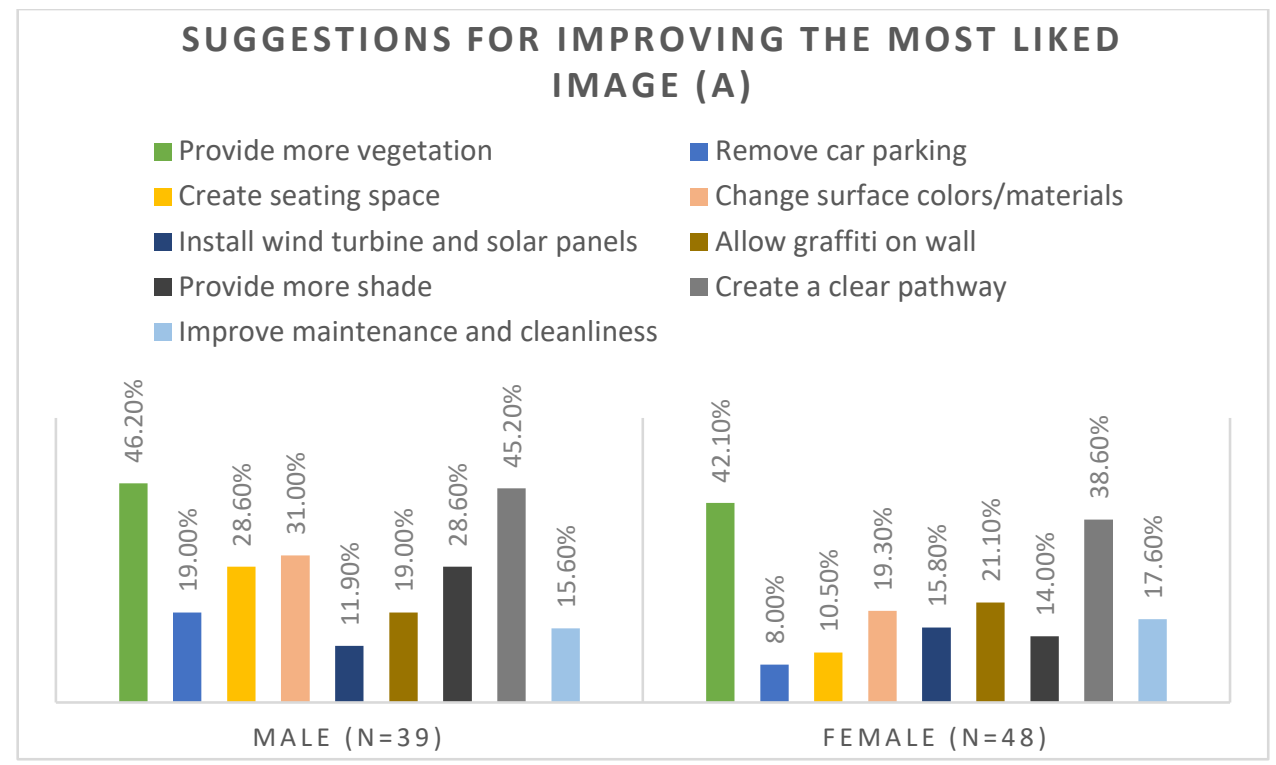

Figure 6.64 Gender preferences for improvement options

\subsection{Space on the rooftop of a building}

For the space on the rooftop of a building, 39 males and 45 females answered the question, with $61 \%$ of males and $52 \%$ of females wanted to see a change in option $B$ (figure 6.65). The most common suggestions from males for changing option $B$ dealt with providing more vegetation (45\%), creating seating space $(38 \%)$, and providing more shade $(31 \%)$, whereas female suggestions were providing more vegetation $(28 \%)$, installing wind turbines and solar panels (19\%), and creating a clear pathway (19\%) (figure 6.66).

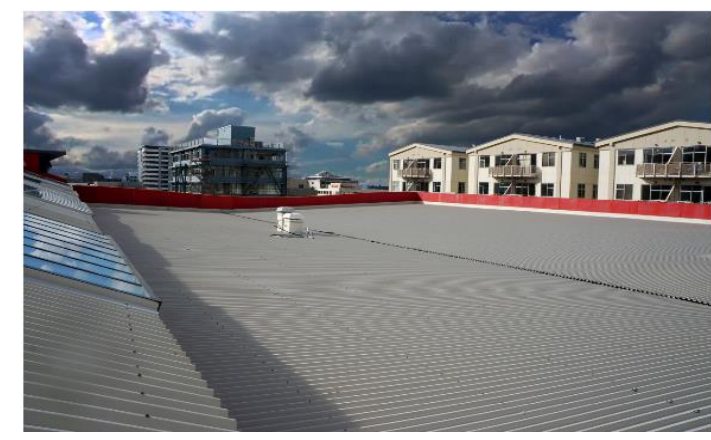

A

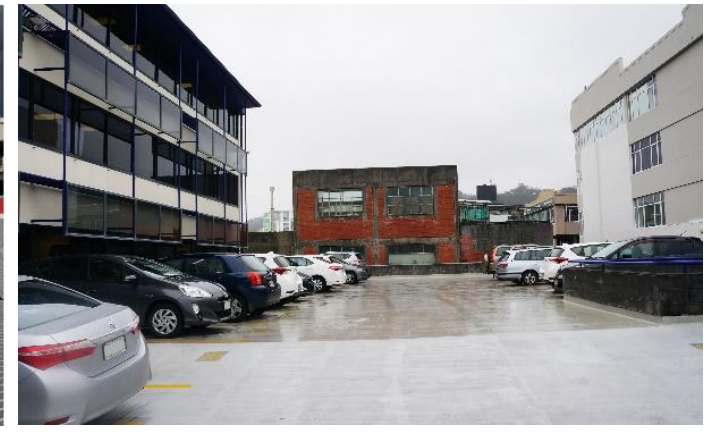

B 


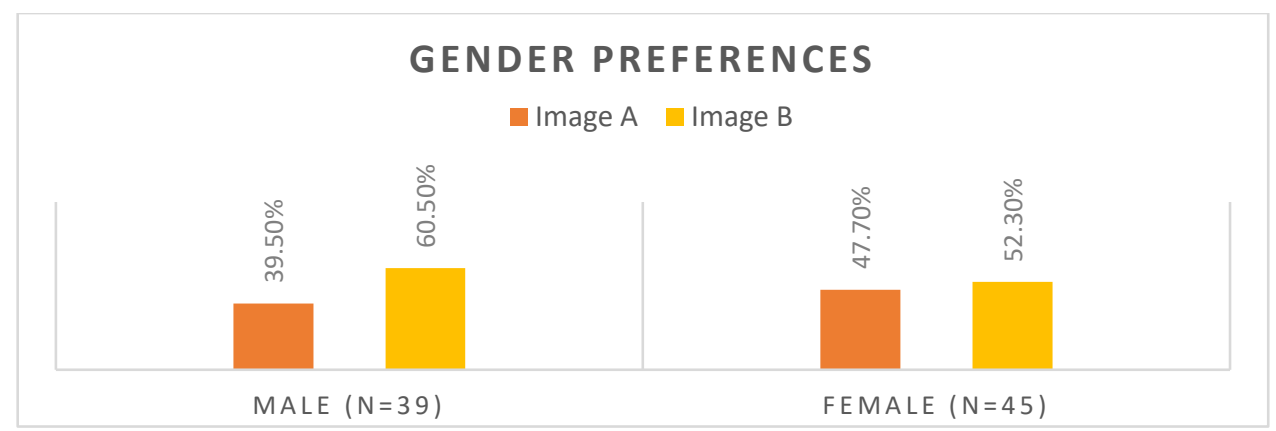

Figure 6.65 Gender preferences for spaces to be improved

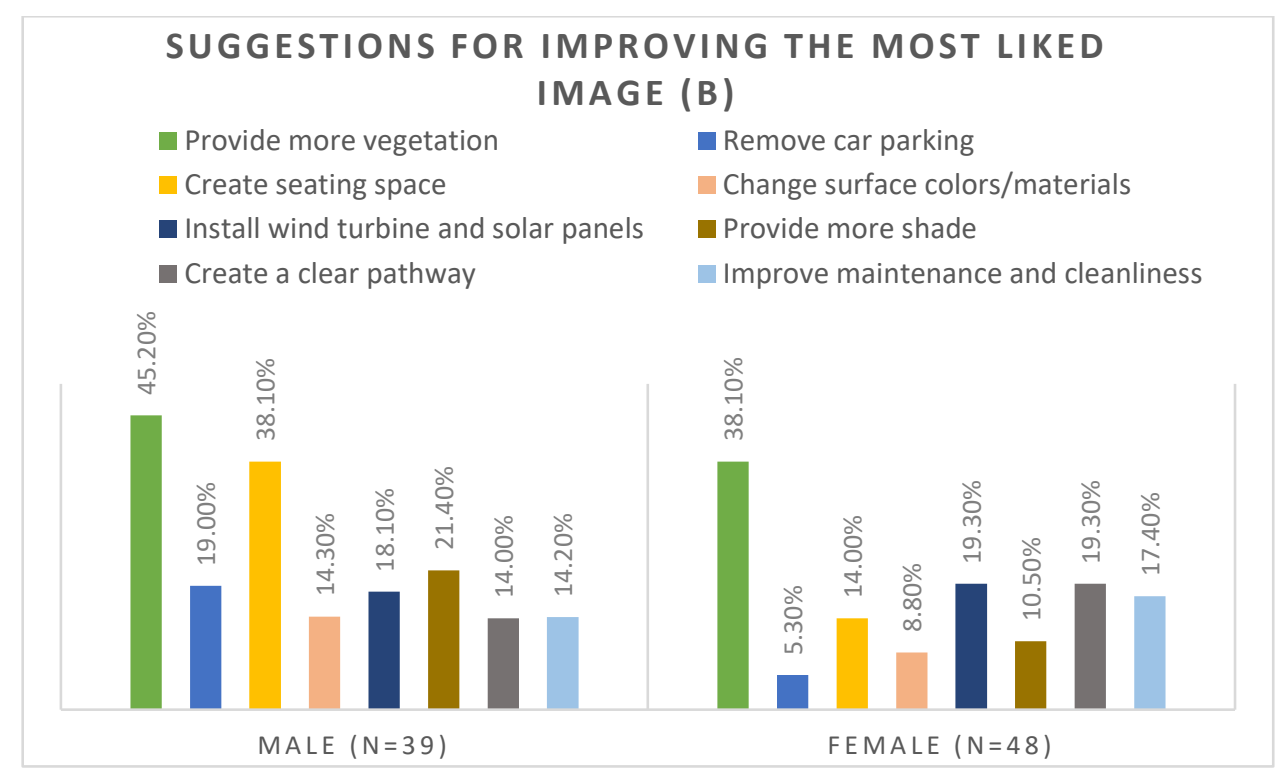

Figure 6.66 Gender preferences for improvement options

6.3.3 Analysis of Stage three: Built Environment (BE) and Non-Built Environment (NBE) Participants

In the third stage of Study One, the responses of 61 (64.2\%) Built Environment (BE) and 38 (38.4\%) Non-Built Environment (NBE) participants were analysed, to look for any differences in the preferences expressed.

\subsubsection{Part One}

The demographic characteristics of participants have already been covered (see section 6.2.1). 


\subsubsection{Part Two}

\subsection{Natural elements in a space}

In the first pair of photographs, an overwhelming $97 \%$ of $\mathrm{BE}$ and $94 \%$ of NBE participants favoured the space with natural features in the built environment (figure 6.67).
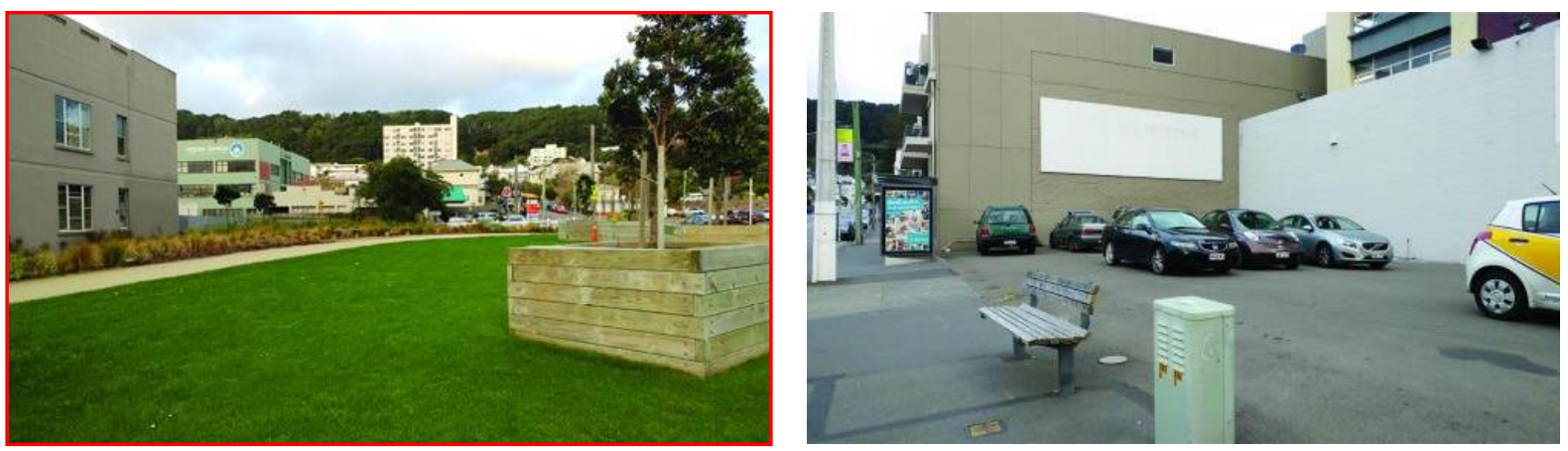

Figure 6.67 Space with natural elements was preferred over space with artificial elements

\subsection{Organised space}

For the second pair of photographs, a high $84 \%$ of BE and an even higher $94 \%$ of NBE participants liked the space that was well organised (figure 6.68).
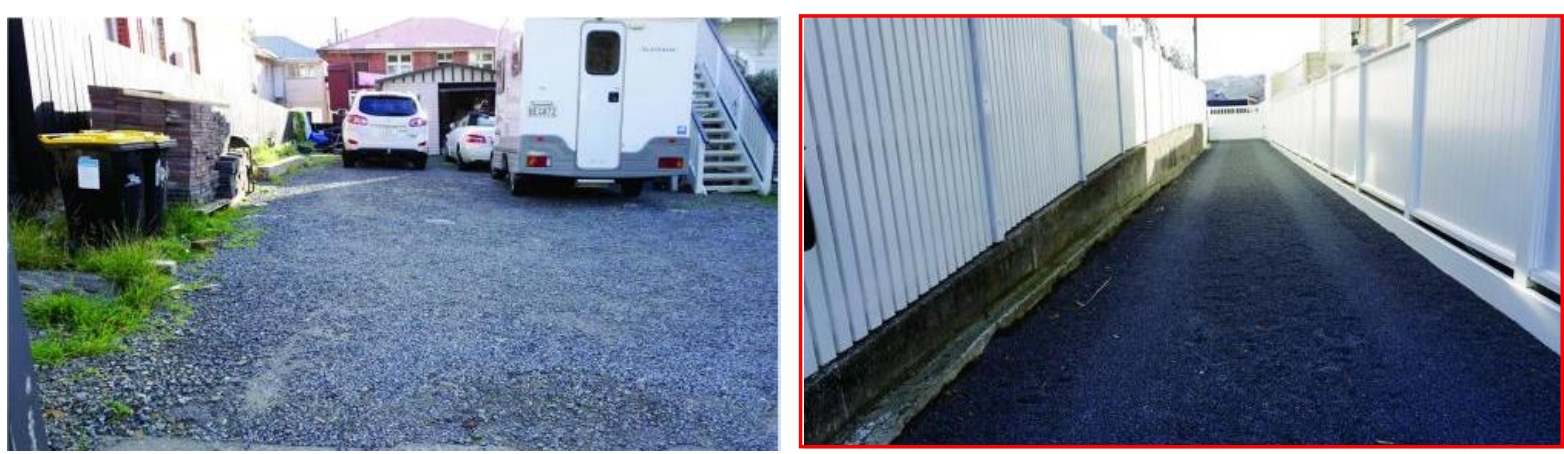

Figure 6.68 The well-organised space was preferred over the disorganised space

\subsection{Open space}

The preference for the space open to the sky was less clear for the BE participants as $60 \%$ preferred it, compared to a higher $71 \%$ of NBE participants (figure 6.69 ). 

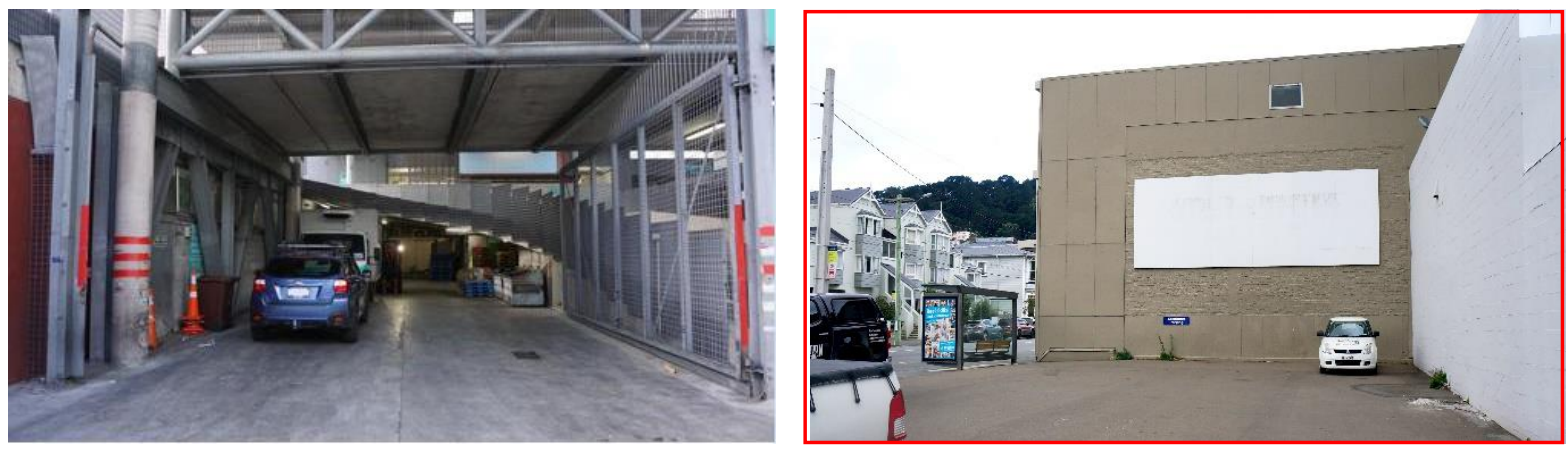

Figure 6.69 Space open to the sky was preferred over an enclosed space

\subsection{Well maintained space}

In the fourth pair of photographs about upkeep, there was high support for the space that looked well maintained with $77 \%$ of BE and $80 \%$ of NBE participants preferring it (figure 6.70).
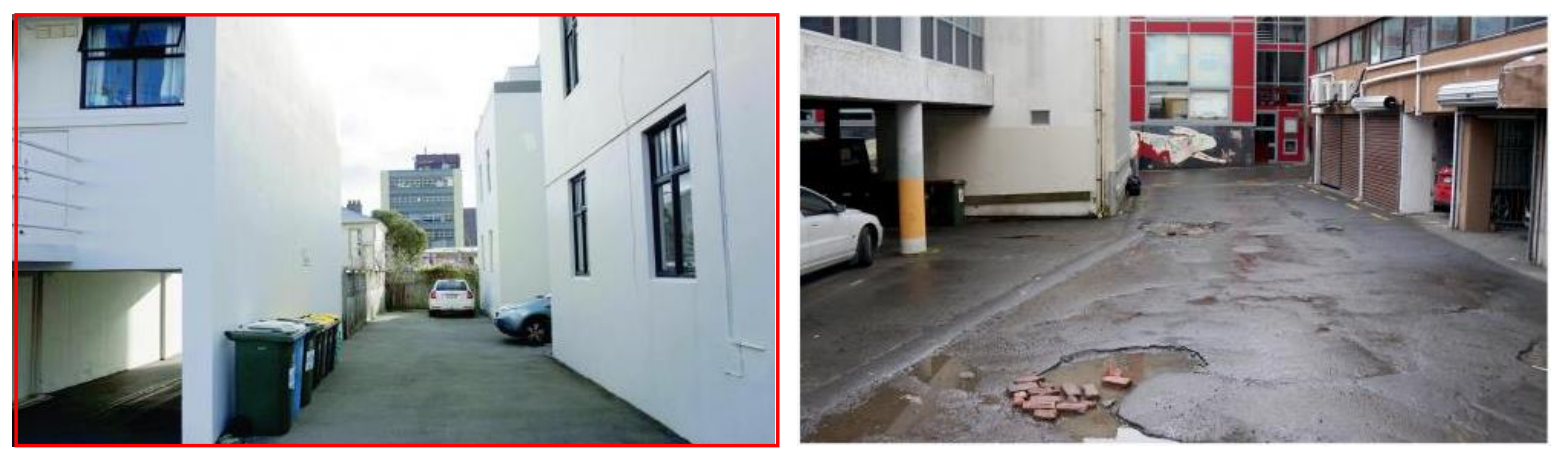

Figure 6.70 Well-maintained space was preferred over space that was not well kept

\subsection{Modern Building style}

In the fifth pair of photographs about building style, there was a slightly higher preference for the modern style from the BE participants (70\%) compared to the NBE (64\%) (figure 6.71).
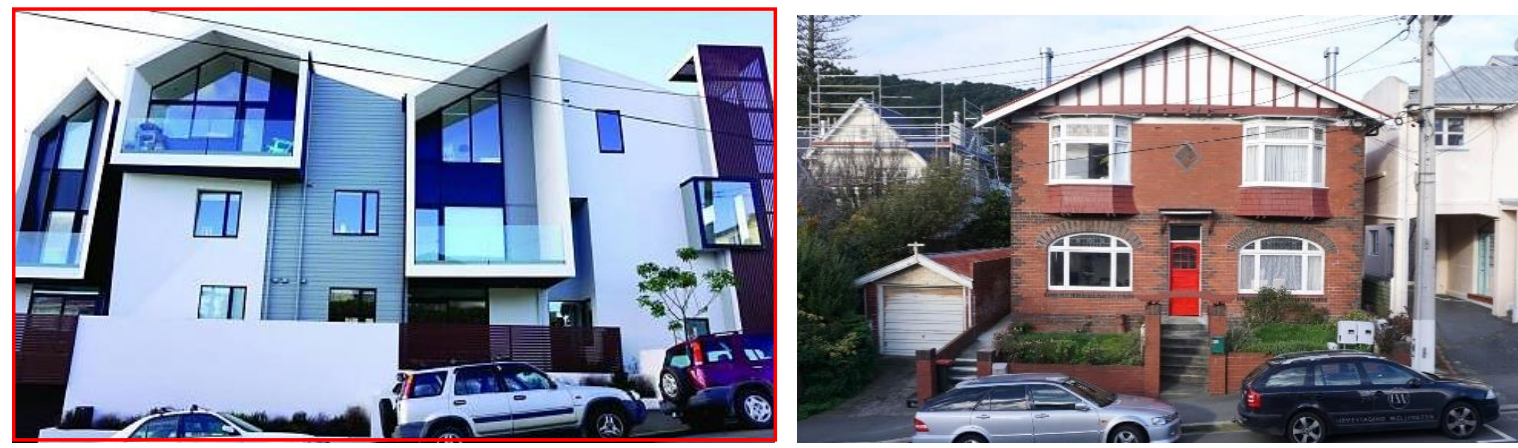

Figure 6.71 Modern building style was preferred over the older building style 
The preferences of BE and NBE participants for the different spaces are presented in figure 6.72 .

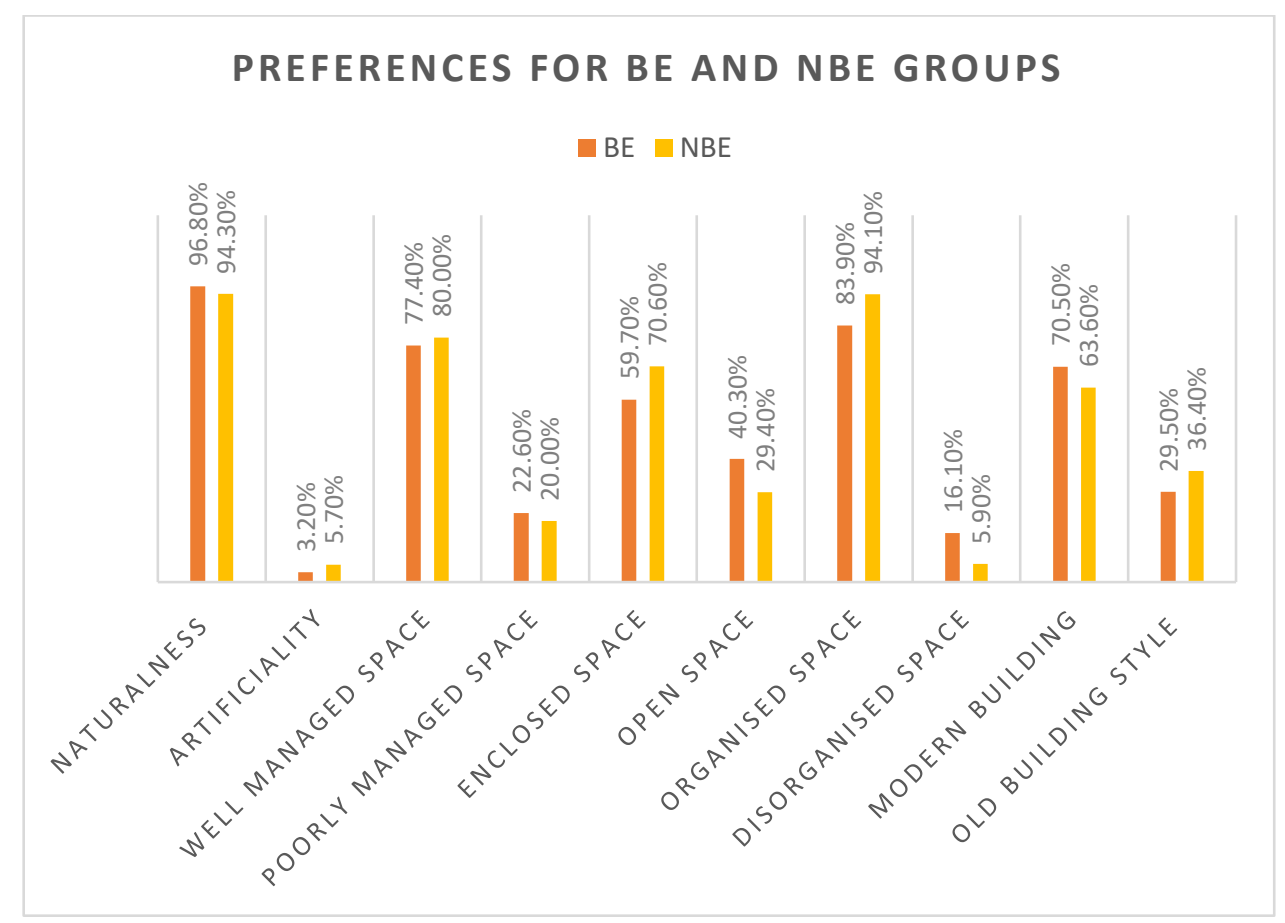

Figure 6.72 Preferences for spaces with different attributes

\subsubsection{Part Three}

Here the results for the most liked image were compared for the two groups of BE and NBE participants. The percentages are calculated for those participants, who preferred the image.

\subsection{Space underneath a building}

For the space underneath the building, $52 \mathrm{BE}$ and $32 \mathrm{NBE}$ participants responded to the question. Option $C$ (see figure 6.73) was overwhelming liked or somewhat liked by $96 \%$ BE and $100 \%$ NBE participants. This option also had the highest mean score of 4.52 with a Standard deviation (SD) of 0.57 for $B E$ respondents and a mean score of 4.72 with an SD of 0.46 for NBE participants. Option A was second being liked/somewhat liked by $23 \%$ BE and $37 \%$ NBE participants. This option received a mean score of 2.60 with an SD of 1.19 for BE participants and a mean score of 3.06 with an SD of 1.12 for NBE respondents. Only $15 \%$ of BE but $44 \%$ of NBE 
liked/somewhat liked option B (figure 6.74), which had a mean score of 2.50 with an SD of 1.11 for BE participants and a mean score of 3.16 with an SD of 1.05 for NBE participants.

A

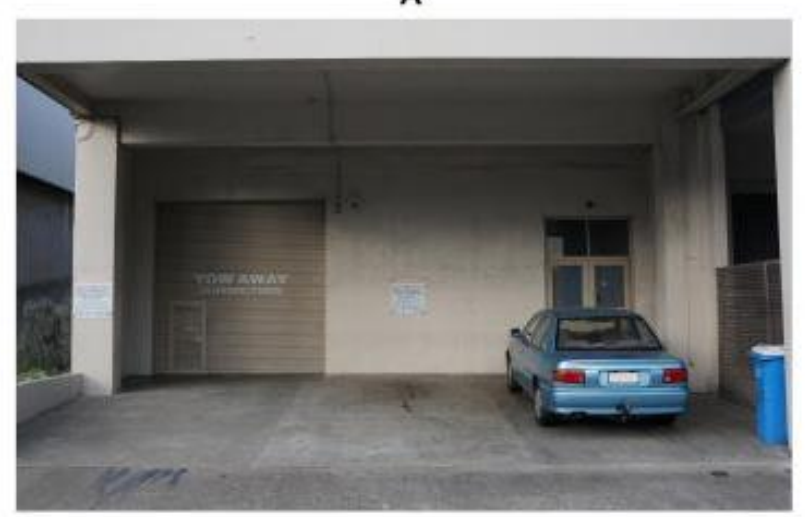

B
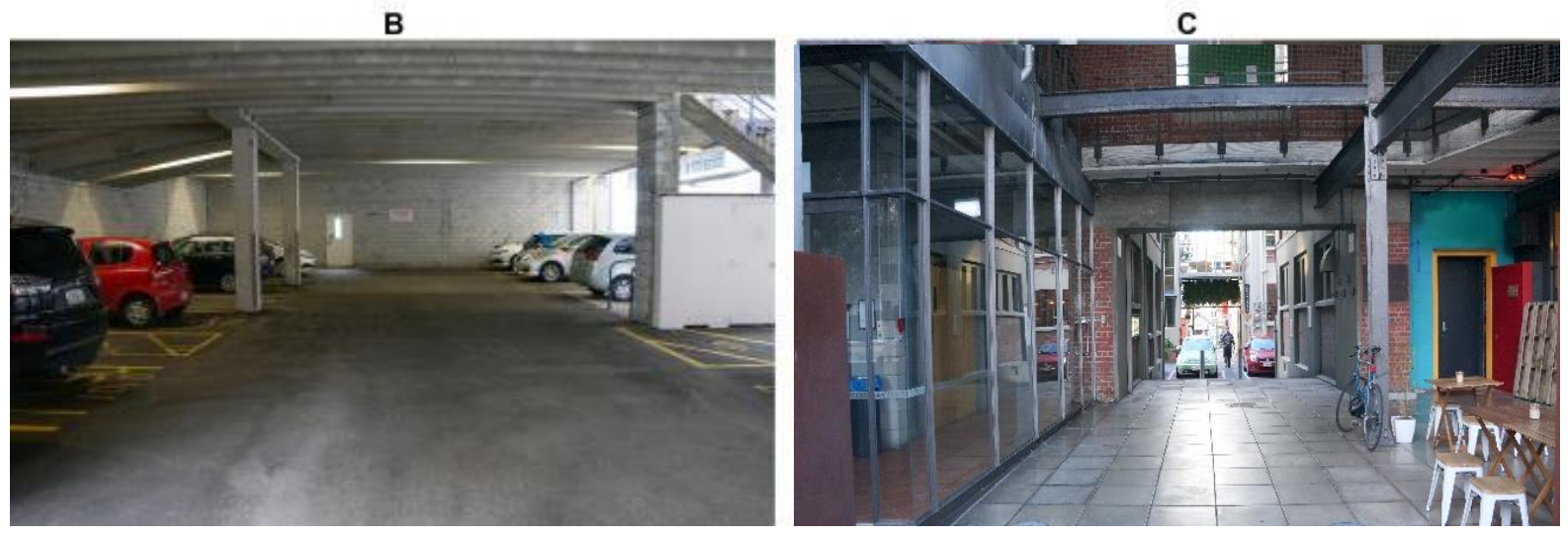

Figure 6.73 Options shown to participants 
Chapter 6 - STUDY ONE: FIRST VISUAL PREFERENCE STUDY

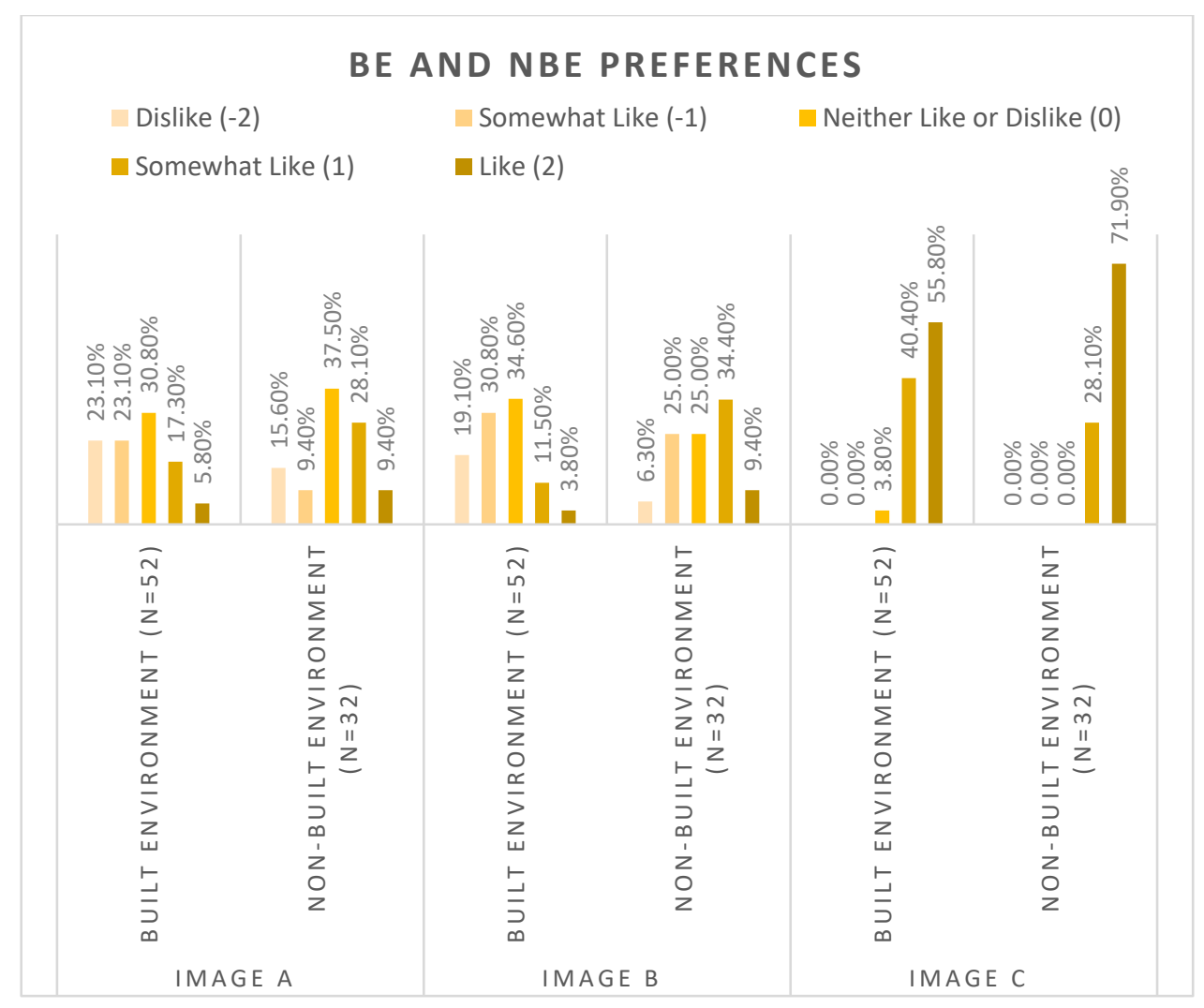

Figure 6.74 Preferences for different options as rated by BE and NBE participants

\subsection{Space in front of a building}

Overall, $61 \mathrm{BE}$ and $34 \mathrm{NBE}$ participants responded to the question on the space in front of a building. Option A (see figure 6.75) was the most preferred with $88 \% \mathrm{BE}$ and $100 \%$ NBE participants liking it. This option also had the highest mean score of 4.71 with a Standard deviation (SD) of 0.46 for BE participants and a mean score of 4.36 with an SD of 0.85 for NBE participants. The second most liked space was option C, which was liked by $40 \%$ of BE and $50 \%$ of NBE participants. This option received the mean score of 3.38 with an SD of 1.13 for $B E$ respondents and a mean score of 3.23 with an SD of 1.17 for NBE participants. Option B was only liked by $21 \%$ BE and a low $9 \%$ NBE participants (figure 6.76). Image B had a mean score of 2.36 with an SD of 1.10 for BE participants and a mean score of 2.56 with an SD of 1.03 for NBE participants. 

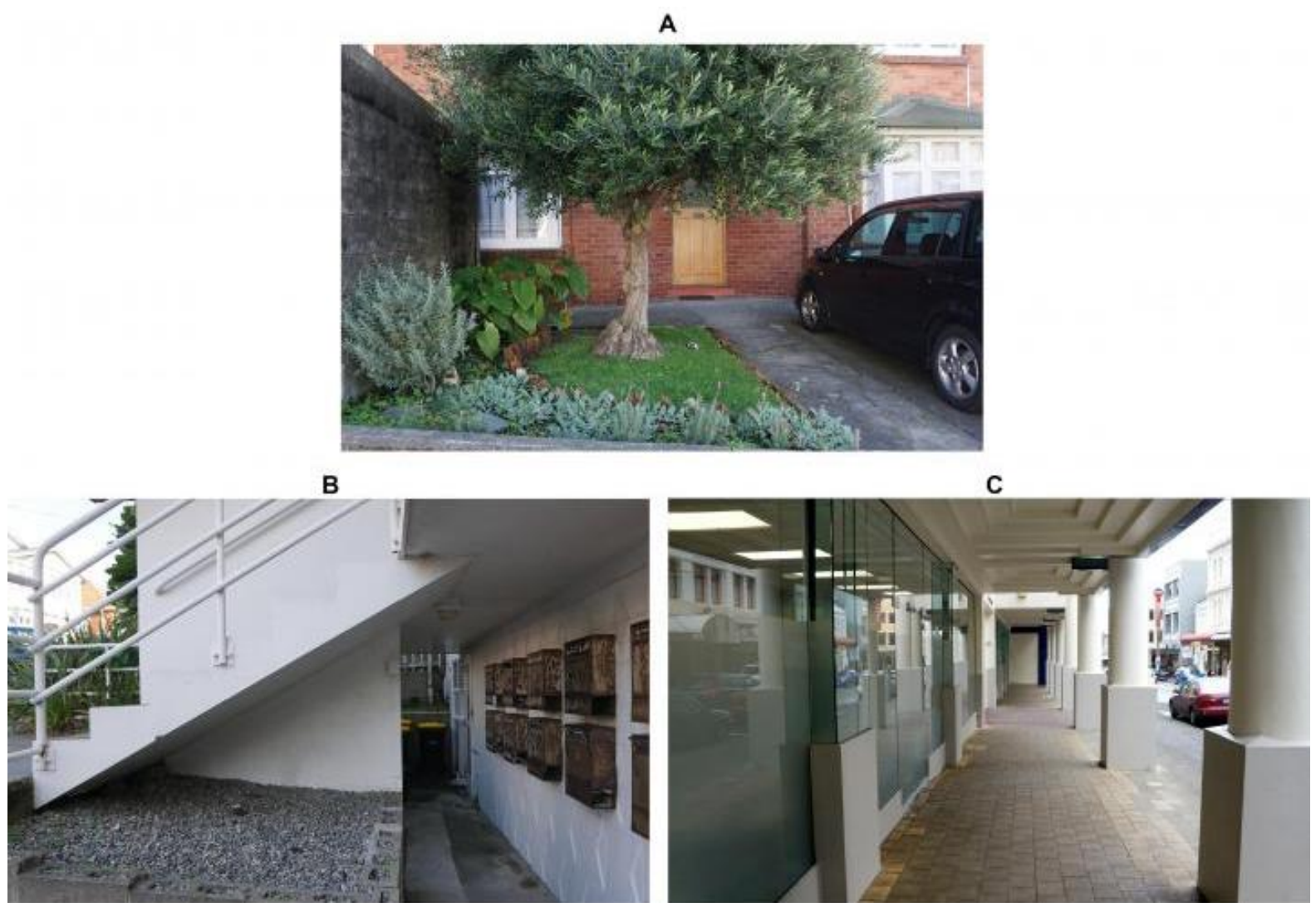

Figure 6.75 Options shown to participants

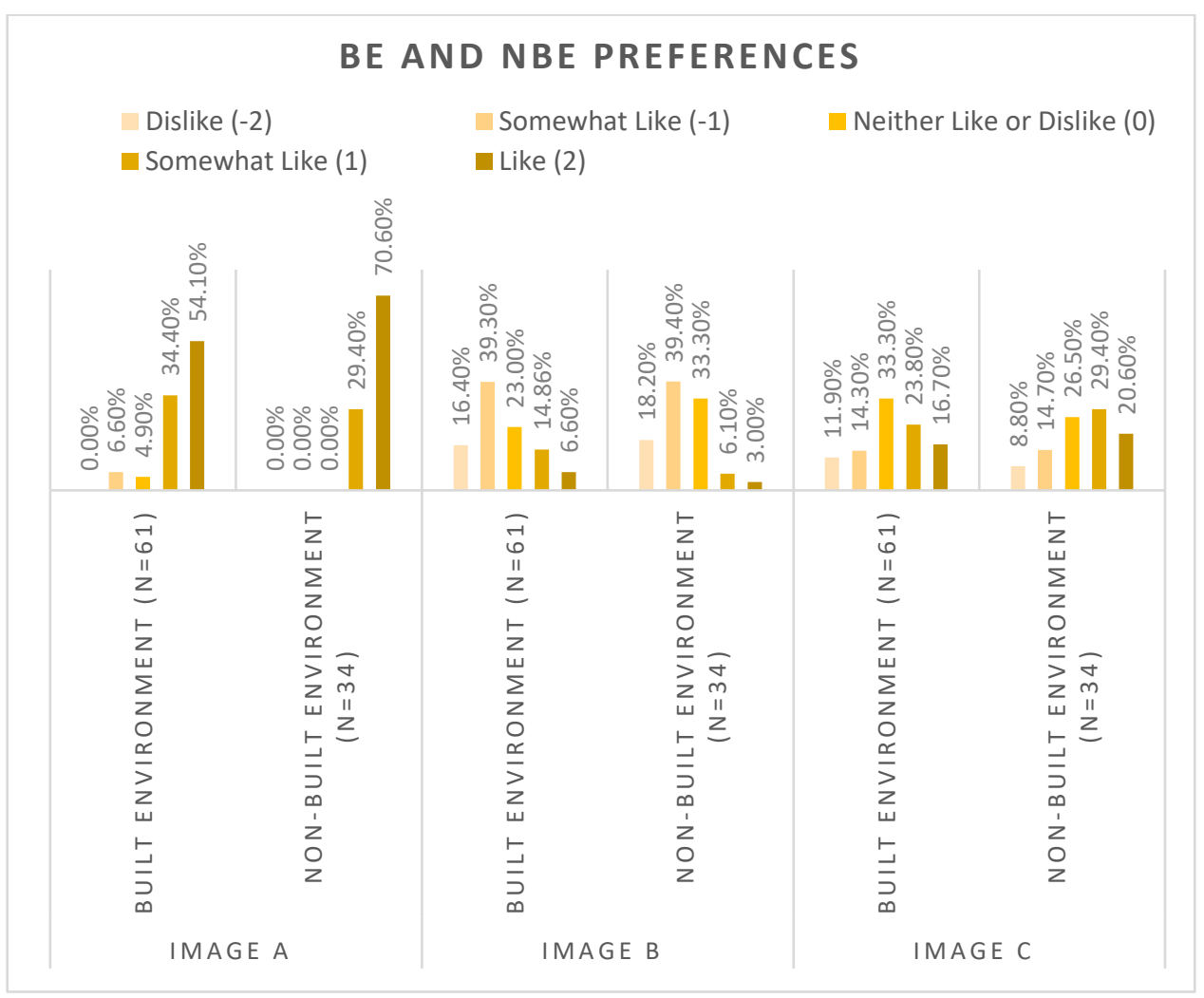

Figure 6.76 Preferences for different options as rated by BE and NBE participants 


\subsection{Space at the back of a building}

For the space at the back of a building (figure 6.77), $58 \mathrm{BE}$, and $33 \mathrm{NBE}$ participants responded to the question, with option $B$ being liked by $55 \%$ BE and $51 \%$ NBE participants. This option also had the highest mean score of 3.41 with a Standard deviation (SD) of 1.10 for BE participants and a mean score of 3.18 with an SD of 1.21 for NBE participants. Option A was the second most liked space, being liked by $41 \%$ BE and $48 \%$ NBE participants. This option had a mean score of 3.14 with an SD of 1.22 for $\mathrm{BE}$ and a mean score of 3.24 with an SD of 1.06 for NBE participants. However, $39 \%$ of BE and $33 \%$ of NBE participants also said they liked option C (figure 6.78). This image had a mean score of 3.05 with an SD of $1.05 \mathrm{BE}$ respondents and a mean score of 3.03 with an SD of 1.01 for NBE participants.

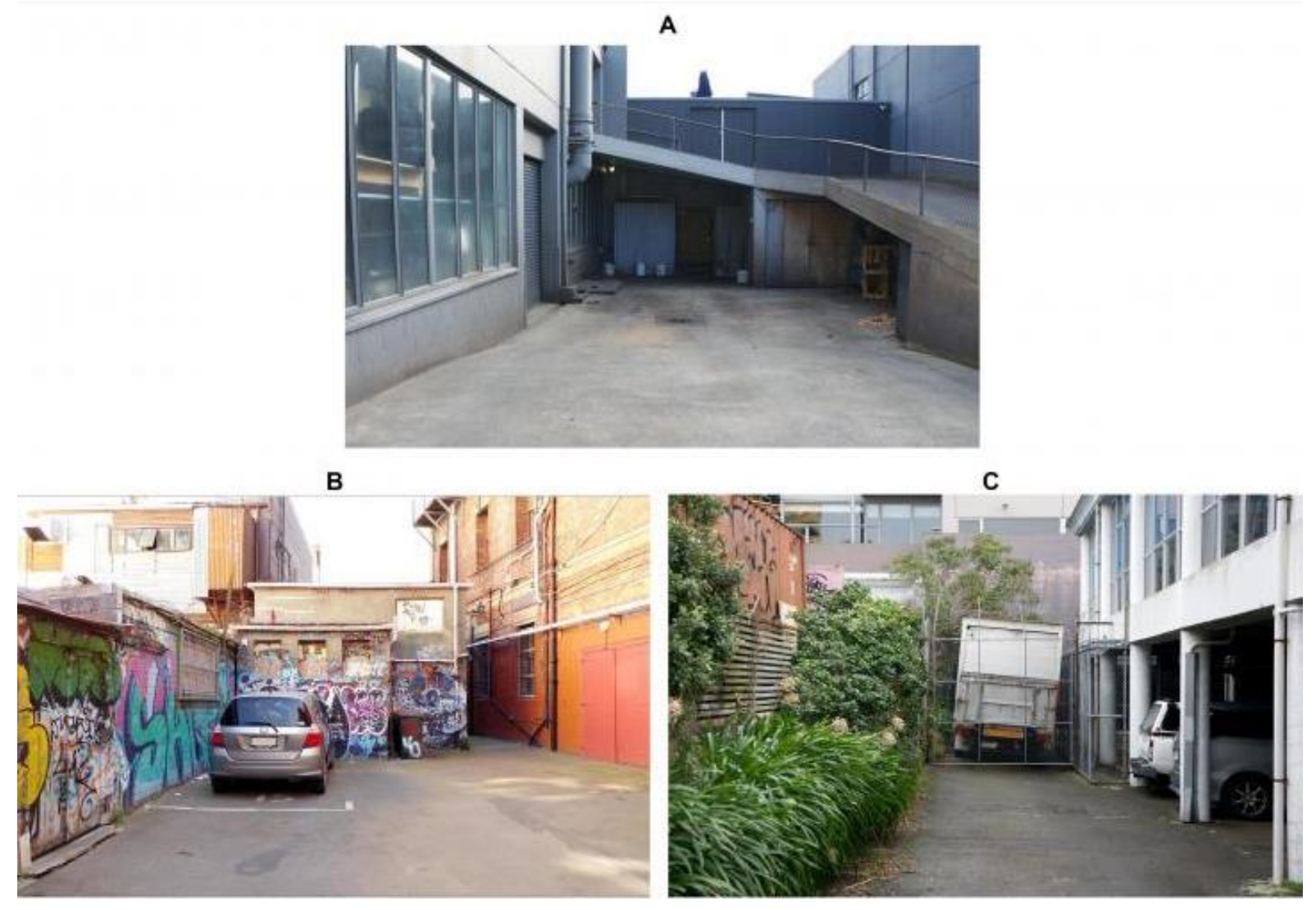

Figure 6.77 Options shown to participants 


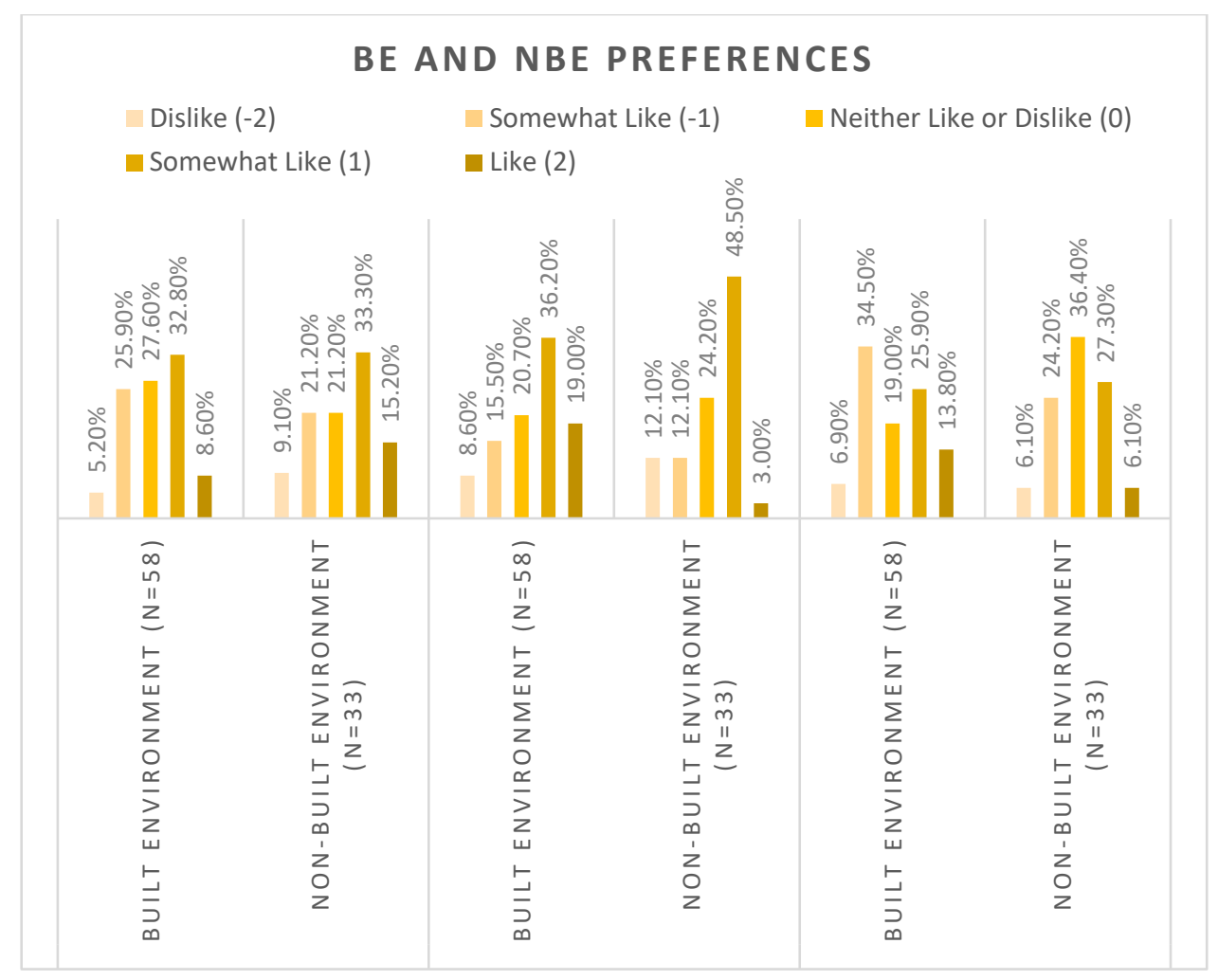

Figure 6.78 Preferences for different options as rated by BE and NBE participants

\subsection{Space enclosed by buildings on three sides}

For the space enclosed by buildings on three sides, $51 \mathrm{BE}$, and 32 NBE participants answered the question, with Option $C$ (figure 6.79) being the most liked by both (77\% $\mathrm{BE}$ and $84 \% \mathrm{NBE}$ ). This option also had the highest mean score of 4.06 with a Standard Deviation (SD) of 0.90 for BE participants and a mean score of 4.25 with an SD of 1.10 for NBE respondents. The second highest liked space was option A, which was liked by $34 \%$ of BE and $62 \%$ NBE participants. This option received a mean score of 2.85 with an SD of 1.22 for BE respondents and a mean score of 3.53 with an SD of 1.13 for NBE participants. Around a quarter of all participants still liked option B (29\% BE and $25 \%$ NBE) (figure 6.80). Image B had a mean score of 2.67 with an SD of 1.16 for $\mathrm{BE}$ respondents and a mean score of 2.66 with an SD of 1.10 for NBE participants. 


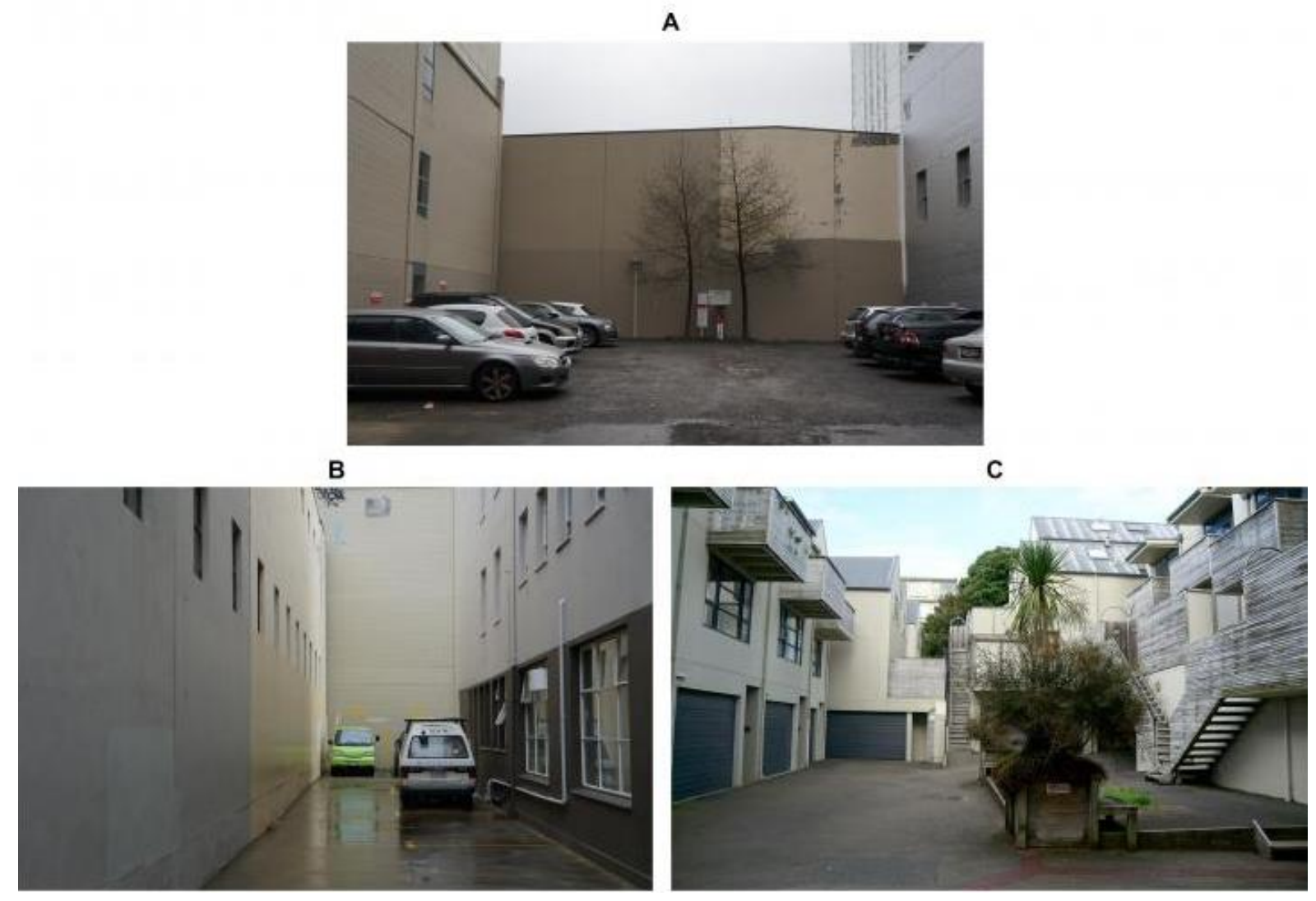

Figure 6.79 Options shown to participants

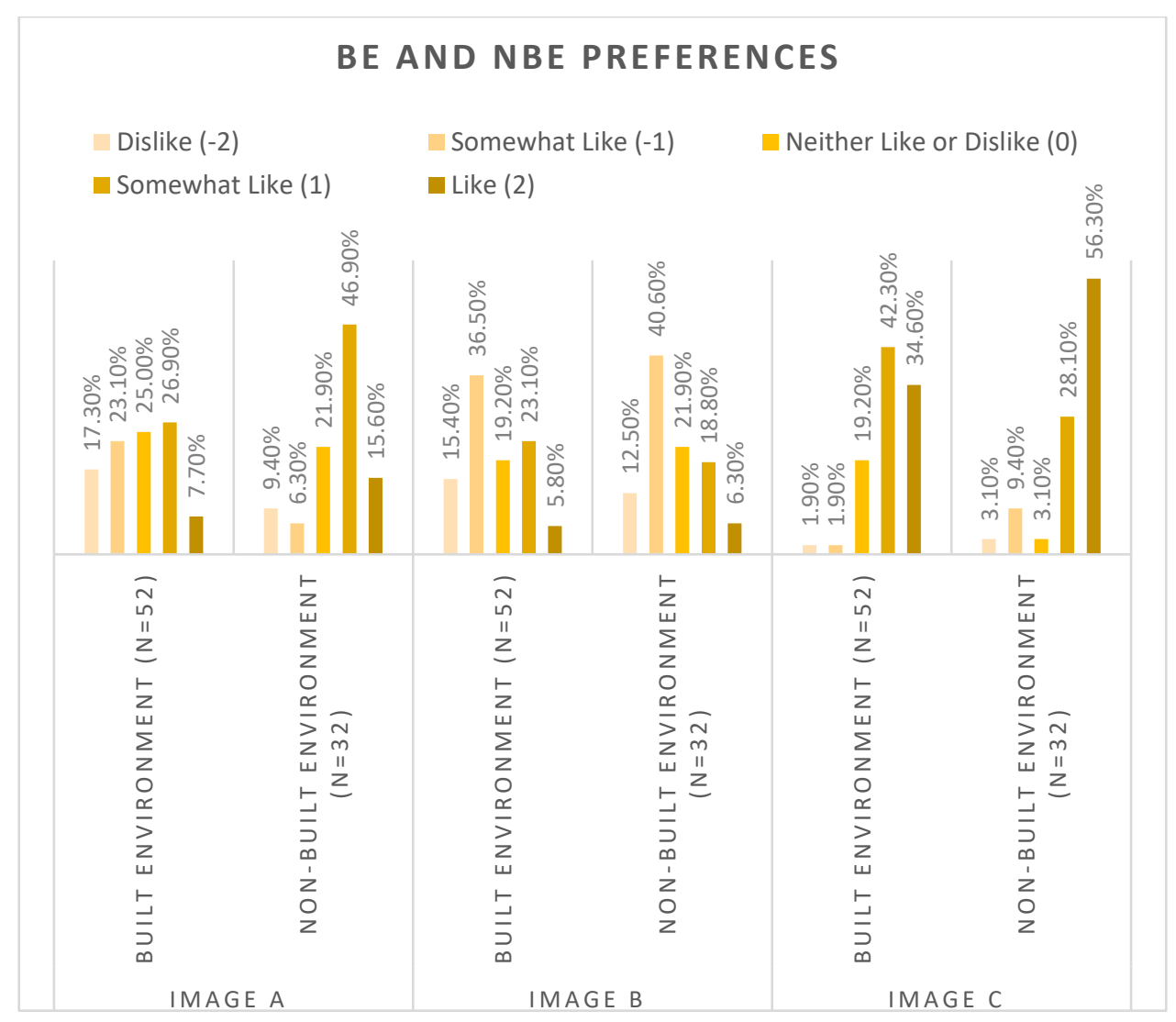

Figure 6.80 Preferences for different options as rated by BE and NBE participants 


\subsection{Space enclosed by a building on two sides}

Overall, $54 \mathrm{BE}$ and $33 \mathrm{NBE}$ participants responded to the question, and option C (figure 6.81 ) was the most liked by $35 \%$ of BE but a higher $51 \%$ of NBE respondents. This option also had the highest mean score of 3.06 with a Standard Deviation (SD) of 1.22 for BE participants and a mean score of 3.27 with an SD of 1.18 for NBE participants. Slightly lower percentages were given for liking option B $(29 \%$ BE and $39 \%$ NBE). This option had a mean score of 2.80 with an SD of 1.15 for BE participants and a mean score of 3.21 with an SD of 1.05 for NBE participants. Although option A was the least liked by BE participants (13\%), it was again liked by $39 \%$ of NBE participants (figure 6.82). Image $A$ had a mean score of 2.43 with an SD of 1.22 for BE participants and a very slightly lower mean score of 3.18 than option $B$ with an SD of 1.12 for NBE participants.

A

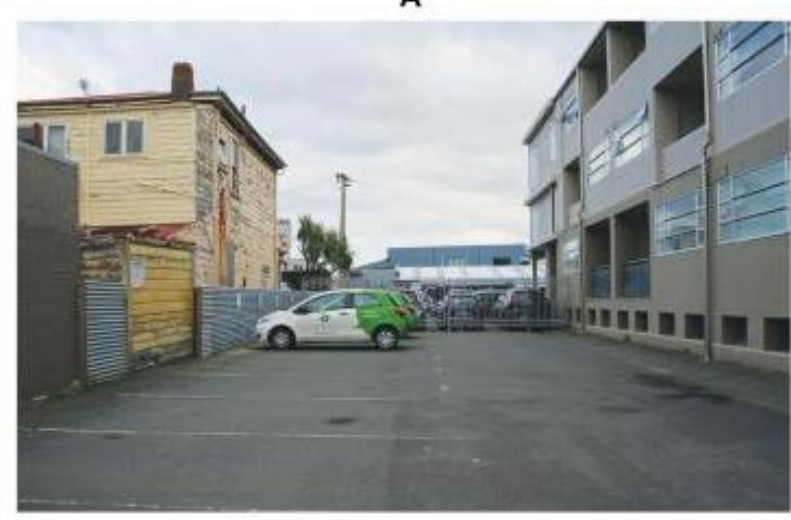

B

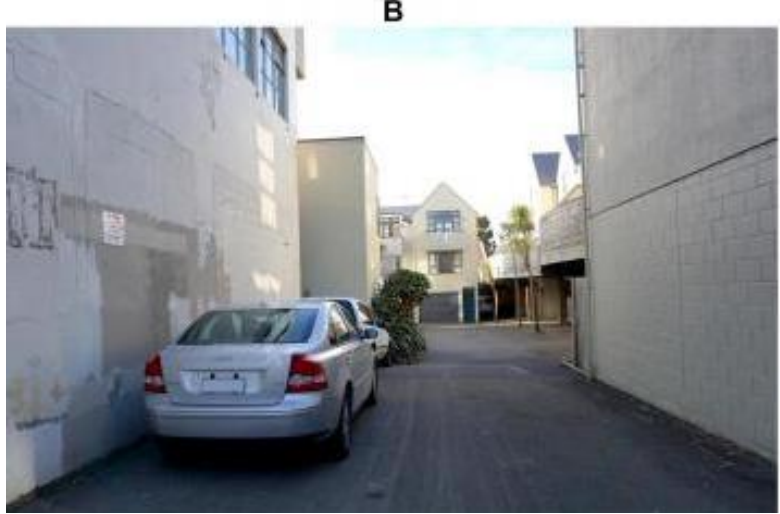

C

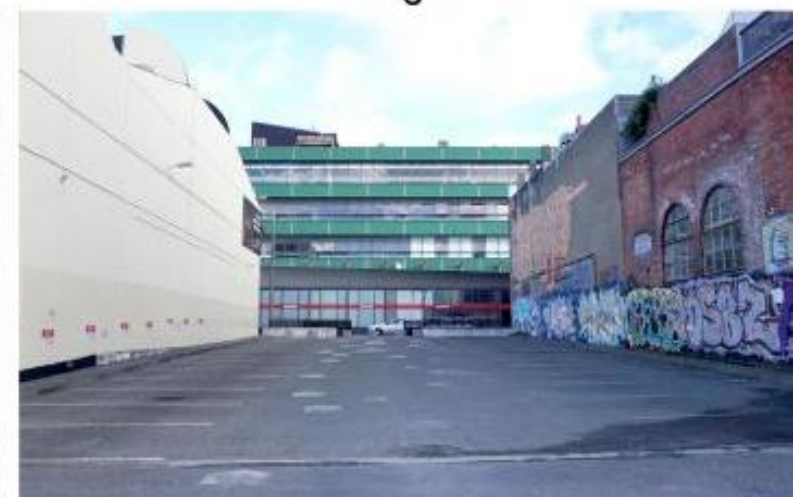

Figure 6.81 Options shown to participants 


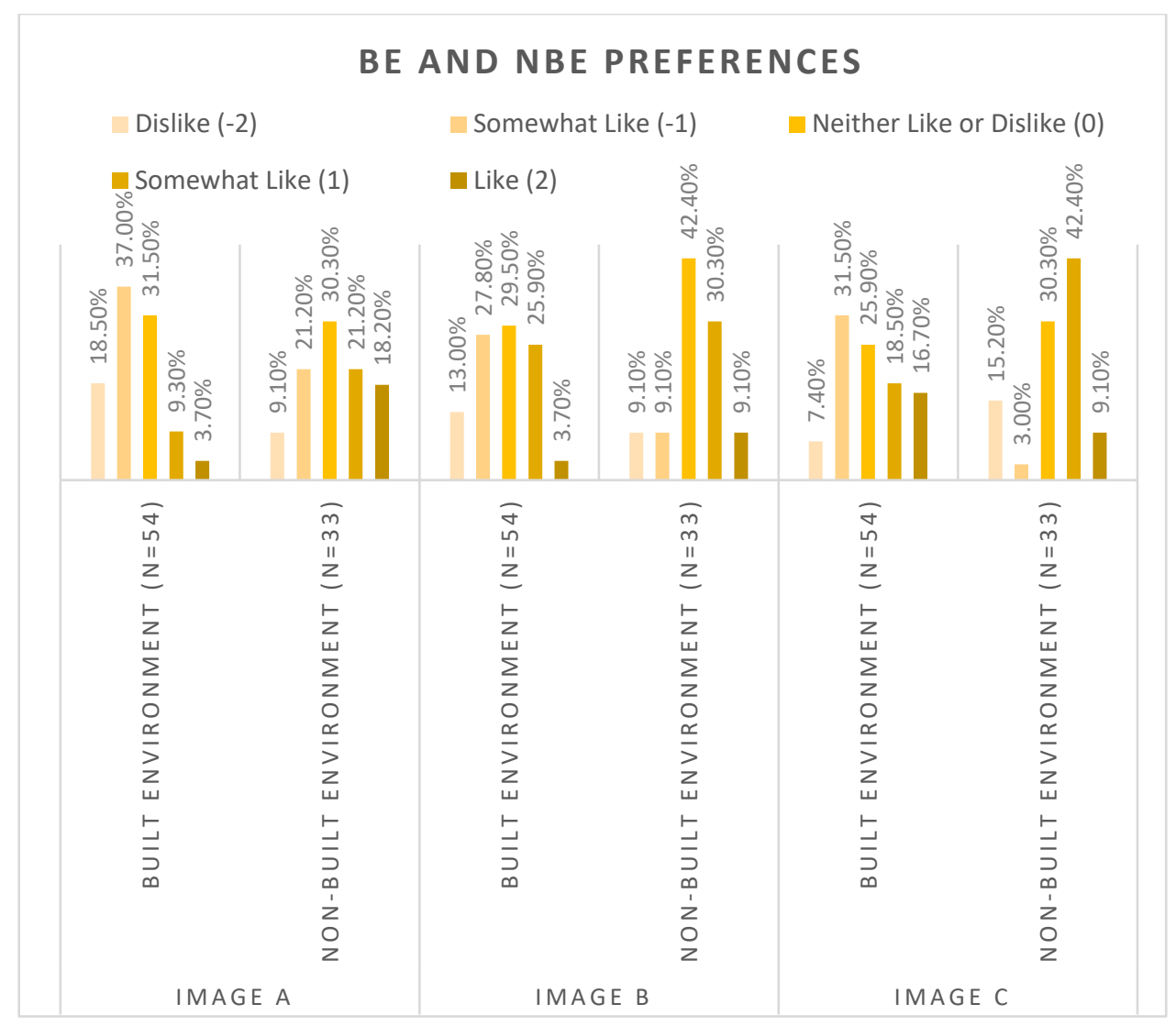

Figure 6.82 Preferences for different options as rated by participants

\subsection{Space on the rooftop of a building}

Overall, $52 \mathrm{BE}$, and $32 \mathrm{NBE}$ participants responded to the question about the rooftop space. Option C (figure 6.83) was the most liked overall (27\% BE and $53 \%$ NBE). This option also had the highest mean score of 2.92 with a Standard Deviation (SD) of 1.13 for BE participants and a mean score of 3.53 with an SD of 1.04 for NBE respondents. However, the likings for option A were similar, with a lower $23 \%$ of $B E$ and a higher $56 \%$ of NBE respondents liking it. This option had a mean score of 2.60 with an SD of 1.14 for BE participants and a slightly lower mean score of 3.34 with an SD of 1.08 than option C for NBE respondents. Option B was less liked (17\% BE and 19\% NBE) (figure 6.84). Image $B$ had a mean score of 2.25 with an SD of 1.20 for BE participants and a mean score of 2.16 with an SD of 1.27 for NBE respondents. 
A
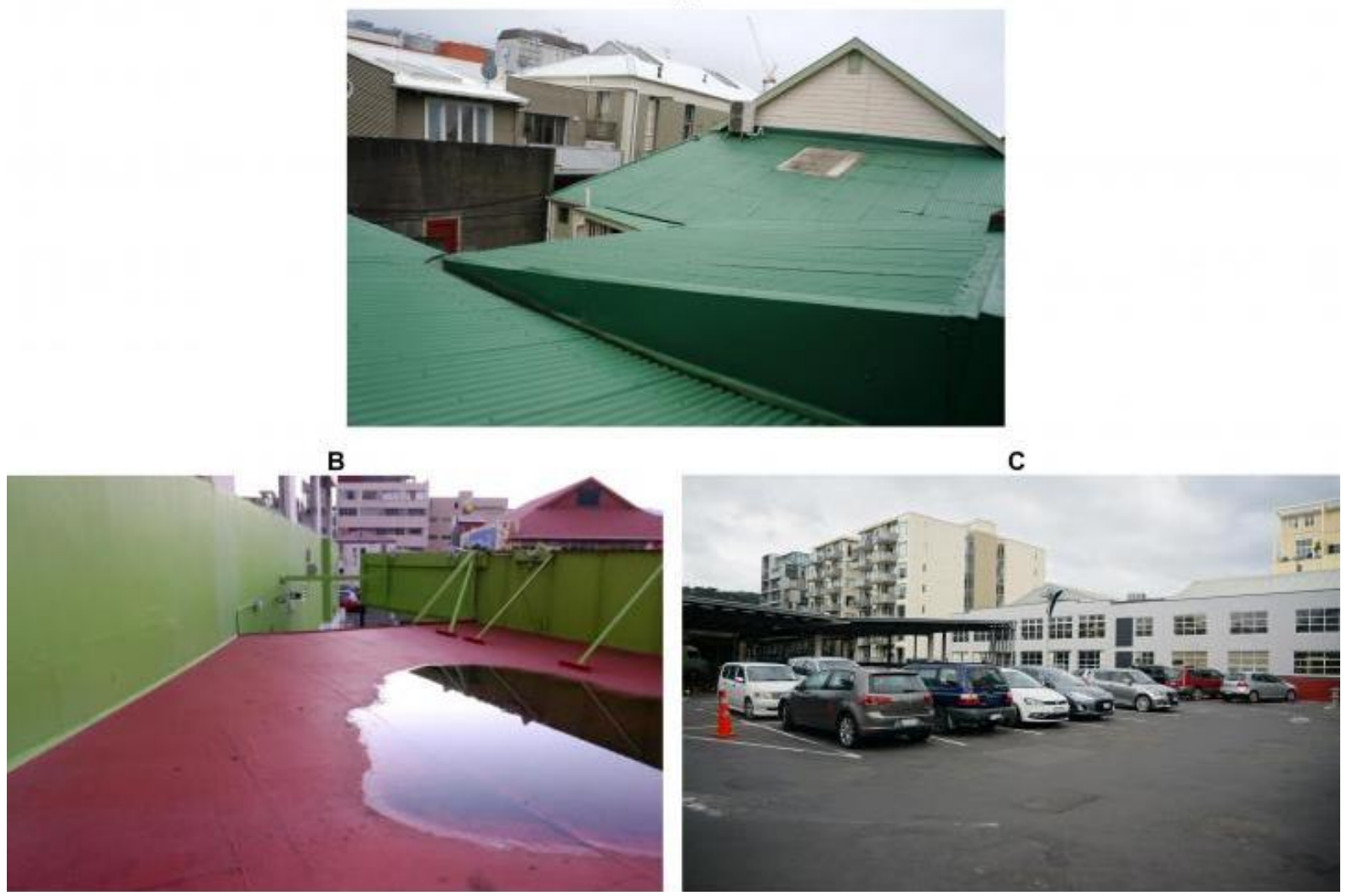

Figure 6.83 Options shown to participants

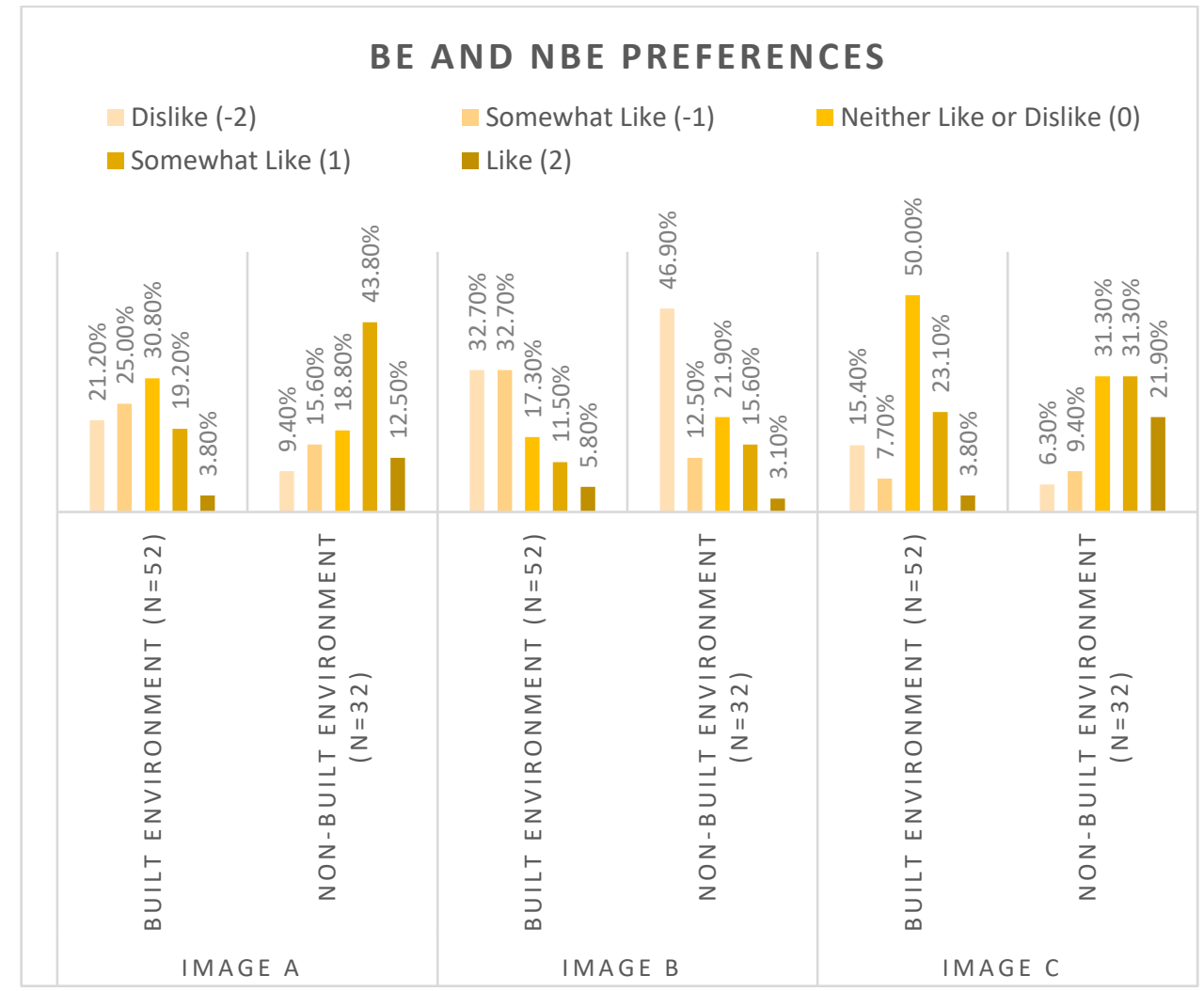

Figure 6.84 Preferences for different options as rated by BE and NBE participants 


\subsubsection{Part Four}

In part 4 of Study One participants were shown pairs of photos and asked to select one out of the two that needed transformation. Participants were given a list of options for improving the aesthetics of the space by adding to and removing things from it. The suggestions for improving the selected image are given below.

\subsection{Space underneath a building}

For the space underneath a building, $51 \mathrm{BE}$, and $32 \mathrm{NBE}$ participants responded to the question, with $82 \% \mathrm{BE}$ and $75 \%$ of NBE participants wanting to change option $\mathrm{A}$ (figure 6.85). The favourite suggestions from $B E$ participants concerned changing the surface materials $(38 \%)$, creating a clear pathway $(36 \%)$, providing more vegetation (35\%), and improving the maintenance and cleanliness (33\%). NBE participants differed, suggesting improving the lighting condition (39\%), improving the maintenance and cleanliness (36\%), creating a clear pathway (33\%), and providing more vegetation (28\%) (figure 6.86).

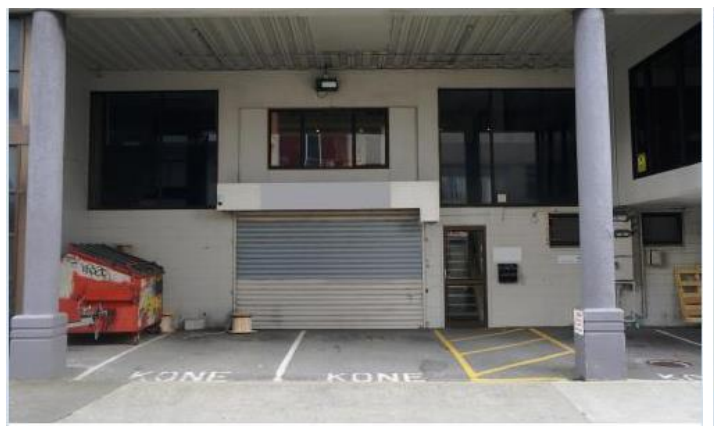

A

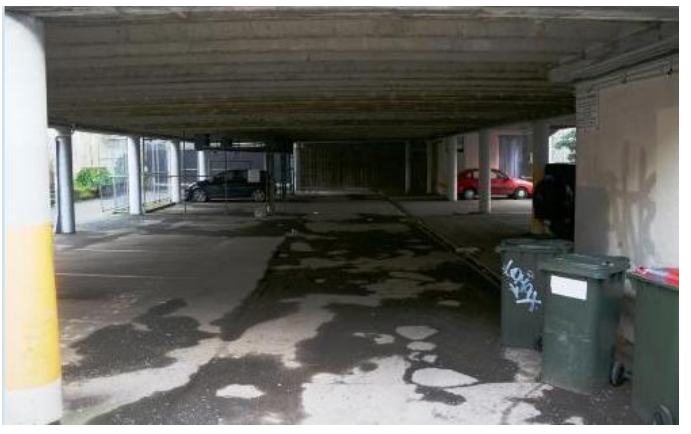

B

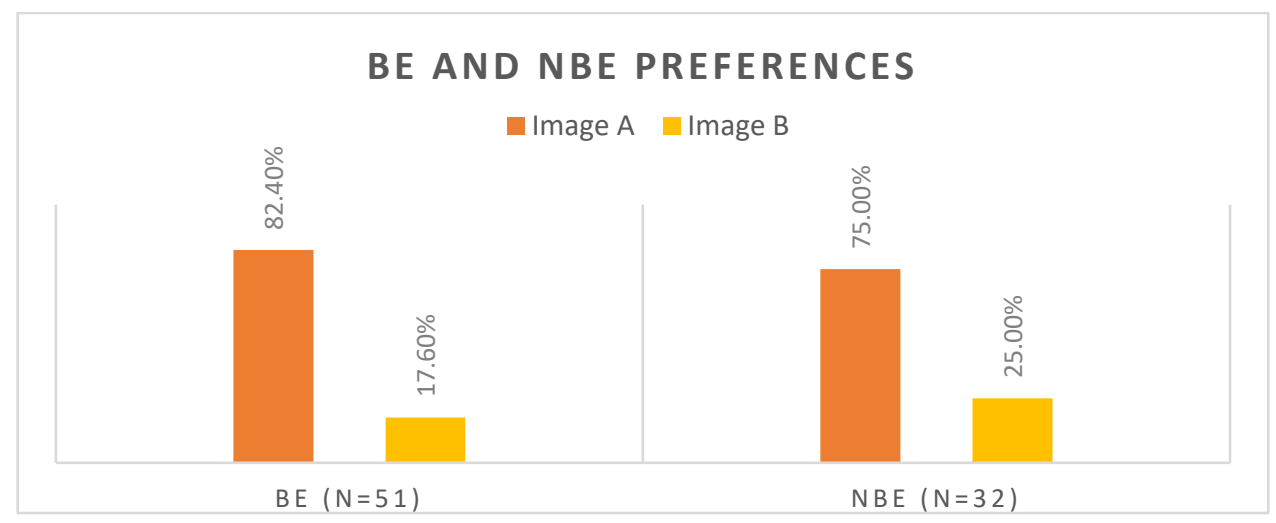

Figure 6.85 Preference for options $A$ and $B$ for BE and NBE participants 


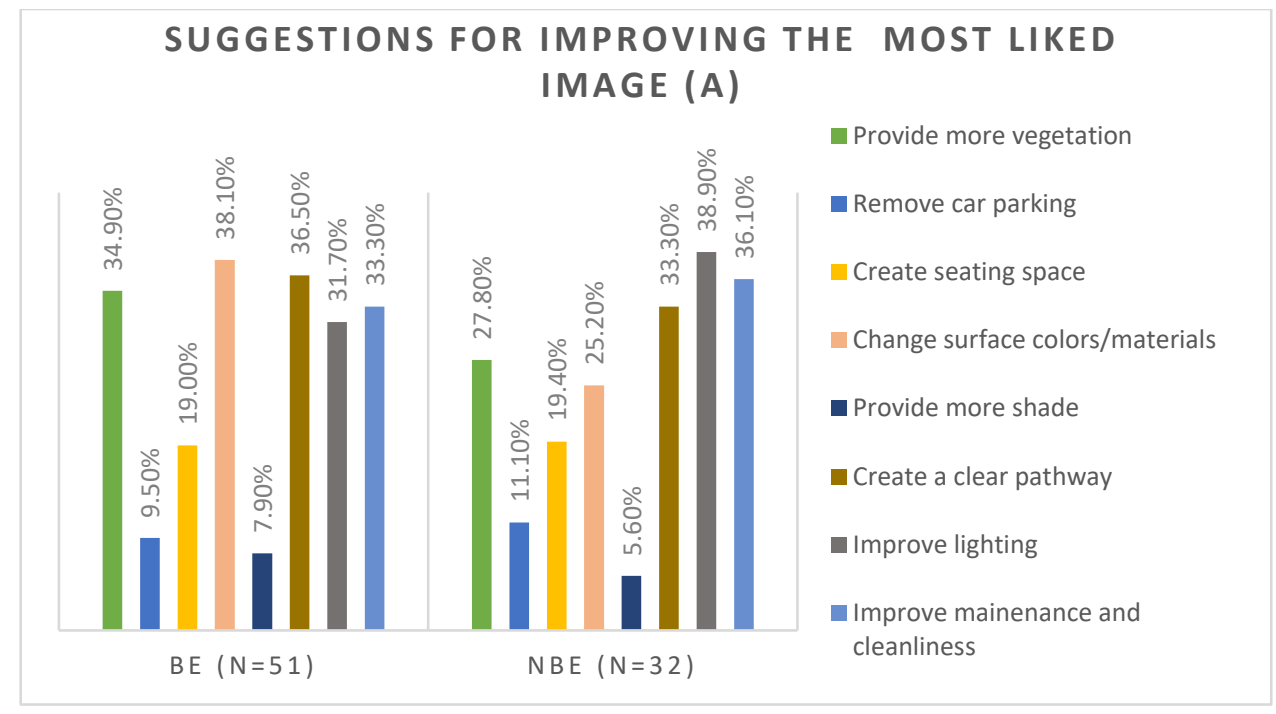

Figure 6.86 Preferences for improvement options as rated by BE and NBE participants

\subsection{Space in front of a building}

Overall $51 \mathrm{BE}$ and $32 \mathrm{NBE}$ participants responded to the question about space in front of a building, with $69 \% \mathrm{BE}$ and $72 \%$ NBE respondents wanting to see a change in option B (figure 6.87). The most common suggestions from BE participants for transforming this space were the removal of boundary walls (40\%), providing more vegetation (35\%), and creating more seating space (21\%). NBE participants agreed with the first two suggestions, opting for providing more vegetation (39\%), removal of boundary walls (36\%), and creating a clear pathway (25\%) (figure 6.88).

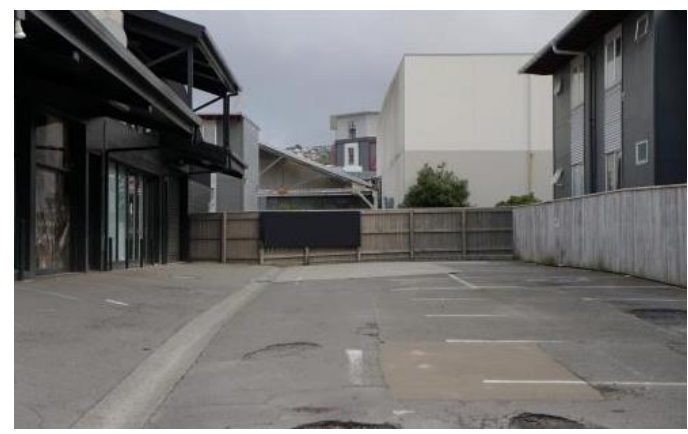

A

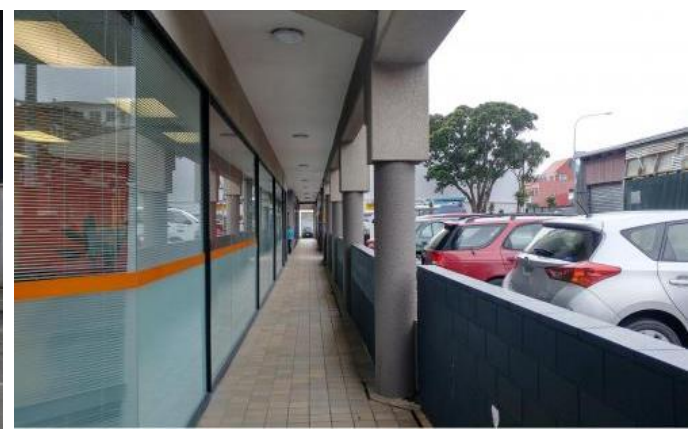

B 


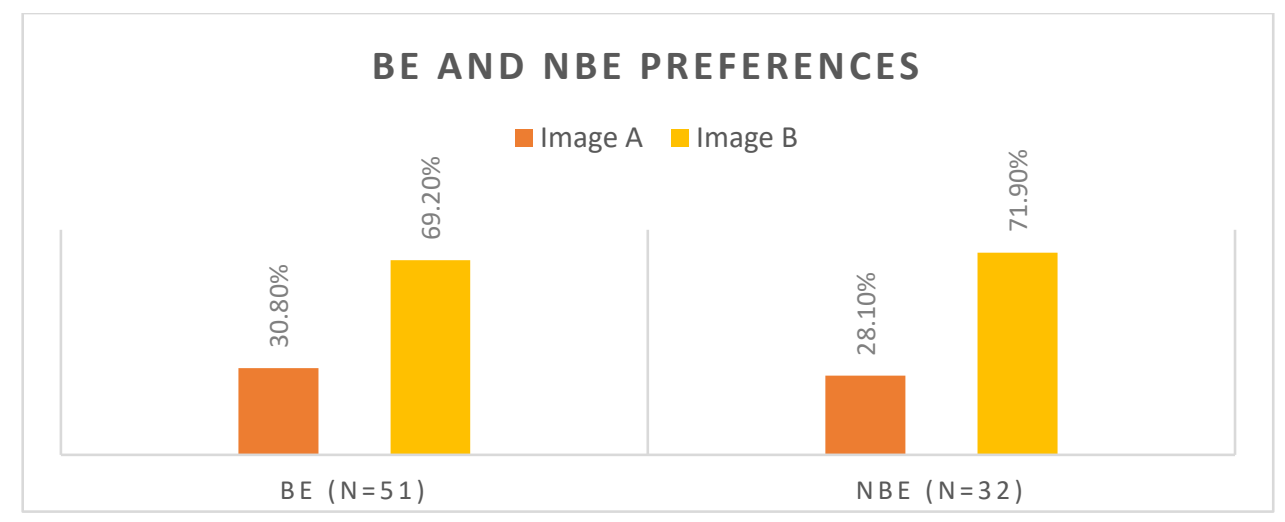

Figure 6.87 Preference for options A and B by BE and NBE participants

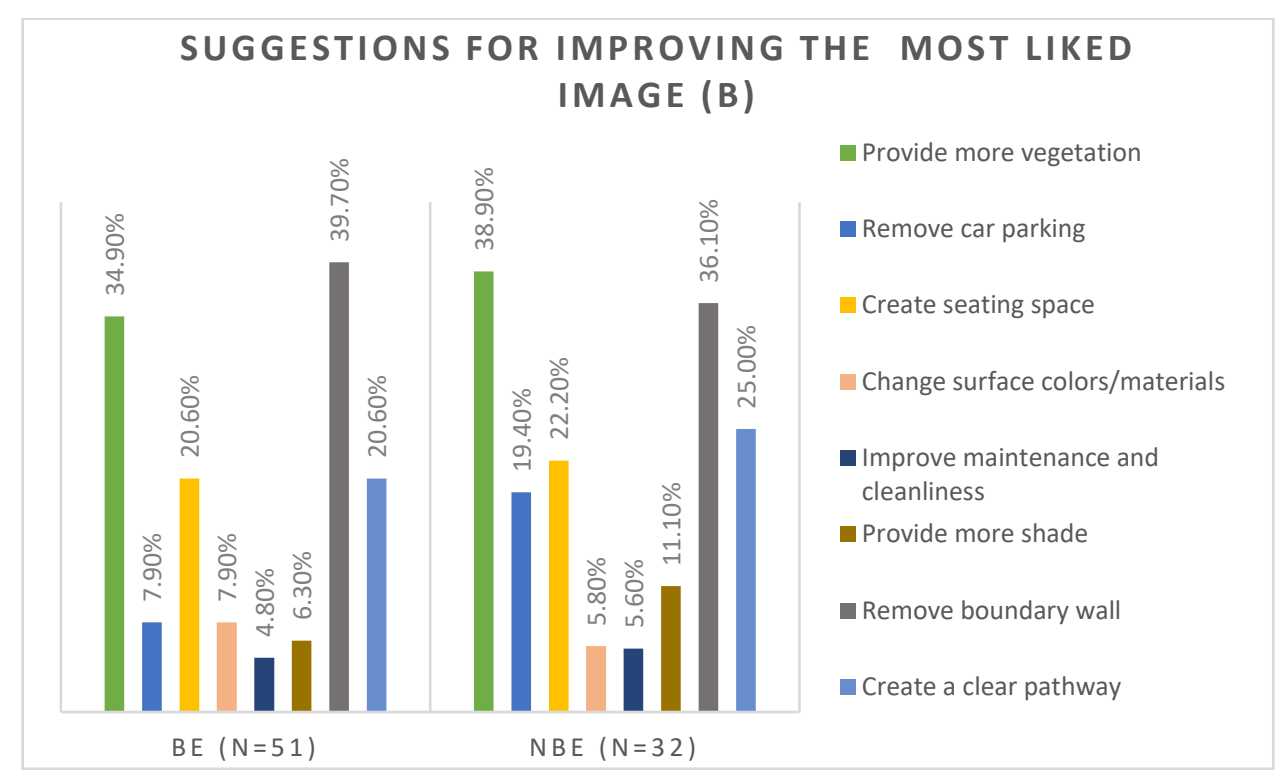

Figure 6.88 Preference for improvement options as rated by BE and NBE participants

\subsection{Space at the back of a building}

For the space at the back of a building, $51 \mathrm{BE}$, and 32 NBE participants responded, and $61 \%$ of BE and $62 \%$ of NBE participants wanted to see a change to option B (figure 6.89). The most common suggestions from BE participants were providing more vegetation $(40 \%)$, changing the surface materials $(30 \%)$, and creating a clear pathway (30\%). NBE participants were similar by wanting to provide more vegetation $(36 \%)$, create a clear pathway (25\%), and change the surface materials (25\%) (figure $6.90)$. 


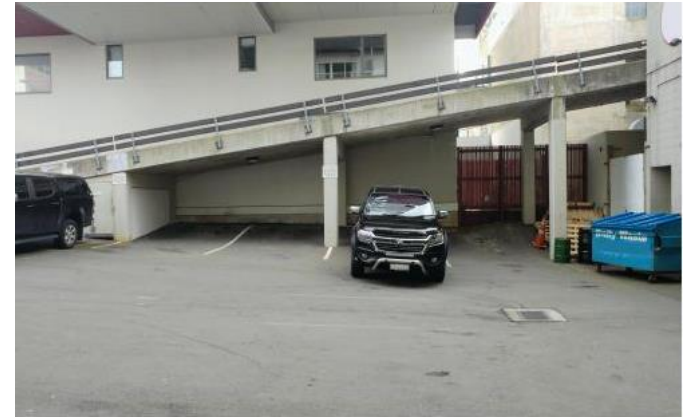

A

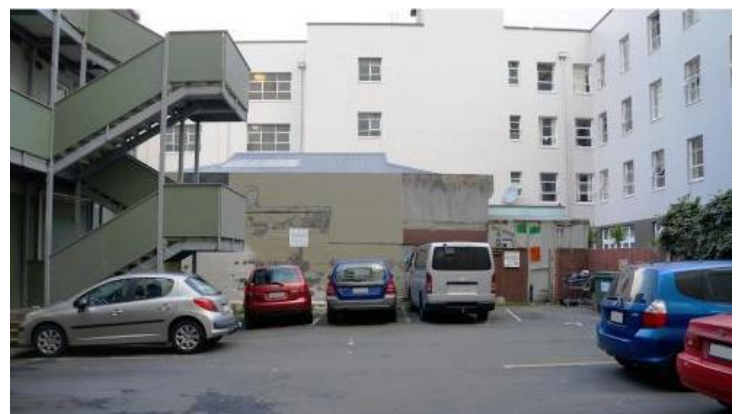

B

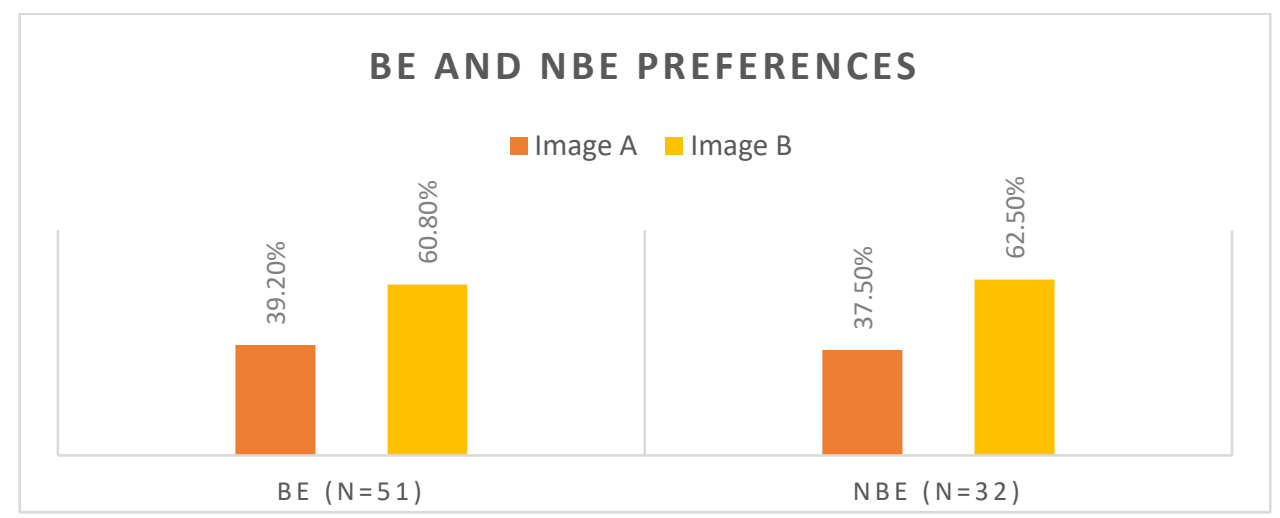

Figure 6.89 Preferences options A and B by BE and NBE participants

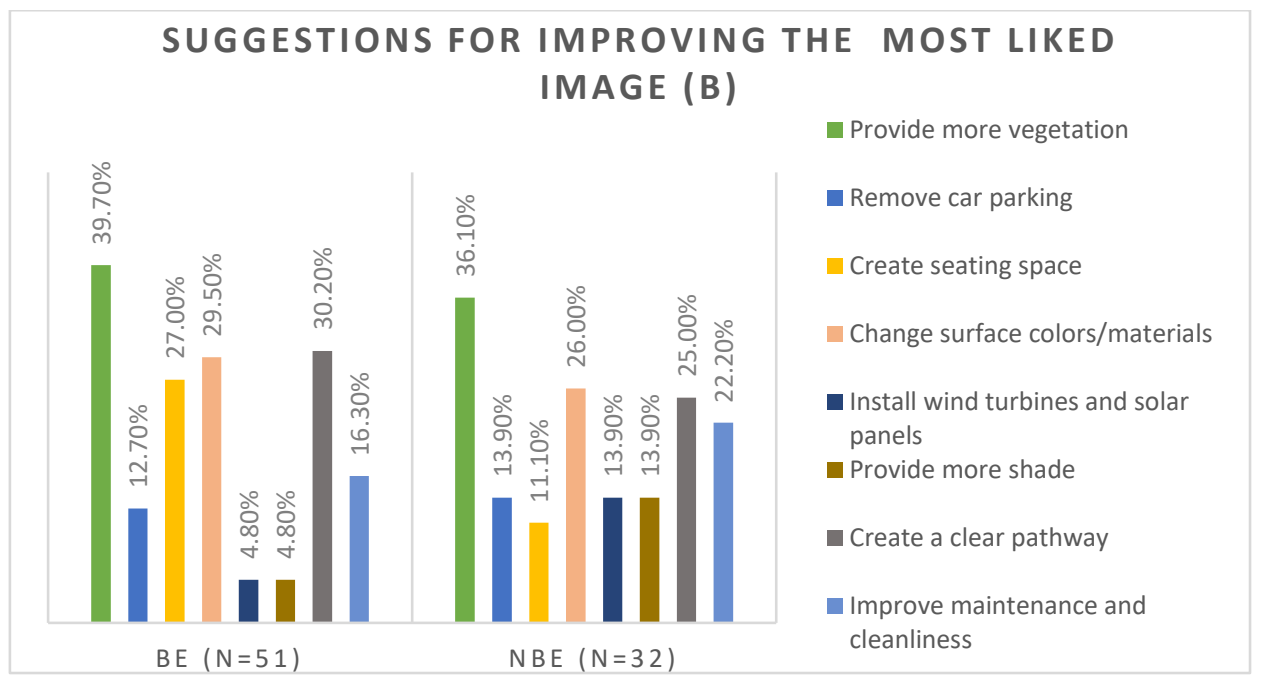

Figure 6.90 Preferences for improvement options as rated by BE and NBE participants

\subsection{Space enclosed by buildings on three sides}

For the space enclosed by buildings on three sides, $51 \mathrm{BE}$, and 32 NBE participants responded with $57 \% \mathrm{BE}$ and $87 \%$ of NBE participants wanting to change option $\mathrm{B}$ (figure 6.91). The most common suggestions from BE participants were providing more vegetation $(33 \%)$, creating a clear pathway $(29 \%)$, and allowing graffiti on the 
wall $(21 \%)$. The first two were strongly echoed by NBE participants with providing more vegetation (47\%) and creating a clear pathway (33\%), followed by changing the surface materials (31\%) (figure 6.92).

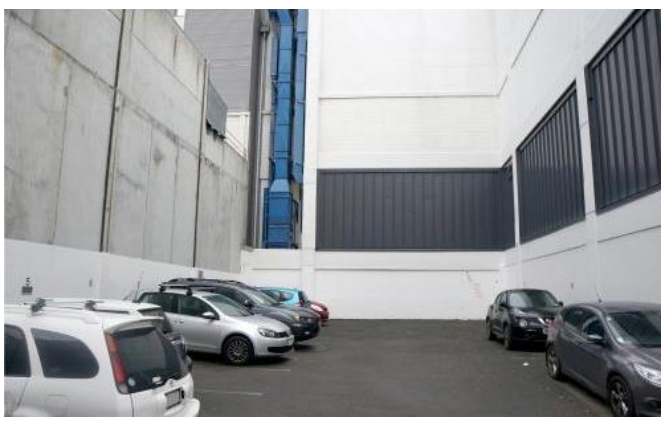

A

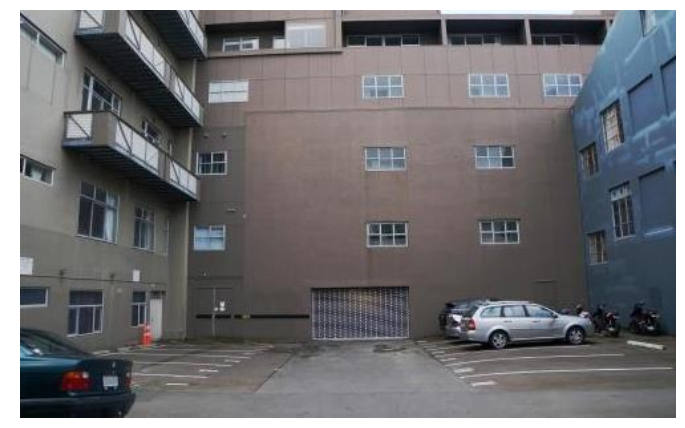

B

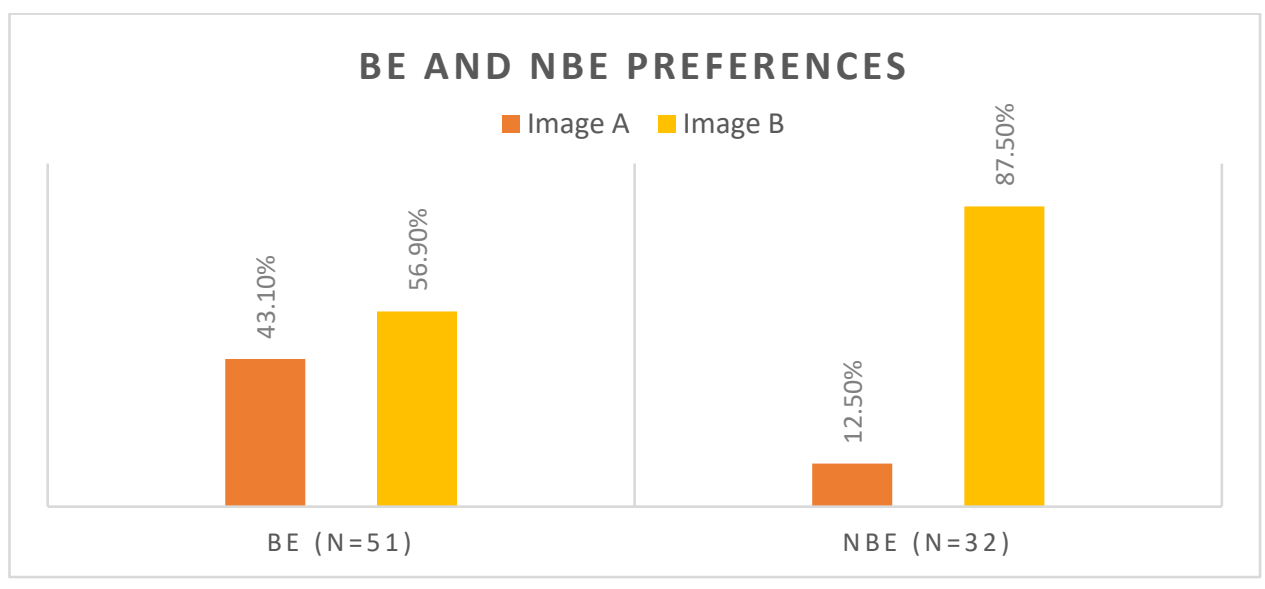

Figure 6.91 Preferences for options A and B by BE and NBE participants

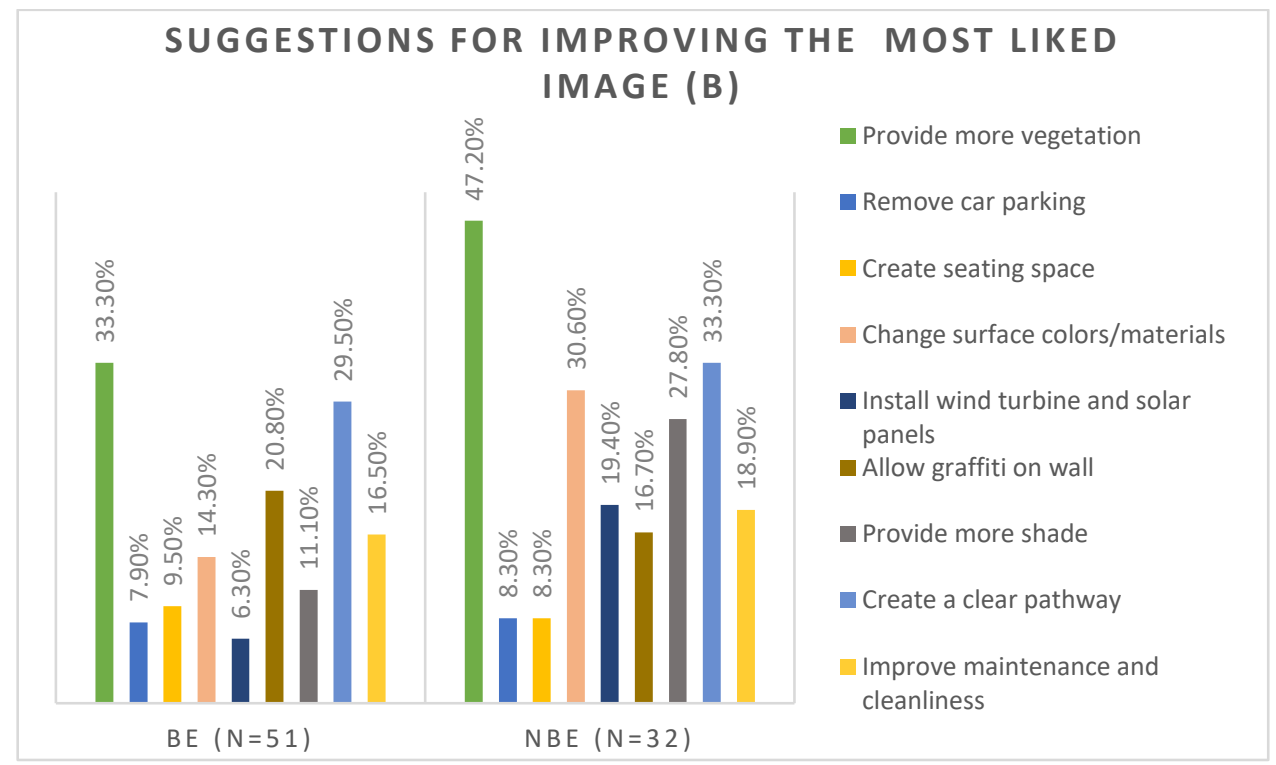

Figure 6.92 Preferences for improvement options as rated by BE and NBE participants 


\subsection{Space enclosed by buildings on two sides}

For the space enclosed by buildings on two sides (answered by $51 \mathrm{BE}$, and 32 NBE participants) $75 \%$ BE and $94 \%$ NBE participants choose to change option A (figure 6.93). The most recurring suggestions among $B E$ participants were providing more vegetation (43\%), creating a clear pathway (38\%), and changing the surface material (23\%). Again, the first two suggestions were echoed by NBE participants with providing more vegetation (44\%) and creating a clear pathway (47\%), followed by providing more shade (31\%) (figure 6.94).

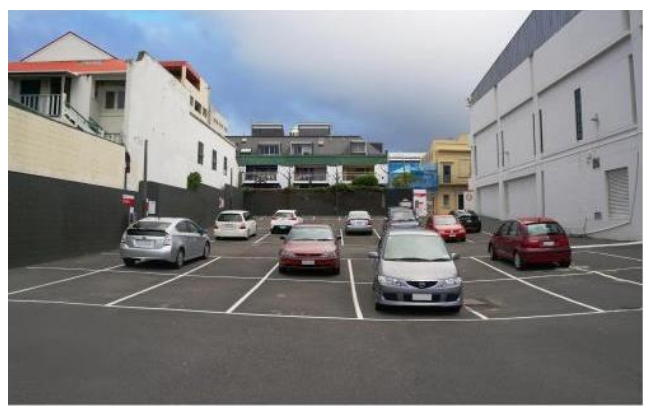

A

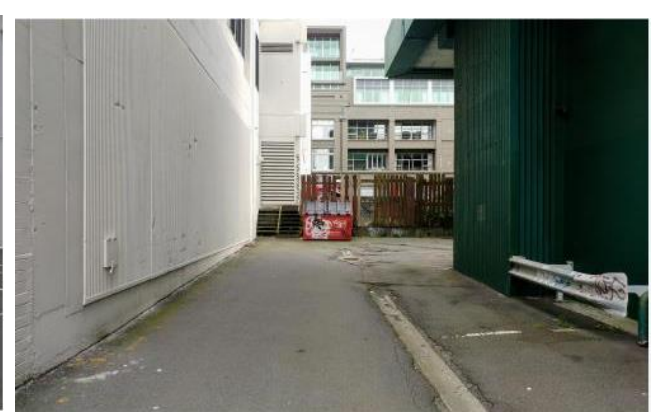

B

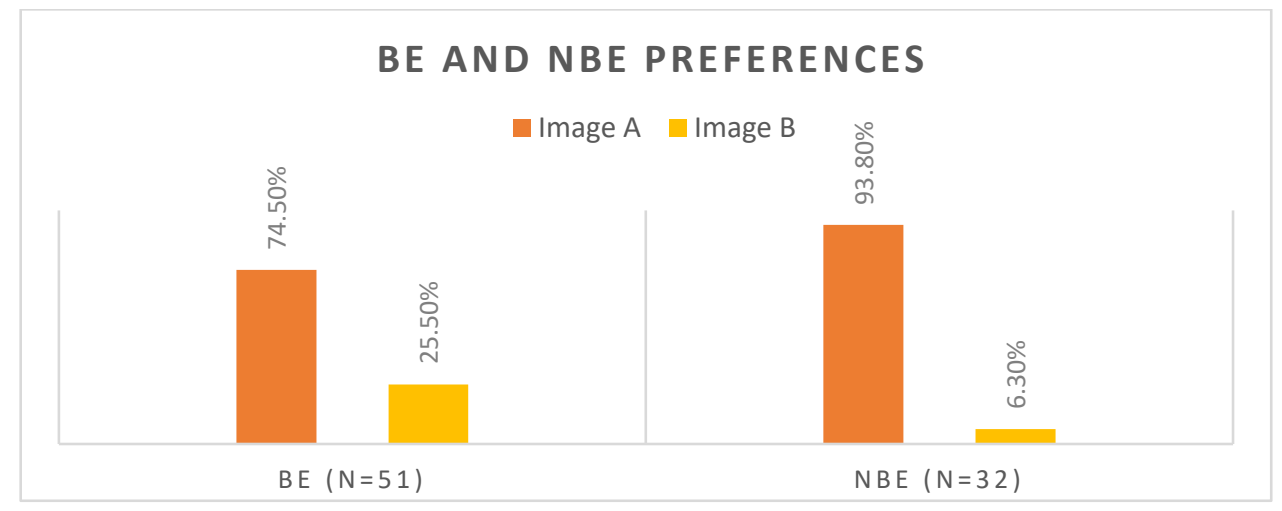

Figure 6.93 Preferences for options A and B by BE and NBE participants 


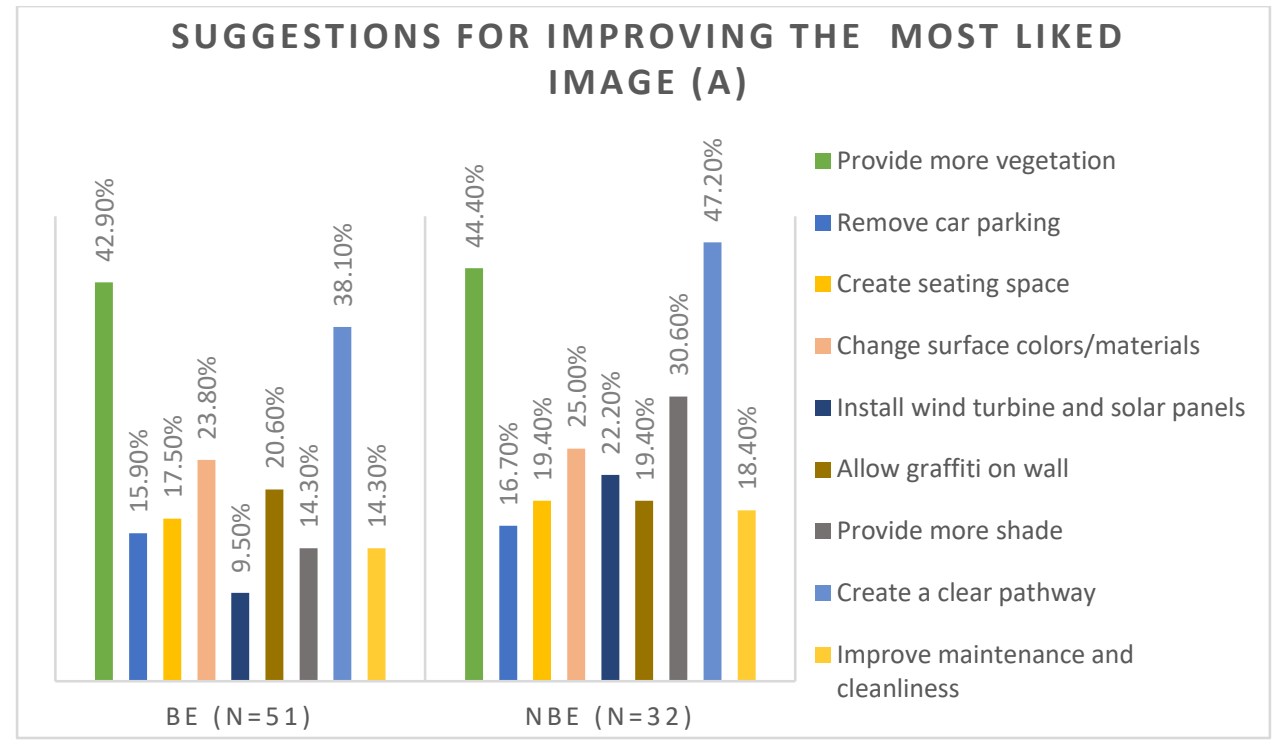

Figure 6.94 Preferences for improvement options as rated by BE and NBE participants

\subsection{Space on the rooftop of a building}

Overall, $51 \mathrm{BE}$ and $32 \mathrm{NBE}$ participant responded to the question about the rooftop space, with $56 \%$ of both BE and NBE participants wanting to change option B (figure 6.95). The most common suggestions from $B E$ participants concerned providing more vegetation $(37 \%)$, creating seating space $(22 \%)$, and creating a clear pathway $(22 \%)$. Again, NBE participants echoed the first two with a change for the third suggestion with providing more vegetation (33\%), creating a seating space (27\%), and installing wind turbines and solar panels (14\%) (figure 6.96).

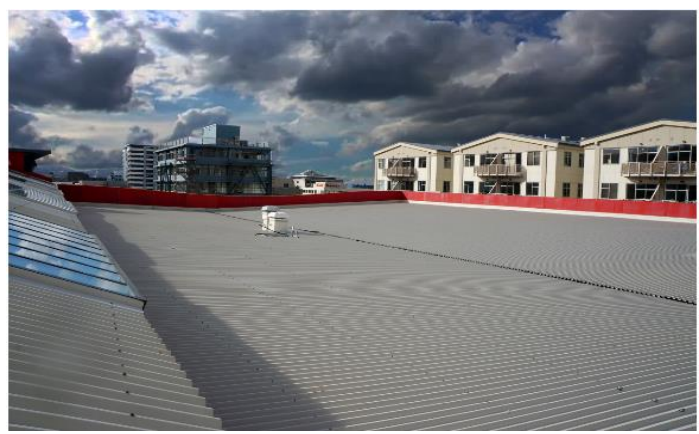

A

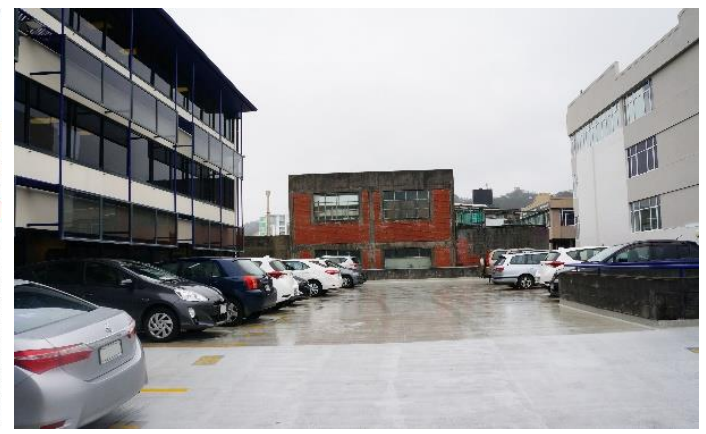

B 


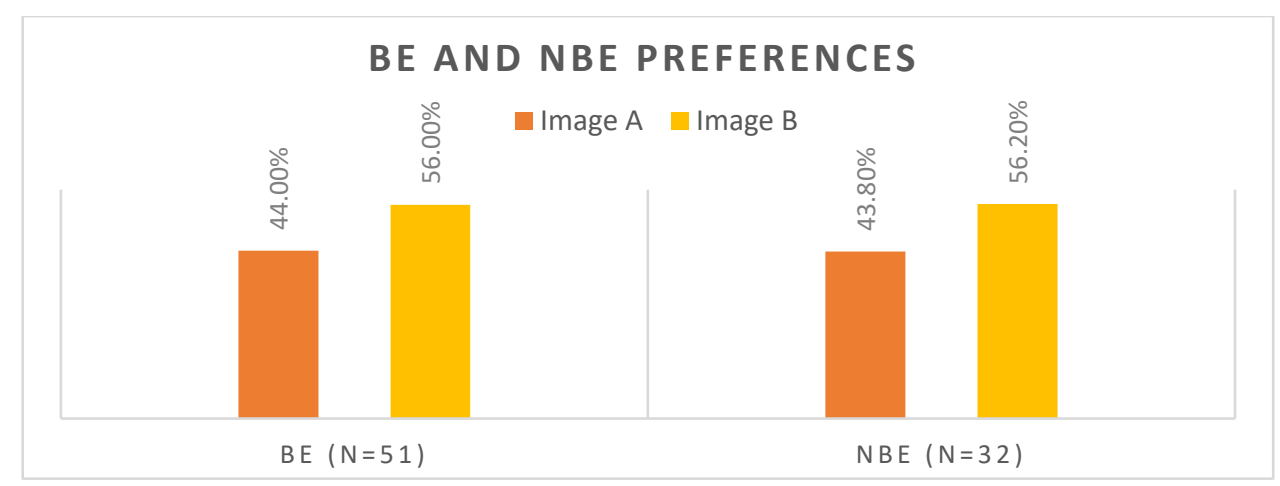

Figure 6.95 Preferences for options A and B by BE and NBE participants

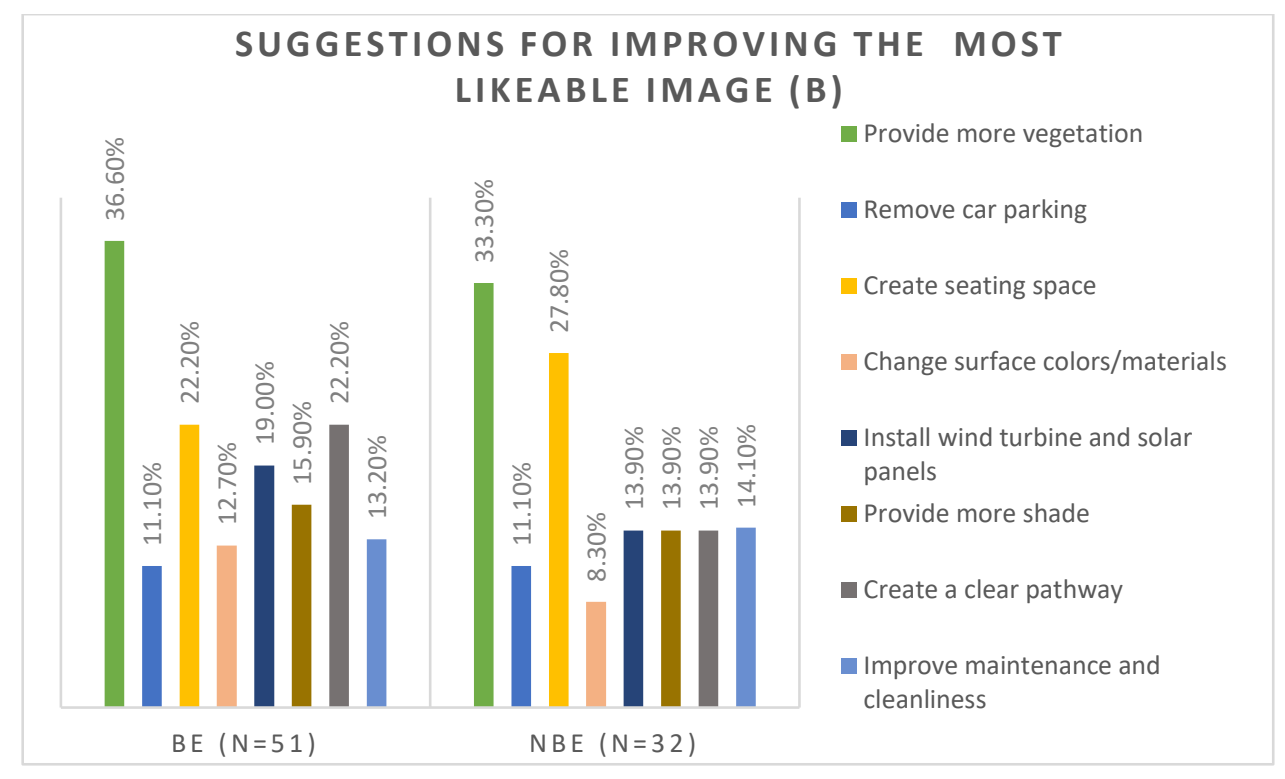

Figure 6.96 Preferences for improvement options as rated by BE and NBE participants

\subsection{Study Findings}

The particular approach taken in Study One was to understand how the sample viewed the selected leftover spaces rather than speculating as to what people see as a problem within a space and how improvements might be made. Study One thus investigated the relationships between different preferences and space attributes. The results showed that people have different opinions, but there are some common liked attributes for different leftover spaces. If these can be categorised, this could help in the redesign of leftover spaces (see section 7.3). 


\subsubsection{Stage one: Whole Sample $(\mathrm{N}=99)$}

The second part of Study One confirmed Nasar's (1998) theory for the four aspects of naturalness, organised space, open space, and well-maintained space. These four aspects were behind the preferences and likes of the participants and is confirmed by the reasons for liking spaces they gave, such as the presence of planting, good organisation, being open to the sky, and having adequate cleanliness (see section 6.3.1.2). The results disagreed with Nasar for the preference for an older style building. People liked the modern style of building more, with their reasons being that the building was constructed with new materials, used bright colours, and explored different geometric shapes.

In the third part of this study, participants were asked to rate each of three photos on a Likert scale from -1 (Dislike) to +2 (Like). This part of Study One was aimed at investigating the preferences for different spaces of the same type but with different attributes in each space. It became evident from the results that the sample preferred spaces, which were visually permeable, open to the sky, clean, constructed with different surface materials, well maintained, had vegetation, graffiti on walls, good lighting, organised parking spaces, a variety of facades, a clear thoroughfare, and different levels. The least liked spaces had attributes such as dead ends, blank walls, poor maintenance, no apparent entrance, and parked cars.

The fourth part of Study One was based on two photos for each type of leftover space out of which participants had to choose the one photo which needed change. It became evident that people wanted to change those scenes that were more chaotic and those that were not being actively used and looked neglected. This part aimed to use the preferred photo, which would then be photomontaged with the attributes suggested the most for further use in Study Two. The overall suggestions for improving the leftover spaces related to providing more vegetation, creating clear pathways, changes to surface materials/colours, creating seating spaces, improving maintenance and cleanliness, providing more shade, removal of car parks, and installing wind turbines and solar panels (figure 6.97). Other less favoured suggestions were the removal of boundary walls or fences and having graffiti on walls. 


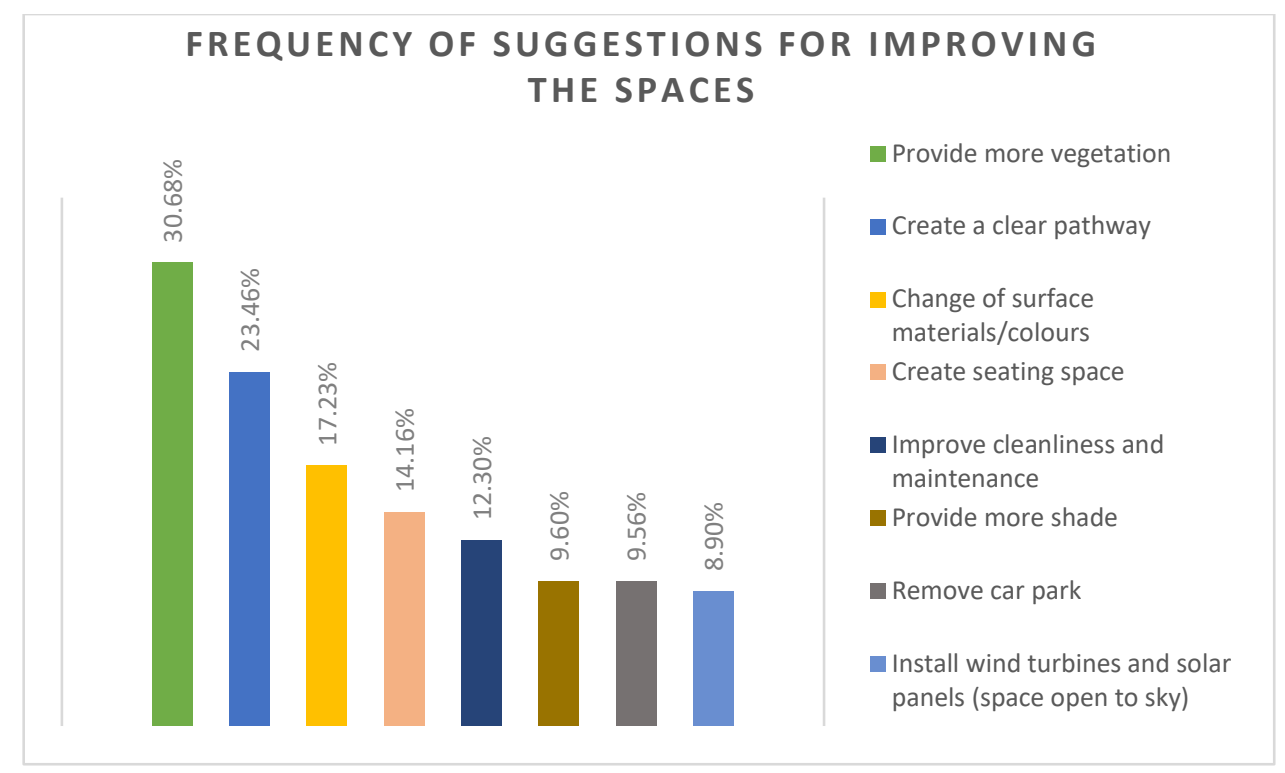

Figure 6.97 Overall modifications suggested by participants for the leftover spaces

\subsubsection{Stage two: Gender analysis}

This stage probed the differences in preferences between males and females, noting the female group was slightly larger than the male group.

The second part affirmed Nasar's (1998) theory for the aspects of naturalness, organised space, open space, and well-maintained space as both gender groups preferred these aspects. However, in one example the males preferred an enclosed space $(49 \%)$ significantly more than females $(24 \%)$, perhaps because space was being actively used. The results again disagreed with Nasar's findings for the attribute of the older style of building. Both genders liked the modern style of building, although females were more in favour of the older style building (35\%) than males $(27 \%)$.

The third part investigated the likings on a Likert scale (1-5) for three photos of different leftover spaces with different attributes. It became evident from the results that the likings of males and females were similar for all spaces. The fourth part was based on two photos for each type of leftover space from which participants selected one photo which they thought needed changing. The suggestions for making changes to the most liked images were similar, as both genders agreed that providing more cleanliness and maintenance by removing garbage bins could transform the spaces. Females favoured providing more vegetation, creating clear pathways, creating seating space, and allowing graffiti on walls more than males, while males preferred removing the car 
parks, making changes to surface materials/colours, providing more shade, improving the maintenance and cleanliness, and installing wind turbines and solar panels more than females (figure 6.98). Other less favoured solutions related to the removal of the boundary walls for spaces in front of a building.

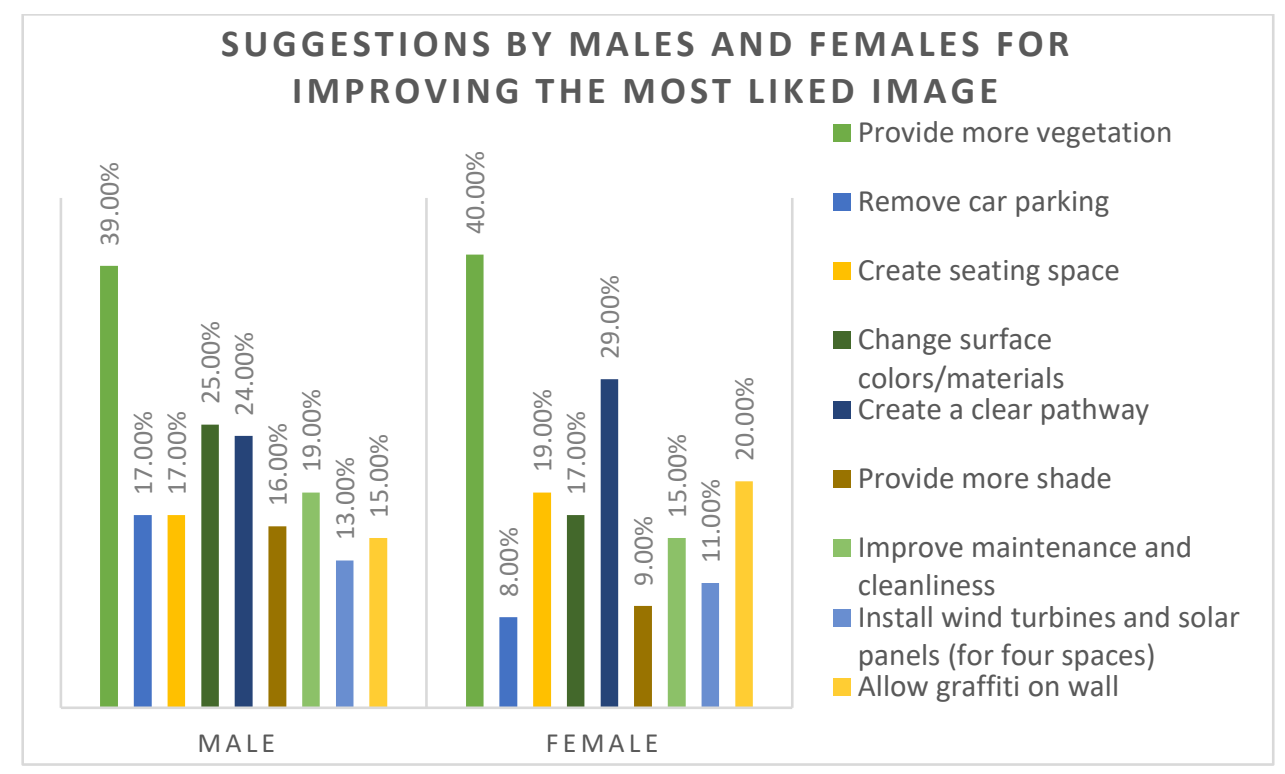

Figure 6.98 Overall modifications suggested by male and female participants

\subsubsection{Stage three: Built Environment (BE) and Non-Built Environment (NBE) Participants}

This stage looked for differences in preferences between those with a built environment background and those with other backgrounds.

Stage three confirmed Nasar's (1998) theory for the aspects of naturalness, organised space, open space, and well-maintained space, for both BE and NBE participants. BE participants preferred the enclosed space $(40 \%)$ slightly more than NBE $(30 \%)$ because of its practical usage. The results again disagreed with Nasar's theory for the attribute of an older style of building as both BE and NBE participants liked the modern style of building. However, BE respondents were slightly more in favour of a modern style of building (71\%) than NBE participants (64\%).

The third part investigated the liking for three photos of different leftover spaces with different attributes on a Likert scale (1-5). It became evident from the results that the likings of BE and NBE participants were very similar for all spaces. The fourth part 
concerned suggestions for improving the selected image and these were again similar for both groups. BE participants wanted to see changes related to providing vegetation, changing surface materials/colours, and allowing graffiti on walls more than NBE participants (figure 6.99). NBE participants were more definite about removing car parks, creating seating space, creating clear pathways, and providing more shade. Other suggestions for improving the spaces related to installing wind turbines and solar panels for the space on the rooftop of a building, providing more cleanliness and maintenance for the space underneath a building, and removing boundary walls for space in front of a building.

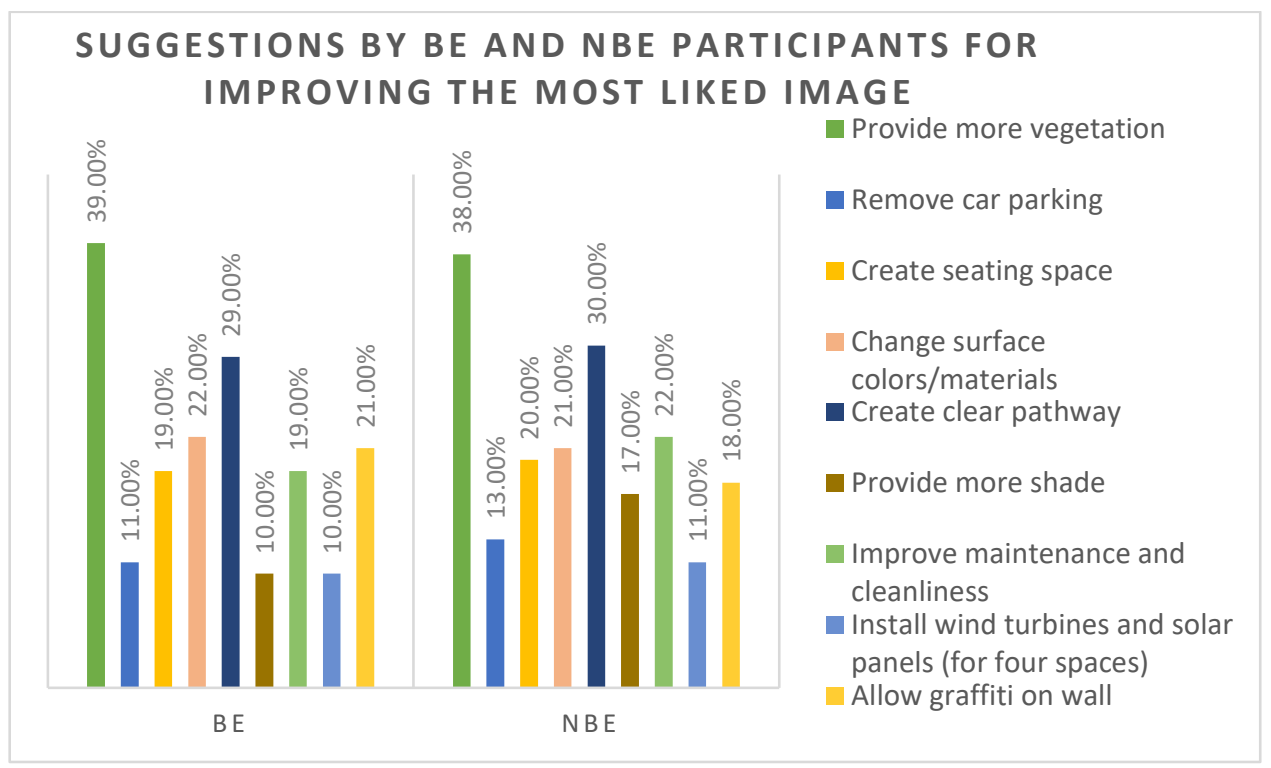

Figure 6.99 Overall modifications suggested by BE and NBE participants

\subsection{Summary}

Chapter 6 has discussed the technique for recruiting participants, conducting Study One with the representation of spaces through using photographs, testing Nasar's theory relating to environmental perception, and collecting information regarding people preferences about different leftover spaces and suggestions for improving these. One exciting finding drawn out from Study One was that both BE and NBE participants suggested modifying spaces by providing more vegetation. This variable was also highly preferred by both male and female participants. Therefore, one aspect of Nasar's theory concerning the liking for naturalness was confirmed by the Study One findings. The next Chapter gives the details of the visual preferences study using 
Chapter 6 - STUDY ONE: FIRST VISUAL PREFERENCE STUDY

photomontages. The photomontages of leftover spaces were designed per the suggestions made by participants in Study One. 


\section{CHAPTER 7}

\section{STUDY TWO: SECOND VISUAL PREFERENCE STUDY}

\subsection{Introduction to Study Two}

Study Two was a follow-up study based on the results of Study One (Chapter 6) in which participants rated design changes made to different types of urban leftover space by envisaging these through photographs. The preferred changes from Study One were photomontaged onto the original images for Study Two. The six different leftover spaces which were depicted as vacant and without any specific function in Study One (underneath a building, in front of a building, behind a building, enclosed by buildings on two sides, enclosed by buildings on three sides, and a rooftop) were represented with the most preferred changes. Following analysis of the data collected from Study One, it became evident that people wanted to see more vegetation and cleanliness in urban leftover spaces. Study Two probed these ideas in more detail.

\subsection{Description of Study Two}

Study Two was divided into three parts (table 7.1). The first part sought information related to demographics, which included age, gender, academic qualification, the field of education/work, and cultural ethnicity.

The second part had three sections. The first presented three design modifications for each of the six leftover spaces, with a Likert scale for rating the modifications ('Dislike 1', 'Somewhat dislike 2', 'Neither like or dislike 3', 'Somewhat Like 4', and 'Like 5'). Numerical values were then assigned for calculating how much each scheme was liked. The second section was related to the semantic differential measures which sought each participant's reaction to the redesigned space through a series of stimulus concepts. The concepts (adjectives) were evaluated through a 5-point bipolar rating scale. This section investigated reactions to the concepts of attractiveness (uglybeautiful), satisfaction (annoying-pleasing), buildable (impossible-realisable), usability (boring-interesting) and mood (constrained-energetic) (Parkes \& Thrift, 1980; Hanyu, 1993). The adjectives were chosen according to how they best fitted the study's aims and were consistent throughout Study Two. The Likert-scale reveals how much people 
agree or disagree with a particular statement, whereas the semantic differential scale decides how much of a trait or quality the item has when rated using a bipolar scale defined by adjectives (Osgood \& Snider, 1969).

The third section collected suggestions from respondents about improving the visual quality of the redesigned spaces and included an empty suggestion box. This part had multiple-choice questions about visual preferences for four different leftover spaces. Participants were asked to select one or two things that could happen to space from a list of options, for example, removing all the parking spaces.

\begin{tabular}{|c|c|c|}
\hline \multicolumn{3}{|c|}{ Study Two } \\
\hline Part 1 & Part 2 & Part 3 \\
\hline $\begin{array}{l}\text { Demographic } \\
\text { Data }\end{array}$ & $\begin{array}{l}\text { Section 1: Likert scale } \\
\text { Section 2: Semantic Differential } \\
\text { Section3: } \\
\text { suggestions for } \\
\text { improvements }\end{array}$ & $\begin{array}{l}\text { Multiple-choice } \\
\text { questions }\end{array}$ \\
\hline
\end{tabular}

Table 7.1 The three parts of the study two

\subsubsection{Photographic representation}

The photomontage visuals in Study Two were produced based on the results of Study One (see section 6.4.1.3), and three alternative design modifications for each type of leftover spaces were produced. According to Stamps $(1990,1992)$, reaction to a photomontage gives similar responses to those experienced in the real world, and Stamps found a correlation of preferences for places represented through photographs with on-site experience. In this research, all one-point perspective photos were treated in Photoshop to reconstruct the spaces after each change with an emphasis on one specific attribute in each leftover space, noting that these attributes changed with the spaces. All the leftover spaces were designed without changing the current usage of the site. The concepts of providing more vegetation, improving maintenance or cleanliness, and changes to the surface materials were photomontaged for the space underneath a building. For the space in front of a building, removing the boundary walls, providing more vegetation, and providing seating space were conceptualised. Changing the surface material, providing more 
vegetation, and creating a clear pathway were introduced for the backyard of a building. The visualisation of having a clear pathway, changes in surface material, and providing more vegetation was created for a space enclosed by buildings on three sides. For the space enclosed by buildings on two sides, the concepts were changes in surface material, providing more vegetation, and creating a clear pathway. For rooftop spaces, the actions visualised were creating seating space, providing more vegetation, and installing solar PV panels and wind turbines.

In Study One, the respondents had suggested various solutions for each type of leftover space, responding to the unique characteristics of each, and these were to be reflected in Study Two. As noted in section 6.4, the most popular design suggestion to emerge from Study One was providing more vegetation.

\subsection{Study Recruitment and Sample}

The Study Two survey was conducted from $13^{\text {th }}$ December 2017 to $15^{\text {th }}$ March 2018. The participants responded to an interactive web-based survey made using Qualtrics. The study was initiated after ethics approval was obtained (25265). The same procedure of conducting the survey was carried out as discussed in section 6.2, which was to send email invitations to participate, and by putting up posters, other invitation emails were sent to those Study One respondents who had agreed to participate Study Two.

By the end of the survey period, data had been collected from 121 individuals. The data were imported into the Statistical Package for the Social Sciences (SPSS) software for analysis. Overall, 96 participants completed, and 15 participants partially completed the survey, and 10 participants failed to complete it. The response rate for each question fluctuated with the number of participant responses. A draw was held on $20^{\text {th }}$ March 2018, and two participants were contacted through email to receive a NZ\$25 voucher. Respondents were also asked to indicate their willingness to participate in future focus group discussions (see Chapter 8.0) and 19 participants (16\%) volunteered for this.

\subsubsection{Sample demographics}

The demographic information about the participants was analysed to see the diversity in their educational and cultural backgrounds, which might reveal different preferences 
among subgroups. Regarding gender, $43 \%$ of respondents were male, $57 \%$ female. A high $56 \%$ of respondents were young (aged 18 to 35 ), and $44 \%$ of participants were aged 36 or above. Participants with built environment knowledge formed $24 \%$ of the sample, with the remaining $76 \%$ being from different fields. Just over half $(53 \%)$, the participants had an NZ European background compared to $47 \%$ who had a different cultural ethnicity. Regarding formal education, $68.0 \%$ of respondents had a postgraduate qualification (see table 7.2).

\begin{tabular}{|l|l|l|l|}
\hline Demographic Distribution \\
\hline Gender & \multicolumn{2}{|l|}{} \\
\hline Male & 42 & $43.2 \%$ \\
\hline Female & 55 & $56.7 \%$ \\
\hline Total & 97 & $100 \%$ & \\
\hline Prefer not to answer & 24 & & \\
\hline Age & & & \\
\hline $18-25$ & 30 & $30.3 \%$ & $55.6 \%$ \\
\hline $26-35$ & 25 & $25.3 \%$ & \\
\hline $36-45$ & 18 & $18.1 \%$ & \multirow{2}{*}{$33.2 \%$} \\
\hline $46-55$ & 15 & $15.1 \%$ & \\
\hline $56-65$ & 06 & $06.1 \%$ & \multirow{2}{*}{$11.2 \%$} \\
\hline 65 Above & 05 & $05.1 \%$ & \\
\hline Total & 99 & $100 \%$ & \\
\hline Missing & 22 & & \\
\hline & & & \\
\hline Field of Study & & & \\
\hline Built Environment & 24 & $24.0 \%$ & $24.0 \%$ \\
\hline Health Science & 03 & $03.0 \%$ & \\
\hline Public Service & 15 & $15.0 \%$ & \\
\hline Trade & 02 & $02.0 \%$ & $76.0 \%$ \\
\hline Finance & 02 & $02.0 \%$ & \\
\hline Science & 48 & $48.0 \%$ & \\
\hline Retired & 04 & $04.0 \%$ & \\
\hline Other & 02 & $02.0 \%$ & \\
\hline & & & \\
\hline & & \\
\hline
\end{tabular}


Chapter 7 - STUDY TWO: SECOND VISUAL PREFERENCE STUDY

\begin{tabular}{|c|c|c|c|}
\hline Total & 100 & \multicolumn{2}{|l|}{$100 \%$} \\
\hline Missing & \multicolumn{3}{|l|}{21} \\
\hline \multicolumn{3}{|c|}{ Academic Qualification } & \multirow{5}{*}{$34.1 \%$} \\
\hline Secondary School & 03 & $03.1 \%$ & \\
\hline High School / College & 04 & $04.1 \%$ & \\
\hline Undergraduate & 25 & $25.9 \%$ & \\
\hline Trade Certificate & 01 & $01.0 \%$ & \\
\hline Postgraduate & 64 & $65.9 \%$ & $65.9 \%$ \\
\hline Total & 97 & \multicolumn{2}{|l|}{$100 \%$} \\
\hline Missing & 24 & & \\
\hline \multicolumn{3}{|l|}{ Cultural Ethnicity } & \\
\hline Asian & 17 & $17.7 \%$ & \multirow{4}{*}{$46.9 \%$} \\
\hline European & 18 & $18.8 \%$ & \\
\hline African & 02 & $02.1 \%$ & \\
\hline Other & 08 & $08.3 \%$ & \\
\hline $\begin{array}{l}\text { NZ European } \\
\text { (Pakeha) }\end{array}$ & 51 & $53.1 \%$ & $53.1 \%$ \\
\hline Total & 96 & & \\
\hline Missing & 25 & & \\
\hline
\end{tabular}

Table 7.2 Demographic details of the Study Two sample

\subsection{Analysis}

Different statistical methods in SPSS were performed to analyse the questions depending on their type and the variables. Descriptive statistics were used to describe the demographic characteristics of the sample (Table 7.2). Different methods of analysis were used to understand the relationships between the different groups. These included a frequency statistics test for determining the means and standard deviations, cross tabulation results for preferences between groups and their subgroups, and the selection of groups for determining differences of opinion between 
subgroups by using independent sample T-tests. Furthermore, Cronbach's alpha ( $\alpha$ ) reliability test was used to check internal consistency in several variables for the semantic differential scale. A Kendall's tau-b $\left(\tau_{\mathrm{b}}\right)$ correlation test was conducted between the most liked images with the respondent's attitude form the semantic differential scale. The Kendall's tau-b $\left(T_{b}\right)$ correlation coefficient calculates the strength and direction of association in a nonparametric measure, such as exists between two variables measured on an ordinal scale (Laerd Statistics, 2016). All these methods were used in the various stages of Study Two.

Numerical values were assigned to investigating the data. The numerical value of 'dislike' equalled 1, and 'like' equalled 5, with other categories assigned a whole number within this range. The averages of each 5 -point Likert scale were then collated to produce a new 3-point scale (figure 7.1) for seeking the differences more accurately and to understand the data more efficiently (1 Dislike, 2 Neutral, 3 Like). Benson (1971) recommended using a 3-point Likert scale for its practical convenience. Researchers have argued that a 3-point Likert scale meets the criteria of test-retest reliability and predictive validity reliabilities for scales with two, three, five, seven, and even with nine response categories. It has been claimed that as few as two response categories might be adequate in practice and simplified scales are easily understood (Bendig, 1954; Jacoby \& Mattell, 1971; Hernandez \& Kaufman, 1990). Lehmann \& Hulbert (1972) claimed that a 2 or 3-point scale should be used if the focus of research is on overall groups. This approach allowed investigation of the preferences for the whole sample and its sub-groups in a more precise way. To determine the differences in preference between subgroups, an independent sample T-test was conducted by taking the averages of the semantic differentials calculated on the 5-point ranking scale. The null hypothesis assigned to each question indicated no preference or difference of opinion in the group or subgroup. The "p" value measured the statistical significance of the comparisons.

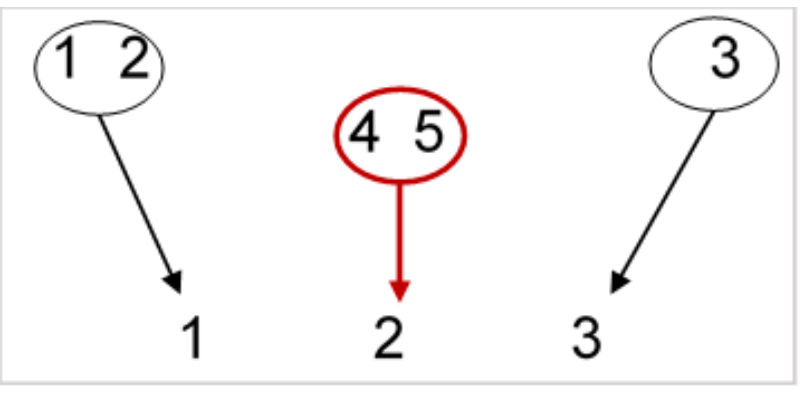

Figure 7.1 5-point Likert scale converted to a 3-point Likert scale 
The first step in an investigation of the subgroups was to test for differences in preference based on gender, age, academic qualification, the field of expertise, and cultural ethnicity. The study sought preferences of al sample group, genders and built and non-built environment participants since no significant difference in preference opinion $(p<0.05)$ was found for age with participants divided into the two age groups of 18-35 and 36+, and for ethnic groups by using T-Test. As a result, a detailed analysis was carried out for Study Two in three stages.

- Stage one analysis was performed for the whole sample group of 121 respondents to identify the most appealing attribute in each redesigned leftover space.

- Stage two analysis was based on the subgroups of 47 males $(42.7 \%)$ and 61 females $(57 \%)$ from the 121 participants to identify differences of opinion in their preferences.

- Stage three analysis investigated the subgroups of 26 (23.6\%) built environment professionals compared to $85(76.6 \%)$ respondents from other occupations, to see again whether there were differences in the preferences expressed.

Stage 1: ALL PARTICIPANTS Stage 2: GENDER PREFERENCES

Stage 3: BUILT ENVIRONMENT AND NON-BUILT ENVIRONMENT PARTCIPANTS STUDY 2
Part 1: Demographic Data

Part 2: i. Likert scale ii: Semantic Differential iii: Suggestions

Part 3: Multiple-choice questions

Figure 7.2 Three stages conducted in Study Two with the three parts in each stage

\subsubsection{Analysis of Stage One}

The first stage of the analysis of general preferences was conducted with 111 participants. The mean preferences for the most and least preferred redesigned photos were measured on the Likert scale (1-3) by using the descriptive frequency test in SPSS. The simple technique of calculating the mean, standard deviation ( $\pm S D)$ and 
percentage of the most preferred design was used. Three different design modifications with one attribute were individually analysed for the six types of leftover space, as discussed in sections 7.3.1.1 to 7.3.1.6. A Kendall's tau-b $\left(T_{\mathrm{b}}\right)$ correlation was tested for the most liked image with its affective appraisals to measure the respondent's association with the most liked redesigned option.

\subsubsection{Space underneath a building}

Out of 111 participants, 96 rated the space underneath a building on the Likert scale. The double height space was a parking space containing the entrance to the building and a garbage bin. The space in option A (figure 7.3) was redesigned with planters and a green wall. Permeable pavers were used to emphasise the entrance to the building. The space in image $B$ was changed by giving the building façade a textured treatment. Lastly, image $C$ was depicted as a clean space by removing the garbage bin and tidying up the wall surface.

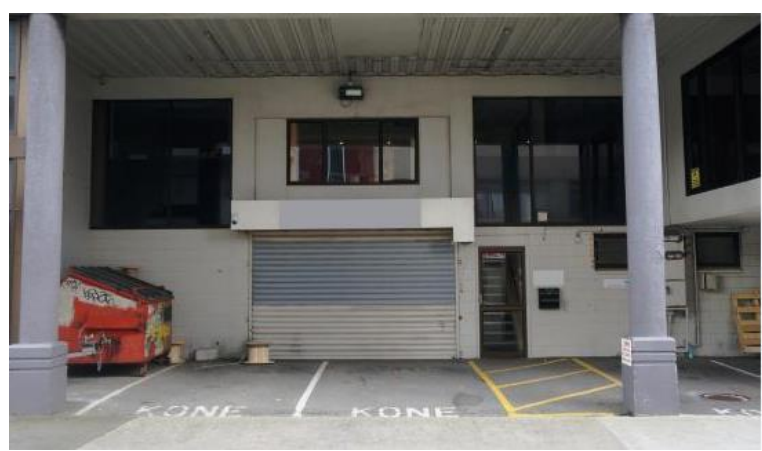

\section{Space underneath a building}

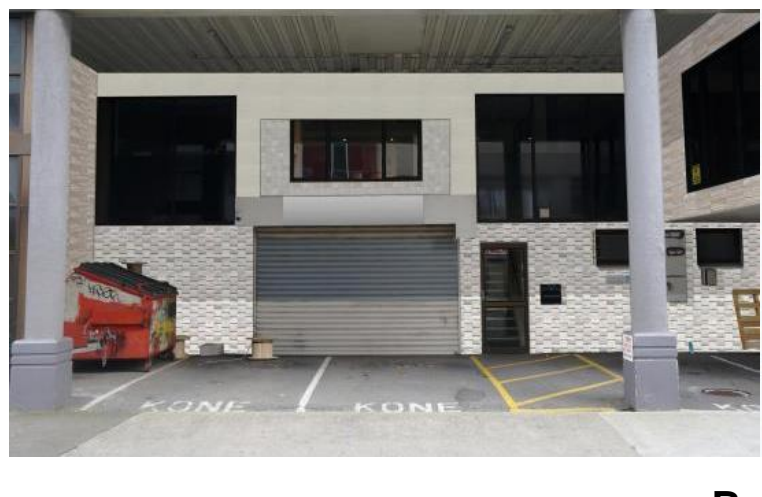

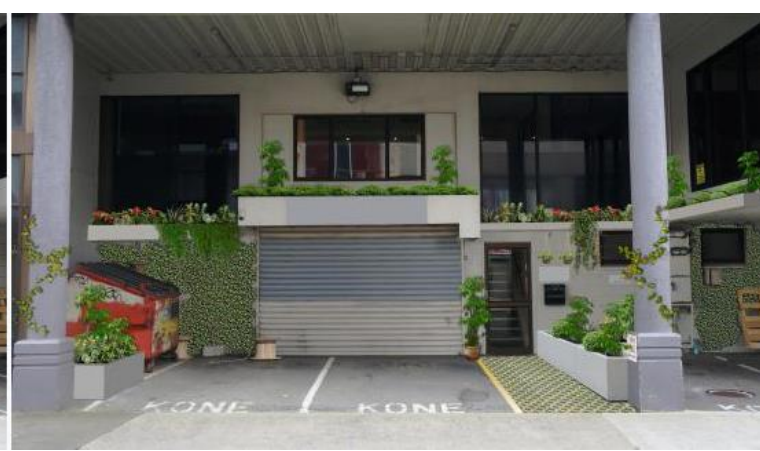

A

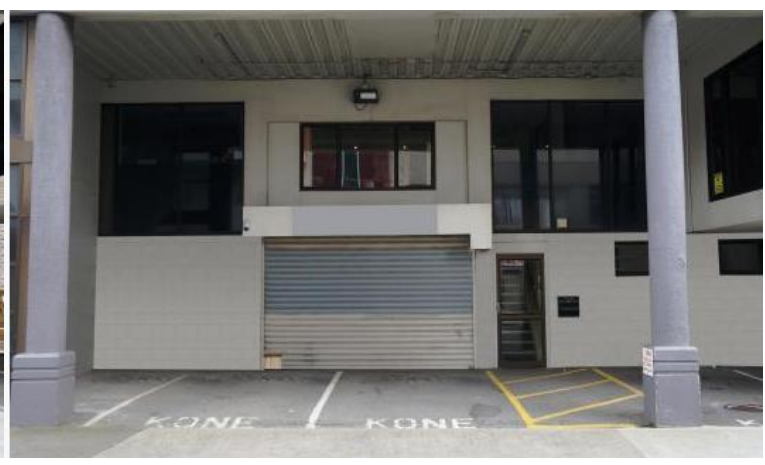

C

Figure 7.3 Options presented to participants in Study Two

The highest mean preference of 4.02 and Standard Deviation (SD) of 0.97 was given to option A with the introduced vegetation. More than $77 \%$ of all respondents liked the 
vegetation, while only $9 \%$ disliked it and $13 \%$ had no preference. On the other hand, change in the surface material, option $B$, with a mean of 2.85 ( $S D=1.02)$, and improving the cleanliness, option $\mathrm{C}$, with a mean of $2.93(\mathrm{SD}=1.00)$, had much lower mean preference scores (see appendix table 7.1 ). Only $24 \%$ and $27 \%$ of participants liked images $B$ and $C$ respectively, whereas $37 \%$ of participants disliked option $B$ and $31 \%$ disliked image C. Almost 38\% respondents were neutral for option B and $40 \%$ for option C (figure 7.4). The results showed that people wanted to see more vegetation in the space underneath a building.

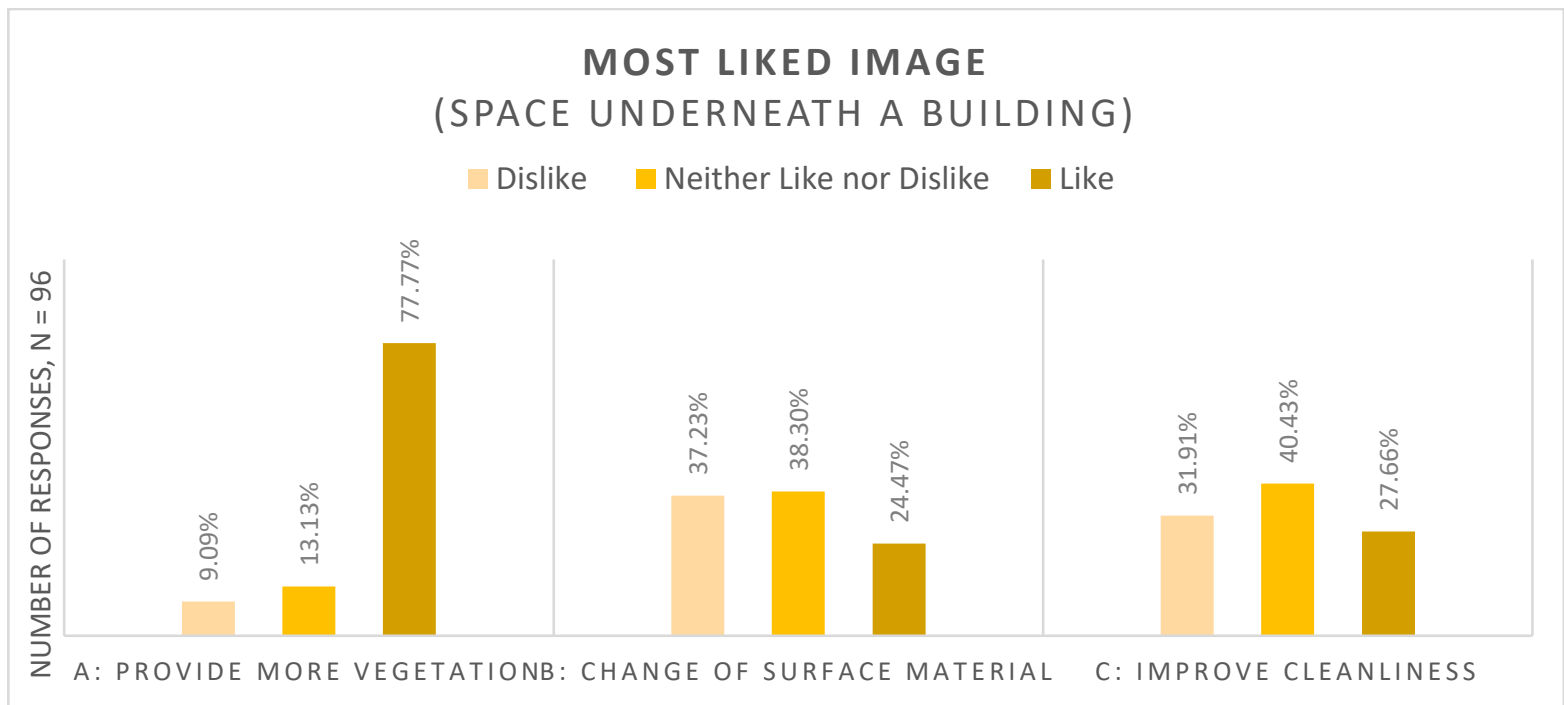

Figure 7.4 Preferences for the space underneath a building $(\mathrm{N}=96)$

In the second part, the preferences for the different options were further investigated through the semantic differential scale from 1 to 5 . The mean values for the semantic differentials were calculated to understand the attitudinal effects on participants. A Kendall's tau-b correlation was run to determine the relationship between the most liked option A and its five semantic differentials. The test revealed a strong, positive association between the image likeability and all semantic differentials except the one bipolar category "impossible to realisable," which was statistically insignificant, $\mathrm{Tb}=$ $.180, p=.076(p>.05)$. This result meant that only one aspect had a weak, negative association, and was not correlated to the preference for image $A$. It became evident that image A had a positive influence on the attitudes of respondents, but whether or not it could happen did not influence the preference scores (figure 7.5). 


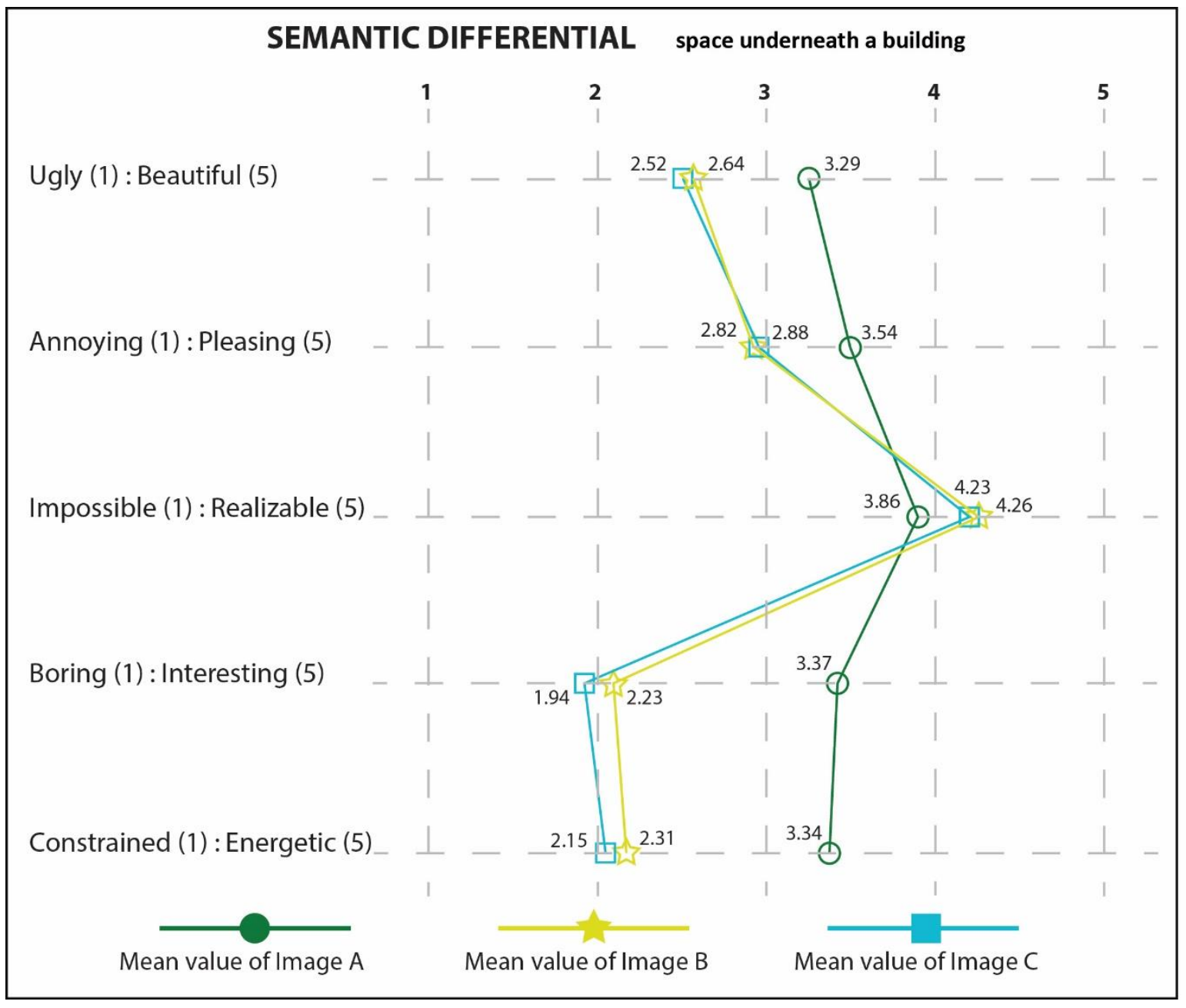

Figure 7.5 Attitudes of respondents to the three options of the space underneath a building

In the third part, other suggestions for improving the visual quality of the space underneath a building were related to changing the configuration of the vegetation, providing variety in textures and surface colours, and keeping the space tidier by removing the garbage bin.

\subsubsection{Space in front of a building}

Out of the study sample, 90 respondents rated the space in front of a building. Pedestrians currently use this space for entering the building from the parking lot and for taking a short cut through to the rear street. The space in option A (figure 7.6) had the boundary walls removed to open up space, and the entrance was made more explicit. The space in image B was redesigned by introducing a green wall along the 
passageway. Lastly, image $\mathrm{C}$ was made by lowering the boundary wall and using the change in level between the car park and building to create seating.

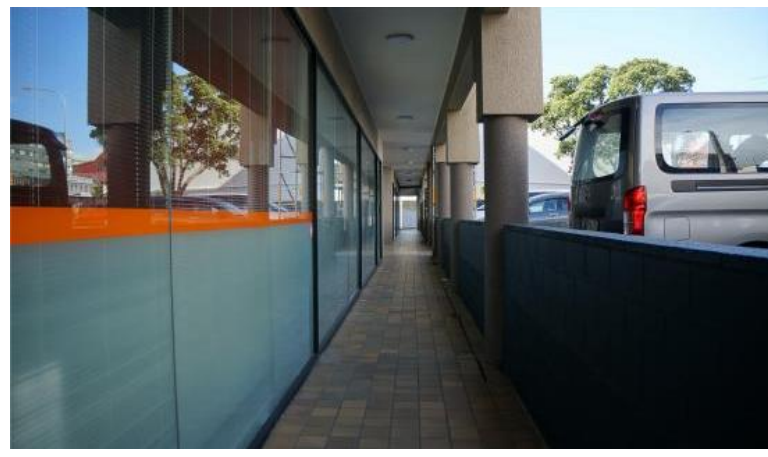

Space in front of a building

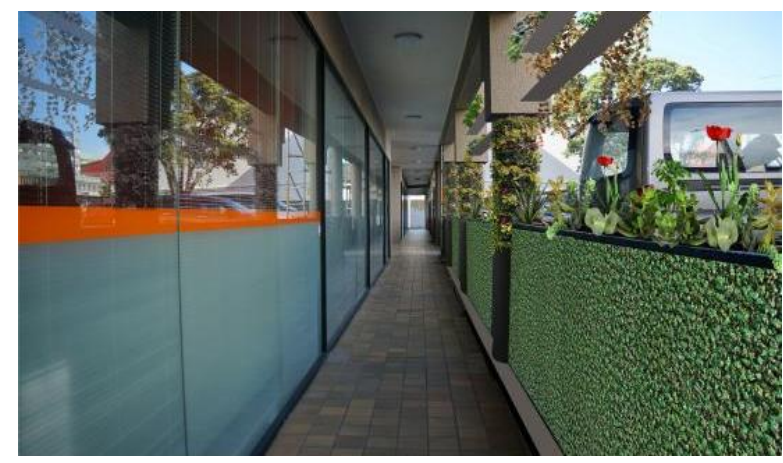

B

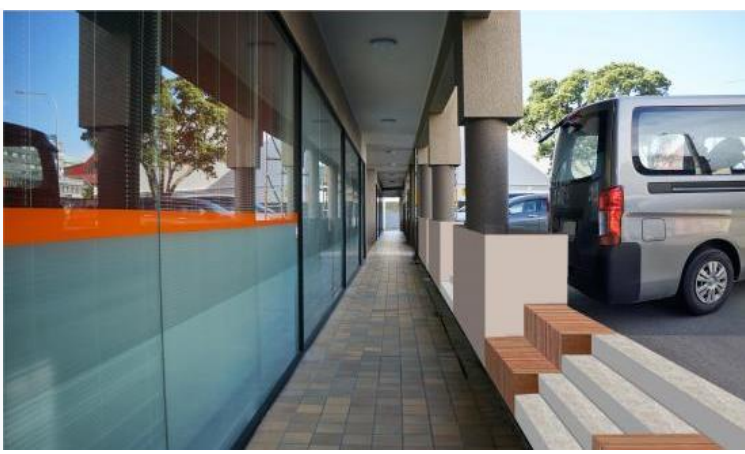

A

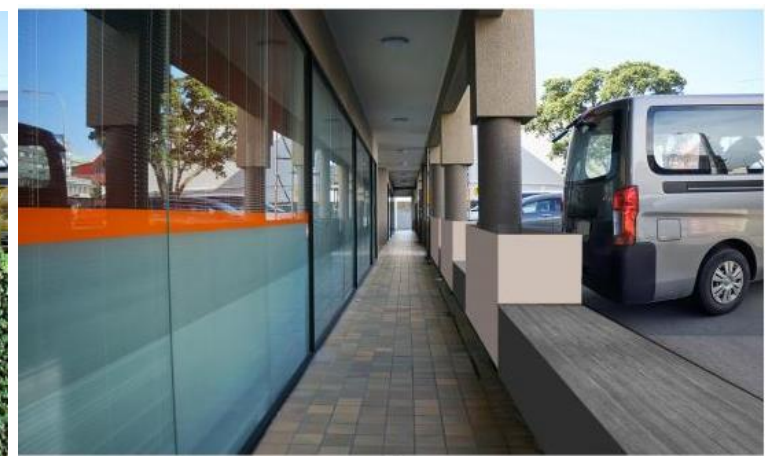

C

Figure 7.6 Options presented to participants in Study Two

The highest mean preference of 4.01 and Standard Deviation (SD) of 0.80 was found for option A where removal of a boundary wall was liked by $79 \%$ of respondents. Only $3 \%$ of participants disliked option $A$, and $18 \%$ had no preference. There was slightly less preference for option $B$, with a mean score of $3.84(S D=1.02)$, and for option $C$, with a mean of $3.62(S D=1.23)$ (see appendix table 7.1$)$. Some $70 \%$ and $58 \%$ of participants liked images B and C, respectively. Additionally, $12 \%$ of participants did not like option $B$, and $8 \%$ did not like $C$, while $18 \%$ and $34 \%$ were neutral when it came to options $B$ and $C$, respectively. These results suggested that people wanted to see more unobstructed and open spaces in front of the building (figure 7.7). 


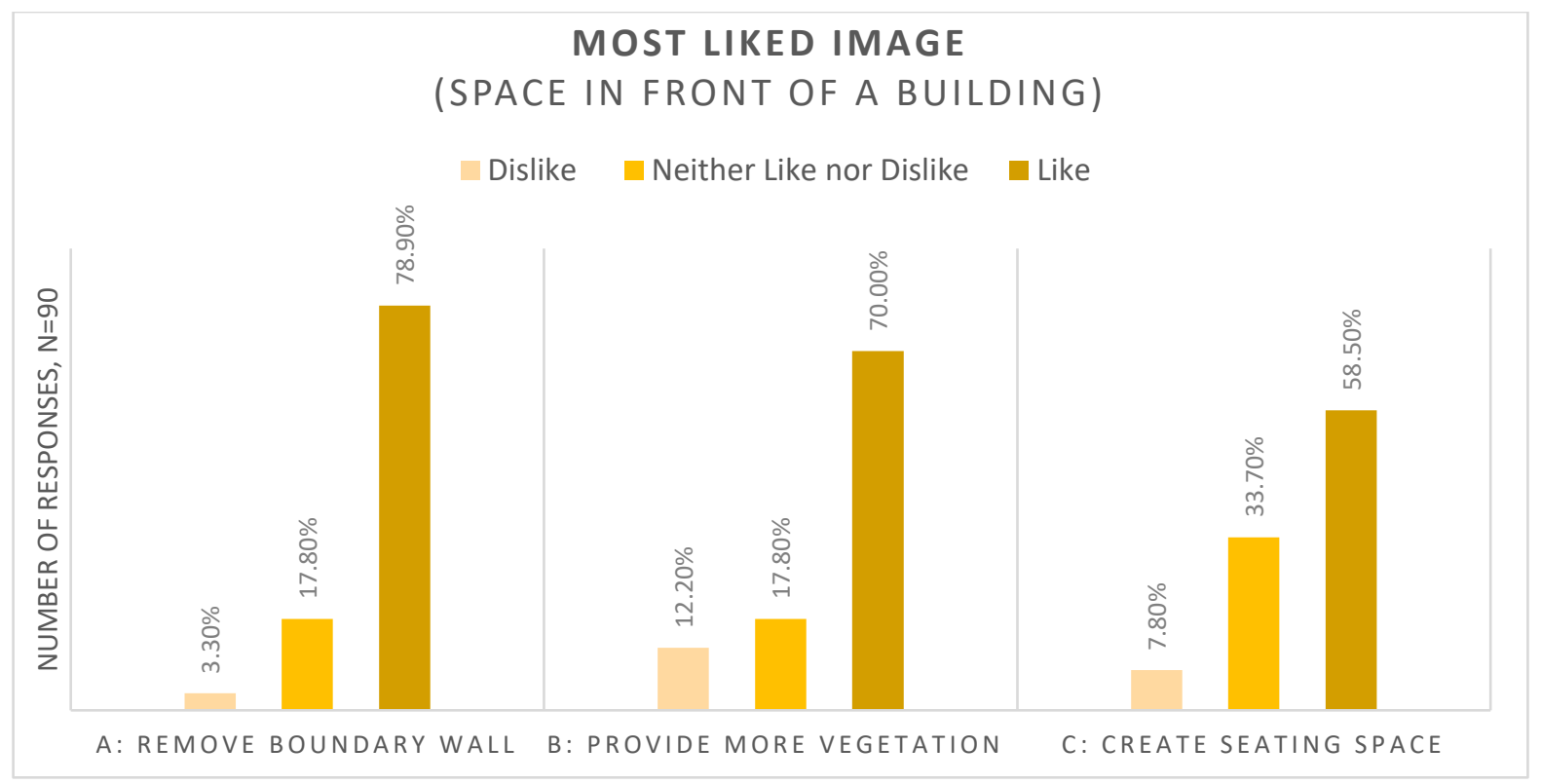

Figure 7.7 Preferences for the space in front of a building $(\mathrm{N}=90)$

The preference for option A was backed up by the semantic scale analysis (figure 7.8). The mean values for the semantic differentials were calculated, which showed image A had a positive influence on the attitudes of respondents, but some aspects like appeal (boring-interesting), attractiveness (ugly-beautiful) and mood (constrainedenergetic) had lower mean values. The Kendall's tau-b correlation determined a relationship between the most liked option $A$ and its five semantic differentials for all respondents. The test revealed a strong, positive association between the likability of the image and all semantic differentials, meaning the attributes were significantly correlated with the likability of image $A$. 


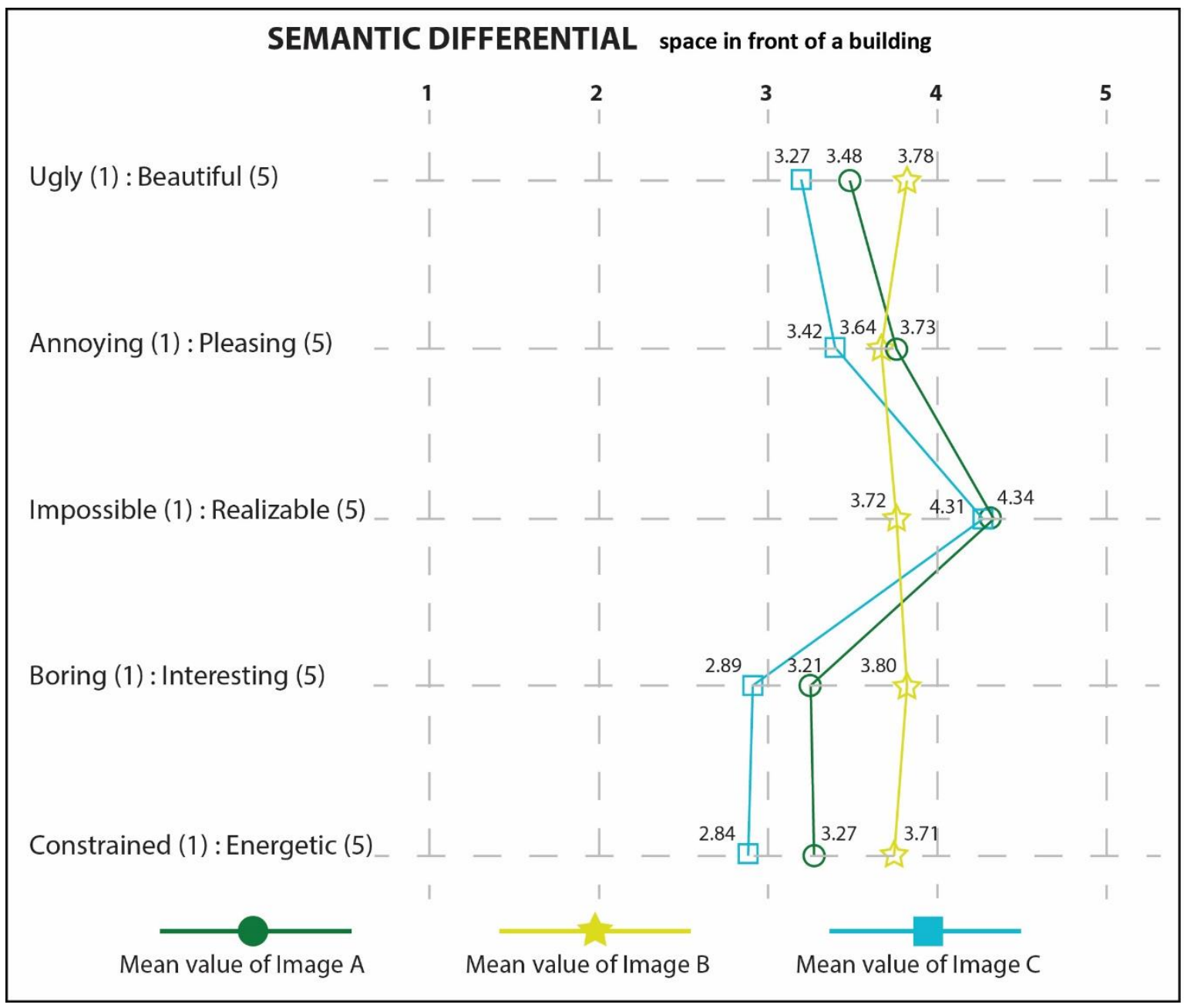

Figure 7.8 Attitudes of respondents to the three options for space in front of the building

Suggestions for improving the visual quality of the space in front of a building were related to enhancing the safety measures by providing a transparent fence, providing more artificial lighting, change of surface colour of the pavement, and having murals along the pathway.

\subsubsection{Space at the back of a building}

Of the sample, 86 participants rated the space at the back of a building. The redesigned space is still used for car parking in the evenings and is hidden from the street. Option C (figure 7.9) designed with the addition of vegetation in the form of a vertical garden and additional planters was the most preferred being liked by $89 \%$ of participants and scoring the highest mean preference of 4.32 with Standard Deviation 
(SD) of 0.84 (see appendix table 7.3 ). Only $7 \%$ of participants were neutral, and $4 \%$ did not like this option.

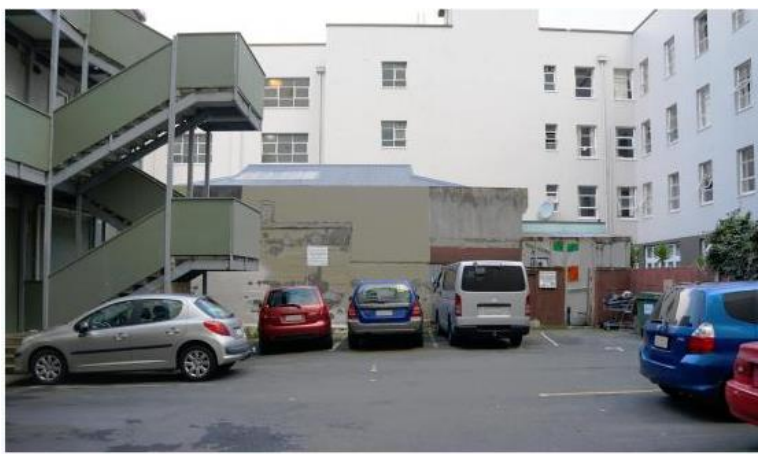

Space at back of a building

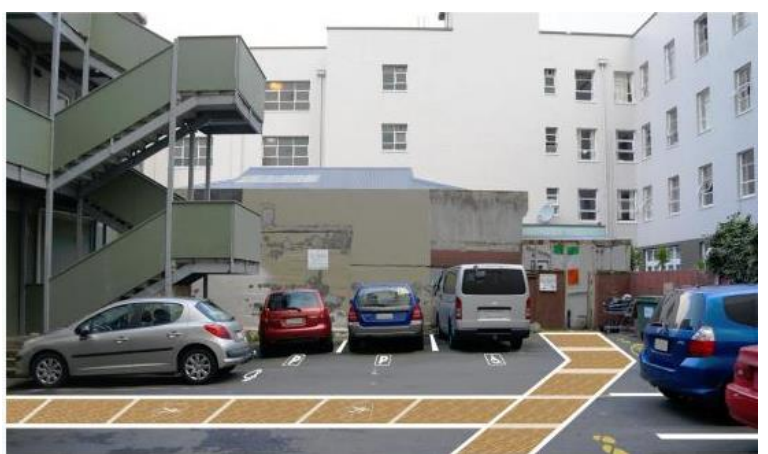

B

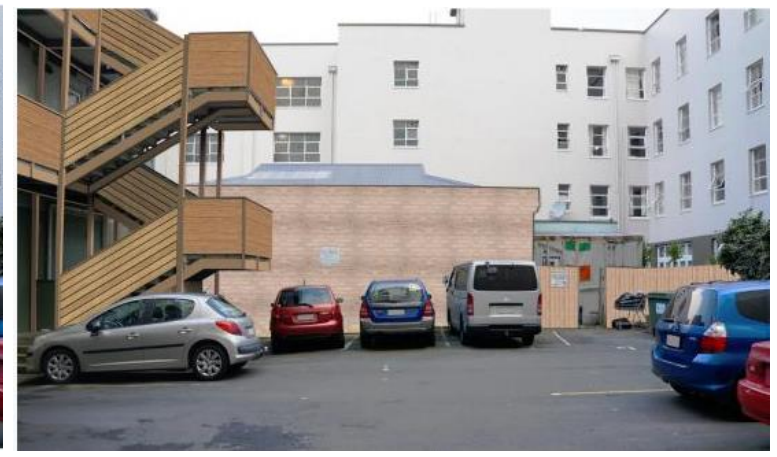

A

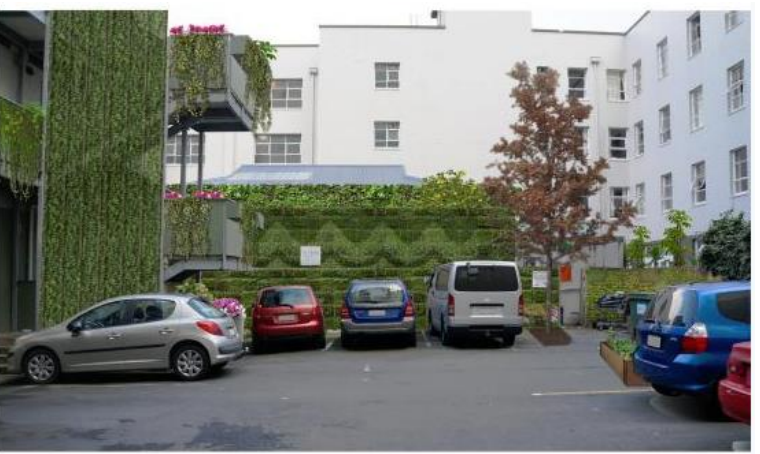

C

Figure 7.9 Options presented to participants in Study Two

Option A with a change of surface material scored the second highest mean preference of 3.50 with SD of 1.18 , as $54 \%$ participants liked it (figure 7.8 ) whereas $35 \%$ were neutral and $14 \%$ disliked it. Only $17 \%$ of participants liked option B with its clear pathway for pedestrians. This option scored the mean preference of 2.59 $(\mathrm{SD}=1.22)$, and $51 \%$ of respondents disliked it, and $33 \%$ were neutral (figure 7.10 ). 


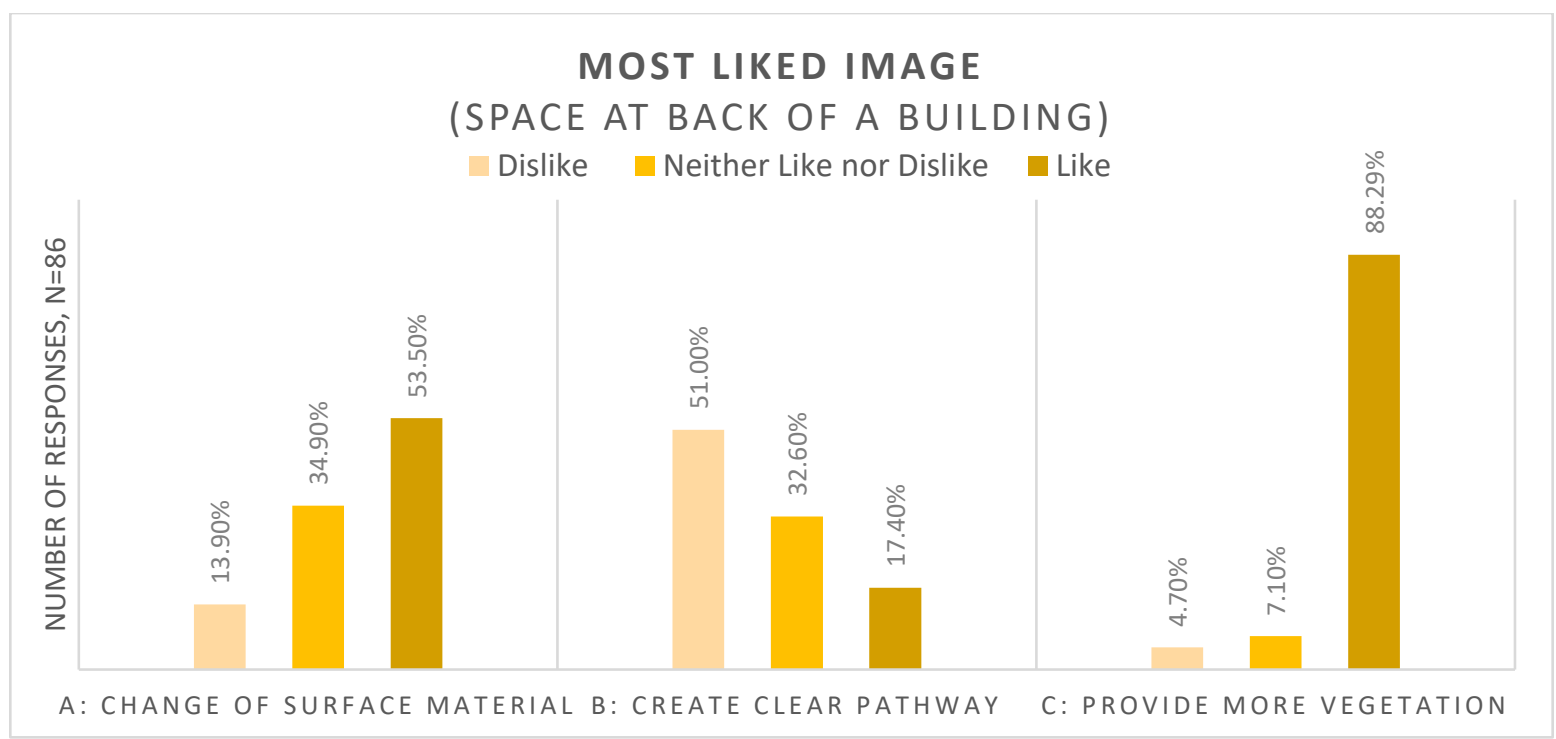

Figure 7.10 Preferences for space at the back of a building ( $N=86)$.

The preference for vegetation in option $C$ was supported through the semantic differentials (figure 7.11). Kendall's tau-b correlation test revealed that image $C$ had a strong, positive association between the liking for image $C$ and all semantic differentials except for the one bipolar aspect of its buildability (impossible-realisable). For this, the test revealed a negative, weak association which was not significantly correlated at $\mathrm{Tb}=-.230, \mathrm{p}=.08(p>.05)$. 


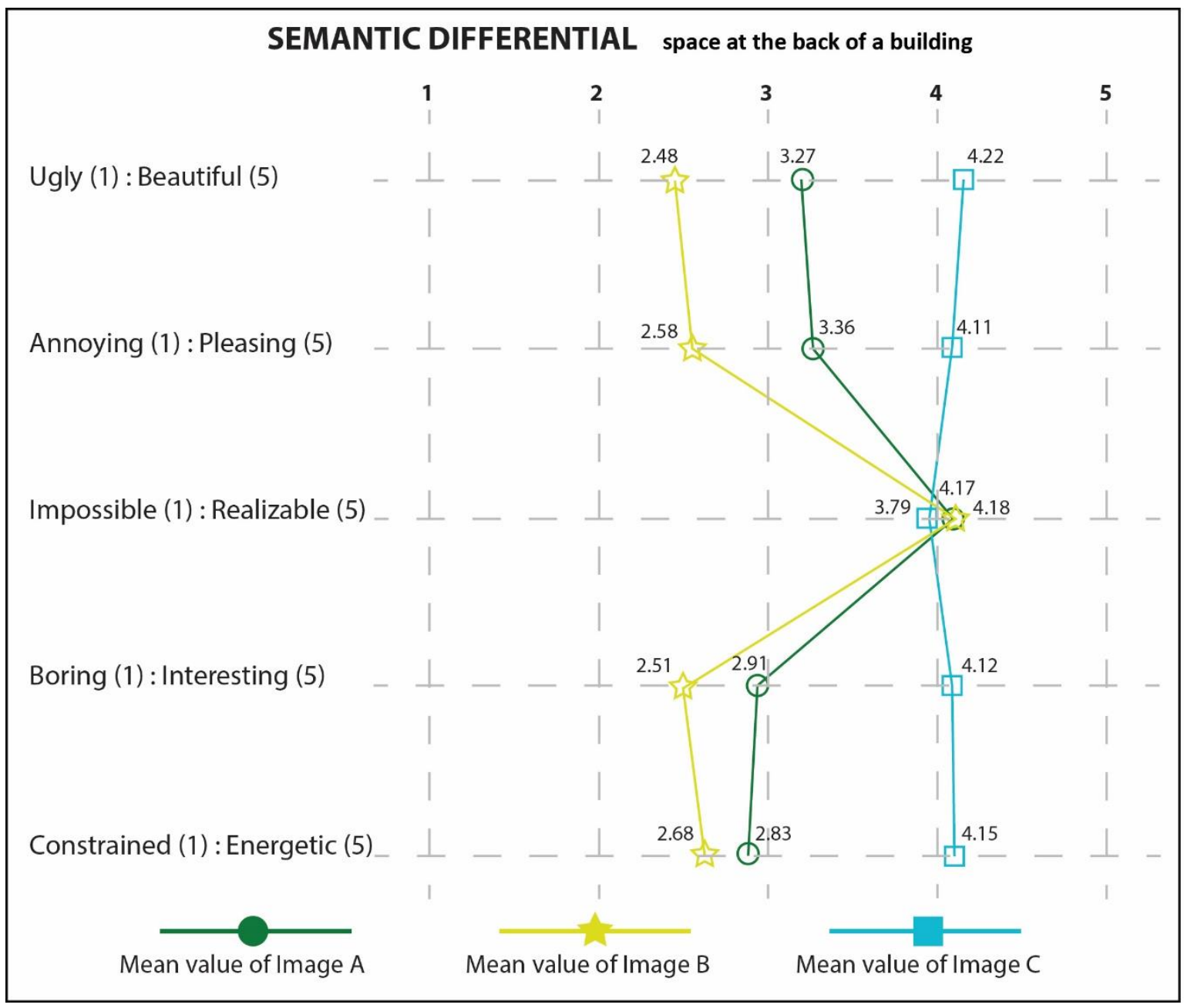

Figure 7.11 Attitudes to the three options for space at the back of a building

Suggestions for improving the visual appearance of the space at the back of a building were related to adding different species of plants and trees, introducing more artificial lighting, and improving its cleanliness and maintenance.

\subsubsection{Space enclosed by buildings on three sides}

Of the initial sample, 85 rated the designs for the space enclosed by buildings on three sides. Space is currently used as a parking lot for people in the surrounding buildings. Option C (figure 7.12) was redesigned with planters and a green wall. In option A there was a change made to the surfaces of the surrounding façades, whereas option $B$ was designed to emphasise a clear pathway for pedestrians. Option $\mathrm{C}$ with the vegetation was given the highest mean preference of 4.12 with a Standard Deviation (SD) of 0.84 (see appendix table 7.1). 

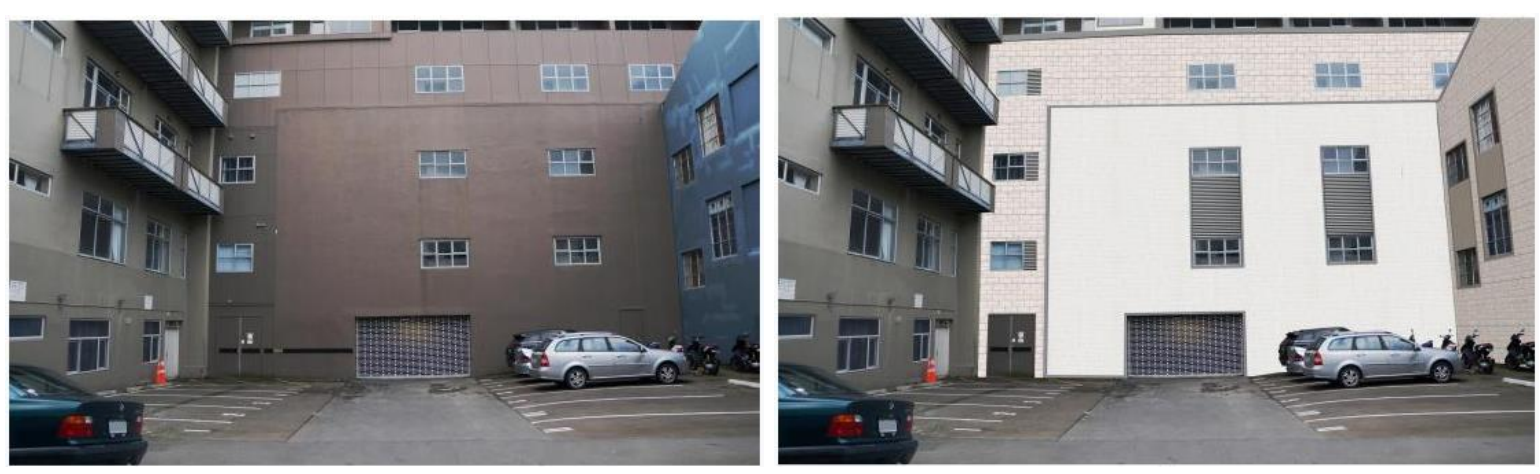

Space enclosed by buildings on three sides

A
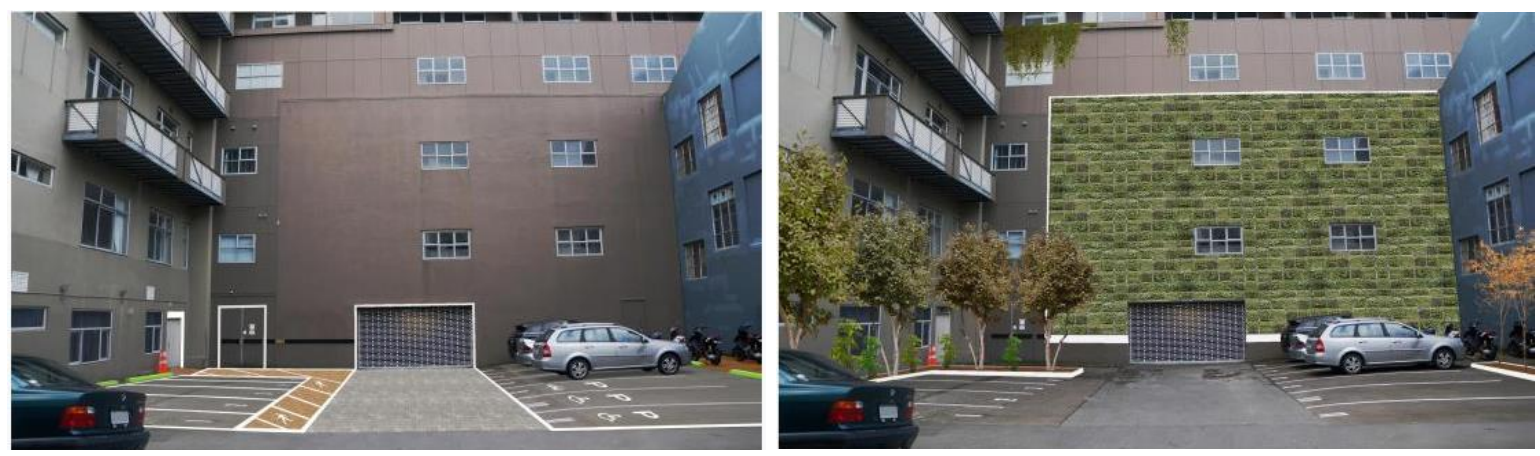

B

C

Figure 7.12 Options presented to participants in Study Two

For all respondents, $86 \%$ liked option C with only $7 \%$ not liking this option and $7 \%$ being neutral (figure 7.13). However, option $A$ with its change in surface material, mean $3.33(S D=1.11)$, and option $B$ with its clear pathway for pedestrians, mean 2.78 ( $S D=1.14$ ), scored much lower. About $43 \%$ of respondents liked option $A$ and $22 \%$ option $B$, whereas, $14 \%$ and more than $37 \%$ of participants did not like options $A$ and $B$ respectively. Almost $43 \%$ of respondents were neutral when it came to option A, and $40 \%$ were neutral about option B. Overall, people wanted to see more vegetation in the space enclosed by buildings on three sides. 


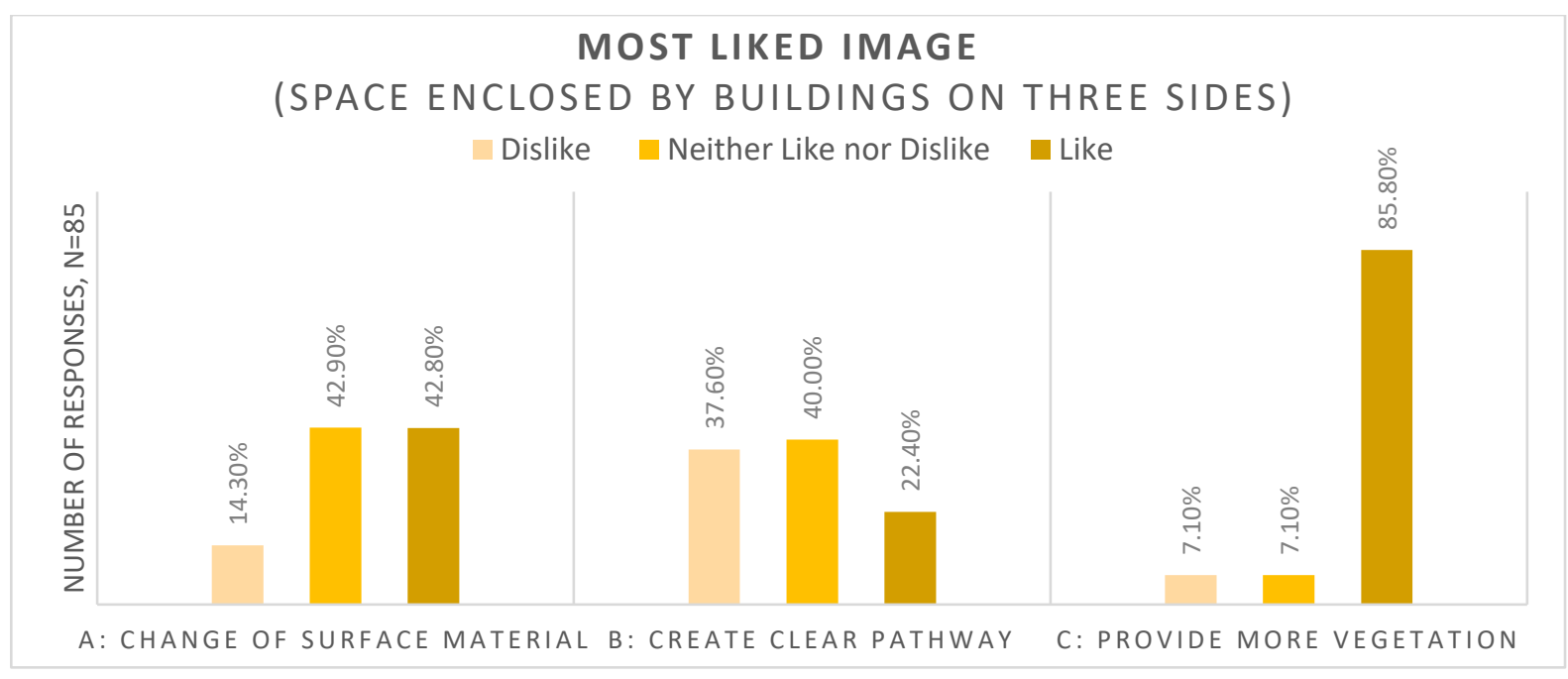

Figure 7.13 Preferences for the space enclosed by buildings on three sides $(\mathrm{N}=85)$

The preferences for image $C$ were also tested using the semantic differential scale from 1 to 5 . This analysis confirmed that image $C$ had a positive influence on the attitudes of respondents except for the bipolar category of buildability (impossiblerealisable), which scored the lowest. The most liked image $C$ was not seen as a practical solution by respondents, although the other options were seen as practical, especially the idea of creating the pathway for pedestrian (figure 7.14). Kendall's tau$\mathrm{b}$ correlation determined the relationship between the most liked option $\mathrm{C}$ to its five semantic differentials for all respondents. The test revealed a strong, positive association between the liked images and four semantic differentials, with only the one aspect of buildability (impossible- realisable) being not statistically significant, $\mathrm{Tb}=$ $.408, p=.10(p>.05)$. 


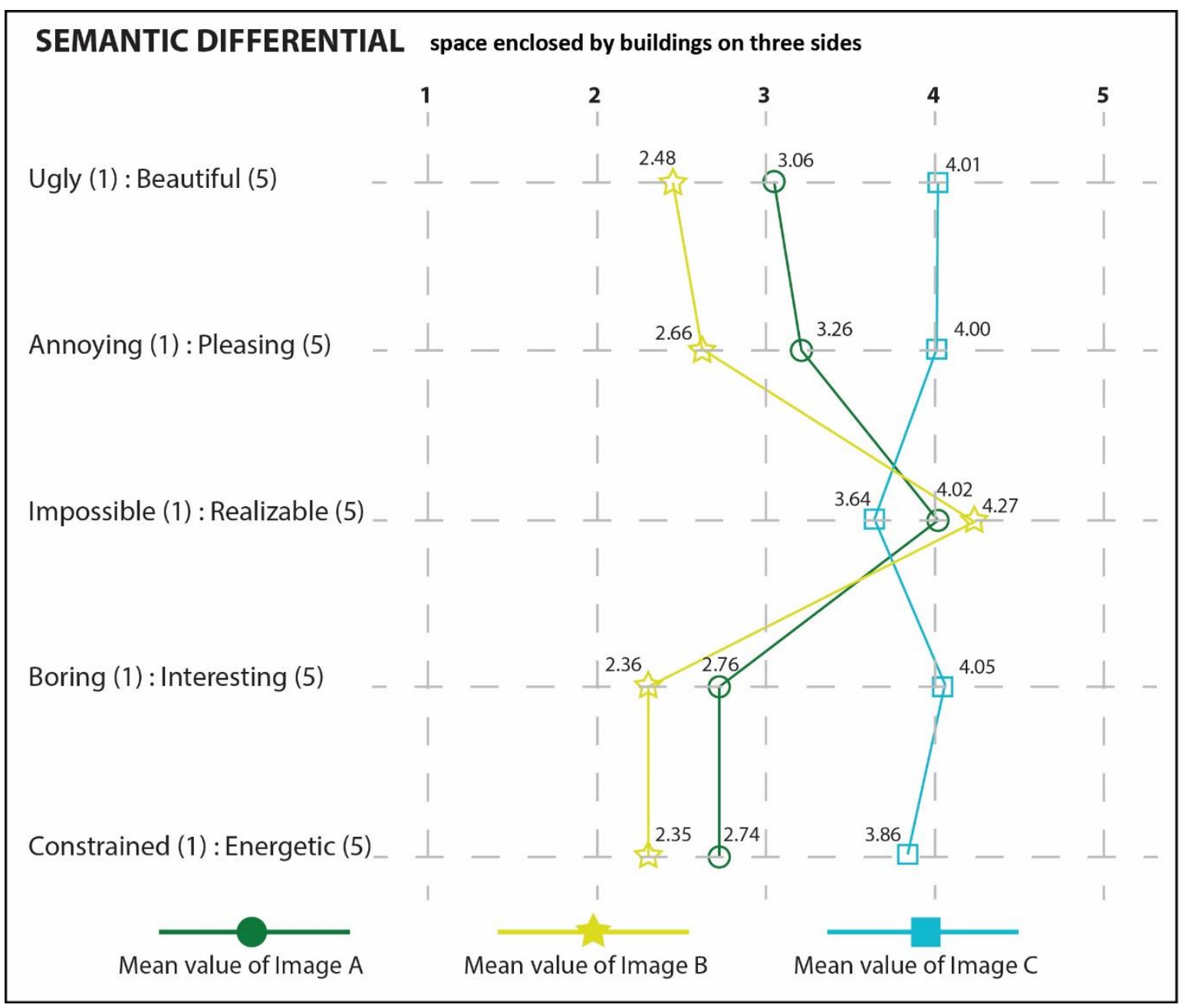

Figure 7.14 Attitudes to the three options for a space enclosed by buildings on three sides

Suggestions for improving the visual appearance of a space enclosed by buildings on three sides were related to improving the maintenance of the space, constructing improved façades with a variety of textures, and providing more lighting.

\subsubsection{Space enclosed by buildings on two sides}

Out of the sample of 111 respondents, 85 ranked the options for a space enclosed by buildings on two sides. Space is currently used as a parking lot for nearby offices and other users only in the daytime. Option B (figure 7.15), which was redesigned with planters, permeable pavers, and a green wall, scored the highest mean preference of 4.26 with Standard deviation (SD) of 0.77 making it the most liked image. 

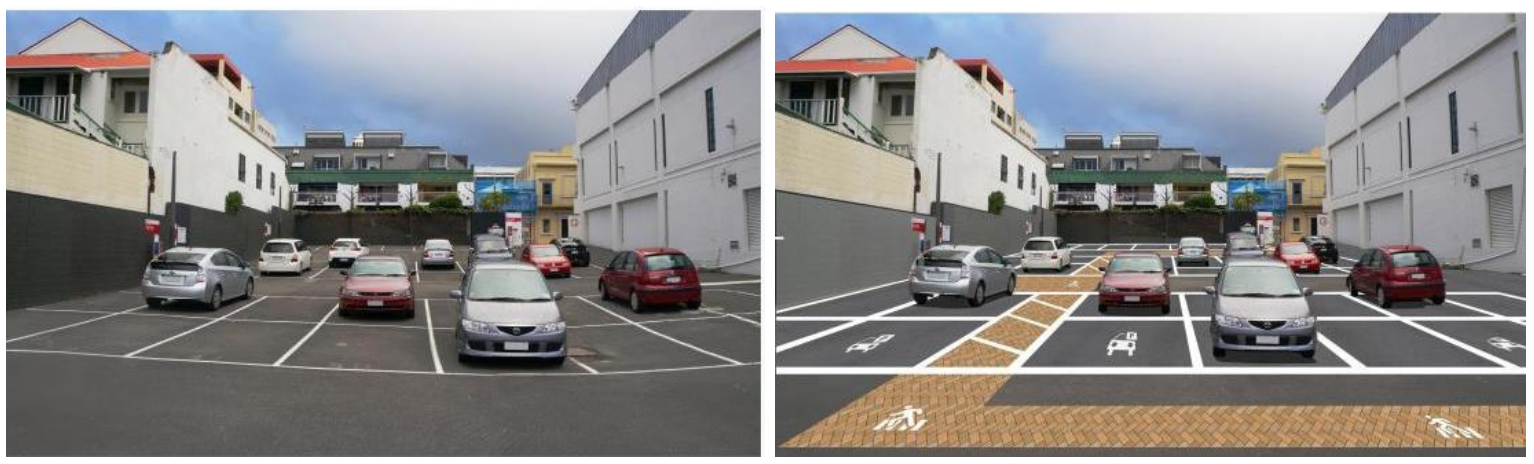

Space enclosed by buildings on two sides

A
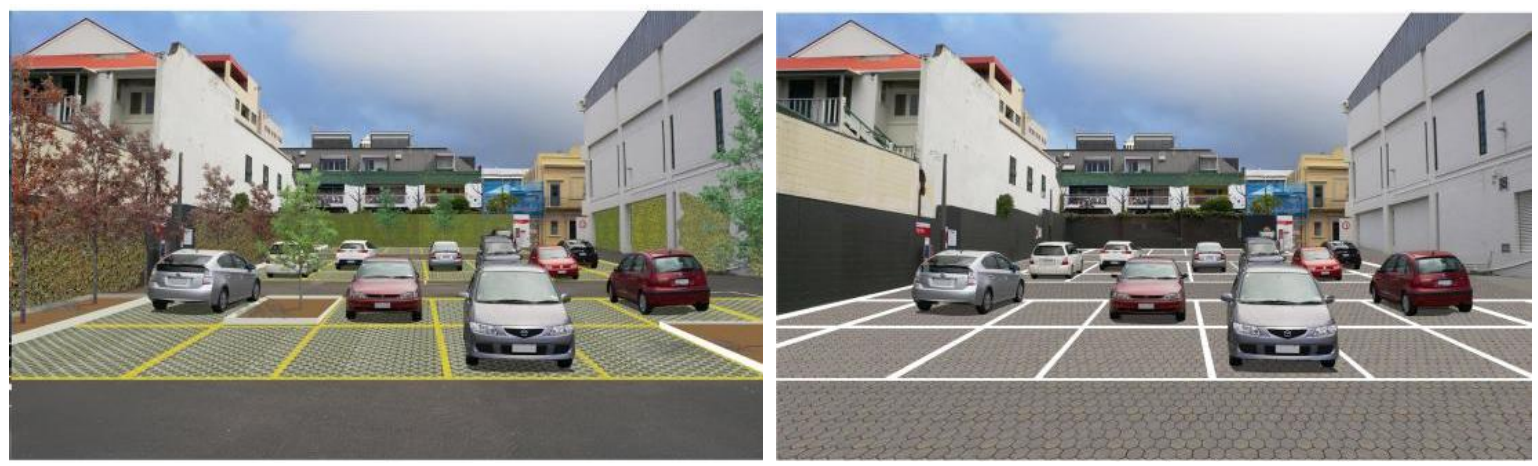

B

C

Figure 7.15 Options presented to participants in Study Two

More than $88 \%$ of respondents liked image $B$ with its vegetation while only $6 \%$ did not like it, and $6 \%$ were neutral (figure 7.16). Creating a clear pathway for pedestrians (option $A)$ scored a lower mean value of $3.08(S D=1.11)$ while a change in the surface material (option $\mathrm{C}$ ) scored a lower mean $3.13(\mathrm{SD}=1.09)$ (see appendix table 7.1).

More than $35 \%$ of participants liked option A and $36 \%$ like option C. However, $27 \%$ and $26 \%$ of participants did not like options $A$ and $B$ respectively. For both $A$ and $B$, $38 \%$ of respondents were neutral. Option $B$ was thus highly preferred, suggesting people wanted to see more vegetation in spaces enclosed by buildings on two sides. 


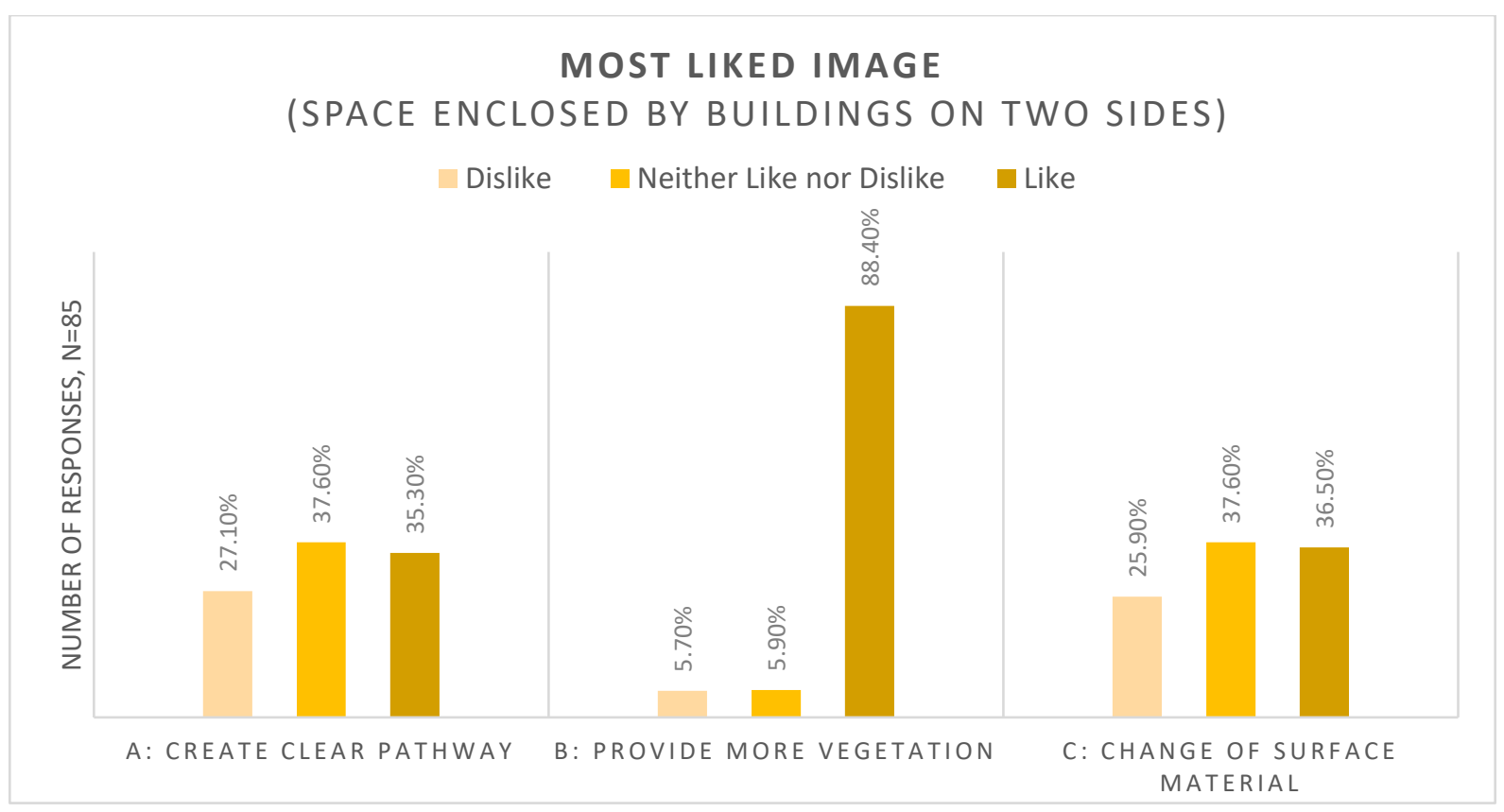

Figure 7.16 Preferences for space enclosed by buildings on two sides $(\mathrm{N}=85)$

The preferences were again tested using the semantic differential scale from 1 to 5 . This analysis confirmed that image B had a positive influence on the attitudes of respondents except for the one aspect of buildability (impossible-realisable) as participants realised that the option was hard to construct (figure 7.17). The Kendall's tau-b correlation tested the relationship between the most liked option $B$ and the five semantic differentials. The test revealed a strong, positive association between the likability of the image and all semantic differentials. In other words, if the participants liked the image, their affective appraisal value increased for the space enclosed by buildings on two sides. 


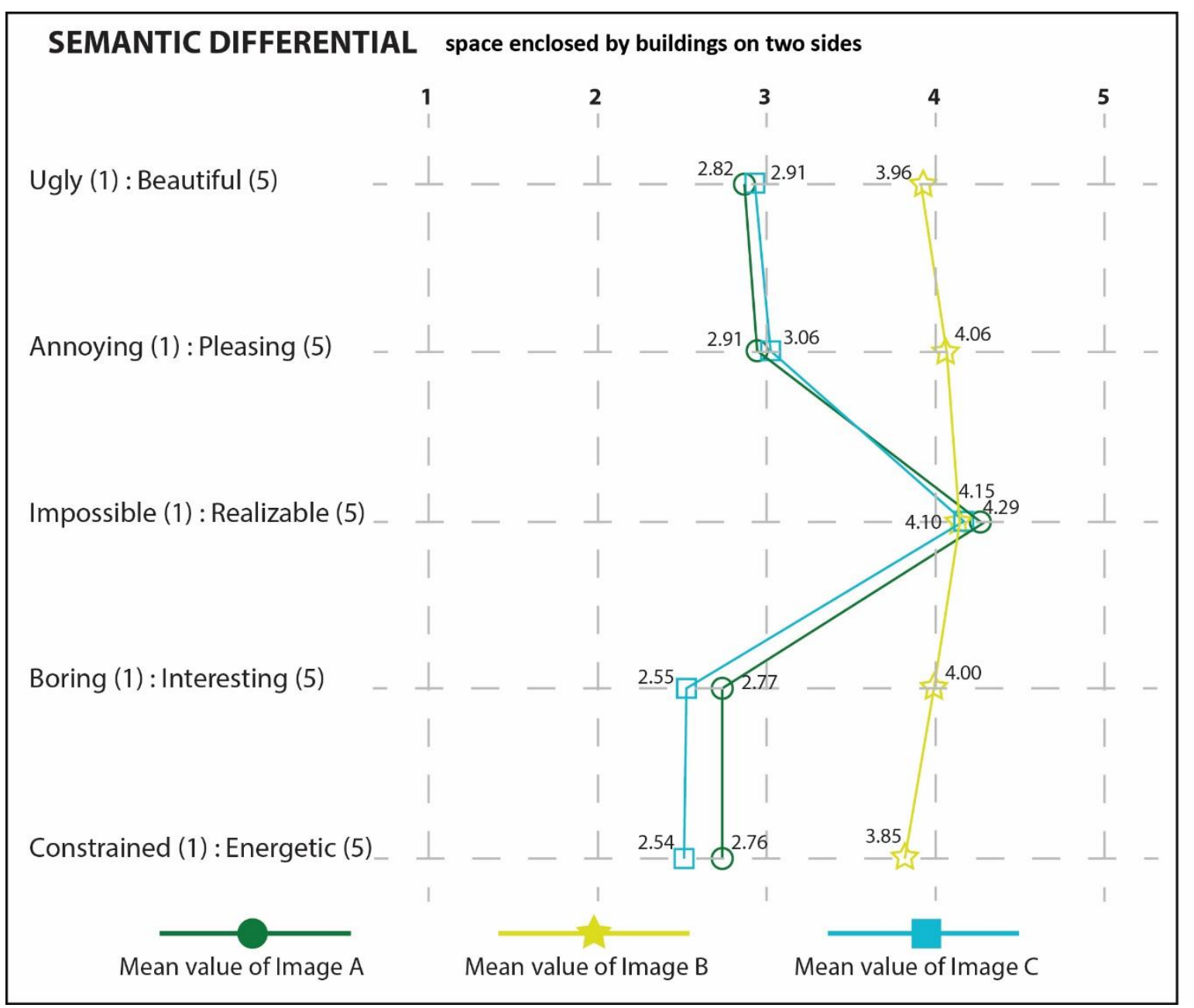

Figure 7.17 Attitudes to the three options for a space enclosed by buildings on two sides

Other suggestions made by participants for improving the visual quality of the space were related to providing more signs, having designed graffiti on walls, providing more pathways leading to the surrounding buildings, and lastly having proper maintenance.

\subsubsection{Space on the rooftop of a building}

Out of the whole sample, 84 respondents rated the images for the space on the rooftop of a building. This space is currently used as a commercial car park and has no use other than in working hours. The space in option A (figure 7.18) was redesigned with planters and a green wall to hide the view of the car park. Option $B$ included new seating areas and option $\mathrm{C}$ concerned the installation of solar panels and vertical axis micro wind turbines. 


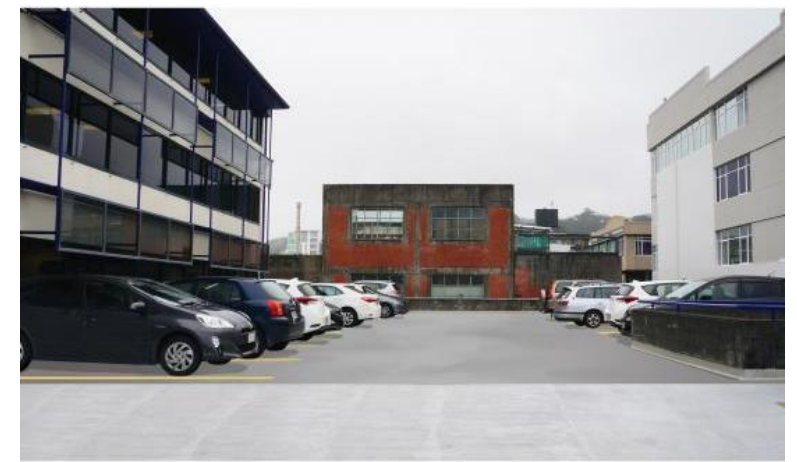

Space on the rooftop of a building

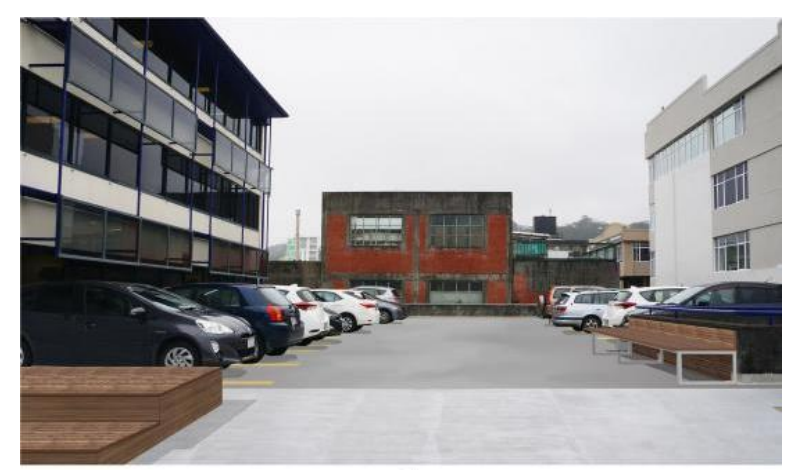

B

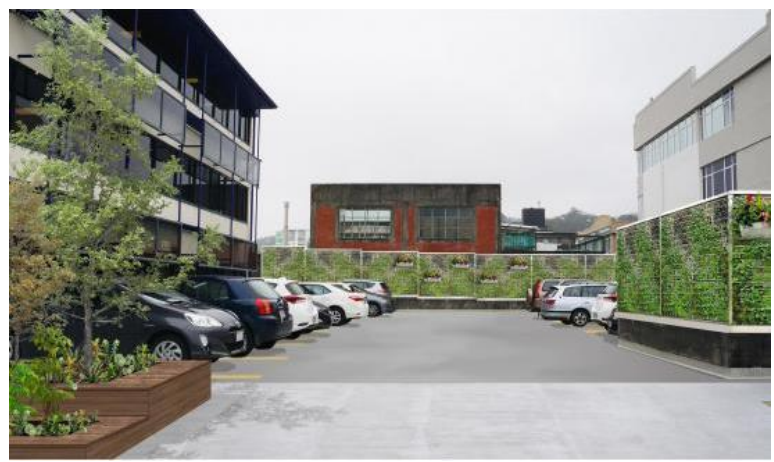

A

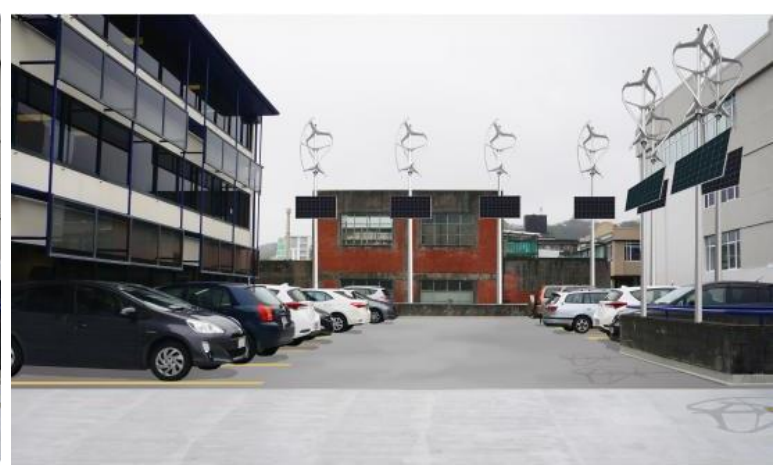

C

Figure 7.180ptions presented to participants in Study Two

Option $A$ with the vegetation scored the highest mean preference of 4.27 with a Standard Deviation (SD) of 0.73 . Of all respondents, $88 \%$ liked option A, and only $3 \%$ disliked it, while $9 \%$ of participants were neutral (figure 7.19). Creating seating spaces in option $B$ scored a mean value of $2.98(S D=1.12)$, while option $C$ with its solar panels and micro wind turbines scored much lower with a mean preference of $3.29(\mathrm{SD}=1.04)$ (see appendix table 7.1). Option B was liked by $31 \%$ of participants and Option $C$ by $46 \%$, while $28 \%$ and $23 \%$ of participants disliked options $B$ and $C$ respectively. Almost $41 \%$ of respondents were neutral when it came to option $B$, and $31 \%$ were neutral about option C. 


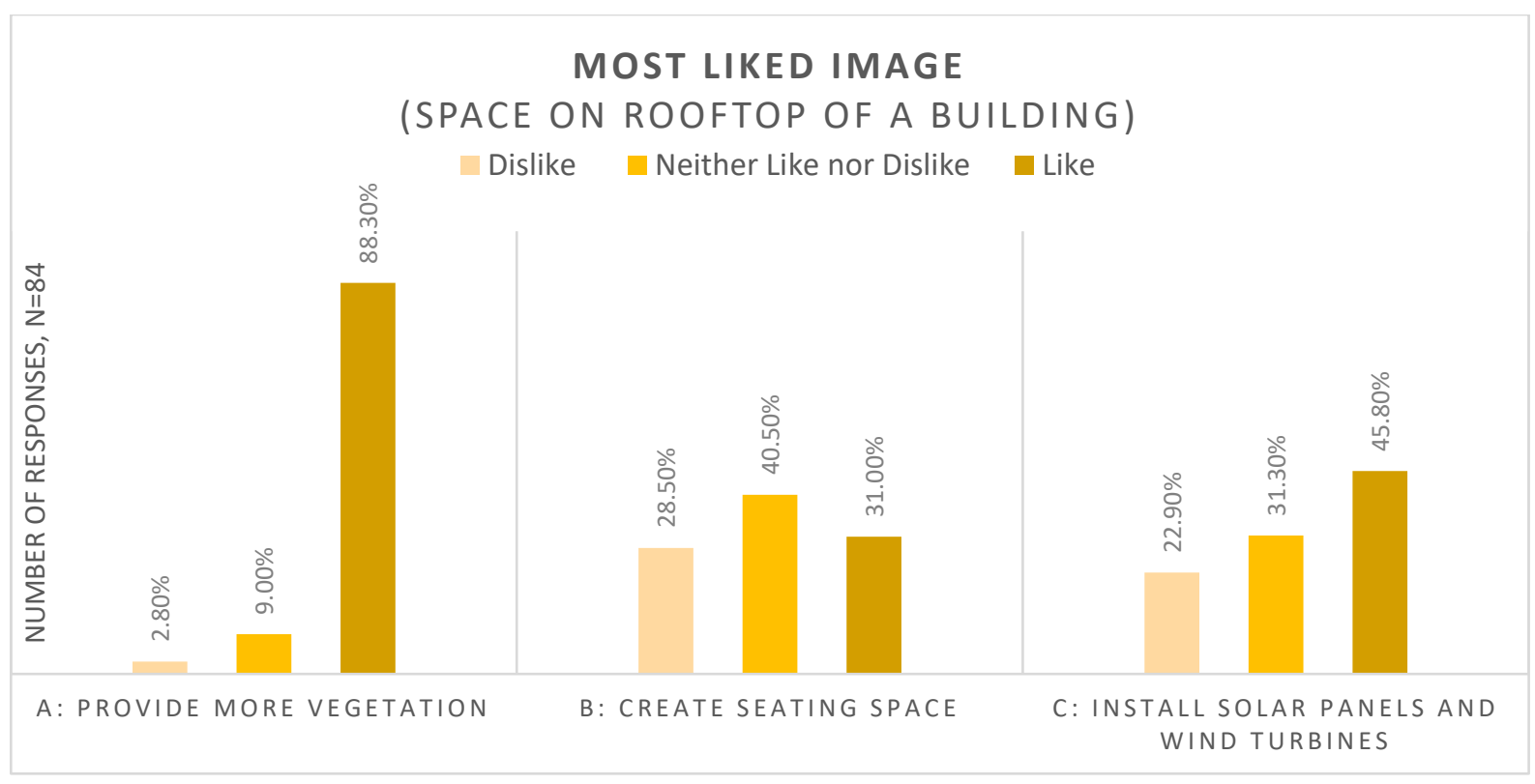

Figure 7.19 Preferences for space on the rooftop of a building $(\mathrm{N}=84)$

Interestingly, this analysis showed people wanted to see more vegetation rather than solar panels or micro wind turbines on the rooftop of a building. The preferences were further analysed using the semantic differential scores on a scale of 1 to 5 . This analysis confirmed that image $A$ had a positive influence on the attitudes of respondents for all aspects, and it also scored a higher mean value (figure 7.20). Kendall's tau-b correlation determined the relationship between the most liked option $A$ and its five semantic differentials. The test revealed a strong, positive association between the liked image and two of the semantic differentials; the other three bipolar categories found not to be statistically significant were the concepts of attractiveness (ugly-beautiful), satisfaction (annoying-pleasing), usability (boring-interesting). These non-significant correlations were measured at, $\mathrm{Tb}=-.412, p=.11(\mathrm{p}>.05) ; \mathrm{Tb}=-$ $.362, p=.06(p>.05) ; \mathrm{Tb}=-.408, p=.07(p>.05)$ respectively. The weak, negative associations indicated that liking option $A$ had no impact on feelings for such aspects. 


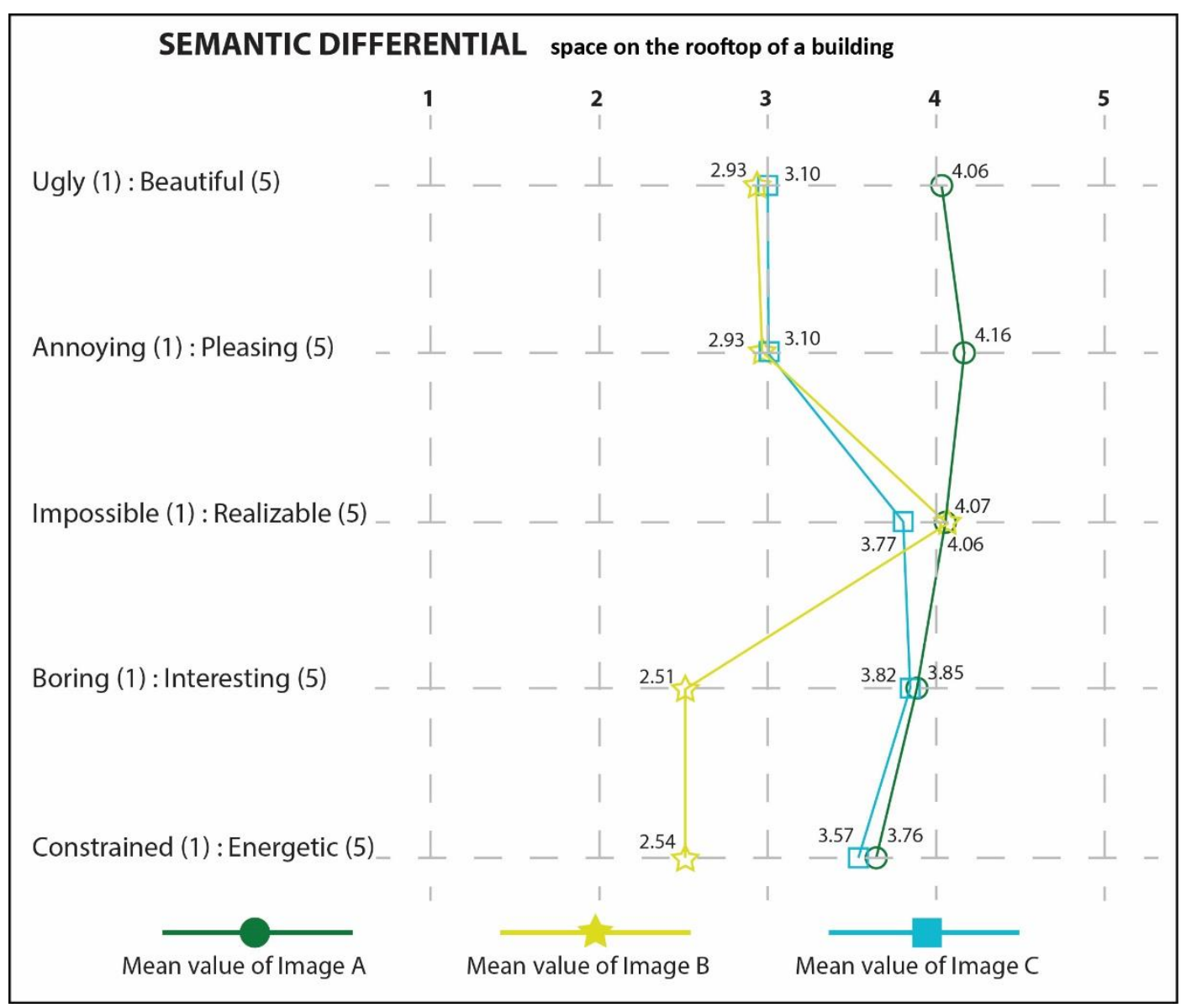

Figure 7.20 Attitudes to the three options for the space on the rooftop of a building

Suggestions from respondents for improving the visual quality of the rooftop space were related to keeping a balance between parking space and planting, and that the vegetation should cover the line of sight and act as a visual barrier.

\subsubsection{Participant selections for improving the leftover spaces}

In this third part of Study Two, four different leftover spaces were shown (figure 7.21), and participants were asked to select at least three recommendations from a given list for providing functions for each leftover spaces assuming the parking had been removed (figure 7.22). 

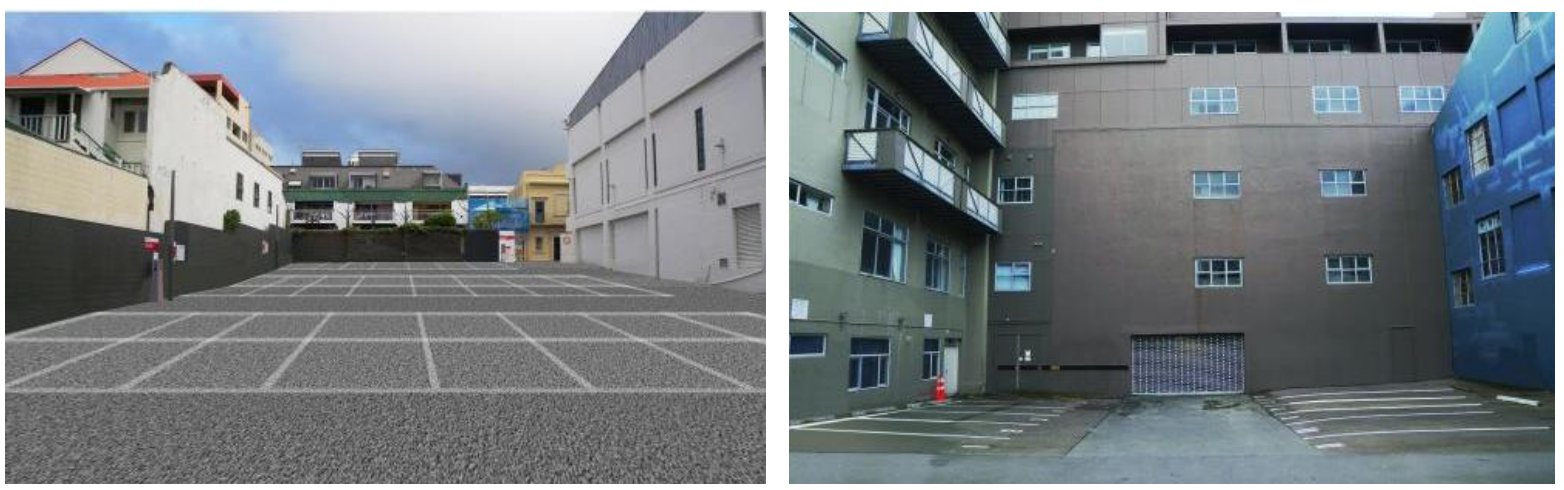

A

B
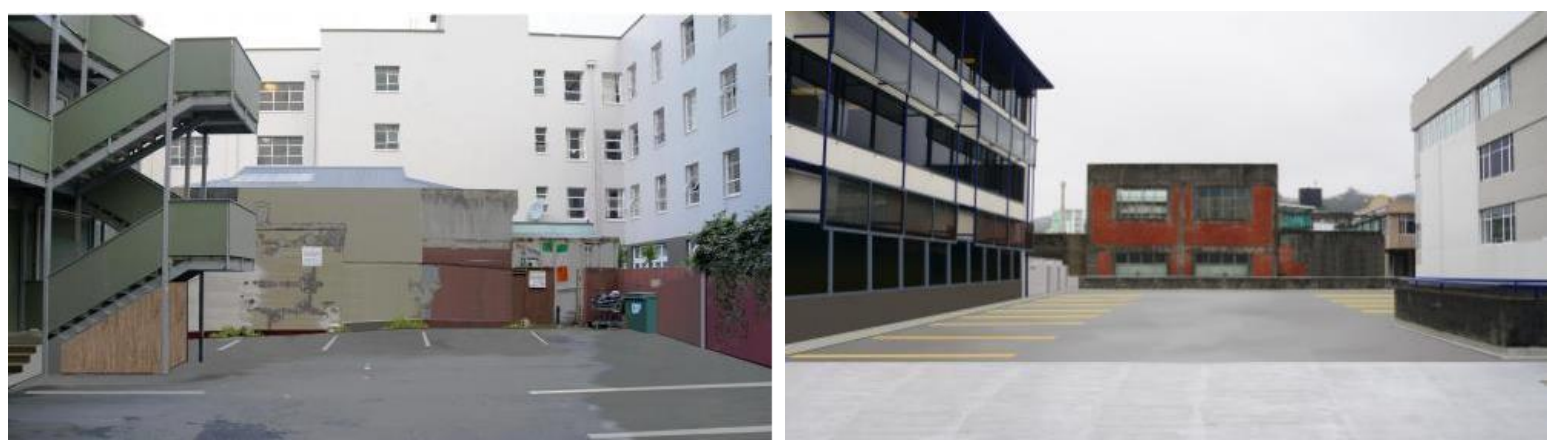

C

D

Figure 7.21 Four options shown to participants in part 3 of Study Two

\section{A—space enclosed by buildings on two sides}

The popular suggestion for improving the visual quality of the space enclosed by buildings on two sides was to create a community garden. This was the highest preference for $36 \%$ of participants and was followed by providing a food market with $24 \%$ of participants preferring this option. Lastly, designing a sports area for the public was preferred by $15 \%$ of participants (figure 7.20 )

\section{$B$-space enclosed by buildings on three sides}

Recommendations for this space were evenly spread out. The suggestions were the creation of graffiti on walls $(20 \%)$, having a food market (19\%), having a community garden (19\%), having a sports area (18\%), and lastly, creating an exhibition space $(17 \%)$.

C-space at the back of a building 
The most preferred suggestion among participants for space at the back of a building was for a community garden, this being preferred by $43 \%$ of participants. The other two recommendations were designing a sports area (16\%) and creating a food market (14\%).

\section{D-space on the rooftop of a building}

Of the available options, $25 \%$ of participants selected installing solar panels on the rooftop of the building, with designing a community garden and installing micro wind turbines chosen by $20 \%$ and $15 \%$ of participants respectively.

Overall the preferred new uses indicated respondents wanted to see community gardens and a food market. The other common usage for the samples of leftover spaces was related to designing an outdoor recreation space.

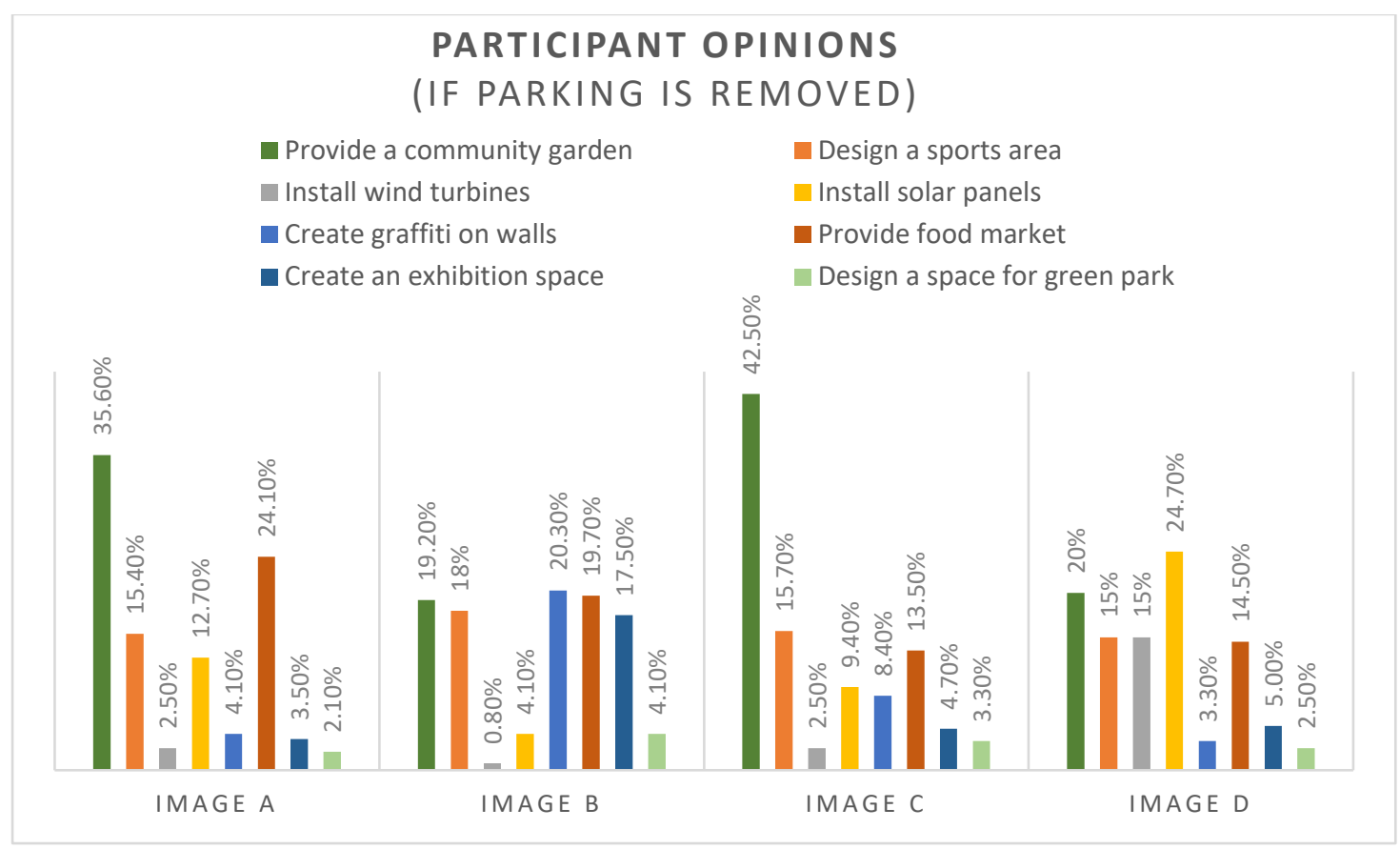

Figure 7.22 Preferred functions for four different leftover spaces

\subsubsection{Analysis of Stage Two}

The second stage of the analysis looked for differences by gender. Gender analysis is vital in a quantitative study as it leads to an understanding of gender roles and relationships within society (Edwards 1990). Tadria (1999) points out the primary purpose of doing gender analysis is to examine the degree of difference in opinions and generate more valid information for planning or to formulate a policy for the 
particular situation (Eagly, 2009). Comparisons lead to the recognition and identification of the strategic, cultural, and biological needs of both males and females. Furthermore, this enhances the understanding of the economic and social roles within a society based on gender while acknowledging their realities. Gender roles and relations are culturally characterised, but they alter with time, place and settings (Tadria, 1999).

The preferences for the different design options were investigated by gender. This meant the data was split into two subgroups (47 male and 61 female). The Cronbach alpha $(\alpha)$ reliability test was used to check the internal consistency of the five bipolar aspects on the semantic scale 1 to 5 before independent sample T-tests could be carried out. Independent sample T-tests were used to identify the differences of opinion regarding preferences by gender. Cronbach's alpha ( $\alpha$ ) was 0.89 , which indicated a high level of internal consistency, as values should be 0.7 or higher to check for significance (Kline, 2005). The averages of the semantic differential responses were combined in order to find the difference in preferences between the male and female subgroups through an independent sample T-test. The latter test confirmed that there were no statistically significant differences in the semantic scale for attitudes between males and females $(p>0.05)$ for all leftover spaces, although there were differences in preference values. These differences are discussed in the next sections.

\subsubsection{Space underneath a building}

For the space underneath a building, 42 males and 55 females responded to the question. Option A, which introduced vegetation into space (see figure 7.3) was the most preferred by both genders, with $71 \%$ of males and $82 \%$ of females preferring it. Only $9 \%$ of both males and females disliked the image (figure 7.23 ), while $19 \%$ of males and $9 \%$ of females neither liked nor disliked option A. For image B with its changed surface textures $37 \%$ of males and $30 \%$ of females liked the space, while $33 \%$ of males and $40 \%$ of females disliked it, and $29 \%$ of males and $30 \%$ of females were neutral. Lastly, image $\mathrm{C}$ represented a well-managed/maintained space. This was liked by $32 \%$ of males and $25 \%$ of females, disliked by $27 \%$ of males and $37 \%$ of females, while $41 \%$ of males and $37 \%$ of females were neutral. The results show 
option A was liked by both genders with more slightly more females than males picking the option.

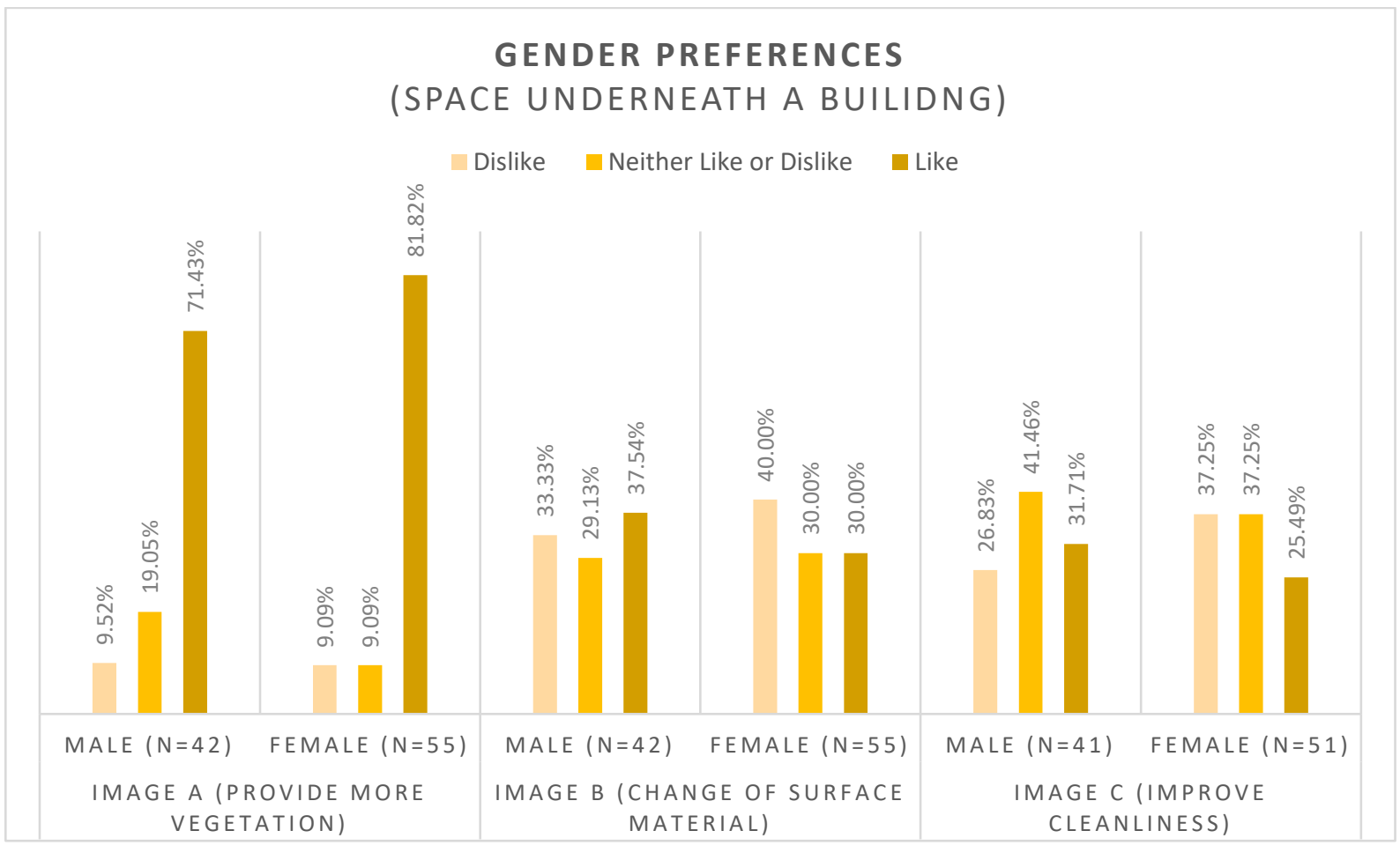

Figure 7.23 Gender preferences for space underneath a building

\subsubsection{Space in front of a building}

Of the sample, 39 males and 49 females answered the question regarding their preferences for the space in front of a building. The most preferred design for both genders was the removal of the boundary wall in option A (see figure 7.6 ), as $77 \%$ of males and $87 \%$ of females preferred this option and only $5 \%$ of males and no females disliked it (figure 7.24 ). Some $18 \%$ of males and $16 \%$ of females neither liked nor disliked option A. Option B with the addition of vegetation was the second most preferred design, being preferred by $64 \%$ of males and $73 \%$ of females. Some $18 \%$ of males and $8 \%$ of females disliked this design, whereas $18 \%$ of both genders were neutral. Lastly, option C, with its seating space created by lowering the boundary wall, was the least liked among the three images. That said, $50 \%$ of males and $67 \%$ of females liked image $\mathrm{C}$. Only $5 \%$ of males and $10 \%$ of females disliked this option, while $44 \%$ of males and $22 \%$ of females were neutral. The comparative results showed both genders and females liked option $A$, but females tended to be more positive towards all options than males. 


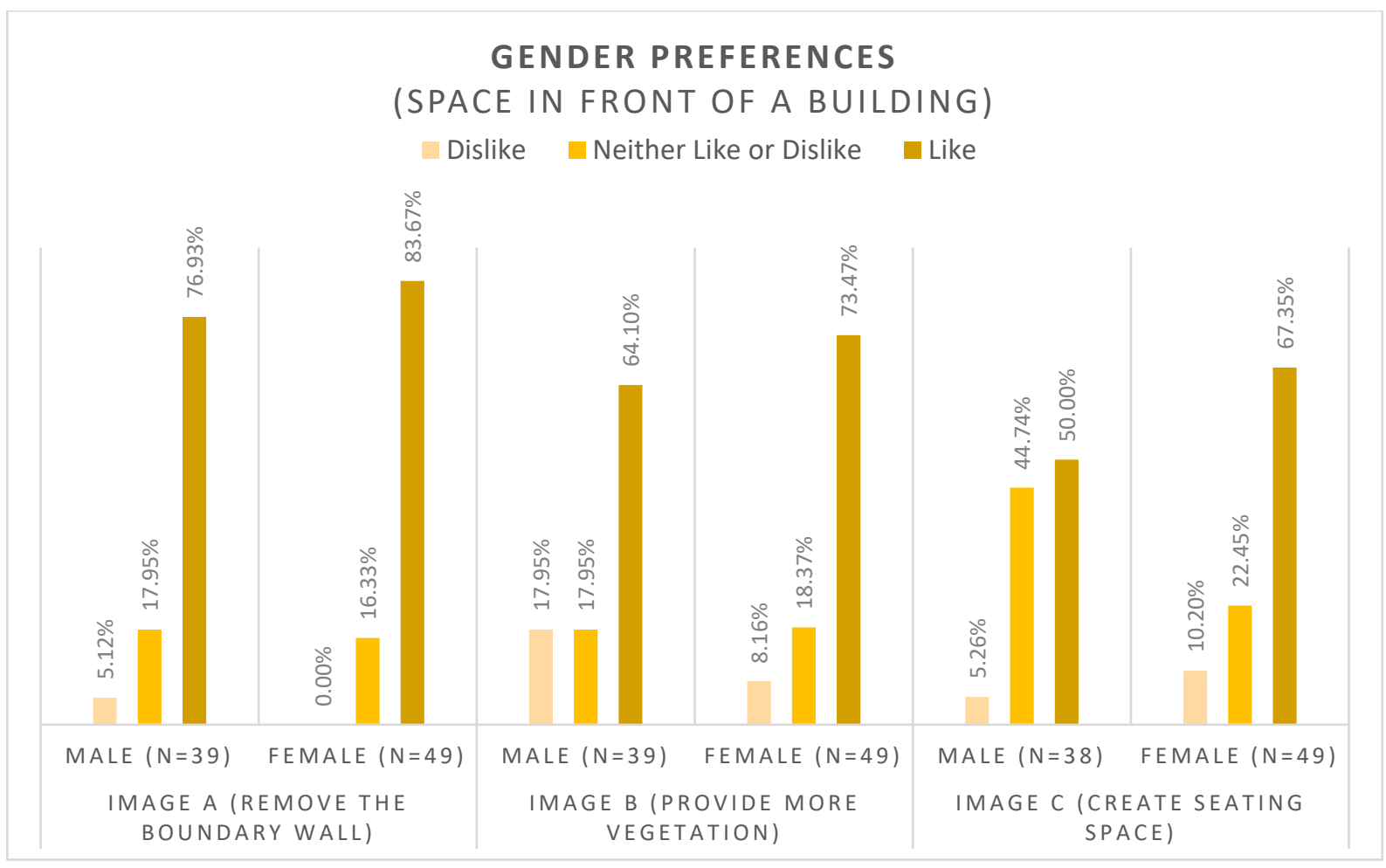

Figure 7.24 Gender preferences for the space in front of a building

\subsubsection{Space at the back of a building}

Of the sample, 36 males and 48 females answered the question regarding space at the back of a building. Option $\mathrm{C}$ for this space was designed with additional vegetation (see figure 7.9). This was the most preferred option for both genders as $94 \%$ of males, and $85 \%$ of females preferred it with only $3 \%$ of males and $6 \%$ of females disliking the image (figure 7.25). Those who were neutral towards it were $3 \%$ males and $8 \%$ females. For option, A with its changes to surface finishes $53 \%$ of males, and $56 \%$ of females liked it, only $8 \%$ of males and $14 \%$ if females disliked it, and $39 \%$ of males and $29 \%$ of females were neutral in their opinions. For image B, which represented having a clear pathway for pedestrians, just $17 \%$ of males and $19 \%$ of females liked it. Those disliking were $53 \%$ male and $48 \%$ female, while $30 \%$ of males and $33 \%$ of females had no preference. The comparative results showed option $\mathrm{C}$ with the addition of vegetation was liked by both genders, but preferred by slightly more males than females. 


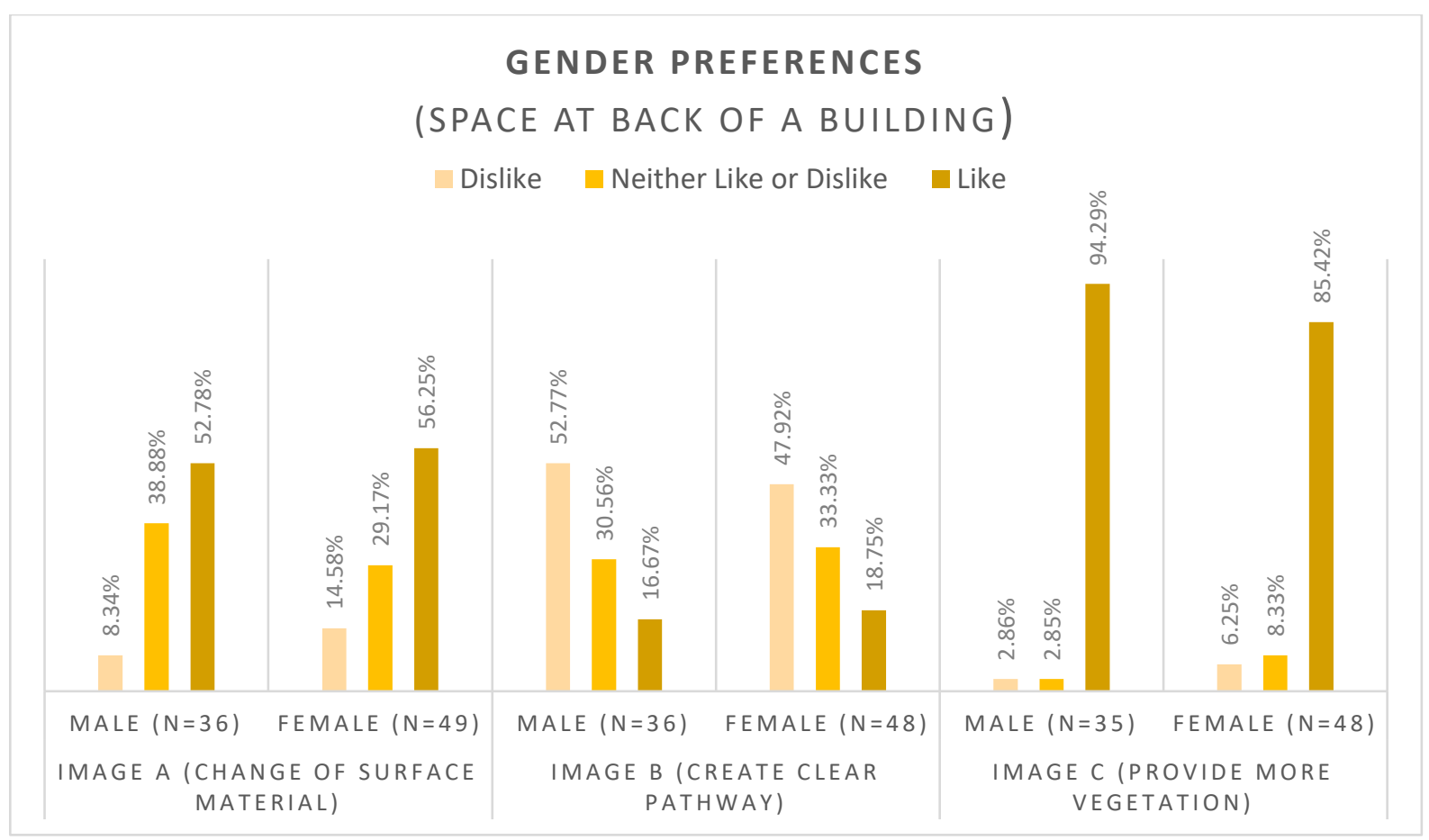

Figure 7.25 Gender preferences for space at the back of a building

\subsubsection{Space enclosed by buildings on three sides}

For this space, 35 males and 48 females answered the question. Option $C$ with the addition of vegetation was again the most preferred by both genders (see figure 7.12), as $83 \%$ of males and $87 \%$ of females preferred it, and only $6 \%$ of males and $8 \%$ of females did not like the image (figure 7.26). Those who were neutral towards it were $11 \%$ male and $4 \%$ female. Those who liked image $A$ with its changed surface materials were $37 \%$ male and $48 \%$ female, whereas $17 \%$ of males and $13 \%$ of females disliked it, and $46 \%$ of males and $41 \%$ of females were neutral towards it. The least liked option was $\mathrm{B}$, with its clear pathway. This was liked by $25 \%$ of males and $21 \%$ of females, while those who disliked it was $37 \%$ male and $35 \%$ female, with $37 \%$ of males and $43 \%$ of females being neutral towards it. The comparative results showed option C with the additional vegetation was preferred by both genders, with very slightly more females preferring it. 


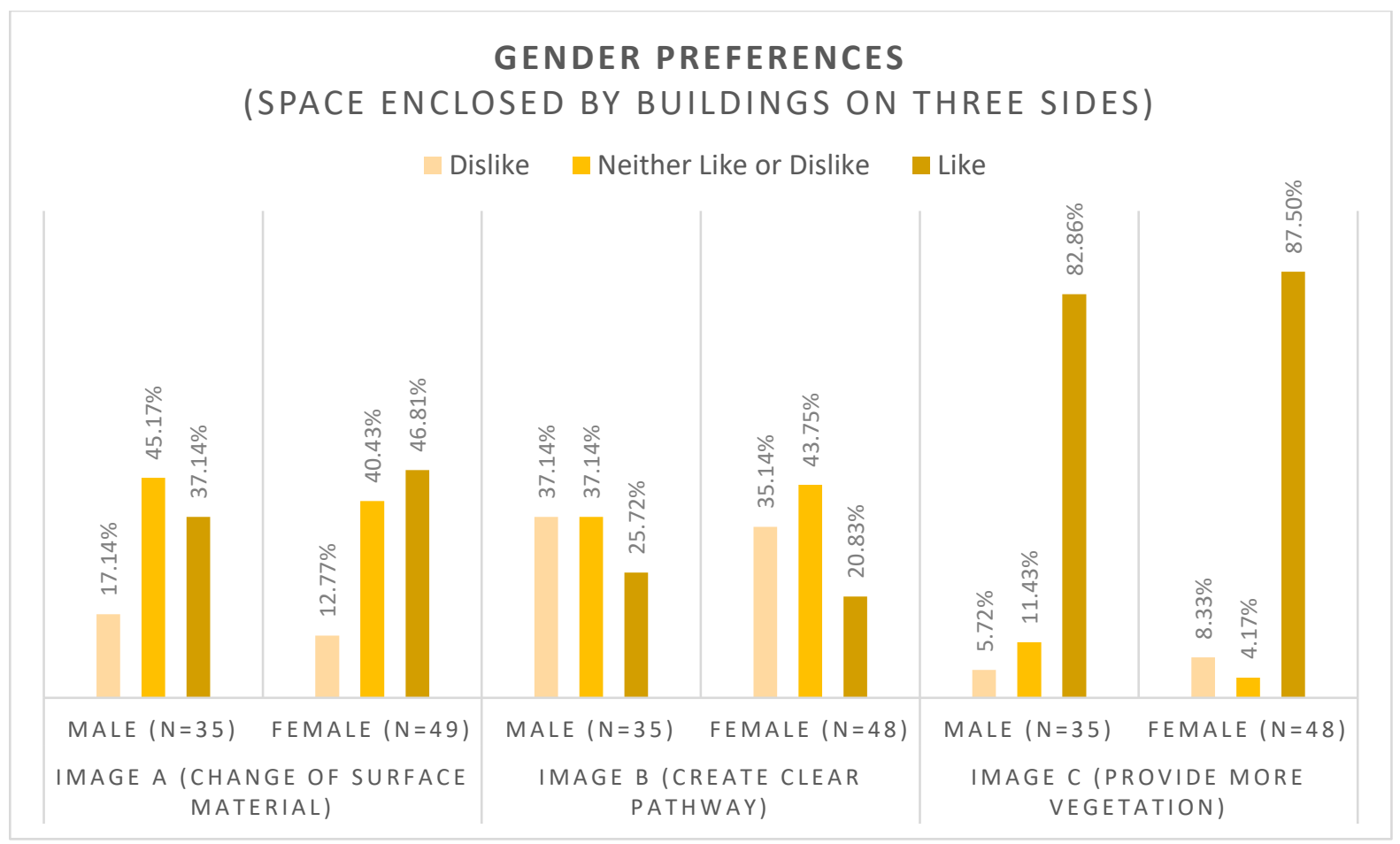

Figure 7.26 Gender preferences for space enclosed by buildings on three sides

\subsubsection{Space enclosed by buildings on two sides}

For the space enclosed by buildings on two sides, 35 males and 48 females answered the question. Option B with its added vegetation was the most preferred choice for both genders (see figure 7.15), being preferred by $91 \%$ of males and $89 \%$ of female, whereas only $3 \%$ of males and $6 \%$ of females did not like it (figure 7.27 ), and only $5 \%$ of males and $4 \%$ of females were neutral to it. The introduction of a clear pathway in option A was liked by $40 \%$ of males and $31 \%$ of females, with $31 \%$ of males and $25 \%$ of females disliking the space, and $28 \%$ of males and $44 \%$ of females feeling neutral towards it. The changes to surface materials in image $C$ was liked by $37 \%$ of both genders, while $20 \%$ of males and $37 \%$ of females disliked this option, and $42 \%$ of males and $25 \%$ of females were neutral towards it. Again the option with the vegetation was the most preferred with more slightly more males than females liking it. 


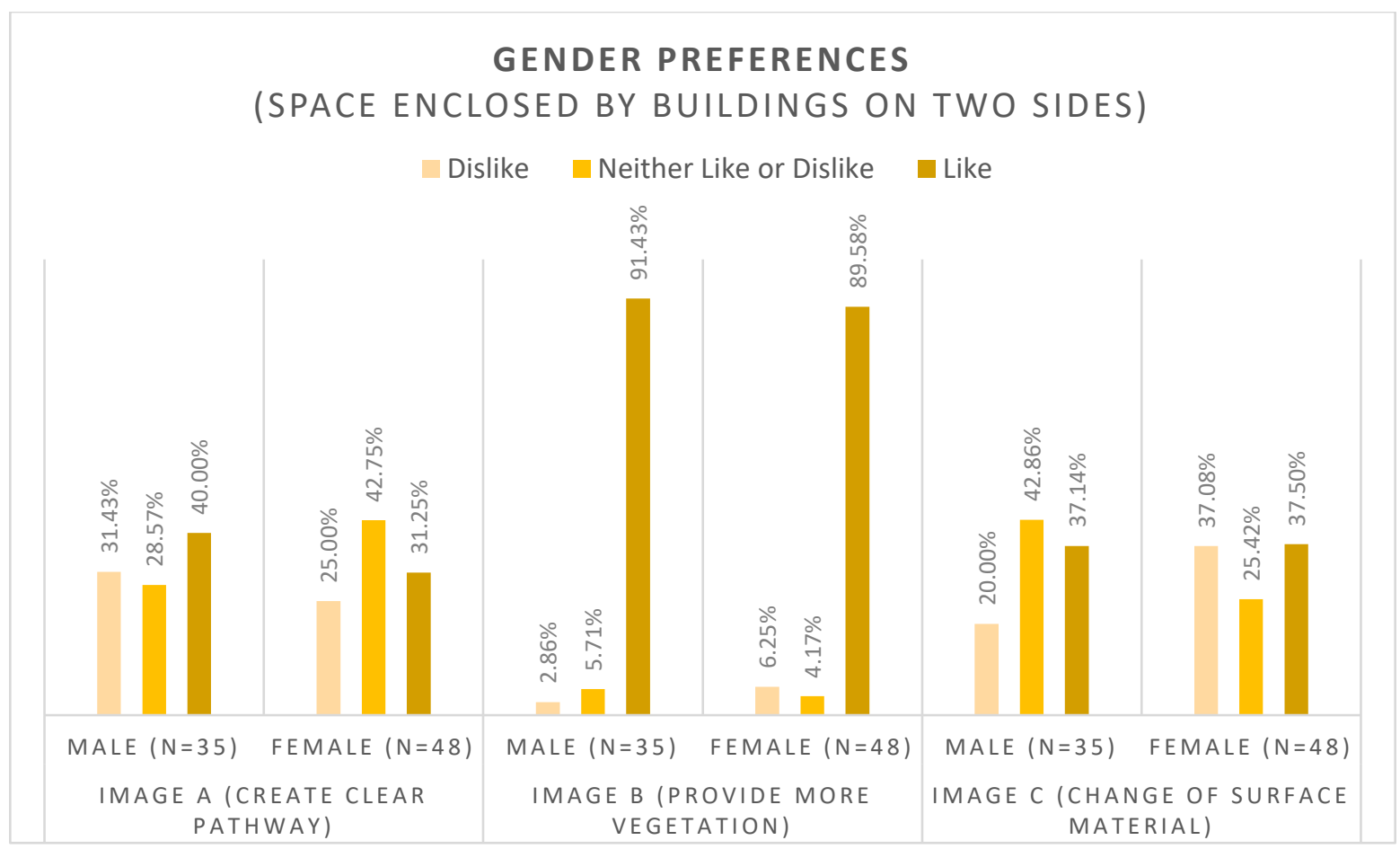

Figure 7.27 Gender preferences for space enclosed by buildings on two sides

\subsubsection{Space on the rooftop of a building}

For this question about designs for the rooftop 35 males and 48 females answered it. Again, option $A$ with its vegetation was preferred, being the choice of $88 \%$ of males and $89 \%$ of females (see figure 7.18 ). Only $3 \%$ of males and no females disliked option A (figure 7.28 ), while $6 \%$ of males and $10 \%$ of females were neutral. Option B with its new seating was liked by $32 \%$ of males and $31 \%$ of females, while $35 \%$ of males and $25 \%$ of females disliked the space, and those who were neutral towards it were $32 \%$ male and $43 \%$ female. Image $C$ with its new solar panels and micro wind turbines, was the second most preferred design, as those who liked it was 50\% male and $42 \%$ female. Those who disliked it was $23 \%$ male and $21 \%$ female and those who were neutral were $26 \%$ male and $36 \%$ female. Again, introducing vegetation is the most preferred option being nearly equally liked by both genders. 


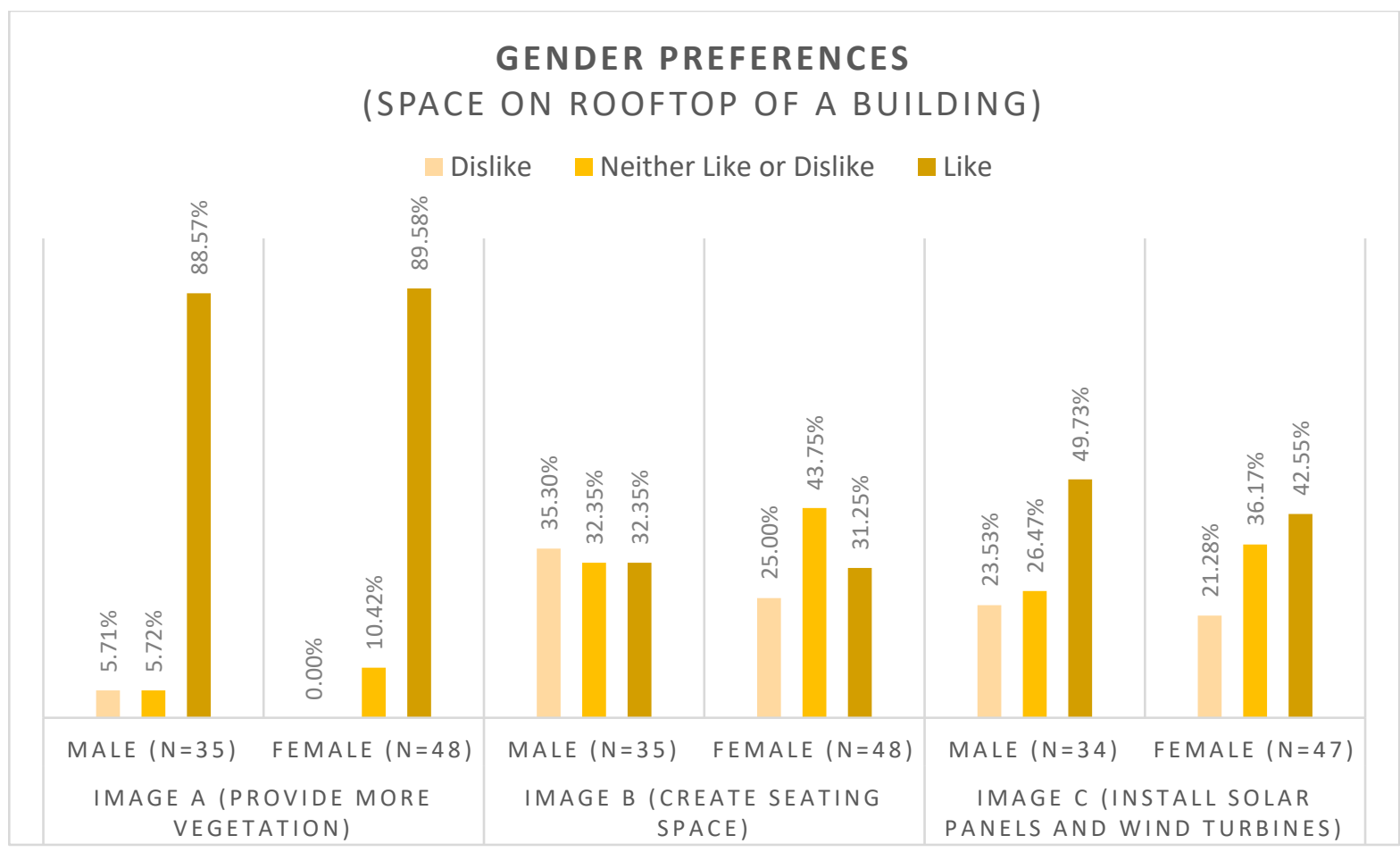

Figure 7.28 Gender preferences for space on the rooftop of a building

\subsubsection{Analysis of Stage Three}

The third stage of analysis investigated the differences in preference between participants who had a Built Environment background $(B E)(N=24)$ with those who did not (NBE) (N=72). Arnheim (1977) points out that BE professionals not only see what a building or a place looks like but also deconstruct the setting to understand how it was built. Rapoport (1982) claims that the pragmatic meaning of a place inferred from a specific style differs for NBE experts. In addition, NBE people seek visual continuity or an associational relevance within a building and setting (Groat, 1988). The Prince of Wales (1984) argued that BE professionals often ignored the sentiments of ordinary people and should play an active role in developing strategies with them.

For this stage of Study Two, the data was split into two subgroups in SPSS, and the preferences were calculated based on the regrouped 3 points Likert scale. The Cronbach alpha $(\alpha)$ reliability test was used to check the internal consistency of the semantic variables on a scale from 1 to 5 before independent sample T-tests could be carried out. Cronbach's alpha ( $\alpha$ ) indicated a high level of internal consistency at 0.86 . The averages of the semantic differential responses were determined to find the differences in preferences between the two groups. Independent sample t-tests for both groups confirmed that there were no statistically significant differences in 
preferences for $B E$ and NBE respondents $(p>0.05)$ for all the redesigned spaces. This meant that NBE participants had approximately the same attitudes to the designs as the BE participants. Hence only the means for the preferences are discussed here.

\subsubsection{Space underneath a building}

This questioned was answered by $24 \mathrm{BE}$ and 72 NBE participants. Option A with introduced vegetation was preferred by all participants (figure 7.3), breaking down into $62 \% \mathrm{BE}$ and $82 \%$ NBE. Of the participants who disliked option $\mathrm{A}, 12 \%$ were $\mathrm{BE}$ and $8 \%$ NBE (figure 7.29), and 25\% BE and 9\% NBE participants were neutral. Option B, with its changes to the surface materials, was liked by only $14 \% \mathrm{BE}$ and $28 \% \mathrm{NBE}$ participants. Lastly, image $C$, with its improved cleanliness, was the second most liked designed option, being preferred by $18 \%$ BE and 30\% NBE participants. Overall, the preference for option $A$ was evident but $B E$ participants were less enthusiastic for it than the NBE respondents.

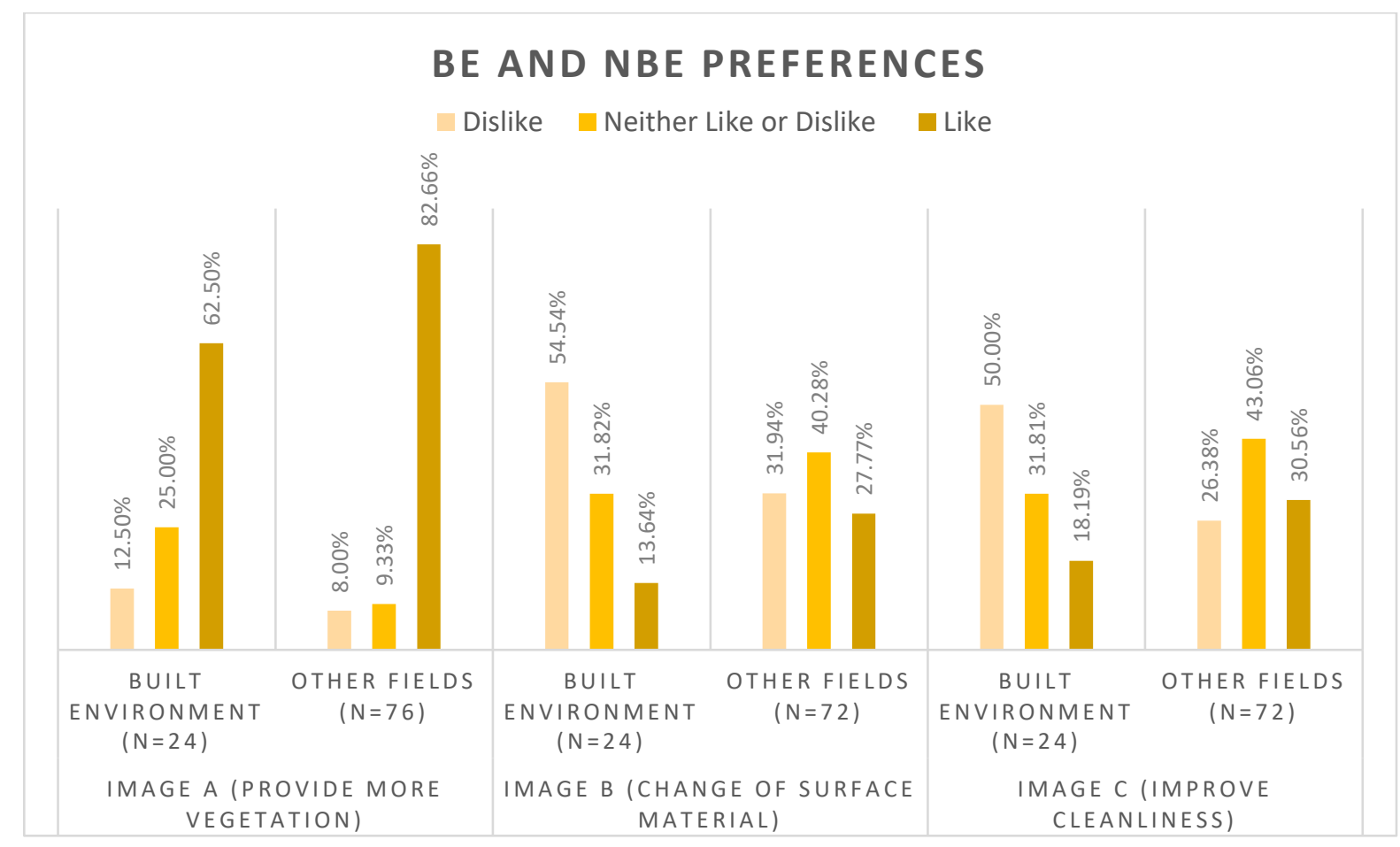

Figure 7.29 BE and NBE preferences for space underneath a building

\subsubsection{Space in front of a building}

This questioned was answered by $23 \mathrm{BE}$ and 63 NBE participants. Option A with the removal of the boundary wall (figure 7.6 ) was the most preferred, being liked by $71 \%$ 
BE and $81 \%$ NBE participants. Few people disliked it (5\% BE and 3\% NBE) (figure 7.30), and $24 \%$ BE and $16 \%$ NBE participants were neutral towards it. Option B with more vegetation was the second most liked with $68 \%$ of $\mathrm{BE}$ and $71 \%$ of NBE participants liking this option. Image $\mathrm{C}$, which was designed to create seating space, was liked by $55 \%$ BE and $59 \%$ NBE respondents. Overall, the preference for option A was evident.

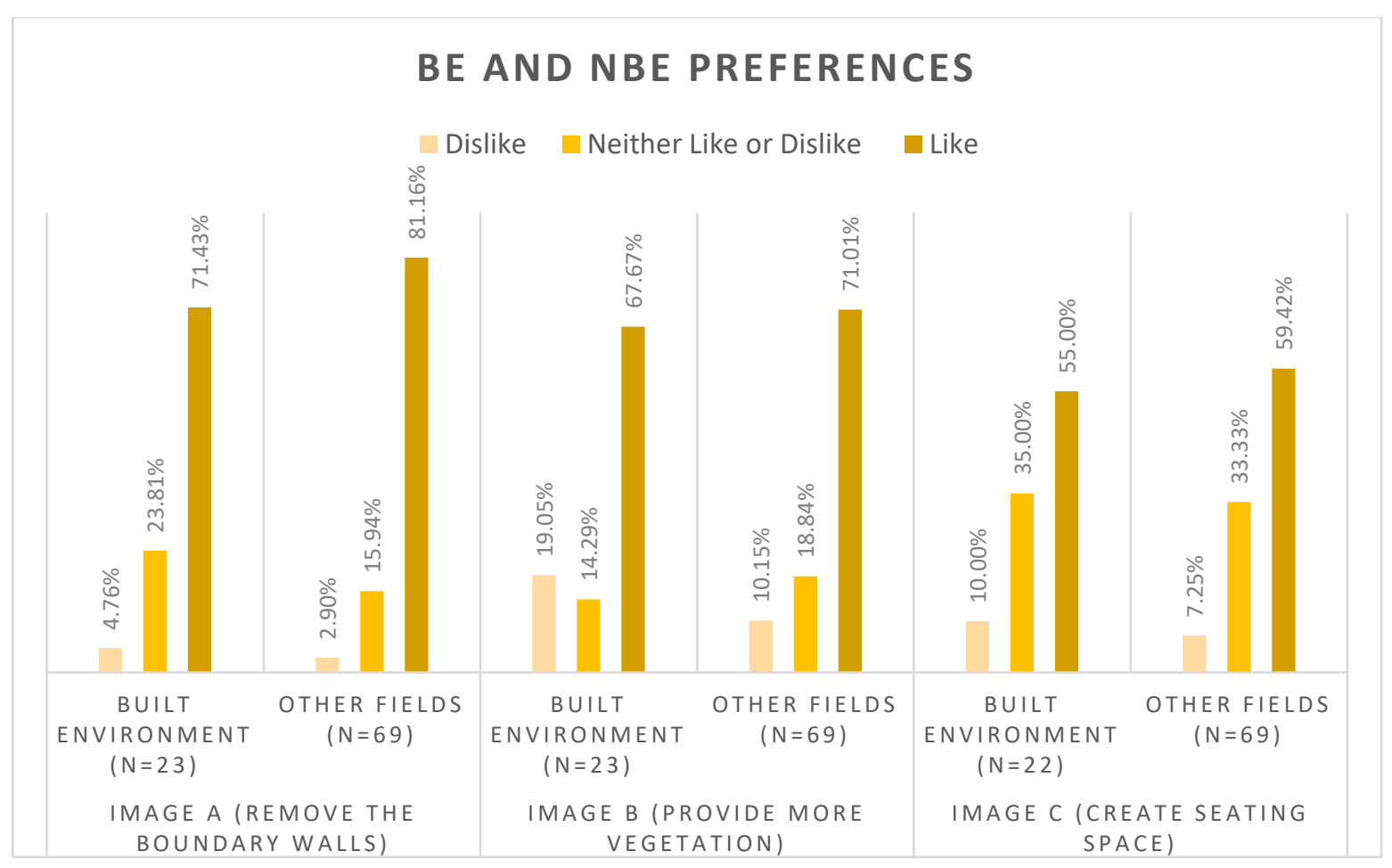

Figure 7.30 BE and NBE participant preferences for space in front of a building

\subsubsection{Space at the back of a building}

There were $22 \mathrm{BE}$ and $66 \mathrm{NBE}$ answers to this question. Option $\mathrm{C}$ with its vegetation (see figure 7.9) was the most preferred design option as $89 \% \mathrm{BE}$ and $88 \% \mathrm{NBE}$ participants preferred it and no BE and only $6 \%$ of NBE participants did not like it (figure 7.31). Some 10\% BE and 6\% NBE participants were neutral in their approach to option $\mathrm{C}$. Option $\mathrm{A}$ with its change of surface material was the second most liked design option, being liked by $45 \%$ BE and $56 \%$ NBE participants. Lastly, image B, which was designed to create a clear pathway, was only liked by $23 \%$ NBE participants and no participants from the BE group. Overall, the preference for option $C$ was prominent, but NBE participants had a wider spread of preferences than the BE group. 


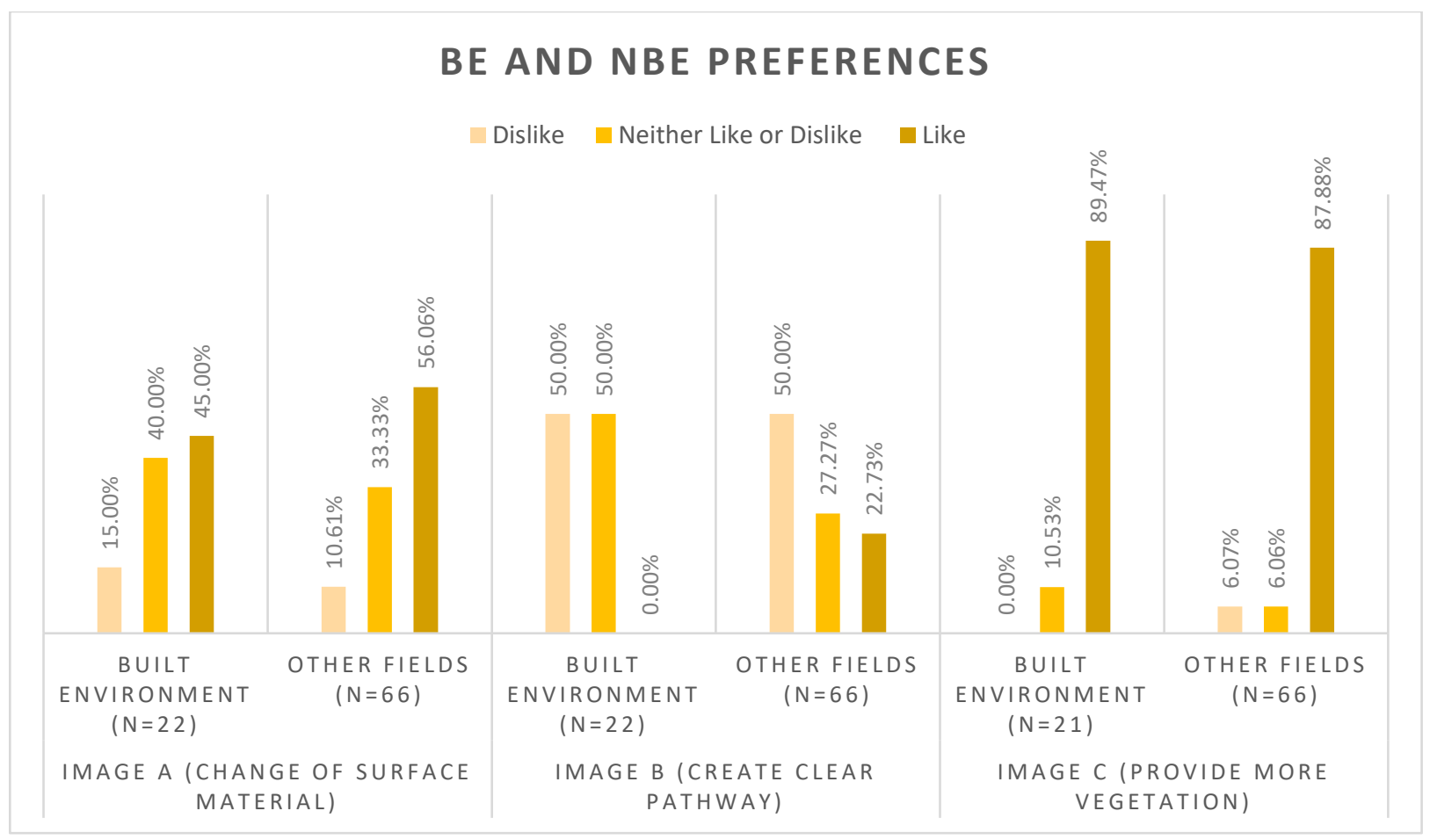

Figure 7.31 BE and NBE preferences for space at the back of a building

\subsubsection{Space enclosed by buildings on three sides}

There were $21 \mathrm{BE}$ and $66 \mathrm{NBE}$ respondents, and Option C with its introduced vegetation (figure 7.12 ) was preferred by both groups as more than $89 \% \mathrm{BE}$ and $85 \%$ NBE participants preferred it, whereas just 5\% BE and $8 \%$ NBE participants did not like the option (figure 7.32), and 5\% BE and $8 \%$ NBE were neutral in their opinion of it. Option $A$ with its changes to surface materials was the second most preferred option, with $42 \%$ BE and $43 \%$ NBE participants liking it. Lastly, image B, with its clear pathway, was liked by $29 \%$ NBE participants, but no participants from the BE group liked this option. Overall, the preference for option $C$ was evident, and NBE participants were again wider in their preferences than the BE group. 


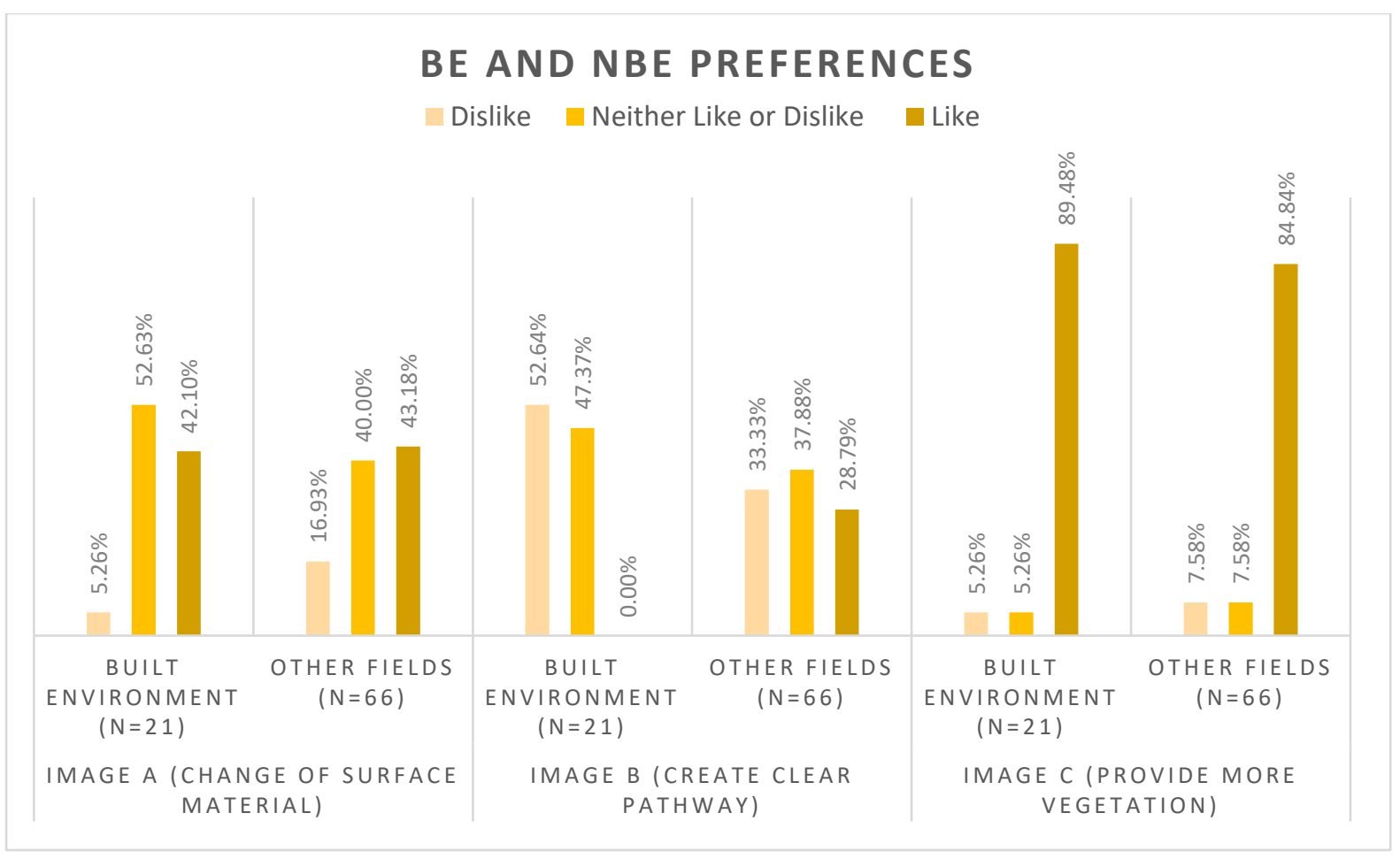

Figure 7.32 BE and NBE preferences for space enclosed by buildings on three sides

\subsubsection{Space enclosed by buildings on two sides}

This question was answered by $21 \mathrm{BE}$ and 66 NBE participants. Option B with its vegetation was again the most preferred (figure 7.15 ) as $95 \% \mathrm{BE}$ and $88 \%$ of NBE participants liked it, while only $6 \%$ of NBE participants disliked it (figure 7.33). No BE participant disliked option B. Those neutral to this design were $5 \% \mathrm{BE}$ and $6 \% \mathrm{NBE}$. Option A with its clear pathway, was liked by $37 \%$ BE and $35 \%$ NBE participants. This was liked more than option $C$ with changes to surface materials, as this option was liked by $26 \%$ BE and $39 \%$ NBE participants. Overall, the preference for option B was apparent. 


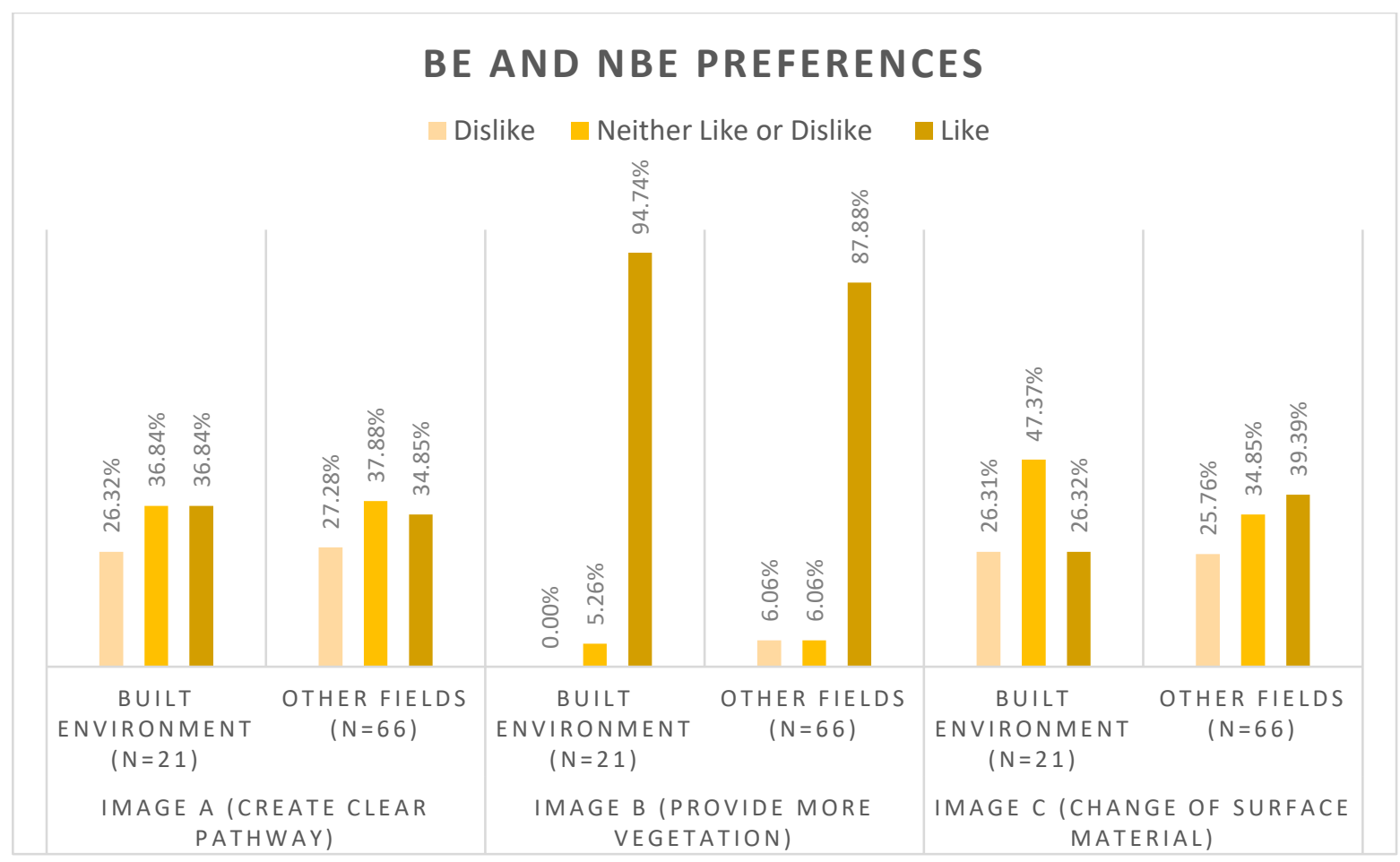

Figure 7.33 BE and NBE preferences for space enclosed by buildings on two sides

\subsubsection{Space on the rooftop of a building}

The question regarding the rooftop space was answered by $21 \mathrm{BE}$ and $66 \mathrm{NBE}$ participants. Option A, the space with added vegetation (figure 7.18 ) was again preferred by both groups. The liking for option $A$ breaks down into $89 \% \mathrm{BE}$ and $88 \%$ NBE participants. Only 5\% BE and 1\% NBE participants did not like this option (figure 7.34), and 5\% BE and 10\% NBE participants were neutral towards it. The second highest preference was for option $\mathrm{C}$ with its solar panels and micro wind turbines, as this was liked by $44 \%$ BE and $46 \%$ NBE participants. Lastly, only $21 \%$ BE and $33 \%$ NBE respondents liked option $B$, which was designed to create a seating space. Overall, the preference for option A with vegetation was very high. 


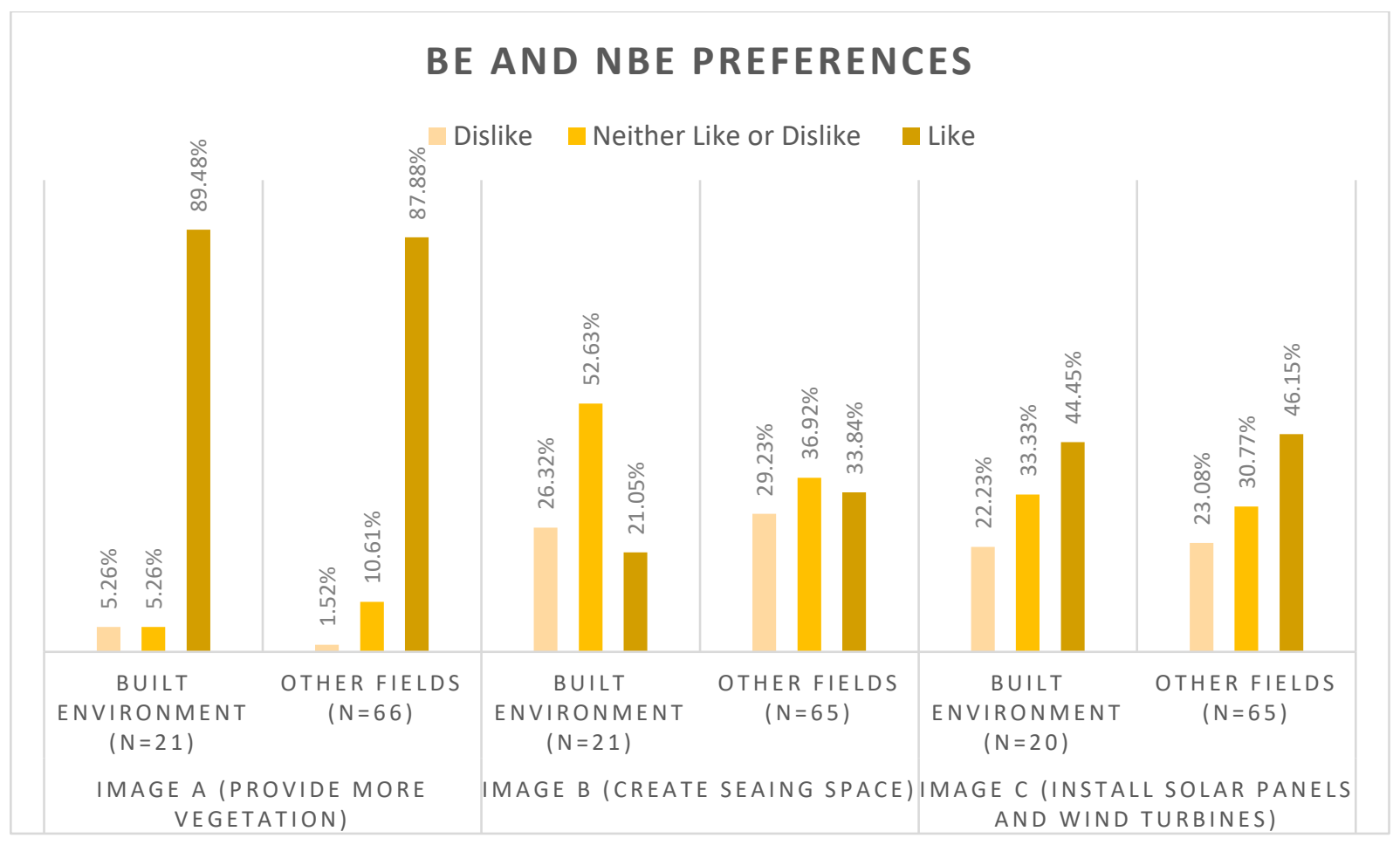

Figure 7.34 BE and NBE preferences for space on the rooftop of a building

\subsection{The Findings}

Study 2 probed people's aesthetic responses to the six different redesigned leftover spaces. Each scene portrayed the space with the introduction of only one attribute. The photographic representations thus sought responses to a variety of design solutions. The design solutions used were suggested by the results of Study One and were limited to three changes to each type of leftover space. A strong preference was found for the spaces that had an element of vegetation in them. Although the level of this preference fluctuated a little for five of the different types of leftover space, only the space in front of a building was scored differently. In that case, the preferred solution was lowering the boundary wall. The results of the various stages of Study 2 are summarised below.

\subsubsection{Stage One: Whole Sample ( $\mathrm{N}=96)$}

The most preferred design solution among all participants was related to adding more vegetation in all leftover spaces (figure 7.35). The space in front of a building had a different preference ranking. The first preference was given to the removal of boundary walls, whereas the second most preferred design was providing more vegetation. The Kendall's tau-b $(T \mathrm{~b})$ correlation test revealed the most liked image (removing the 
boundary walls) in front of a building had a weak, negative association with the one bipolar affective appraisal category of "boring-interesting".

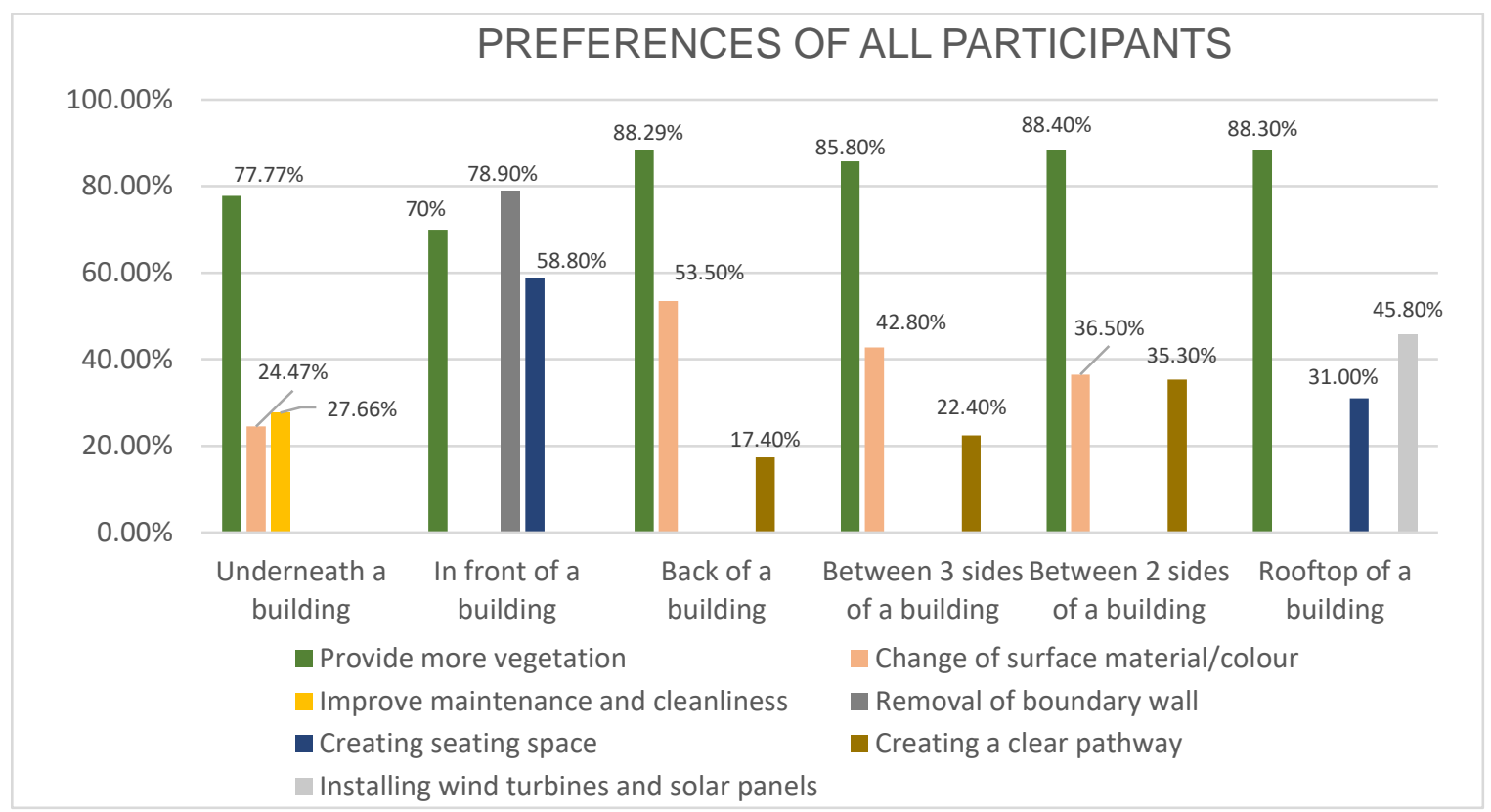

Figure 7.35 Preferences for redesigned leftover spaces for all 96 respondents

A Kendall's tau-b $\left(T_{b}\right)$ correlation test for all designed spaces with vegetation revealed that the most liked image had a strong, positive association and correlated with all affective appraisals (semantic differentials) except for the bipolar category of 'impossible-realisable'. This suggested this category was perhaps independent of the association and was not influenced by the image's likability. The results inferred that designing a space with vegetation is something the public would like, but at the same time, this is not seen as a practical solution. The suggestions for improving the spaces were about providing amenities for the public, such as providing community gardens, designing a recreation space, and providing a food market.

\subsubsection{Stage Two: Gender}

This stage probed the differences in preferences expressed by males and females, noting that the female group was larger than the male. The most liked options for males and females were those that had an element of vegetation in them (figure 7.36). Female preference percentages for providing more vegetation were generally higher than those of males (for spaces underneath a building, in front of a building, enclosed by buildings on three sides, and the rooftop of a building). 


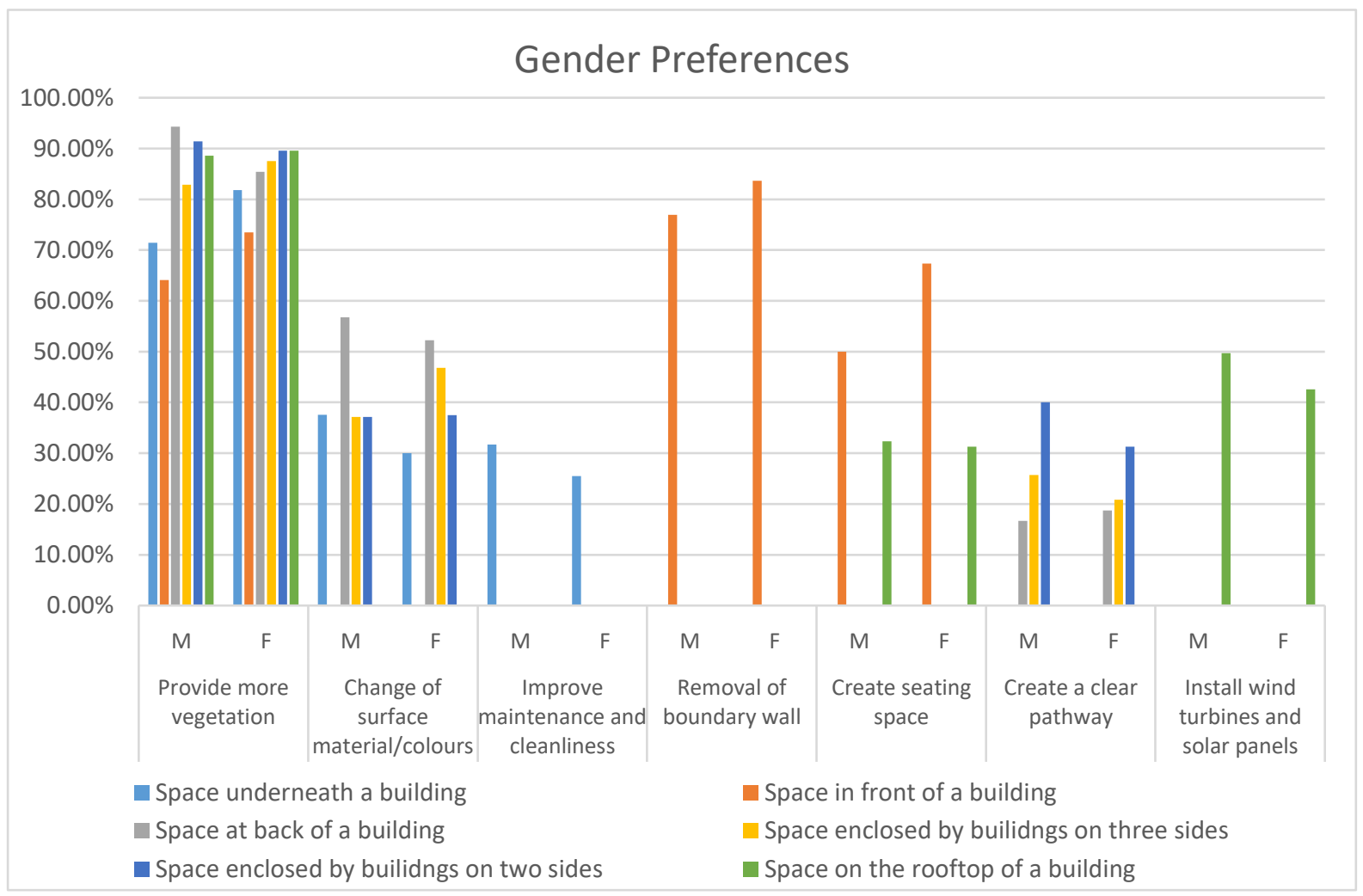

Figure 7.36 Preferences by Gender group

The space in front of a building with the removal of the boundary wall was unanimously liked by both gender groups, again with more females than males preferring it. An independent sample T-test for both groups confirmed that there was no statistically significant difference in opinions between male and female participants $(p>0.05)$ for all redesigned leftover spaces.

\subsubsection{Stage Three: Built Environment (BE) and Non-Built Environment (NBE) participants}

This stage looked for differences in preferences between those with a built environment background and those with other backgrounds. The most robust agreement for both groups was for spaces that had an element of vegetation in them (figure 7.37). The space at the back of a building, enclosed by the buildings on three 
sides, enclosed by the buildings on two sides, and a rooftop space all with the introduction of vegetation were valued higher by BE than NBE participants.

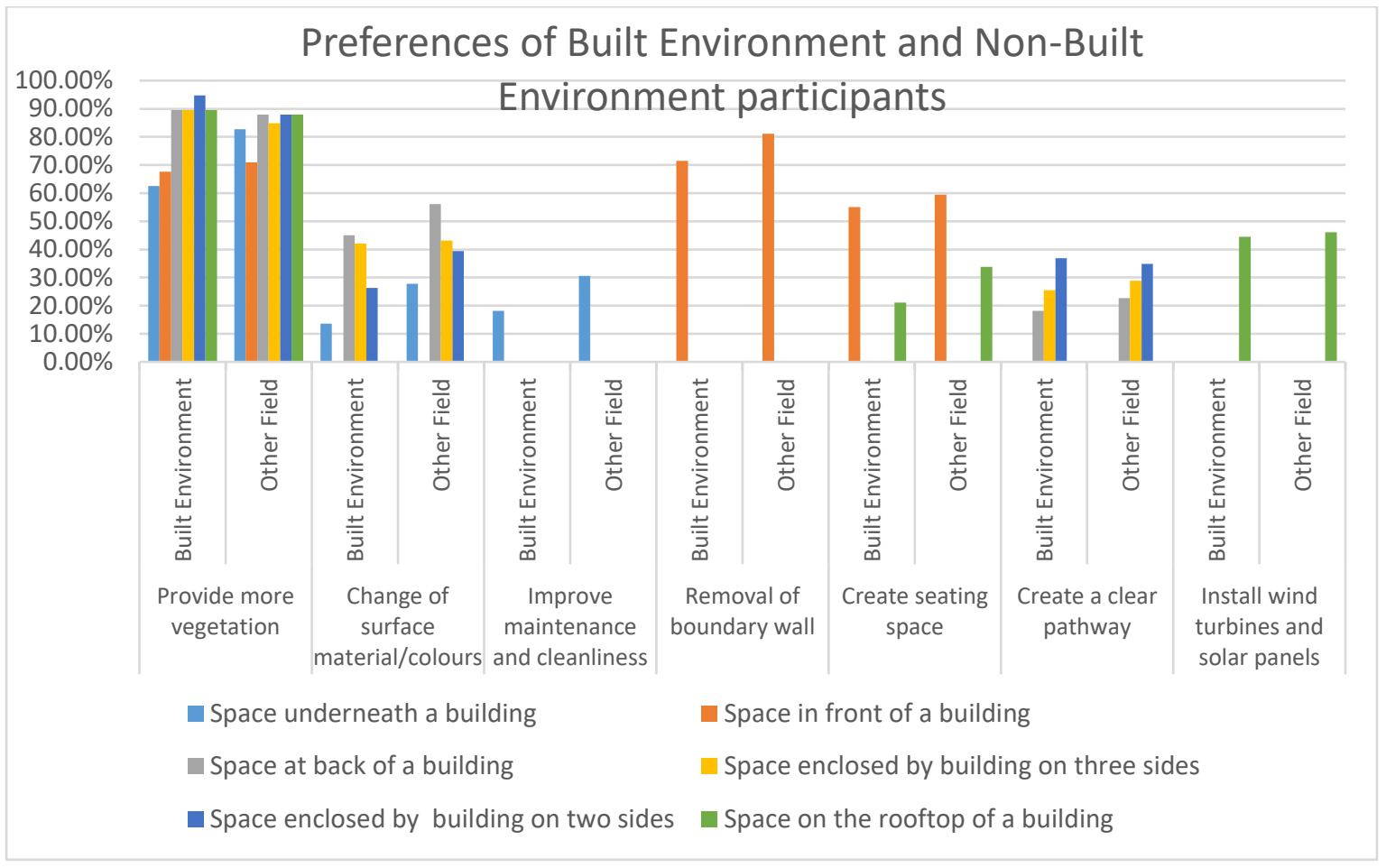

Figure 7.37 Preferences of BE and NBE participants

The space in front of a building with the removal of boundary walls was liked best by both groups. The option with vegetation was the second most liked design for both $\mathrm{BE}$ and NBE participants. An independent sample T-test for both groups confirmed that there were no statistically significant differences in opinions between the BE and NBE participants $(p>0.05)$ for all redesigned leftover spaces.

\subsection{Summary}

This Chapter discussed the significance of the visual preference study that forms Study Two, the technique for conducting the study and collecting the information regarding people's preferences for different redesigned leftover spaces. Each leftover space was photomontaged with three design alternatives based on Study One and people rated each photograph on a Likert scale. Another 5-point Likert scale was used for measuring the attitudes of participants related to their emotions through semantic differentials for the photomontages. It became evident that the respondents preferred 
spaces that were designed with vegetation in them. Participants also suggested providing a community garden if parking was to be removed from the leftover spaces (see section 7.3.1.7). Chapter 8 focuses on conducting focus group discussions with $\mathrm{BE}$ and NBE participants to probe more deeply into whether there are any differences in preferences between participants. Another purpose of Study Three is to enable comparisons between the results of the quantitative studies (Studies One and Two) and the qualitative results of the focus group discussion. This work forms the basis of Chapter 9. 


\section{CHAPTER 8}

\section{STUDY THREE: FOCUS GROUP DISCUSSION}

\subsection{Introduction to Study Three}

This Chapter details the qualitative method used for Study Three, which concerned the focus group discussions and the results of the analysis. Study Three followed Studies One and Two (see Chapter 6 and 7). Study Three investigated the perceptions and experiences of two focus groups and the redesigns for leftover spaces prepared by the participants. The first group comprised people who had a working life and qualifications to do with the built environment $(\mathrm{BE})$, and the second group were people without these experiences (NBE). The focus groups were undertaken to compare the earlier quantitative survey results with those obtained from using a qualitative method in order to highlight the similarities and differences in preferences between the BE and NBE groups.

Scheer (1994) and Stamps (2000) report that BE and NBE people seem to perceive the natural and built environment in different ways. These differences include aspects of contextual compatibility and cognitive complexity. Studies by Hershberger (1988) and Brown \& Gifford (2001) have shown BE people judge the appearance of built spaces differently from NBE people. However other studies by Carmen et al. (2011) and Ghomeshi \& Jusan (2012) concluded that there is no significant difference in opinions between architects and others, in terms of emotional attributes for selecting an area and physical cues related to building aspects, including glass cladding, colour uniformity and stepped storeys. Thus, the overall aim was to confirm the attributes that could potentially improve the usability and visual appearance of leftover spaces by investigating participants from these two fields of study.

\subsection{Importance of Focus Groups}

Focus groups are defined as supervised discussions for exploring a single topic in a specified population (Ward et al., 1991). Full acceptance of focus groups has evolved, and these are now recognised as an established method of collecting data (Kruger, 1994). Focus groups were initially limited to health studies but have since become 
popular in having a general understanding of human behaviour in depth. The idea of group discussions could be said to have been reinvented by psychologists concerned with finding a better way to conceptualise and analyse human personalities (Desvouges \& Frey, 1989). Krueger (1994) argued that focus groups are more vital for topics that have to do with perception, intuition, and experiences. Carnaghi (1992) claimed that a focus group is a technique for research brainstorming that can be of use in many domains, including educational, professional, and business. This method of data collection is widely popular as the results are quick to appear and easy to assimilate, and both specialists and non-specialists can understand the outcomes of this approach. The accumulation of new data from scholars and non-experts is a core basis for participatory research (Krueger \& King, 1998; Veale, 2005). Most importantly, focus groups have been observed to produce effective results (Kreuger, 1994). Other conveniences of this way of setting up a study include the ease of data analysis and the fact that little expertise is required (Bertrand, 1992).

Focus groups are often used in combination with quantitative methods. For many years the latter has been used effectively by researchers to collect and analyse data. However, the language of a quantitative study can be difficult for a practitioner to comprehend and can create discomfort for those not trained in statistics (Krueger, 1994). Lederman (1990) emphasised that researchers need to investigate the information and reasons behind people's preferences, and this is where focus groups are useful.

Researchers like Calder (1977), Carnaghi (1992), Brown (1999) and Krueger (1994) have argued that decision making is done by gaining information about an individual's experience, attitudes, and perceptions, and a focus group is a way of understanding how individuals think and feel. Focus groups can be the sole research tool for data collection, although this was not the case in this research. Krueger (1994) discussed the benefits of choosing focus groups, including the fact that they are more dynamic, refreshing, appealing, and reveal diverse experiences while still obtaining information. The results are easily understandable, the respondents generally relish the chance to participate and be a part of a discussion with their peers, and lastly holding a focus group creates the positive impression for the public that their ideas for change, improvements, and future needs are noted. However, the most common constraint of the focus group is that a smaller group of participants does not necessarily represent 
the views of the whole population where this is central to the topic of study. Ryan (1993) has termed this way of data collection a mere 'snapshot' and not capable of being generalised for a larger population. According to Krueger (1994), systematic analysis is necessary for a focus group, since this helps to examine and challenge researcher assumptions. This linear method of handling a focus group involves six things. These are sequencing the questions to be asked, capturing and handling the focus group data, verification with respondents, debriefing with an assistant moderator, data coding, and lastly sharing the report. However, Boyatzis (1998) and Braun \& Clark (2006) opted to call this process a tool of learning for research as they believe it is not tied to a particular epistemological or theoretical perspective.

\subsection{Description of Study Three}

The aim of Study Three was to analyse two separate focus groups, one for BE and one for NBE participants (Table 8.1). For both, participants were welcomed with a brief introduction and given the ground rules for the discussions. These included sharing honest opinions, respecting each other's views, speaking one at a time, keeping the information confidential, and using 'l' instead of 'You' statements.

Each focus group was in two stages. The first sought design information through a charrette in which participants were given six pictures of the different types of urban leftover space. According to Roggema (2014), a design charrette is a collaborative meeting in which a mixed group of participants sketch their ideas on the given sheet or paper. The main idea of this stage of Study Three was to explore possible creative concepts or strategies for the future of the leftover spaces used in the previous studies and then share these visions within the group meeting. The photographs of leftover spaces were taken from Study Two (see section 7.4.1). Participants worked in pairs to re-design the six types of unused spaces and sketch or note their ideas on the photographs (appendix 8.1). The aim was to improve the usability and visual appearance of each space, and respondents were told to do any changes they wanted as if they were the owner of the space. The use of photographs for representing natural and built environments is widely accepted, and there is a high correlation between preferences gained through photographic representations and building visits (Hershberger \& Cass, 1998). 
Stage Two investigated the design decisions developed in Stage One through focused group discussions. All participants were asked to compare the pictures one by one at the start with the new ideas for each leftover space. The questions for stage two were related to the visual enhancements, their functionality, and how the designed schemes might be implemented. The interview guide for this stage is in appendix 8.2.

\subsection{Study Recruitment and Sample}

The two focus group sessions of NBE and BE were conducted on 27th August 2018 and 10th September 2018, respectively, after ethics approval was obtained (25265). Both sessions were confidential, which meant the participants agreed not to communicate any details about the session, including family members and close friends. Consent was obtained to record their discussions with audio. Invitations were sent through email to those participants who indicated their willingness to take part in focus group discussions in the earlier Study Two. Other invitation emails were sent to Wellington City Council, architectural firms, and Registered Engineering Associates (REA). Invitation posters (appendix 9.2) were set up in a local café, Wellington City Library, and Victoria University's School of Architecture. These efforts were made to generate responses from people with diverse fields of knowledge.

Both studies took 60-70 minutes (20-30min for the design charrette and 30-40 min for the discussions). Although arrangements were made to have six people in each group, only five people participated in the NBE group and four in the BE group. King \& Horrocks (2010) state there are no set rules for group size since it depends upon the individuals who are a part and the aim of the study. Other researchers (Fern, 1982; Kitzinger, 1995; Krueger \& Casey, 2009) feel that four to six participants are sufficient for a focus group to be conducted. To investigate and understand diverse perspectives and validate the data, a study requires data collection from different types of individuals, groups, families, or communities (Carter et al., 2014). In the end, Study Three was conducted with participants who had not been part of Study Two, and although unplanned, this spread the individuals involved in the data collection.

The focus group discussions were held in the School of Architecture, Victoria University of Wellington. Two private rooms with little chance of interruption were booked for both sessions on different dates. In both, the chairs were organised in a circle around a table. Coffee and snacks from a separate table were served in both 
sessions. Apart from the researcher, an assistant was present to take notes and help with serving refreshments and other small tasks. The notes described the physical nonverbal cues. A NZ\$10 gift was given to each person who participated in this study. At the end of each session, the audio recording was transcribed by the researcher into Microsoft Word for analysis and checked with the help of the assistant. Figure 8.1 outlines the steps taken in Study Three.

Those who had previously undertaken Study Two and shown their willingness to participate further were sent a letter of invitation with a timeline link (doodle.com). Invitation posters were put up in various locations (see appendix 8.3).

When people responded positively, an information sheet was sent (appendix 3).

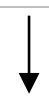

A convenient time and a location were set for the participants. Invitation reminders were sent out one week before the scheduled focus group times.

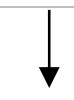

After arrival at the location and before the focus group, participant consent was gained (appendix 9.4). Afterwards, an introduction was given to the research project, and the focus group rules were explained, including the importance of confidentiality.

The two phases of the focus group (mini-design charrette and discussions) were briefly explained. Participants were asked to work in pairs.

Before recording, participants were asked to introduce themselves and describe their work role.

The design charrette (phase 1) began with the distribution of the photographs of leftover spaces. The voice recorder was turned on for the focus group discussion (phase 2). The focus groups lasted 60-75 minutes.

Participants were debriefed and asked if there was anything they wanted to add. 
Participants were given NZ $\$ 10$ cash before they left.

\section{Figure 8.1 Procedures taken in Study Three}

\subsubsection{Sample demographics}

The demographic information given by the participants was analysed (Table 8.1) to see if the diversity in education and age could be linked to different opinions. The invited participants had different study background levels that included undergraduate, post-graduate and doctoral. The age group of participants represented a broad section of society, especially for the non-built environment participants.

\begin{tabular}{|l|l|l|l|l|}
\hline $\begin{array}{l}\text { Focus group } \\
\text { Session and } \\
\text { their Group } \\
\text { codes }\end{array}$ & $\begin{array}{l}\text { Individual } \\
\text { Codes }\end{array}$ & Gender & Age Group & $\begin{array}{l}\text { Work } \\
\text { area/Profession }\end{array}$ \\
\hline NBE & A1 & Male & $26-35$ & $\begin{array}{l}\text { Agriculture } \\
\text { (professional) }\end{array}$ \\
\hline NBE & A2 & Female & $56-65$ & $\begin{array}{l}\text { Accountant } \\
\text { (retired) }\end{array}$ \\
\hline NBE & B1 & Male & $46-55$ & $\begin{array}{l}\text { Computer Science } \\
\text { (professional) }\end{array}$ \\
\hline NBE & B2 & Female & $36-45$ & $\begin{array}{l}\text { Education \& } \\
\text { Management (PhD }\end{array}$ \\
\hline NBE & B3 & Female & $18-25$ & $\begin{array}{l}\text { Nutritional Science } \\
\text { (professional) }\end{array}$ \\
\hline BE & X1 & Male & $26-35$ & $\begin{array}{l}\text { Digital Architecture } \\
\text { (Master's student) }\end{array}$ \\
\hline BE & X2 & Female & $26-35$ & $\begin{array}{l}\text { Landscape } \\
\text { Architect } \\
\text { (Student + } \\
\text { professional) }\end{array}$ \\
\hline & & & & \\
\hline
\end{tabular}




\begin{tabular}{|l|l|l|l|l|}
\hline $\boldsymbol{B E}$ & Y1 & Male & $18-25$ & $\begin{array}{l}\text { Architect (Master's } \\
\text { student) }\end{array}$ \\
\hline $\boldsymbol{B E}$ & Y2 & Male & $36-45$ & $\begin{array}{l}\text { Civil Engineer } \\
\text { (PhD student) }\end{array}$ \\
\hline
\end{tabular}

Table 8.1 Demographic details of the Study Three sample

\subsection{Analysis}

The focus group sessions involved two stages, as discussed below:

\subsubsection{Stage 1: Mini-design Charrette}

The design charrette is a crucial method for exchanging ideas and can be used from the pre-design phase through to the realisation of a project. The charette aims to resolve complex problems and generate a buildable scheme involving the public (Lindsey, Todd, Hayter, \& Ellis, 2009). The primary intent of Study Three mini design charrette at the beginning of each focus group was to engage the participants and extract possible design solutions through a collaborative decision-making process. Participants were asked to provide input in the form of design concepts that would improve the usability and aesthetics of the space. The design teams worked in pairs (with one group of three in the NBE group). This brainstorming session began by handing over six different photographs to the participants in a sequence, this being from underneath a building, in front of a building, at the back of a building, space enclosed by buildings on three sides, space enclosed by buildings on two sides, and lastly the rooftop of a building. The respondents spent approximately five to seven minutes on each photograph and created realistic design schemes. It was noted that participants spent more time in understanding and elaborating on their diverse opinions for the first two photographs but gave little time to the other four pictures.

All the participants liked the idea of sketching with different colours and felt excited about it (see figure 8.2). The design decisions depicted through sketches and brainstorming notes written on the photographs suggested that participants preferred to add to or subtract one or more attributes relevant to the different leftover spaces. Non-verbal observational notes were made for both stages. Conversation analysis is a way of understanding a participant's engagement and responses in social interaction (Sacks, Schegloff, \& Jefferson, 1974). Gorden (1980) concluded that nonverbal communication data compromises kinesics (i.e. behaviour related to body 
movements), proxemics (i.e. behaviour related to research and participant relationships), chronemics (i.e. behaviour related to transient discourse such as shyness, nervousness, quietness), and paralinguistic (i.e. behaviour related to vocal articulation). Heritage (1984) argued for the importance of participant behaviour during a conversation, and that paying attention to body movements, nervousness, pauses, and volume of the voice can enhance the interpretation. Furthermore, it helps to acknowledge the most or least stressed ideas within a conversation that relates to the question being asked (Krueger, 1994; Braun \& Clarke, 2006). Non-verbal communication, such as body language is a possible way to extract essential meanings (Graham \& Argyle, 1975; Holler, Shovelton, \& Beattie, 2009). The nonverbal gestures of participants during both sessions suggested their experiences were constructive. All participants felt comfortable though it became evident that $\mathrm{BE}$ participants were more enthusiastic and confident than NBE at presenting their concepts.
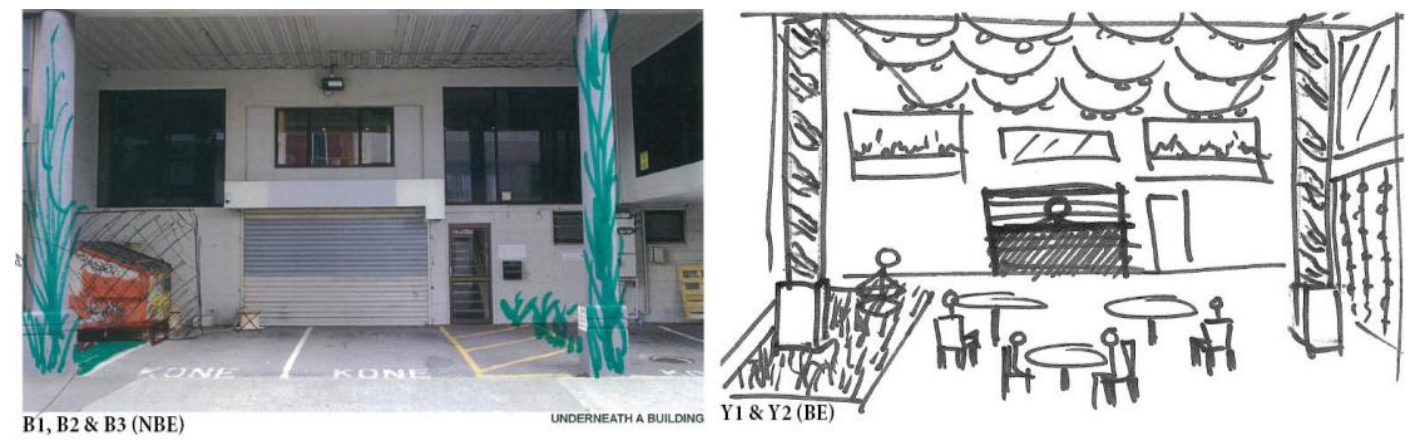

Figure 8.2 Images sketched and doodled by focus group participants

\subsubsection{Stage 2: Design discussions}

Researchers Riessman (1993), Lapadat \& Lindsay (1999) and Bird (2005) claims the process of transcription is a crucial phase in the interpretation of a dataset as this is where a researcher becomes familiar with the information and interpretations are created. The focus group transcription was analysed by assigning labels to the most occurring themes in the data. This process is known as coding. A useful technique for retrieving a piece of information so it can be assembled differently from the original version is known as 'Axial Coding' (Kruger, 1994). Krueger (1994) and Braun \& Clark (2006) argue that 'thematic coding' is the core skill in qualitative analysis. It is a technique for identifying, analysing and reporting different or reoccurring themes or patterns within data where the perceptions of the participants are mostly unknown 
(Braun \& Clarke, 2006). Furthermore, it is essential to note that coding in any study should be free from any prior framework or personal interest. A theme is a core idea that identifies something critical within a dataset as being related to a question in the study (Krueger, 1994). Participant ideas become the core of interpretation (Braun \& Clarke, 2006). When having an overview of the structure of a study, Boyatzis (1998) and Braun \& Clarke (2006) identified two theme levels. The first was the semantic or explicit level and the second the interpretative level. The semantic level calls for viewing the results explicitly and not taking into consideration anything other than what the participant has remarked on or suggested. Effectively this is only looking at the surface level of the data, and the focus is not yet on interpreting or explaining any outcomes. In contrast, the interpretative level calls for the researcher to dig a little deeper into the 'why' and 'how' of the ideas. The researcher then conceptualises the underlying reasons behind the remarks of participants and makes assumptions accordingly.

In Study Three, the researcher first transcribed the data, reading and then re-reading it. Coding the features of the data was the second step. The third was the search for recurring themes and assembling the codes. The frequency and length of regular codes were identified (Lewis et al., 2007). In the following stage, a thematic delineation was drafted. These refined themes were then named in the fifth stage, followed by the final step of composing a thematic report. Typically, the thematic analysis allows a researcher to catalogue a study's results and investigate the official data and assess its intrinsic value to the research topic. However, Boyatzis (1998) and Attride-Stirling (2001) have argued that the limitation of thematic analysis lies within its descriptions and the process. As such, a conceptual idea should be categorised into themes and sub-themes to suggest an overall theme rather than attempting any quantification of themes.

\subsubsection{Built Environment (BE) and Non-Built Environment (NBE) Participants}

The difference in opinions between NBE and BE respondents was tested by asking the same four questions for the six types of leftover space. The only question asked in the mini-design charrette was "If you were to become the owner of these different 
urban leftover spaces shown in the pictures, what things would you want to add or subtract in order to improve or increase the usability and visual aesthetics of these spaces?" Following the mini-design charrette, the participants were asked to present their design schemes to each other and then compare them. In stage 2, the three questions given to participants were;

1) How could such spaces enhance usability and visual aesthetics?

2) Would it be easy to apply these suggested modifications or to implement the designs?

3) What are the most critical aspects that the participants can draw out of the discussion, which could improve the urban leftover spaces?

The overall preferences drawn out of the focus group discussions for improving the leftover spaces were similar for both groups. These were about providing more vegetation, changing the surface material, installing screens, removing the car parking, introducing a café or restaurant, creating seating space, removing the boundary walls, improving cleanliness, and installing murals/sculptures. The suggestions varied with the type of urban leftover space, but an overall preference was towards providing vegetation and changing the surface material. These preferences are reflected in the bubble diagrams (figures 8.4.3.1 - 8.4.3.6). These were developed after doing the thematic analysis to find the most recurring attributes.

\subsubsection{Space underneath a building}

Overall all the focus group participants wanted to see more vegetation in the space underneath a building (figure 8.5). The second most preferred aspect was changing the surface materials, followed by installing new screens to hide the garbage bins. Other attributes that appeared essential to participants but were less significant concerned creating seating space and achieving more cleanliness. The NBE participant group thought having to redesign the space was demanding, and $\mathbf{A} 1$ stated, "it is quite a sort of challenging...probably the most difficult one." B1 agreed to assert, "yes, it is the most difficult one." However, the BE participants saw a design opportunity in this space, and one claimed, "I love the fact that it frames the middle shed, not the garage." BE participant, X1 agreed and argued, "what appealed to me 
is the double height space." The most preferred design attributes, as suggested by NBE and BE participants, are mentioned in order below.

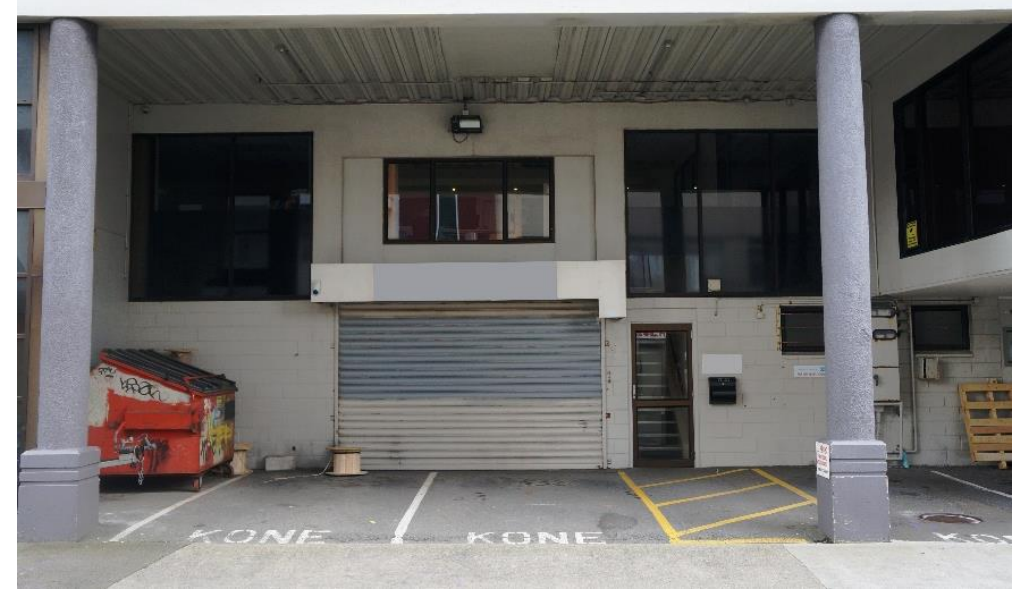

Figure 8.3 Image shown to focus group participants
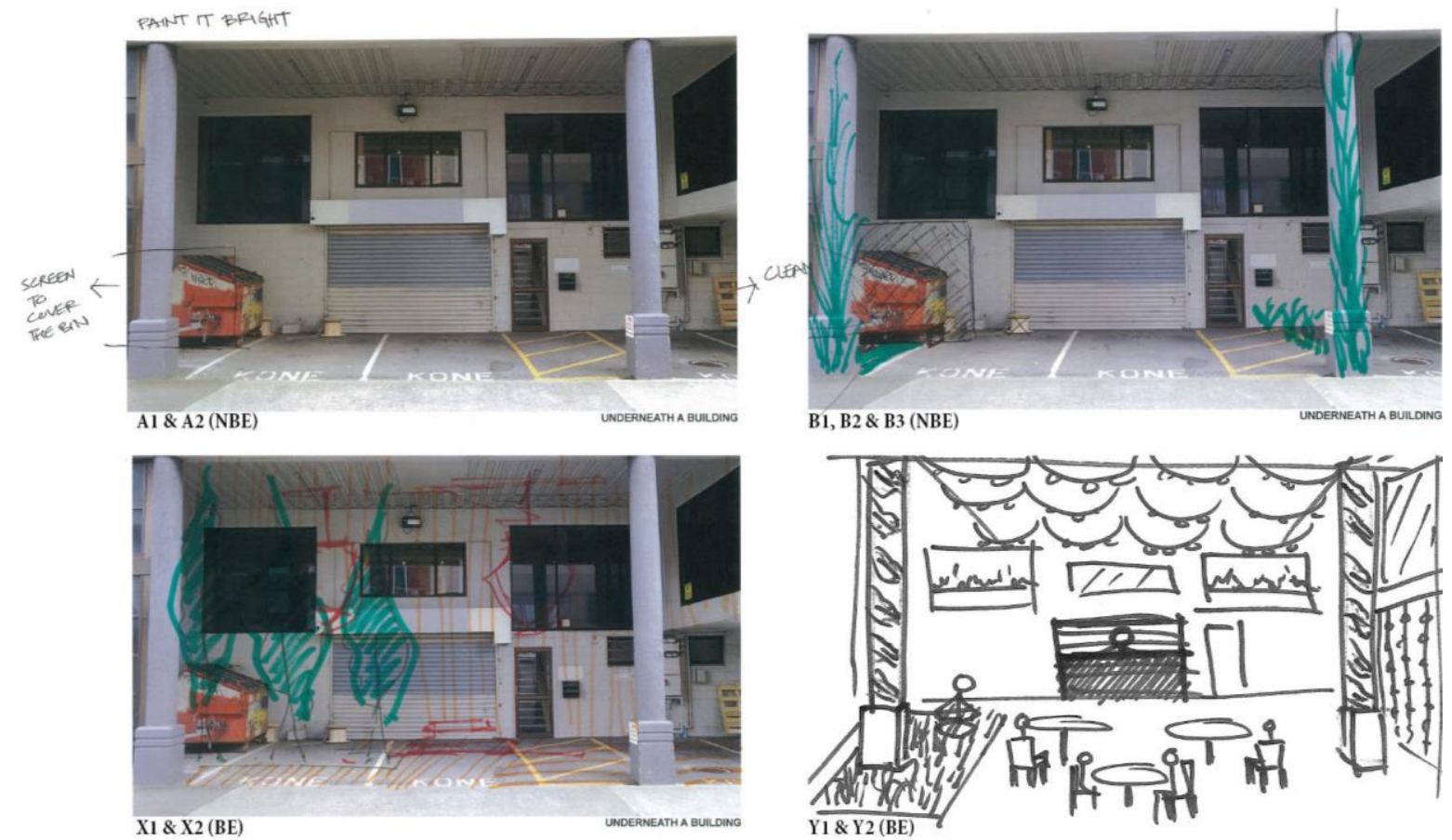

Figure 8.4 Sketches drawn by focus group participants 


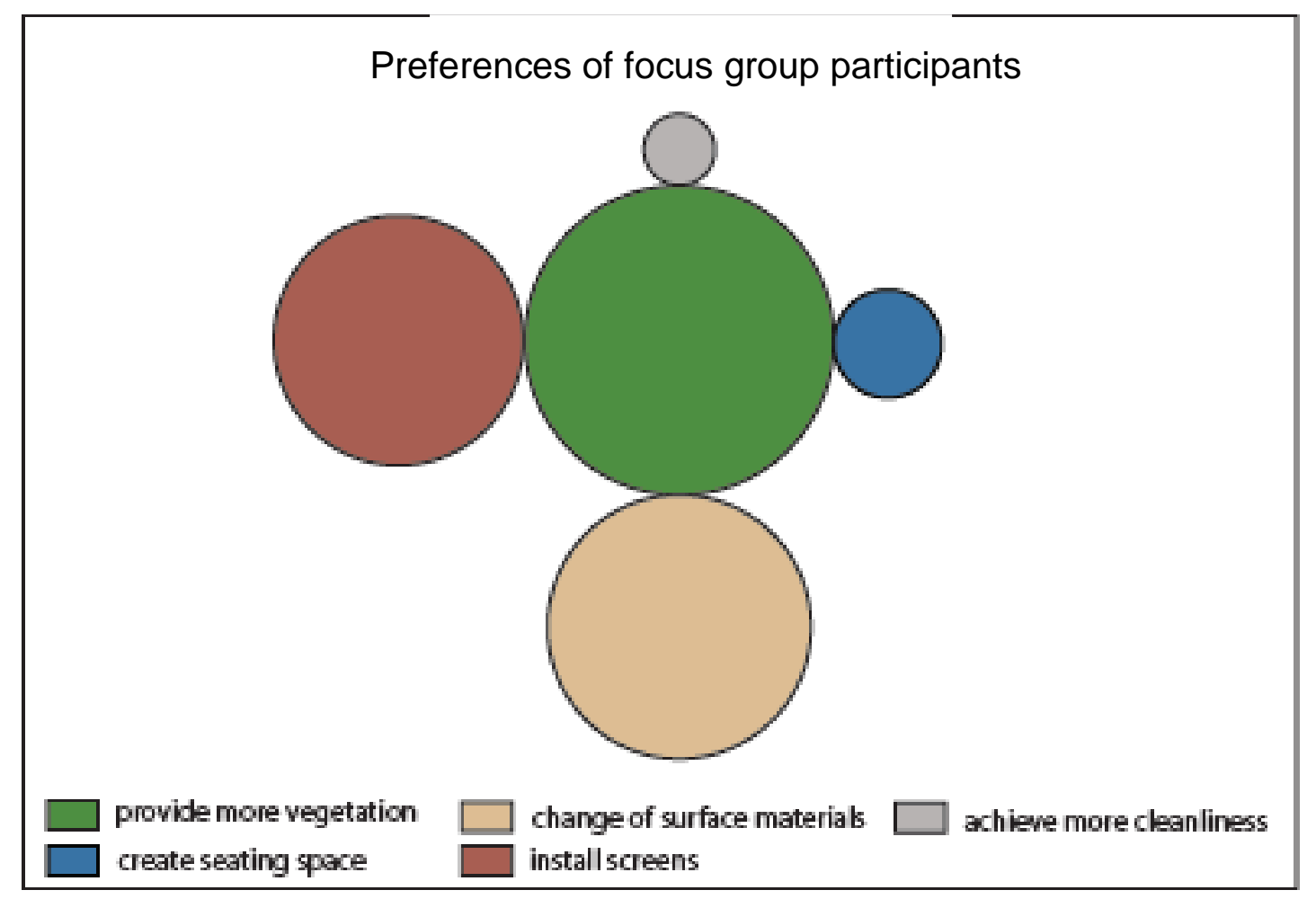

Figure 8.5 Design preferences of focus group participants

\subsection{Provide more vegetation}

Both the groups were unanimous about providing more vegetation. BE participants laid more stress on the importance of vegetation in the space, claiming it would make it feel more natural (figure 8.6). However, the NBE group emphasised that vegetation can have a significant visual impact and make the space more inviting. B3 felt that "putting more plants looks pleasing to an eye and [also] attracting some sunlight." X1 highlighted the significance of providing more vegetation by suggesting, "my intervention calls for vegetation." Furthermore, the participant continued by emphasising, "vegetation to improve [space] maybe if trees or some planters are added, to make use of double height space." X2 claimed, "[the concepts are] quite similar regarding vegetation...adding in vegetation to increase that nice feeling because everyone loves plants, don't they?" Y1 agreed and stated, "add creeping plants around the heightened column...then accentuate one of the columns [to make a] scenic space." 


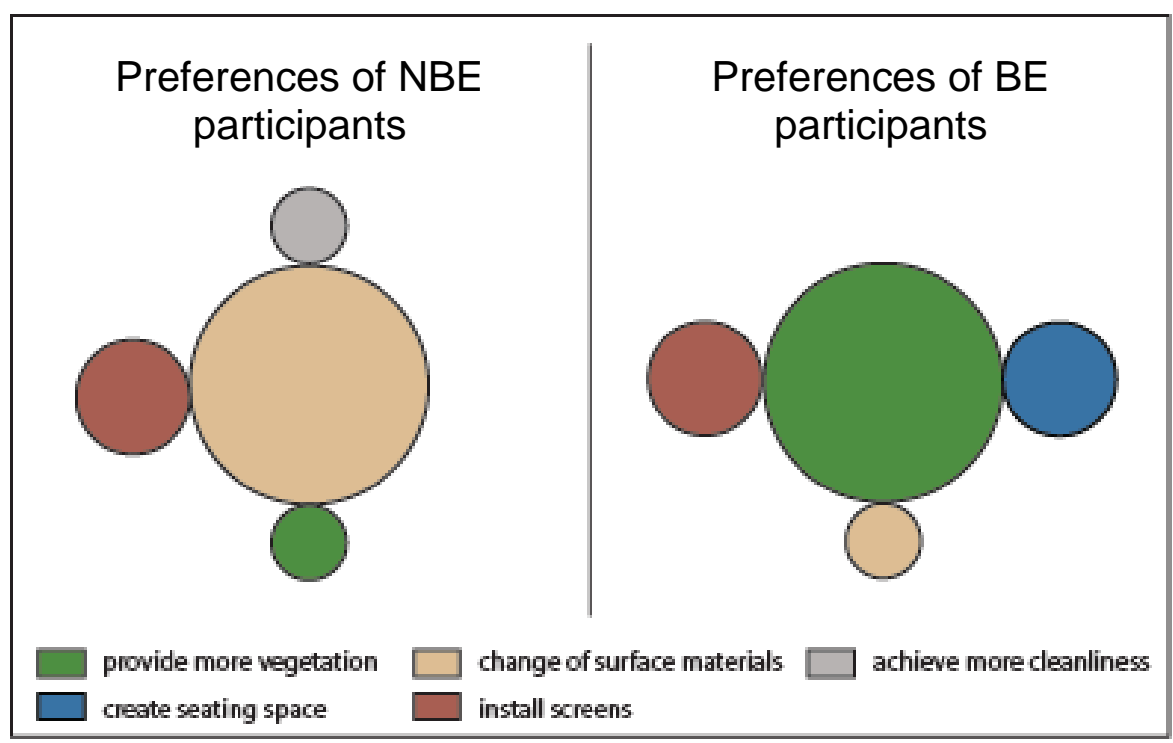

Figure 8.6 Preferences of NBE and BE participants for the space underneath a building

\subsection{Change of surface material}

Surprisingly, NBE participants were more enthusiastic about the idea of changing the surface material than BE participants. The focus was on brightening up the spaces by the combinations of colours and materials. Both BE and NBE respondents recommended painting surfaces white and adding timber. NBE linked the idea of changing the surface material with its visual impact on people, and $\mathbf{A} 2$ claimed, "doing some painting...maybe with kind of bright colours that brightens up the whole area [and creates] more pleasant visual effect." B3 agreed and claimed that "painting all of this white, you know it looks nice to eyes... maybe repainting the pillars a little brighter colour." The BE group emphasised the dark and gloomy aspect of the space and X2 asserted, "the tones of the space are quite cold...using more materials such as timber could make it feel a lot warmer."

\subsection{Install more screens}

Both NBE and BE groups highlighted the significance of installing more screens in the space underneath a building as this would noticeably reduce the unpleasant aspects by hiding the garbage bins. However, NBE participants placed more stress on installing screens in the space underneath a building than BE. A2 suggested to "improve the street visual [with] some screens [and] to cover that horrible dumper." B3 agreed by suggesting "putting it [rubbish bin] inside or in the building will make space 
for the people." The BE group also stressed the importance of hiding the garbage bins with $\mathbf{X} 2$ exclaiming that "the main thing, I have noticed is that there is rubbish everywhere, concealing them but still making them accessible would be nice." This suggestion was validated by $\mathbf{X} \mathbf{1}$ who emphasised the need to make space appear less busy by mentioning that "there is too much happening at the moment, too much irregularity and [it] needs to be simplified." Y1 had similar views for proposing an "additional array of changes to simplify the visual appearance". Here, the two participants stressed the need to "screen the dumper" and "relocate and cover" the rubbish bin.

\subsection{Achieve more cleanliness}

It was interesting to note that the only additional suggestion from the NBE participants was to achieve more cleanliness in the space. The "horrible dumper thing" and "little rubbish thing" were expressions used by A2 and B3, respectively. NBE participants laid more stress on the maintenance of the space, with $\mathbf{A} 2$ suggesting, "to clean up or cover up." B3 acknowledged the idea of "cleanliness of space" and also "focused upon getting rid of the little rubbish thing."

\subsection{Create seating space}

BE participants highlighted the importance of creating seating space for the public to make them feel a part of the setting. $\mathbf{X} \mathbf{1}$ argued in favour of seating space to attract people and claimed, "obviously creating that space here to attract...someone can sit down...stop by and have a break." Furthermore, X2 affirmed the idea and said, "[providing] some green seating space [for] people [who] are passing for cigarette breaks, a space for courtesy of community so that people do not have to stand out in the cold environment." Y2 agreed and claimed, "it [the proposed seating] is looking terrific."

\subsubsection{Space in front of a building}

The prevalent feeling for changes to space in front of a building was removing the boundary walls to make it look more visually inviting or welcoming (figure 8.9). No significant difference was found for this preference between BE and NBE participants. This design preference was followed in order by providing vegetation, changing the surface materials, and removal of the parking space. 
Chapter 8 - STUDY THREE: FOCUS GROUP DISCUSSION

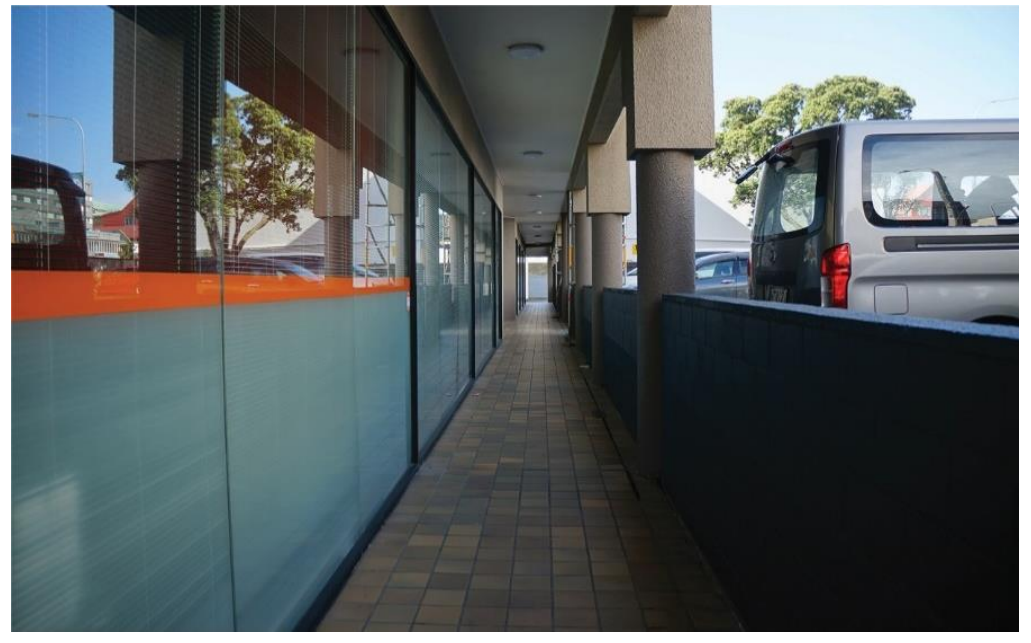

Figure 8.7 Image shown to focus group participants

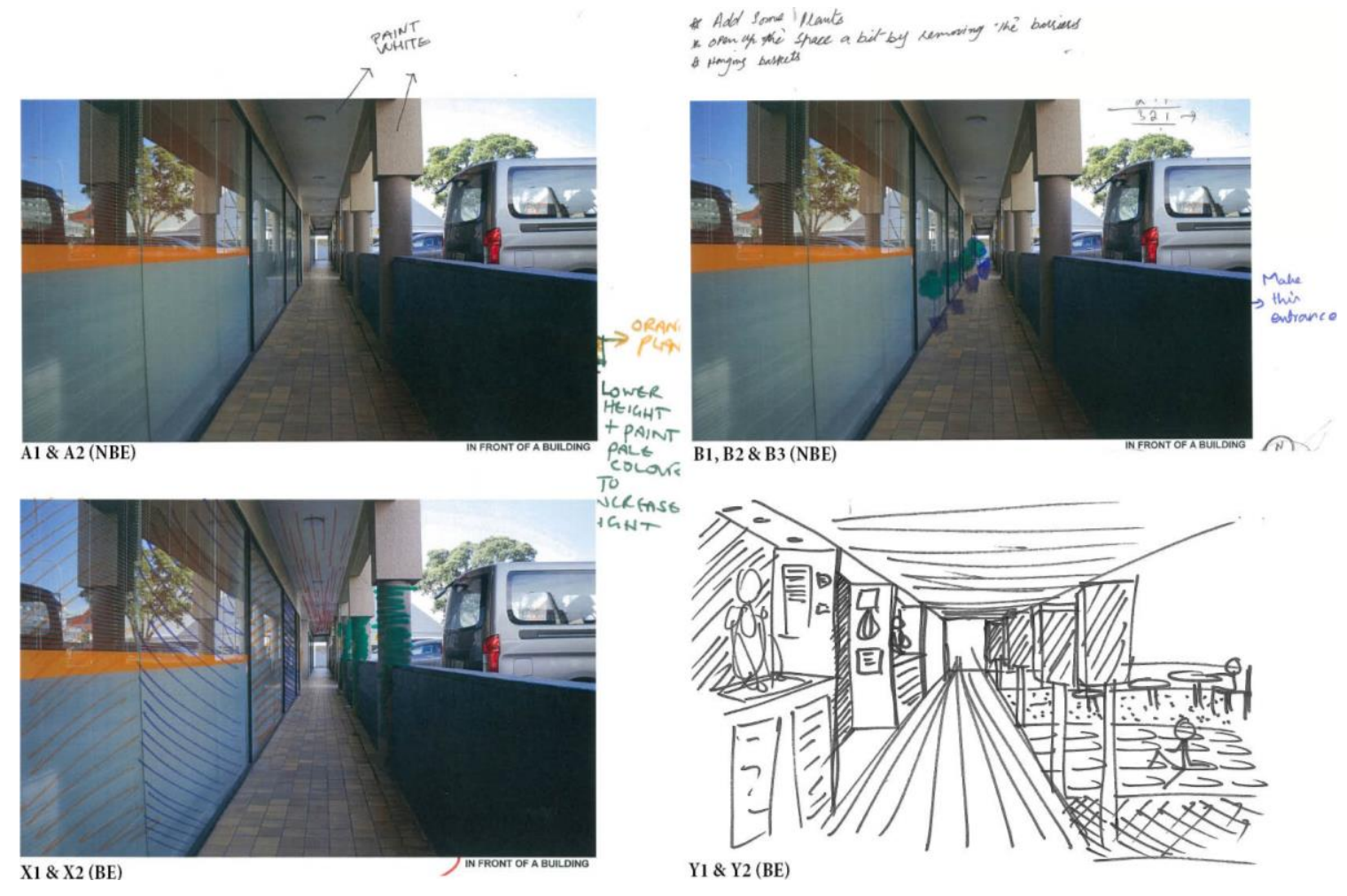

Figure 8.8 Sketches drawn by focus group participants 


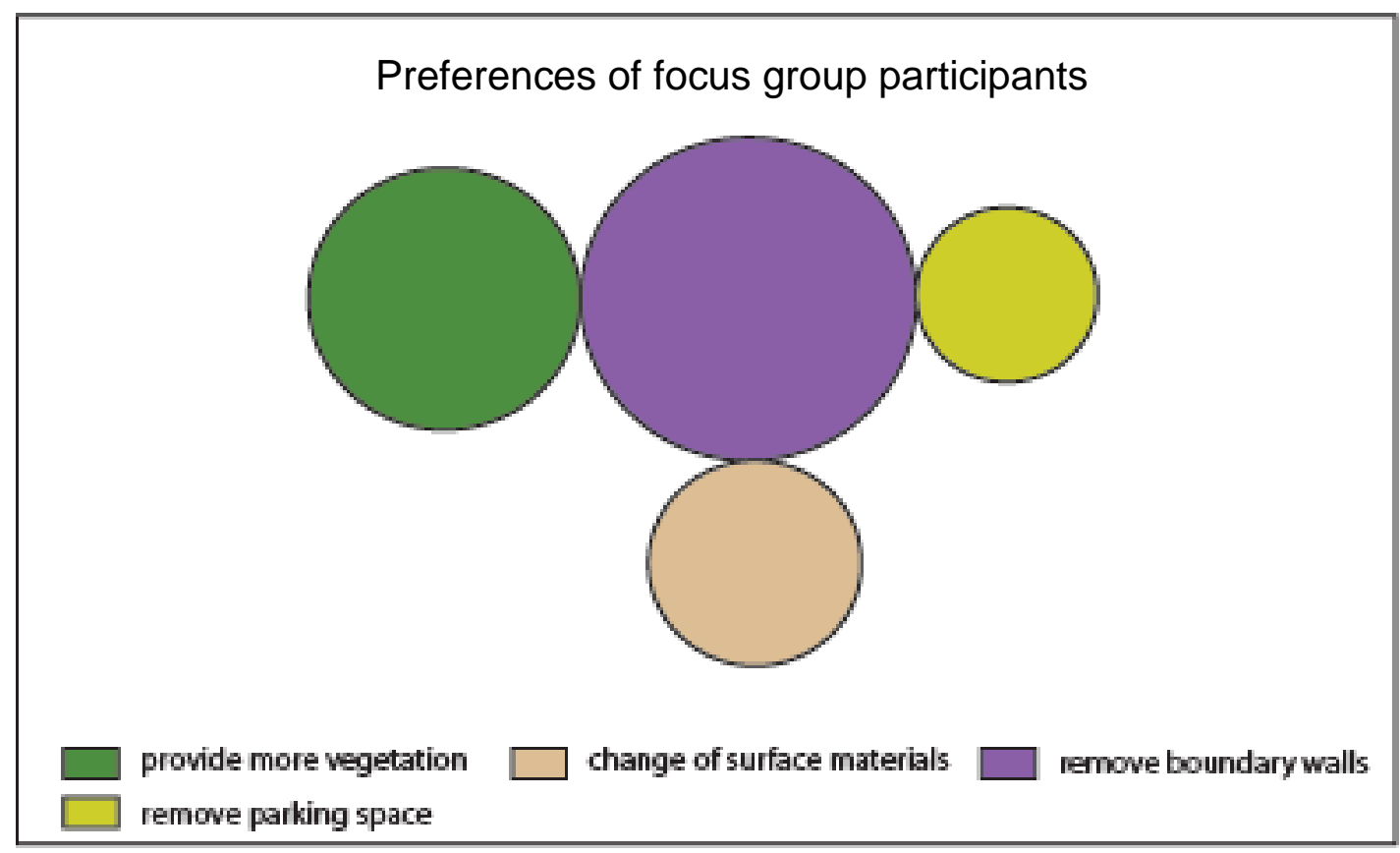

Figure 8.9 Design preferences of focus group participants

\subsection{Removing boundary walls}

The high preference for the idea of removing the boundary wall was examined by BE and NBE participant groups. Both groups proposed physical changes to expand the space. Overall, there was a general similarity of ideas (figure 8.10), although reducing the height of the boundary wall was also proposed. NBE participants were more realistic when it came to taking out the boundary wall. As A1 stressed, "lowering the height or take it out because of the safety [issue] as you know, these cars would come back." B2 emphasised how the wall in front of the entrance doors acted as a barrier and suggested, "taking down this [boundary] wall" on several occasions. Furthermore, B2 argued that the building "does not have a nice entrance opening out into space". BE preferences were quite similar to NBE for removing the boundary walls. X1 insisted on "removing these [boundary] walls. They are quite unnecessary... it does not make sense, but then maybe the first step is the need of removing the walls." X2 agreed and stated, "get rid of the wall. It is quite unnecessary". Y1 affirmed the views of X2 and agreed, "with everyone's view regarding removing those walls." Y2 also liked the idea of "reducing the height of the wall." 


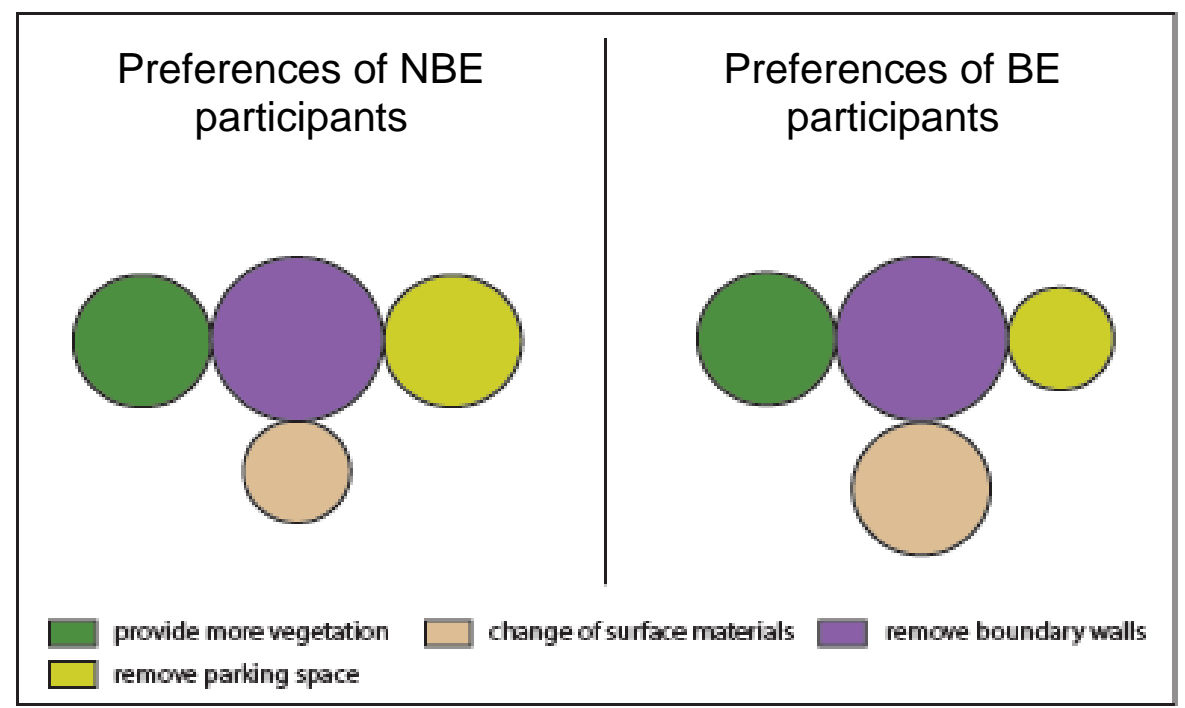

Figure 8.10 Preference of NBE and BE participants for the space in front of a building

\subsection{Provide more vegetation}

The analysis revealed that the second highest preference was for providing more vegetation in front of the building. NBE participants were unanimous about beautifying the space through vegetation. A1 discussed the importance of "placing the plants [so they are] reflected in the glass", and A2 wanted "to place bright orange planters." B1 argued about, "putting some plants along here [along the wall] hang some hanging the baskets from these pillars, so try to lift it a bit." BE also discussed ways to improve the aesthetics of the space by providing more vegetation. $\mathbf{X} \mathbf{1}$ proposed "growing some planters until this point [where the columns end]." and $\mathbf{X} 2$ added that "it would be great to place a little garden [that] swells and acts a sort of barrier." Y2 raised the importance of vegetation by "making it grassier."

\subsection{Change of surface material}

Opinions of BE and NBE participants did not differ from each other about changing the surface material. However, BE participants paid much more attention to the details of spaces and one suggested that "it might be good to design the glass façade in different ways." NBE participants discussed the importance of brightening up space with light and bright colours. A1 wanted to "add more bright colours on the ceiling and columns. Also, mimicking the bright orange colour." A2 suggested, "paint everything white to brighten up space". On several occasions, B1 also emphasised, "brightening up particular parts of the area." BE participants stressed changing several surface 
materials in the space. X1 wanted to, "do something with the ceiling" and on another occasion discussed changing, "[the] glass façade that is covered with blinds behind". $\mathbf{X} \mathbf{2}$ argued for, "replacing the materials with more creative design and give it a more timber surface [for] warmth." Y1 agreed to say, "keeping some of the actual textures...create a beautiful flow which hides the column."

\subsection{Remove parking space}

Another mutually-agreed design option was about removing the parking space, although analysis revealed that NBE participants laid more stress on this compared to BE participants. The NBE group wanted to make the entrance more spacious by removing the car park. A1 suggested that "it would be nice if we can move the car parking" and A2 wanted, "not to have parking immediately outside." B1 argued about removing the parking on several occasions and agreed with A1 by wanting, "to remove parking space immediately in front of that wall." B2 also proposed, "to take out one or two parking spaces." BE participants felt removing the car park would make the space more welcoming. $\mathbf{X} \mathbf{1}$ stated that "if you do not want to remove the boundary walls then remove the car park" and $\mathbf{X} 2$ agreed with $\mathbf{X} 1$ by suggesting to have, "few car parks [that] would open this space a lot more, also will make it warmer and more welcoming." However, Y2 wanted to "have little stores between the car park and the office space."

\subsubsection{Space at the back of a building}

The third figure was an image of space at the back of a building. Two common themes that emerged from the data concerned providing more vegetation and changing the surface material, with the former the highest rated idea (figure 8.13). Furthermore, NBE participants also suggested installing screens and achieving more cleanliness. Contrary to expectation, BE participants gave two different suggestions, these being removing the parking space and creating seating space. 


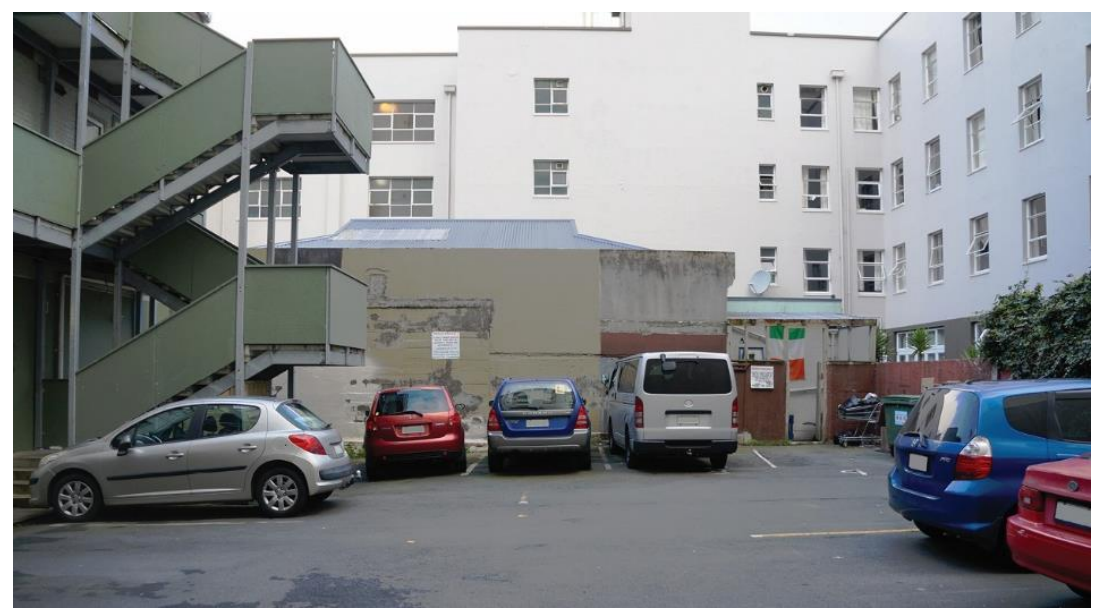

Figure 8.11 Image shown to focus group participants
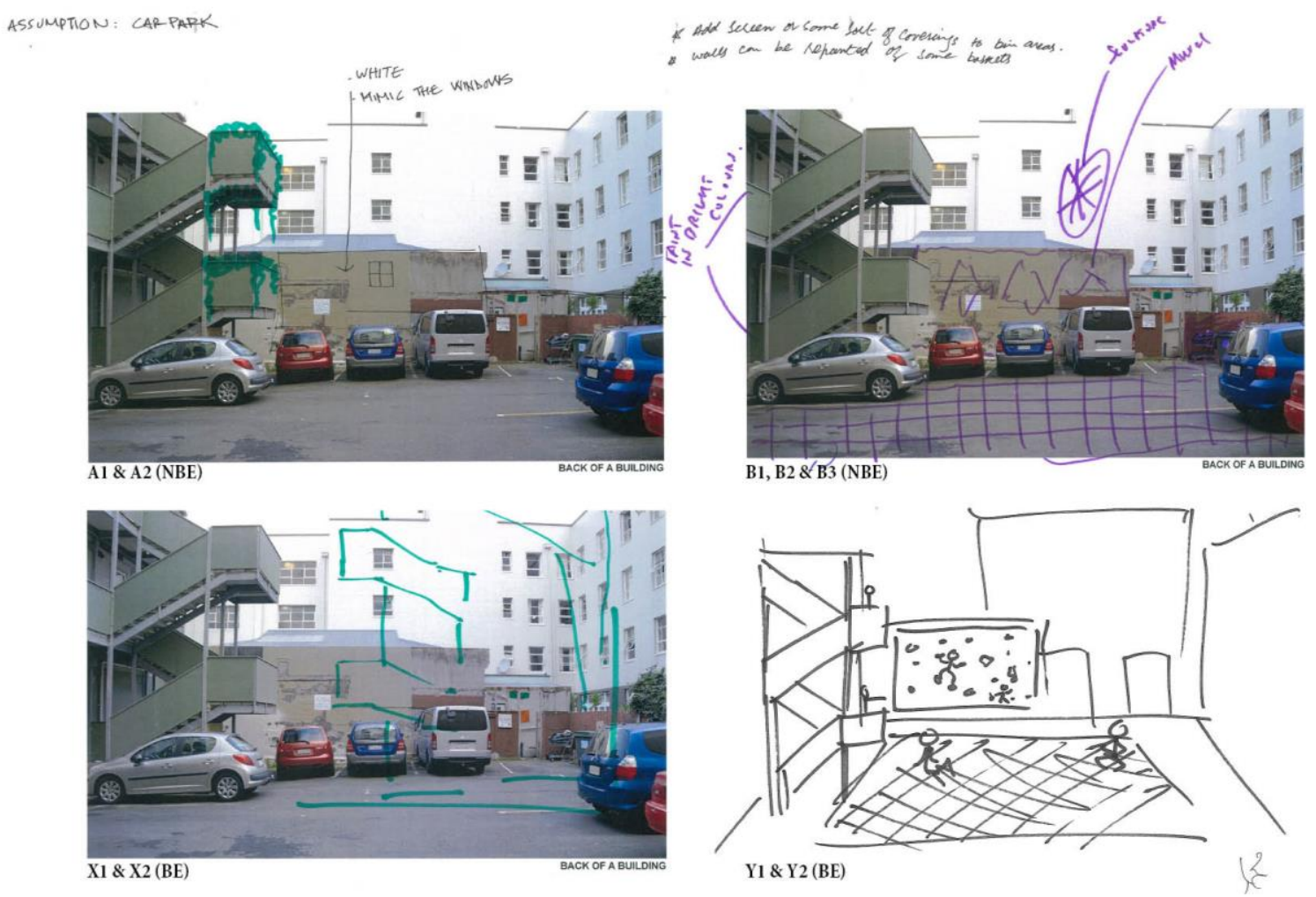

Figure 8.12 Sketches drawn by focus group participants 


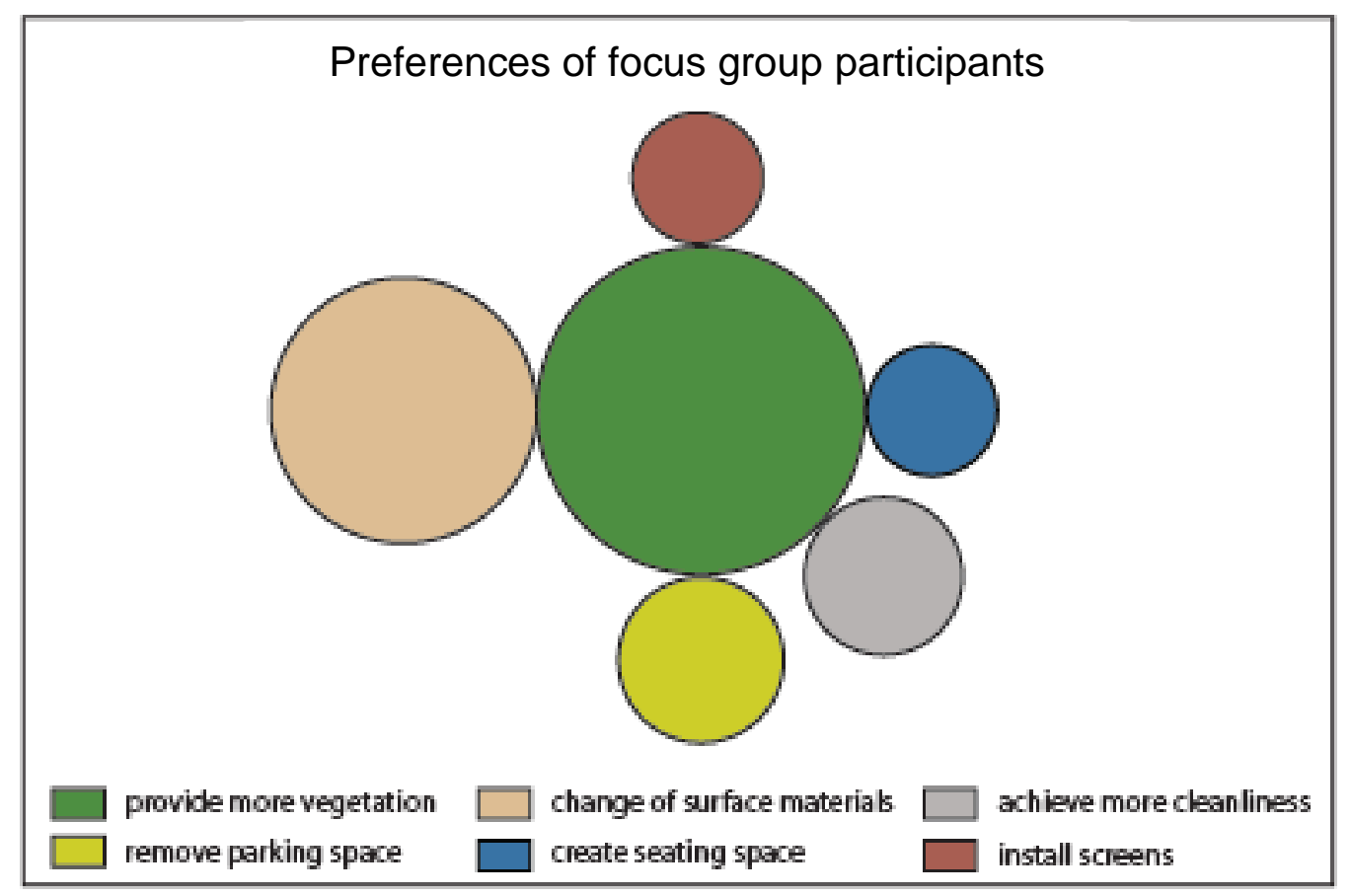

Figure 8.13 Design preferences of focus group participants

\subsection{Provide more vegetation}

The prevalent idea from both NBE and BE groups was to create a natural ambience by providing more vegetation in the space (figure 8.14). NBE participants thought this would improve visual aesthetics. A1 wanted vertical planting on the façade of the staircase, stating that the "staircase as the best feature of this area...should is turned into green [vegetation]." B2 claimed that making "some changes related to landscape...should give a good impression." BE participants presented solutions that created a natural environment and increased the biodiversity of the area. X2 argued that "having something green on the other wall [and] creating a space for the community with grass and trees." Y1 also shared the same view and suggested: "to place some grass in the area." While Y2 was also in favour of creating a "natural environment." 


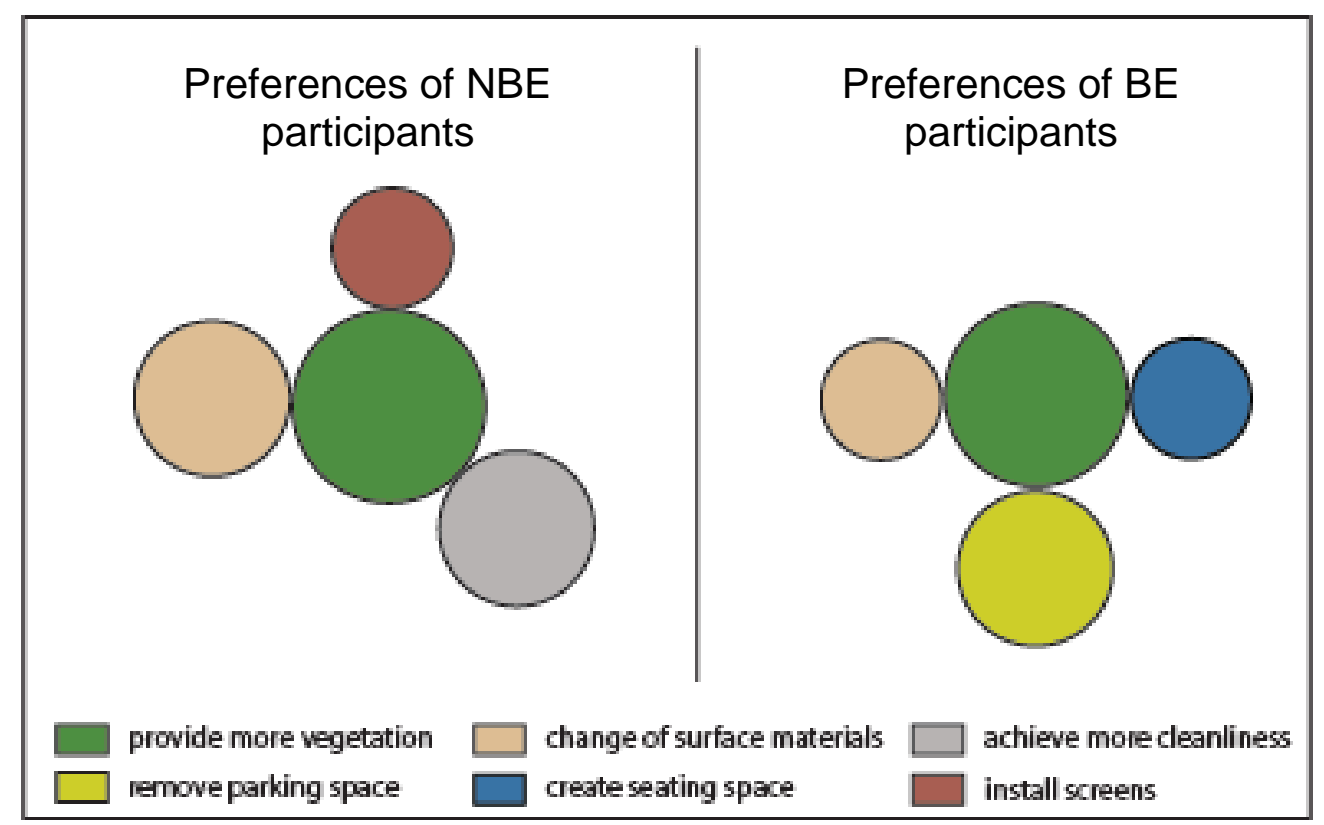

Figure 8.14 Preferences of NBE and BE participants for space at the back of a building

\subsection{Change surface material}

The discussions revealed some discrepancy between NBE and BE participants. NBE participants emphasised painting the wall white, while BE participants wanted to change the design of the space. A1 wanted "to paint the ugliest building in the picture [the olive coloured building at the back] with white colour or by adding fake windows", and A2 agreed with these changes. B2 focused on "painting [the wall] white and [putting] some sorts of brick pathways that would give a good impression." However, B3 wished, "to repaint the surfaces in flashy sort of colours." Rather than changing the surface material, $\mathbf{X} \mathbf{1}$ wanted "to see a building [in the space]" whereas $\mathbf{X} \mathbf{2}$ desired, "to add textures to the big building behind." $\mathbf{Y} 1$ fancied, "to put a rock-climbing wall to make it an engaging space instead of a blank wall."

\subsection{Remove parking space}

Another discernible pattern emerged through the idea of removing the parking spaces as proposed by both NBE and BE participants, although the latter was more enthusiastic about this. A1 suggested that "instead of seeing parking, people can use the whole space." All NBE participants agreed with this idea. BE participants asserted that removal of the parking space and finding another use would benefit the public. $\mathbf{X} 1$ suggested that "removing the parking space, make a building there." While X2 wanted, 
"removal [of] the parking." Y1 proposed that "removing of the car park and create a little private space for the people living in the building." However, Y2 proposed, "not to remove car parking space entirely since a car park is a necessity in the city."

\subsection{Achieve more cleanliness and Install more screens}

The discussion revealed that achieving more cleanliness and installing screens was the critical design attribute for NBE participants. B2 said to make the space, "neat, clean and tidy.

Furthermore, B2 argued for, "putting some screens in this corner that hide or camouflage this bin area, which is an issue." A1 agreed with cleaning up space in, "pretty much the same way...covering this bin area."

\subsection{Create seating space}

The analysis demonstrated that BE participants were more in favour of creating a seating space than NBE participants. X2 wanted, "to create seating space...for a lunch break, maybe, sit in a nice setting along with trees." Whereas, Y1 asserted that "the staircase is such a beautiful object to have. So, keeping that as a viewing and seating platform."

\subsubsection{Space enclosed by buildings on three sides}

The most significant result to emerge from the discussions was to provide more vegetation in the space enclosed by buildings on three sides (figure 8.17). The second most preferred design attribute was changing the surface materials, and the third was installing murals/sculptures. Further comparison between NBE and BE participant preferences revealed subtle differences. The NBE group showed more preference for changing the surface material, whereas the BE group wanted to see more vegetation. 


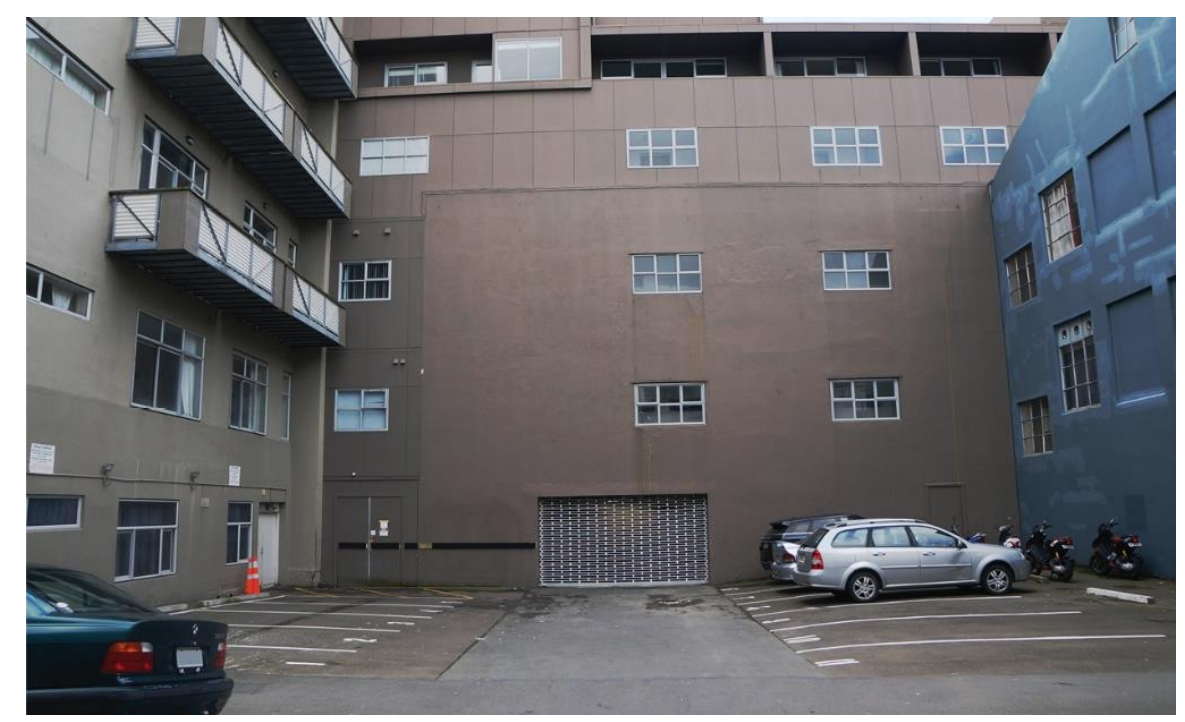

Figure 8.15 Image shown to focus group participants
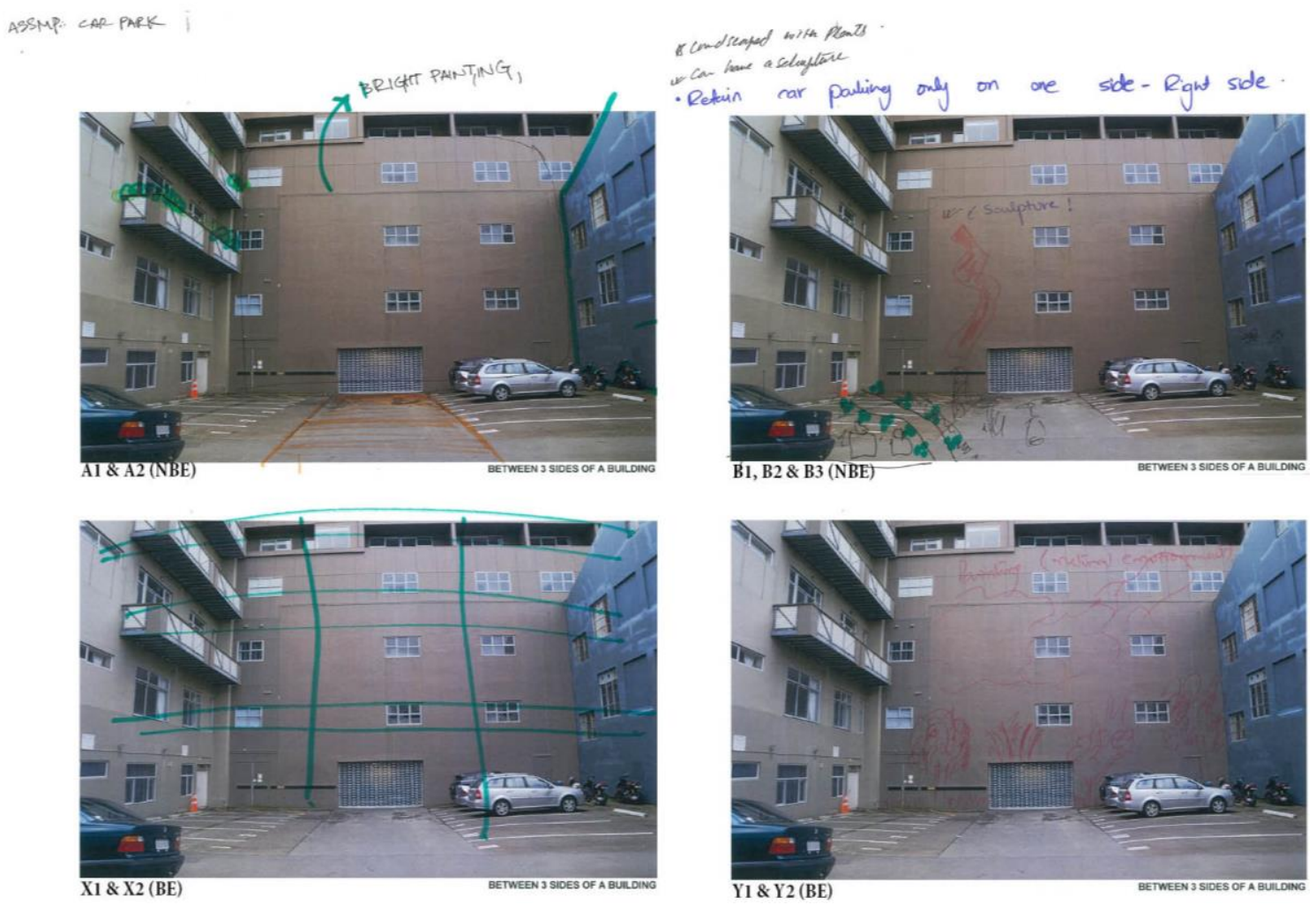

Figure 8.16 Sketches drawn by focus group participants 


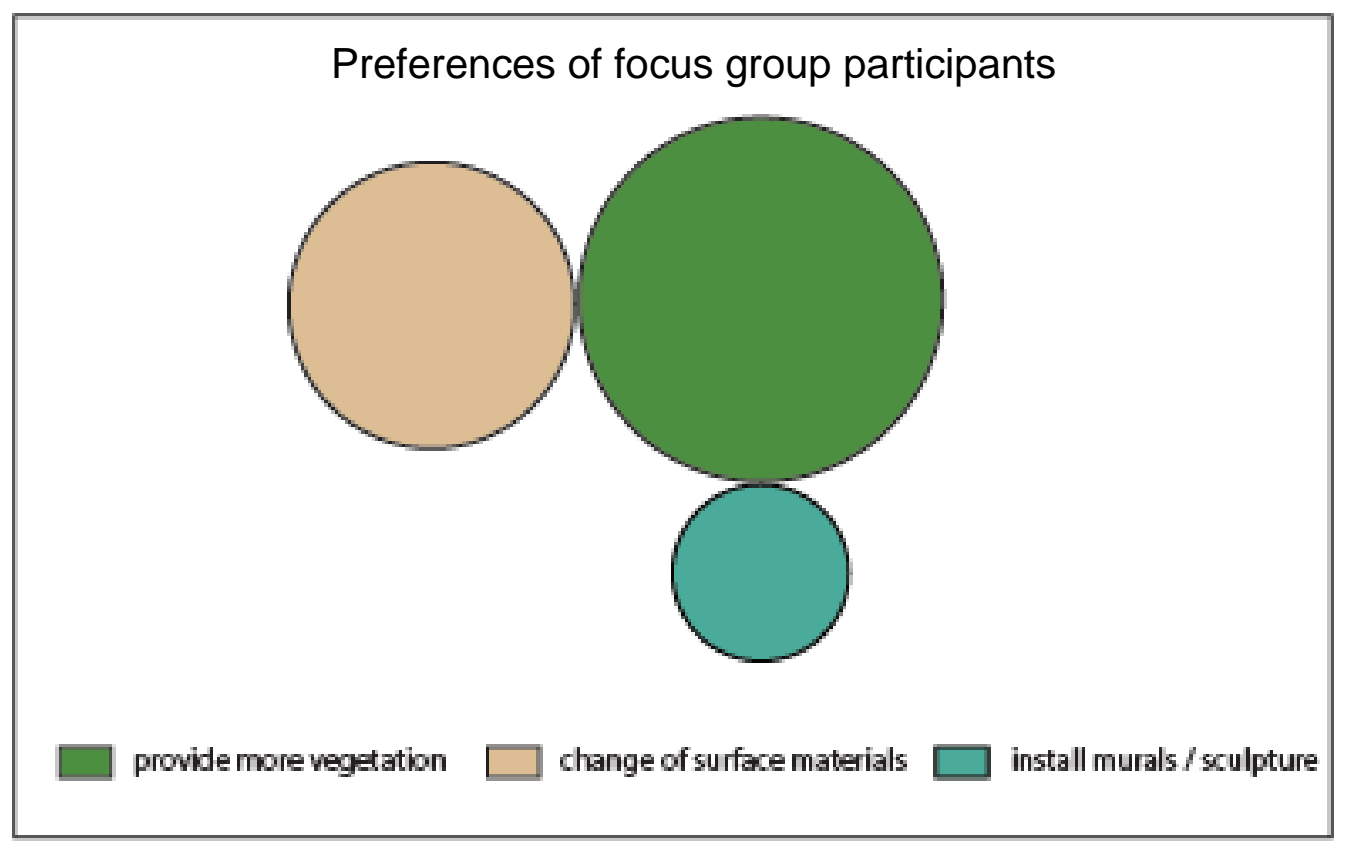

Figure 8.17 Design preferences of focus group participants

\subsection{Provide more vegetation}

Providing more vegetation was the most critical attribute for NBE and BE participants (figure 8.18). NBE participants argued in favour of putting vegetation in small parts of the space and $\mathbf{A} 2$ wished, "to put planting or plant-like screens on the balconies." B3 wanted, "to place some greenery". Interestingly, BE participants laid more stress on the importance of providing more vegetation. $\mathbf{X} \mathbf{1}$ insisted on "[doing] something with planting [to gain attention]", and X2 proposed "[providing] some green spaces."

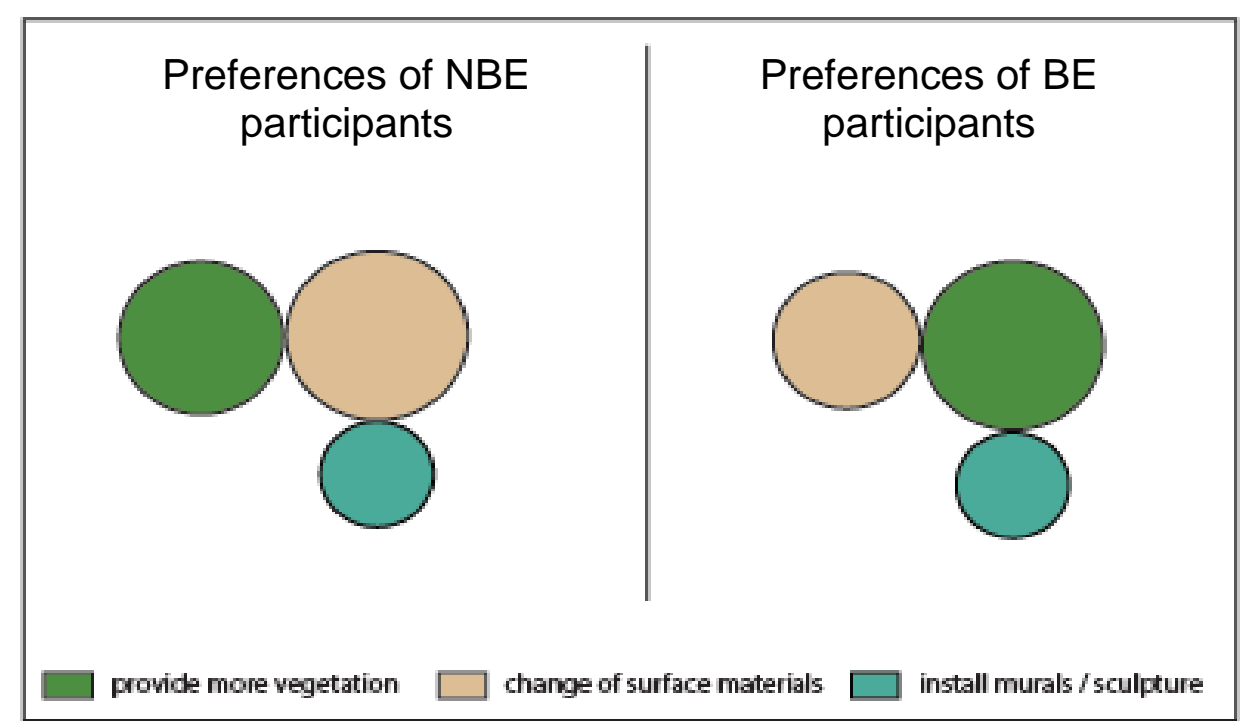

Figure 8.18 Preferences of NBE and BE participants for the space enclosed by buildings on three sides 


\subsection{Change of surface material}

Higher preference for changing the surface material came from the NBE participants. Overall participants varied in the changes they wanted to see, and no distinct patterns were seen except for vegetation. NBE participants emphasised the idea of changing the materials in certain parts of the area. A2 suggested that "putting a new texture in the pedestrianised bits of the car park to differentiate but to lighten the whole setting."

Furthermore, A2 wanted "to paint the balconies in light and bright." B3 had similar ideas of "painting the areas in light shades of grey, or white to look [a] little brighter." Y1 was the only participant in the BE group with similar ideas, stated that, "improving the colour, maybe brighten the space".

\subsection{Install murals and sculptures}

There was no significant difference in opinions for installing murals/sculptures among NBE and BE participants. NBE respondents proposed placing sculptures in the space and murals on the walls to attract the attention of the public. A2 desired "to place a sculpture [and] some artistic like piece of paintings on the big wall to give a bit of focus and liven it up." A2 also felt, "it would be nice if people could look at something more attractive." B2 wanted "to install a sculpture", and B3 also proposed installing "a big sculpture." BE participants also recommended the idea of installing murals and sculptures. X1 wanted "to draw a connection between three empty facades." Whereas, Y2 suggested that, "some paintings [on the wall] and recreation [facilities] would be nice."

\subsubsection{Space enclosed by buildings on two sides}

The fifth image shown to participants was a space enclosed by buildings on two sides (figure 8.19). The analysis showed that on average, all focus group subjects wanted more vegetation (figure 8.21). The other preferences were to create small interventions in the space to increase its usability for both residents and the public. In contrast with earlier findings, here changing the surface material and removing the parking space was less preferred by all participants. 
Chapter 8 - STUDY THREE: FOCUS GROUP DISCUSSION

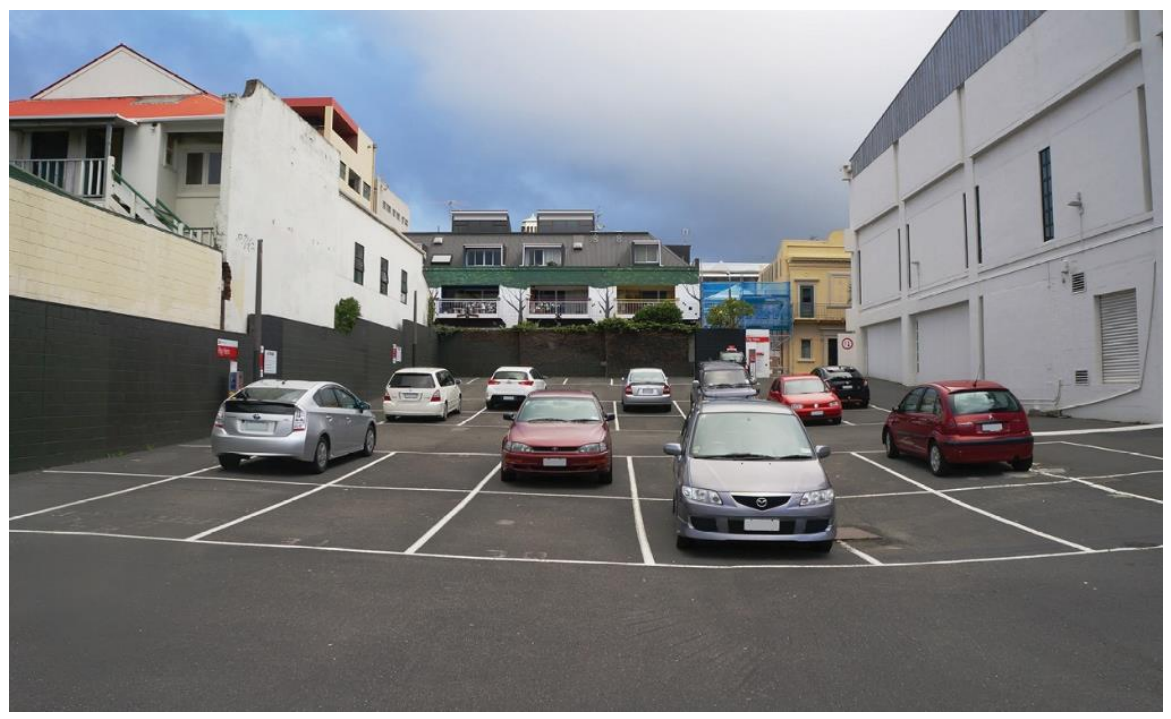

Figure 8.19 Image shown to focus group participants

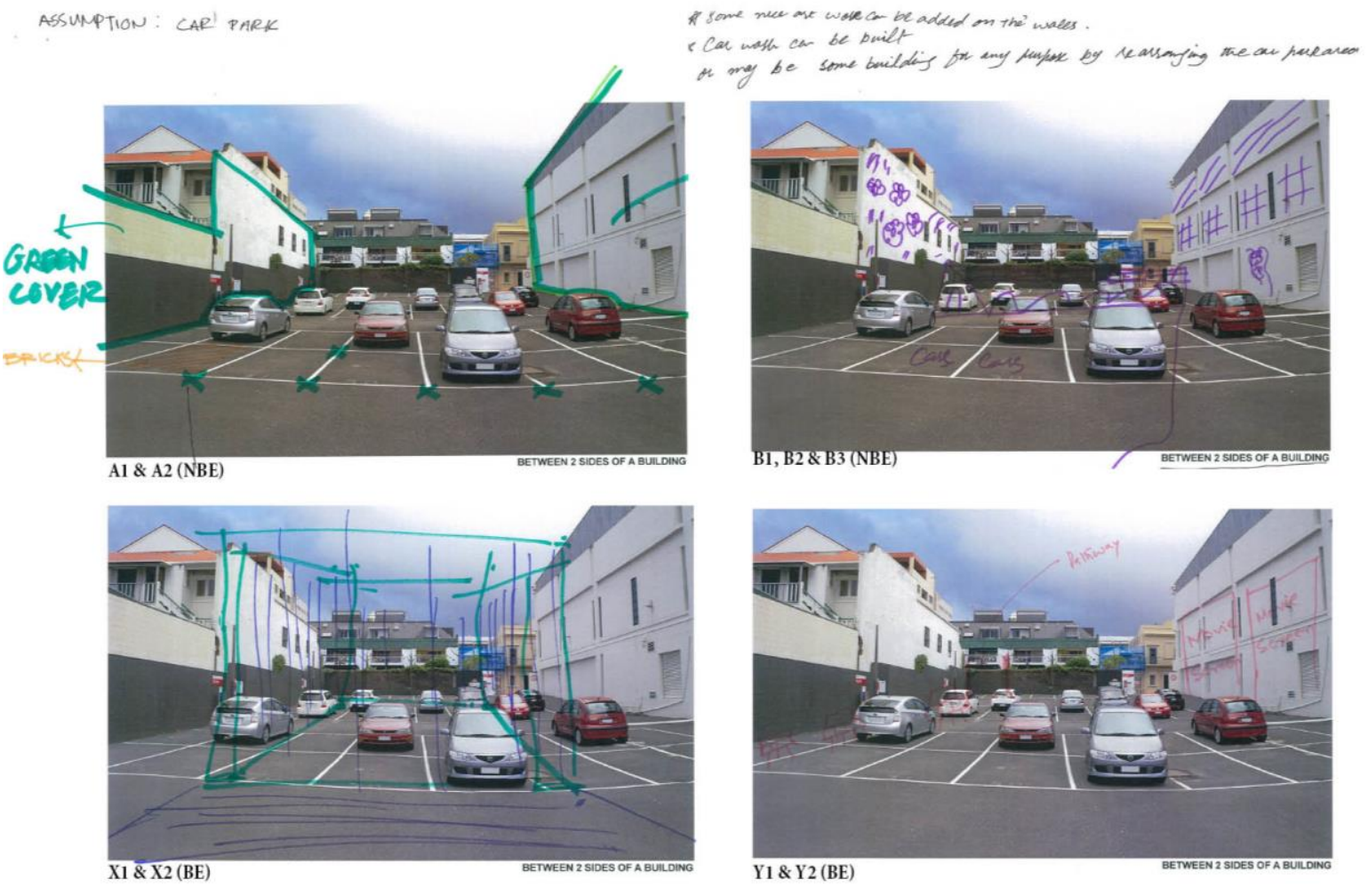

Figure 8.20 Sketches drawn by focus group participants 


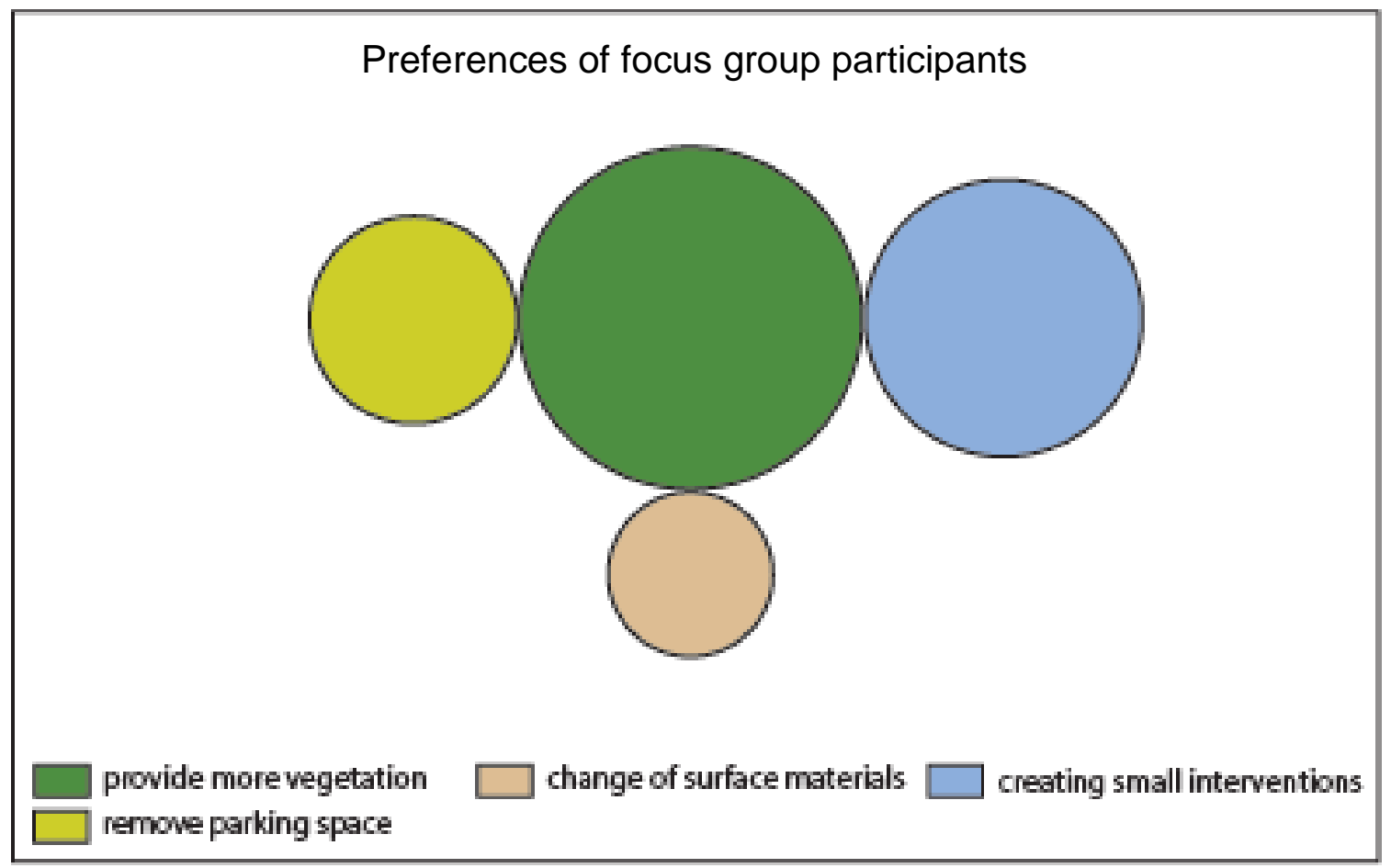

Figure 8.21 Design preferences of focus group participants

\subsection{Provide more vegetation}

The most prevalent idea among NBE and BE participants was to create a natural environment by providing more vegetation in the space enclosed by buildings on two sides

(figure 8.22). NBE participants wanted to see more vegetation on walls and the ground. A1 suggested by "adding lines of green...to turn these sides as green walls, maybe add a green cover adding a fence and then hang something over it." B2 agreed and concluded that "nice looking landscapes would be an addition." BE respondents stressed the need for vegetation for local people. X1 suggested "lots of comfortable outdoor green spaces." Y1 felt to "create a skate park would work." 


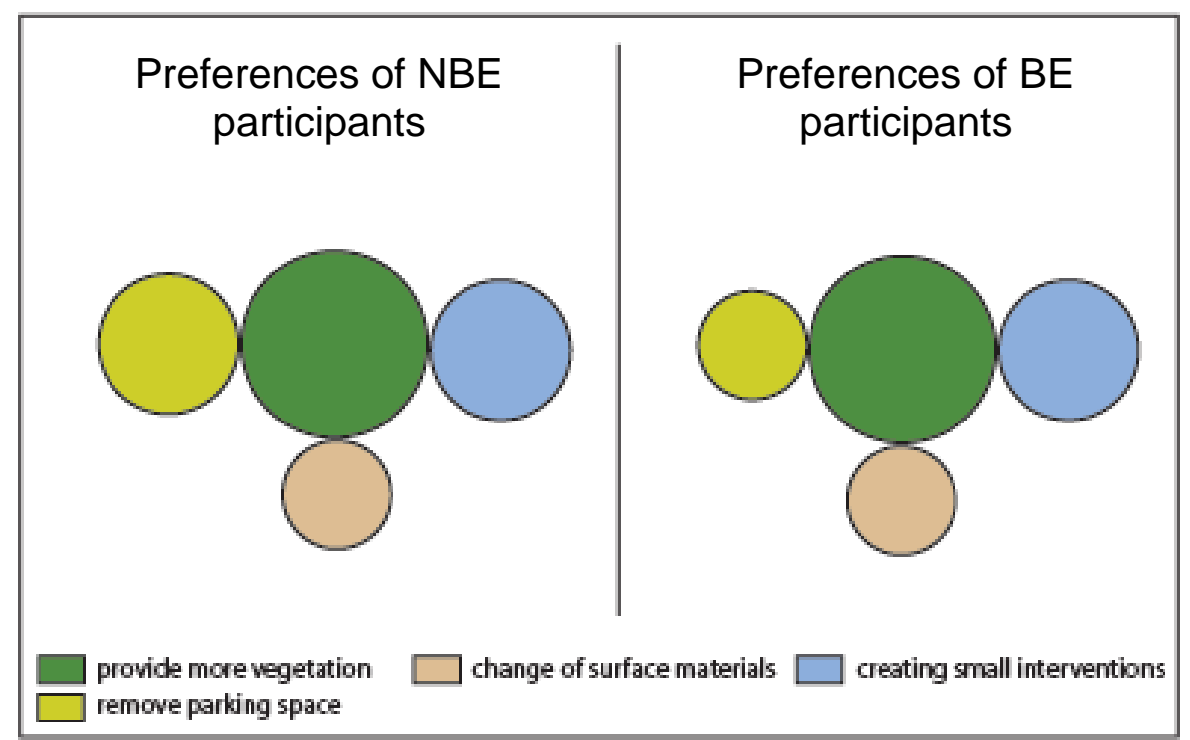

Figure 8.22 Preferences of NBE and BE participants for the space enclosed by buildings on two sides

\subsection{Creating small interventions}

Creating small interventions was suggested by both BE and NBE participants. NBE participants thought creating small interventions could lead to some new uses. B3 started the conversation and emphasised having "nice small buildings with different purposes," while A1 agreed and wanted to implement "the same strategy." BE participants were more proactive about creating small interventions. X1 wanted "to create a building....an opportunity for architecture to overpower everything around it." X2 wished "to create something with an indoor-outdoor flow... with lots of outdoor seating". Lastly, Y2 wanted to "fix two-three movie screens with seating arrangements."

\subsection{Change of surface materials}

There was no significant difference in preferences between BE and NBE participants for changing the surface materials. NBE respondents thought different textures should be integrated into the surface to facilitate the public. A1 thought that "we can play with the texture, with car park and then a walking area." B3 suggested "the walls around should be painted or maybe have something that gives them a nice coverage for being similar to both the sides. Maybe paint in the same colours or two". However, BE participants were more enthusiastic over changing the whole façade. X1 argued for something that "this time contrasting to existing façade...I prefer something very 
transparent." Y2 agreed and proposed to "put something there, as there is nothing on the back side of this wall."

\subsection{Removing parking space}

NBE participants preferred removing the parking space more than BE participants. NBE respondents agreed to minimise or remove some of the car parking space. B2 wanted "to minimise the car park area [or] adjust the car park to one side", whereas A1 suggested, "we can play with the texture, the car park area". X1 was the only participant who proposed "not to give a big amount of space to car parks."

\subsubsection{Space on the rooftop of a building}

The last image was of space on the rooftop of a building. The analysis revealed that providing more vegetation was the most favoured aspect (figure 8.25). Most respondents felt the need to remove the parking space, followed by adding vegetation. The other attributes for improving the aesthetics of the space concerned changing the surface materials, creating seating space, and having small interventions. The NBE group stressed the utility of creating seating spaces and modifying surface materials, whereas BE respondents wanted to create small interventions.

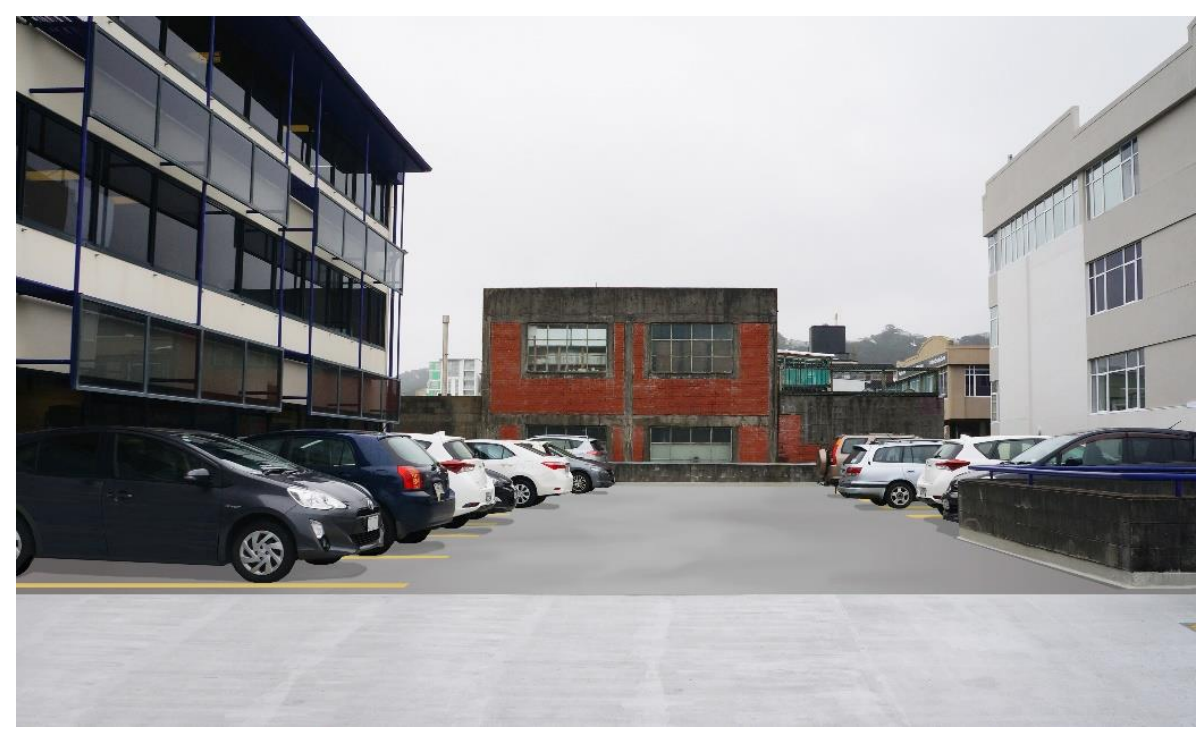

Figure 8.23 Image shown to focus group participants 


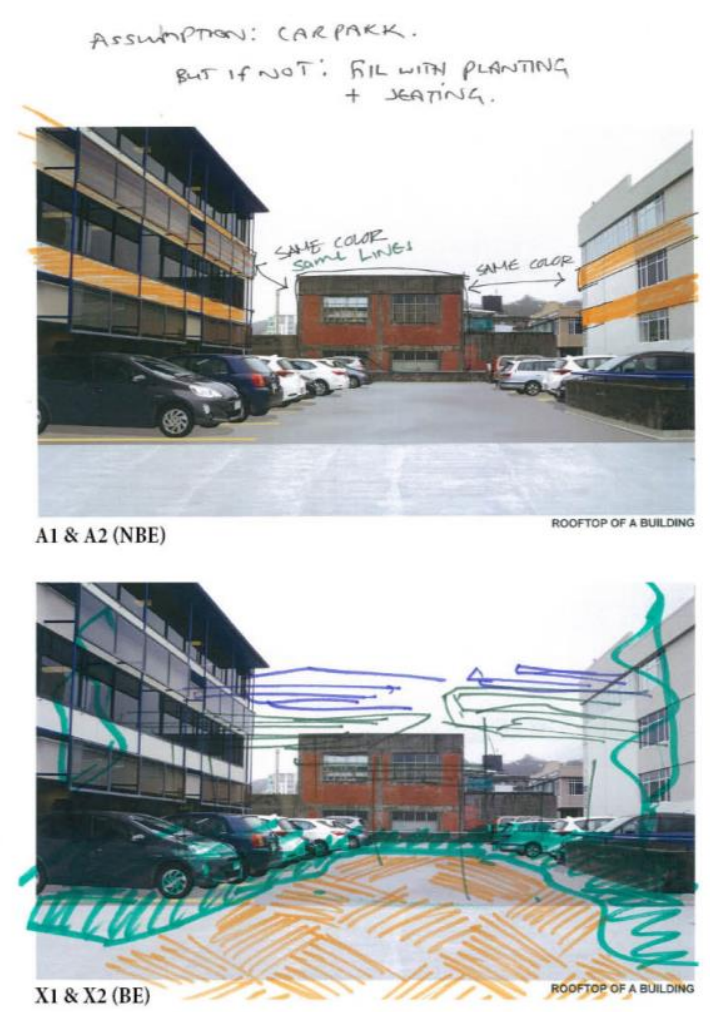

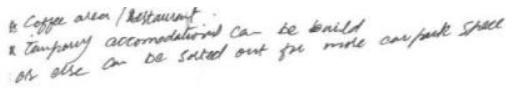
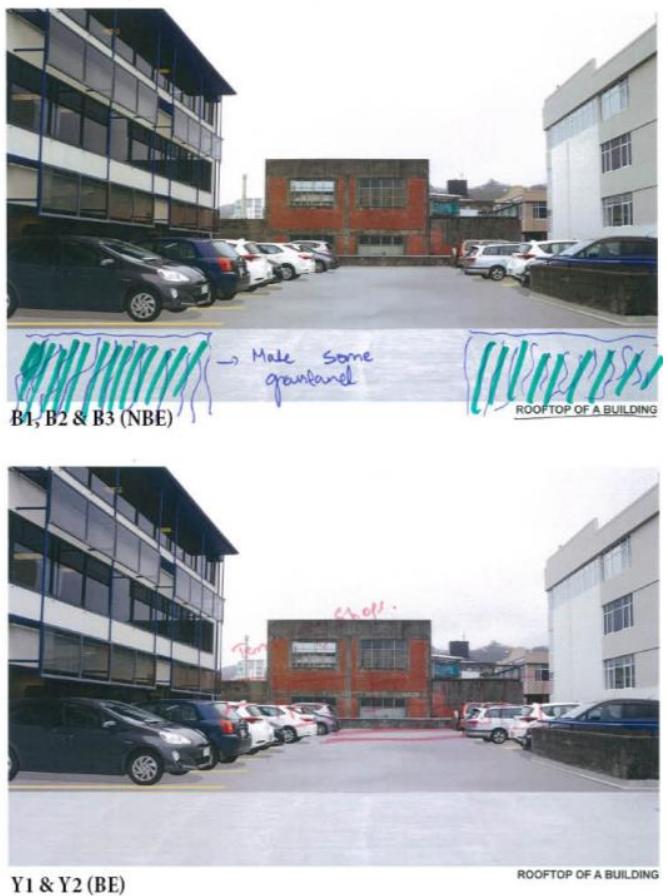

Figure 8.24 Sketches drawn by focus group participants

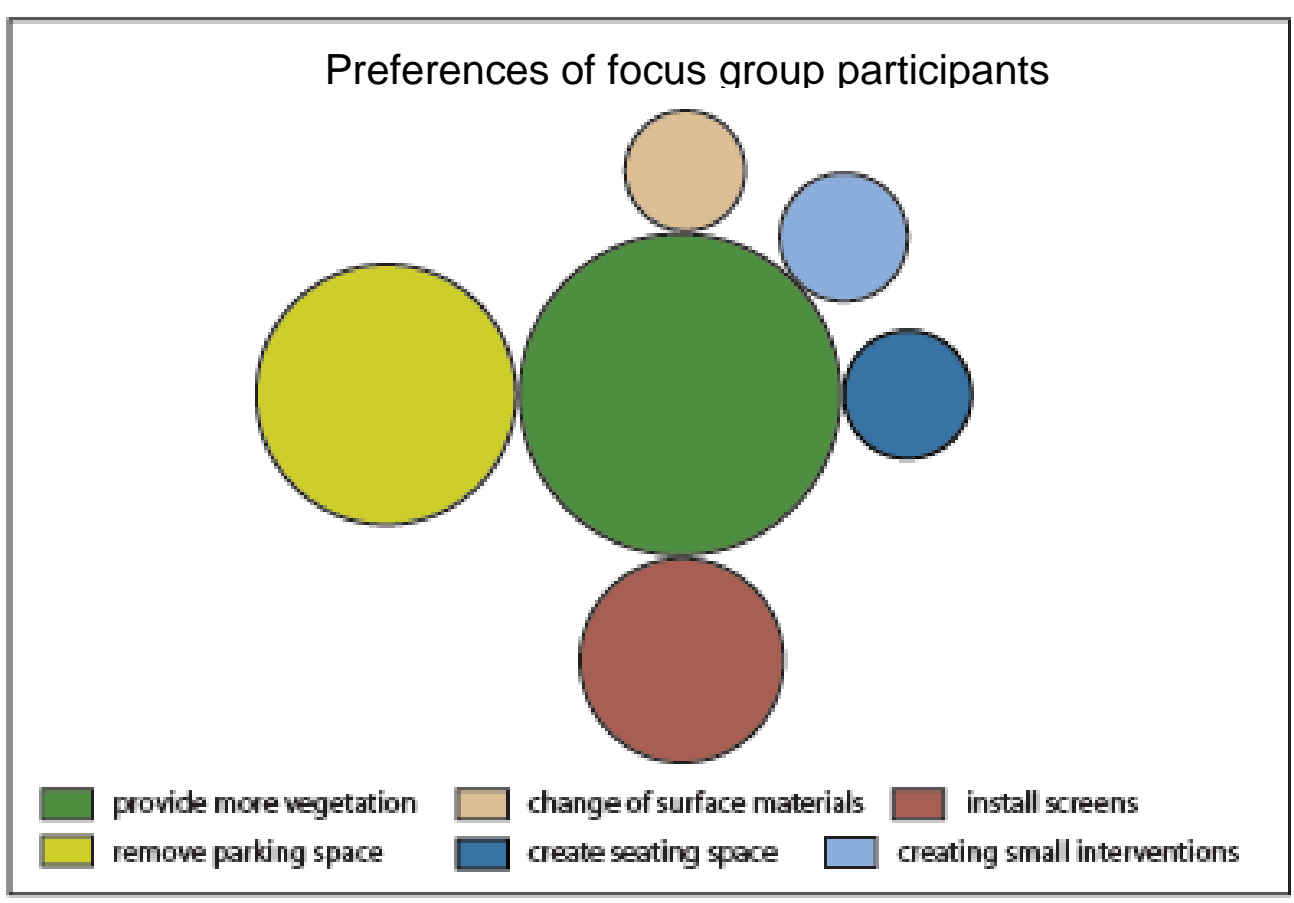

Figure 8.25 Design preferences of focus group participants

\subsection{Provide more vegetation}

No significant differences between BE and NBE participants were observed over providing more vegetation (figure 8.26). NBE participants agreed unanimously on 
turning the rooftop into a "garden, planting the area." The Participants also agreed "to use the space for people who are living there but also for people in the area." BE respondents wanted to "vacate the space and turn it "into a proper garden or something." A2 argued that "the area could be planted" and B1 proposed to have "some grassland...grass is turning into some roof garden." X2 wanted "to make a nice outdoor environment."

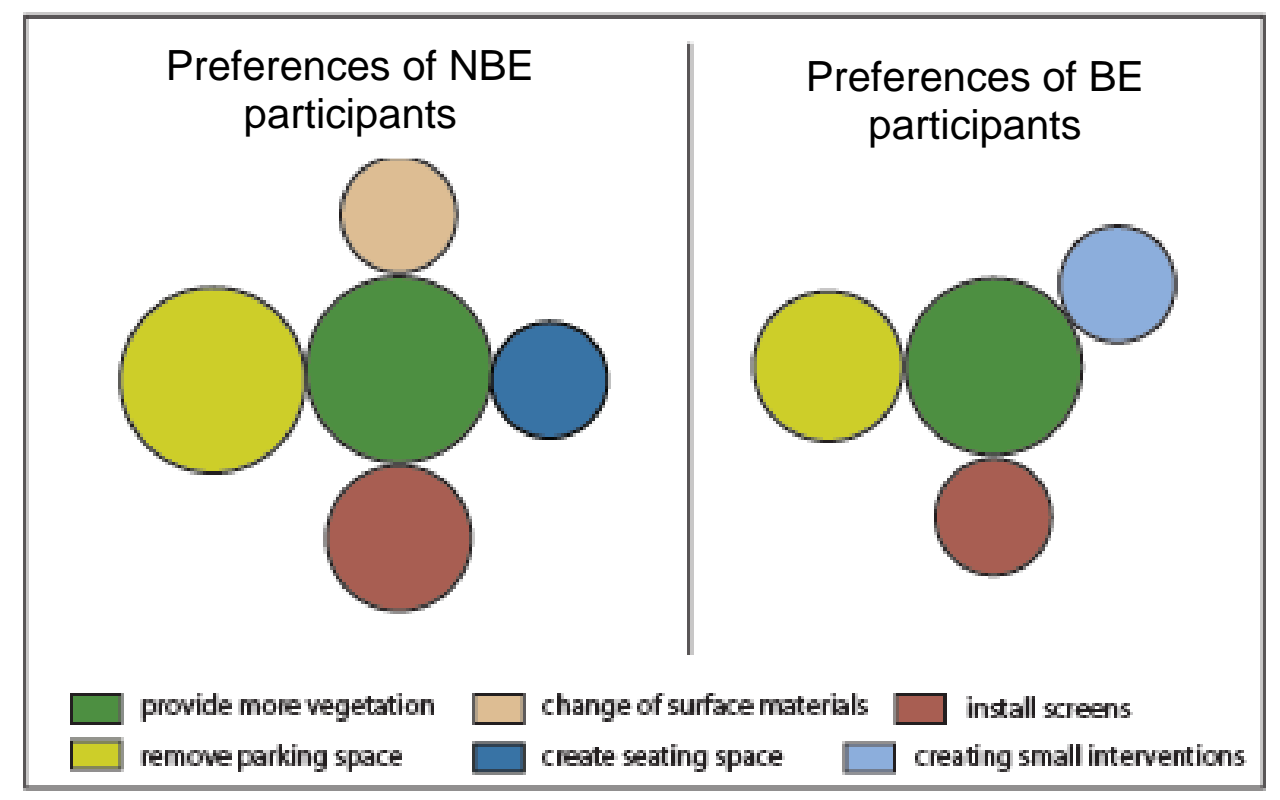

Figure 8.26 Preferences of NBE and BE participants for space on the rooftop of a building

\subsection{Removing parking space}

There was little difference in preferences between NBE and BE participants over removing the parking space. NBE participants were keener on making this change, but all participants wanted to utilise the space by promoting a rooftop culture. NBE argued in favour of creating a space for the public. A2 acknowledged the fact that the car park belonged to the adjacent building users and argued that "this car park is no clear place for people to walk...this could not be a car park." B1 wanted "to get rid of those cars" on more than one occasion. $\mathbf{X} 1$ wished "to create a rooftop culture for nice sunny days... since the cars are at the top, I imagine all the floors below are car parks." X2 added, "get rid of the cars." 


\subsection{Install screens}

All the participants proposed installing more screens on the periphery of the space to enclose it and make it more private to attract people. NBE participants were more positive about this change. For NBE participants, the concept of screening was related to hiding the garbage and making such spaces less visible. A2 wanted "to block or screen the area which is messy." B1 also emphasised putting screens around "as this rooftop has not got any views." BE participants had a different approach as they wanted to utilise screens for protection. $\mathbf{X} \mathbf{1}$ was aware of the practical issue of wind in Wellington and proposed creating "...screens to divert wind." The other participants approved this idea.

\subsection{Create small interventions}

Both NBE and BE participants agreed on creating small interventions having removed the car park, although BE participants were more enthusiastic about this. NBE participants stressed the "usage of space for people who are living there but also for the people in the area." B1 started the discussion by wanting "to intervene in the space with a coffee place or some café with outdoor seating." A2 agreed with this suggestion and focused on "keeping the beautiful building and nice seating." BE participants wanted to see a rooftop restaurant. $\mathbf{X} \mathbf{1}$ claimed that "we need more restaurants on them [rooftops]. I am a big fan." Y2 argued in favour of "shops or cafés for lunch."

\subsection{Changing surface material and creating seating space}

The analysis revealed that NBE participants preferred to create seating spaces and to change the surface material. Neither of these ideas was preferred by the BE participants group. A2 argued that "the area could be bricked and turned into a nice seating space...we could mimic these in lovely modernist lines by painting in light and blacks...putting colour variations in the parking and delineating the pedestrianised bit." The idea was acknowledged by B1, who also wanted "to have some outdoor seating."

\subsubsection{Participant Opinions}

At the end of each focus group discussion, the participants were asked about the most important aspects that needed to be changed or transformed from among all the suggestion made earlier. There was not an appreciable difference in the answers of both focus groups. 


\subsection{The Non-Built Environment Group}

NBE respondents identified four significant areas of concern. In order of decreasing importance for them, these were lack of vegetation, car parking spaces, the surface materials/colours, and untidy spaces. This group emphasised the need to add vegetation to make spaces "look less ugly, user-friendly." Both sub-groups generally preferred making spaces "visually enjoyable" for people. The NBE participants added, "Green spaces can be artwork, look less draggy and attractive to the eye." They also wanted "planting and replacing bins" and "even one-colour of the same palette."

A1 claimed, "parking lots are required by residences and would be okay to have some...make these spaces user-friendly, less ugly." A2 argued that "this is about the political will and ownership...will need to fight some battles." Furthermore, the participants talked about sustainable solutions and discussed that "the direction for cities is to remove cars-that could be done through pool cars. Also, the direction should be to create beautiful spaces and do not destroy them...the word 'enjoyment' that I use, and you see a little gap, and there is something special about it." B1 commented, "if you can remove the parking space from the rooftop and turn it into a space with seating [it] will look nice...the combination of different things." B2 agreed, "small changes mean a lot...adding up something is good, a value addition." Lastly, B3 argued about ownership of space and claimed, "...the usability of spaces can be questioned in those pictures because we do not know if it is easy for residents to park their cars on one side or remove them."

\subsection{The Built Environment Group}

In contrast to the NBE participants, BE participants responded in a more heterogeneous pattern. The overall results showed three significant areas of concern for the $\mathrm{BE}$ respondents. In order of decreasing importance, these were adding more vegetation, adding seating space, and eliminating the car parks. Both BE groups strongly favoured getting rid of cars to improve the visual aesthetics of leftover spaces.

X1 positively appreciated "getting rid of cars", but another respondent Y2 argued against this by giving more preference to the "usability of the spaces" than just their aesthetics. Y2 validated this concern by saying he could not "remove car parking entirely; it is a necessity in the city." $\mathbf{X} \mathbf{1}$ suggested that "cars in the city is not a problem 
but hassle." X2 wanted to "create a nice outdoor environment...create more creative design." Y1 argued, "you cannot wait for people to change, you must change their context and for them to realise that there will be a change for them to react eventually." Lastly, Y2 claimed, "real implementations more than ideal implementation."

\subsection{Differences of Opinion within the Built Environment Group}

This group was made up of three architects and one engineer. It was observed that the designers were more critical, enthusiastic, vocal, and provocative than the engineer participant, whose concepts were more linked to the existing situation in the real world. The first conflict was about having more car parking space in the city. The engineer participant argued "we cannot propose removing car parking. I do not think people in the city prefer to compromise on car ownership. A car park is a necessity in the city...the hassle is not for the car owners, but a hassle for those who do not [have cars]." The second dispute between the designers and the engineer was related to the practical usage of the space. The engineer participant wanted "to make the best utilisation of the property, by getting more earning and making money from it." The last difference in opinion was about the feasibility of the design. The engineer respondent argued, "consider the psyche of humans, proposing underground parking is not so easy to implement."

\subsection{Study Findings}

It became evident from the mini-design charrette that BE participants paid more attention to overall space, and their drawings were more dynamic and bolder than those of NBE respondents. Also, BE participants looked to change the entire space rather than changing or improving just one or two attributes in an area. The overall preference among all participants was to provide more vegetation in the leftover spaces (figure 8.27). NBE respondents shared positive notions regarding the implementation of their vegetation plans, even though the day-to-day maintenance seemed to be a concern. BE respondents accentuated design more than the usability of space. These participants introduced new ideas, but vegetation for them still outweighed the other concepts. All the focus group participants preferred to provide more vegetation in leftover spaces except for in front of a building where removal of 
the boundary wall was the most preferred, and vegetation the second most preferred design attribute.

The participants agreed that visual quality could be improved by designing small interventions or taking little steps that are easy to implement and that have an effect on usability, like providing more vegetation or creating a pop-up cafe. This highlighted how people-friendly environments could be built in small spaces in the city. The second most preferred choice among all participants was to change the surface material or colours. Even though there was some inconsistency in the details of this, there was overall agreement among all respondents. NBE participants suggested the third most preferred choice of installing more screens to hide the garbage bins. BE participants paid minor attention to cleanliness. The BE participants emphasised the redesign of the leftover spaces more than their usability. This group felt that parking space was mismanaged land that could be reused for good. NBE participants were less concerned with revising the design altogether. However, it should be noted that these findings were based on a limited number of participants, and the results from such analyses should be treated with some caution.

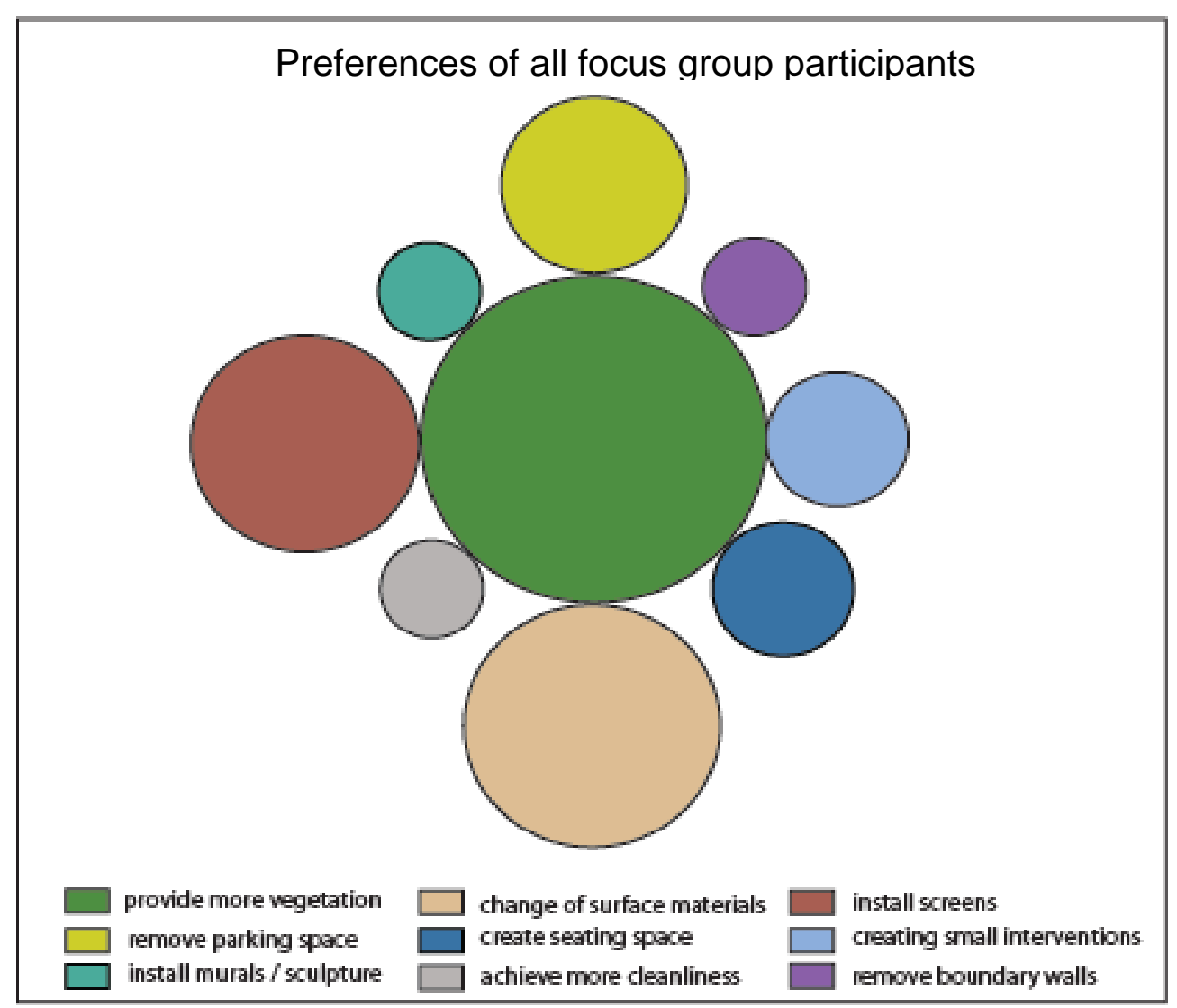

Figure 8.27 Overall preferences of all participants 


\subsection{Summary}

This Chapter has discussed the significance, methods, and findings of focus group discussions, and the similarities and differences in preferences between the $\mathrm{BE}$ and NBE groups. The two sessions were held separately with four BE and five NBE participants from different age groups. The focus group session was divided into two stages, and in each session, participants were paired up. The first stage was a minidesign charrette in which the pairs were given six photographs of different types of urban leftover space, using the same photographs as in Study One. The participants were asked to draw/doodle on the photographs and come up with a design scheme by adding or removing attributes from each space. These designs were used as the basis for discussion in stage two. The most evident finding was related to providing more vegetation as both BE and NBE participants wanted this. Chapter 9 interprets the results of all three studies and discusses the usefulness of the research findings. 


\section{CHAPTER 9}

\section{INTERPRETATION OF THE RESULTS}

\subsection{Introduction}

This Chapter highlights the critical findings of Studies One, Two and Three, which together have probed design solutions for urban leftover space in the context of Wellington. The similarities and differences in the responses of the participants in all three studies are compared (see section 9.2). Chi-square tests have been performed to check the strength of association between two categorical variables. These are the preference for the attributes and the age of participants and their field of study.

The aim of Study One was to probe how much participants liked the different types of leftover space with their diverse characteristics. A photographic representation of each space was used, and participants picked their most preferred spaces and the attributes of these, as well as stating what they thought needed to be transformed. The pattern of responses in part two of Study One confirmed Nasar's (1998) theory of what people prefer for the aspects of naturalness, organised space, open space, and wellmaintained space. Nasar's prediction that people would like an older-style building did not hold accurate and participants preferred the photo showing a modern style building over the older style one. However, Nasar referred to a historic building, and there was no example of this in a left-over space in the Wellington case study area so this may not be a valid comparison with Nasar's findings. In part three of Study One, participants preferred those spaces with vegetation, good lighting, organised parking, and that had visual permeability, openness to the sky, different materials/colours, and graffiti on the walls. The participants wanted to see changes in spaces that did not appear to be actively used, or that looked neglected and disorganised. The fourth part included two different photographs of each leftover space and participants chose the space they felt the most required transformation. From a list that was provided, the most common suggestions for transforming the space were providing more vegetation, creating pedestrian pathways, changing the surface materials/colours, creating seating spaces, improving the lighting, improving maintenance and cleanliness, and removing the garbage bins. 
The purpose of Study Two was to understand the preferences for the spaces both before and after they had been changed using the most popular suggestions for change from Study One (see section 6.3). Study Two was thus built on part four of Study One as the photographs of the spaces identified as most needing changes were photomontaged to create three redesigned alternatives. The photomontages were rated by participants on a 5-point Likert scale. However, to understand the preference opinions more comprehensively, the 5-point Likert scale was converged to make a simplified 3-point Likert scale (Benson, 1971). A semantic differential scale measured the emotions of participants towards the different design solutions also on a 5-point Likert scale. This found the most preferred design option was adding vegetation to space, but this was also negatively associated with maintenance and the difficulty of making such a change (whether the change was impossible, somewhat impossible, neutral, somewhat realisable, realisable). Changing the surface materials/colours was negatively associated with satisfaction with the design (where it fell in the range annoying-pleasing), suggesting people wanted to see changes in these but did not like the suggestion for doing this in the photomontage. Creating seating space was also negatively associated with mood (whether this was boring to interesting), again suggesting people did not like the specific type of seating spaces chosen for the new design.

In the same way improving the maintenance and removal of a boundary wall was negatively associated with being visually appealing (whether these actions were boring to interesting), and creating a clear pathway and installing wind turbines and solar panels were negatively associated with attractiveness (ugly to beautiful) and satisfaction (annoying to pleasing) respectively. The most attractive and recurring solutions were about providing more vegetation and changing the surface materials/colours, while the other solutions were related to creating seating space, creating a clear pathway, removal of boundary walls, and improving maintenance and cleanliness.

The objective of Study Three was to understand the similarities or differences in perception and design preferences of the built environment (BE) participants and others (NBE) for different leftover spaces. Sheer (1994) argued that professional opinions should be considered essential for designing a transformation in an 
environment. Study Three (see section 8.4.3) consisted of focus group discussions with BE and NBE participants who shared their opinions and their perceptions and experiences when redesigning leftover spaces. The respondents were shown the photographs from part four of Study One, these being the spaces that most needed change. Design suggestions from participants included providing more vegetation, changing the surface materials/colours, installing screens, removal of parking spaces, creating seating space, creating small interventions such as pop up cafes, installing murals/sculptures, removal of boundary walls, and having better maintenance and cleanliness.

Rapoport (1982) claimed that the designer perceives and designs the built environment in accordance with the meanings learnt from and associations reinforced by their professions, whereas non-designers respond and perceive the built environment mainly through cognitive associations. Other researchers have added to this view by referring to a combination of ideas. According to Symes et al., (1995), often clients and society seek the advice of design professionals on matters relating to aesthetics and taste. Moreover, the study by Stamps (1999) found that designers and non-designers have similarities when evaluating building design or natural space, but have a difference in their aesthetic stimulus. Therefore, with the results of the three studies, a useful comparison can be drawn. This requires evaluating the aesthetic preference for design attributes between BE and NBE participants from different age groups. In this Chapter, the aesthetic perception of young BE (18-35 aged) is tested for a relationship with young (18-35), middle-aged (36-55) and older (56 plus) NBE participants. The aim is to identify the differences in preferences between young $B E$ participants, whether designers or engineers, who have some knowledge of the industry and the client group, which is usually from a NBE background. For this purpose, a chi-square test was performed to understand if preferences for design attributes between these groups have a relationship.

\subsection{Data Comparison}

The most preferred design attributes of leftover spaces as identified by participants in Study One (part four) and Study Two were compiled in SPSS (appendix 9.5), and the results were compared with those of Study Three (Table 9.1). To find out if BE and 
groups of NBE participants by age think similarly about different design attributes, a chi-square test of the results of Studies One and Two was performed for young (1835 aged) BE participants with young (age 18-35), middle-aged (age 36-55) and older (age 56 plus) NBE participants. A Spearman correlation test would have been ideal had there been a much larger sample size (Toebe et al., 2015).

\subsubsection{Most preferred attributes}

The most preferred design attributes from the three studies are listed in table 10.1 and are discussed in detail in the following sub-sections.

\begin{tabular}{|l|c|c|c|c|c|}
\hline \multicolumn{1}{|c|}{ Study One } & \multicolumn{2}{c|}{$\begin{array}{c}\text { Study Two } \\
\text { N=99 }\end{array}$} & $\begin{array}{c}\text { Study } \\
\text { Three }\end{array}$ \\
N=9
\end{tabular}




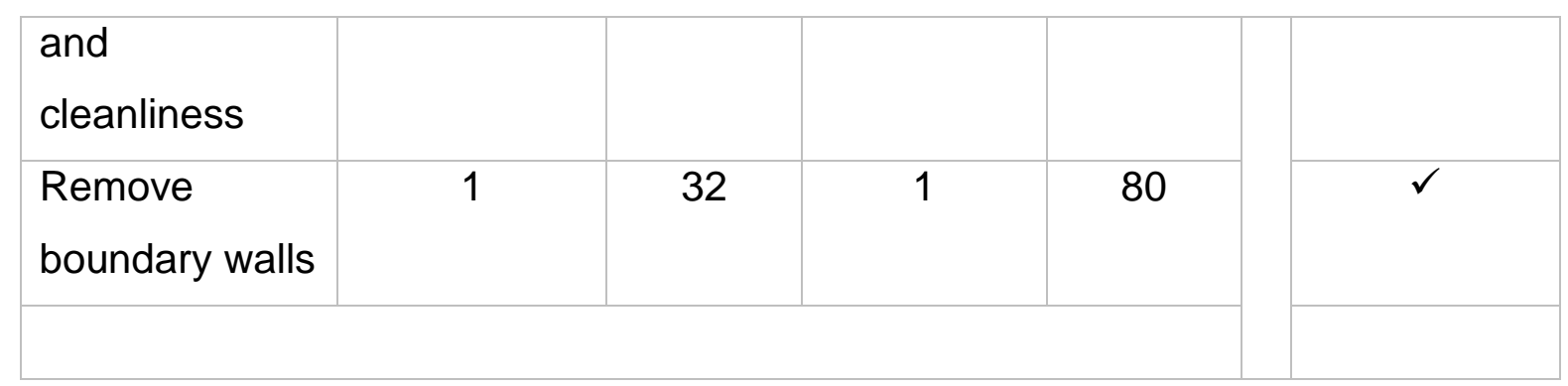

Table 9.1 Most preferred design attributes of different leftover spaces

${ }^{*} A$ tick indicates that the attribute emerged as a design theme in the analysis of the focus group discussions

The result of Study One and Study Two about preferred attributes were combined and divided by the number of variables to calculate the average value. This process was found helpful to comprehend the data into a single value that also demonstrates the variability around a single value within the given dataset (Student Learning Development, 2019). The process of calculating the average values was repeated for recurring attributes that appeared in Studies One and Two (see section 9.2.1.19.2.2.4).

\subsubsection{Provide more vegetation}

The results of the three studies showed that the participants wanted to see more vegetation in leftover spaces, this being the most preferred attribute. According to Thanyer \& Atwood (1978), people prefer natural spaces over human-made features, and their liking is increased if the vegetation in a particular setting is increased. Douglas et al. (2018) found that vegetation was a critical measure of satisfaction for assessing perceptions within society. Much earlier, Lynch (1960) noted that people received pleasure from and cared about vegetation, and this also strengthened the imageability of a place.

The results of both studies revealed that in average, females (32\%) liked having vegetation marginally more than males (30\%). Krenichyn (2006) also affirmed that vegetation is an essential attribute of an environment and that females prefer natural green space in the built environment more than males. Their preferences for green spaces can be correlated with their physical activities. Similarly, Magde (1997) and Jansson et al. (2013) found that parks are mainly used by females, although areas in a park with poor lighting are avoided due to concerns over safety. The analysis in this research also found that young female participants (54\%) liked having vegetation more 
than old male participants (44\%) (see appendix table 9.1). Participants aged 36+ liked vegetation (26\%) more than young participants aged 18-35 (24\%). The research of Bjerke et al. (2006) found that densely vegetated spaces were liked more by participants who were more than 30 years old. In this study, no difference in opinion was found between BE and NBE participants who equally liked this attribute $(60 \%)$. Study Three also confirmed that all participants wanted to create a more natural urban outdoor environment by providing more vegetation so that it would look more attractive and be more inviting and pleasing for the community. BE respondents were more assertive over providing vegetation than NBE participants, to the point of preferring vegetation over the usability of the space (figure 9.1). It was observed from the mini design charrette that the first response of $B E$ participants was to add vegetation to space.
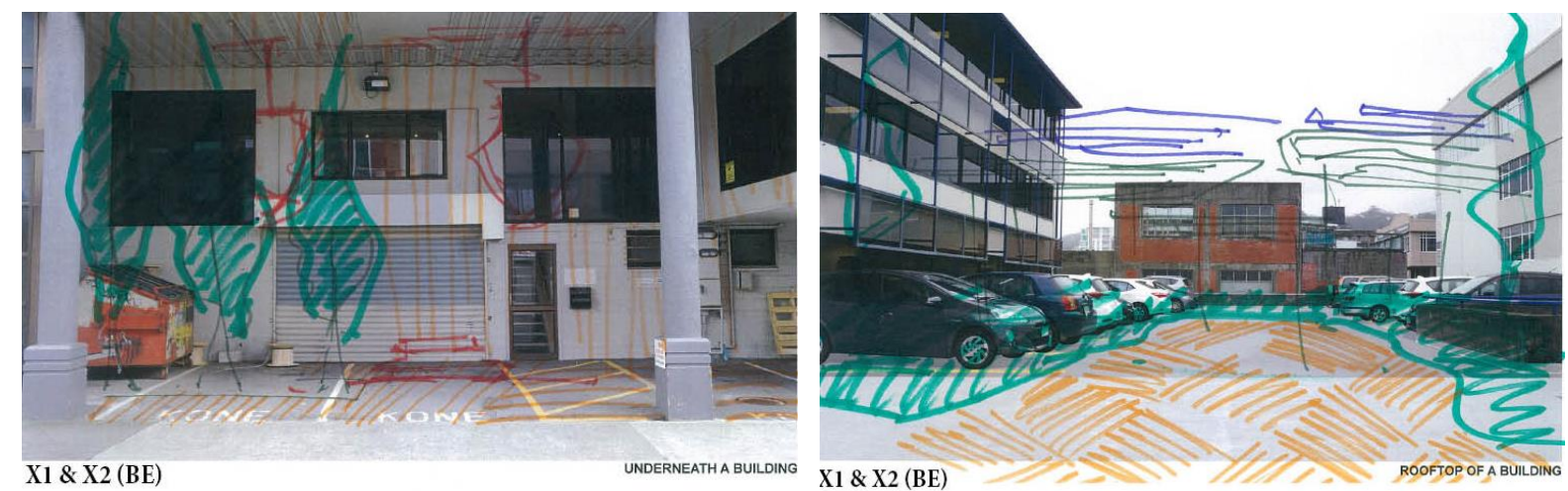

Figure 9.1 Built environment participant sketches with an emphasis on providing more vegetation (in green)

The Chi-square test examined the relationship between young BE participants for the desire to have more vegetation with NBE participants of all age groups (table 9.2). The first test revealed that the young (aged 18-35) BE and young (aged 18-35) NBE participants have no difference in opinion over providing more vegetation. The result was found statistically significant at $X^{2}(3)=04.17, p(.021)<0.05$, and $51 \%$ of young BE participants supported providing more vegetation as compared to young NBE participants (48\%). The second test found that the young (aged 18-35) BE and middleaged (aged 36-55) NBE participants have similar thoughts for providing more vegetation. The result was found statistically significant at $X^{2}(3)=07.12, p(.043)$ $<0.05$, and $53 \%$ of the middle-aged (aged 36-55) NBE participants supported the idea of providing more vegetation compared to slightly fewer young BE participants $(47 \%)$. 
The third test looked at finding the preference association between young BE (aged 18-35) and older (aged 56+) NBE participants. The association between the two sets of variables was again found statistically significant at $X^{2}(3)=05.32, p(.039)<0.05$, and $52 \%$ of older NBE participants preferred the design attribute compared to $48 \%$ of young BE participants. All these results confirmed that providing more vegetation is unanimously seen as the most important response when redesigning leftover spaces.

\begin{tabular}{|c|c|c|c|c|}
\hline \multicolumn{5}{|c|}{ Providing more vegetation } \\
\hline & Value & df & $\begin{array}{l}\text { Asymp. Sig. (2 } \\
\text { sides) }\end{array}$ & $\begin{array}{c}\text { Association } \\
\text { between } \\
\text { variables }\end{array}$ \\
\hline \multicolumn{5}{|c|}{ Young (aged 18-35) BE and Young (aged 18-35) NBE participants } \\
\hline Pearson Chi-Square & 04.17 & 03 & 0.021 & $\checkmark$ \\
\hline \multicolumn{5}{|c|}{$\begin{array}{l}\text { Young (aged 18-35) BE and Middle-aged (aged 36-55) NBE } \\
\text { participants }\end{array}$} \\
\hline Pearson Chi-Square & 07.12 & 03 & 0.043 & $\checkmark$ \\
\hline \multicolumn{5}{|c|}{ Young (aged 18-35) BE and Older (aged 56+) NBE participants } \\
\hline Pearson Chi-Square & 05.32 & 03 & 0.039 & $\checkmark$ \\
\hline
\end{tabular}

Table 9.2 Participant groups by age from Studies One and Two have a similar preference for wanting vegetation.

One more test was undertaken to check if there was a relationship between NBE respondents from different age groups (those aged 18-35 and aged 36+) and want to see vegetation in leftover spaces. This test was performed for only this attribute of providing more vegetation since a positive association was found between $\mathrm{BE}$ and NBE of different age group. The relationship between these variables was also found statistically significant at $X^{2}(3)=03.85, p(.049)<0.05$. This means that although both NBE groups prefer a space with more vegetation in it, being more than 36 years old means you are more likely to prefer vegetation in a leftover space. Some $60 \%$ of NBE participants (aged $36+$ ) liked spaces with vegetation in them compared to just $30 \%$ of young NBE participants (aged 18-35). The same test was performed for BE participants in different age groups (those aged 18-35 and aged 36+) to check if the age group was the predictor of liking vegetation. The relation between these variables was also found statistically significant at $X^{2}(3)=04.33, p(.041)<0.05$, meaning that 
both $\mathrm{BE}$ groups prefer a space with more vegetation in it. However, this test revealed that young BE participants (aged 18-35) have more preference for providing vegetation $(55 \%)$ than the participants aged $36+(45 \%)$.

\subsubsection{Change of surface material/colours}

Change in materials/colours was the second most preferred action for redesigning urban leftover spaces. According to Babin et al., (2003), behaviour and mood are directly influenced by the colour and the texture of a material in a person's surroundings. Courtis (2004) recognised that colour affects interpretation, recognition, and can change a person's perception of a place. Similarly, Cernin (2003) and Mehta et al. (2009) asserted that red and blue colours improve both memory and the functional capability of the brain, as red enhances detail-oriented tasks while blue is beneficial for creative tasks. Yildirim et al. (2007) and Huchendorf (2007) concluded that colour arouses emotional feeling and affects behaviour.

The results of Studies One and Two revealed that on average 34\% of male participants liked the idea of changing the surface materials/colours more than female participants (29\%), and younger (aged 18-35) participants liked the solution (14\%) more than the participants aged 36 plus (11\%). Lastly, $20 \%$ of both NBE and BE participants had a similar preference for changing the surface materials/colours. Study Three confirmed that all participants wanted to see a change in surface materials and colours as they suggested this was a way of differentiating the pedestrian pathways, and that putting surface textures and painting on the walls with light colours would make the areas look brighter. NBE respondents wanted to change the surface colours/materials more than BE participants, as the former wanted to make the spaces aesthetically appealing by hiding the flaws by painting the surfaces with lighter colours. Ainsworth et al. (1993) claimed that colour affects work performance, and much earlier, Acking \& Kuller (1972) found that different colours affect both breathing rate and blood pressure. While intense or saturated colours encourage a sense of excitement, weak or less saturated colours promote a feeling of calmness. Similarly, NBE participants in Study Three preferred calm and soothing colours, such as shades of grey, while BE participants wanted warm colours to make an impact on the surroundings and so the difference could be seen. 
The Chi-square test looked for the association of preference for changing the surface materials/colours between young BE participants (aged 18-35) and NBE participants of all age groups (table 9.3). The first test revealed that the young BE and young NBE participants (aged 18-35) do not have the same opinions for changing the surface materials/colours. The result was not found to be statistically significant at $X^{2}(3)=$ $5.67, p(2.81)>0.05$, although $53 \%$ of young BE participants supported the change in surface materials/colours as compared to $47 \%$ of young NBE participants. The second test found that the young (aged 18-35) BE and middle-aged (aged 36-55) NBE participants have similar thoughts for changing the surface materials/colours. The result was statistically significant at $X^{2}(3)=03.92, p(.039)<0.05$, and $51 \%$ of young BE participants (aged 18-35) supported the idea of changing surface materials/colours as compared to slightly fewer middle-aged (aged $36-55)$ NBE participants (49\%). The third test looked at finding the relationship between young BE (aged 18-35) and older (aged 56+) NBE participants. The association between the two variables was not statistically significant at $X^{2}(3)=08.21, p(4.73)>0.05$, which means that young BE and older NBE do not think similarly, as $59 \%$ of young BE participants (age 18-35) preferred the design attribute than the older (aged 56+) NBE participants $(41 \%)$. Although participants have a preference for the attribute of changing the surface materials/colours, there was a difference in opinion between young and older NBE participant and young BE participants.

\begin{tabular}{|c|c|c|c|}
\hline Change of surface materials & colo & & \\
\hline Value & $\mathrm{df}$ & $\begin{array}{c}\text { Asymp. Sig. (2 } \\
\text { sides) }\end{array}$ & $\begin{array}{c}\text { Association } \\
\text { (between } \\
\text { variables) }\end{array}$ \\
\hline
\end{tabular}

Young (aged 18-35) BE and Young (aged 18-35) NBE participants \begin{tabular}{|l|l|l|l|l} 
Pearson Chi-Square & 5.67 & 03 & 2.81
\end{tabular}

Young (aged 18-35) BE and Middle-aged (aged 36-55) NBE participants

\begin{tabular}{|c|c|c|c|c|}
\hline Pearson Chi-Square & 03.92 & 03 & 0.039 & $\checkmark$ \\
\hline \multicolumn{5}{|c|}{ Young (aged 18-35) BE and Older (aged 55+) NBE participants } \\
\hline Pearson Chi-Square & 08.21 & 03 & 4.73 & - \\
\hline
\end{tabular}


Table 9.3 Participant groups by age from Studies One and Two and preferences for a change in surface materials/colours

\subsubsection{Create seating space}

Creating a seating space was the third most preferred design attribute for urban leftover spaces. According to Chen et al. (2016), having seating space invites more people to use an area. Gehl $(1987,2010)$ mentioned the importance of stationary activities such as sitting, standing, and waiting, as these are significant contributors to social life and better opportunities for seating are desirable as a means of enhancing this. Whyte (1980), perhaps not unexpectedly, claimed that people use seats more often if there are places to sit. However, people can become frustrated if there are no places to sit (Cattell et al., 2008). The results of Studies One and Two revealed that both male and female participants on average equally liked this design attribute (32\%), and participants (aged 36+) liked the attribute (17\%) more than young (aged 18-35) participants (15\%). Thompson et al. (2013) found that seating spaces are essential for older people since they have less stamina for standing or walking, although it has to be said that being aged $36+$ is not the same as being old in Thompson's study. Spooner (2014) found a more general correlation between seating space and social activity. Lastly, a little difference in the preference for creating a seating space was found between NBE (17\%) and BE participants (16\%). However, in Study Three, only $B E$ participants wanted to create outdoor seating space to attract and look visually appealing to the public.

The Chi-square test was performed for finding a relationship between young $\mathrm{BE}$ participants (aged 18-35) for creating seating space with NBE participants of all age groups (table 9.4). The first test revealed that young (aged 18-35) BE and NBE participants do not have similar opinions for creating seating spaces. The result was not statistically significant at $X^{2}(3)=08.26, p(05.22)>0.05$, and $54 \%$ of young $B E$ participants supported the idea of creating seating spaces compared to $46 \%$ of young NBE participants. The second test also found that young (aged 18-35) BE and middleaged (aged 36-55) NBE participants do not have similar thoughts for creating seating space. The result was not statistically significant at $X^{2}(3)=06.21, p(03.25)>0.05$, and $54 \%$ of young BE participants supported creating seating space compared to fewer 
middle-aged (aged 36-55) NBE participants (46\%). The third test looked at finding the relationship between young BE (aged 18-35) and older (aged 56+) NBE participants. This relationship was statistically significant at $X^{2}(3)=02.54, p(0.49)<0.05$ which means that the young BE and older NBE participants think similarly, as $58 \%$ of older (aged 56+) NBE participants preferred the design attribute compared to $42 \%$ of young BE participants (aged 18-35). Although participants have a preference for the attribute of creating seating space, there was a difference in opinion between young and middle-aged NBE participants and young BE participants.

\begin{tabular}{|c|c|c|c|c|}
\hline \multicolumn{5}{|l|}{ Create seating space } \\
\hline & Value & df & $\begin{array}{l}\text { Asymp. Sig. }(2 \\
\text { sides })\end{array}$ & $\begin{array}{c}\text { Association } \\
\text { (between } \\
\text { variables) }\end{array}$ \\
\hline \multicolumn{5}{|c|}{ Young (aged 18-35) BE and Young (aged 18-35) NBE participants } \\
\hline Pearson Chi-Square & 08.26 & 03 & 05.22 & - \\
\hline \multicolumn{5}{|c|}{$\begin{array}{l}\text { Young (aged 18-35) BE and Middle-aged (aged 36-55) NBE } \\
\text { participants }\end{array}$} \\
\hline Pearson Chi-Square & 06.21 & 03 & 03.25 & - \\
\hline \multicolumn{5}{|c|}{ Young (aged 18-35) BE and Older (aged 55+) NBE participants } \\
\hline Pearson Chi-Square & 02.54 & 03 & 0.49 & $\checkmark$ \\
\hline
\end{tabular}

Table 9.4 Participant groups from Studies One and Two and preferences for creating seating space

\subsubsection{Improve maintenance and cleanliness}

According to Herzog et al. (1976) and Nasar (1994), the most critical aspect for the human perception of a space is its cleanliness and maintenance. Rapoport (1982) also emphasised that cleanliness is a primary criterion for assessing the aesthetic preferences for space. In New Zealand, Gjerde (2017) found the public had a strong liking for buildings that are well maintained. Spaces that are dirty and a visual nuisance are those least remembered by the public (Lynch, 1960; Maran, 1976). Jacobs (1961) also argued that places which are not appropriately managed would have a higher tendency of encouraging crime. The results of Studies One and Two revealed that on average $17 \%$ of male participants liked the design attribute compared to fewer female 
participants (14\%), and participants aged $36+$ liked the attribute $(15 \%)$ more than young participants aged 18-25 (13\%). Medina (2009) also concluded that older people perceive a safe and maintained area as being more liveable compared to young people and would prefer to live there. Lastly, $23 \%$ of NBE participants preferred the design attribute compared to $15 \%$ of BE participants. Study Three also confirmed that it was the NBE participants who laid more stress on improving the maintenance and cleanliness of a leftover space by getting rid of rubbish bins, hiding a dumpster with screens, and cleaning up space. In contrast, BE participants did not separate improving maintenance as a problem as they were more interested in the overall design scheme.

A Chi-square test was performed for the preference association between young $B E$ participants (aged 18-35) for improving maintenance and cleanliness with NBE participants of all age groups (table 9.5). The first test revealed that the young BE and young NBE participants (aged 18-35) do not have the same opinion for improving maintenance and cleanliness. The result was not statistically significant at $X^{2}(3)=$ 17.77, $\mathrm{p}(09.91)>0.05$, and although $62 \%$ of young NBE participants (aged 18-35) supported the idea of improving the maintenance and cleanliness, only $38 \%$ of young BE participants wanted to do this. The second test found that young (aged 18-35) BE and middle-aged (aged 36-55) NBE participants have similar thoughts about improving maintenance and cleanliness. The result was statistically significant at $X^{2}(3)=02.36$, $p(0.044)>0.05$, although $57 \%$ of middle-aged (aged 35-55) NBE participants wanted to improve maintenance and cleanliness compared to fewer young BE participants $(43 \%)$. The third test looked at finding a relationship between young BE (aged 18-35) and older (aged 56+) NBE participants, but this was not statistically not significant at $X^{2}(3)=07.43, p(03.21)>0.05$, which means that young BE and older NBE do not think similarly, as $62 \%$ of older (aged 56+) NBE participants preferred the design attribute compared to $38 \%$ of young BE participants. The results confirmed that although participants have a preference for the attribute for improving maintenance and cleanliness, there was a difference in opinion between young and older NBE participants and young BE participants. 


\begin{tabular}{|c|c|c|c|c|}
\hline \multicolumn{5}{|c|}{ Improve maintenance and cleanliness } \\
\hline & Value & $\mathrm{df}$ & $\begin{array}{l}\text { Asymp. Sig. (2 } \\
\text { sides) }\end{array}$ & $\begin{array}{c}\text { Association } \\
\text { (between variables) }\end{array}$ \\
\hline \multicolumn{5}{|c|}{ Young (aged 18-35) BE and Young (aged 18-35) NBE participants } \\
\hline Pearson Chi-Square & 17.77 & 03 & 09.91 & - \\
\hline \multicolumn{5}{|c|}{ Young (aged 18-35) BE and Middle-aged (aged 36-55) NBE participants } \\
\hline Pearson Chi-Square & 02.36 & 03 & 0.044 & $\checkmark$ \\
\hline \multicolumn{5}{|c|}{ Young (aged 18-35) BE and Older (aged 55+) NBE participants } \\
\hline Pearson Chi-Square & 07.43 & 03 & 03.21 & - \\
\hline
\end{tabular}

Table 9.5 Participant groups from Studies One and Two and a similar preference for improving maintenance and cleanliness

\subsubsection{Remove boundary walls}

According to Sennett (2004), the boundary is an edge or a threshold where a wall or fence acts as an aspect of division or separation regarding the activity in and purpose of the space with which it relates (Klein, 2005). The security of space and who owns it also marks a boundary (Falah \& Newman, 1995). The results of Studies One and Two revealed that an average $66 \%$ of female participants liked this design attribute, which was more than male participants (54\%), and young (aged 18-35) liked the attribute $(30 \%)$ more than participants aged $36+(35 \%)$. Lastly, $59 \%$ of NBE participants liked the design attribute, which was slightly more than BE participants (55\%). Study Three also confirmed that both NBE and BE participants wanted to expand and open up space. The design suggestions were related to either lowering the height of the wall or removing the wall from the space in front of the building. However, Atlas and LeBlanc (1994) claimed that people and potential property buyers feel safer in living in or buying a place with a boundary.

The same Chi-square tests were performed to look for a relationship between young BE participants (aged 18-35) with NBE participants of all age groups for removing boundary walls (table 9.6). The first test revealed that young BE and young NBE participants (aged 18-35) have the same opinions for removing boundary walls. The result was statistically significant at $X^{2}(3)=03.35, p(0.048)<0.05$, and $53 \%$ of young 
BE participants supported removing boundary walls compared to $47 \%$ of young NBE participants. The second test found that young (aged 18-35) BE and middle-aged (aged 36-55) NBE participants do not have similar thoughts about the removal of boundary walls. The result was statistically found not significant at $X^{2}(3)=06.97, p$ (04.54) $>0.05$, although $52 \%$ of young (aged 18-35) BE participants supported the idea of removing the boundary walls as compared to $48 \%$ of middle-aged (aged $35-55$ ) NBE participants. The third test was not statistically significant at $X^{2}(3)=12.22, p$ (07.87) $>0.05$, which means that young BE and older NBE do not think similarly about removing the boundary wall, as $62 \%$ of young BE participants preferred the design attribute compared to $38 \%$ of older (aged $56+$ ) BE participants. The results confirmed that although participants have a preference for the attribute of removing the boundary walls, there was a difference in opinion between middle-aged and older NBE participants and young BE participants.

\begin{tabular}{|c|c|c|c|c|}
\hline \multicolumn{5}{|c|}{ Remove boundary walls } \\
\hline & Value & df & $\begin{array}{l}\text { Asymp. Sig. }(2 \\
\text { sides })\end{array}$ & $\begin{array}{c}\text { Association } \\
\text { (between } \\
\text { variables) }\end{array}$ \\
\hline \multicolumn{5}{|c|}{ Young (aged 18-35) BE and Young (aged 18-35) NBE participants } \\
\hline Pearson Chi-Square & 03.35 & 03 & 0.048 & $\checkmark$ \\
\hline \multicolumn{5}{|c|}{$\begin{array}{l}\text { Young (aged 18-35) BE and Middle-aged (aged 36-55) NBE } \\
\text { participants }\end{array}$} \\
\hline Pearson Chi-Square & 06.97 & 03 & 04.54 & - \\
\hline \multicolumn{5}{|c|}{ Young (aged 18-35) BE and Older (aged 55+) NBE participants } \\
\hline Pearson Chi-Square & 12.22 & 03 & 07.87 & - \\
\hline
\end{tabular}

Table 9.6 Participant groups from Studies One and Two and preference for removing boundary walls

\subsubsection{Other Preferred Attributes}

The other less preferred attributes are listed in table 9.7. A Chi-square test was performed for the two aspects that appeared in both Studies One and Two (i.e. create a clear pathway and install wind turbines and solar panels). 


\begin{tabular}{|c|c|c|c|c|c|}
\hline \multirow[t]{2}{*}{$\begin{array}{c}\text { Design } \\
\text { Attributes }\end{array}$} & \multicolumn{2}{|c|}{$\begin{array}{l}\text { Study one } \\
\qquad N=99\end{array}$} & \multicolumn{2}{|c|}{$\begin{array}{l}\text { Study two } \\
\qquad N=111\end{array}$} & \multirow[t]{2}{*}{$\begin{array}{c}\text { Study three } \\
\qquad N=9\end{array}$} \\
\hline & $\begin{array}{c}\text { Number of } \\
\text { pictures (six } \\
\text { types of } \\
\text { leftover } \\
\text { spaces) } \\
\text { chosen with } \\
\text { the attribute }\end{array}$ & $\begin{array}{c}\% \text { of } \\
\text { those } \\
\text { liking } \\
\text { attribut } \\
\text { e }\end{array}$ & $\begin{array}{l}\text { Number of } \\
\text { pictures } \\
\text { (six types } \\
\text { of leftover } \\
\text { spaces) } \\
\text { chosen } \\
\text { with the } \\
\text { attribute }\end{array}$ & $\begin{array}{l}\% \text { of } \\
\text { those } \\
\text { liking } \\
\text { attribut } \\
\mathrm{e}\end{array}$ & \\
\hline $\begin{array}{l}\text { Create a } \\
\text { clear } \\
\text { pathway }\end{array}$ & 6 & 23 & 03 & 56 & $\mathrm{~N} / \mathrm{A}$ \\
\hline $\begin{array}{l}\text { Install wind } \\
\text { turbines } \\
\text { and solar } \\
\text { panels }\end{array}$ & 4 & 11 & 01 & 64 & $\mathrm{~N} / \mathrm{A}$ \\
\hline $\begin{array}{l}\text { Remove } \\
\text { car park }\end{array}$ & 6 & 09 & $\mathrm{~N} / \mathrm{A}$ & $\mathrm{N} / \mathrm{A}$ & $\checkmark$ \\
\hline $\begin{array}{l}\text { Allow } \\
\text { graffiti on } \\
\text { the wall }\end{array}$ & 02 & 12 & $\mathrm{~N} / \mathrm{A}$ & $\mathrm{N} / \mathrm{A}$ & $\checkmark$ \\
\hline
\end{tabular}

Table 9.7 Other liked design attributes

${ }^{*}$ A tick indicates that the attribute emerged as a design theme in the analysis of focus group discussions

\subsubsection{Create a clear (pedestrian) pathway}

According to Broadbent (1990), pedestrian movement in cities is essential since people perceive their surroundings more when walking than when travelling in a car. Similarly, much earlier Jacobs (1961) emphasised that walkable streets are essential 
in making cities and that pedestrians know more about a city than drivers. The results of Studies One and Two revealed that on average $21 \%$ of female participants liked the design attribute compared to $18 \%$ of male participants, and $12 \%$ of participants aged $36+$ liked the attribute more than young participants aged 18-35 (10\%). However, McDonald (2008) found that pedestrian students have a positive relationship with the physical facilities near home and school, such as the presence of a park. Hill (1982) claimed that routes are chosen subconsciously, and pedestrians chose the shortest paths; however, females prefer fewer complex routes than males. Duncan \& Mummery (2007) found that safety was a critical aspect of route preference. Cheng \& Chen (2015) asserted that pathways should be designed to be continuous as people prefer direct routes. Abley (2005) claimed that the pedestrians have a higher preference for using streets with wider pathways, those where there are fewer vehicles and driveway conflicts, and those with a good surface material to walk on and with crossing opportunities. In this research, $29 \%$ of NBE participants preferred this design attribute compared to $21 \%$ of BE participants. However, Study Three failed to raise any design suggestions related to this attribute.

Chi-square tests were performed for the relationship between young BE participants (aged 18-35) for creating a clear pathway and NBE participants of all age groups (table 9.8). The first test for young BE and young NBE participants (aged 18-35) was statistically not significant at $X^{2}(3)=07.88, p(05.43)>0.05$, although $55 \%$ of young NBE participants (aged 18-35) supported the idea of creating a clear pathway compared to $45 \%$ of young BE participants. The second test for young (aged 18-35) BE and middle-aged (aged 36-55) NBE participants were also not statistically significant at $X^{2}(3)=10.56, p(6.67)>0.05$., although $62 \%$ of middle-aged (36-55) NBE participants supported the idea of creating a clear pathway compared to only $38 \%$ of young BE participants. The third test between young BE (aged 18-35) and older (aged $56+)$ NBE participants was also not statistically significant at $X^{2}(3)=08.56, p(07.33$ $>0.05$. This means young BE and older NBE participants do not think similarly, as $58 \%$ of older NBE participants preferred the design attribute compared to $42 \%$ of young BE participants. 


\begin{tabular}{|c|c|c|c|c|}
\hline \multicolumn{5}{|c|}{ Create a clear pathway } \\
\hline & Value & $d f$ & $\begin{array}{l}\text { Asymp. Sig. (2 } \\
\text { sides) }\end{array}$ & $\begin{array}{c}\text { Association } \\
\text { (between } \\
\text { variables) }\end{array}$ \\
\hline \multicolumn{5}{|c|}{ Young (aged 18-35) BE and Young (aged 18-35) NBE participants } \\
\hline Pearson Chi-Square & 07.88 & 03 & 05.43 & - \\
\hline \multicolumn{5}{|c|}{$\begin{array}{l}\text { Young (aged 18-35) BE and Middle-aged (aged 36-55) NBE } \\
\text { participants }\end{array}$} \\
\hline Pearson Chi-Square & 10.56 & 03 & 6.67 & - \\
\hline \multicolumn{5}{|c|}{ Young (aged 18-35) BE and Older (aged 55+) NBE participants } \\
\hline Pearson Chi-Square & 08.56 & 03 & 07.33 & - \\
\hline
\end{tabular}

Table 9.8 Participant groups from Studies One and Two and preference for creating a clear pathway

\subsubsection{Install wind turbines and solar panels}

This attribute probed participant preferences for using environmentally friendly, renewable energy sources in cities (Dihrab \& Sopian, 2010). Kanase-Patil et al. (2010) argue that a sustainable approach to energy consumption in cities is essential for not depleting the earth's resources. In this regard, solar and wind energy have the potential for becoming the primary urban sources (Juaidi et al., 2016), although wind does not work well at a small scale in urban areas since the wind velocity is reduced by neighbouring buildings (Shahrestani et al., 2015). Pedersen \& Persson (2007) found that wind turbines are appreciated as an environmentally friendly technology, which is seen as necessary and efficient but also as ugly and as a potential noise annoyance. However, Toja-Silva (2013) claimed that Vertical Axis Wind Turbines (VAWT) characteristics are applicable to urban settings since they look aesthetically pleasing, generate less noise, and are not influenced by a change in wind direction. According to Mohajeri (2018), photovoltaic (PV) equipment and thermal solar collectors have low maintenance and no noise and are useful for producing clean energy in cities as they can be placed on buildings. The data from Studies One and Two revealed that on average $31 \%$ of male participants liked the design attribute 
compared to $26 \%$ of female participants, and $37 \%$ of participants aged $36+$ liked it compared to $35 \%$ of young participants aged $18-35$. Lastly, $29 \%$ of NBE participants liked this design attribute compared to $27 \%$ of BE participants. However, Study Three did not raise this attribute as an issue.

The same Chi-square tests were performed (table 10.6). The first test revealed that young BE and young NBE participants (aged 18-35) do not have similar opinions for installing wind turbines and solar panels, as the test was not statistically significant at $X^{2}(3)=07.77, p(4.79)>0.05$. However, $55 \%$ of young NBE participants supported the idea of installing wind turbines and solar panels compared to $45 \%$ of young $B E$ participants. The second test was also not statistically significant at $X^{2}(3)=06.89, p$ (3.36) $>0.05$. However, $58 \%$ of middle-aged (aged 36-55) NBE participants supported installing wind turbines, and solar panels compared to $42 \%$ of young BE participants. The third test was also not statistically significant at $X^{2}(3)=09.32, p(6.69)>0.05$, which means that young BE and older NBE participants do not think similarly, as $53 \%$ of older NBE participants preferred the design attribute compared to $47 \%$ of young BE participants.

\begin{tabular}{|l|l|l|l|l|}
\hline Installing wind turbines and solar panels \\
\hline & Value & do & $\begin{array}{c}\text { Asymp. Sig. (2 } \\
\text { sides) }\end{array}$ & $\begin{array}{c}\text { Association } \\
\text { (between } \\
\text { variables) }\end{array}$ \\
\hline Young (aged 18-35) BE and Young (aged 18-35) NBE participants \\
\hline Pearson Chi-Square & 07.77 & 03 & 4.79 & - \\
\hline Young (aged 18-35) BE and Middle-aged (aged 36-55) NBE \\
pearson Chi-Square & 06.89 & 03 & 3.36 \\
\hline Young (aged 18-35) BE and Older (aged 55+) NBE participants \\
\hline Pearson Chi-Square & 09.32 & 03 & 6.69 & - \\
\hline
\end{tabular}

Table 9.9 Participant groups from Studies One and Two and preferences for installing wind turbines and solar panels 


\subsubsection{Remove car park}

The European Commission (2004) has affirmed that the quality of life in cities is negatively associated with the usage of vehicles, as these create noise, vibrations, visual intrusion, congestion, air pollution, local temperature rises, loss of urban living space, health-related issues, and roadside accidents. They also found cars to be inefficient in economic terms. Putnam (2000) argued that higher vehicle usage lowers interaction through the loss of contacts in the public realm.

Similarly, Homer et al. (2004) found that the use of an increased number of cars in the city has a positive correlation with land use and temperature rise. Study One of this research revealed that $17 \%$ of male participants preferred removing a car park from the leftover space compared to $8 \%$ of female participants, while $7 \%$ of participants aged 36+ liked the attribute more than young (aged 18-35) participants (06\%). Lastly, $13 \%$ of NBE participants preferred the design attribute compared to $11 \%$ of $\mathrm{BE}$ participants. The European Commission (2004) also found that air pollution is significantly less in areas that had a road closure. Nieuwenhuijsen et al. (2014) argued that cities are overly burdened with cars and car park spaces that could be strategically used to provide more vegetation and that this could improve the health and well-being of people. Study Three also found that both NBE and BE participants laid stress on removing the car parks to create more space for the public. The participants suggested making small car parks for fewer cars or merely getting rid of the car parks.

\subsubsection{Allow graffiti on the wall}

Coleman (1985) argued that graffiti is a kind of vandalism that diminishes the value of a property and promotes crimes, whereas Tristan (2010) called urban graffiti art. In this research, graffiti could be seen as a form of street art but also as a form of vandalism (Ivenson, 2010). As an artistic expression of inquiry, graffiti can represent emotions about current affairs through the medium of art (Madison, 2005). Feireiss (2010) claimed that the artists who make these interventions create their own space and environment within the city. Study One revealed that $20 \%$ of female participants liked this attribute compared to $15 \%$ of male participants, and $60 \%$ of young (aged 1835) participants liked the attribute more than participants aged $36+(40 \%)$. Lastly, $21 \%$ of BE participants liked the design attribute compared to $18 \%$ of NBE participants. 
Study Three also confirmed that BE participants were keener to allow graffiti on walls than NBE participants. BE participants suggested leaving some walls for graffiti while NBE participants wanted to murals on walls and sculptures in empty spaces.

\subsection{Discussion}

Lynch (1981) claimed that a good city is one in which the continuity of the complex environment is maintained while dynamic change is allowed. A report by the Prosperity Index (2016) showed that cities that enhance the quality of life for their citizens tend to have higher levels of prosperity; they are likewise potentially able to make more progress in terms of sustainability. Florin (1989) suggested that citizen participation in community organisations has been an important method for improving the quality of the physical environment, enhancing services, preventing crime, and improving social conditions. Wellington's success as a city relates closely to the quality of its built environment (Wellington City Council, 2014). Wellington Future 2040 (2015) has strategised the concept of urban development with Wellington City Council in order to make a smart capital that includes the themes of being an eco-city through making a low-carbon future based on 'green' innovations, a connected city, a people-centred city, and a dynamic central city. However, the value of leftover spaces that could become an asset for strategic use has been ignored.

As this research has demonstrated, the design attributes preferred by participants were related to providing more vegetation, changing the surface material or colours, creating seating space, and improving the maintenance and cleanliness of leftover spaces. These emerged as the most preferred attributes for five of the six leftover spaces, while for the space in front of a building removing the boundary walls was the most preferred. However, to generate appropriate design proposals, it is essential to overcome the barrier of user rights as someone must own these leftover spaces, and to reference the context. The ownership and stewardship of semi-private leftover spaces could become an essential consideration once car parking in the city is limited, which must happen if the city is to move towards a 'green' future.

The findings of this research that people want more vegetation represents an opportunity to create restorative environments (RE) within cities. The significance of vegetation can be dated back to ancient times such as horticulture being used to calm 
human senses (Shepard, 1991) and garden walks being used to recover from mental illness (Sachs, 1999). Ulrich et al. (2008) argued that natural healing spaces improve the recovery of patients. Natural green spaces have beneficial effects on multiple dimensions of health and wellbeing, including reducing mental fatigue (Hartig et al., 1991) and rates of mortality (Mitchell \& Popham, 2008).

Restorative environments relate to different levels of naturalness in a setting, which influences human preferences and behaviours. The two concepts of RE were developed some 30 years ago by Ulrich (1983) and Kaplan \& Kaplan (1989), these being respectively the psycho-evolutionary approach and attention restoration approach. The psycho-evolutionary concept posits that stress is reduced in individuals by a natural environment that also creates feelings. These feelings are tied to aesthetics and visual preferences for natural settings that also affect human behaviour (Ulrich 1993). Furthermore, the parameters that affect the aesthetic responses to a scene are related to depth, surface material/texture, security/threat, focal point, complexity and structural elements (Ulrich, 1993). Ulrich (1993) claimed that positive emotions are aroused in the nervous system, and the emotional state of a human being is improved in a natural restorative environment. Park et al. (2007) concluded that respondents who spent time in a forest environment became more relaxed and stress-free than those in a built environment. Pals et al. (2009) examined the relationship between restorative environmental components and landscape preferences in a zoo. Visitors were asked to rate their preferences of restorative sights in a Netherland's zoological park. His findings confirmed that people preferred spaces with a natural setting that was visible to them.

The attention restoration concept is concerned with the two interlinked psychological processes of perception and attention (Kaplan \& Kaplan, 1989). Human attention is subjective, and environments that raise stress levels affect decision-making capabilities (Kaplan 1995). Thus, the two terms of direct attention and involuntary attention, also known as 'fascination', are vital in understanding the attention restoration concept. Direct attention is when a person focuses by resisting ongoing distractions, while involuntary attention is caused without any effort and relates to excitement or arousal of a person's feelings. The feelings are the result of visual stimuli (Kaplan, 1995). Attention restoration postulates that an individual who experiences a restorative environment by spending time there can perform and focus better. 
Although restorative environments can vary and can happen in a built setting, natural environments are generally superior restorative environments. Tennessen \& Cimprich (1995) concluded that students living in a dormitory room with a natural view from their windows outperformed those who could only see a built context. Herzog et al. (2003) rated 70 different settings in his study and concluded that the participants had a higher mean rating for a natural environment than for an urban setting. Laumann et al. (2001) investigated the two different environments of natural and cityscape on a set of rating scale measures, and found the natural setting yielded higher preference scores than the built environment. Both theories were developed separately, but the importance of them is that they show visual stimuli play a significant role in natural restorative environments as a means of arousing feelings and promoting the human-nature association to support health and well-being.

This research has shown that leftover spaces could be used effectively and contribute positively to the 'image' of the city rather than being seen as empty and neglected as at present. They have the possibility of being used in diverse ways by the general public. Therefore, it is imperative to analyse and capture the complex spatial condition of places and the spatial relationship between these and their environment. Accessibility is a fundamental aspect of determining the quality of public space (Langstraat \& Van Melik, 2013). Jacobs \& Appleyard (1987) have declared that good urban space is space that provides excellent accessibility to the public. Carr et al. (1992) claimed that there are three types of accessibility and these are related to visual, physical, and symbolic access. According to Pirie (1979), accessibility changes human behaviour in terms of the use of space and is vital to generate functions. Gehl (1987) argued that space fulfils its purpose if it is welcoming and accessible to people. The usability of space is dependent on how people perceive space as being accessible, readable, safe, comfortable, and functional Bittencourt et al. (2015). Nissen (2008) posited that the usability of public-private spaces is a concern for cities. Similarly, Madanipour (2003) emphasised the need to blur the boundaries of publicprivate realms through their usage, arguing that this presents a challenge for developing cities. However, private spaces that are accessible to the public can enhance social networks and the quality of urban living (Kayden, 2000). The concept of easements or covenants (Cross et al., 2011) could be applied where the owner of the private land agrees with another party to allow the property to be used in a 
particular manner. Easements can be struck to enable the full potential of land or a roof to be realised by an interested party while ownership is not affected. Public-private spaces within cities could be controlled and designed by residents, associated local users, and the city council.

Through urban regeneration, Wellington City Council could make effective use of leftover spaces by enabling 'grow-in' strategies. Ways to enhance urban productivity as a means of promoting sustainability could include energy production and community gardening in leftover spaces, but unless the public see these as positive interventions, they are unlikely to be successful. According to the tactical urbanism movement, when people improve urban design and bring about a positive change in their surroundings, cities become more sustainable (Planning Tank, 2016). Small changes to urban leftover spaces might be the first step in this. This approach could become a deliberate, phased approach to instigating change by offering local ideas for local planning challenges, short-term commitment and realistic outcomes, and is low risk but with possibly high reward (Pfeifer, 2013).

The tactical approach offers low cost, flexible processes whereby the urban landscape becomes an urban laboratory in which people can test out their diverse ideas. Making gradual changes in leftover voids could create an opportunity for people and planners to explore and experiment with multiple ideas. This approach has mainly two types. Temporary interventions are lighter, quicker, and cheaper but remain practical tools for producing better spaces within cities (Turner, 2013). Although many of these projects may be small in scale, such as makeshift seating along streets and tiny parks, others have changed entire parking lots and roads into temporary green spaces providing people with a vision of what is possible (Voigt, 2015). It is fair to say these bottom-up approaches may prove to be a coordination challenge for municipalities and other agencies, but these approaches could potentially coincide with long-term planned strategies and exist for at least or less than year.

The second type is the 'planned' approach, which implies strategically transforming spaces and focuses on the enhancement of projects such as infill development and the creation of guidelines. However, the budgets for permanent projects run much higher and require a longer and more complex process. One of the biggest challenges faced by the strategically planned method is how to engage the community effectively 
(Voigt, 2015). The concept is mainly a collective one in which local people need to identify the options for taking small steps or initiatives rather than bringing in fundamentally new forms of transformation on a larger scale. Projects like Letting space, City Lab, Street-Plans New York, Do-It-Yourself urbanism, and City repair all emphasise the importance of empty spaces by designing spaces which can contribute to making places that are more enjoyable for the public. As such, the urban landscape should not be viewed as something static, but as something vital with the need to achieve a coherent design solution at each change point that is considered beneficial for the community.

Although many leftover spaces represent a threat to safety and security, they also represent a positive potential if they can be appropriately incorporated into an active urban realm. Benedict \& McMahon (2003) claimed that the two concepts that are crucial in strategising the notion of green infrastructure towards more sustainable cities are the physical context and visual connectivity. Bishop \& William (2012) supported the idea of improving life in cities through activities and physical attributes that could be carried out in unused or leftover spaces to make them appealing. This research has built on this idea by focussing on participant preferences for different attributes in the design of leftover spaces. The researchers Tinsley et al. (2002), Hull \& Michael (1995), Wilkinson (1991), Shanahan et al. (2015), Godbey et al. (1992), Tyrvainen et al. (2014) and Saraev (2012) have all argued that spaces with vegetation provide significant benefits as these have a positive impact on both the environment and health-related problems, improve physical activities, reduce mental stress, boost economic development, build social cohesion, and enhance biodiversity. A study by Ulrich (1983) found that natural environments are usually evaluated as having a higher rate of aesthetic quality over built environments. At the same time, Ulrich's study also suggested that incorporating vegetation on a horizontal surface or ground is not always practical and can be expensive since it comes with maintenance and care or stewardship issues. Bjerre (2011) also pointed out that the green vertical wall system does not require the soil but an optimum storage of water and minerals, which is required to be maintained appropriately through consistent inspection. This research also raised the same problem of managing spaces with vegetation in them. Wellington City Council has an initiative of planting 2 million trees in Wellington city by 2025. The strategy is about providing free plants to local communities and local partners to grow 
trees on the town belts, roadsides and reserves. Perhaps urban leftover spaces could be part of this initiative?

The visual quality of a space can influences a person's experience significantly because people react to what appears before them, and the use of colours can affect their mood (Weller \& Livingston, 1988). Rapoport (1982) argued that people see differences in an environment through colour contrast. This research found that a dull and gloomy space was the least preferred (see section 6.3.1.3.2) whereas the transformation of the same space with new materials or colours was rated higher by participants in Study Two (see section 7.4.1). Although the opinions differed over the choice of colour or material, the participants agreed that leftover spaces could become more attractive, energetic, and less tedious with the application of new materials or colours. Another approach would be using living wall system, this being a green façade that requires less space as it is vertical planting and can even be in the form of lightweight, permeable screen (Manso \& Castro-Gomes, 2015). Alternatively, climbers could be planted to grow up the elevations of buildings. This would provide an aesthetic improvement, and with the right choice of the plant could lead to sound reduction and better insulation of the building envelope (Rakhshandehroo et al., 2015).

Whyte's (1980) study of a plaza in Vancouver found that seating space is the most significant attribute of public space. Siu \& Wong (2015) asserted that social interaction is enhanced by providing street furniture. Similarly, Lesan (2015) asserted that the sense of comfort and relaxation in an environment is supported by its physical attributes, including street furniture such as seating, the width of the pedestrian pathway, shade, and natural features. Hass-Klau et al. (1999) claimed user needs and desires should be met for relocating and orienting seating space, as some people might want to be hidden away while others might want to be people watchers. Gehl (2010) claimed that adults and senior people are more decisive and concerned about sitting in a comfortable space for a short or long time, whereas children and young people can sit anywhere regardless of comfort levels or choice of material.

Furthermore, Gehl (2010) found that people prefer sitting if space has an excellent microclimate, the seating has a backrest, and there is a good visibility and low noise pollution. Although seating spaces depend on the users and their preferences for different activities, providing benches, steps, and ledges in semi-private leftover 
spaces could be a way of providing social and psychological comfort (Carr et al., 1992). Leftover spaces could act as a connecting element, retaining and building local patterns of the use of open, in-between space.

Tibbalds (2001) pointed out that areas like car parks, paved surfaces, street furniture, building facades, and parks need to be maintained for a healthy lifestyle. Nasar (1988) confirmed that up-keep is a primary physical aspect and that the public prefers maintenance over safety concerns. Spaces that are not maintained are undesirable. A report by UN-Habitat Urban October (2015) claimed that well-maintained spaces improve safety and enhance the physical and mental well-being of the public. The issue of maintenance and keeping the space clean is associated with municipalities, as it is often seen as their responsibility. It is also crucial for the communities or citizens to work with local government and the private sector to attract investment and increase property values and revenue by being part of the upkeep of leftover spaces.

Respondents in this research also exhibited preferences for visual openness, as evidenced by the scores for the removal of a boundary wall in front of a building. This suggests that building entrances should be designed to be open and inviting and that areas that are perceived as claustrophobic should be avoided. Grant \& Mittelsteadt (2004) emphasised that boundaries serve many functions in a society and also form a critical element of the city as they create a visual barrier, act as a means of privacy or identifying a property line, make a social barrier, and constrain an entrance through having high or short walls. Replacing boundary fences with a permeable material can have an impact on the use of leftover space by making it look broader, as Hilty et, al. (2006) have argued that impermeable fences can reduce connectivity and the ecological processes that go with this.

Accessibility is the basic and essential aspect of determining the quality of public space (Langstraat \& Van Melik, 2013). Similarly, Gehl (1987) and Jacobs \& Appleyard (1987) have declared that good urban space is the one, which provides excellent accessibility and can be used by the public. Carr et al. (1992, pp. 138-151) claimed that there are three types of accessibility are related to visual, physical, and symbolic access. The application of distinct colours with appropriate materials and the use of different signage could define and revitalise leftover spaces. Carmona et al. (2010) 
argued for creating accessible pathways which enable the public to stop for certain activities along the way and provide for casual or formal interactions.

Energy Gardens also have potential since they generate new biomass through the planting, but whether this would be appropriate for small-scale leftover spaces is an issue. Renewable energy sources, except large scale hydro, are widely dispersed compared with fossil fuels. Hence, renewable energy must be used either in a distributed manner or concentrated in a form that meets the energy requirements of the urban sector, including industrial areas (Climate Change, 2007). The Global Energy Report (2017) stated that the building sector in the city consumes $35 \%$ of global energy and emits $40 \%$ of energy-related carbon emissions. According to the International Energy Agency (IEA) (2009), micro-grids have a high potential for generating electricity for each building according to its need. Local-scale energy production is more cost-effective than large scale projects. Integrating solar photovoltaic (PV) panels with the roofs and facades of buildings at the design-andbuild stage is a growing development in many cities, providing such areas are in full sun and not overshadowed. PV systems capture the solar radiation and generate electricity, and any surplus can be fed into the grid, which can generate revenue. The IEA (2009) found that $10-30 \%$ of the total electricity demand can be met by installing PV systems on the rooftop of a building within urban centres. These solar PV cells vary in efficiency, performance, and price, and their annual output depends on the local mean annual solar radiation levels. Wind energy also has potential in cities, and vertical axis wind turbines on poles could be placed on rooftops or in spaces open to the sky. Micro wind turbines have the capacity of providing enough electricity for a single dwelling (IEA 2009) but not generally in an urban context.

European cities are aiming to reduce car usage and encourage commuters to shift to more energy efficient transport modes (Steg, 2007). Similarly, Loukopoulos et al. (2004) argued that vehicles should be prohibited from entering specific areas of a city and putting limitations on car parking spaces can have a positive impact on health and the environment. Car-pooling or car sharing is desirable as this can decrease congestion, reduce the demand for car parks, and reduce urban Green House Gas (GHG) emissions. According to the Wellington City Council Resident Monitoring Survey (2013), Wellington is compact, but almost $33 \%$ of people who work in the central city use private transport, i.e. car or bikes, for commuting. Currently, the council 
is planning to make the city more pedestrian friendly. In 2013, for every 100 Wellingtonians taking a car into the city, only 90 people used public transport, walked, or biked (Foster, 2015). Developers are incentivised to provide adequate parking in order to maximise the value of their investment. However, car ownership is also decreasing on a per-capita basis across the city (Information Demographics, 2019). These figures imply a usage pattern in which leftover spaces are currently being under-utilised for car parking during the day and then remain empty at night. This means they do have the potential for different use. The highest concentration of nocar households is in the central business district and the immediate surrounding suburbs. This research has shown that the removal of car parking completely or limiting car parking was something participants found desirable.

Another use of leftover spaces could be related to collecting or storing rainwater, which can be further used to cool buildings in hot periods, or for watering urban vegetation. Urban areas tend to exhibit higher temperatures than their rural surroundings, which can, in certain conditions, lead to thermal discomfort and impacts on human cardiovascular and respiratory systems. During the day, as air temperatures increase in poorly ventilated buildings, more energy is consumed to cool these spaces. In cities, the micro-urban hot spots are formed at parking lots, asphalt roads, and on nonreflective roofs. Hence, assessment of this so-called Urban Heat Island (UHI) effect and development of strategies by which leftover spaces can be used to lower local temperatures could be helpful. For Wellington, two main UHI reduction strategies could be applied to leftover spaces. The first is to increase surface reflectivity to reduce radiation absorption. This can be done by placing light coloured panels or paint on existing surfaces. This technique can be applied roofs to reduce energy demand for cooling as well on pavements.

The second is to increase vegetation cover to control temperature rise. Trees form a beautiful canopy, shading the area and have a cooling effect. Trees are also a mediator between nature and human beings while increasing biodiversity within cities. Another way is to create community gardens, which is a small scale and low investment method of growing organic vegetables and fruit. By installing more trees and creating community gardens in leftover spaces, the environment could be cooled while allowing biodiversity to flourish. Nordh et al. (2009) also found that small urban parks provide an opportunity for psychological restoration with a positive association 
between nature and health. To make a coherent design scheme, it is also possible to use attributes together. However, the most recurring design attribute of providing vegetation should be given priority in any future strategy for Wellington. As Nelson and Stolterman (2003) have argued, the environment is flexible enough to accommodate any design change; however, discovering the 'right' solution is critical. The solutions suggested in this Chapter could make a positive difference and contribute to the environment while enhancing the aesthetics of the city.

\subsubsection{Similarities with Nasar's framework}

Nasar (1998) evaluated people's responses to improving the visual appearances of the two cityscapes of Knoxville and Chattanooga, Tennesse. His concept of the evaluative image of the city is an extension of the work of Lynch (1960) on cognitive maps. Nasar's evaluation incorporated the psychological domain of feelings about environments. His research involved two variables in measuring the subjective quality through the visual representation of different aspects and evaluative responses. The evaluative responses referred to "favourable emotions and meanings experienced about the environment". His theoretical framework draws out the two concepts of cognition and perception. This notion aligns with Study One of this research, where the perception of built environment features and cognition (affective appeals) are studied, and in Study Two it plays a crucial role when assessing spaces visually (see section 3.5).

Nasar's theory highlighted five significant environmental features that participants liked. These were order, upkeep, historical significance, openness, and naturalness. In this research, Study One evaluated built environmental features based on Nasar's theory, which resulted in the same responses except for historical significance. Study One confirmed the validity of visual representation and environmental features. The key theme of this research aligns with Nasar's theory regarding aesthetics and likeability as environmental preferences. According to Nasar, the preferred attribute of participants (nature) may hold a connotative meaning. Nasar argued that natural (resortative) elements could be predicted and the increase in built elements is a disliked feature of cities. This finding correlates with the results of this study, where participants preferred to see vegetation in leftover spaces, followed by their cleanliness. 


\subsubsection{Differences with Nasar's framework}

The scale of this research differs from that of Nasar since this research has focused on private-public leftover micro scale spaces within Wellington City, whereas Nasar's comparison was an evaluation of responses regarding the perception of two cities on an urban scale with no clear distinction between public or private spaces. Additionally, his studies of two cities can be generalised to the whole population due to the substantial number of responses, whereas this is not possible with the limited responses in this research.

Comparing the methodologies, there is a noticeable difference in the approach to collecting and analysing the responses. Nasar's method focused on extracting oral and written descriptions from respondents and conducting telephones interviews. The descriptions were then transformed into individual evaluative maps. Finally, all maps were amalgamated to make a composite map to indicate how likeability could change the evaluative image of a city. However, the method used for this research focuses on using photographs (like Nasar's research) that were then photomontaged in a second study as a predictor of preferences for different design attributes. Participants in this research were not asked to respond by writing their preferences in the form of descriptions but rather to select from alternatives, with information being collected using online surveys and focus group discussions.

\subsection{Summary}

Chapter 9 has compared the results of all three studies and tested the strength of liking for an attribute based on the different age groups. It appears that providing vegetation is seen as the most necessary design attribute for leftover spaces. The Chapter also highlighted the significance of attributes for the design of leftover spaces that could make Wellington more sustainable and could become part of the 'Smart Capital Strategy' in future. Chapter 10 discusses the conclusions that are drawn out from this research by answering the research question and discusses the contribution to knowledge contribution made by this research. 


\section{CONCLUSIONS}

\subsection{Introduction}

This research has aimed to identify the most preferred design attributes for different types of leftover space in Wellington. The research was based on the idea of making better use of semi-public leftover spaces in an urban context and the importance of people's perceptions of such spaces when it comes to redesigning them. QamaruzZaman et al. (2012) claim that the adequate use of planned or unplanned space is crucial in a city as such use contributes to a sense of security and surveillance and defines interactions within a society. To fulfil the research aims a survey of what people felt about semi-left-over spaces and their design attributes were undertaken. This survey was split into a pilot study and three main investigations, each building on the results of the previous one. The research also investigated the consistency of preferences between gender and the built environment and non-built environment participants. In this Chapter, the findings are outlined as they relate to answering the research question, the significance of the research and the results of the three studies are explained, and how these results contribute to the theory of environmental psychology. The limitations of this research are discussed together with recommendations for further research.

\subsection{Answers to the Research Questions}

10.1.1 What are people's visual preferences for urban leftover spaces in Wellington City?

According to Kaplan \& Kaplan (1983) and Nasar (2000), the cognitive process involves the perception of a visual attribute based on which an individual evaluates the environment. Consequently, this research has concentrated on finding those attributes that could enhance the aesthetics and usability of space from the public's perspective using appropriate photographs and photomontages. The studies found that people prefer spaces that are clean, well-maintained, open to the sky, organised, have natural elements, and that has a modern outlook. However, the participants had issues with 
spaces were, in order of decreasing preference, there was no vegetation, there was a car park, space had dull surface materials or colours, and spaces were untidy and dark with dead ends. The research showed that the participants wanted to see small changes that could become a valuable addition in the underutilised spaces in Wellington City through their evaluation of different attributes for designing leftover spaces. Participants wanted spaces with attributes that would make the space less ugly and look more user-friendly. They wanted to see more creativity in the design of such spaces to make them visually attractive and enjoyable, including adding vegetation and seating. Participants also wanted to remove the car parks from the city as they see it as a hassle. However, a concern was raised regarding the ownership of spaces as thus may mean residents will want the car parks kept.

The following three sub-questions were formed in support of the main question;

\subsubsection{What attributes do people prefer for the design of urban leftover} spaces?

As noted above, the studies revealed that people have a common preference for providing more vegetation. This attribute was the most recurring design solution for each type of leftover space. Studies Two and Three confirmed that installing different types of plants such as trees, shrubs, climbers, and ground cover was liked by all participants. Participants recommended designing a community garden with grass and trees in the spaces enclosed by buildings on two or three sides and a natural green space for people to sit in for space at the back of a building once the car parks were removed. The only concern related to more vegetation (both for planters and green walls) was about maintaining the space. The solutions of providing more vegetation was attractive however, the maintenance of public-private spaces needs to be acknowledged that depends on the owner's good will.

Providing more vegetation also could help in mitigating adverse climate change effects and enhancing urban life by providing environmental and social services (Robinson \& Lundholm, 2012). Ulrich (1986) found that vegetation affects the psychological and perceptual function of the brain. Nelson (1976) argued that the form, colour, and texture of urban vegetation affects the emotional qualities of people when it comes to aesthetic preferences. The participants saw having vegetation as a way of increasing 
the user's feelings for space, gaining the attention of users and alleviating negative visual impacts through having properly designed schemes.

Participants also wanted to change the surface materials/colours, which was the second most mentioned attribute for the redesign of leftover spaces. The participants wanted to paint the surfaces with light but bright colours and replace the general hard and dull surface materials with timber to give texture and warmth to the feeling of the space. Moughtin et al. (1995) argued that the form and colour of a façade affect its perception. Gjerde (2015) found that the surface qualities of a cladding material give an insight into the durability of a building. Gatz \& Achterberg (1976) claimed that the exterior of a building must be associated with its context since the emotional quality of happiness is attached to it. However, participants had different views over applying materials or colours, so no one solution would satisfy all their desires. The next most preferred attributes were related to creating seating spaces, improving the maintenance and cleanliness of the space, and removing boundary walls from the space in front of a building. Participants mentioned that having seating spaces would freshen up their mood while improving the maintenance and cleanliness would attract more people to use the space. Removing the boundary walls would open space and even replacing the walls with visually permeable fences would work as participants could see what activities were happening at the end of the street or inside the building. The spaces that were disliked had dead ends, blank walls, unorganised car parks, unmanaged space, and were dark spaces without lighting.

\subsubsection{Are there any differences in design preferences between male and female participants?}

The male-female dichotomy is crucial for any society as understanding this can lead to information processing strategies for creating and maintaining living conditions (Bem 1981). Gary (1992) postulated that the genders have different approaches, needs, motivations, and values that need to be acknowledged.

\subsection{Findings related to Female Participants}

This research found the most recurring preferred design attributes for female participants related to providing more vegetation, creating a clear pathway and removal of boundary walls (appendix table 9.1). The study by Roe et al. (2013) found 
that green spaces are necessary for females to lower their stress levels in the built context. Females are also more concerned about environmental health (Mohai, 1992; Hunter et al., 2004) and its negative effect on their families (Seager, 1993). A good atmosphere provided by a variety of planting and attractive seating spaces in a park are perceived highly by females (Harth, 2014). Females are also affected emotionally by the attractiveness of parks and spend more time in a park for exercise than males Krenichyn (2006). Furthermore, Gladkikh et al. (2015) in a study of public squares, found a large percentage of female participants preferred spaces that had vegetation in them, and this was more than male participants.

A report by Women's Safely in Public Transport (WSPT, 2015) highlighted the importance of clear and continuous routes for females to travel safely. Studies by Galea \& Kimura (1993) and McGuiness \& Sparks (1983) highlighted the capability of males to understand a more complicated route accurately, whereas female participants follow direct routes. Ahmed (2014) and Krenichyn (2006) argued for enhancing visibility in parks for female users to overcome safety threats related to crime and harassment. Nordh \& Otsby (2013) found that female respondents chose good seating and a calmer atmosphere with views as an important element within a context of the park. The findings of this research regarding female preferences align with the existing literature, since they preferred vegetation and a clear pathway.

\subsection{Findings related to Male Participants}

This research concluded that the most design attributes for male participants related to change of surface materials/colours and to improved maintenance and cleanliness (appendix table 9.1). According to Stamps (2000), three characteristics of the built environment that arouse interest relate to silhouette, surface texture and the relationship of form. The surface colour and the textured pattern of a material arouses feelings for liking something on both cognitive and subconscious levels (Gjerde, 2010). Moser et al. (1985) found that males have a higher tendency of detecting colour anomalies and are more assertive for wanting changes (Mccoby \& Jacklin, 1974). Abramov et al. (2012) concluded that males are more visually sensitive to hues and prefer blues when it comes to colour choice (Ellis \& Ficek,2001).

However, the fact males in this study preferred the design attribute of improving maintenance and cleanliness is in conflict with the existing literature. Kwiringira et al. 
(2014) concluded that both males and females have equal preference for cleanliness. However, females are more concerned about hygiene and cleaning (Ocal et al. 2018), but males are more efficient when it comes to mechanical repairs and maintenance (Hannan et al., 2002). Similarly, O'Brien (2005) reported that females feel safer to walk in spaces that are well managed and organised. Jiggins (1994) deduced that women play a more significant role in caring for the environment and maintaining nature and farmlands. Moreover, Akwa (2009) argued that the female concern for cleanliness could be seen as the result of day-to-day household duties, which still tend to be done more by women than men. The difference between the study results and the literature could have occurred since maintenance is about repairing and fixing (Pukitea, 2017), while cleanliness is about maintaining hygiene. Other issues of the small sample size and the skewed distribution of the demographics towards the young might have resulted in this difference of opinion.

\subsubsection{Are there any differences in design preference between} participants with a built environment background and those without?

According to Gifford et al. (2000), architects have a different approach to perceiving and differentiating the physical environment from non-built environment professionals. Similarly, a study by Nasar and Devlin (1989) found that architects and other professionals have a difference in opinion when it comes to liking the design of houses. However, the findings of this research showed that the built environment and non-built environment participants have the same preferences for designing leftover spaces. Gjerde (2015) also found that the opinions of lay people and built environment professional overlapped with each other. However, in this research, there were differences in their priorities for designing a space, as built environment participants were more enthusiastic about transforming the overall space. The built environment participants were concerned about the appropriate usage and aesthetics of the space, whereas the non-built environment participants wanted to add or remove small objects from within the space and were concerned about making space more visually pleasing. It was also found that both groups wanted to combine more than one attribute for achieving a better design solution and hence, aesthetics of the space.

The most desirable solution for redesigning leftover spaces was related to providing more vegetation and changing the surface materials/colours. Built environment 
participants had a higher preference for the attribute of providing more vegetation than non-built environment participants while changing the surface materials/colours were preferred equally. Built environment participants wanted to see bold colours whereas non built environment preferred lighter and calmer colour tones.

Study One results showed that built environment participants have a higher preference for enclosed spaces to ensure their usability, and for modern buildings that were of good quality materials and suitable colours. The same group also preferred the attributes of creating graffiti on walls and removing boundary walls to make spaces more inviting. However, non-built environment participants had a higher preference for spaces that were well managed and organised. The other design attributes that were rated higher by non-built environment participants were related to removing the car parks, creating seating spaces, providing more shade, improving the cleanliness and maintenance by putting up screens, and creating clear pathways. The attribute of installing more vertical axis wind turbines and solar panels in open spaces and on the rooftop of the building was also rated higher by non-built environment participants. Hoffman (2008) found that people support installing small-scale community based or individual household wind turbines, whereas Khan (2018) reported that most people prefer using solar panels on the roof of their home. However, the cost of panels and lack of information are the main factors behind the low usage of this technology (Zhou et al., 2107).

\subsection{Contribution to knowledge}

\subsubsection{General Findings}

This research adds to the understanding of leftover spaces, which is a term used in this thesis for spaces created in sprawling cities. These spaces are underutilised and create semantic confusion with different names associated with them such as liminal, terrain vague or interzone. The literature focused on discussing the issues related to leftover spaces and the potential of designing these spaces, but no previous knowledge exists regarding the preferences the public might have when it comes to the better utilisation of these spaces. In this research, the leftover spaces were divided into the two levels of macro and micro, to define spaces that exist between and underneath or on the rooftop of a building. Furthermore, the research categorised the 
leftover spaces at a micro level into six different types found in Wellington City. These were underneath a building, in front of a building, at the back of a building, enclosed by buildings on both two and three sides, and lastly on the rooftop of a building. The study focused on semi-private spaces that exist between the private and public realms of social space and that are defined through the symbolic and physical borders of the building and street. These spaces exist as a threshold or intermediate area and are underutilised in Wellington City mainly due to existing property rights or just lack of interest.

This research into exploring the potential of urban leftover spaces has probed people's preferences for designing such spaces in the context of Wellington City. It has shown which design attributes people like and which are less favoured. Differences and similarities in people's perceptions of the built environment can be found in the literature (see 3.5), but little is known about the perception of leftover spaces and what attributes of such spaces people find favourable. This research also compares the preferences of people with built environment training or experience with people who do not have this and found that the design preferences for different attributes align with each other. The research findings disagreed with Nasar's (1998) theory related to the preference for historic buildings over modern ones, but this result may have been skewed by the fact the sample had few older people in it and the building in the photograph was an older building rather than a historic one, as there were no leftover spaces with historic buildings in Wellington City.

The significance of creating a new healthy environment is vital for promoting emotional wellbeing and the physical health of people (Social Report, 2003). Kaplan et al., 1972 argued that the quality of a space has a relationship with the emotion of an individual that can make it more pleasurable for them to be there. The quality of a space can also affects the behaviour of the person using it (Ulrich, 1983). By using semantic differentials, this research also found that people had emotional reactions to urban leftover spaces, something that has not been investigated before. The studies make a case for making modifications in leftover spaces that will make them into places people can like. The visual preference study was initiated to reveal the design problems of such spaces and probe the ideas of their potential users, making their redesign a bottom-up rather than a top-down process. 
The studies found common opinions about the attributes needed for designing leftover spaces, out of which the most recurring was related to providing more vegetation. Lynch (1960) claimed that the imageability of a city is strengthened by providing views of nature. Kaplan and Kaplan (1989) argued that vegetation could be appropriately managed to achieve visual coherence. The findings confirmed that people want to see more vegetation and that having natural features affects the stimuli and is seen as a permanent design solution. Just as vegetation was seen as desirable by both genders, with females having a slightly higher preference for seeing this than males, the T-tests in Study Two and Chi-square tests confirmed that built environment and non-built environment participants have the same preferences for the design attribute of providing more vegetation, and their preferences align with each other. The Chisquare tests also found that the preference of young (age 18-35) BE participants who are in the early stage of their professional career have similar preferences to those of NBE participants who represent the client group of all ages.

\subsubsection{Findings related to the method}

Lowenthal \& Riel (1972) found that experiencing a place and using images of the same place to evoke an experience of it have similar results. The photographs help to identify aspects of a place and establish a spatial relationship with the viewer (Canter, 1983). This research was conducted by using the Visual Preference Study (VPS) technique in which photographs of current leftover spaces in Study One were shown to the participants and suggestion for improvements were made by the latter. According to Mullen (1998), photographs can be studied in more detail than looking at the real scene as when an individual perceives the environment, they are constantly changing their focus. In Study Two, the spaces were redesigned according to the suggestions made by the participants in Study One and presented as photomontages. The technique of making designs through photomontage for Study Two took considerable time but was an effective way of communicating the concepts and ensuring sufficient data was collected for analysis. Study Three used the same photographs from Study One to probe the built environment and non-built environment participants in a mini design charrette. This research has, therefore, confirmed the usefulness of photographs when it comes to perceptions of the built environment. Although not a 
new finding (Gjerde, 2015), this research has added to the validation of the photographic method.

\subsubsection{Usefulness of the findings}

The knowledge gained from this research could be applied in the transformation of urban leftover spaces. Wellington City Council is taking measures to make the city sustainable, and in that effort, this research could play a valuable part in suggesting how leftover spaces could be used more effectively. As Nasar (1989) has argued, architects do not necessarily understand what non-architects want or prefer. However, this research can help BE professionals to design and develop leftover spaces in accordance with the preferences of those without an education in the built environment field.

\subsection{Research Limitations}

The mixed-use approach used in this research has the potential for being used as a tool to promote participatory urban design and planning consultations in support of the design of urban policies. Denzin \& Lincoln (1994) claimed that the triangulation of a mixed methodology overcomes the problem of a single theory method and its potential bias by involving two approaches to confirm the findings. This research found that the mixed-method approach is time-consuming and needs expertise. Another constraint of using a mixed-methods approach and inherently limiting the number of participants in each study, is that the results from the limited number of participants might have affected the robustness of findings, as these may not represent the opinions of the broader population. Study One had 119, and Study Two 121 participants. The original intention was to generalise the findings for the population in Wellington, but the sample was younger (aged 18-35) than the overall population of Wellington, as Studies One, Two, and Three had $91 \%, 66 \%$ and 56\% young participants respectively. In 2013 the total population of Wellington City was 197,500 of which $34.8 \%$ were aged $20-34$ (Information Demographics (2019a), these being the nearest age ranges available for comparison. However, the data compiled was sufficient to conduct a statistical analysis and achieve useful results. The pilot study raised concerns about the survey approach, specifically the need to provide more options for participants to select, having closed rather than open-ended questions, and having clear photos with consistent lighting. These issues were addressed in later studies. However, the 
questionnaire could have been shortened, and even more options for choosing answers from a list could have been helpful, and thus have encouraged a higher number of participants leading to more generalisable findings.

Study One took approximately 21 minutes on average, and Study Two approximately 18 minutes, and this might have affected the response and completion rates. Study One was completed by $80 \%$ of participants, and Study Two by $82 \%$ of respondents. According to Revilla and Ochoa (2017), a web survey should not exceed more than 20 minutes because the average attention span of a human being is only 20 minutes (Cape \& Philips, 2015). The research would also have benefitted from conducting more focus group discussions, for example, with the property owners and city council professionals. Although Wellington City Council professionals were invited to participate in the focus group discussion in Study Three, no-one was able to attend. Had they taken part this could have provided an insight into the extent the solutions could have been initiated in the real world.

Danied \& Meitne (2001) argued that visualisation is inconsistent with real-life experiences, but where it does depict a real setting and arouses positive or negative feelings, this increases public participation (Lange \& Hehl-Lange, 2005). The photomontage technique in Study Two adequately communicated the design attributes but would have benefitted from better and more balanced compositions for representing the design attributes, which would, in turn, have gained a more insightful set of results from the participants.

\subsection{Possible improvements for further studies}

The qualitative Study Three could have involved more respondents by organising a World Cafe workshop to discuss design guidelines with the wider public. This would have given a better insight into what people expect of each type of leftover space. Also, the participation of Wellington City Council executives and other stakeholders could have enriched the study results. Thus, future studies should involve decisionmaking groups in discussions of possible design changes and future urban policies.

The use of internet technology is different within a geographic and demographic group (Reip, 2002). Researchers Stanton \& Rogelberg (2001) and Kaplowitz et al. (2004) found that web questionnaires are mostly preferred by younger than older respondents who prefer paper-based surveys (Galea \& Tracy, 2007), but these studies are quite 
old. There is thus room for updating knowledge about the attitude of older people, many of whom will have used computers all their working lives, to web-based surveys. In this research, younger and adult participants answered the online web-based survey, but whether this was due to the survey type or the method of recruitment is not clear. Therefore, a mixed-mode (paper and web) method for conducting surveys might be useful in similar studies.

The expectation was that Study Three (focus group discussions) would help to explain the results of Study Two (visual preferences for photomontages). However, in order to make future studies more productive, a focus group(s) could precede the investigation of visual preferences using photomontages, so that the transformations of leftover spaces would be based on what people wanted to see in them.

\subsection{Further research}

The research and its findings have opened new directions in extending knowledge about urban leftover spaces. The context of this research was based on Wellington City, which has a temperate zone marine climate, being colder, wetter, windier and generally sunnier than other parts of New Zealand (New Zealand Tourism Guide, 2019). One possible area to further the research would be to design solutions for leftover spaces in different contexts and climates and to see whether the same attributes still emerge as preferences. As noted above, it would also be useful to extend the research in Wellington City to include property owners and council officials and thus address ownership rights issues. The notion of environmental easements and implementation of designs on privately held land creates a possible future research effort.

Another issue that needs further exploration is the level of light and sunlight when it comes to measuring preferences. In this study, only one view of each space was given, with an attempt to have a similar level of light in each. Ephemeral qualities, such as light level, affect emotional responsiveness. It would, therefore, be useful to explore preferences for design attributes at different times of the day and seasonally as light levels change.

Although the research validated the use of photographs, other experiments could be initiated by using Hybrid Virtual Environment software to create an immersive environment. The virtual settings could also engage multiple people who could 
Chapter 10 - CONCLUSIONS

simultaneously make design changes by doing 3D-sketching in the virtual environment.

Another possible area for further research to enhance knowledge in the field of landscape urbanism is research into the best types of vegetation for small spaces, and those that require the least maintenance to keep them in a tidy condition. 


\section{BIBLIOGRAPHY}

A

Abbaszadeh, S. (2006). Occupant Satisfaction with Indoor Environmental Quality in Green Buildings, Proceedings of Healthy Buildings,3(1), 365-370.

Aberson C. (2010). Applied power analysis for the behavioural sciences. New York: Routledge.

Abley, S. (2005). Walkability scoping paper (report no. Sa001 3523). Accessed on: 04.03.2019

Retrieved from http://levelofservice.com/walkability-research.pdf

Abramov, I., Gordon, J., Feldman, O. \& Chavarga, A. Sex \& vision I: Spatio-temporal resolution. Biological Sex Differences, 3, pp. 20-21.

Acking, C. \& Kuller, R. (1972). The Perception of an Interior as a Function of its Colour. Ergonomics, 15(6), 645-654.

AECOM (2016). Community Greenhouse Gas Inventory for Wellington City and the Greater Wellington Region 2000-2015, Wellington City Council. Accessed on 22.09.2019, Retrieved from http://www.gw.govt.nz/assets/Climate-change/CommuntityGHGInventoryWlgtnCityRegion2016.pdf

Ahmad. (2014). The relationship between landscape planting patterns and perceived safety in urban parks in Tabriz, Iran. Journal of Environmental Science and Technology, 107-113.

Ainsworth, R., Simposon, L. \& Cassell, D. (1993). Effects of three colours in an office interior on mood and performance. Journal of Perceptual and Motor Skills, 76(1), 235-241.

Akalin, A. \& Yildirim, K. (2009). Problems related to the dimensions of curved areas in the main living rooms of apartment housing. Journal of Architectural and Planning Research, 26(1), 70-87.

Akkerman, A. \& Cornfeld, A. (2009). Greening as an Urban Design Metaphor: Looking for the City's Soul in leftover space, Structurist 2009/2010, 30-35.

Akwa, L. (2009). Women involvement in Environmental protection and Management, A case of Nasarawa State. Journal of Sustainable Development in Africa, 10(4). pp. 123-143.

Alexander, C., Ishikava, S. \& Silverstein, M. (1977). A Pattern Language: Towns, Buildings, Construction. New York: Oxford University Press.

Alexander, C. (1997). A Pattern Language, Oxford: Oxford University Press.

Altman, I. (1975). The Environment and Social Behaviour: Privacy, Personal Space, Territory, and Crowding. London: Brooks/Cole Publishing Company

Allan, P. \& Bryant, M. (2011). The role of open space in recovery. Journal of Landscape Architecture, 6(2), 34-45.

Amin, M. (2002). Urban Quality and Designing of Spaces. International Planning Congress, Athens. Accessed on 12.02.2019, Retrieved from http://www.isocarp.net/Data/case_studies/145.pdf

Appleyard D. (1976). Planning a Pluralist City: Conflicting Realities in Ciudad Guayana, Cambridge Massachusetts: M.I.T. Press,

Armitage, A. (2007). Mutual Research Designs: Redefining Mixed Methods Research Design, paper presented at the British Educational Research Association Conference, 5-8 Sep 2007, London, United Kingdom.

Armstrong, H. (2006). Time, Dereliction and Beauty: An argument for Landscapes of Contempt. The Landscape Architect, IFLA Conference Papers (May 2006). Queensland University, 116 -127.

Arnheim, R. (1971). Art and Visual Perception. A Psychology of the Creative Eye. Los Angeles: University of California Press. 
Arnheim, R. (1977). The Dynamics of Architectural Form. Los Angeles: University of California Press.

Arnold, C. \& Gibbons, C. (1996). Impervious surface coverage: Emergence of a key environmental indicator, Journal of the American Planning Association, 62 (2) 243-258.

Arriaza, M., Canas-Ortega, J.F., Canas-Madueno, J.A., Ruiz-Aviles, P., (2004). Assessing the visual quality of rural landscapes. Landscape Urban Plann. 69, 115-125.

Aruninta. A. (2004). Controversies in public land management decision-makings: the case study of land utilization in Bangkok, Thailand, Doctoral dissertation, Chulalongkorn University, Bangkok.

Atlas, R. \& LeBlanc, W. (1994). The impact on crime of street closures and barricades: A Florida case study. Security Journal 5, 140-145.

Attride-Stirling, J. (2001). Thematic networks: an analytic tool for qualitative research. Qualitative Research, 1(3), 385-405.

Auge, M. (1995). Non-Places: Introduction to an Anthropology of Supermodernity, Social Science and Urban, London: Verso.

Australian Bureau of Statistics (2016). Women are outnumbering men in Australia, Australian Demographic Statistics. Accessed on 20.10.2016 Retrieved from http://www.abs.gov.au/ausstats/abs@.nsf/lookup/3101.0Media\%20Release1Dec\%202015

Azhar, J. \& Gjerde, M. (2016). Re-thinking the role of Urban In-Between Spaces. In (Eds.) J. Zuo, L. Daniel, V. Soebarto, Fifty years later: Revisiting the role of architectural science in design and practice: 50th International Conference of the Architectural Science Association (7-9 Dec), 279-288.

B

Babin, B., Hardesty, D. \& Suter, T. (2003). Colour and shopping intentions: The intervening effect of price fairness and perceived effect. Journal of Business Research, 57, 541-551.

Bar, M. \& Nata, M. (2006). Humans prefer curved visual objects. Psychological Science, 17(8), 645648.

Barker, R. (1968). Ecological Psychology: Concepts and methods for studying the environment of human behaviour. Stanford, CA: Stanford University Press.

Barron, P. \& Mariani, M. (2013). Terrain Vague: Interstices at the Edge of the Pale, London: Routledge Press.

Basiago, A. (1999). Economic, Social and Environmental Sustainability in Development Theory and Urban Planning Practice, Environmentalist 19(2),145-161.

Baum, A., Mapp, K., \& Davis, G. E. (1978). Determinants of residential group development and social control. Environmental psychology and nonverbal behaviour, 2(3), 145-160

Bechtel, R. \& Churchman, A. (2002). Handbook of Environmental Psychology. New York: John Wiley.

Beck, H., 2009. Linking the quality of public spaces to quality of life. Journal of Place Management and Development, 2(3), 240-248.

Beer, A. (1994). Urban greenspace and sustainability, Proceedings of PRO/ECE-Workshop on Sustainable Urban Development: Research and Experiments. Dordrecht. Netherlands.

Beierle, T. C. (2002). Democracy in Practice: Public Participation in Environmental Decisions, New York: Routledge.

Belot, M., Bhaskar, V., van de Ven, J. (2007). Is Beauty Only Skin Deep? Disentangling the Beauty Premium on a Game Show, Discussion Paper, Department of Economics, University of Essex. 
Bell, S. (1999). Landscape. Pattern, Perception and Process. London, E\&FN Spon.

Bell, P., Greene T., Jeffery F. \& Baum A. (2005). Environmental Psychology. $5^{\text {th }}$ Ed, Philadelphia: Psychology Press.

Bem, S. (1981). Gender Schema Theory: A Cognitive Account of Sex Typing, Psychological Review, 88, 354-364.

Bently, I., Alcock, A., Murrain, P., McGlynn, S.\& Graham, S. (1987). Responsive Environment: a manual for desginers. London: Elsevier, Architectural Press.

Bendig, A. W. (1954). Reliability and the number of rating scale categories. The Journal of Applied Psychology, 38, 38-40.

Benedict, M. \& McMahon, E. (2003). How cities use parks for green infrastructure. City parks forum briefing papers 5, American Planning Association, Chicago. Accessed on 03.04.2019, Retrieved from https://www.planning.org/publications/document/9148673/

Benson, P. H. (1971). How Many Scales and How Many Categories Shall We Use in Consumer Research? A Comment, Journal of Marketing, 35, 59-61.

Bentley, I., Alcock, A., Murrain, P., McGlynn, S., \& Smith, G. (1985). Responsive Environments a Manual for Designers. London: Architectural Press.

Berleant, A. (1998). Environmental Aesthetics. The Encyclopedia of Aesthetics. Accessed on 01.05.2019 Retrieved from https://www.academia.edu/15169737/Environmental_Aesthetics

Berlyne, D. (1967). Arousal and reinforcement. Nebraska Symposium on Motivation, University of Nebraska, Lincoln, 15, 1- 110.

Berlyne D. (1974). Studies in the New Experimental Aesthetics. New York: Taylor \& Francis.

Bertrand, J. (1992). Techniques for analysing focus group data. Evaluation Review, 16(2), 198-209.

Bird, C. (2005). How I stopped dreading and learned to love transcription. Qualitative Inquiry, 11(2), 226-248.

Bitner, M. (1992). Servicescapes: The Impact of Physical Surroundings on Customers and Employees, Journal of Marketing, 56(1), 57-71.

Bittencourt, M., Pereira, V.\&. Junior, W. (2015). The Usability of Architectural Spaces: Objective and Subjective Qualities of Built Environment as Multidisciplinary Construction, Procedia Manufacturing, 3(1), pp. 6429-6436.

Bjerre, L. (2011). Green Walls. Denmark.

Bjerke, T., Ostdahl, T., Thrane, C. \& Strumse, E. (2006). Vegetation density of urban parks and perceived appropriateness for recreation. Urban Forestry \& Urban Greening, 35-44.

Blackburn, S. (1994). Dictionary of philosophy, Oxford: Oxford University Press.

Blanc, N. (2013). Aesthetic engagement in the City. Contemporary Aesthetics, volume 11. Accessed on 20.05.2019, Retrieved from

https://www.contempaesthetics.org/newvolume/pages/article.php?articlelD=683

Blair, A., Ross, N., Gariepy, G. \& Schmitz, N. (2014). How do neighborhoods affect depression outcomes? A realist review and a call for the examination of causal pathways. Social Psychiatry and Psychiatric Epidemiology. 49, 873-887.

Blaxter, L., Hughes, C. \& Tight, M. (2001). How to Research. $2^{\text {nd }}$ Ed., Philadelphia, USA: Open University Press.Blumer, H. (1969). Symbolic interactionism: perspective and method, Berkeley: University of California Press.

Boone, H. \& Boon, D. (2012). Analysing Likert Data. Journal of Extension, 50(2), Article 2T0T2. Accessed 20 Jan 2019, Retrieved from: https://www.joe.org/joe/2012april/pdf/JOE_v50_2tt2.pdf 
Bolton, R. \& Foxon, T. (2011). Governing Infrastructure Networks for a Low Carbon Economy: CoEvolution of Technologies and Institutions in UK Electricity Distribution Networks, Competition and Regulation in Network Industries, 12(1), 02-26.

Boyatzis, R. (1998). Transforming Qualitative Information. Cleveland: Sage publications.

Bramley G. \& Power S. (2009). Urban form and Social sustainability: the role of density and housing type, Journal of Environment and Planning. 36, 30-48.

Brandlhuber, A. 2010. Semi-Private Space, writing on Architecture, Urbanism \& Art, Brunnenstrasse, Berlin. Accessed on 12.04.2016, Retrieved from https://waua.wordpress.com/tag/semi-private-space/

Brebner, J. (1998). Happiness and personality. Personality and Individual Differences, 25(2), 279-296.

Brizee, A. (2003). Teaching visual literacy and document design in the first-year composition.

(Masters of Art thesis). Virginia Polytechnic Institute and State University, Blacksburg, Virginia, US. Accessed 19 Dec 2018, Retrieved from http://scholar.lib.vt.edu/theses/available/etd05192003110024/unrestricted/Thesis.pdf

Braun, V. \& Clarke, V. (2006). Using thematic analysis in psychology. Qualitative Research in Psychology 3: $77-101$.

Broadbent, G. (1990). Emerging Concepts in Urban Space and Design, London: Routledge.

Brook, A. (2017). What is Urban development. Non profit. Accessed on 19.05.2019, retrieved from https://bizfluent.com/about-4728387-what-urban-development.html

Brower, S. (1980). Territory in urban settings, In I. Altman, A. Rapaport, \& J. Wohwill (Eds.), Human behavior and environment, New York: Plenum Press.

Brown, J. (1999). 'The use of focus groups for clinical research'. In B.F. Crabtree \& W. L. Miller (Eds.), Doing qualitative research, Thousand Oaks, CA: Sage publications, 109-124.

Brown, G. \& Gifford, R. (2001). Archiects predict Lay Evaluations of Large Contemporary Buildings: Whose conceptual properties? Journal of Environmental Psychology, 21(1), 93-99.

Brown, J. (2011). Likert items and scales of measurement? SHIKEN:JALT Testing and Evaluation. SIG Newsletter, 15(1), 09-14.

Buchanan, P. (1988). What city? A plea for place in the public realm, Architectural Review, 184(1101), $31-41$.

Buchner A, Erdfelder E \& Faul F. (1997). How to use GPower. Heinrich-Heine- Universitat:

Düsseldorf. Accessed 07 Jan 2019, Retrieved from: www.psycho.uni-duesseldorf.de/aap/projects/ gpower/how_to_use_gpower.html

Bundy, A., Lane, S., \& Murray, E. (2002). Sensory integration: Theory and practice. Philadelphia: F.A. Davis Company.

Burnston, D. (2017). Is aesthetic experience evidence for cognitive penetration?, New Ideas in Psychology, 47 (1), 145-156.

Burroughs, W. (1989). Interzone. New York: Viking Press.

Byers, P. \& Wilcox, J. (1991). Focus groups: A qualitative opportunity for researchers, The Journal of Business Communication, Vol. 28 (1), 63-78.

C

Calder, B. (1977). Focus Groups and the Nature of Qualitative Marketing Research. Journal of Marketing Research, 14, 353-364. 
Camaghi, J. (1994). Diverse Methods for Research and Assessments of College Students. Boston: ACPA publications.

Campari, I. (1996). Uncertain boundaries in urban space. In P. Burrough, \& A. Frank, (Eds) Geographic objects with indeterminate boundaries, London, Bristol: Taylor \& Francis Inc., 57-70.

Canfin, P. (2016). Mankind Has Eaten into its Year's Supply of Natural Resources - in Just Seven Months. World news, The Independent. Accessed on 15.07.2016, Retrieved from http://www.independent.co.uk/news/world/year-supply-natural-resources-seven-months-earthovershoot-day-a7177636.html

Canter, D. (1983). The purposive evaluation of Places: A facet approach. Environment and Behaviour, 15(6), 659-698.

Cape, P. \& Phillips, K. (2015). Questionnaire length and fatigue effects: the latest thinking and practical solutions. White paper. Accessed on 21.04.2019, Retrieved from www.surveysampling.com/site/ assets/files/1586/questionnaire-length-and-fatigue-effects-the-latestthinking-and-practicalsolutions.pdf

Craik, K. (1973). Environmental Psychology. Annual review of Psychology, 24(1), 403-422.

Carmen L., Antoni M., \& Elena N. (2011). Differences in Architects and Non-architects, perception of Urban Design: An Application of Kansei Engineering Techniques. Urban Studies Research,1,13.

Carmona, M., Tiesdell, S., Heath, T., \& Oc, T. (2010). Public places, Urban spaces (2nd Ed.). Oxford: Elsevier Ltd.

Carmona M. (2010). Contemporary Public Space, Critique and Classification, Part one: critique, Journal of Urban Design, 15(1), 151-156.

Carmona, M. (2013). Does urban design add value? Urban Design, 126: 47-49.

Carnaghi, J. (1992). 'Focus groups: Teachable and educational moments for all involved'. In F. K. Stage (Eds.), Diverse methods for research and assessment of college students, American College Personnel Association, Alexandria, 105-121.

Cerosaletti, C., \& Loui, A. (2009). Measuring the perceived aesthetic quality of photographic images. 2009 International Workshop on Quality of Multimedia Experience, 47-52.

Carr, S., Francis, M., Rivlin, L., \& Stone, A. (1992). Public Space. Cambridge: Cambridge: University Press.

Carter N., Bryant-Lukosius D., DiCenso A., Blythe J. \& Neville A. (2014). The use of triangulation in qualitative research. 41(5):545-7.

Casey, E. (1997). The Fate of Place: A Philosophical History, Berkeley: University of California Press

Cassarino, M. \& Setti, A. (2015). Environment as 'brain training': a review of geographical and physical environmental influences on cognitive ageing. Ageing Research Reviews, 23, 167-182.

Cassidy, T. (1997). Environmental psychology: Behavior and experience in context. London: Psychology Press.

Cattell, V., Dines, N., Gesler, W. \& Curtis, S. (2008). Mingling, observing, and lingering: Everyday public spaces and their implications for well-being and social relations. Health \& Place, 14, 544-561.

Cernin, R., Keller, B. \& Stoner, J. (2003). Colour vision in Alzheimer's patients: Can we improve object recognition with colour cues? Ageing, Neuropsychology, and Cognition, 10, 255-267.

Cerosaletti, C.D., \& Loui, A.C. (2009). Measuring the perceived aesthetic quality of photographic images. 2009 International Workshop on Quality of Multimedia Experience, 47-52.

Chatterjee, A. (2011). Neuroaesthetics: A Coming of Age Story. Journal of Cognitive Neuroscience, 23 (1), 53-62. 
Chen F. (2014). Urban Morphology and Citizens' Life. In: Michalos A.C. (Eds.) Encyclopedia of Quality of Life and Well-Being Research. Dordrecht: Springer.

Chen, Y., Liu, T. \& Liu, W. (2016). Increasing the use of large-scale public open spaces: A case study of the North Central Axis Square in Shenzhen, China. Habitat International 53, 66-77.

Cheng, Y. \& Chen, S. (2015). Perceived accessibility, mobility, and connectivity of public transportation systems, Transportation Research Part A: Policy and Practice, 77, 386-403.

Ching, F. (2007). Architecture - form, space and order, $3^{\text {rd }}$ edition. Hoboken, New Jersey: J. Wiley \&Sons, Inc.

Clavera, A. (2017). The world of design, a designed world: The relevance of aesthetics for everyday life. University of Barcelona, UB research Unit, 25(2). DOI: 10.20287/ec.n25.v2.a05

Climate Change (2007) Mitigation of Climate Change: Contribution of Working Group III contribution to the 4th Assessment Report. Intergovernmental Panel on Climate Change, New York: Cambridge University Press. Retrieved from:

https://www.ipcc.ch/site/assets/uploads/2018/03/ar4_wg3_full_report-1.pdf

Clinton Foundation, W. (2008). Annual Report: Clinton climate initiative, Clinton Foundation. Accessed on 14.05.2016, Retrieved from https://www.clintonfoundation.org/files/2008_AR.pdf

Coleman, A. (1985) Utopia on Trial: Vision and Reality in Planned Housing, London: Hilary Shipman.

Collis, J. \& Hussey, R. (2003). Business Research: a practical guide for undergraduate and postgraduate students, second edition. Basingstoke: Palgrave Macmillan.

Cook, M. (2010). Energy supply and use - Renewable energy, Te Ara - the Encyclopedia of New Zealand. Accessed on 15.07.2016, Retrieved from: http://www.teara.govt.nz/en/energy-supply-anduse/page-9

Courtis, J. (2004). Colour as visual rhetoric in financial reporting. Accounting Forum, 28, 265-281.

Cowan, R., Rogers, L., \& Hall, P. G. (2005). The dictionary of Urbanism, 67, Tisbury: Streetwise Press.

Cresswell, T. (1996). In place/out of place: geography, ideology, and transgression,

Minneapolis, London: University of Minnesota Press.

Creswell, J., \& Clark, P. (2007). Designing and conducting mixed methods research. Thousand Oaks, CA: Sage.

Creswell, J. (2009). Research Design: qualitative, quantitative and mixed approaches, third edition. Thousand Oaks, CA: Sage.

Creswell, J., Clark, P., Gutmann, M., \& Hanson, W. (2003). Advanced mixed methods research designs. In Tashakkori \& C. Teddlie (Eds.), Handbook of mixed methods in social and behavioural research, 209-240, Thousand Oaks, CA: Sage.

Crisman, P. (2009). Inhabiting the In-between: Architecture and Infrastructure Intertwined, Masters Thesis of Landscape Architecture. School of Architecture, University of Virginia.

Croasmun, J. \& Ostrom, L. (2011). Using Likert-Type Scales in the Social Sciences. Journal of Adult Education, 40. Accessed on 10.03.2018, Retrieved from https://files.eric.ed.gov/fulltext/EJ961998.pdf

Crosby, A., (1997). The Measure of Reality: Quantification and Western Society. Cambridge: Cambridge University Press.

Cross, J., Keske, C., Lacy, M., Hoag, D., \& Bastian, C. (2011). Adoption of conservation easements among agricultural landowners in Colorado and Wyoming: The role of economic dependence and sense of place. Landscape and Urban Planning, 101 (2), pp. 75-83.

Crotty, M. (2003). The Foundations of Social Research: Meaning and Perspectives in the Research Process, third edition. London: Sage. 
Cullen, G. (2007). Introduction to the concise townscape. In Larice, M., Macdonald, E. (Eds.), The urban design reader, 167-173, London, England: Routledge.

Cupchik G \& Winston A. (1996). Handbook of perception \& cognition: Cognitive ecology. San Diego, CA: Academic Press.

Cupers, K. \& Miessen, M. (2002). Spaces of Uncertainty. Berlin: Muller and Bussmann.

Cuthbert, A. R. (2006). The form of cities, political economy and urban design. Oxford, UK: Blackwell Publishing.

Cybriwsky, R. (1999). Changing patterns of urban public space: Observations and assessments from the Tokyo and New York metropolitan area, Cities, 16(4), 223-231.

Czech, B., Krausmann P., \& Devers P. (2000). Economic associations among causes of species endangerment in the United States. BioScience, 50, 593-601.

D

Daniel, T \& Meitner, M. (2001). Representational validity of landscape visualisations: The effects of graphical realism on perceived scenic beauty of forest vistas. Journal of Environmental Psychology, 21(1), pp. 61-72.

Dassah, E.., \& Nimlyat. P. (2010). The role and responsibilities of Professionals in the Built Environment in contributing to Sustainable Development in Nigeria Dassah. E. T. and Nimlyat, P. S. Journal of Sciences and Multidisciplinary Research, 40-47.

De-Girolamo, F. (2013). Living Landscapes: Landscapes for Living Time and Regeneration: Temporary reuse in Lost Spaces. The Journal of Urbanism. Planum.

Denzin, N. \& Lincoln, Y. (1994). Handbook of Qualitative Research (4th Edition). New York: Sage Production, Inc.

Denzin, N. \& Lincoln, Y. (2005). Introduction: The Discipline and Practice of Qualitative Research. In Denzin \& Lincoln (Eds.), The Sage handbook of qualitative research, 10-11, Thousand Oaks, CA: Sage.

Department of Interior Affairs (2015). Setting the Scene, Te Tari Taiwhenua: building a safe, prosperous and respected a nation. New Zealand. Accessed on 12.07.2016 Retrieved from https://www.dia.govt.nz/diawebsite.nsf/wpg_URL/Resource-material-Sustainable-UrbanDevelopmentSetting-the-Scene?OpenDocument

Deleuze, G. \& Guattari, F. (1988). A Thousand Plateaus: Capitalism and Schizophrenia, translation by Brian Massumi, London: Athlone.

Desvousges, W. \& Frey, J. (1989). 'Integrating focus groups and surveys: examples from environmental risk studies'. Journal of Official Statistics, 5(1), 349-363.

Dewey, J. (1958). Experience and Nature. 2nd ed., paperback. New York: Dover.

Dihrab, S. \& Sopian, K. (2010). Electricity generation of hybrid PV/wind systems in Iraq, Renewable Energy, 35, 1303-1307.

Dijkstra, N., Zeidman, P., Ondobaka, S., Van Gerven, M. \& Friston, K. (2017). Distinct Top-down and Bottom-up Brain Connectivity during Visual Perception and Imagery. Scientific Reports, 7(1), 56775690.

Doron, G. (2000). The Dead Zone and Architecture of Transgression: CITY analysis of Urban Trends, Culture, Theory, Policy, Action, 4, 247-263.

Doron, G. (2006). The derelict land and the Elephant, the field journal, 1, 10-23. 
Douglas, O., Russell, P., \& Scott, M. (2018). Positive perceptions of green and open space as predictors of neighbourhood quality of life: implications for urban planning across the city region. Journal of Environmental Planning and Management, 1-21. Accessed on 30.01.2019, Retrieved from https://www.tandfonline.com/doi/full/10.1080/09640568.2018.1439573

Dovey, K. \& Polakit, K. (2007). Urban Slippage: Smooth and Striated streetscapes in Bangkok. In Loose Space (Eds) Karen. A. Frank and Quentin Stevens, 113-131, New York: Routledge.

Driver, J. \& Spence, C. (2000). Visual Capture of Touch: Out-of-the-Body Experiences with Rubber Gloves. Psychological Science, 11(5), 353-359. Accessed 09 Jan 2019, Retrieved from http://www.jstor.org/stable/40063541

Duany, A., Plater-Zyberk, E. \& Speck, J. (2000). Suburban Nation: The Rise of Sprawl and the Decline of the American Dream, New York: North Point Press.

Duncan M. \& Mummery W. (2007). GIS or GPS? A comparison of two methods for assessing route taken during active transport. American Journal of Preventive Medicine, 33(1), 51-53.

Durlak, J. (2009). How to Select, Calculate, and Interpret Effect Sizes, Journal of Pediatric Psychology, 34 (9), 917-928, Accessed on 02.01.2019, Retrieved from https://doi.org/10.1093/jpepsy/jsp004

Durrheim, K. (2004). Research design. In M. Terre Blanche, \& K. Durrheim (Eds.), Research in practice: Applied methods for the social sciences, 29-53, Cape Town: University of Cape Town.

\section{E}

Eagly, A. H. (2009). The his and hers of prosocial behaviour: An examination of the social psychology of gender. American Psychologist, 64, 644-658.

Edensor, T. (2005). Industrial Ruins. New York: Berg Publishers.

Edwards, Rosalind (1990). Connecting method and Epistemology: A white women [sic] interviewing black women, Women's Studies International Forum, 13, 477-90.

Ekman, P. (1992) An argument for basic emotions, Cognition and Emotion, 6(1), 169-200.

Freedberg, D. \& Gallese, v. (2007). Motion, emotion and empathy in esthetic experience, Trends in Cognitive Sciences, 11(5), 197-203.

Elkin, T., McLaren, D. \& Hillman, M. (1991). Reviving the City: Towards Sustainable Urban Development, London: Friends of the Earth.

Ellis, L. \& Ficek, C. (2001). Color preferences according to gender and sexual orientation. Personality and Individual Differences. 31, 1375-1379.

Ellison, D. \& Woodward, I. (2010). Aesthetic experience, transition al objects, and the Third space: the Fusion of audience and Aesthetic objects in the performing arts. Thesis Eleven, 103(1), 45-53.

European Commission (2004). Reclaiming city streets for people: chaos or quality of life? Accessed on 03.03.2019 Retrieved from http://ec.europa.eu/environment/pubs/pdf/streets_people.pdf

European Commission. (2011). Cities of Tomorrow: challenges, visions, way forward. European Union Regional Policy, Brussels. accessed on 11.01.2018, Retrieved from https://ec.europa.eu/regional_policy/sources/docgener/studies/pdf/citiesoftomorrow/citiesoftomorrow_f inal.pdf

European Commission. (2012). The Multifunctionality of Green Infrastructure. Science for Environmental Policy. Accessed on 27.08.2016, Retrieved from www.ec.europa.eu/environment/nature/ecosystems/docs/Green_Infrastructure.pdf

Evan, G. \& Garling, T. (1991). Environment, cognition and Action: An integrated Approach. New York: Oxford University Press. 
Evan, G. (2003). The built environment and mental health. Journal of Urban Health, 80(4), 536-555.

Evans, J. \& Jones, P. (2008). Rethinking sustainable urban regeneration: ambiguity, creativity, and shared territory. Environment and Planning, 40, 1416-1434.

Evans, L., Maio, G., Corner, A., Hodgetts, C., Ahmed, S. \& Hahn, U. (2013). Self-interest and proenvironmental behaviour. Nature Climate Change, 3 (2), 122-125.

$\mathbf{F}$

Falah, G., \& Newman, D. (1995). The spatial manifestation of threat: Israelis and Palestinians seek a 'Good' border. Political Geography, 689-706.

Fechner, G. T. (1876) Vorschule der Asthetik, Leipzig: Breitkopf \& Härtel.

Feireiss, L. (2010). Living in the City: The Urban Space as Creative Challenge, Urban Interventions: Personal Projects in Urban Space, (Eds.) Robert Klanten and Matthias Hubner, Berlin: Die Gestalten, 3-8.

Fern, E. (1982). 'The use of focus groups for idea generation: The effects of group size, acquaintanceship and moderation on response quantity and quality'. Journal of Marketing Research, 19, 1-13.

Fiske, S. \& Taylor, S. (1991). Social Cognition, 2nd ed., New York: McGraw-Hill

Fleming, R., Wiebel, C., \& Gegenfurtner, K. (2013). Perceptual qualities and material classes. Journal of Vision 13(8), 9.

Florin, P. (1989). Nurturing the grassroots: Neighbourhood volunteer organisations and American cities, Citizens Committee for New York City. New York: Graphic Exchange.

Foddy, W. (1994). Constructing questions for interviews and questionnaires: Theory and practice in social research. Cambridge: Cambridge University Press.

Folke, C., Jansson, J., Larsson, M. \& R. Costanza. (1997). Ecosystem appropriation by cities. Ambio, 26, 167-172.

Fossey, E., Harvey, C., McDermott, F., \& Davidson, L. (2002). Understanding and evaluating qualitative research. Australian and New Zealand Journal of Psychiatry, 36(6), 717-732.

Foster, A. (2015). The plan for Wellington's next 30 years, The Dominion Post. New Zealand. Acessed on 15.09.2016, Retrieved from http://www.stuff.co.nz/dominionpost/comment/72336482/Theplan-for-Wellingtons-next-30-years

Franck, K and Steven, Q. (2007). Loose Space: Possibility and Diversity in Urban Life, London: Routledge Press.

Franz, M., Pahlen, G., Nathanail, P, Okuniek, N. \& Koj A. (2006). Sustainable development and brownfield regeneration. What defines the quality of derelict land recycling?, Environmental Sciences, 3 (2), 135-151, DOI: 10.1080/15693430600800873

G

Galea, L. \& Kimura, D. (1993). Sex differences in route learning. Personal Individual. Differences. 14, pp. 53-65. 
Galea S. \& Tracy M. (2007). Participation rates in epidemiologic studies. Ann Epidemiol. 17(9), pp. 643-653. doi: 10.1016/j.annepidem.2007.03.013.

Gallagher, J. (2010). Reimagining Detroit: Opportunities for Redefining an American city. Michigan: Wayne State University Press.

Garcia-Domenech, S. (2015). Urban Aesthetic and Social function of actual Public Space: a desirable balance. Theoretical and Empirical researchers in Urban Management, 10(4), 54-65.

Gatz, K. \& Achterberg, G. (1967). Colour and Architecture. London: Batsford.

Gearin, E. \& Kahle. C., (2006). Teen and Adult Perceptions of Urban Green Space Los Angeles. Children, Youth and Environments, 16(1), 25-48.

Gehl, J. (1987). Life between buildings: Using public space. New York: Van Nostrand Reinhold.

Gehl, J. (2010). Cities for People. Washington, DC: Island Press.

Ghomeshi, M., \& Jusan, M. (2012). Indoor and Built uilt Environment Investigating Different Aesthetic Preferences Between Architects and Non-architects in Residential Façade Designs. Indoor and Built Environment, 1-13.

Gibson, J. (1977). The Theory of Affordances. In R. Shaw and J. Bransford (Ed.), Perceiving, Acting, and Knowing, 67-82, New York: Lawrence Erlbaum.

Gieseking, J. (2014). Environmental Psychology. In T. Teo, M. Barnes, Z. Gao, M. Kaiser, R. Sheivari, and B. Zabinski (Eds). International Encyclopedia of Critical Psychology. 587-593, New York: Springer.

Gifford, R. (2002). Making a difference: Some ways environmental psychology has improved the world. In R. Bechtel \& A. Churchman (Eds.), Handbook of environmental psychology, 323-334, New Jersey: John Wiley \& Sons Inc.

Gifford, R. (2007). Environmental psychology: Principles and practice. Colville: Optimal Books.

Gifford, R. (2008). Psychology's essential role in climate change. Canadian Psychology, 49, 273-280.

Gifford, R., Hine, D., Muller-Clemm, W., \& Shaw, K. (2000, 2002). Why architects and laypersons judge buildings differently: Cognitive properties and physical bases. Journal of Architectural and Planning Research, 19(2), 131-148.

Gjerde, M. (2010). Visual aesthetic perception and judgement of urban streetscapes. In: P. Barrett (Eds.) Building a Better World: CIB World Congress, Salford, UK: CIB Press.

Gjerde, M. (2015). Street perceptions: A study of visual preferences for New Zealand streetscapes, Doctoral thesis. Victoria University of Wellington, Wellington, New Zealand.

Gjerde, M. (2017). Informing Design Review: Discussion of the Findings of a Visual Preference Study in New Zealand, Procedia Engineering, 198, 562-69.

Gladkikh, T., Liz Betancourt, L. \& Vargas, Y. (2015). Green versus Gray: Attitudes toward Vegetation in a Tropical Metropolitan Square Luis Santiago. Environment and Natural Resources Research, 5(2). 67-82.

Global Energy Report (2017). Towards a zero-emission, efficient and resilient building and construction sector. Internal Energy Agency and UN Environment. Accessed on 13.05.2019 Retrieved from: https://www.worldgbc.org/sites/default/files/UNEP\%20188_GABC_en\%20\%28web\%29.pdf

Gobster, P. \& Chenoweth, R. (1989). The dimensions of aesthetic preference: a quantitative analysis. Journal of Environment Management, 29 (I),47-72.

Godbey, G., Graefe, A., \& James, J. (1992). The benefits of local recreation and park services: A nationwide study of the perceptions of the American public. Washington, DC: National Recreation and Parks Association. 
Goldkuhl, G. (2012). Pragmatism vs interpretivism in qualitative information systems research, European Journal of Information Systems, (21) 2, 135-146.

Goldkuhl, G. (2004). Meanings of pragmatism: Ways to conduct information systems research, In Proceedings of the 2nd International Conference on Action in Language, Organisations and Information Systems, Linköping University, ALOIS.

Gorden, R. (1980). Interview strategy techniques and tactics. Homewood: Dorsey Publiations

Gosling, S., Vazire, S., Srivastava, S. \& John, O. (2004). Should we trust web-based studies? American Psychologist 59(2), 93-104.

Graham, J. \& Argyle, M. (1975). 'A cross-cultural study of the communication of extra-verbal meaning by gestures'. International Journal of Psychology, 10(1), 57-67.

Graham, P. (2009). Building Ecology: First principles for a sustainable Built Environment. 25-27, New Jersey: Wiley-Blackwell.

Grant, J. \& Mittelsteadt, L. (2004). Types of Gated communities, Environmental and Planning B Planning and Design, 31, 913-930.

Groat, L. (1982). Meaning in post-modern architecture: An examination using the multiple sorting task. Journal of Environmental Psychology, 2, 3-22.

Green, R. (1999). Meaning and Form in Community Perception of Town Character, Journal of Environmental Psychology, 19, 311-329.

Greenberg, M., Pooper, F. \& West, B. (1990). The TOADS: A New American Urban Epidemic. Urban Affairs Quarterly, 25, 435-54.

Greene, J., Caracelli, V., \& Graham, W. (1989). Toward a conceptual framework for mixed-method evaluation designs. Educational Evaluation and Policy Analysis, 11(3), 255-274.

Greenberg, M., Popper, F., \& West, B. (1990). The TOADS: A New American Urban Epidemic. Urban Affairs Quarterly, 25(3), 435-454.

Greenway, R. (2010). What is ecopsychology? Gatherings: Journal of the International Community for Ecopsychology. Accessed 27 Dec 2018, Retrieved from http://www.ecopsychology.org/journal/gatherings/what.html

Grimmond, S. (2007). Urbanisation and Global Environmental Change: Local Effects of Urban Warming, The Geographical Journal, 173, 83-88.

Grinde, B., \& Patil, G. (2009). Biophilia: Does Visual Contact with Nature Impact on Health and WellBeing? International journal of environmental research and public health, 6(9), 2332-2343.

Groat, L. (1988). Contextual compatibility in Architecture: An issue of personal taste?, In J. Nasar (Eds.). Environmental Aesthetics: Theory, Research, and Applications. 120-253. New York: Cambridge University Press.

Groat, L., \& Despres, C. (1990). The significance of architectural theory for environmental design research. In E. H. Zube \& G. T. Moore (Eds.), Advances in environment, behaviour, and design: Vol. 4, New York: Plenum

Groat, L. \& Wang, D. (2013). Architectural Research Methods, second edition. New Jersey: John Wiley \& Sons.

Groth, J. \& Corijn, E. (2005). Reclaiming Urbanity: Indeterminate Spaces, Informal Actors and Urban Agenda Setting. Journal of Urban Studies, 1, 503-526.

Guba, E. \& Lincoln, Y. (1989). Fourth Generation Evaluation. Newbury Park, CA: Sage.

Guba E. \& Lincoln Y. (1994). Competing paradigms in qualitative research. In Denzin N. and Lincoln Y. (Eds), Handbook of qualitative research, 105-117, Thousand Oaks, CA: Sage. 
Guest, G. (2013). Describing mixed methods research: An alternative to typologies. Journal of Mixed Methods Research, 7(2), 141-151.

\section{H}

Habe, R. (1989). "Public Design Control in American Communities: Design Guidelines/Design Review." The Town Planning Review 60 (2): 195-219.

Habibi, A. (2016). Environmental Aesthetics: The share of Environmental Aesthetics indices in the cognition of Urban Landscape, Art, Nature, City in Manzar issue, 35, 50-55. Accessed on 18.02.2019, Retrieved from http://www.manzar-

sj.com/?_action=showPDF\&sc=1\&article=43489\&_ob=71004f8e1e0968b0afdc3ce0ddaaefc5\&fileNa me=full_text.pdf

Hagerhall, C., Laike, T., Taylor, R., Kuller, M., Kuller, R., \& Martin, T. P. (2008). Investigations of human EEG response to viewing fractal patterns. Perception, 37, 1488-1494.

Hajer, M \& Reijndorp, A. (2001). In Search of New Public Domain: Analysis and Strategy. Rotterdam: NAI Publishers.

Handszuh, H. (1991). Tourism trends and patterns. In. Travel Medicine 2. (Eds.) Lobel, H.O., Steffen, R., and Kozarsky, P.E. Georgia, USA: International Society of Travel Medicine, 7-12.

Hannan, C. \& Andersson, I. (2002). Gender perspective on ecological sanitation. Ecological Sanitation Research (EcoSanRes) Stockholm. Accessed on 29.10.2019, Retrieved from: www.ecosanres.org/pdf_files/Nanning_PDFs/Eng/

Hanyu, K (1993). The affective meanings of Tokyo: Verbal and non-verbal approaches. Journal of Environmental Psychology, 13, 161-172.

Harth, A. (2014). Open Space and Gender - Gender-Sensitive Open-Space Planning. Deutsches Institute for Urbanistik. Accessed on 26.10.2019. Retrieved from: https://difu.de/publikationen/openspace-and-gender-gender-sensitive-open-space.html

Hartig, T., \& Staats, H. (2006). The need for psychological restoration as a determinant of environmental preferences. Journal of Environmental Psychology, 26, 215-226.

Hartig, T., Mang, M. \& Evans, G. (1991). Restorative effects of natural environment experiences, Environment and Behavior, 23 (1), 3-26.

Hass-Klau, C., Crampton, G., Dowland, C., \& Nold, I. (1999). Streets as Living Space: Helping public places play their proper role. London: Landor

Heidegger, M. (1971). Building, Dwelling, Thinking in Poetry, Language, Thought. New York: Harper Colophon, pp. 143-154.

Heritage, J. (1984). A change-of-state token and aspects of its sequential placement. In M. Atkinson and J. Heritage (Eds.), Structures of Social Action, Cambridge University Press, 299-347.

Hershberger, R G (1988). A study of meaning and architecture, In Jack Nasar. (Eds.) Environmental Aesthetics: theory, research and applications, Cambridge University Press, Cambridge, 175-194.

Hershberger, R., \& Cass, R. (1998). Predicting user responses to buildings. In Jack Nasar, (Eds.), Environmental Aesthetics: Theory, research and applications, Cambridge, UK: Cambridge University Press, 195-211.

Herzog, T. (1989). A cognitive analysis of preference for urban nature. Journal of Environmental Psychology, 9, 27-43. 
Herzog, T., Kaplan, S. \& Kaplan, R. (1976). The prediction of preference for familiar urban place. Environment and Behaviour, 8(1), 627-645.

Herzog, T., Maguire, C., \& Nebel, M. (2003). Assessing the restorative components of environments. Journal of Environmental Psychology, 23(2), 159-170.

Hill, M. (1982). Spatial structure and decision-making of pedestrian route selection through an urban environment. PhD dissertation, University of Nebraska.

Hilty, J., Lidicker, J., W. \& Merenlender, A. (2006). Corridor Ecology: The Science and Practice of Linking Landscape for Biodiversity Conservation. London: Island Press.

Homer, C., Huang, C., Yang, L., Wylie, B., \& Can, M. (2004). Development of a 2001 National Land Cover Database for the United States. Photogrammetric Engineering and Remote Sensing 70, 829840.

Hoffman, M. (2008). Is small beautiful? Attitudes towards community-owned wind energy in Waitati, BSc thesis. University of Otago, Dunedin, New Zealand.

Holbrook, M. (1999). Introduction". In Consumer value: A framework for analysis and research (Eds.) Holbrook, M., New York: Routledge.

Holgate, A. (1992). Aesthetics of built form. Oxford: Oxford University Press.

Holler, J., Shovelton, H., \& Beattie, G. (2009). Do iconic gestures really contribute to the semantic information communicated in face-to-face interaction? Journal of Nonverbal Behaviour, 33, 73-88.

Howe, K. (1988). Against the Quantitative-qualitative Incompatibility Thesis or Dogmas die Hard. Educational Researcher 17(8): 10-16.

Huchendorf, L. (2007). The effects of colour on memory. Journal of Undergraduate Research. Accessed on 21 Jan 2019, retrieved from http://www.uwlax.edu/urc/juronline/PDF/2007/huchendorf.pdf

Hudson, J. \& Shaw, P. (2012). Contested uses within the 'leftover' spaces of the city, As Found Conference Paper Research Group for Landscape architecture and urbanism, Nordic Journal of Architectural Research.

Hughes, W. (2010). Built Environment education, research and practice: Integrating diverse interests to make an impact. In: Laryea, S., Leiringer, R. and Hughes, W. (Eds.) Procs West Africa Built Environment Research (WABER) Conference, Accra, 1-8.

Huffschmid, A. (2012). From the City to lo Urbano: Exploring Cultural Production of Public Space in Latin America, Doctoral dissertation, Ibero-American University.

Hunter, L., Hatch, A. \& Johnson, A. (2004). Cross-national gender variation in environmental behaviours. Social Science Quarterly 85(3):677-694

Hull, R. \& Michael, S. (1995). Nature-based Recreation, mood change, and stress restoration, Leisure Sciences, 17, 1-14.

I

Information Demographics (2019). Wellington City: number of cars per household. ID community, Demograhic resources, New Zealand. Accessed on 13.05.2019, Retrieved from https://profile.idnz.co.nz/wellington/car-ownership

Information Demographics (2019a). Wellington City: population forecast, Demograhic resources, New Zealand. Accessed on 17.05.2019, Retrieved from https://forecast.idnz.co.nz/wellington/populationage-structure 
International Energy Agency (2009). Cities, Towns \& Renewable Energy: Yes in my front yard. International Energy Agency, 65-90, Paris. Accessed on 11.04.2019, Retrieved from https://webstore.iea.org/cities-towns-and-renewable-energy-yes-in-my-front-yard

Ishizu T, Zeki S. (2013). The brain's specialized systems for aesthetic and perceptual judgment. European Journal of Neuorscience. 37(9), 1413-1420.

Iveson, K. (2010). Graffiti, Street Art and the City, City 26, 14(1).

$J$

Jackson, J. (1984). The American public space. Winter 1984, 52-65. Accessed on 10.05.2019, Retrieved from https://www.nationalaffairs.com/public_interest/detail/the-american-public-space

Jacobs, A., \& Appleyard, D. (1987). Toward an Urban Design Manifesto. Journal of the American Planning Association, 53(1), 112-120.

Jacobs, J. (1961, 1993). The Death and Life of Great American Cities. New York: Random House

Jacoby, J. \& Matell. M. (1971). Three-Point Likert Scales Are Good Enough, Journal of Marketing Research, 7 (November 1971), 495-500.

Jalaian, Y. (2015). City Infrastructure and Fractured Space: creating continuity in a Fractured Urban Fabric, Masters Thesis, Virginia Polytechnic Institute and State University

James W. (1907) Pragmatism, Dover: New York.

Jamieson, S. (2004). Likert scales: How to use them. Medical Education, 38, 1212 -1218. doi:

10.1111/j.1365-2929.2004.02012.x

Janssens, J. (2001). Facade colours not just a matter of personal taste. Nordisk Arkitekturforskning, $2,17-21$.

Jansson, M., Lindgren, H. \& Wistrom, B. (2013). Perceived personal safety in relation to urban woodland vegetation - A review. Urban Forestry \& Urban Greening, 127-133.

Jarnang, M. (2010). Stadens Odefmierade rum (English translation). Sveriges Lantbruksuniversitet.

Jenks, M., Burton, E. \& Williams, K. (1996). The Compact City: A Sustainable Urban Form? London: E.\&FN Spon.

Jick, T. (1979). Mixing Qualitative and Quantitative Methods: Triangulation in Action. Administrative Science Quarterly, 24, 602-611.

Jiggins, J. (1994). Changing the boundaries. Women-centred perspectives on population and the environment. Washington, DC: Island Press.

Johnson, B. \& Onwuegbuzie, A. (2004). Mixed Methods Research: A Research Paradigm Whose Time Has Come. Educational Researcher, 33(7), 14-26.

Johnson, B. and Gray, R. (2010). History of philosophical and theoretical issues for mixed methods research, 2 nd Ed. in Tashakkori, A., Teddlie, C. (Eds), Handbook of Mixed Methods in Social and Behavioral Research, Thousand Oaks, CA: Sage.

Juaidi, A.; Montoya, F., Ibrik, I. \& Manzano-Agugliaro, F. (2016). An overview of renewable energy potential in Palestine. Renewable Sustainable Energy, 65, 943-960. 
Kanase-Patil, A., Saini, R. \& Sharma, M. (2010). Integrated Renewable Energy Systems for Off-Grid Rural Electrification, Renewable Energy, 35, 1342-1349.

Kant, I. (2000). Critique of the Power of Judgment. Cambridge: Cambridge University Press.

Kaplan, R. (1974). Some psychological benefits of an outdoor challenge program. Journal of Environment and Behaviour, 6, 101-116.

Kaplan, R. (1985). Nature at the doorstep: Residential satisfaction and the nearby environment. Journal of Architectural and Planning Research, 2, 115-127.

Kaplan, S. \& Kaplan, R. (1983). Cognition and environment: functioning in an uncertain world. New York: Praeger.

Kaplan S. \& Kaplan R. (1989). The Experience of Nature: a psychological perspective, New York: Cambridge University Press

Kaplan S., Kaplan R. \& Wendt J. (1972). Rated preference and complexity for natural and urban visual material, Perception and Psychophysics, 12, 354-356.

Kaplowitz, M., Hadlock, T., \& Levine, R. (2004). A comparison of Web and mail survey response rates. Public Opinion Quarterly, 68, pp. 94-101. doi:10.1093/poq/nfh006

Kayden, J. (2000). Privately owned public space: The New York City experience. New York: John Wiley \& Sons.

Khan, A. (2018). Why say no to Solar Energy? An exploration of residential reluctance towards Solar Energy. Oslo Centre for Research on Environmentally Friendly Energy, 42. Accessed on 22.04.2019, retrieved from

https://www.cree.uio.no/publications/CREE_working_papers/pdf_2018/khan_solar_energy_cree_wp0 4_2018.pdf

Kenko, J., 1998. Essays in Idleness: The Tsurezuregusa of Kenko, New York: Columbia University Press.

Keniger, L., Gaston, K., Irvine, K., Fuller, R. (2013). What are the benefits of interacting with nature? International Journal of Environmental Research and Public Health 10, 913-935

Kent, J. and Thompson, S. (2014). The three domains of urban planning for health and well-being. Journal of Planning Literature 29(3), 239-256.

King, N. \& Horrocks, C. (2010). Interviews in qualitative research, London: Sage publications.

Kirsch, L., Urgesi, C. \& Cross, E. (2016). Shaping and reshaping the aesthetic brain: Emerging perspectives on the neurobiology of embodied aesthetics, Neuroscience \& Biobehavioral Reviews, 62(1),56-68.

Kitzinger J. (1995). Qualitative Research: Introducing focus groups. BMJ 311:299-302.

Klein, M. (2005). Old and New Walls in Jerusalem, Political Geography 24, 53-76

Kline, R. B. (2005). Principles and practice of structural equation modelling (2nd Ed.). New York: Guildford.

Kloppenberg, J. (1996). An old name for ways of thinking? The Journal of American History, 83(1), 100-138.

Korpela, M., Hartig, T., Kaiser, F., \& Fuhrer, U. (2001). Restorative experience and self-regulation in favorite places. Environment and Behaviour, 3, 572-589.

Kraft, H. (1994). A history of architectural theory: From Vitruvius to the present (Ronald Taylor, Elsie Callander, and Antony Wood, Trans). New York: Princeton Architectural Press.

Krenichyn, K. (2006). The only place to go and be in the city: women talk about exercise, being outdoors, and the meanings of a large urban park. Health \& Place, 631-643. 
Krier, L. (2009). The architecture of the community. Washington: Island Press.

Kropf, K. (2017). The handbook of Urban Morphology. Chichester: John Wiley.

Krueger, R. (1994). Focus Groups: A Practical Guide for Applied Research. $2^{\text {nd }}$ Edition, Thousand Oaks, CA: Sage publications.

Krueger, R., \& Casey, M. (2009). Participants in a Focus Group. Focus Groups: A Practical Guide for Applied Research, 63-84. Accessed on 28.11.2018, Retrieved from https://www.sagepub.com/sites/default/files/upm-binaries/24056_Chapter4.pdf

Krueger, R. \& King, J. (1998). Involving community members in focus groups. Thousand Oaks, CA: Sage publications.

Krupat, E. (1985). People in cities: The urban environment and its effects. New York, NY, US: Cambridge University Press.

Kubovy, M. (1999). Well-being: The foundations of hedonic psychology. Pleasures of the mind. New York: Russell Sage.

Kuo, F. \& Sullivan, W. (2001). Aggression and violence in the inner city: Effects of environment and Behaviour, 33(4), 543-571.

Kyro, P. (2003). Revising the concept and forms of benchmarking. Benchmarking: An International Journal, (10) 3, 210-225.

$\mathbf{L}$

La Varra, G. (2003). Post-it city: the other European public spaces, Use-Uncertain States of Europe, Milan: Skira.

Laerd Statistics (2016). Kendall's tau-b using SPSS Statistics. Statistical tutorials and software guides. Accessed on 21.08.2018, Retrieved from https://statistics.laerd.com/

LaGasse, A. (2013). Pilot and feasibility studies: Application in Music Therapy Research. Journal of Music Therapy, 50 (4): 304-20. doi: 10.1093/jmt/50.4.304

Lakoff, G. \& Johnson, M. (1999). Philosophy in the flesh. The embodied mind and its challenge to western thought. New York: Basic Books.

Lang, J. (1987). Creating architectural theory. New York: Van Nostrand Reinhold.

Lang, J. (1988). Symbolic aesthetics in architecture: Toward a research agenda. In Jack Nasar (Eds.), Environmental aesthetics: Theory, research, and applications. New York: Cambridge University Press.

Lang, J. (2003). Aesthetic theory. In A. R. Cuthbert (Eds.), Designing cities: critical readings in urban design, 275-284, Oxford, UK: Blackwell Publishing.

Lange, E. \& Hehl-Lange, S. (2005). Combining a participatory approach with a virtual landscape model for the siting of wind turbines. Journal of Environmental Planning and Management, 48(6), pp. 833-852.

Langstraat, F. \& Van Melik, R. (2013). Challenging the 'End of Public Space': A Comparative Analysis of Publicness in British and Dutch Urban Spaces. Journal of Urban Design, 18(3), pp.429-448.

Lapadat, J. C. and Lindsay, A. C. (1999). Transcription in research and practice: from standardization of technique to interpretive positioning. Qualitative Inquiry, 5, 64-86.

Langstraat, F., \& Van Melik, R. (2013). Challenging the 'End of Public Space': A Comparative Analysis of Publicness in British and Dutch Urban Spaces. Journal of Urban Design, 18(3), 429-448. 
Laumann, K., Garling, T., \& Stormark, K. (2001). Rating scale measures of restorative components of environments. Journal of Environmental Psychology, 21(1), pp. 31-44. doi:10.1006/jevp.2000.0179

Lazarus, R., Kanner, A., \& Folkman, S. (1980). Emotions: A cognitrve-phenomenological analysis. In R. Plutchik \& H. Kellerman (Eds.), Theories of emotion, 189-217, New York: Academic Press

Lederman. L. (1990). Assessing educational effectiveness: The focus group interview as a Technique for data collection. Communication Education, 38(4): 117- 127.

LeDoux, J. (1996). The emotional brain: The mysterious underpinnings of emotional life. New York, NY: Simon \& Schuster.

Leech, N. \& Onwuegbuzie, A. (2006). A typology of mixed research designs. Quality and Quantity. Accessed Dec 22, 2018, Retrieved from: doi: 10.1007/s11135-007-9105-3

Lee, S. J., Hwang, S., \& Lee, D. (2015). True Smart and Green City?, International Forum on Urbanism Urban Voids : As a Chance for Sustainable Urban Design. Accessed on 17.08.2016, retrieved from doi.org/10.3390/ifou-D007.True

Lefebvre, H. (1991). The Production of Space, Translated by Donald Nicholson-Smith. Oxford: Blackwell.

Lehmann, D. R., \& Hulbert, J. (1972). Are Three-Point Scales Always Good Enough? Source Journal of Marketing Research, 9(4), 444-446. Accessed on 11.08.2018, Retrieved from http://www.jstor.org/stable/3149313

Lennertz, B. \& Lutzenhiser, A. (2006). The Charette Handbook. Chicago: American Planning Association.

Lenth, R. (2001). Some practical guidelines for effective sample size determination. The American Statistician, 55, 187-193.

Lerup, L. (1977). Building the unfinished: architecture and human action; introduction by Roger Montgomery, Beverly Hills : Sage Publications.

Lesan, M. (2015). Public streets for multicultural use: exploring the relationship between Cultural Background, Built Environment, and Social Behaviour. Doctoral Thesis. Victoria University of Wellington, New Zealand.

Lewis, I., Watson, B., White, K., \& Tay, R. (2007). Promoting public health messages: Should we move beyond fear-evoking appeals in road safety?. Qualitative Health Research, 17(1), 61-74.

Lindsey, G., Todd, J., Hayter, S. \& Ellis, P. (2009), 'A Handbook for Planning and Conducting Charrettes for High-Performance Projects', Accessed on 26.10.2018, Retrieved from www.nrel.gov/docs/ fy09osti/44051.pdf

Linde, P \& Karin, B. (2014). Performing the City: Exploring the Bandwidth of Urban Place-Making through New-Media Tactics, Making Futures, Cambridge: MIT Press.

Lock, D. (1995). Room for more within city limits? Town and Country Planning, 64(7), 173-176.

Loukaitou-Sideris, A. (1996). Cracks in the city: Addressing the constraints and potentials of urban design, Journal of Urban Design, 1, 91-103.

Loukopoulos, P., Jakobsson, C., Garling, T., Schneider, C. M., \& Fujii, S. (2004). Car user responses to travel demand management measures: Goal setting and choice of adaptation alternatives.

Transportation Research 9, 263-280.

Lowenthal, D. \& Riel, M. (1972). The nature of perceived and imagined environments. Environment and Behaviour, 4(2), 189-207.

Luz, A. (2001). Places In-Between: The Transit(ional) Locations of Nomadic Narratives. Place and location. Studies in environmental aesthetics and semiotics 5, 143-165. 
Lyman, S. \& Scott, M. (1967). Territoriality: A neglected sociological dimension. Social problems, 15(2) 236- 249.

Lynch, K. (1960). The Image of the City. Cambridge: MIT Press.

Lynch, K. (1981). Good City Form. Cambridge: MIT Press.

\section{M}

Maccoby, E. \& Jacklin, C. (1974). The psychology of sex differences. Stanford, CA: Stanford University Press.

Madanipour, A. (1996). Design of Urban Space: An Inquiry into a Socio-Spatial process. New York: Wiley.

Madanipour, A. (2003). Public Private Spaces of the City. London: Psychology Press.

Madison, D. (2005). Critical Ethnography: Method, Ethics, and Performance. Sage: Calif, Thousand Oaks.

Magde, C. (1997). Public parks and the geography of fear. Journal of Economic and Social Geography, 88, 237-250.

Manco, T. (2010). Street Sketchbook. San Francisco, CA: Chronicle Books.

Manning, P. (1991). Environmental Aesthetic Design: Identifying and achieving desired environmental effects, particularly "image" and "atmosphere". Building and environment, 26(4), 331-340.

Manso, Maria \& Castro-Gomes, João. (2015). Green wall systems: A review of their characteristics. Renewable and Sustainable Energy Reviews. 41. 863-871. Accessed on 05.09.2019, Retrieved from https://www.researchgate.net/publication/266078897_Green_wall_systems_A_review_of_their_chara cteristics

Mansouri, S. A. (2005). An introduction to the aesthetics of Iranian Garden. Journal of Bagh-e-Nazar, 2(3): 58-63.

Marans, R. (1976). Perceived quality of residential environments: some methodological issues, in (Eds.) K. Craik \& E. Zube, Perceiving Environmental Quality: Research and Applications. New York: Plenum.

Maron, R. \& Spreckelmeyer, K. (1982). Meaning of overall Architecture Quality: a component of building evaluation. Environment and Behaviour 14(1), 652-669.

Marcus, I., (2010). Spatial Capital. 'A proposal for an Extension of Space Syntax into a More General Urban Morphology'. In the Journal of Space Syntax,1, 30-40.

Martindale C. (1990). The Clockwork Muse: The Predictability of Artistic Change. New York: Basic Books.

Matell, M. \& Jacoby, J. (1971). Is there an optimal number of alternatives for Likert scale items? Study 1: Reliability and validity. Educational and Psychological Measurement, 31, 657-674.

Matlin, M. \& Foley, H. (1992). Sensation and perception (5 $5^{\text {th }}$ Edition). New York: Routledge.

Maxcy, S. (2003). Pragmatic threads in mixed methods research in the social sciences. The search for multiple modes of inquiry and the end of the philosophy of formalism, In Tashakkori, A., Teddlie, C. (Eds), Handbook of Mixed Methods in Social and Behavioral Research, Thousand Oaks, CA: Sage.

McDonald, N. (2008). Critical factors for active transportation to school among low-income and minority students - evidence from the 2001 national household travel survey. American Journal of Preventive Medicine, 34(4), 341-344. 
McDonnell, M. \& Pickett, S. (1990). Ecosystem structure and function along urban-rural gradients: An unexploited opportunity for ecology. Ecology,71, 1232-1237.

McGuiness, D. \& Sparks J. (1983). Cognitive style and cognitive maps: sex differences in representations of familiar terrain. Journal of Mental Imagery, 7, pp. 91-100.

McKelvie, S. (1978). Graphic rating scales-How many categories? British Journal of Psychology, 69, 185-202.

McKenzie, S. (2004). Social Sustainability: Towards Some Definitions, Working Paper Series No. 27, Hawke Research Institute. The University of South Australia. McGill

McKinney, M. (2002). Urbanisation, biodiversity and conservation. BioScience 52, 883-890.

McCrea, R., Shyy, T., \& Stimson, R. (2006). What is the strength of the link between objective and subjective indicators of urban quality of life? Applied Research in Quality of Life, 1, 79-96.

McWhinnie, H. (1968). A review of research on aesthetic measure, Acta Psychologica 28, 363-375.

Medina, M. (2009). Social dimensions of livability in a housing project. Philippine Geographical Journal, 53, 16-22.

Mehta, V. (2009). Look closely and you will see, Listen carefully and you will hear: Urban design and social interaction on streets. Journal of Urban Design, 14, 20-64.

Mehta, R. \& Zhu, R. (2009). Blue or Red? Exploring the effect of colour on cognitive task performances (Report 1169144), Vancouver, Canda:Science express. Retrieved from: https://www.uvm.edu/pdodds/files/papers/others/2009/mehta2009a.pdf

Menzies, E. (n.d.). Progress vs Preservation: a history of TeAro Wellington. Accessed on 25.07.2017, Retrieved from http://www.nzta.govt.nz/assets/projects/wicb/resources/pdf/Te-Aro-History.pdf

Meyer, W. \& Turner, B. (1992). Human population growth and global land-use/cover change. Annual Review of Ecological Systems. 23, 39-61.

MFE, (2015). New Zealand Greenhouse Inventory 1990-2013, Ministry for the Environment. Accessed on 06.08.2016, Retrieved from https://www.mfe.govt.nz/publications/climate-change/newzealands-greenhouse-gas-inventory-1990-2013

MFEa, (2015). About the New Zealand Emissions Trading Scheme, Ministry for the Environment. Accessed on 26.08.2016, Retrieved from: http://www.mfe.govt.nz/climate-change/reducinggreenhouse-gas-emissions/about-nz-emissions-trading-scheme

Mhatre, P. (2013). Vacant and Abandoned Lands: A Theory Paper, Neighborhood Revitalization. Accessed on 13.04.2018, Retrieved from: https://pratikmhatre99.files.wordpress.com/2013/11/vacantand-abandoned-lands.pdf

Millennium Project, (2008). State of the Future, Summary. Accessed on 22.07.2016, Retrieved from: www.eldis.org/go/home\&id=42523\&type=Document

Mills, J., Bonner, A., \& Francis, K. (2006). The development of constructivist grounded theory. International Journal of Qualitative Methods, 5(1), 1-10.

Ministry of Economic Development (2007). New Zealand Energy Strategy to 2050: powering our future, towards a sustainable low emissions energy system, Ministry of Economic Development. Accessed on 12.04.2016, Retrieved from http://www.mcguinnessinstitute.org/wpcontent/uploads/2016/08/nzenergystrategyto2050.pdf

Ministry of Business, Innovation and Employment, (2015). Energy in New Zealand. Modelling and Sector Trends. Accessed on 22.07.2016, Retrieved from: https://www.mbie.govt.nz/assets/3318904e46/energy-in-new-zealand-2014.pdf

Mitchell, D. \& Staeheli, L. (2006). Clean and Safe? Property Redevelopment, Public Space, and Homelessness in Downtown. In Politics of Public Space (Eds.) Setha Low and Neil Smith. New York: Routledge. 
Mitchell, R., Popham, F. (2007). Greenspace, urbanity and health: relationships in England, Journal of Epidemiology and Community Health. 61(8). 681-683.

Modell, S. (2009). In Defence of Triangulation: A Critical Realist Approach to Mixed Methods Research in Management Accounting. Management Accounting Research 20 (3), 208-221.

Mohai, P. (1992). Men, women, and the environment: An examination of the gender gap in environmental concern and activism. Society and Natural Resources, 5(1), pp.1-19.

Mohajeri, N., Assouline, D., Guiboud, B., Bill, A., Gudmundsson, A. \& Scartezzini, J. (2018). A cityscale roof shape classification using machine learning for solar energy applications. Renewable Sustainable Energy, 121, 81-93.

Montanana, A., Llinares, C. \& Navarro, E. (2013). Architects and non-architects: differences in perception of property design. Journal of Housing and the Built Environment, 28(2), 273-291.

Montgomery, J. (2008). The New Wealth of Cities: Urban Dynamics and the Fifth Wave, London: Routledge.

Moos, H. \& Insel, P. (1974). Psychological environments: Expanding the scope of human ecology. American Psychologist, 29(3), 179-188.

Morgan, D. (2006). Connected contributions as a motivation combining qualitative and quantitative methods. In L. Curry, R. Shield, \& T. Wetle (Eds.), Applying qualitative and mixed methods in ageing and public health research. Washington, DC: American Public Health Association.

Morgan, D. (2007). Paradigms lost and pragmatism regained: Methodological implications of combining qualitative and quantitative methods. Journal of Mixed Methods Research, 1(1), 48-76.

Morse, J. (1991). Approaches to qualitative-quantitative methodological triangulation. Nursing Research, 40, 120-123.

Morse, J. \& Niehaus, L. (2009). Mixed Method Design: Principles and Procedures. Walnut Creek, CA: Left Coast Press Inc.

Moser, J., Wozniak, W., Naleway C., \& Ayer W. (1985) Color vision in dentistry: A survey Journal of American Dental Association, 110(4), pp. 509-510.

Moughtin, C., Oc, T. \& Tiesdell, S. (1995). Urban design: Ornament and decoration. Oxford: Butterworth.

Mullen, L. (1998). Truth in Photography: Perception, Myth and Reality in the postmodern world. Master's thesis. University of Florida, Florida, US. Accessed on 10.04.2019, Retrieved from http://etd.fcla.edu/UF/amd0040/Leslie.pdf

Muller, V \& Busmann (2002). Spaces of Uncertainty, In K. Cupers \& M. Miessen, Wuppertal: Muller und Busmann.

\section{$\mathbf{N}$}

Nasar, J. (1988). Environmental Aesthetics: Theory, Research, and Applications, Cambridge: Cambridge University Press.

Nasar, J. (1989). Symbolic meanings of house styles. Environment and Behavior 21(3), 235-242.

Nasar, J. \& Devlin, K. (1989). The beauty and the beast: Some preliminary comparisons of "high" versus "popular" residential architecture and public versus architecture judgments of same. Journal of Environmental psychology, 9, 333-334.

Nasar, J. (1990). The Evaluative Image of the City, Journal of the American Planning Association, 56, 41-53, DOI: 10.1080/01944369008975742 
Nasar, J. (1994) Urban design aesthetics: The evaluative qualities of building exteriors. Environment and Behavior, 26(3), 377-401. Accessed on 11.07.2018 Retrieved from DOI:

$10.1177 / 001391659402600305$

Nasar, J. (1998). The evaluative image of the city. Thousand Oaks, California: Sage Publication.

Nasar, J. (2000). The evaluative image of Places. In W. Walshm K. Craik, and R. Price (Eds.) $2^{\text {nd }}$ Edition, Person-environment psychology: New directions and perspectives, 117-168, New Jersey: Lawrence Erlbaum Associates.

Nasar, J. \& Devlin, K. (1989). The beauty and the beast: Some preliminary comparisons of "high" versus "popular" residential architecture and public versus architect judgments of same. Journal of Environmental Psychology, 9, 333-344.

National Vacant Properties Campaign. (n.d.). National Vacant Properties Campaign". Accessed on 22.10.2016, Retrieved from http://www.vacantproperties.org

Newman. O. (1972). Design Guidelines for Creating Defensible Space. Wikipedia. Accessed on 13.09.2016, Retrieved from http: www/en.wikipedia.org/wiki/DefensibleSpaceTheory

Nayak, B. (2010). Understanding the relevance of sample size calculation. Indian journal of ophthalmology, 58(6), 469-70.

Nelson, W. (1976). Aesthetic consideration in the selection and use of trees in the urban environment. Symposium Proceeding: Better trees for the Metropolitan Landscapes. USDA General Technical Report, NE-22, 13-29.

Nevarez, J., (2007). Central Park, the Aesthetics of Order and the Appearance of Looseness. In Loose Space (Eds.) Karen. A. Frank and Quentin Stevens, 154-170, New York: Routledge.

Newman, O. (1972). Defensible space: Crime prevention through urban design. New York: Macmillan.

New York State Department of State. (2009). Opportunities are waiting to happen. Local Government and Community Sustainability, Office of Coastal. Accessed on 13.09.2017, Retrieved from: https://www.dos.ny.gov/opd/programs/pdfs/Guidebooks/ab/AbandonedBuildings.pdf

New Zealand Tourism Guide (2019). Wellington weather and climate. Christchurch. Accessed on 09.05.2019, Retrieved from: https://www.tourism.net.nz/new-zealand/about-new-zealand/wellingtonweather.html

Nielson, T. (2002). The Return of Excessive: Superfluous Landscape. The Aarhus School of Architecture. Copenhagen: Sage Publication.

Nieuwenhuijsen, M., Khreis, H., Verlinghier, E. \& Rojas-Rueda, D., (2016). Transport and health: a marriage of convenience or an absolute necessity. Environment International. 88, 150-152. Accessed on 05.03.2019, Retrieved from http://www.smithsonianmag.com/ist/?next=/smartnews/hamburgplans-become-car-free-2034-180949780

Nipesh. 2012. Urban Voids \& Shared Spaces, deep within an exploration. Accessed on 12.04.2016, Retrieved from https://nipppo.wordpress.com/2012/05/07/urban-voids/

Nissen, S. (2008). Urban Transformation from Public and Private Space to Spaces of Hybrid Character. Czech Sociological Review, 44(6), pp. 1129-1149.

Nordh, H., Hartig, T., Hagerhall, C. \& Fry, G. (2009). Components of small urban parks that predict the possibility for restoration. Urban Forestry and Urban Greening 8, 225-235.

Nordh, H., \& Otsby, K. (2013). Pocket parks for people- a study of park design and use. Urban Forestry \& Urban Greening, 12, pp. 12-17.

Norman, D. (2004). Emotional Design: Why We Love (or Hate) Everyday Things. New York: Basic Books.

Northam, R. (1971). Vacant Urban Land in the American City. Land Economics, 47(4), 345-355. doi: $10.2307 / 3145071$ 
NZUDP, (2005). New Zealand Urban Design Protocol. Ministry for the Environment. Accessed on 13.08.2016, retrieved from https://www.mfe.govt.nz/sites/default/files/urban-design-protocol-colour.pd

0

O'Cathain, A., Murphy, E., \& Nicholl, J. (2008). The quality of mixed methods studies in health services research. Journal of Health Services Research and Policy, 13(2), 92-98.

Ocal, A., Yigittir, S., Kyburiene, L. \& Navickiene, G. (2018). The Value of Cleanliness in the view of the Students of Two Higher Education Institutions. Advances in Higher Education. 2. 1.

Doi:10.18686/ahe.v2i1.1059.

Ojo, B. \& Kayode, F. (2006). The role of Colour in Environmental Beautification and Urban Aesthetics: the Nigerian example. Indoor Built Enivoronment, 15(6), 543-550.

Oke, T. (1981). Canyon geometry and the urban heat island: Comparison of the scale model and field observations. International Journal of Climatology, 1, 237-254.

Oke, T. (1997). Urban climates and global environmental change. In R.D. Thompson and A. Perry (ed.) Applied climatology: Principles and practice. London: Routledge.

Onwuegbuzie, A. \& Leech, N. (2007). A call for qualitative power analyses. Quality \& Quantity, 41, 105-121.

Orr, D. (2002). Four Challenges of Sustainability. Conservation Biology, 16(6), 1457-1460.

Osborne, H. (1979). Some Theories of Aesthetic Judgment. The Journal of Aesthetics and Art Criticism, 38(2), 135-144.

Osgood, C. E., \& Snider, J. (1969). Semantic Differential technique: A sourcebook. Chicago: Aldine Publications.

Ottosson, J. \& Grahn, P. (2005). A comparison of leisure time spent in a garden with leisure time spent indoors: On measures of restoration in residents in geriatric care. Landscape Research, 30(1), 23-55.

$\mathbf{P}$

Pagano, A., \&. Bowman. A. (2000). Vacant Land in Cities: An Urban Resource. The Brookings Institution Survey Series: $1-9$.

Panerai, P., Castex, J. \& Depaule, J. (2004). Urban Form: The Death and Life of the Urban Block. Architectural Press: Oxford: Architectural Press.

Park, B., Tsunetsugu, Y., Kasetani, T., Hirano, H., Kagawa, T., Sato, M., \& Miyazaki, Y. (2007). Physiological effects of shinrin-yoku (taking in the atmosphere of the forest) using salivary cortisol and cerebral activity as indicators. Journal of Physiological Anthropology, 26(2), pp. 123-128. doi:10.1016/0306-4530(94)90013-2

Parkes, D. \& Thrift, N. (1980). Times, spaces and places: A chrono-geographic perspective. New York: John Wiley.

Patton, M. Q. (1990). Qualitative evaluation and research methods. Thousand Oaks, CA: Sage.

Pedersen, E. \& Persson, W. (2007). Wind turbine noise, annoyance and self-reported health and wellbeing in different living environments. Occupational and Environmental Medicine, 64(7), 480-486. 
Pena, M. 2009. Examination of the land surface temperature response for Santiago, Chile. Photogrammetric Engineering \& Remote Sensing, 75(10), 1191-1200.

Pfeifer, L. (2013). The Planner's Guide to Tactical Urbanism. Masters Thesis, McGill School of Urban Planning, Montreal, Canada.

Pheasant, S., Haslegrave, C. (2006) Bodyspace: Anthropometry, Ergonomics and the Design of Work, $3^{\text {rd }}$ Edition, London: Taylor \& Francis.

Philipp, R., Philipp, E. \& Thorne, P. (1999). The importance of intuition in clinical occupational medicine consultation. Occupational Medicine, 49(1), 37-41.

Pihlstrom, S. (1998). Pragmatism and Philosophical Anthropology: Understanding Our Human Life in a Human World. New York: Peter Lang.

Piccinno, G. \& Elisa L. (n.d.). Spatial Design for New Typologies of Places: In-between Urban Spaces, inter-disciplinary global conference. Accessed on 21.10.2016, Retrieved from https://www.academia.edu/2171941/Spatial_Design_for_new_typologies_of_places_inbetween_urban_spaces

Pirie, G. (1979). Measuring accessibility: a review and proposal. Environment and Planning, 11, pp. 299-312.

Planning Tank (2016). Learn about Tactical Urbanism. Happy, Healthy and Sustainable Human Settlements. Accessed on 13.02.2017 Retrieved from: http://planningtank.com/urbanisation/learntactical-urbanism

Pomeranz, D. (1980). Environmental Psychology. Chapter 3 in Krasner, Leonard. (Eds). Environmental Design and Human Behaviour: A Psychology of the Individual in Society. Sydney: Pergamon Press.

Portas, N., Domingues, A. \& Cabral, J. (2003). Urban Issues, Trends, Strategies and Opportunities, Lisbon: Politicas Urbanas.

Porteous, D. (1996). Environmental Aesthetics: ideas, politics and planning. New York: Routledge.

Pradinie, K., Navastara, A. \& Martha, E. (2015). Who's own the public space? The adaptation of limited space in Arabic Kampong. Journal of Social and Behavioural Sciences 227(1), 693- 698.

Prince of Wales (1984). Speech at the Royal Institute of British Architects Gala Evening, Hampton Court. Accessed on 04.04.2019, Retrieved from https://www.princeofwales.gov.uk/speech/speechhrh-prince-wales-150th-anniversary-royal-institute-british-architects-riba-royal-gala

Privitera, G. J. (2012). Statistics for the Behavioral Sciences. Thousand Oaks, California: Sage.

Prosperity Index (2016). The Lagatum Prosperity index: Bringing Prosperity to Life (Tenth Edition). Lagatum Institute, London. Retrieved from https://www.prosperity.com/download_file/view_inline/2857

Putnam, R. (2000). Bowling Alone: The Collapse and Revival of American Community. New York: Simon \& Schuster.

\section{Q}

Qamaruz-Zaman, N., Samadi, Z. \& Azhari, F. (2012). Opportunity in Leftover Spaces: Activities under the flyovers of Kuala Lumpur. Social and Environment-Behaviour Studies 68, 451-463.

Scherer, K. (2001). Appraisal considered as a process of multilevel sequential checking. In K. Scherer, A. Schorr, \& T. Johnstone (Eds.), Appraisal processes in emotion, 95-118. New York: Oxford University Press. 
Radford, S. (2007). Have you seen the new model? Visual design and product newness, PhD Dissertation, Faculty of the Graduate School, University of Missouri-Columbia.

Rahmann, H., \& Jonas, M. (2014). Tokyo Void: Possibilities in Absence. Berlin: Jovis

Rakhshandehroo, M., Yusof, M., Johari, M., Najd, D. \& Meysam. (2015). Green Façade (Vertical Greening): Benefits and Threats. Applied Mechanics and Materials. 747. Accessed on 04.04.2019, Retrieved from 10.4028/www.scientific.net/AMM.747.12.

Rapoport, A. (1970). The study of spatial quality. Journal of Aesthetic Education, 4(4), 81-95.

Rapoport, A. (1977). Human aspects of urban form, Oxford: Pergamon Press.

Rapoport, A. (1982). The Meaning of the Built Environment. A Nonverbal Communication Approach, Beverly Hills, Canada: Sage.

Rapoport, A. (1990). History and precedent in environmental design. New York: Plenum.

Rapoport, A. (1999). A FRAMEWORK FOR STUDYING VERNACULAR DESIGN. Journal of Architectural and Planning Research, 16(1), 52-64.

Redies,C. (2015). Combining universal beauty and cultural context in a unifying model of visual aesthetic experience. Frontiers in Human Neuroscience, 9(218), 1-20.

Rees, W. \& Wackernagel, M. (1994). Ecological footprints and appropriated carrying capacity: Measuring the natural capital requirements of the human economy. Focus, 6, 121-130.

Reips, U. (2002). Standards for Internet-based experimenting. Experimental Psychology, 49, pp. 243256. doi:10.1026//1618-3169.49.4.243

Revilla, M. \& Ochoa, C. (2017). The ideal and maximum length for a web survey. International Journal of Market Research, 59 (5), 557-565.

Riessman, C. (1993). Qualitative research methods. Narrative analysis. Thousand Oaks, US: Sage Publications.

Robinson, J. (2005). Deeper than Reason: Emotion and its role in literature, music, and art. New York: Oxford University Press.

Robinson, S. \& Lundholm, J. (2012). Ecosystem services provided by spontaneous urban vegetation. Urban Ecosystem, 15, 545-557.

Roe, J., Thompson C. \& Aspinall, P. (2013). Green Space and Stress: Evidence from Cortisol Measures in Deprived Urban Communities. International Journal of Environment Residential Public Health, 10, pp. 4086-4103.

Roggema R. (2014). The Design Charrette. In: Rob Roggema (Eds.) The Design Charrette. Dordrecht: Springer.

Roseman, I. \& Smith, C. (2001). Appraisal theory: Overview, assumptions, varieties, controversies. In R. Scherer, A. Schorr, \& T. Johnstone (Eds.), Appraisal processes in emotion: Theory, methods, research, 4-17. New York: Oxford University Press.

Rose, L. (1989). Urban land supply: Natural and contrived restrictions. Journal of Urban Economics, 25(3), 325-345.

Rossman, G. B., \& Wilson, B. L. (1985). Numbers and words: Combing quantitative and qualitative methods in a single large-scale evaluation study. Evaluation Review, 9, 627-643.

Ryan, J. (1993). Shed a little light: eight illuminating axioms of market research. Currents, 19(2), 40-42.

Ryan, R., Weinstein, R., Bernstein, J., Brown, K., Mistretta, L. \& Gagne, M. (2010). Vitalising effects of being outdoors and in nature, Journal of Environmental Psychology, 30(2), 159-168. 
Sachs, N. (1999). Psychiatric hospitals. In C. Marcus \& M. Barnes (Eds.), Healing gardens: Therapeutic benefits and design recommendations. New York: John Wiley \& Sons.

Sacks, H., Schegloff, E. \& Jefferson, G. (1974). A simplest systematics for the organization of turntaking for conversation. Language 50(4), 696-735.

Saito, Y. (2008) Everyday Aesthetics. London: Oxford University Press. Accessed on 04.05.2019, Retrieved from https://www.oxfordscholarship.com/view/10.1093/acprof:oso/9780199278350.001.0001/acprof9780199278350-chapter-2

Saleski, C. (1972). Color, light and shade matching Journal of Prosthetic Dentistry, 27(3), pp. 263268.

Samadi, Z \& Hasbullah. M. (2008). The Enhancement of Space in between Urban Recreation Development. Accessed on 22.11.2016, Retrieved from //www.malaysianpublications.blogsport. com/

Sanoff, H., 1991. Visual research methods in design New York: Van Nostrand Reinhold

Saraev, V. (2012). Economic benefits of green space: A critical assessment of evidence of net economic benefits. (Forestry Commission Research Report). Forestry Commission: Edinburgh, Scotland.

Sargeant, J. (2012). Qualitative research, Part II Analysis and Quality insurance. The Journal of Medical Education, 4(1), 1-3.

Satterthwaite, D., McGranahan \& Tacoli, C. (2010). Urbanisation and its implication for food and farming. Philosophical Transactions of the Royal Society B, Biological Sciences, 365 (1554).

Accessed on 12.04.2016, Retrieved from https://doi.org/10.1098/rstb.2010.0136

Saunders, C. (2003). The emerging field of environmental psychology. Human Ecology review, 10(2), 137-149.

Scannell, L., \& Gifford, R. (2010). Defining Place Attachment: A Tripartite Organizing Framework. Journal of Environmental Psychology,30(1), 1-10.

Schaffnit-Chatterjee, C., \& Kahn, B. (2011). Mitigating climate change through agriculture - an untapped potential, 06-08, Frankfurt: Deutsche Bank Research.

Scheer, C. (1994). Introduction: The debate on design review. In B. C. Scheer \& W. F. E. Preiser (Eds.), Design review: challenging urban aesthetic control, Chapman \& Hall, New York, 1-10.

Schmidt, G. (2005). Ecology \& Anthropology: A Field without a Future? Ecological and Environmental Anthropology, 1(1), 13-15.

Schneekloth, L. \& Shibley, R. (2000). Implacing Architecture into the Practice of Placemaking, Journal of Architectural Education, 130-140.

Schrader, B. (2010). City Planning- Early settlement planning, Te Ara-the Encyclopedia of New Zealand. Accessed on 21.09.2016, Retrieved from http://www.teara.govt.nz/en/city-planning/page-2

Scoffham, E. \& Vale, B., (1996). How compact is sustainable - how sustainable is compact?, In Jenks, Burton and Williams (Eds.) The Compact City: a sustainable urban form, 66-73, London: E.\&FN. Spoon.

Scruton, R. (1979). The aesthetics of architecture. Princeton, New Jersey: Princeton University Press.

Seamon, D. (2010). Merleau Ponty, Perception, and Environmental Embodiment: Implications for Architectural and Environmental Studies. In McCann, R. \& Locke, P. M. (Eds.), Carnal echoes, Merleau-Ponty and the flash of architecture, New York: Routledge. 
Seager, J. (1993). Earth Follies: Coming to Feminist Terms with the Global Environmental Crisis. New York: Routledge.

Sennett, R., (2003). Respect in a World of Inequality. New York: W.W. Norton and Company.

Sennett, R. (2007). The City as an Open System. Leverhulme International Symposium 2004, The Resurgent City, London School of Economics, Themed Session: The Habitable City. Accessed 25 Jan. 2019, retrieved from http://www.Ise.ac.uk/collections/resurgentCity/Papers/richardsennett.pdf

Serageldin, I. (1993). Developmental Partners: Aid and Cooperation in the 1990s, Stockholm: Swedish International Development Cooperation Agency.Publisher? place?

Shaftel, H. (2016). Responding to Climate Change. Accessed on 06.10.2016, retrieved from http://climate.nasa.gov/solutions/adaptation-mitigation/

Shanahan, D., Lin, B., Bush, R., Gaston, K., Dean, J., Barber, E. \& Fuller, R. (2015). Towards improved public health outcomes from urban nature. American Journal of Public Health 105, 470477.

Shahrestani, M., Yao, R., Luo, Z., Turkbeyler, E. \& Davies, H. (2015). A field study of urban microclimates in London. Renewable Energy 73, 3-9.

Shahreen, F. \& Voghera, A. (2016). Urban planning and design methods for sustainable development. Politecnico di Torino. Accessed on 12.06.2018, Retrieved from:

https://www.researchgate.net/publication/265922689_Urban_planning_and_design_methods_for_sus tainable_development

Sharifi, A. (2019). Resilient Urban Forms: A macro-scale analysis, Cities, 85, 1-14.

Shea, J., \& Fortna, G. (2002). Psychometric methods. In G. Norman, C. van der Vleuten \& D. Newble (Eds.), International Handbook of Research in Medical Education, Vol. 1, 97-126. Dordrecht: Kluwer Academic Publishers.

Shepard, P. (1991). Man in the landscape (2 $2^{\text {nd }}$ edition). College Station: Texas A\&M University Press.

Stanton, J. \& Rogelberg, S. (2001). Using Internet/intranet Web pages to collect organisational research data. Organizational Research Methods, 4, pp. 200-217. doi:10.1177/109442810143002

Shochat E., Warren P., Faeth S., McIntyre N. \& Hope D. (2006). From patterns to emerging process in mechanistic urban ecology. Trends in Ecology and Evolution. 21(4), 186-91.

Shukla, D. (2013). Reconsidering residual spaces: a boon or Bane for the City? CEPT University. Accessed on 14.01.2018, Retrieved from

https://www.academia.edu/4172353/17_4.2013_final_presentation_recosidering_residual_spaces?aut o=download

Siegel, A. \& White, S. (1975). The development of spatial representations of large-scale environments. In H. W. Reese (Ed.) Advances in child development and behaviour, 10-40, New York: Academic Press.

Siemens (n.d). What is Urban Sustainability? The crystal. Accessed on 03.04.2016 Retrieved from https://www.siemens.co.uk/education/pool/teachers/crystal/downloads/what_is_urban_sustainability_v 1.pdf

Siu, K. \& Wong, K. (2015). Flexible design principles: Street furniture design for transforming environments, diverse users, changing needs and dynamic interactions. Facilities, 33(10), 588-662.

Smet, A. (2008). Learning Form Tactical Approaches to Urban Voids. Doctoral dissertation. KU Leuven and Sint-Lucas Architecture, Brussel.

Smith, R. \& Diener, E. (1975). Personality research: Components of variance attributable to the person and the situation. Journal of Personality and Social Psychology, 32(2), 199-204.

Smith, N \& Low, S. (2006). The imperative of public space. In S Low and N Smith (Eds) The Politics of Public Space, New York: Routledge 
Snieska, V. \& Zykiene, I. (2015). City attractiveness for investment: characteristics and underlying factors. Journal of Social and Behavioural Science, 213, 48-54.

Soja, E. (2000). Thirdspace: Expanding the scope of the geographical imagination, In Architecturally Speaking: Practices of Art, Architecture and the Everyday (Eds). Alan Read. London: Routledge.

Sola-Morales, D. (1995). Terrain Vague. In Cynthia Davidson (Ed.) Anyplace. Cambridge: MIT Press.

Sommer, R. (1969). Personal Space: The Behavioural Basis of Design. Englewood Cliffs, New Jersey: Prentice-Hall.

Sousa, M. (2009). Urban Landscape: Interstitial Spaces. Landscape Review, 13, 61-71.

Spooner, D. (2014). Enhancing Campus Sustainability through sites and socially equitable design.

Planning for Higher Education Journal 42(4), 30-45.

Stamps, A. (1990). Use of photographs to stimulate environments. A meta-analysis. Perceptual and Motor skills, 71, 907-913.

Stamps, A. (1992) Perceptual and preferential effects of photomontage simulations of environments. Perceptual and Motor skills, 74, 675-688.

Stamps, A., (2000). Psychology and the aesthetics of the built environment, Dordrecht, NL: Kluwer Academic Publishers Group.

Stanton, J. \& Rogelberg, S. \& (2007). Introduction: Understanding and Dealing with Organizational Survey Nonresponse. Organizational Research Methods. 10. 195-209. 10.1177/1094428106294693.

Statistics New Zealand (2014). National Population Estimates, Accessed on 30.07.2016 Retrieved from

http://www.stats.govt.nz/browse_for_stats/population/estimates_and_projections/NationalPopulationE stimates_HOTPAt30Jun14.aspx

Steg, L. \& Garling, T. (2007). Threats to the quality of urban life from car traffic: problems, causes, and solutions. Amsterdam: Elsevier

Stem, D. \& Noazin, S. (1985). The effects of a number of objects and scale positions on graphic position scale reliability. In: R.E. Lusch et al. (Eds.) AMA Educators' Proceedings, 370-373, Chicago: Marketing Association.

Sternberg, R. (1991) The urban aesthetic in comparative perspective. In: Environmental, Urban and Geographic Studies, pp. 70-79. Upper Montclair, NJ, Montclair State College.

Stevens, S. (1951). Mathematics, measurement, and psychophysics. In S.S. Stevens (Ed.), Handbook of experimental psychology. New York: John Wiley Press.

Study Learning Development (2019). Measures of variability: the range, inter-quartile range and standard deviation. University of Leicester. Accessed on 21.04.2019, Retrieved from https://www2.le.ac.uk/offices/ld/resources/numerical-data/variability

Suttles, G. (1984). The Cumulative Texture of Local Urban Culture. American Journal of Sociology, 90(2), 283-304.

Svabo, C. \& Ekelund, K. (2015). Environmental Aesthetics: Notes for Design Ecology. Design Ecologies. Accessed on 13.06.2019, Retrieved from

https://pdfs.semanticscholar.org/80f2/8e5db41b409e627896e7e2912681fc4fedf1.pdf

\section{$\mathbf{T}$}

Tadria, H. (1999). Balancing the Scales - Participants' manual. Retrieved August 13, 2018, from http://www.nzdl.org/gsdlmod 
Tahir, O. \& Roe, M. (2006). Sustainable Urban Landscapes: Making a case for the Development of an Improved Management System. International Journal on Sustainable Tropical Design Research \& Practice, 1 , pp. 17-23

Tashakkori, A., \& Teddlie, C. (1998). Mixed Methodology: Combining Qualitative and Quantitative Approaches, Thousand Oaks, CA: Sage.

Tashakkori, A., \& Teddlie, C. (2003). Handbook of mixed methods in social and behavioural research (Eds.). Thousand Oaks, CA: Sage.

Taylor, K., Salamoura, A., Randall, B., Moss, H., Tyler, L. (2008). Clarifying the nature of the distinctiveness by domain interaction in conceptual structure: comment on Cree, McNorgan, and McRae, Journal of Experimental Psychology: Learning, Memory, and Cognition, 34 (1), 719-725.

Teddlie, C., \& Tashakkori, A. (2009). Foundations of mixed methods research: Integrating quantitative and qualitative approaches in the social and behavioural sciences. Thousand Oaks, CA: Sage.

Tennessen, C., \& Cimprich, B. (1995). Views to nature: Effects on attention. Journal of Environmental Psychology, 15(1), pp. 77-85.

Thayer, R. \& Atwood, B. (1978). Plants, complexity, and pleasure in urban and suburban environments. Journal of Nonverbal Behaviour, 3, 67. Accessed on 11 Jan 2019, Retrieved from https://doi.org/10.1007/BF01135604

Thompson, M., Medley, A., \& Teran, S. (2013). The validity of the Sitting Balance Scale in older adults who are non-ambulatory or have limited functional mobility. Clinical Rehabilitation, 27(2), 166-173. Accessed on 10.03.2019, retrieved from https://doi.org/10.1177/0269215512452879

Thumfart S., Jacobs R., Lughofer E., Eitzinger C., Cornelissen F. W., Groissboeck W. (2011). Modelling human aesthetic perception of visual textures. ACM Transactions on Applied Perceptions 8(1), 1-29.

Tinsley, H. Tinsley, D. Croskeys, C. (2002). Park usage, social milieu and psychological benefits of park use reported by older urban park users from four ethnic groups. Leisure Science 24, 199-218.

Tibbalds, F. (2001). Making People-Friendly Towns: Improving the Public Environment in Towns and Cities, Spon Press: London.

Toebe, M., Cargnelutt, F., Lopes, J., Cláudia, B., Silveira, T., \& Casarotto, G. (2015). Sample size in the estimation of correlation coefficients for corn hybrids in crops and accuracy levels. Bragantia, 74(1), 16-24. Accessed on 21.05.2019, Retrieved from https://dx.doi.org/10.1590/1678-4499.0324

Toja-Silva, F., Colmenar-Santos, A. \& Castro-Gil, M. (2013). Urban wind energy exploitation systems: behaviour under multidirectional flow conditions - opportunities and challenges, Renewable Sustainable Energy 24, 364-378.

Trancik, R. (1986). Finding lost space. Theories of urban design. New York: Van Nostrand Reinhold.

Trenberth K., Willebrand, J. \& Zwiers, F. (2007). Climate Change: The Physical Science Basis. Contribution of Working Group to the Fourth Assessment Report of the Intergovernmental Panel on Climate Change. Accessed on 13.07.2016, Retrieved from https://www.ipcc.ch/report/ar4/wg1/

Triandis, H. (1989). The self and social behaviour in differing cultural contexts. Psychological Review, 96(3), 506-520.

Tsai, Y. (2005). Quantifying Urban Form: Compactness versus "Sprawl". Urban Studies, 42(1), 141161.

Turner, V. (1967). Betwixt and Between: The Liminal Period in Rites de Passage, The Symbolic Analysis of Ritual. In the forest of symbols: aspects of Ndembu ritual, Ithaca: Cornell University Press. 
Turner, V. (2013). Sustainable Urbanism: An Integrative Analysis of Master Planned Developments as a Vehicle for Urban Environmental Sustainability. Doctoral Thesis, Arizona State University, Arizona, United States.

Tversky, B., Agrawala, M., Heiser, J., Lee, P., Hanrahan, P., Phan, D., Stolte, C., \& Daniel, M. (2006) Cognitive design principles: From cognitive models to computer models. In L Magnani (Editor), Modelbased reasoning in science and engineering, 227-247, London: King's College.

Tyrvainen L., Ojala A., Korpela, K., Lanki, T., Tsunetsugu, Y. \& Kagawa, T. (2014). The influence of urban green environments on stress relief measures: A field experiment. Journal of Environmental Psychology 38, 1-9.

U

Ulrich, R. (1981). Natural versus urban scenes: some psychophysiological effects. Environment and Behaviour. 13, 523-556.

Ulrich, R. (1983). Aesthetic and affective response to the natural environment. in Altman \& Wohlwill (Eds.), Human behaviour and environment: Advances in theory and research, 85-125, New York: Plenum.

Ulrich, S. (1983). Aesthetic and affective response to natural environment, behaviour and the natural environment. Human Behaviour and Environment, 6, 85-125.

Ulrich, S. (1986). Human responses to Vegetation and Landscapes. Journal of Landscape and Urban Planning 13, 29-44.

Ulrich, R. (1993). Biophilia, biophobia, and natural landscapes. In S. Kellert \& E. Wilson (Eds.), The biophilia hypothesis. Washington, D.C.: Island Press.

Ulrich, R., Simons, R., Losito, B., Fiorito, E., Miles, M., \& Zelson, M. (1991). Stress recovery during exposure to natural and urban environments. Journal of Environmental Psychology, 11(3), 201-230. Accessed on 23.03.2019, Retrieved from http://dx.doi.org/10.1016/S0272-4944(05)80184-7

Ulrich, R., Zimring, C., Zhu, X., DuBose, J., Seo, H. \& Choi, Y. (2008). A review of the research literature on evidence-based healthcare design. Health Environments Research \& Design Journal, 1(3), 61-125.

Urban October (2015). Public Spaces for All, Designed to Live Together. Background Paper, UNHabitat. Retrieved from: http://www.urbanoctober.org/downloads/UrbanOctoberBackgroundPaper.pdf

UNEP (n.d.), Global Initiative for Resource Efficient Cities. Accessed on 13.06.2016, Retrieved from www.unep.org/pdf/Gl-REC_4pager.pdf

UN-Habitat, 2016. Energy: Un-Habitat for a Better Urban Future. Accessed on 24.09.2016, Retrieved from: http://unhabitat.org/urban-themes/energy/

United Nations World Commission on Environment and Development (1987). Our common future, Environment and Development. New York: Oxford University Press.

United Nations (2014). World Urbanization Prospects: The 2014 Revision. UN Department of Economic and Social Affairs, Population Division, New York.

V

Vale, B. \& Vale, R. (2010). Is the high-density city the only option? In Ng (ed) Designing high-density cities for social and environmental sustainability, 19-26 needs publisher etc. this is a book chapter. 
Van der Merwe, H. (1996). The research process: Problem statement and research design. In Garbers, J.G. (Ed.). Effective research in the human sciences, 278-291, Pretoria: Van Schaik.

Van Nes, A. \& Lopez, M (2010). Macro and micro scale spatial variables and the distribution of residential burglaries and theft from cars: an investigation of space and crime in the Dutch cities of Alkamaar and Gouda. Journal of Space Syntax, 2, 296-314.

Van Teijlingen, E. \& Hundley, V. (2002). The importance of pilot studies. Journal of Nursing Standard, 16 (40), 33-6. Accessed on 15.04.2018, Retrieved from

http://dx.doi.org/10.7748/ns2002.06.16.40.33.c3214

Varela, F., Thompson, E. \& Rosch, E. (1991). The embodied mind. Cognitive science and human experience. Cambridge: MIT Press.

Veale, A. (2005), Creative methodologies in Participatory Research with Children. In S. Greene \& D. Hogan (Eds.), Researching Children's Experience: Approaches and Methods, Sage Publications Ltd, London, 253-272.

Viradiya, P. (2014). Challenges Posed by Rapid Urbanization and Scarce Resources, International Proceedings of Economics and Development. Accessed on 12.04.2016, Retrieved from DOI: 10.7763/IPEDR

Voigt, A. (2015). The power of the economic in view of nature and Landscape: A discussion of the ecosystem service approach. In S. Kost and A. Schoenwald (Eds.), Landscape Change - Change of power structures, 201-219, Wiesbaden: Springer

Von Grot, R., Friedemann, R. \& Sander, R. (1988). Urban development without landscape consumption, options for clear assurance through Internal Development city, Berlin.publisher?

W

Waite, M. \& Modi, V. (2016). Modelling wind power curtailment with increased capacity in a regional electricity grid supplying a dense urban demand. Application Energy, 183, 299-317.

Walheim, C. (2006). The Landscape Urbanism reader, New York: Princeton Architectural Press.

Ward V., Bertrand J. \& Brown L. (1991). The comparability of focus group and survey results: three case studies. Evaluation Review 15: 266-283.

WCP. (2015). Wellington City Council Profile: key facts about the city, Wellington City Council. Accessed on 27.09.2016, Retrieved from http://wellington.govt.nz/ /media/aboutwellington/profile/files/wellington-city-profile.pdf

Weinreich, P. (2009). Enculturation, not acculturation: Conceptualising and assessing identity processes in migrant communities. International Journal of Intercultural Relations, 33, 124-139

Weller, L., \& Livingston, R. (1988). Effect of the colour of the questionnaire on emotional responses. Journal of General Psychology, 115, 433-440.

Wellington City Council Resident Monitoring Survey (2013). Wellington City Council 2014/2015 annual report. Accessed on 21.01.2019, Retrieved from https://wellington.govt.nz/ /media/yourcouncil/plans-policies-and-bylaws/plans-and-policies/annualreport/2014-15/2014-15-annual-report.pdf

Wellington City Council (2014). Wellington City Housing and Residential Growth Study: Final Planning Assessment and Recommendations. The Property Group New Zealand. Accessed on 16.10.2016, Retrieved from http://wellington.govt.nz/ /media/your-council/projects/files/housing-choicesupply/wcc-residential-study-final-report.pdf 
Wellington Future 2040. (2015). Reshaping Wellington Future, Wellington City Council. Accessed on 17.10.2016, Retrieved from http://www.wgtn2040.govt.nz/smart-green-wellington/dynamic-centralcity/strategic-urban-development

Wellington, J. (2000). Educational Research: Contemporary Issues and Practical Approaches. London: Continuum.

Wellington Urban Growth Plan (2014). Wellington Urban Growth Plan 2014-2043, Absolutely positively Wellington City Council. Accessed on 12.06.2016, Retrieved from http://wellington.govt.nz/ /media/have-your-say/public-input/files/consultations/2014/09-wellingtonuban-growth-plan/draft-wugp-2014-2043.pdf?la=en

Wells, N., \& Evans, G. (2003). Nearby nature: A buffer of life stress among rural children. Environment and Behaviour 35(3), 311-330.

Welman, J. \& Kruger, S. (1999). Research methodology for the business and administrative sciences. Johannesburg, SA: International Thompson.

Whyte, W.H. (1980). The Social Life of Small Urban Spaces. Project for Public Places, pg.125, New York: Project for Public Spaces.

Widgery, R. (1982). Satisfaction with the Quality of Urban Life: A Predictive Model, American Journal of Community Psychology, 10(1), 37-48.

Wikstrom, T. (2004). Connecting the Suburban Landscape - The significance of informal footpaths in Flemingsberg, Sweden. Paper presented at Cities for People Conference, Copenhagen.

Wilkinson, K. (1991). The community in rural America. New York: Greenwood Press.

Wilkinson, L. (2011). Vacant Property: Strategies for Redevelopment in the Contemporary City. Accessed on 09.06.2016 Retrieved from https://smartech.gatech.edu/bitstream/handle/1853/40778/LukeWilkinson_Vacant\%20Property.pdf

Williams, K (n.d). Can Urban Intensification Contribute to Sustainable Cities?. An International Perspective. City Matters. Accessed on 06.05.2017 Retrieved from http://eprints.uwe.ac.uk/9233

Williams, K. (1999). Urban Intensification Policies in England: Problems and Contradictions, Land Use Policy, 16(3), 167-178.

Williams, K., Burton, E. \& Jenks, M. (2000). Achieving Sustainable Urban Form, London: Routledge Press.

Wilson, G., \& Baldassare, M. (1996). Overall "sense of community" in a suburban region: The effects of localism, privacy, and urbanization. Environment and Behavior, 28, 27-43

Wohlwill, J. F. (1966). The physical environment: A problem for the psychology of stimulation. Journal of Social Issues, 22, 29-38.

Wood J., Troutbeck R. The effect of restriction of the binocular visual field on driving performance. Ophthalmic and Physiological Optics, 12(3), 291-298.

Woolley, H. (2003). Urban open spaces. London, England: Spon Press.

World Bank (n.d.). Social Inclusion. The World Bank. Accessed on 12.01.2018, Retrieved from https://www.worldbank.org/en/topic/social-inclusion

Worpole, K. Knox, K. (2007). The social value of Public Spaces, New York: Joseph Rowntree F oundation.

WSPT (2015). Women's Safely in Public Transport: A pilot initiative in Bhopal. The WRI Ross Center for Sustainable Cities. Accessed on 28.10.2019, Retrieved from https://wrirosscities.org/sites/default/files/Final_Report_30072015.pdf 
Yanarella, E. \& Levine, R., (1992). Does sustainable development lead to sustainability, Futures 24 (8), 759-774

Yildirim, K., Akalinbaskaya, A. \& Hidayetoglu, M. (2007). Effects of indoor colour on mood and cognitive performance. Building and Environment, 42, 3233-3240. Accessed on 20.02.2019, Retrieved from http://linkinghub.elsevier.com/retrieve/pii/S036013230600229

\section{Z}

Zangwill, N. (2003). Aesthetic Judgment, Stanford Encyclopedia of Philosophy. Accessed on 12.06.2019, Retrieved from https://plato.stanford.edu/entries/aesthetic-judgment/

Zeki, S. (1999). Inner vision: An exploration of art and the brain. Oxford: Oxford University Press.

Zeki, S., \& Bartels, A. (1999). Toward a theory of visual consciousness. Consciousness and Cognition: An International Journal, 8(2), 225-259.

Zhou, D., Shah, T., Jebran, K., Ali, S., Ali, A., Ali, A., \& Abdullah (2017). Acceptance and willingness to pay for the solar home system: Survey evidence from the northern area of Pakistan. Energy Reports, 3, 54-60. doi:10.1016/j.egyr.2017.03.00

Zubin, J. \& Spring, B. (1977). Vulnerability-a new view of schizophrenia. Journal of Abnormal Psychology, 86(2), 103-126. 


\section{APPENDICES}

\section{Chapter 6: Initial Visual Preference Study}

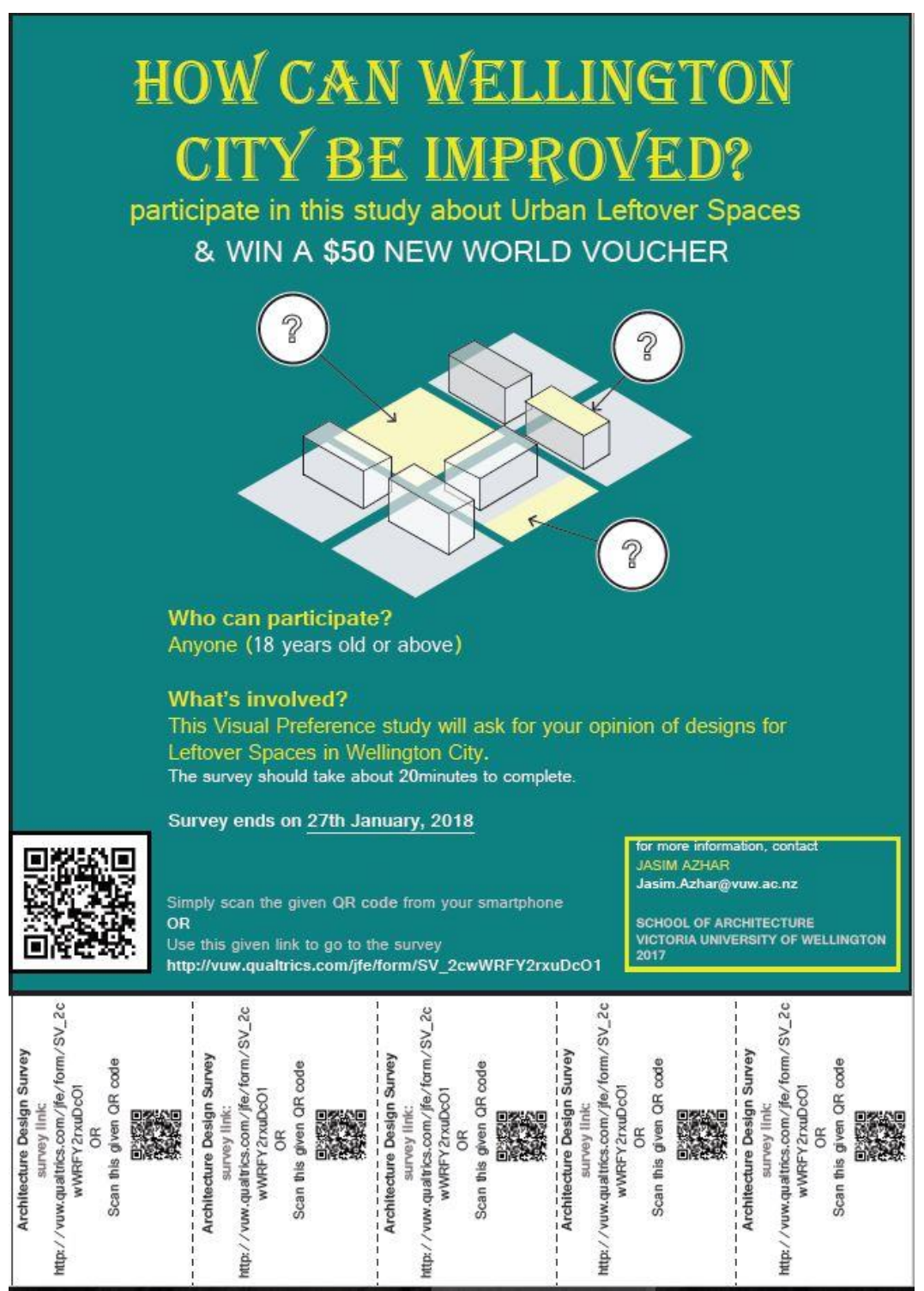

Appendix Figure 6.1 Invitation poster of Study 2 (date changed to $15^{\text {th }}$ Oct 2017) 


\begin{tabular}{|l|l|l|}
\hline & Mean & $\begin{array}{c}\text { Standard } \\
\text { Deviation (SD) }\end{array}$ \\
\hline Preferences of all Participants & & \\
\hline Space underneath the building & & 1.20 \\
\hline Image A & 2.77 & 1.21 \\
\hline Image B & 2.75 & $\underline{0.54}$ \\
\hline Image C & $\underline{4.60}$ & \\
\hline Space in front of the building & & $\underline{0.75}$ \\
\hline Image A & $\underline{4.48}$ & 1.21 \\
\hline Image B & 2.49 & 1.09 \\
\hline Image C & 3.28 & 1.14 \\
\hline Space at the back of the building & & $\underline{1.01}$ \\
\hline Image A & 3.19 & 1.21 \\
\hline Image B & $\underline{3.32}$ & \\
\hline Image C & 3.00 & 1.19 \\
\hline Space enclosed by the buildings on & & 1.13 \\
\hline three sides & & 1.18 \\
\hline Image A & & \\
\hline Image B & 3.11 & 1.27 \\
\hline Image C & 2.67 & \\
\hline Space enclosed by the buildings on & $\underline{0.97}$ \\
\hline two sides & & \\
\hline Image A & & \\
\hline Image B & & \\
\hline Image C & 2.70 & \\
\hline Space at the rooftop of the building & & \\
\hline Image A & & \\
\hline Image B & & \\
\hline Image C & & \\
\hline Gender Preferences & & \\
\hline Space underneath the building & & \\
\hline Image A & & \\
\hline
\end{tabular}




\begin{tabular}{|l|l|l|}
\hline Male & 2.87 & 1.17 \\
\hline Female & 2.85 & 1.19 \\
\hline Image B & & 1.22 \\
\hline Male & 2.85 & 1.26 \\
\hline Female & 2.67 & \\
\hline Image C & & 0.49 \\
\hline Male & 4.62 & 0.58 \\
\hline Female & 4.58 & \\
\hline Space in front of the building & & 0.70 \\
\hline Image A & & 0.79 \\
\hline Male & 4.52 & 1.22 \\
\hline Female & 4.45 & 1.02 \\
\hline Image B & & 1.25 \\
Male & 2.44 & 1.23 \\
\hline Female & 2.53 & 1.20 \\
\hline Image C & & 1.15 \\
\hline Male & 3.19 & 1.22 \\
\hline Female & 3.36 & \\
\hline Space at the back of the building & & \\
\hline Image A & 3.08 & \\
Male & 3.13 & \\
\hline Female & 3.18 & \\
\hline Image B & 3.18 & \\
Male & & \\
\hline Female & & \\
\hline Image C & & \\
Male & & \\
\hline Female & & \\
\hline Space enclosed by the buildings on & & \\
three sides & & \\
\hline Image A & & \\
Male & & \\
Female & & \\
\hline
\end{tabular}




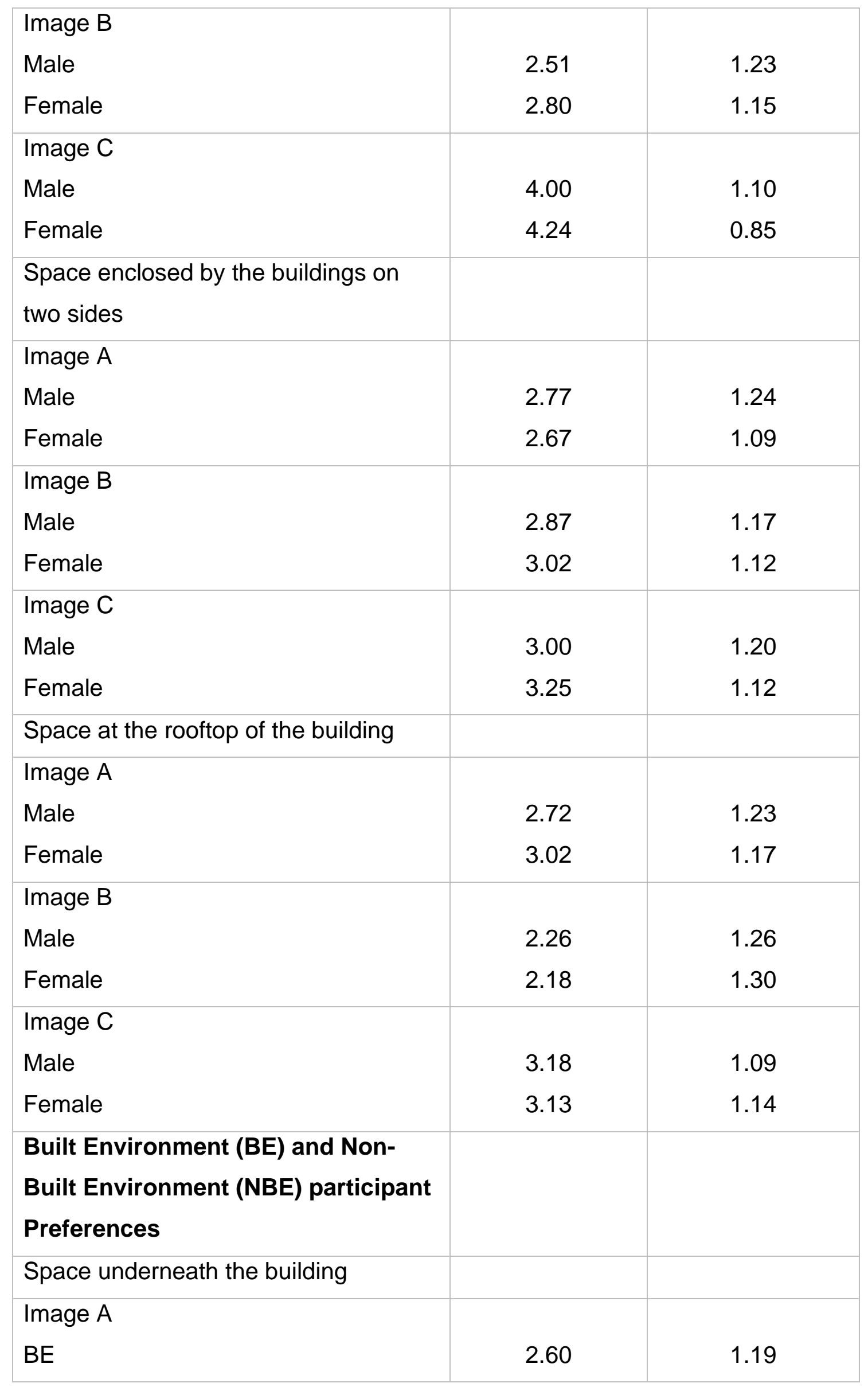




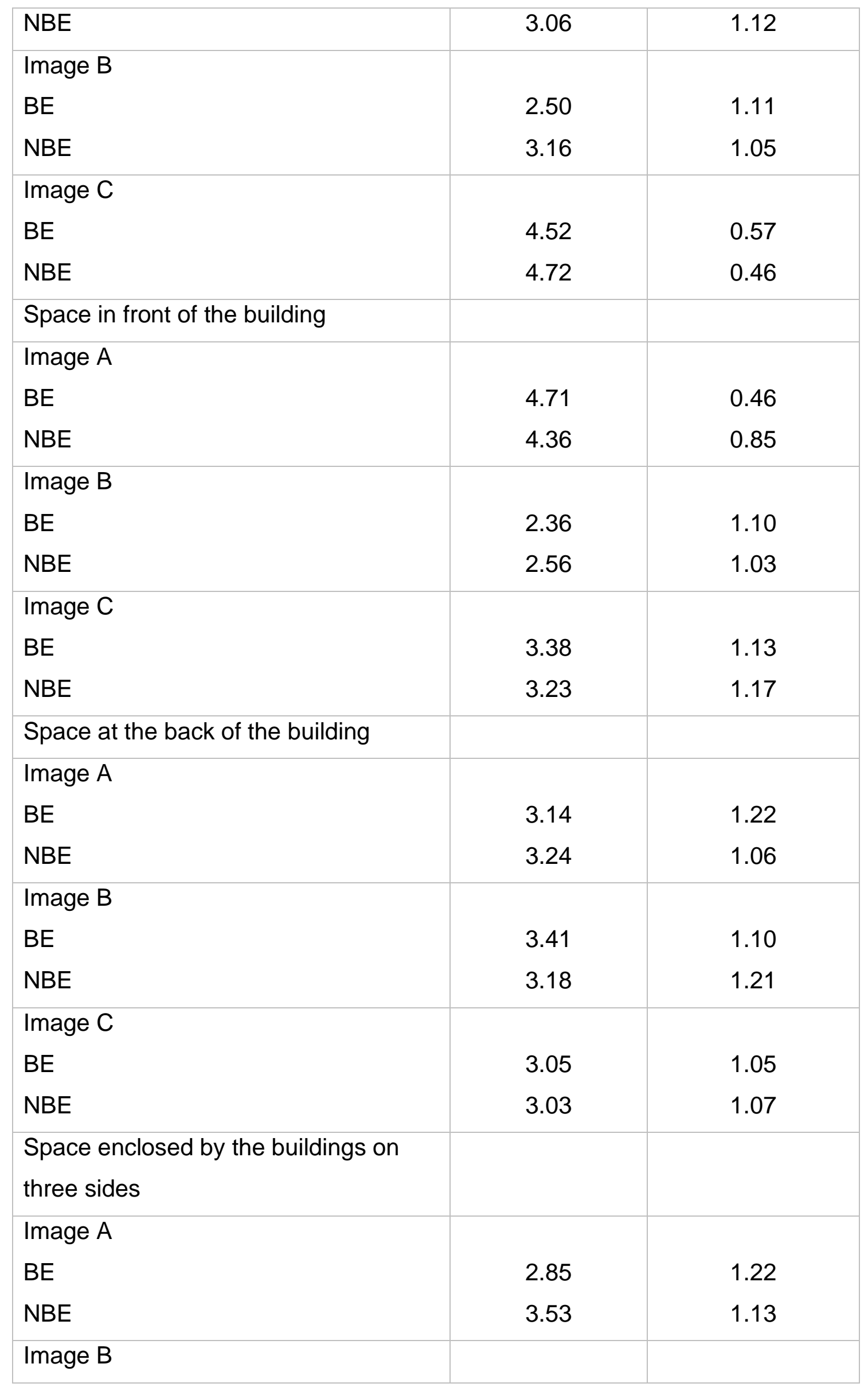




\begin{tabular}{|c|c|c|}
\hline $\begin{array}{l}\mathrm{BE} \\
\mathrm{NBE}\end{array}$ & $\begin{array}{l}2.67 \\
2.66\end{array}$ & $\begin{array}{l}1.12 \\
1.16\end{array}$ \\
\hline \multicolumn{3}{|l|}{ Image C } \\
\hline $\mathrm{BE}$ & 4.06 & 1.10 \\
\hline NBE & 4.25 & 0.90 \\
\hline \multicolumn{3}{|c|}{$\begin{array}{l}\text { Space enclosed by the buildings on } \\
\text { two sides }\end{array}$} \\
\hline \multicolumn{3}{|l|}{ Image A } \\
\hline $\mathrm{BE}$ & 2.43 & 1.22 \\
\hline NBE & 3.18 & 1.12 \\
\hline \multicolumn{3}{|l|}{ Image B } \\
\hline $\mathrm{BE}$ & 2.80 & 1.15 \\
\hline NBE & 3.21 & 1.05 \\
\hline \multicolumn{3}{|l|}{ Image C } \\
\hline $\mathrm{BE}$ & 3.06 & 1.22 \\
\hline NBE & 3.27 & 1.18 \\
\hline \multicolumn{3}{|c|}{ Space at the rooftop of the building } \\
\hline \multicolumn{3}{|l|}{ Image A } \\
\hline $\mathrm{BE}$ & 2.60 & 1.14 \\
\hline NBE & 3.34 & 1.08 \\
\hline \multicolumn{3}{|l|}{ Image B } \\
\hline $\mathrm{BE}$ & 2.25 & 1.20 \\
\hline NBE & 2.16 & 1.27 \\
\hline \multicolumn{3}{|l|}{ Image C } \\
\hline $\mathrm{BE}$ & 2.92 & 1.13 \\
\hline NBE & 3.53 & 1.04 \\
\hline
\end{tabular}

Appendix table 6.1 Mean preferences of different options shown to participants 


\section{Study 1: Initial Visual Preference Study (Note: some images are not stored) O INVESTIGATING THE ROLE OF URBAN LEFTOVER SPACES \\ INFORMATION SHEET FOR PARTICIPANTS}

Thank you for your interest in this project. Please read this information before deciding whether or not to take part. If you decide to participate, thank you. If you decide not to take part, thank you for considering my request. Who am I?

I am Jasim Azhar (Jasim.Azhar@vuw.ac.nz) a Ph.D. Candidate at the School of Architecture, Victoria University of Wellington, working under the supervision of Dr. Morten Gjerde (Morten.Gjerde@vuw.ac.nz), Head of the School of Architecture. This survey is work towards my Ph.D. thesis.

\section{What is the aim of the project?}

My research examines the potential for the positive use of public and privately owned urban leftover spaces. The aim is to use leftover spaces in ways that can help meet future needs.

This phase 1 study surveys how people think about such spaces in terms of their appearance. All spaces are situated in Wellington city whereas this study investigates people's preferences when it comes to their physical and spatial features. This research has been approved by the Victoria University of Wellington Human Ethics Committee [24915].

\section{How can you help?}

If you agree to take part in this online survey which forms phase 1 of the project in which I will show you a series of images and ask questions relating to those. The survey will take approximately $15-20$ minutes to be completed.

You can save your survey and come back to it at any point until 30 minutes after you have started. You can also go back to edit any answer up to the point you submit the survey. You can withdraw from the survey by contacting me before 10 October 2017. If you withdraw, any information you have provided will be destroyed.

\section{What will happen to the information you give?}

This research is confidential. This means that I and my supervisor will be aware of your identity but the research data will be aggregated and your identity will not be discoverable in any reports, presentations, my thesis, or other public documentation. The survey data will be kept securely and destroyed 3 years after my thesis is submitted.

Personal details will only be collected if you wish to enter the prize draw or request a copy of the final report or become a part of doing Survey 2 . All personal details come to me separately from the survey data and are kept confidential.

\section{What will the project produce?}

The information from my research will be used in my Ph.D. dissertation, academic publications, and conferences.

If you accept this invitation, what are your rights as a research participant?

You do not have to accept this invitation if you don't want to. If you decide to participate, you have the right to:

- choose not to answer any question;

- withdraw from the study before 30 OCTOBER 2017;

- ask any questions about the study at any time;

- have access to any writings related to this survey by emailing me to request a copy.

If you have any questions or problems, who can you contact?

If you have any questions, either now or in the future, please feel free to contact either:

Researcher Name: Jasim Azhar

Email: Jasim.Azhar@vuw.ac.nz

Supervisor Name: Dr. Morten Gjerde

Role: Head of School

School: Architecture

Phone: 044636233

Email: Morten.Gjerde@vuw.ac.nz

\section{Human Ethics Committee information}


If you have any concerns about the ethical conduct of the research you may contact the Victoria University HEC Convener: Associate Professor Susan Corbett. Email susan.corbett@vuw.ac.nz or telephone +64-4-463 5480.

Yes, I have read the information sheet and wants to continue (1)

No, I don't want to continue (2)

0.1 Participant Consent Form This consent form will be held for 3 years after the completion date of this project unless the participant withdraws from this study before 30.10.2017 when it will be destroyed.

I give consent to my participation in the research project as explained in the information statement. In giving my consent, I acknowledge that: The procedures required for the project and the time involved have been explained to me, and any questions I have about the project have been answered to my satisfaction.

I
have read the Participant Information Statement and have been given the opportunity to discuss the information and my involvement in the project with the researcher. I understand that I can choose not to participate in the study, without affecting my relationship with the researcher now or in the future. The information I have provided will be destroyed 3 years after the research is finished. Any information I provide will be kept confidential to the researcher and the supervisor. I understand that my name will not be included in this thesis or any other form of reports and will be kept confidential.

I understand that the results will be used for a Ph.D. thesis and a summary of the results may be used in academic reports and/or presented at conferences or in journal articles.

Yes, I agree to participate in the above study by providing my name (as a signup) in the given tab (12)

No, I don't want to participate (13)

Q1.0 Would you like to receive a copy of the final report through an email?

Yes (1)

No (2)

\section{Q1.1 Gender}

Male (1)

Female (2) 
Q1.2 Age

$18-25(1)$

26-35 (2)

36-44 (3)

45-54 (4)

56-65 (5)

$66+(6)$

Q1.3 Cultural Ethnicity

Maori (1)

Pacific Islander (2)

Asian (3)

European (4)

African (5)

NZ European (Pakeha) (7)

Other (6) 
Q1.4 Education Level

High School (5)

Undergraduate (2)

Post Graduate (3)

Doctorate (4)

Trade certificate (6)

Other (7)

Q1.5 Field of Study / Work

Health Science (1)

Finance (2)

Education (3)

Built Environment (4)

Science (5)

Other (6)

End of Block: General Data Collected

Start of Block: SURVEY STARTS

Page Break 
Q2 This survey is divided into three parts.

In Part 1, you will be shown two images and will be asked to select the one you find more appealing. There is no right or wrong answer so please tick one box.

Visually which image you prefer (select one option)?

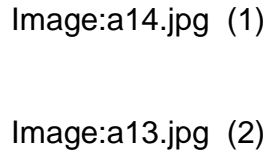

Q2.1 Can you give one reason for your selection?

Planting in space (4)

Other (3)

Hard construction material (9)

Q3 Visually which image you prefer (select one option)?

Image:Unmanaged (1)

Image:upkeep (2)

Q3.1 Can you give one reason for your selection?

Disorganised Space (5)

Other (4)

Cleanliness of Space (3)

Q4 Visually which image you prefer (select one option)?

Image:re1.jpg (1)

Image:open1 (2) 
Q4.1 Can you give one reason for your selection?

Covered space (5)

Other (6)

Openness of space (4)

Q5 Visually which image you prefer (select one option)?

Image:order (1)

Image:1324.jpg (2)

Q5.1 Can you give one reason for your selection?

Unmanaged Space (3)

Other (6)

Properly Maintained Space (4)

Q6 Visually which image you prefer (select one option)?

Image:modern (1)

Image:historical (2) 
Q6.1 Can you give one reason for your selection?

Construction material (3)

Other (6)

Color of facade (5)

Page Break

Q7 In Part 2 of this survey, you have to rate three different images in terms of how each image appeals to you.

Rate how visually appealing you find the images $A-C$ at in front of a building

A

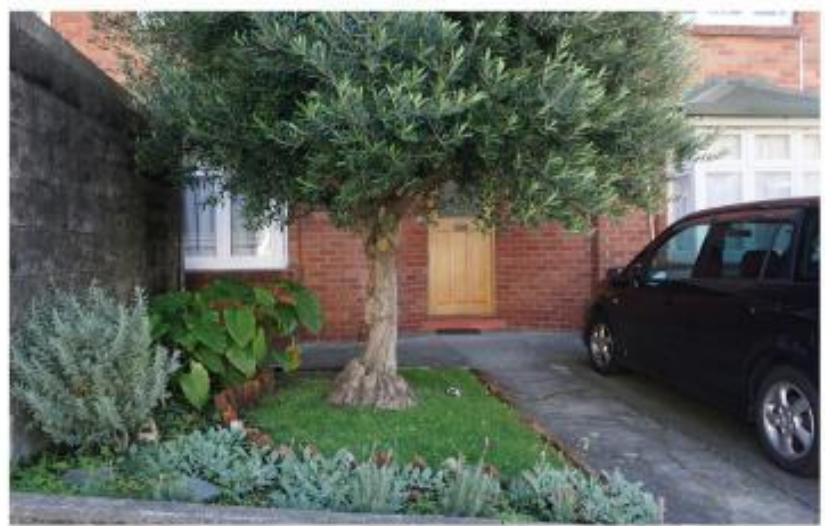

B

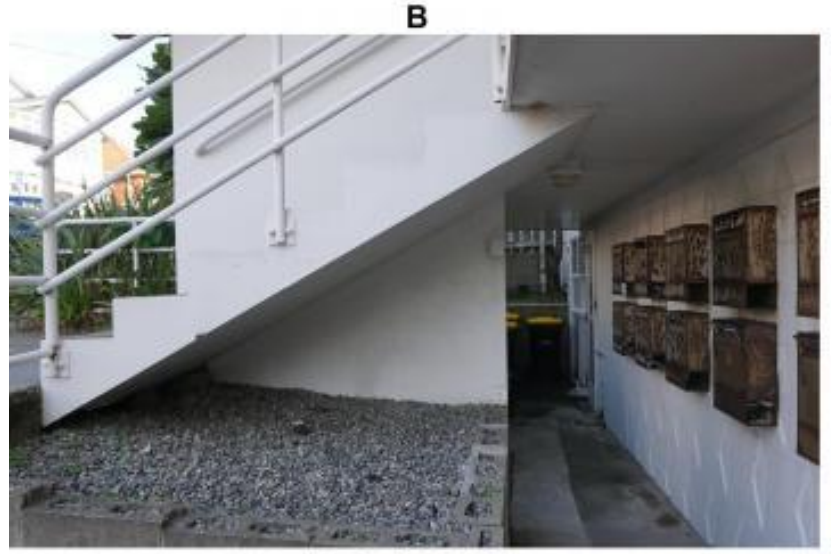

C

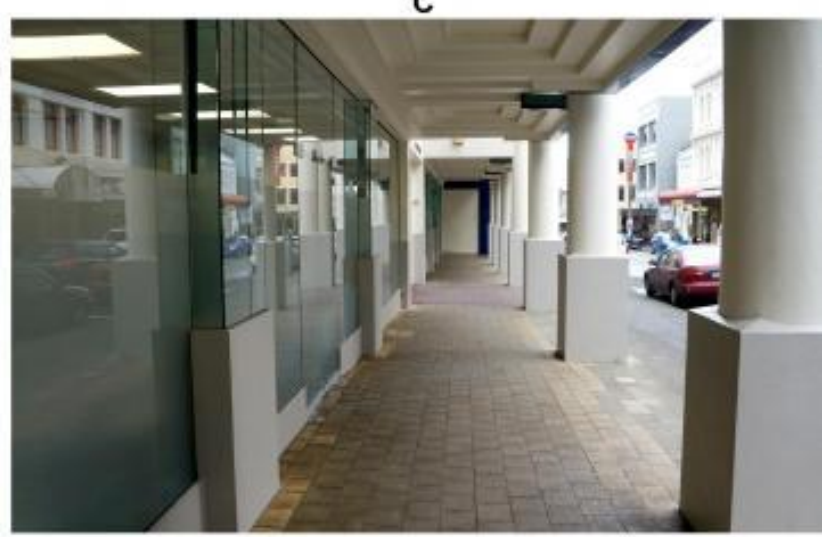


Q7.1 Please score each image from the scale -2 (Dislike) to 2 (Like)

$\begin{array}{ccccc}\text { Dislike } & \text { Somewhat } & \text { Neither Like or } & \text { Somewhat Like } & \text { Like } \\ (-2)(1) & \text { Dislike } & \text { Dislike } & (1)(4) & (2)(5)\end{array}$
A (1)
$\mathrm{B}(2)$
C (3)

Q7.2 Provide one reason behind the selection of your score for Image A (Please click one icon below or write an alternative reason in Box $A$ )

\begin{tabular}{|c|c|c|c|c|c|}
\hline & Planting (1) & $\begin{array}{c}\text { Organised } \\
\text { parking space } \\
\text { (2) }\end{array}$ & $\begin{array}{l}\text { Cleanliness of } \\
\text { the space (8) }\end{array}$ & $\begin{array}{c}\text { Clear entrance } \\
\text { (9) }\end{array}$ & $\begin{array}{c}\text { Different } \\
\text { Materials / } \\
\text { Colors (13) }\end{array}$ \\
\hline A (2) & & & & & \\
\hline
\end{tabular}

Q7.3 Provide one reason behind the selection of your score for Image B (Please click one icon below or write an alternative reason in Box B)

$\begin{array}{ccccc}\text { Dark space (1) } & \begin{array}{c}\text { Decoration on } \\ \text { wall (2) }\end{array} & \begin{array}{c}\text { Clear } \\ \text { thoroughfare (8) }\end{array} & \text { Dead end (9) } & \begin{array}{c}\text { Different } \\ \text { Materials / } \\ \text { Colors (13) }\end{array}\end{array}$


Q7.4 Provide one reason behind the selection of your score for Image C (Please click one icon below or write an alternative reason in Box $\mathrm{C}$ )

\begin{tabular}{l|ccccc} 
Visual & $\begin{array}{c}\text { Clear } \\
\text { Permeability } \\
\text { (view through to } \\
\text { street) (1) }\end{array}$ & $\begin{array}{c}\text { Sense of } \\
\text { openness (8) }\end{array}$ & $\begin{array}{c}\text { Reflection on } \\
\text { thoroughfare (9) }\end{array}$ & $\begin{array}{c}\text { Use of dull } \\
\text { materials } / \\
\text { colours (14) }\end{array}$ \\
\hline C (2) & & &
\end{tabular}

Page Break 
Q8 Rate how visually appealing you find the images $A-C$ at back of a building

A

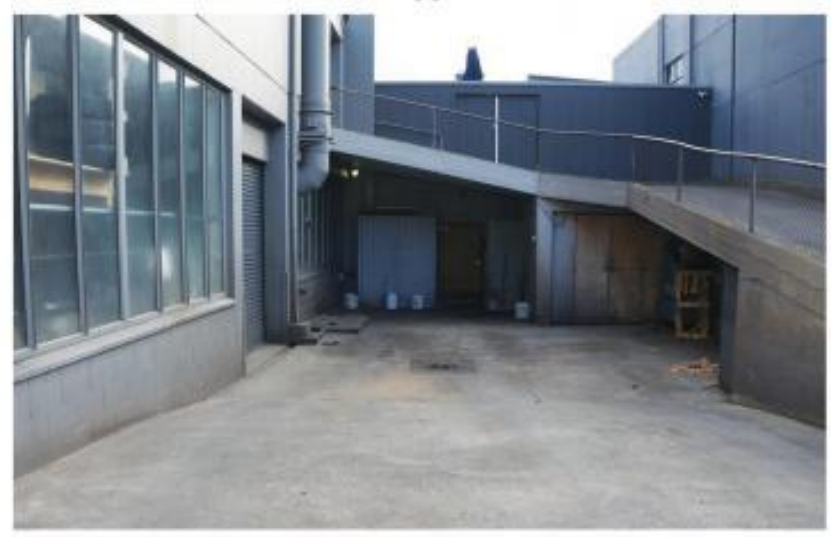

B

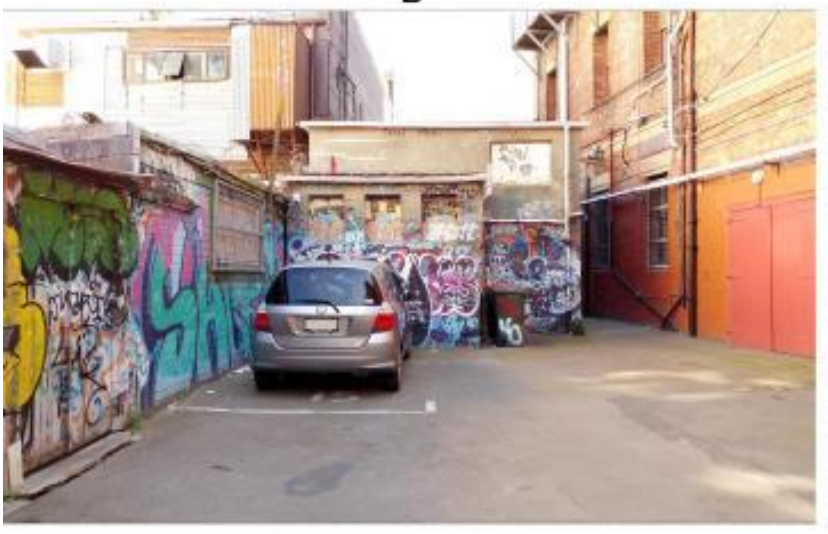

C

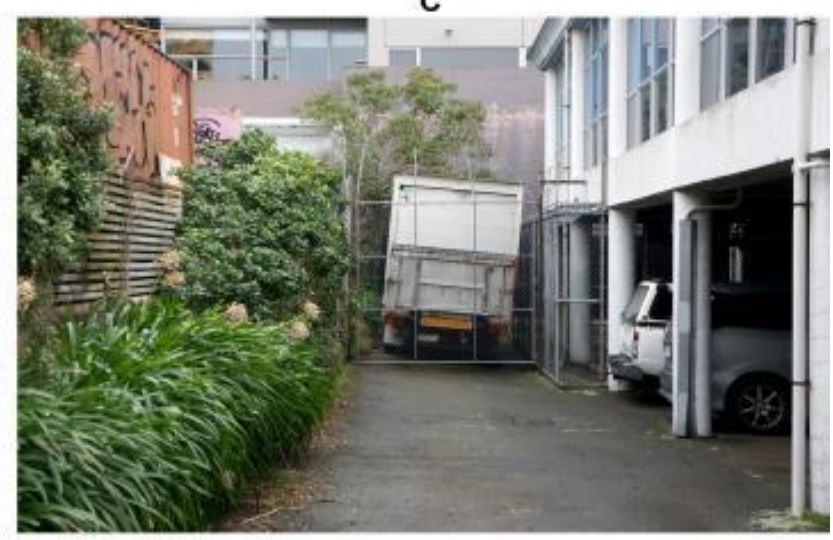

Q8.1 Please score each image from the scale -2 (Dislike) to 2 (Like)

$\begin{array}{ccccc}\text { Dislike } & \text { Somewhat } & \text { Neither Like or } & \text { Somewhat Like } & \text { Like } \\ (-2)(1) & \text { Dislike } & \text { Dislike } & (1)(4) & (2)(5)\end{array}$

\begin{tabular}{l|l}
$A(1)$ & \\
$B(2)$ & \\
$C(3)$ &
\end{tabular}


Q8.2 Provide one reason behind the selection of your score for Image A (Please click one icon below or write an alternative reason in Box A)

\begin{tabular}{c|ccccc} 
Poor & $\begin{array}{c}\text { Storage space } \\
\text { under ramp (2) }\end{array}$ & $\begin{array}{c}\text { Openness to } \\
\text { Sky (8) }\end{array}$ & $\begin{array}{c}\text { Clear entrance } \\
\text { to building (9) }\end{array}$ & $\begin{array}{c}\text { Use of dull } \\
\text { materials / } \\
\text { colours (13) }\end{array}$
\end{tabular}

A (2)

Q8.3 Provide one reason behind the selection of your score for Image B (Please click one icon below or write an alternative reason in Box B)

\begin{tabular}{|c|c|c|c|c|c|}
\hline & $\begin{array}{c}\text { Wires and } \\
\text { Pipes on wall } \\
\text { (1) }\end{array}$ & $\begin{array}{l}\text { Graffiti on wall } \\
\text { (2) }\end{array}$ & Parked car (8) & $\begin{array}{l}\text { Lack of clear } \\
\text { Entrance (9) }\end{array}$ & $\begin{array}{c}\text { Different } \\
\text { Materials / } \\
\text { Colors (13) }\end{array}$ \\
\hline B (2) & & & & & \\
\hline
\end{tabular}

Q8.4 Provide one reason behind the selection of your score for Image C (Please click one icon below or write an alternative reason in Box C)

\begin{tabular}{|c|c|c|c|c|c|}
\hline & Planting (1) & Parked car (2) & $\begin{array}{l}\text { Well organised } \\
\text { space }(7)\end{array}$ & Dead End (8) & $\begin{array}{c}\text { Different } \\
\text { Materials / } \\
\text { Colors (13) }\end{array}$ \\
\hline C (2) & & & & & \\
\hline
\end{tabular}

\section{Page Break}


Q9 Rate how visually appealing you find the images $A-C$ for a space enclosed by buildings on two sides.

A

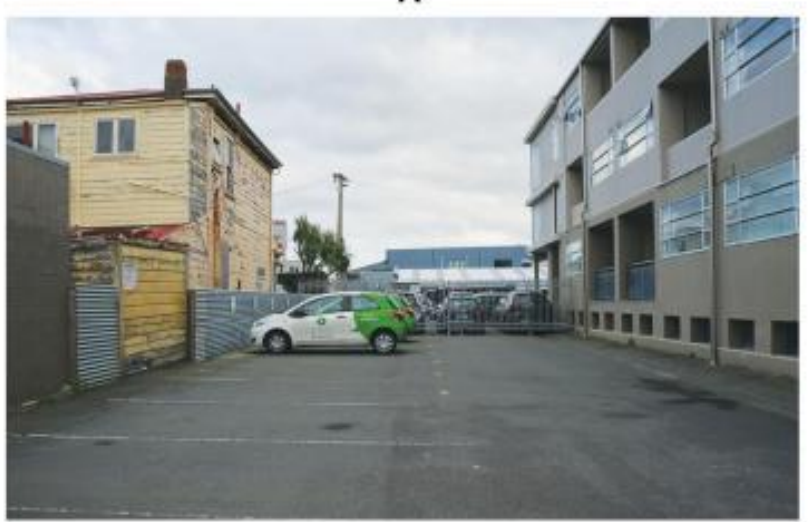

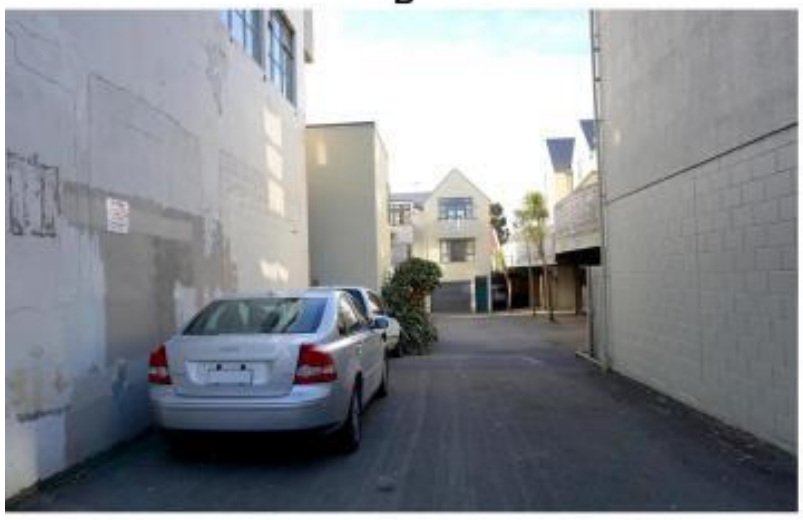

C

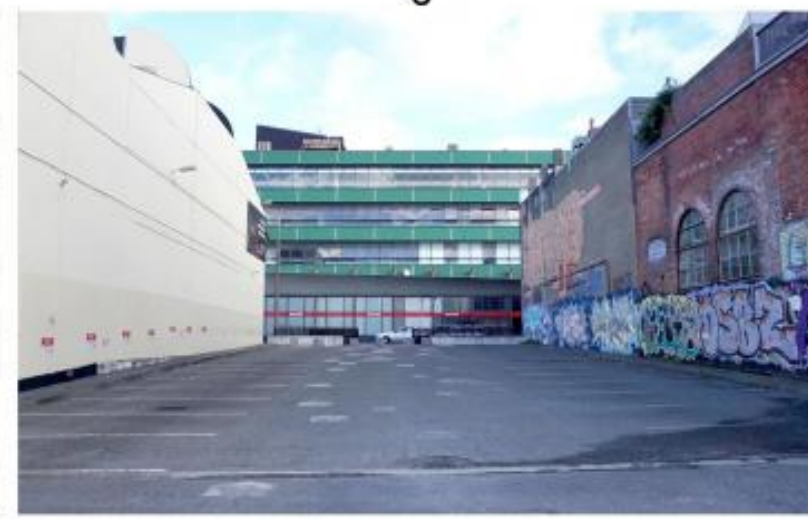

Q9.1 Please score each image from the scale -2 (Dislike) to 2 (Like)

\begin{tabular}{|c|c|c|c|c|c|}
\hline & $\begin{array}{l}\text { Dislike } \\
(-2)(1)\end{array}$ & $\begin{array}{l}\text { Somewhat } \\
\text { Dislike } \\
(-1)(2)\end{array}$ & $\begin{array}{l}\text { Neither Like or } \\
\text { Dislike } \\
\text { (0) (3) }\end{array}$ & $\begin{array}{l}\text { Somewhat Like } \\
\text { (1) (4) }\end{array}$ & $\begin{array}{l}\text { Like } \\
\text { (2) (5) }\end{array}$ \\
\hline \multicolumn{6}{|l|}{$A(1)$} \\
\hline \multicolumn{6}{|l|}{$B(2)$} \\
\hline C (3) & & & & & \\
\hline
\end{tabular}


Q9.2 Provide one reason behind the selection of your score for Image A (Please click one icon below or write an alternative reason in Box $A$ )

$\begin{array}{ccccc}\begin{array}{c}\text { Variety of } \\ \text { boundary Walls } \\ \text { / fences }(1)\end{array} & \begin{array}{c}\text { Different style } \\ \text { of facade (2) }\end{array} & \begin{array}{c}\text { Openness to } \\ \text { sky (8) }\end{array} & \text { Parked cars (9) } & \begin{array}{c}\text { Different } \\ \text { Materials / }\end{array} \\ \text { Colors (13) }\end{array}$

A (2)

Q9.3 Provide one reason behind the selection of your score for Image B (Please click one icon below or write an alternative reason in Box B)

\begin{tabular}{c|cccc} 
& $\begin{array}{c}\text { Clear } \\
\text { Thoroughfare } \\
(2)\end{array}$ & Planting (7) & Parked Car (8) & $\begin{array}{c}\text { Different } \\
\text { Materials / } \\
\text { Colors (13) }\end{array}$ \\
\hline B (2) & & &
\end{tabular}

Q9.4 Provide one reason behind the selection of your score for Image C (Please click one icon below or write an alternative reason in Box C)

\begin{tabular}{c|ccccc} 
Gaffiti on a & $\begin{array}{c}\text { Variety of } \\
\text { facades (2) }\end{array}$ & $\begin{array}{c}\text { Well maintained } \\
\text { space (7) }\end{array}$ & $\begin{array}{c}\text { Openness to } \\
\text { Sky (9) }\end{array}$ & $\begin{array}{c}\text { Different } \\
\text { Materials / } \\
\text { Colors (13) }\end{array}$ \\
\hline C (2) & & &
\end{tabular}

Page Break 


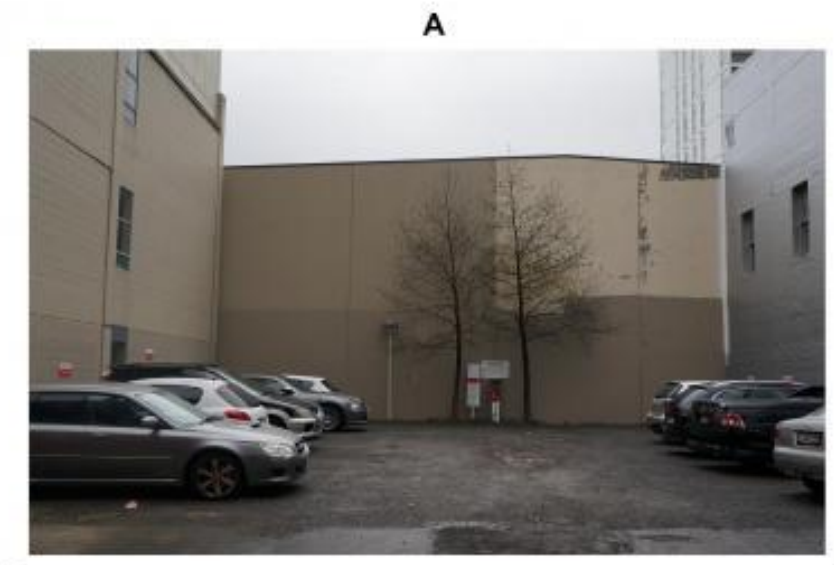

B
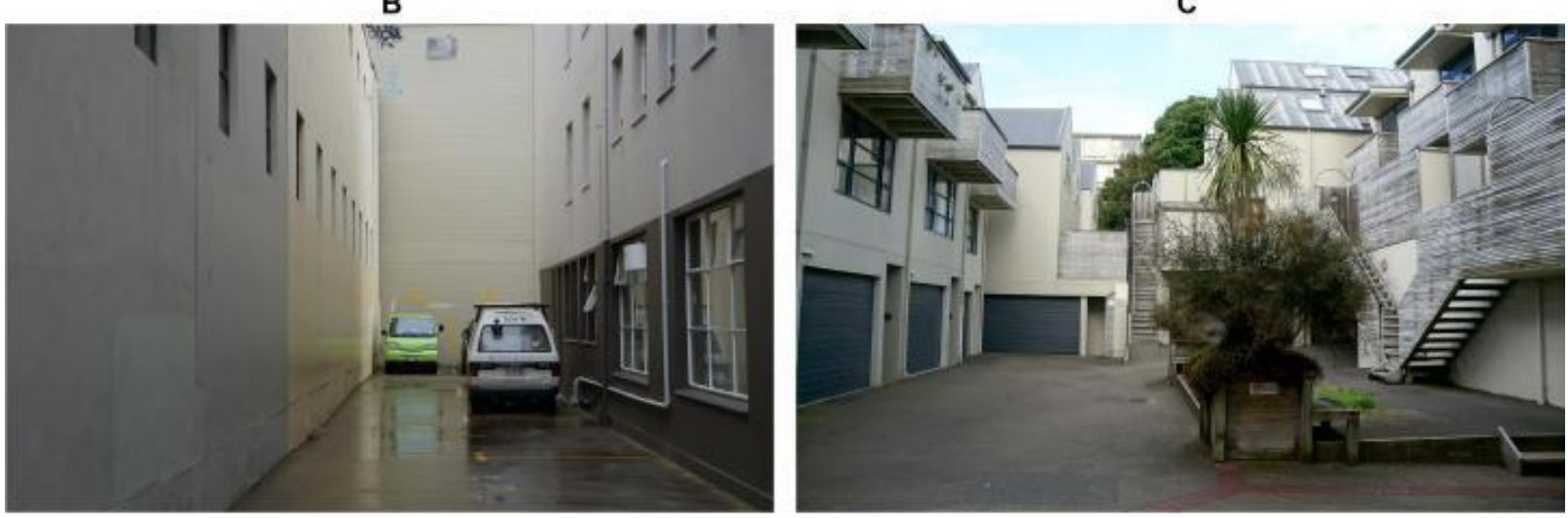

Q10.1 Please score each image from the scale -2 (Dislike) to 2 (Like)

\begin{tabular}{|c|c|c|c|c|c|}
\hline & $\begin{array}{l}\text { Dislike } \\
(-2)(1)\end{array}$ & $\begin{array}{l}\text { Somewhat } \\
\text { Dislike } \\
(-1)(2)\end{array}$ & $\begin{array}{l}\text { Neither Like or } \\
\text { Dislike } \\
\text { (0) (3) }\end{array}$ & $\begin{array}{c}\text { Somewhat Like } \\
\text { (1) (4) }\end{array}$ & $\begin{array}{l}\text { Like } \\
\text { (2) (5) }\end{array}$ \\
\hline$A(1)$ & & & & & \\
\hline B (2) & & & & & \\
\hline C (3) & & & & & \\
\hline
\end{tabular}


Q10.2 Provide one reason behind the selection of your score for Image A (Please click one icon below or write an alternative reason in Box A)

\begin{tabular}{l|cccc} 
& Planting (1) & Dead end (7) & $\begin{array}{c}\text { Openness to } \\
\text { Sky (8) }\end{array}$ & $\begin{array}{c}\text { Different } \\
\text { Materials / } \\
\text { Colors (13) }\end{array}$ \\
\hline A (2) & & &
\end{tabular}

Q10.3 Provide one reason behind the selection of your score for Image B (Please click one icon below or write an alternative reason in Box B)

\begin{tabular}{c|cccc}
$\begin{array}{c}\text { Well Maintained } \\
\text { Space (1) }\end{array}$ & Dead End (2) & Parked Car (8) & $\begin{array}{c}\text { Openings in } \\
\text { Walls (9) }\end{array}$ & $\begin{array}{c}\text { Different } \\
\text { Materials / } \\
\text { Colors (13) }\end{array}$ \\
\hline
\end{tabular}

B (2)

Q10.4 Provide one reason behind the selection of your score for Image C (Please click one icon below or write an alternative reason in Box $\mathrm{C}$ )

$\begin{array}{cccc}\text { Similar Style of } & \text { Planting (2) } & \begin{array}{c}\text { Change in } \\ \text { Level (7) }\end{array} & \begin{array}{c}\text { Openness to } \\ \text { Construction (1) }\end{array}\end{array} \quad \begin{gathered}\text { Dead End (13) }\end{gathered}$

\section{C (2)}

Page Break 
Q11 Rate how visually appealing you find the images $A-C$ of spaces underneath a building.

A
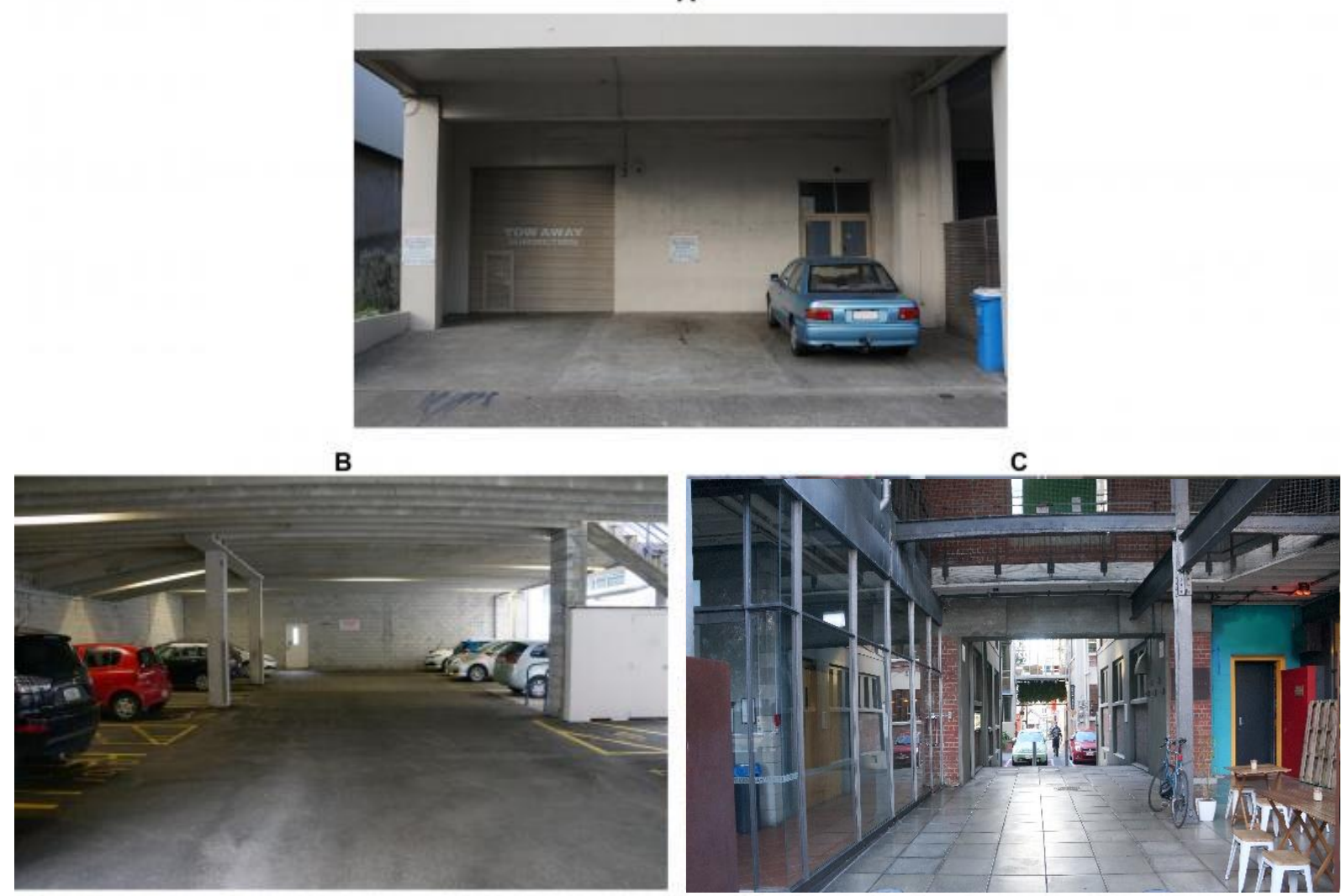

Q11.1 Please score each image from the scale -2 (Dislike) to 2 (Like)

\begin{tabular}{|c|c|c|c|c|c|}
\hline & $\begin{array}{l}\text { Dislike } \\
(-2)(1)\end{array}$ & $\begin{array}{l}\text { Somewhat } \\
\text { Dislike } \\
(-1)(2)\end{array}$ & $\begin{array}{l}\text { Neither Like or } \\
\text { Dislike } \\
\text { (0) (3) }\end{array}$ & $\begin{array}{c}\text { Somewhat Like } \\
\text { (1) (4) }\end{array}$ & $\begin{array}{l}\text { Like } \\
\text { (2) (5) }\end{array}$ \\
\hline$A(1)$ & & & & & \\
\hline $\mathrm{B}(2)$ & & & & & \\
\hline C (3) & & & & & \\
\hline
\end{tabular}


Q11.2 Provide one reason behind the selection of your score for Image A (Please click one icon below or write an alternative reason in Box $A$ )

$\begin{array}{lcccc}\text { Cleanliness of } & \text { Clear Entrance } & \begin{array}{c}\text { Sense of } \\ \text { Openness (7) }\end{array} & \text { Shade (8) } & \begin{array}{c}\text { Parked Cars } \\ \text { the Space (1) }\end{array} \\ (2) & \text { Op) }\end{array}$

A (2)

Q11.3 Provide one reason behind the selection of your score for Image B (Please click one icon below or write an alternative reason in Box B)

\begin{tabular}{|c|ccccc} 
& $\begin{array}{c}\text { Cleanliness of } \\
\text { the Space (2) }\end{array}$ & $\begin{array}{c}\text { Well Maintained } \\
\text { Space (7) }\end{array}$ & $\begin{array}{c}\text { Organised car } \\
\text { park (8) }\end{array}$ & $\begin{array}{c}\text { Different } \\
\text { Blank Walls (9) }\end{array}$ & $\begin{array}{c}\text { Materials/ } \\
\text { Colors (13) }\end{array}$ \\
\hline B (2) & & 0 &
\end{tabular}

Q11.4 Provide one reason behind the selection of your score for Image C (Please click one icon below or write an alternative reason in Box $\mathrm{C}$ )

\begin{tabular}{c|ccccc} 
& $\begin{array}{c}\text { Visual } \\
\text { Permeability (2) }\end{array}$ & $\begin{array}{c}\text { Well Maintained } \\
\text { Space (7) }\end{array}$ & $\begin{array}{c}\text { Change in } \\
\text { Level (8) }\end{array}$ & $\begin{array}{c}\text { Good Lighting } \\
(9)\end{array}$ & $\begin{array}{c}\text { Different } \\
\text { Materials / } \\
\text { Colors (13) }\end{array}$ \\
\hline C (2) & & & &
\end{tabular}

Page Break 
Q12 Rate how visually appealing you find the images $A-C$ of spaces at rooftop of the buildings (image $C$ is a rooftop parking)

\section{A}
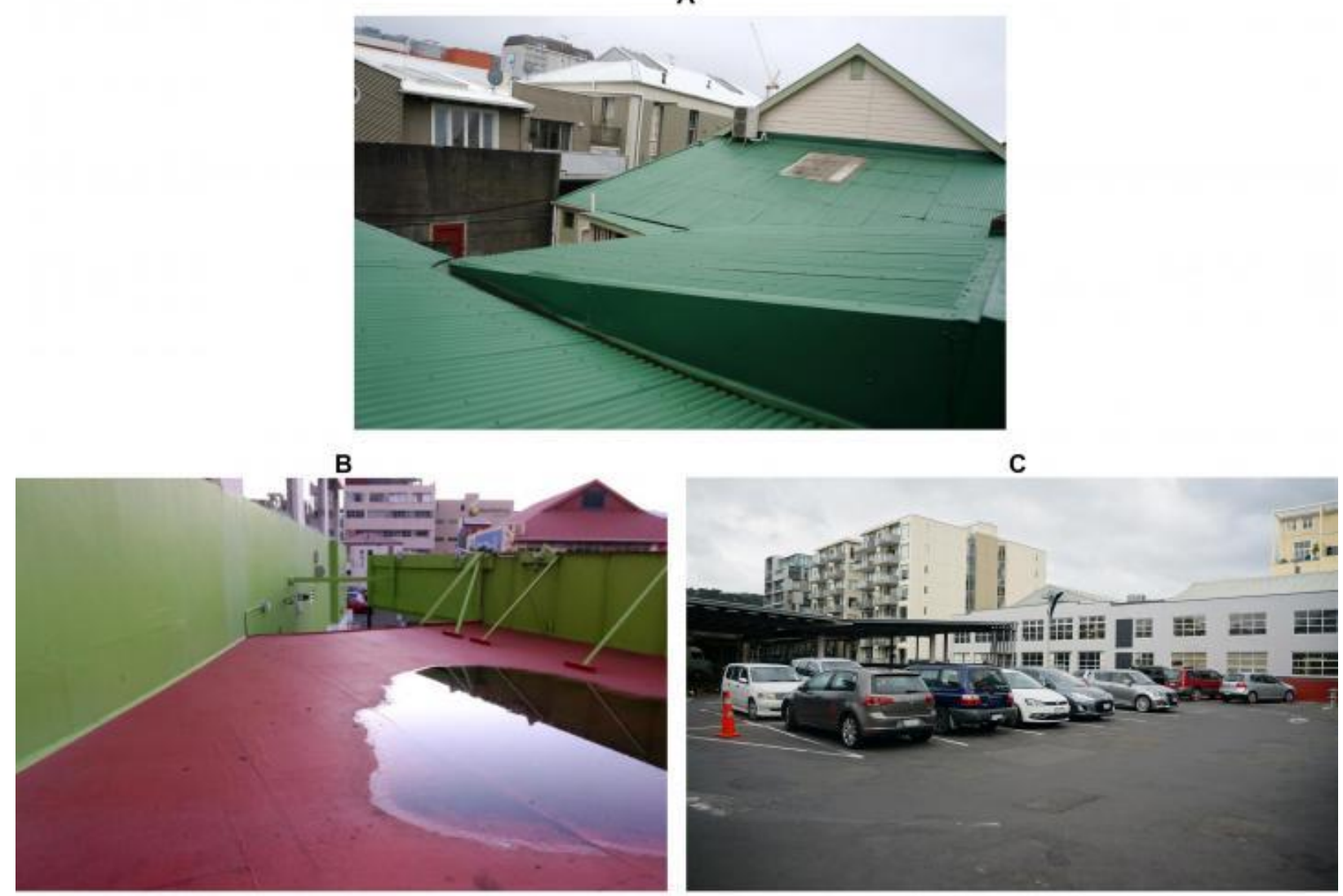

Q12.1 Please score each image from the scale -2 (Dislike) to 2 (Like)

$\begin{array}{ccccc}\text { Dislike } & \text { Somewhat } & \text { Neither Like or } & \text { Somewhat Like } & \text { Like } \\ (-2)(1) & \text { Dislike } & \text { Dislike } & (1)(4) & (2)(5)\end{array}$

A (1)
B (2)
C (3) 
Q12.2 Provide one reason behind the selection of your score for Image A (Please click one icon below or write an alternative reason in Box $A$ )

\begin{tabular}{c|ccccc} 
Different & Rooftop & Well Maintained & Different & Different \\
Angles of Slope & $\begin{array}{c}\text { elements (a/c } \\
\text { unit; duct) (2) }\end{array}$ & Space (7) & Heights (9) & Materials/ \\
Colors (13)
\end{tabular}

A (2)

Q12.3 Provide one reason behind the selection of your score for Image B (Please click one icon below or write an alternative reason in Box B)

\begin{tabular}{c|ccccc} 
Water on & Openness to & Poor & View of & Different \\
Surface (1) & Sky (2) & Maintenance (7) & Adjacent & Materials / \\
& & & Buildings (8) & Colors (13)
\end{tabular}

B (2)

Q12.4 Provide one reason behind the selection of your score for Image C (Please click one icon below or write an alternative reason in Box $\mathrm{C}$ )

$\begin{array}{ccccc}\text { View of } & \text { Well Maintained } & \text { Organised } & \text { Openness to } & \text { Different } \\ \text { Adjacent } & \text { Space (7) } & \text { Parking (8) } & \text { Sky (9) } & \text { Materials / } \\ \text { Buildings (2) } & & & & \text { Colors (13) }\end{array}$

C (2)

Page Break

Q13 In Part 3, you are shown two images. You have to select an image that visually appeals you. Afterwards, you will be asked to make a suggestion for its improvement.

Which image do you prefer visually for the spaces underneath a building (select one option)?

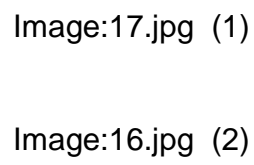




\section{If Q13 = Image:17.jpg}

Q13.1 From the list below tick up to three things which you feel need to be removed or added to improve the space

Provide more vegetation (1)

Remove car parking (2)

Create seating space (7)

Change surface colours / materials (6)

Insert change in level (4)

Create a clear pathway (8)

Improve lighting (9)

Improve maintenance and cleanliness (5)

Other (10)

Display This Question:

\section{If Q13 = Image:16.jpg}

Q13.1 From the list below tick up to three things which you feel need to be removed or added to improve the space

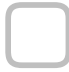

Provide more vegetation (1)

Remove car parking (2)

Create seating space (9)

Change surface colours / materials (10)

Create a clear pathwAY (3)

Improve maintenance and cleanliness (8) 
Improve lighting (11)

Insert change in level (13)

Other (12)

Page Break

Q14 Which image do you prefer visually for the spaces at the back of a building (select one option)?

Image:186.jpg (1)

Image:19.jpg (2)

Display This Question:

If Q14 = Image:186.jpg 
Q14.1 From the list below tick up to three things which you feel need to be removed or added to improve the space

Provide more vegetation (1)

Remove car parking (2)

Create seating space (3)

Change the surface colors / materials (8)

Install wind turbines and solar panels (6)

Improve maintenance and cleanliness (9)

Create a clear pathway (11)

Provide more shade (7)

Other (12) 
Q14.1 From the list below tick up to three things which you feel need to be removed or added to improve the space

Provide more vegetation (1)

Remove car parking (2)

Create seating space (3)

Change the surface colors / materials (9)

Install wind turbines and solar panels (7)

Create a clear pathway (11)

Provide more shade (13)

Improve maintenance and cleanliness (8)

Other (12)

Page Break

Q15 Which image do you prefer visually for the spaces in front of buildings (select one option)?

Image:13.jpg (1)

Image:115.jpg (2)

\section{Display This Question:}

If Q15 = Image:13.jpg

Q15.1 From the list below tick up to three most important things which you think could be added or removed to improve the appearance of the space 
Provide more vegetation (1)

Remove car parking (2)

Create seating space (3)

Change the surface colors / materials (5)

Improve maintenance and cleanliness (8)

Provide more shade (15)

Remove the boundary fence (10)

Create a clear pathway (11)

Other (12)

Q15.1 From the list below tick up to three things which you feel need to be removed or added to improve the space 
Provide more vegetation (1)

Remove car parking (in front of building) (2)

Create seating space (3)

Change the surface colors / materials (5)

Improve maintenance and cleanliness (7)

Provide more shade (4)

Remove the boundary wall (10)

Create clear pathway (9)

Other (11)

Page Break

Q16 Which image do you prefer visually for the spaces between buildings on 2 sides (select one option)?

Image:113.jpg (1)

Image:1862.jpg (2)

\section{Display This Question:}

If Q16 = Image:113.jpg 
Q16.1 From the list below tick up to three things which you feel need to be removed or added to improve the space

Provide more vegetation (1)

Remove car parking (2)

Create seating space (3)

Change the surface colors / materials (4)

Improve maintenance and cleanliness (5)

Allow graffiti on wall (7)

Provide more shade (11)

Create a clear pathway (14)

Other (13) 


\section{If Q16 = Image:1862.jpg}

Q16.1 From the list below tick up to three things which you feel need to be removed or added to improve the space

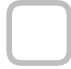

Provide more vegetation (1)

Remove car parking (4)

Provide seating space (10)

Change the surface colors / materials (5)

Install wind turbines \& solar panels on open space (3)

Allow graffiti on wall (11)

Create a clear pathway (6)

Improve maintenance and cleanliness (14)

Other (13)

Page Break

Q17 Which image do you prefer visually for the spaces between buildings on 3 sides (select one option)?

Image:14.jpg (1)

Image:15.jpg (2)

\section{Display This Question:}


Q17.1 From the list below tick up to three things which you feel need to be removed or added to improve the space

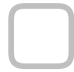

Provide more vegetation (1)

Remove car parking (2)

Create seating space (7)

Change the surface colors / materials (5)

Improve maintenance and cleanliness (3)

Create a clear pathway (4)

Allow graffiti on blank wall (9)

Provide more shade (10)

Install wind turbines \& solar panels (11)

Other (12)

\section{Display This Question:}


Q17.1 From the list below tick up to three things which you feel need to be removed or added to improve the space

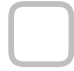

Provide more vegetation (1)

Remove car parking (2)

Create seating space (7)

Change the surface colors / materials (5)

Install wind turbines \& solar panels (3)

Provide more shade (8)

Improve maintenance and cleanliness (4)

Create a clear pathway (10)

Allow graffiti on wall (11)

Other (12)

Page Break

Q18 Which image do you prefer visually for the spaces on the rooftop of a building (select one option)?

Image:100.jpg (1)

Image:1002.jpg (2) 
Q18.1 From the list below tick up to three things which you feel need to be removed or added to improve the space

Provide more vegetation (1)

Provide car parking (2)

Create seating space (3)

Change the surface colors / materials (8)

Install wind turbines and solar panels (4)

Improve maintenance and cleanliness (6)

Provide more shade (7)

Remove air conditioning outlet units (9)

Other (12) 
If Q18 = Image:1002.jpgQ18.2 From the list below tick up to three things which you feel need to be removed or added to improve the space

Provide more vegetation (1)

Remove car parking (2)

Create seating space (3)

Change the surface colors / materials (5)

Install wind turbines and solar panels (4)

Provide more shade (8)

Create a clear pathway (10)

Improve maintenance and cleanliness (12)

Other (13)

Page Break

Q19 Will you like to take part in the follow-up Survey 2 which will study the potential design schemes (before and after) for Urban Leftover spaces?

Yes (1)

No (3) 
Q20 Will you like to participate in the lucky draw?

Yes ( you will redirected to new page to sign up) (1)

No (2)

Chapter 7: Main Visual Preference Study

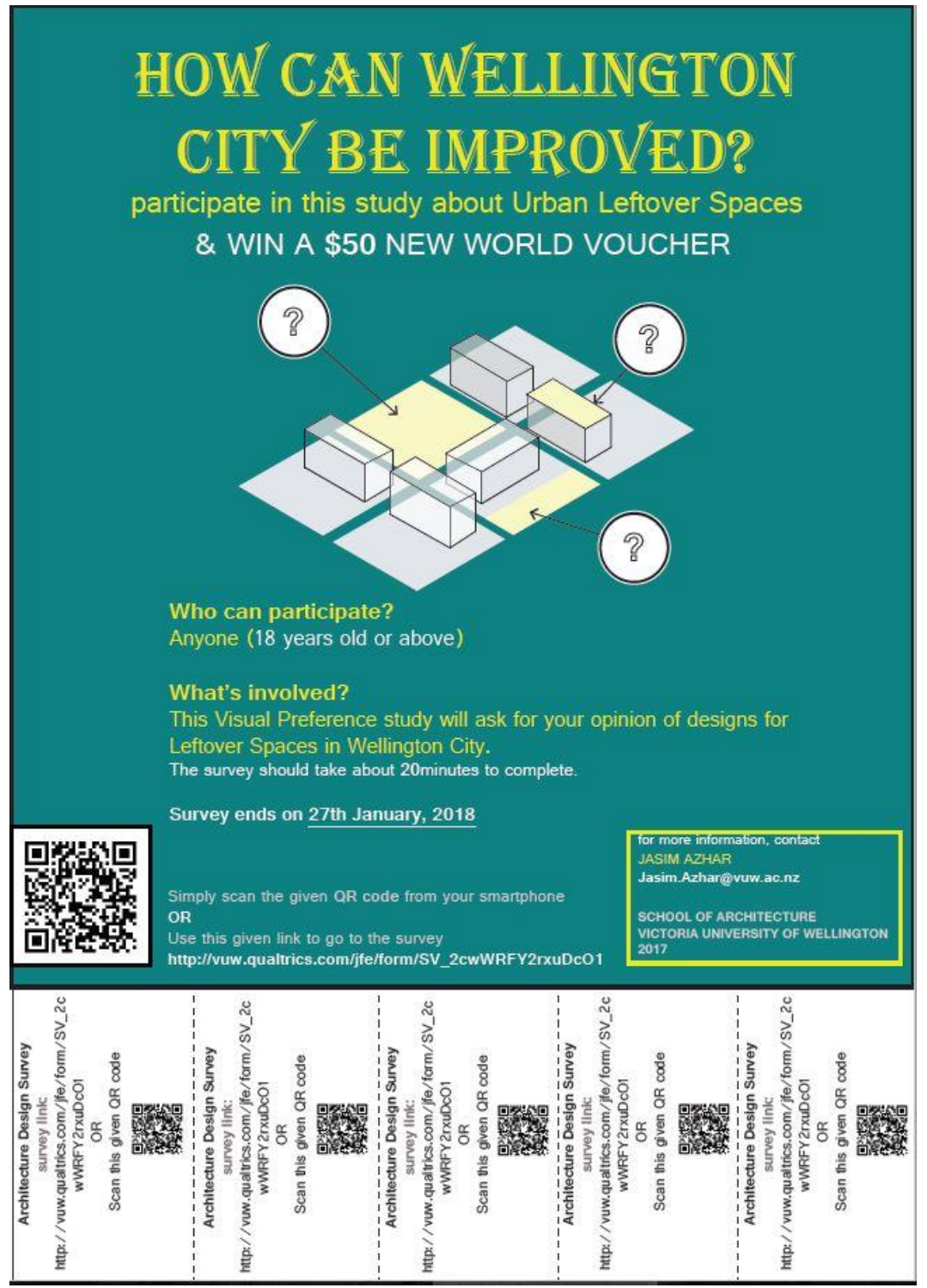

Appendix Figure 7.1 Invitation poster of Study 2

\begin{tabular}{|l|l|l|l|l|l|l|}
\hline Image & $\begin{array}{l}\text { Number of } \\
\text { Respondents } \\
(\mathbf{n})\end{array}$ & $\begin{array}{l}\text { Mean on } \\
\text { Likert } \\
\text { Scale }\end{array}$ & $\begin{array}{l}\text { Standard } \\
\text { Deviation }\end{array}$ & $\begin{array}{l}\text { Negative } \\
\%\end{array}$ & $\begin{array}{l}\text { Neutral } \\
\%\end{array}$ & $\begin{array}{l}\text { Positive } \\
\%\end{array}$ \\
\hline SPACE UNDERNEATH A BUILDING \\
\hline A & 96 & 4.02 & 0.97 & 09.09 & 13.13 & $\underline{77.77}$ \\
\hline
\end{tabular}




\begin{tabular}{|l|l|l|l|l|l|l|}
\hline B & 96 & 2.85 & 1.02 & 37.23 & $\underline{38.30}$ & 24.47 \\
\hline C & 96 & 2.93 & 1.00 & 31.91 & $\underline{40.43}$ & 27.66 \\
\hline
\end{tabular}

SPACE IN FRONT OF A BUILDING

\begin{tabular}{|l|l|l|l|l|l|l|}
\hline A & 90 & 4.01 & 0.80 & 03.30 & 17.80 & $\underline{78.90}$ \\
\hline B & 90 & 3.84 & 1.02 & 12.20 & 17.80 & $\underline{70.00}$ \\
\hline C & 90 & 3.62 & 1.23 & 07.80 & 33.70 & $\underline{58.50}$ \\
\hline
\end{tabular}

SPACE AT THE BACK OF A BUILDING

\begin{tabular}{|l|l|l|l|l|l|l|}
\hline A & 86 & 3.50 & 1.18 & 13.90 & 34.90 & $\underline{53.50}$ \\
\hline B & 86 & 2.59 & 1.22 & $\underline{51.00}$ & 32.60 & 17.40 \\
\hline C & 86 & 4.32 & 0.84 & 04.70 & 07.10 & $\underline{88.29}$ \\
\hline
\end{tabular}

\section{SPACE ENCLOSED BY BUILDINGS ON THREE SIDES}

\begin{tabular}{|l|l|l|l|l|l|l|}
\hline A & 85 & 3.33 & 1.06 & 14.30 & $\underline{42.90}$ & 42.80 \\
\hline B & 85 & 2.78 & 1.19 & 37.60 & $\underline{40.00}$ & 22.40 \\
\hline C & 85 & 4.12 & 0.84 & 07.10 & 07.10 & $\underline{85.80}$ \\
\hline
\end{tabular}

\section{SPACE ENCLOSED BY BUILDINGS ON TWO SIDES}

\begin{tabular}{|c|c|c|c|c|c|c|}
\hline A & 85 & 3.08 & 1.11 & 27.10 & $\underline{37.60}$ & 35.30 \\
\hline B & 85 & 4.26 & 0.77 & 05.90 & 05.90 & $\underline{88.40}$ \\
\hline C & 85 & 3.13 & 1.09 & 25.90 & $\underline{37.60}$ & 36.50 \\
\hline \multicolumn{7}{|c|}{ SPACE AT THE ROOFTOP OF A BUILDING } \\
\hline A & 84 & 4.27 & 0.73 & 02.80 & 9.00 & $\underline{88.30}$ \\
\hline B & 84 & 2.98 & 1.12 & 28.50 & 40.50 & 31.00 \\
\hline C & 84 & 3.29 & 1.04 & 22.90 & 31.30 & $\underline{45.80}$ \\
\hline
\end{tabular}

Appendix table 7.1 Preference scores for all six types of leftover space with the mean score, and distribution of positive, neutral and negative ratings

\section{STUDY 2: Main Visual Preference Study}

INVESTIGATING THE ROLE OF URBAN LEFTOVER SPACES

\section{INFORMATION SHEET FOR PARTICIPANTS}

You are invited to take part in this research. Please read this information before deciding whether or not to take part. If you decide to participate, thank you. If you decide not to participate, thank you for considering this request.

\section{Who am I?}

My name is Jasim Azhar (Jasim.Azhar@vuw.ac.nz) and I am a Doctoral student in Architecture at Victoria University of Wellington. This research project is work towards my Ph.D. thesis.

What is the aim of the project?

This project examines the potential for the positive use of public and privately owned urban leftover spaces. This research investigates people's visual preferences when it comes to their physical and spatial features. 
This survey deals with a Visual Preference Study (VPS) of leftover spaces situated in Wellington city after they have been redesigned in accordance with preferences that emerged in an earlier survey. The aim is to understand the parameters that affect the usability of spaces.

This research has been approved by the Victoria University of Wellington Human Ethics Committee [25265].

\section{How can you help?}

This survey invites you to rate some designed spaces and will take approximately 20 minutes to complete. You can save your survey and come back to it at any point until 45 minutes after you started. You can also go back to edit any answer up to the point you submit the survey.

\section{What will happen to the information you give?}

This research is anonymous. This means that nobody, including the researchers, will be aware of your identity. By answering it, you are giving consent for us to use your responses in this research. Your answers will remain completely anonymous and unidentifiable. Once you submit the survey, it will be impossible to retract your answer. Please do not include any personally identifiable information in your responses.

Personal details will be collected only for those who wish to enter the prize draw/request a copy of the final report. All personal details will be received separately from the survey data. This ensures that your answers to the survey questions are anonymous.

\section{What will the project produce?}

The information from my research will be used in my Ph.D. dissertation, academic publications, and conferences.

\section{If you have any questions or problems, who can you contact?}

If you have any questions, either now or in the future, please feel free to contact either:

$$
\begin{aligned}
& \text { Researcher } \\
& \text { Name: Jasim Azhar } \\
& \text { University Email address: } \\
& \text { Jasim.azhar@vuw.ac.nz } \\
& \text { Supervisor: } \\
& \text { Dr. Morten Gjerde } \\
& \text { Role: Head of School } \\
& \text { School: Architecture } \\
& \text { Phone: } 044636233 \\
& \text { Morten.Gjerde@vuw.ac.nz }
\end{aligned}
$$

Human Ethics Committee information If you have any concerns about the ethical conduct of the research you may contact the Victoria University HEC Convener: Associate Professor Susan Corbett. Email susan.corbett@vuw.ac.nz or telephone +64-4-463 5480.

Yes, I have read the information sheet and want to continue (1)

No, I don't want to continue 
0.1 Gender

Male (1)

Prefer not to answer (2)

Female (3)

0.2 Age

$18-25(1)$

26-35 (2)

36-44 (3)

45-55 (4)

56-65 (5)

$65+(6)$

0.3 Cultural Ethnicity

Maori (1)

Asian (2)

Pacific Islander (3)

European (4)

African (5)

NZ European (Pakeha) (7)

Other (6) 
0.4 Please indicate your highest Academic Qualification

High School / College (5)

Undergraduate (2)

Post Graduate (3)

Trade Certificate (4)

Other (6)

\subsection{Field of Study / Work}

Built Environment (4)

Health Science (1)

Public Service (2)

Retired (3)

Trade (6)

Finance (7)

Science (8)

Other (5)

Q1 In PART 1 of this survey, you are asked to rate the visual appeal of each of the changes that are proposed in the images. 
Rate how visually appealing you find image A for space underneath a building

\section{IMAGE BEFORE}

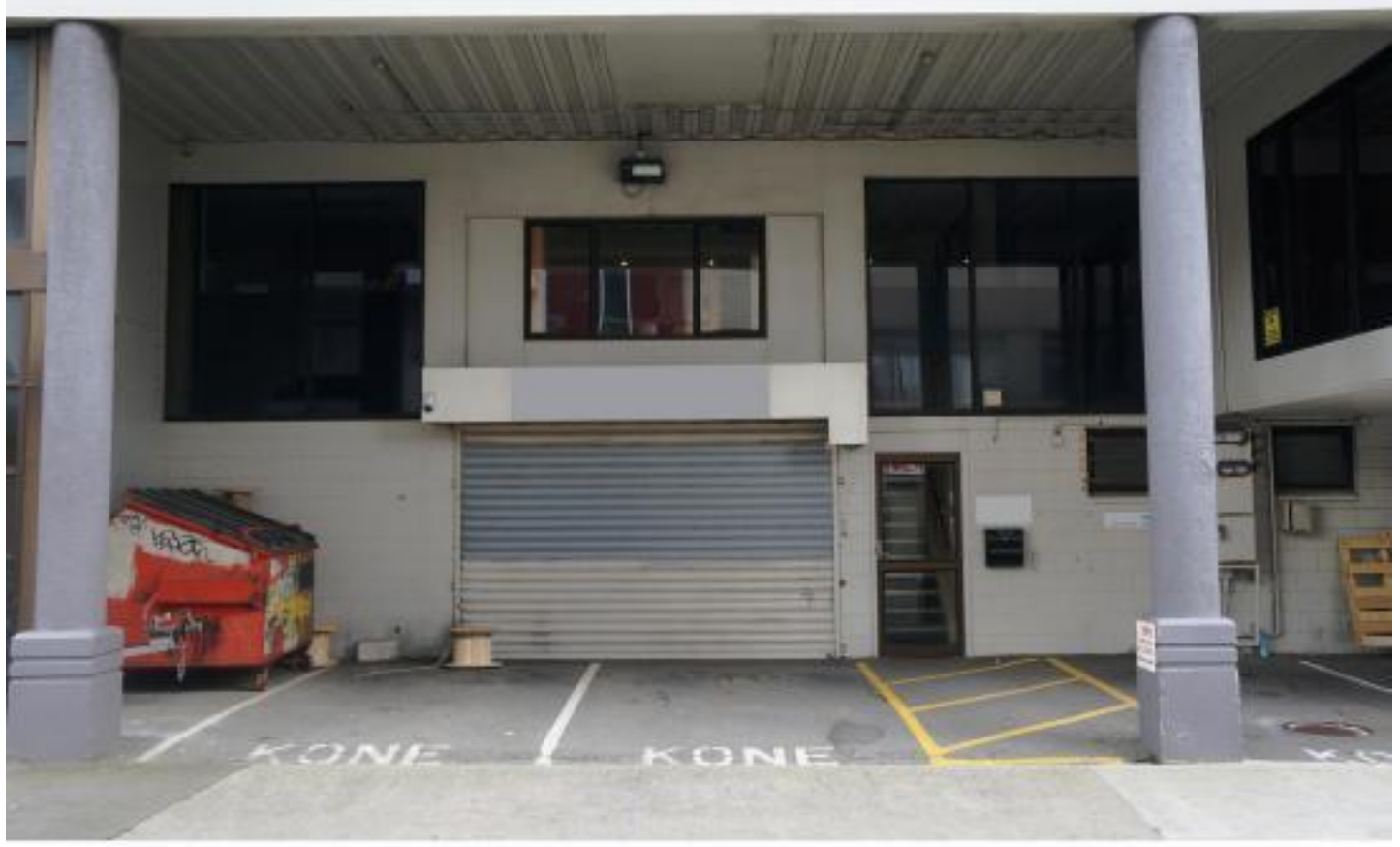

\section{IMAGE AFTER}

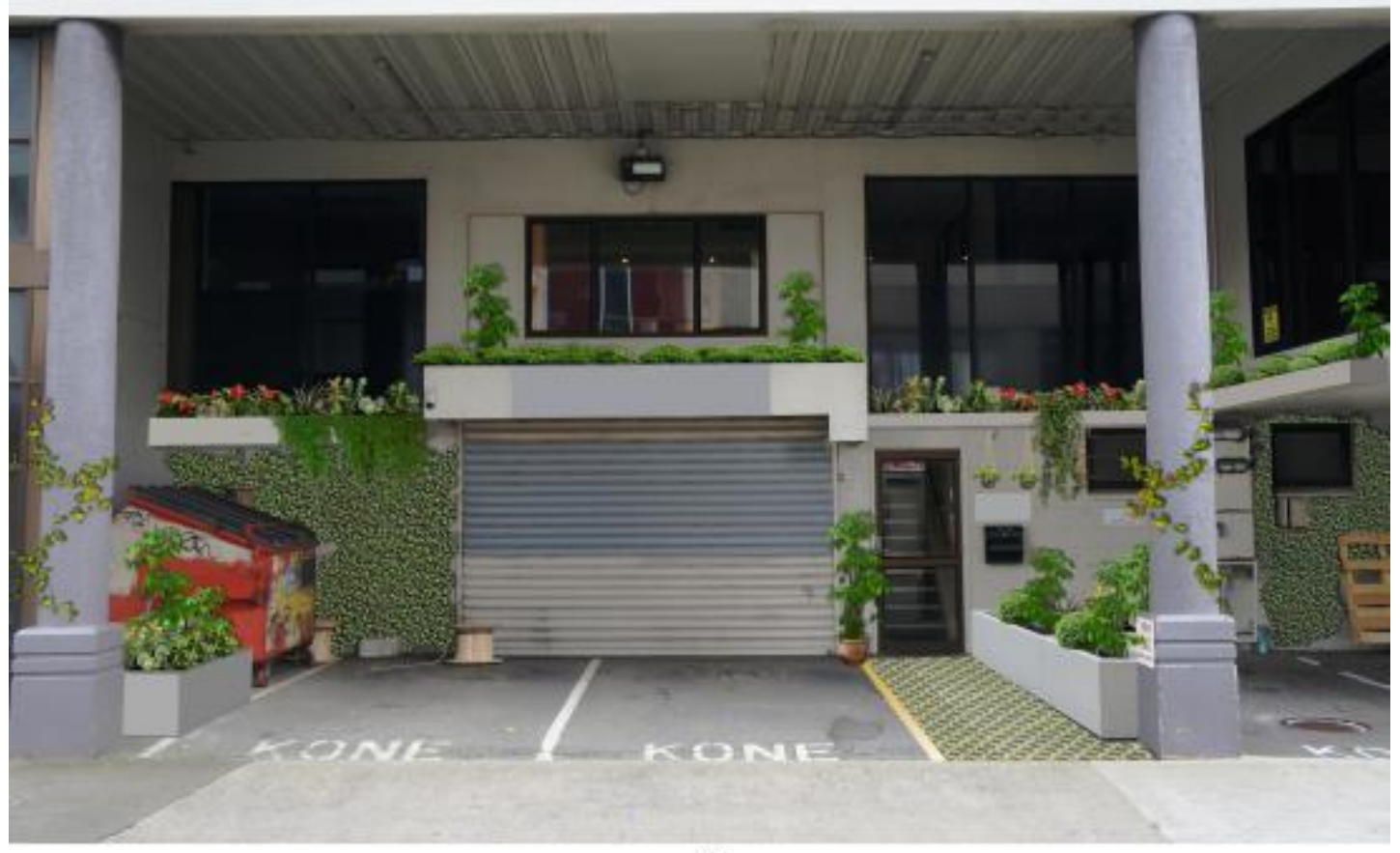

A 
Q1.1 Please score IMAGE A from scale -2 (Dislike) to 2 (Like)

\begin{tabular}{|c|c|c|c|c|c|}
\hline & $\begin{array}{c}\text { Dislike } \\
(-2)\end{array}$ & $\begin{array}{l}\text { Somewhat } \\
\text { Dislike } \\
(-1)\end{array}$ & $\begin{array}{l}\text { Neither Like nor } \\
\text { Dislike } \\
(0)\end{array}$ & $\begin{array}{c}\text { Somewhat Like } \\
\text { (1) }\end{array}$ & $\begin{array}{r}\text { Like } \\
(2)\end{array}$ \\
\hline Image A (2) & & & & & \\
\hline
\end{tabular}

Q1.2 How would you describe 'Image A' on the following scale from 1 to 5 (1 being the lowest and 5 being the highest)

\begin{tabular}{|c|c|c|c|c|c|c|}
\hline & 1 & 2 & 3 & 4 & 5 & \\
\hline & $1(1)$ & $2(2)$ & (3) & (4) & (5) & \\
\hline Ugly & & & & & & Beautiful \\
\hline Annoying & & & & & & Pleasing \\
\hline Impossible & & & & & & Realizable \\
\hline Boring & & & & & & Interesting \\
\hline Constrained & & & & & & Energetic \\
\hline
\end{tabular}

Q1.3 Do you have any suggestion for improving the visual quality of image A (you can type in the blank box)?

Yes (1)

No (2)

Page Break 
Q2 Rate how visually appealing you find image $B$ for space underneath a building

\section{IMAGE BEFORE}

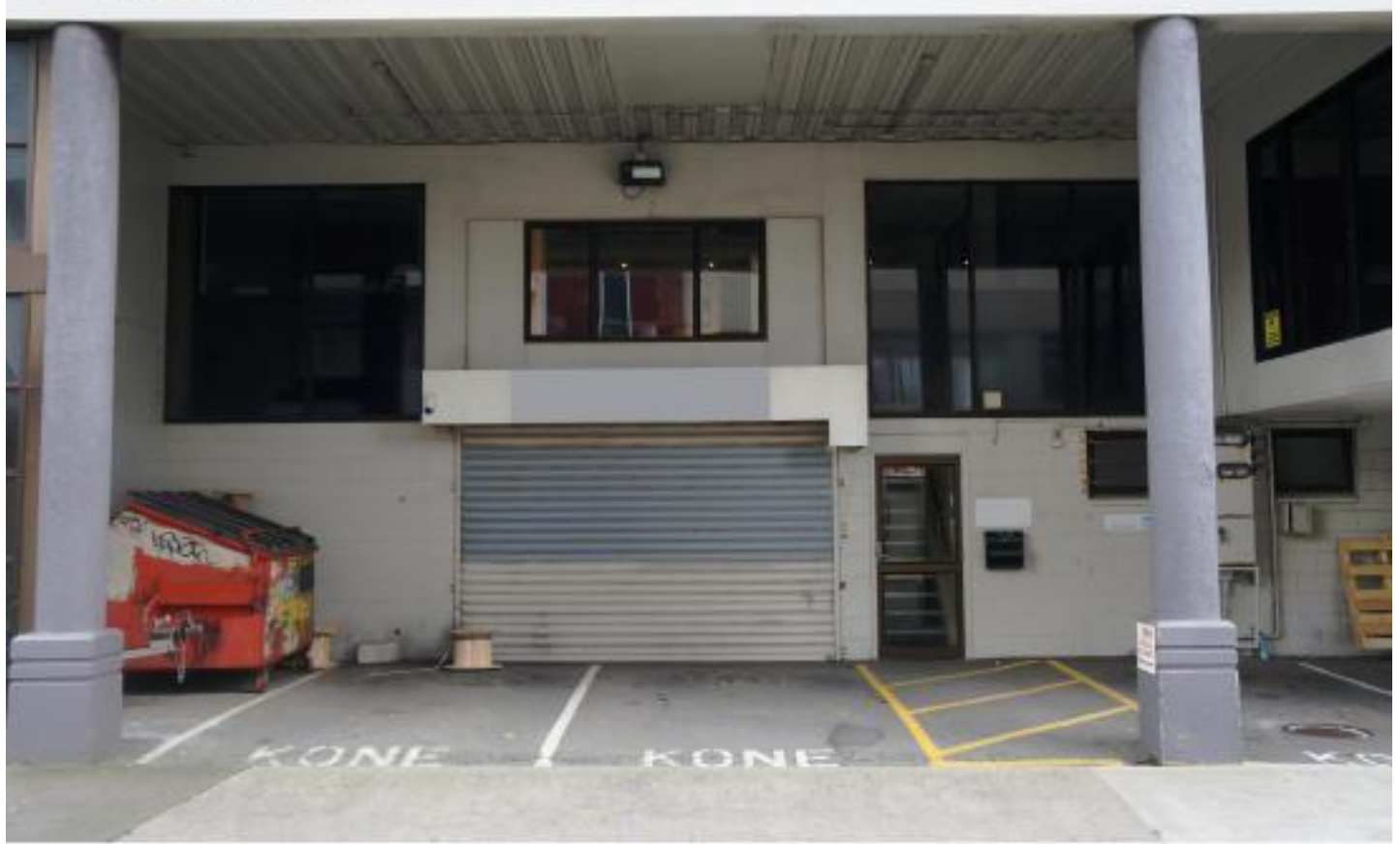

\section{IMAGE AFTER}

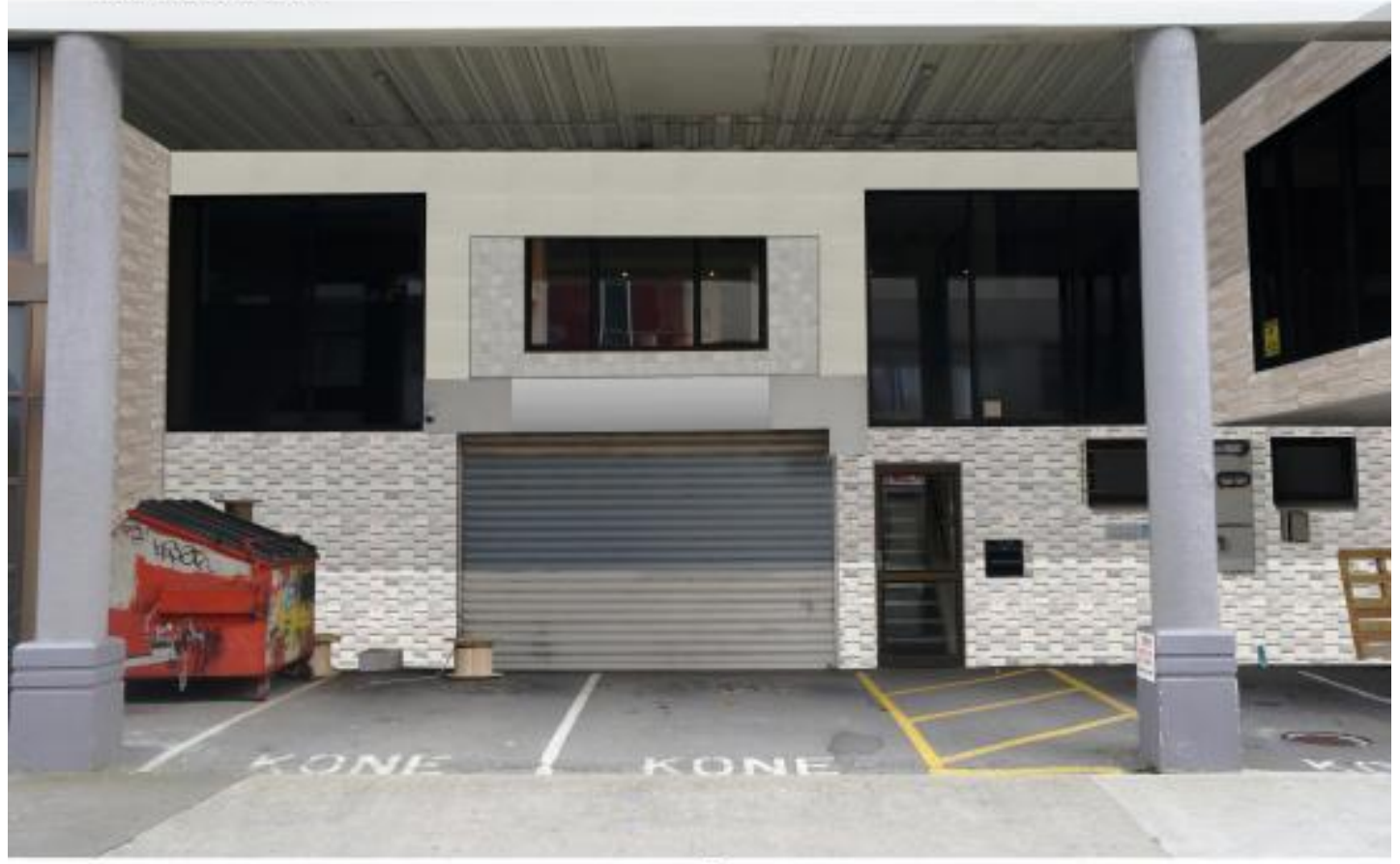

B 
Q2.1 Please score IMAGE B from scale -2 (Dislike) to 2 (Like)

\begin{tabular}{|c|c|c|c|c|c|}
\hline & $\begin{array}{c}\text { Dislike } \\
(-2)\end{array}$ & $\begin{array}{l}\text { Somewhat } \\
\text { Dislike } \\
(-1)\end{array}$ & $\begin{array}{l}\text { Neither Like nor } \\
\text { Dislike } \\
(0)\end{array}$ & $\begin{array}{c}\text { Somewhat Like } \\
\text { (1) }\end{array}$ & $\begin{array}{r}\text { Like } \\
(2)\end{array}$ \\
\hline Image B (2) & & & & & \\
\hline
\end{tabular}

Q2.2 How would you describe 'Image B' on the following scale from 1 to 5 (1 being the lowest and 5 being the highest)

\begin{tabular}{|c|c|c|c|c|c|c|}
\hline & 1 & 2 & 3 & 4 & 5 & \\
\hline & $1(1)$ & $2(2)$ & (3) & (4) & (5) & \\
\hline Ugly & & & & & & Beautiful \\
\hline Annoying & & & & & & Pleasing \\
\hline Impossible & & & & & & Realizable \\
\hline Boring & & & & & & Interesting \\
\hline Constrained & & & & & & Energetic \\
\hline
\end{tabular}

Q2.3 Do you have any suggestion for improving the visual quality of image B (you can type in the blank box)?

Yes (1)

No (2)

Page Break 
Q3 Rate how visually appealing you find image C for space underneath a building

\section{IMAGE BEFORE}

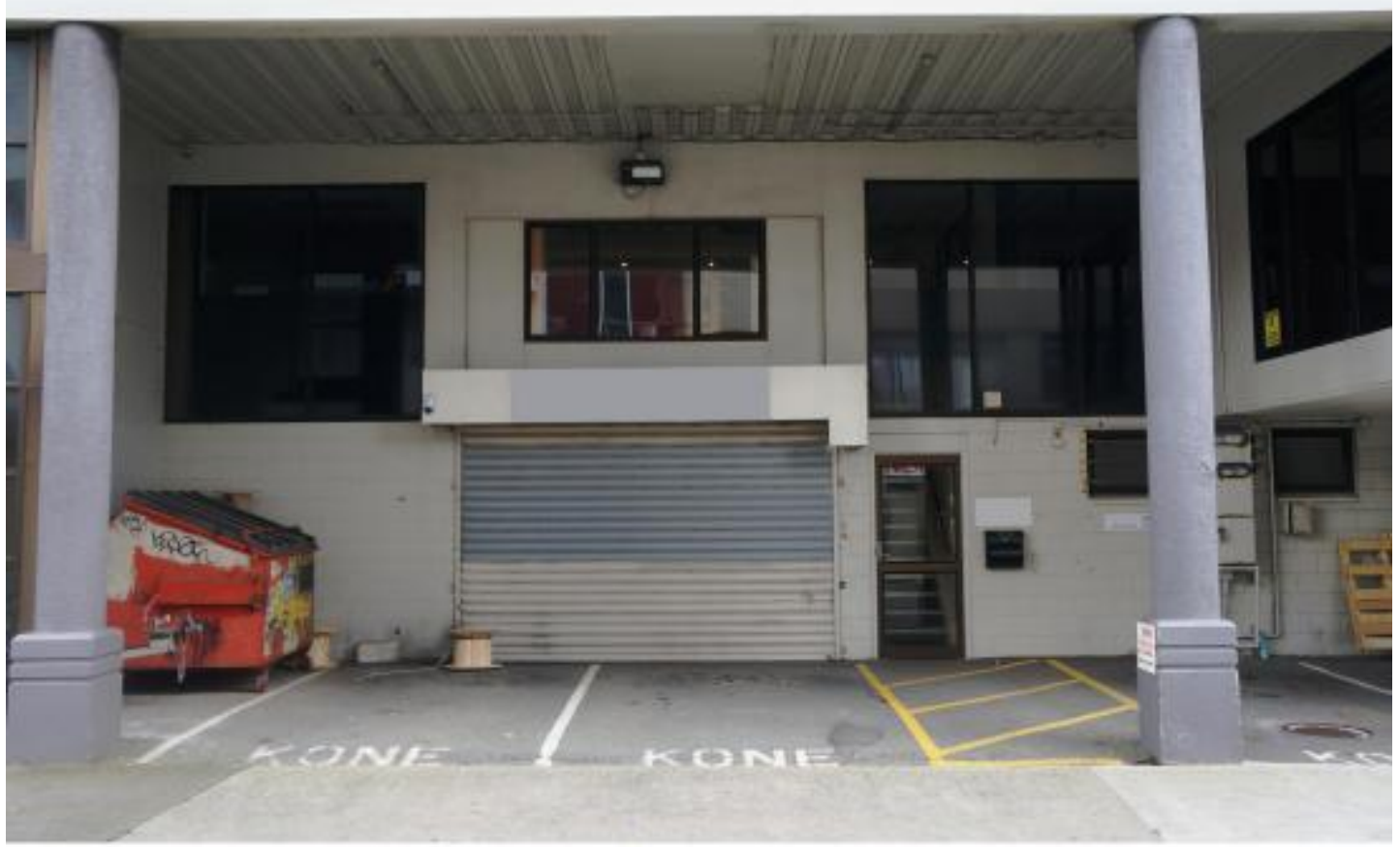

\section{IMAGE AFTER}

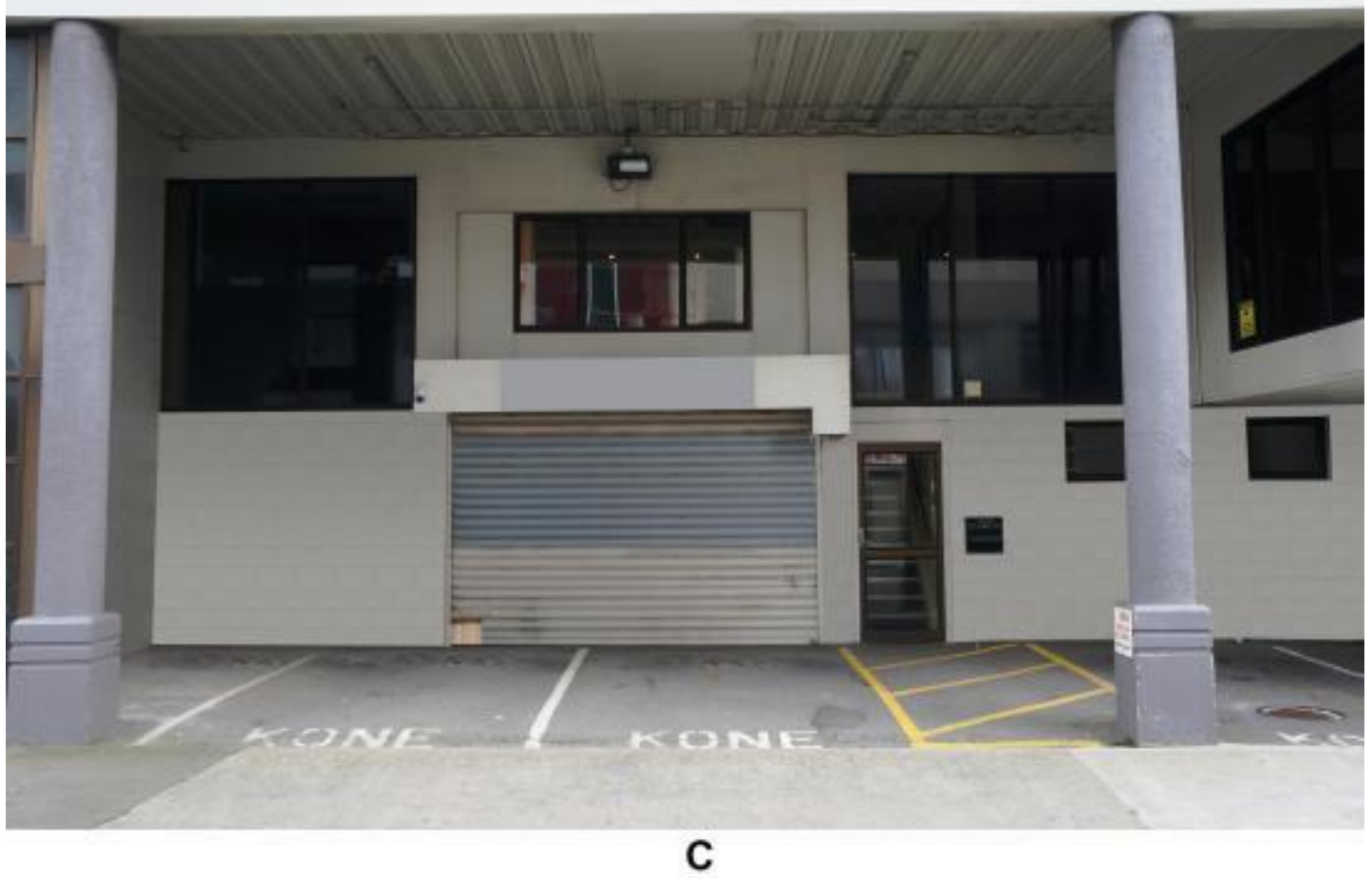


Q3.1 Please score IMAGE C from scale -2 (Dislike) to 2 (Like)

\begin{tabular}{|c|c|c|c|c|c|}
\hline & $\begin{array}{c}\text { Dislike } \\
(-2)\end{array}$ & $\begin{array}{l}\text { Somewhat } \\
\text { Dislike } \\
(-1)\end{array}$ & $\begin{array}{l}\text { Neither Like nor } \\
\text { Dislike } \\
(0)\end{array}$ & $\begin{array}{c}\text { Somewhat Like } \\
\text { (1) }\end{array}$ & $\begin{array}{r}\text { Like } \\
(2)\end{array}$ \\
\hline lage $C(2)$ & & & & & \\
\hline
\end{tabular}

Q3.2 How would you describe 'Image C' on the following scale from 1 to 5 (1 being the lowest and 5 being the highest)

\begin{tabular}{|c|c|c|c|c|c|c|}
\hline & 1 & 2 & 3 & 4 & 5 & \\
\hline & $1(1)$ & $2(2)$ & (3) & (4) & (5) & \\
\hline Ugly & & & & & & Beautiful \\
\hline Annoying & & & & & & Pleasing \\
\hline Impossible & & & & & & Realizable \\
\hline Boring & & & & & & Interesting \\
\hline Constrained & & & & & & Energetic \\
\hline
\end{tabular}

Q3.3 Do you have any suggestion for improving the visual quality of image C (you can type in the blank box)?

Yes (1)

No (2)

Page Break 
Q4 Rate how visually appealing you find image A for a space in front of the building

\section{IMAGE BEFORE}

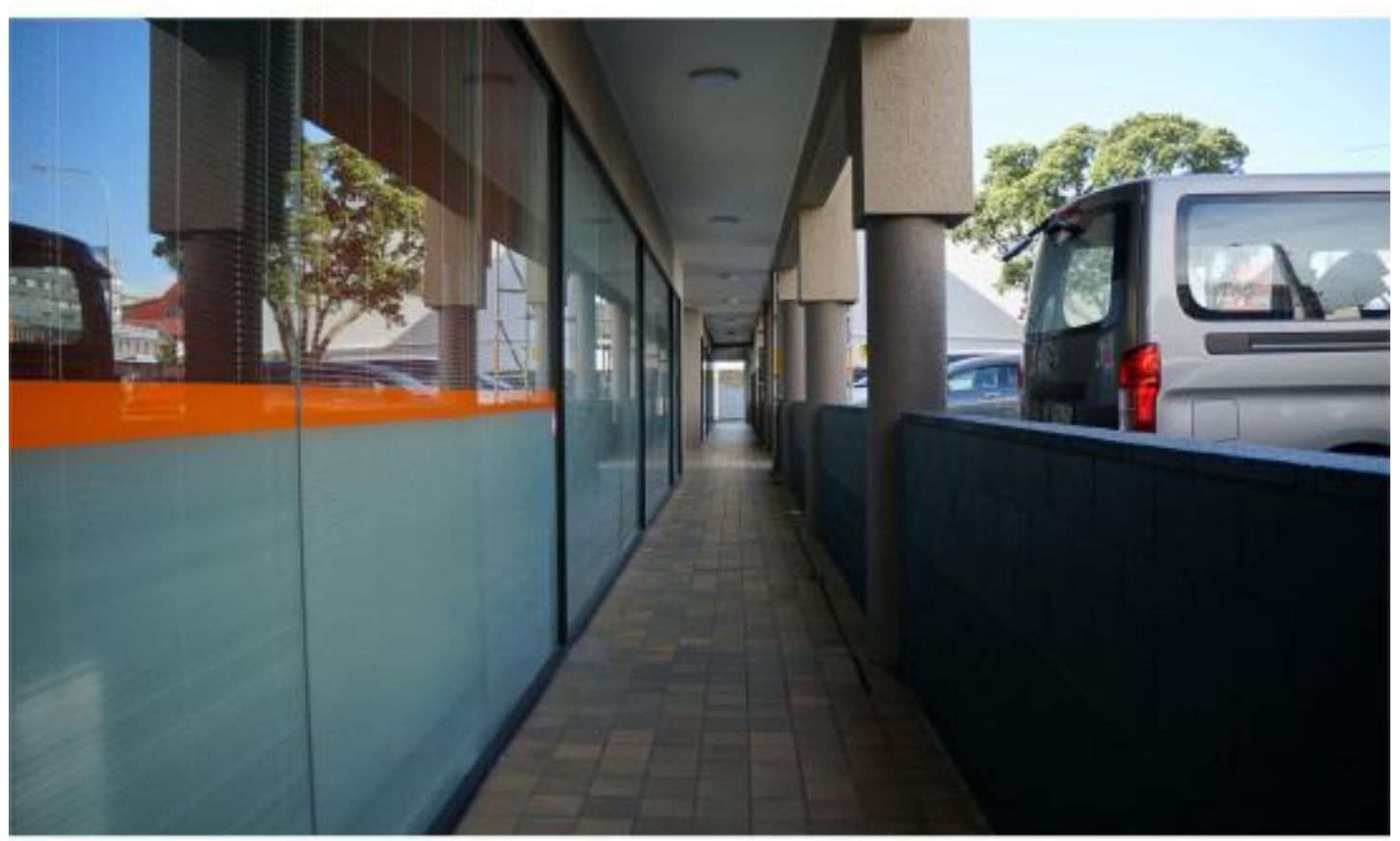

\section{IMAGE AFTER}

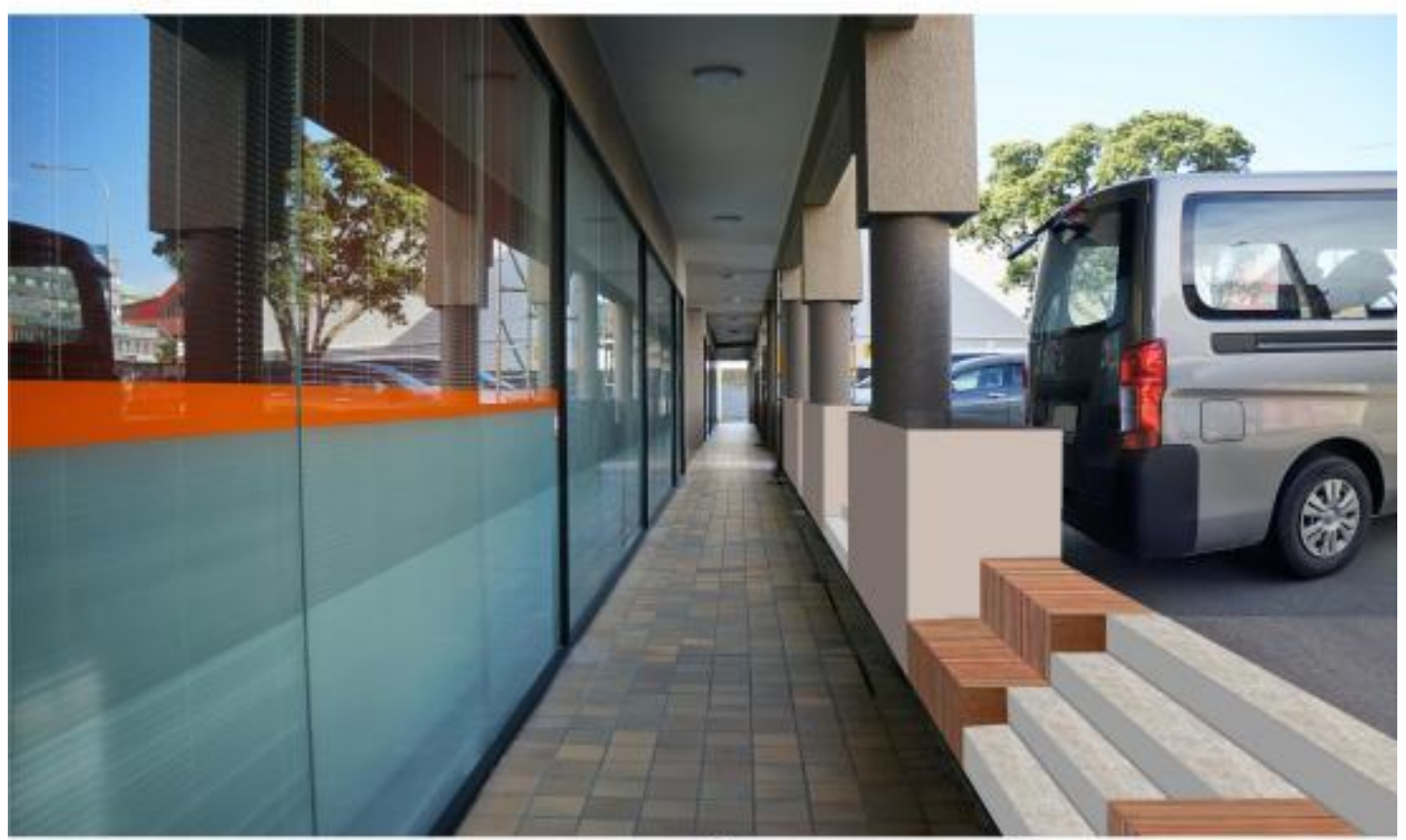

A 
Q4.1 Please score IMAGE A from scale -2 (Dislike) to 2 (Like)

\begin{tabular}{|c|c|c|c|c|c|}
\hline & $\begin{array}{c}\text { Dislike } \\
(-2)\end{array}$ & $\begin{array}{l}\text { Somewhat } \\
\text { Dislike } \\
(-1)\end{array}$ & $\begin{array}{l}\text { Neither Like nor } \\
\text { Dislike } \\
(0)\end{array}$ & $\begin{array}{l}\text { Somewhat Like } \\
\text { (1) }\end{array}$ & $\begin{array}{r}\text { Like } \\
(2)\end{array}$ \\
\hline Image A (2) & & & & & \\
\hline
\end{tabular}

Q4.2 How would you describe 'Image A' on the following scale from 1 to 5 (1 being the lowest and 5 being the highest)

\begin{tabular}{|c|c|c|c|c|c|c|}
\hline & 1 & 2 & 3 & 4 & 5 & \\
\hline & $1(1)$ & $2(2)$ & (3) & (4) & (5) & \\
\hline Ugly & & & & & & Beautiful \\
\hline Annoying & & & & & & Pleasing \\
\hline Impossible & & & & & & Realizable \\
\hline Boring & & & & & & Interesting \\
\hline Constrained & & & & & & Energetic \\
\hline
\end{tabular}

Q4.3 Do you have any suggestion for improving the visual quality of image A (you can type in the blank box)?

Yes (1)

No (2)

Page Break 
Q5 Rate how visually appealing you find image B for a space in front of the building

\section{IMAGE BEFORE}

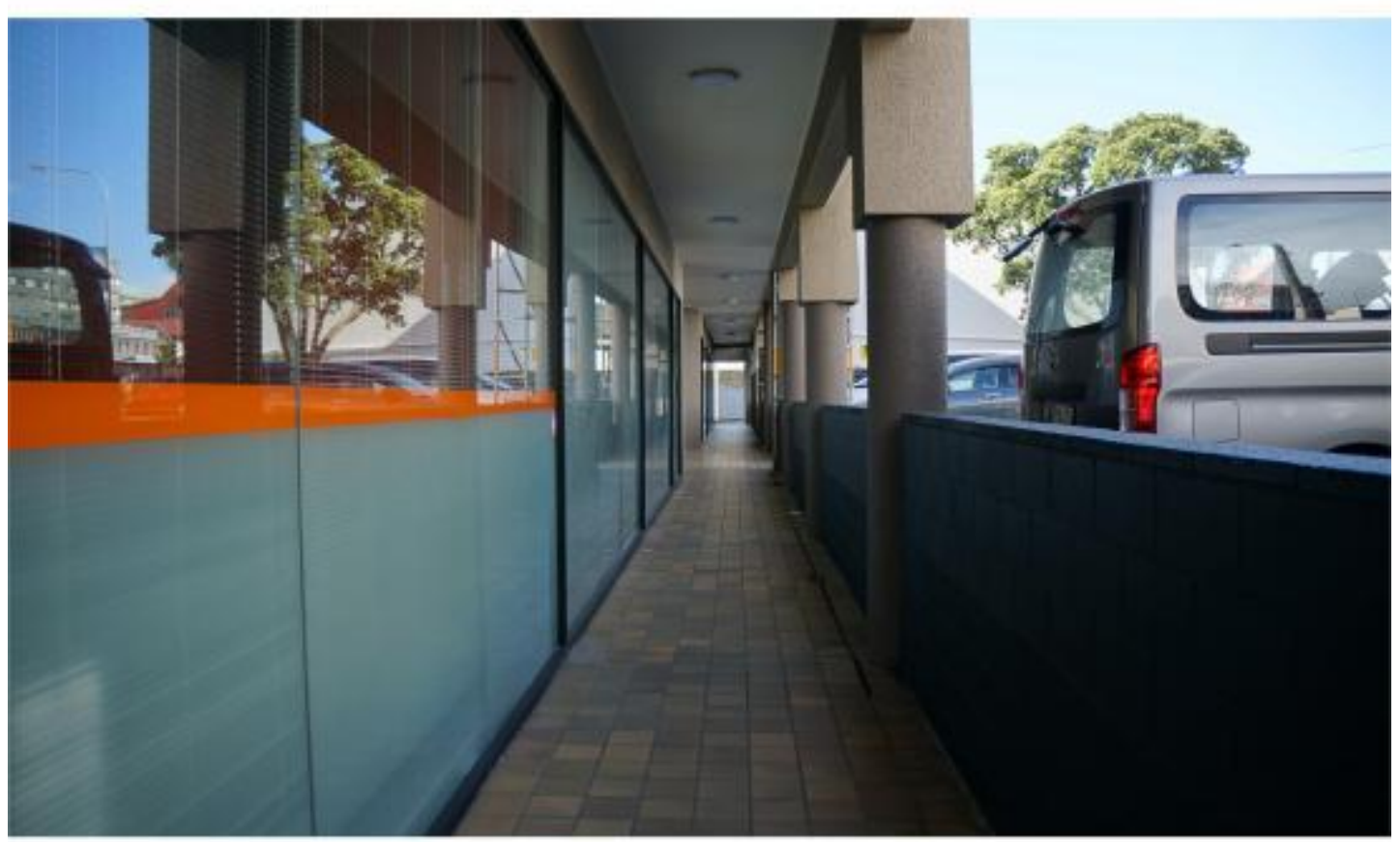

\section{IMAGE AFTER}

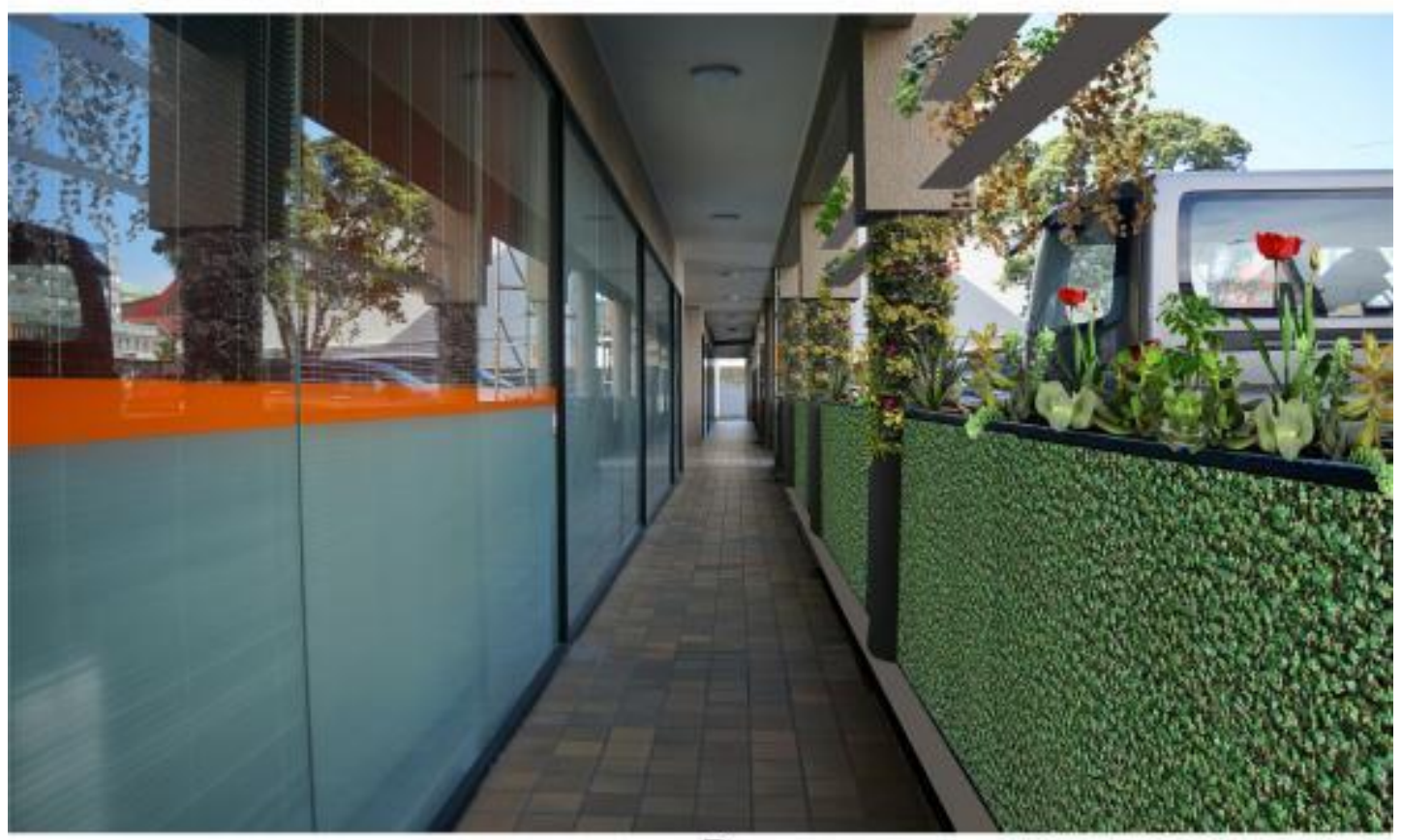

B 
Q5.1 Please score IMAGE B from scale -2 (Dislike) to 2 (Like)

\begin{tabular}{c|ccccr} 
& Somewhat & Neither Like nor & Somewhat Like & Like \\
& Dislike & $(-2)$ & $(0)$ & $(1)$ & $(2)$ \\
\hline Image B (2) & & & &
\end{tabular}

Q5.2 How would you describe 'Image B' on the following scale from 1 to 5 (1 being the lowest and 5 being the highest)

\begin{tabular}{|c|c|c|c|c|c|c|}
\hline & 1 & 2 & 3 & 4 & 5 & \\
\hline & $1(1)$ & $2(2)$ & (3) & (4) & (5) & \\
\hline Ugly & & & & & & Beautiful \\
\hline Annoying & & & & & & Pleasing \\
\hline Impossible & & & & & & Realizable \\
\hline Boring & & & & & & Interesting \\
\hline Constrained & & & & & & Energetic \\
\hline
\end{tabular}

Q5.3 Do you have any suggestion for improving the visual quality of image B (you can type in the blank box)?

Yes (1)

No (2)

Page Break 
Q6 Rate how visually appealing you find image $C$ for a space in front of the building

\section{IMAGE BEFORE}

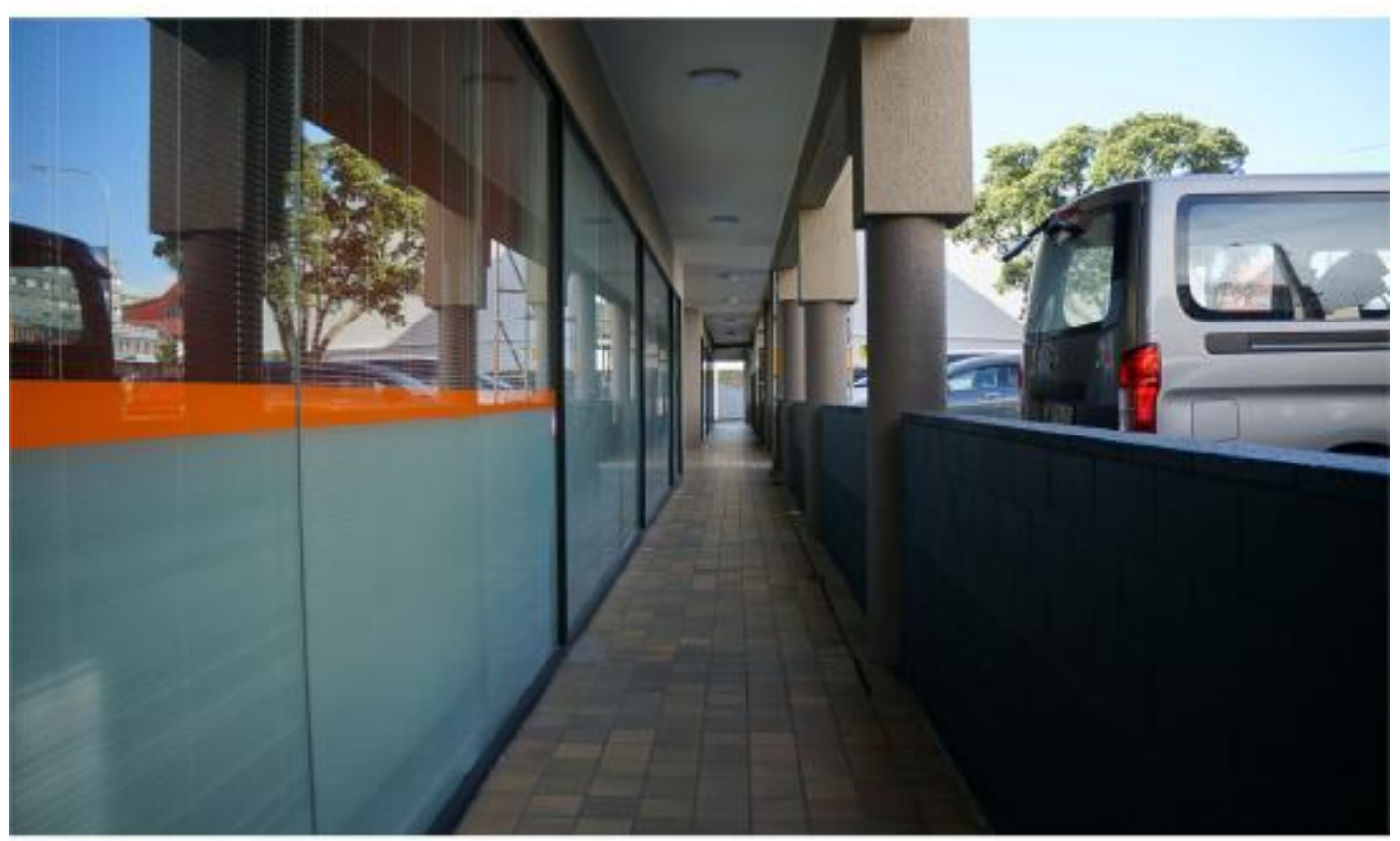

\section{IMAGE AFTER}

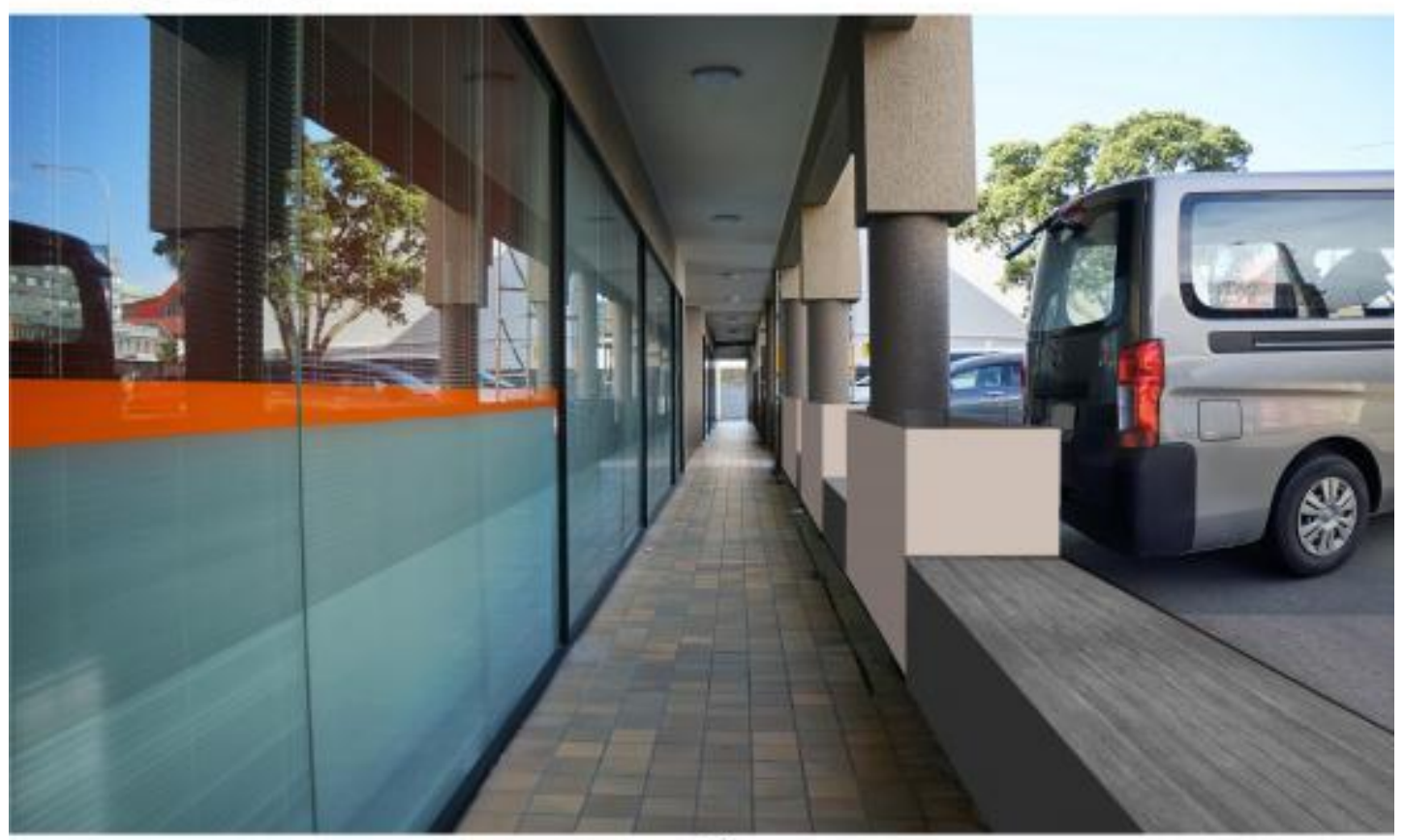

C 
Q6.1 Please score IMAGE C from scale -2 (Dislike) to 2 (Like)

\begin{tabular}{|c|c|c|c|c|c|}
\hline & $\begin{array}{c}\text { Dislike } \\
(-2)\end{array}$ & $\begin{array}{l}\text { Somewhat } \\
\text { Dislike } \\
(-1)\end{array}$ & $\begin{array}{l}\text { Neither Like nor } \\
\text { Dislike } \\
(0)\end{array}$ & $\begin{array}{c}\text { Somewhat Like } \\
\text { (1) }\end{array}$ & $\begin{array}{r}\text { Like } \\
(2)\end{array}$ \\
\hline lage $C(2)$ & & & & & \\
\hline
\end{tabular}

Q6.2 How would you describe 'Image C' on the following scale from 1 to 5 (1 being the lowest and 5 being the highest)

\begin{tabular}{|c|c|c|c|c|c|c|}
\hline & 1 & 2 & 3 & 4 & 5 & \\
\hline & $1(1)$ & $2(2)$ & (3) & (4) & (5) & \\
\hline Ugly & & & & & & Beautiful \\
\hline Annoying & & & & & & Pleasing \\
\hline Impossible & & & & & & Realizable \\
\hline Boring & & & & & & Interesting \\
\hline Constrained & & & & & & Energetic \\
\hline
\end{tabular}

Q6.3 Do you have any suggestion for improving the visual quality of image C (you can type in the blank box)?

Yes (1)

No (2)

Page Break 
Q7 Rate how visually appealing you find image A for a space that forms the backyard of the building

\section{IMAGE BEFORE}

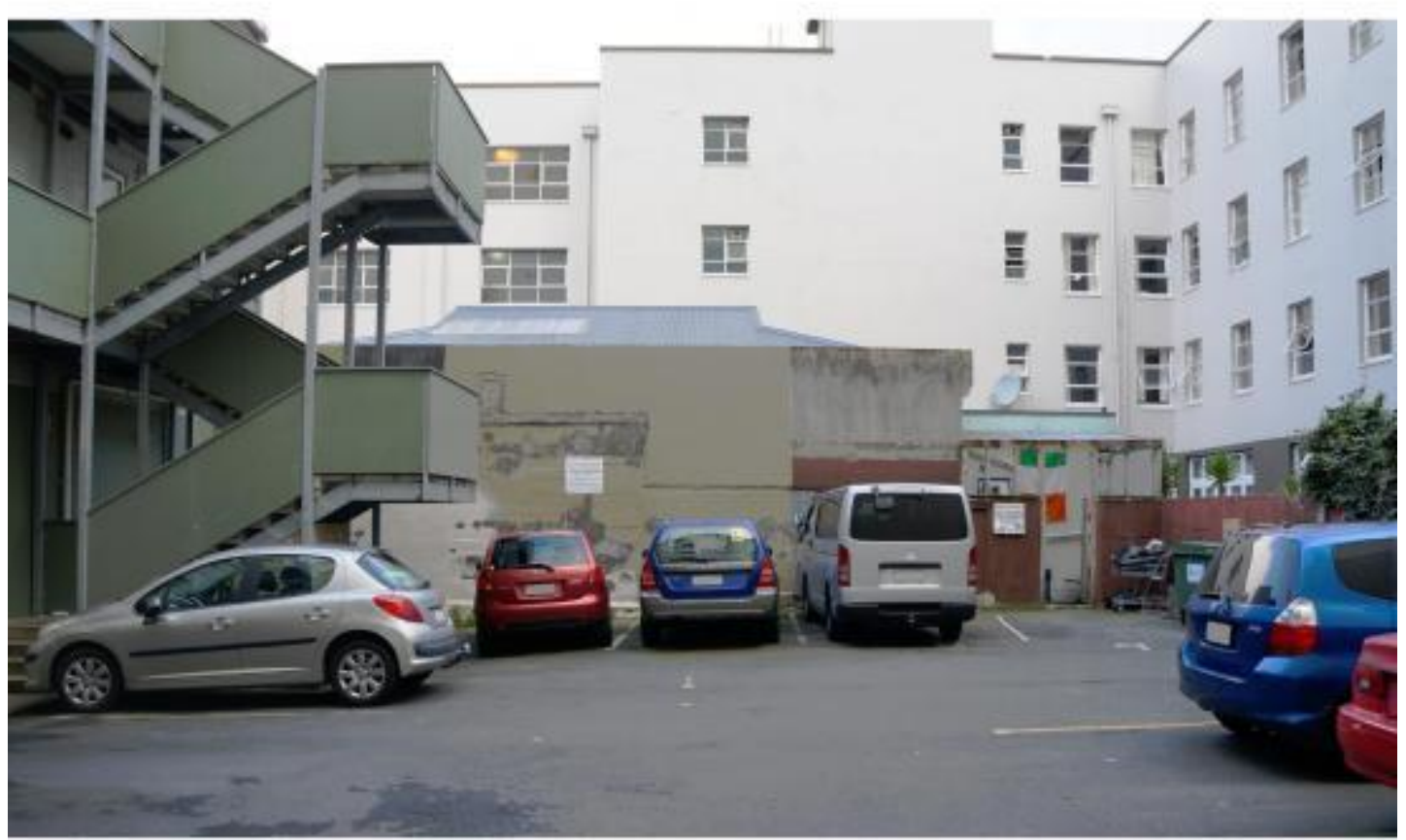

\section{IMAGE AFTER}

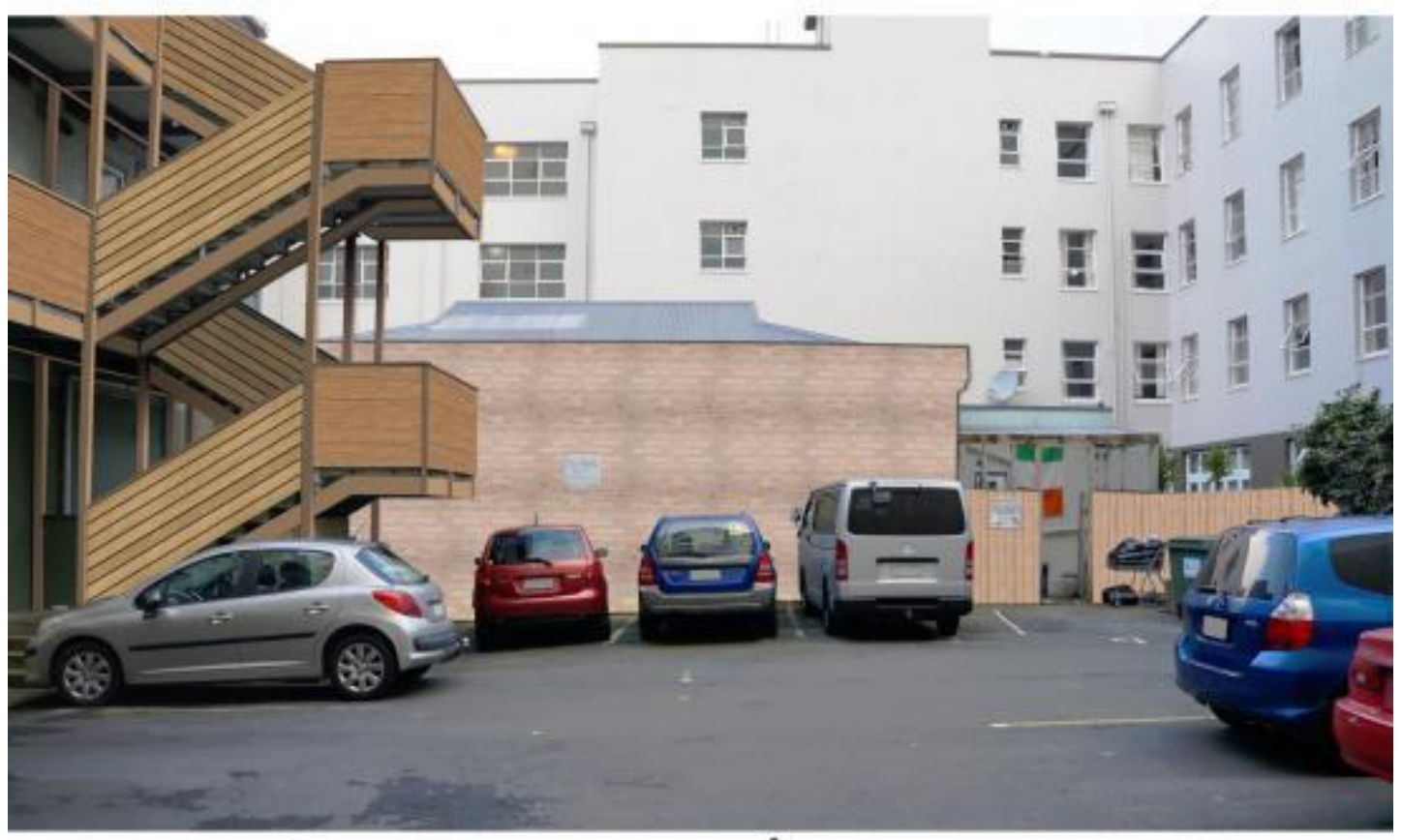

A 
Q7.1 Please score IMAGE A from scale -2 (Dislike) to 2 (Like)

\begin{tabular}{|c|c|c|c|c|c|}
\hline & $\begin{array}{c}\text { Dislike } \\
(-2)\end{array}$ & $\begin{array}{l}\text { Somewhat } \\
\text { Dislike } \\
(-1)\end{array}$ & $\begin{array}{l}\text { Neither Like nor } \\
\text { Dislike } \\
(0)\end{array}$ & $\begin{array}{l}\text { Somewhat Like } \\
\text { (1) }\end{array}$ & $\begin{array}{r}\text { Like } \\
(2)\end{array}$ \\
\hline Image A (2) & & & & & \\
\hline
\end{tabular}

Q7.2 How would you describe 'Image A' on the following scale from 1 to 5 (1 being the lowest and 5 being the highest)

\begin{tabular}{|c|c|c|c|c|c|c|}
\hline & 1 & 2 & 3 & 4 & 5 & \\
\hline & $1(1)$ & $2(2)$ & (3) & (4) & (5) & \\
\hline Ugly & & & & & & Beautiful \\
\hline Annoying & & & & & & Pleasing \\
\hline Impossible & & & & & & Realizable \\
\hline Boring & & & & & & Interesting \\
\hline Constrained & & & & & & Energetic \\
\hline
\end{tabular}

Q7.3 Do you have any suggestion for improving the visual quality of image A (you can type in the blank box)?

Yes (1)

No (2)

Page Break 
Q8 Rate how visually appealing you find image B for a space that forms the backyard of the building

\section{IMAGE BEFORE}

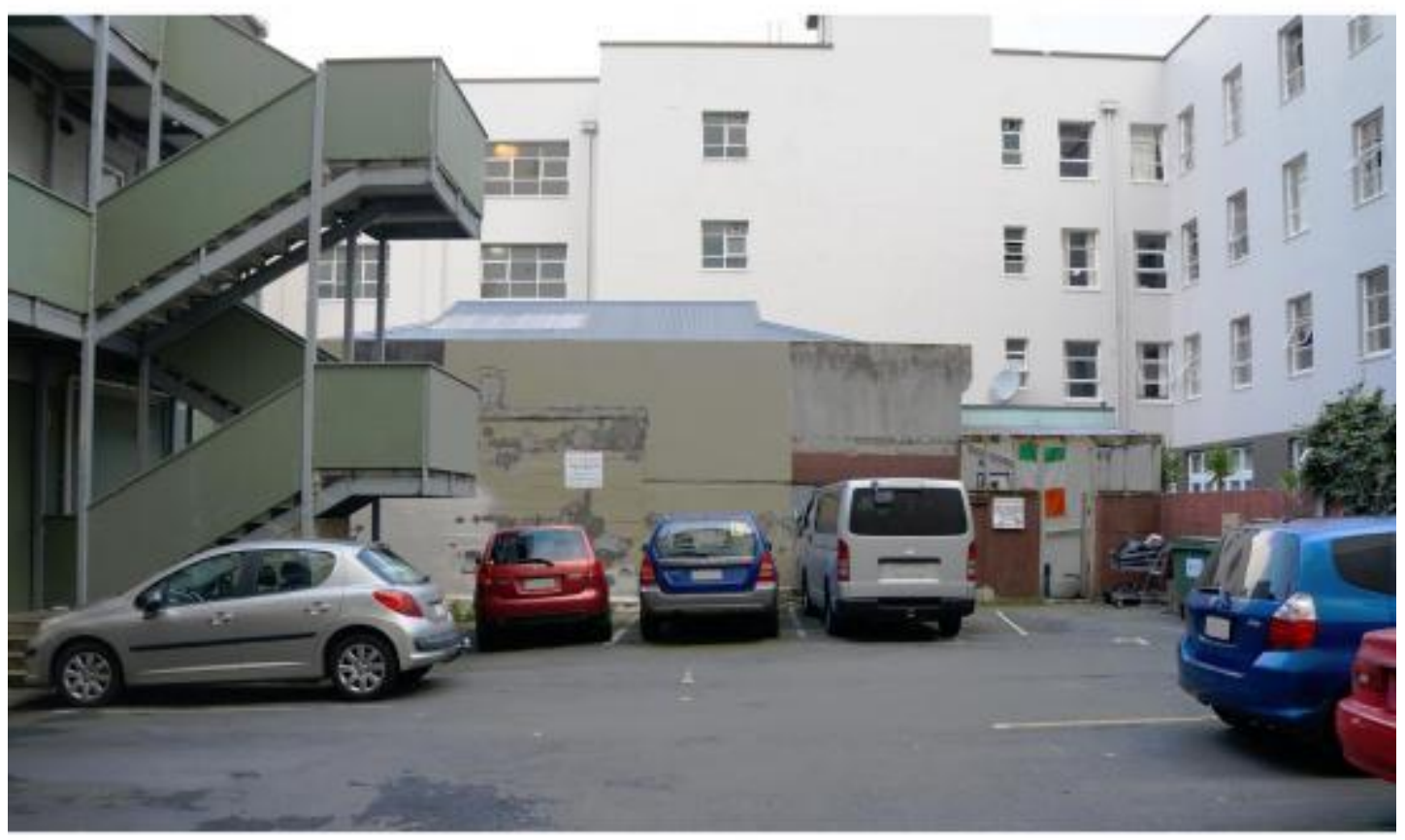

\section{IMAGE AFTER}

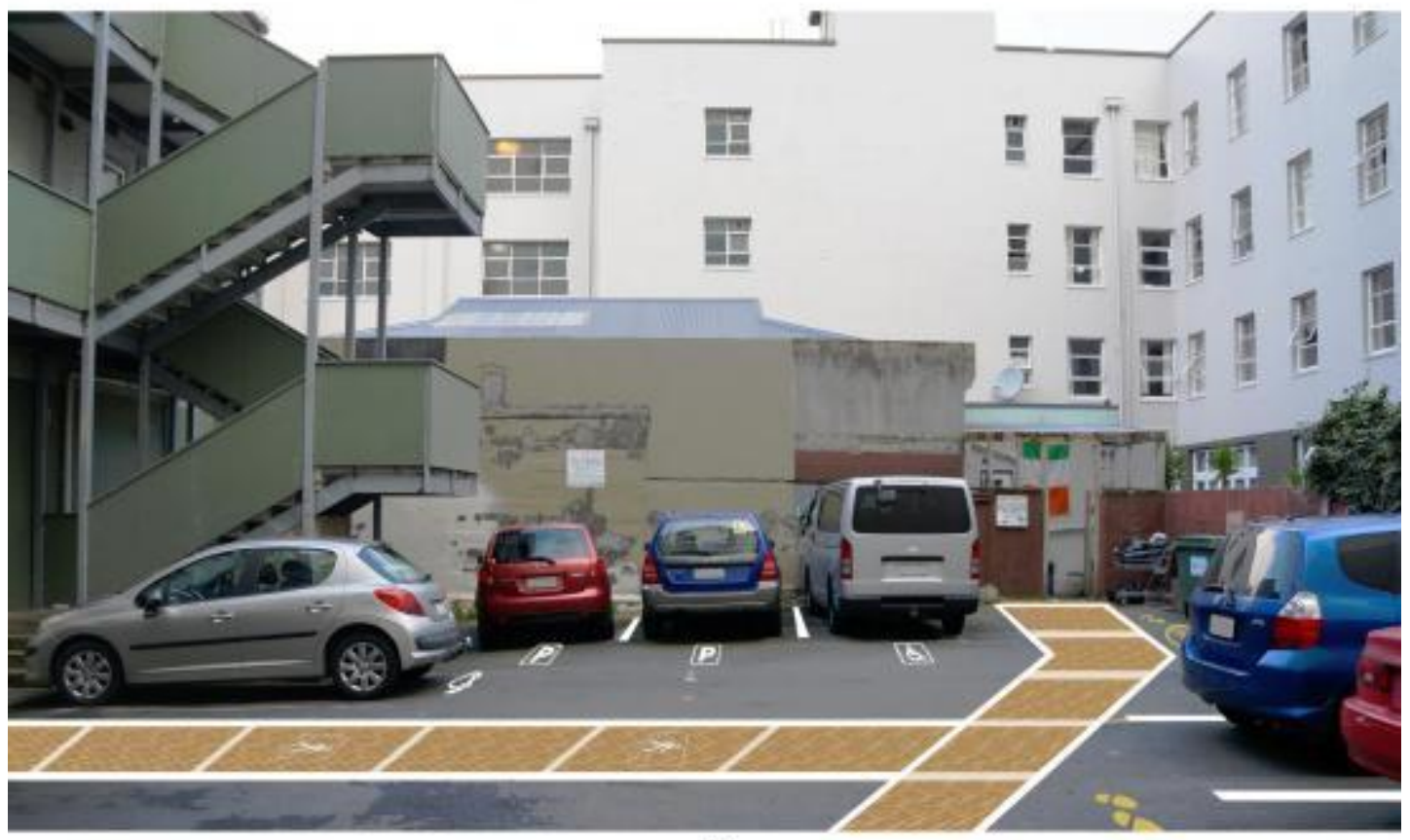

B 
Q8.1 Please score IMAGE B from scale -2 (Dislike) to 2 (Like)

\begin{tabular}{c|ccccr} 
& Somewhat & Neither Like nor & Somewhat Like & Like \\
& Dislike & $(-2)$ & $(0)$ & $(1)$ & $(2)$ \\
\hline Image B (2) & & & &
\end{tabular}

Q8.2 How would you describe 'Image B' on the following scale from 1 to 5 (1 being the lowest and 5 being the highest)

\begin{tabular}{|c|c|c|c|c|c|c|}
\hline & 1 & 2 & 3 & 4 & 5 & \\
\hline & $1(1)$ & $2(2)$ & (3) & (4) & (5) & \\
\hline Ugly & & & & & & Beautiful \\
\hline Annoying & & & & & & Pleasing \\
\hline Impossible & & & & & & Realizable \\
\hline Boring & & & & & & Interesting \\
\hline Constrained & & & & & & Energetic \\
\hline
\end{tabular}

Q8.3 Do you have any suggestion for improving the visual quality of image B (you can type in the blank box)?

Yes (1)

No (2)

Page Break 
Q9 Rate how visually appealing you find image $C$ for a space that forms the backyard of the building

\section{IMAGE BEFORE}

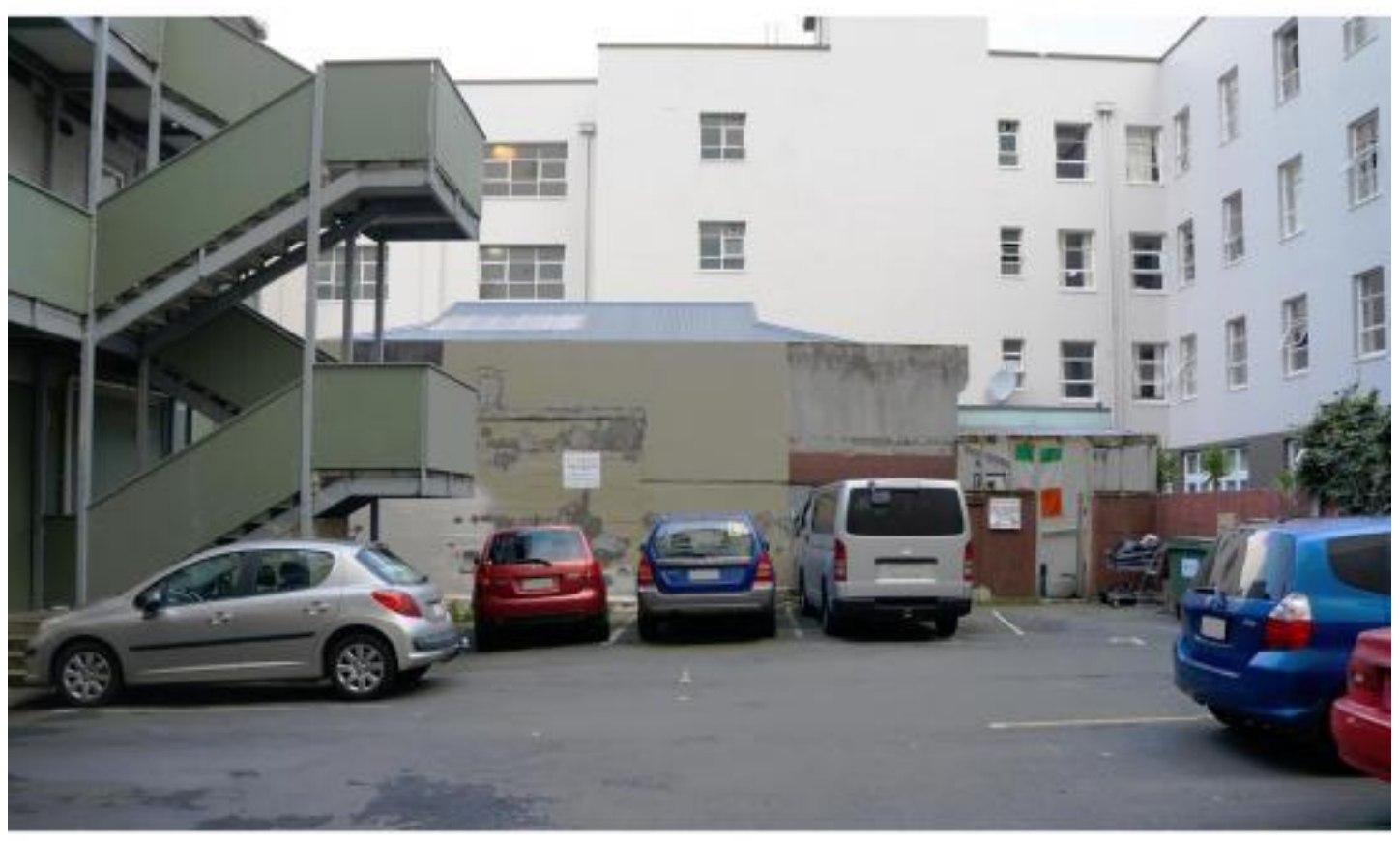

\section{IMAGE AFTER}

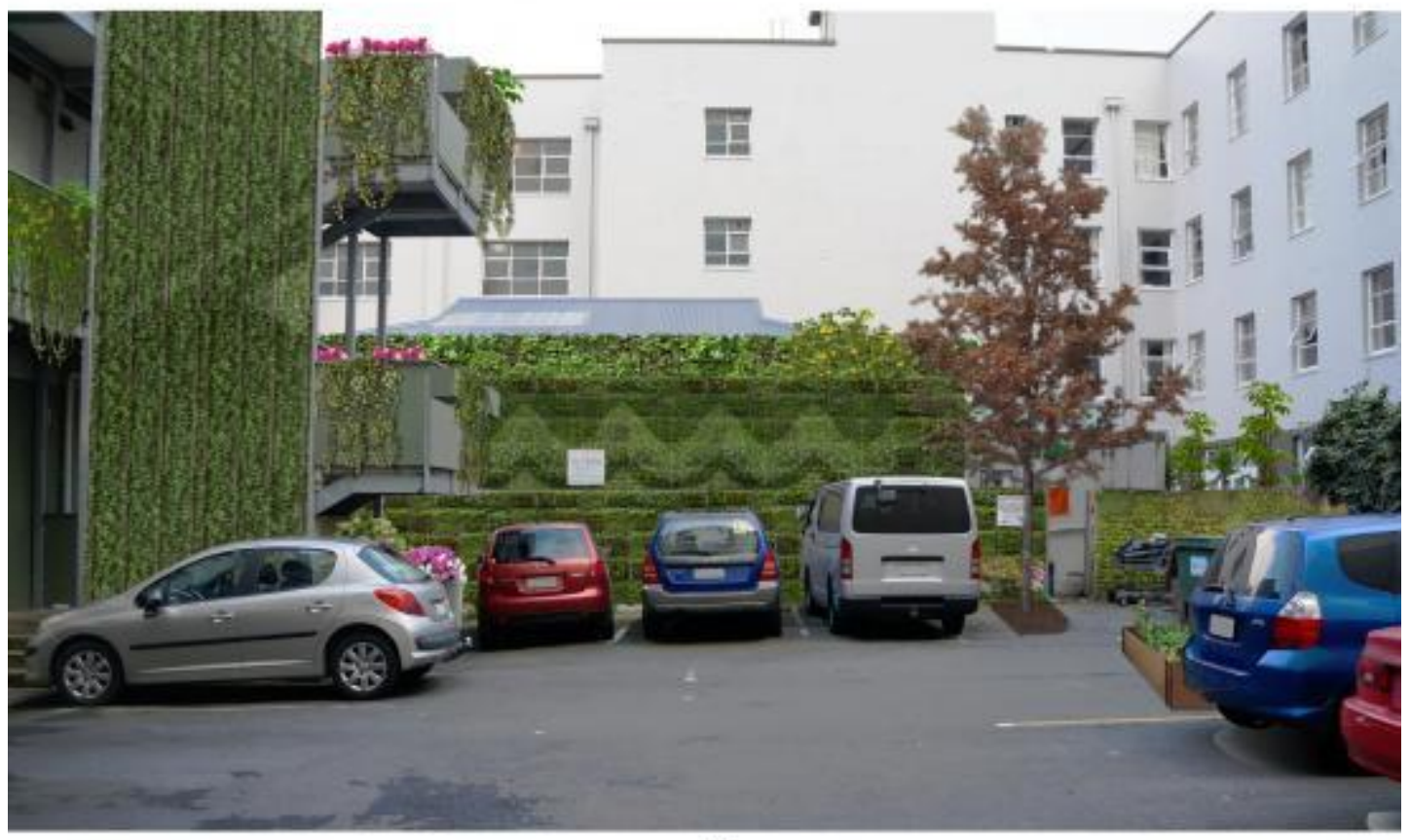

C 
Q9.1 Please score IMAGE C from scale -2 (Dislike) to 2 (Like)

\begin{tabular}{|c|c|c|c|c|c|}
\hline & $\begin{array}{c}\text { Dislike } \\
(-2)\end{array}$ & $\begin{array}{l}\text { Somewhat } \\
\text { Dislike } \\
(-1)\end{array}$ & $\begin{array}{l}\text { Neither Like nor } \\
\text { Dislike } \\
(0)\end{array}$ & $\begin{array}{l}\text { Somewhat Like } \\
\text { (1) }\end{array}$ & $\begin{array}{r}\text { Like } \\
(2)\end{array}$ \\
\hline Image C (2) & & & & & \\
\hline
\end{tabular}

Q9.2 How would you describe 'Image C' on the following scale from 1 to 5 (1 being the lowest and 5 being the highest)

\begin{tabular}{|c|c|c|c|c|c|c|}
\hline & 1 & 2 & 3 & 4 & 5 & \\
\hline & $1(1)$ & $2(2)$ & (3) & (4) & (5) & \\
\hline Ugly & & & & & & Beautiful \\
\hline Annoying & & & & & & Pleasing \\
\hline Impossible & & & & & & Realizable \\
\hline Boring & & & & & & Interesting \\
\hline Constrained & & & & & & Energetic \\
\hline
\end{tabular}

Q9.3 Do you have any suggestion for improving the visual quality of image C (you can type in the blank box)?

Yes (1)

No (2)

Page Break 
Q10 Rate how visually appealing you find image $A$ for a space surrounded with building on 3 sides

\section{IMAGE BEFORE}

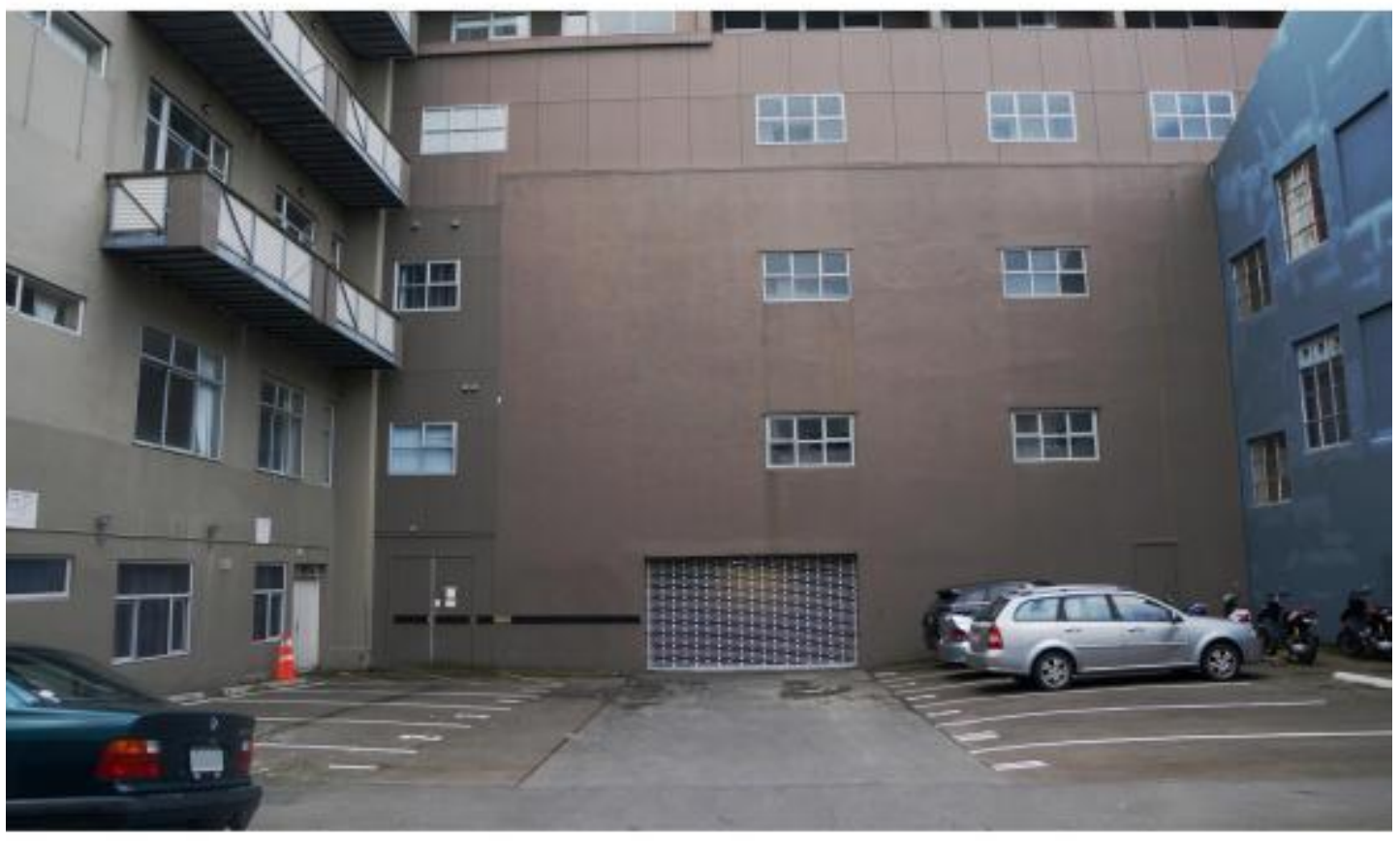

IMAGE AFTER

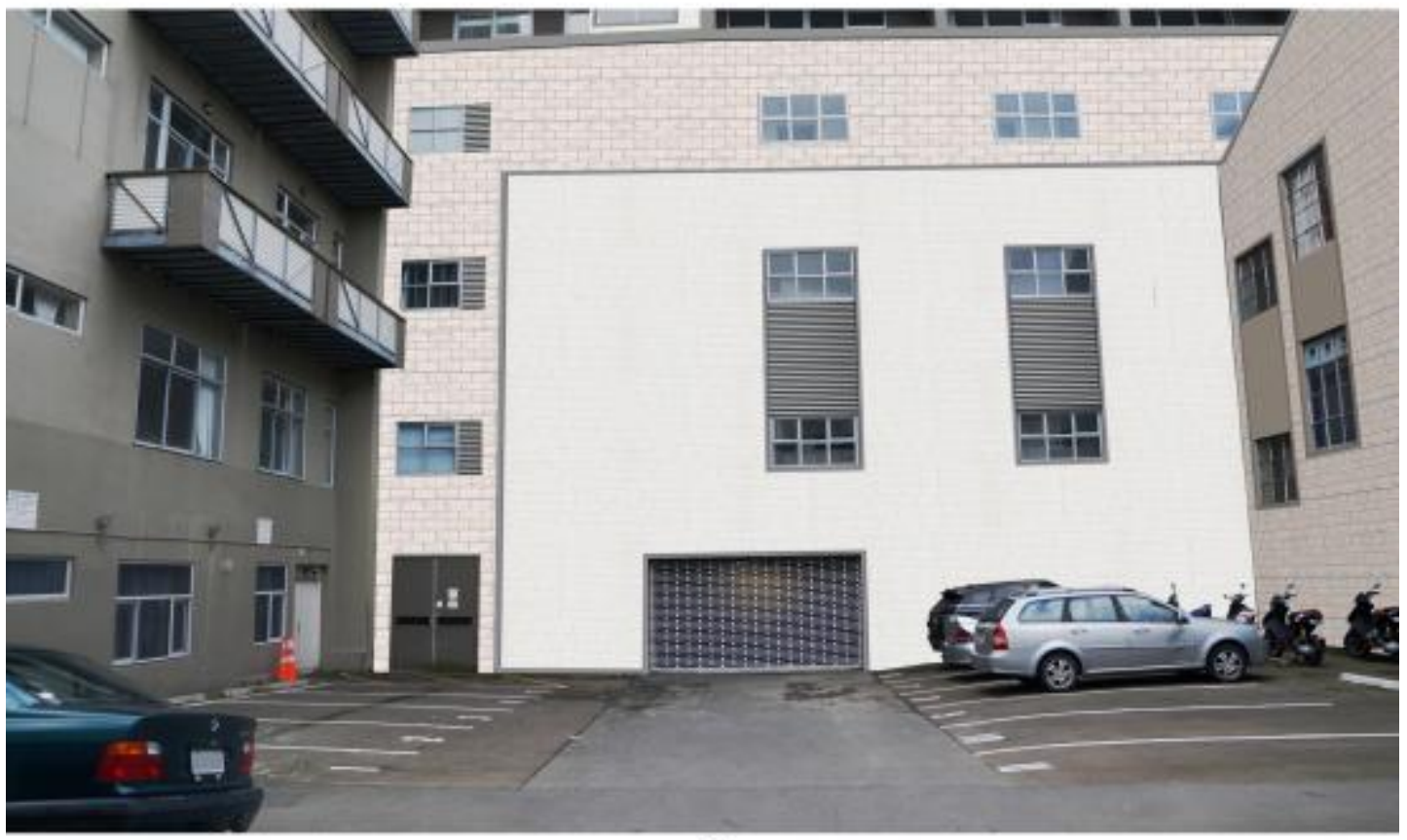

A 
Q10.1 Please score IMAGE A from scale -2 (Dislike) to 2 (Like)

\begin{tabular}{|c|c|c|c|c|c|}
\hline & $\begin{array}{c}\text { Dislike } \\
(-2)\end{array}$ & $\begin{array}{l}\text { Somewhat } \\
\text { Dislike } \\
(-1)\end{array}$ & $\begin{array}{l}\text { Neither Like nor } \\
\text { Dislike } \\
(0)\end{array}$ & $\begin{array}{c}\text { Somewhat Like } \\
\text { (1) }\end{array}$ & $\begin{array}{r}\text { Like } \\
(2)\end{array}$ \\
\hline age A (2) & & & & & \\
\hline
\end{tabular}

Q10.2 How would you describe 'Image A' on the following scale from 1 to 5 (1 being the lowest and 5 being the highest)

\begin{tabular}{|c|c|c|c|c|c|c|}
\hline & 1 & 2 & 3 & 4 & 5 & \\
\hline & $1(1)$ & $2(2)$ & (3) & (4) & (5) & \\
\hline Ugly & & & & & & Beautiful \\
\hline Annoying & & & & & & Pleasing \\
\hline Impossible & & & & & & Realizable \\
\hline Boring & & & & & & Interesting \\
\hline Constrained & & & & & & Energetic \\
\hline
\end{tabular}

Q10.3 Do you have any suggestion for improving the visual quality of image A (you can type in the blank box)?

Yes (1)

No (2)

Page Break 
Q11 Rate how visually appealing you find image $B$ for a space surrounded with building on 3 sides

\section{IMAGE BEFORE}

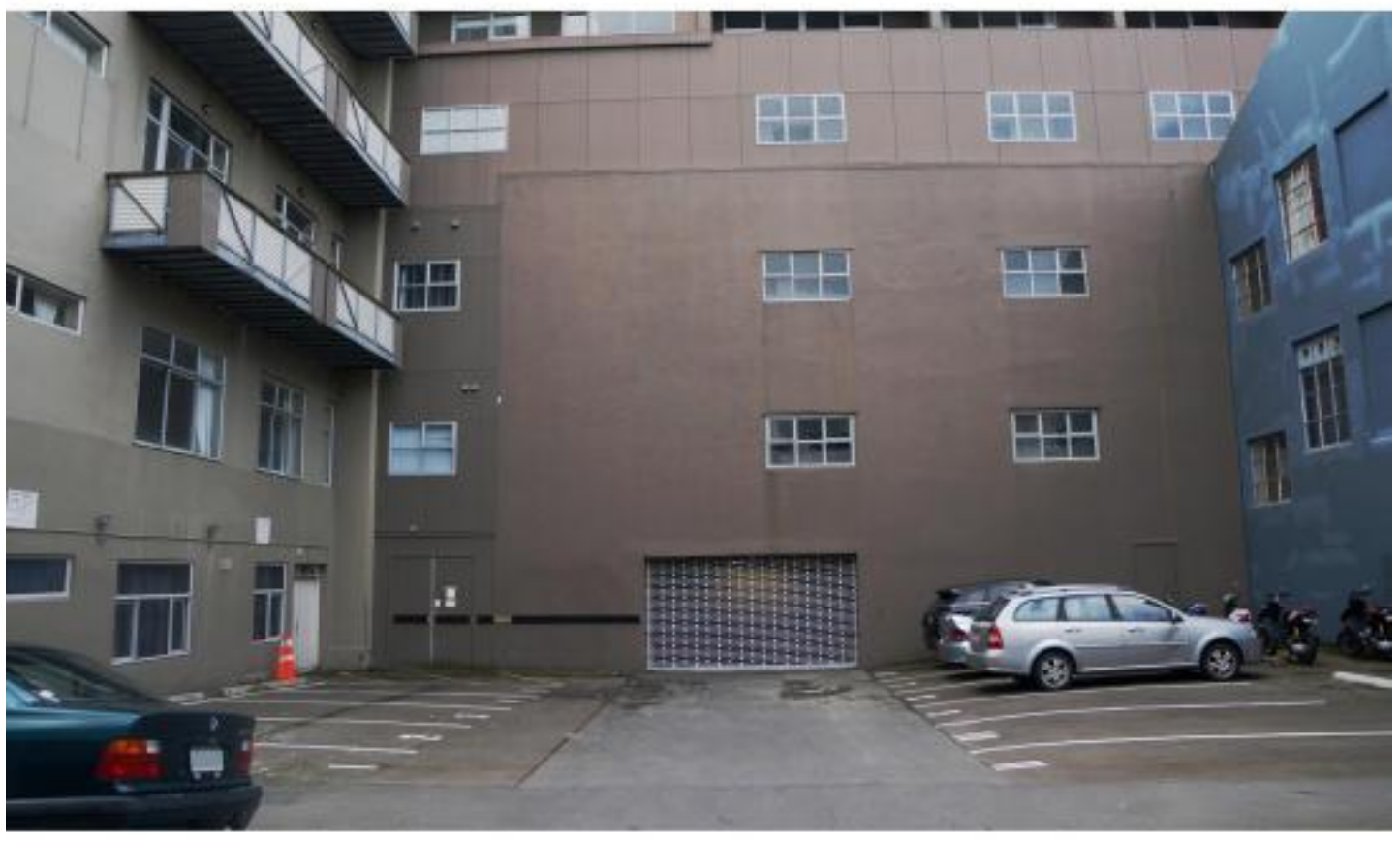

\section{IMAGE AFTER}

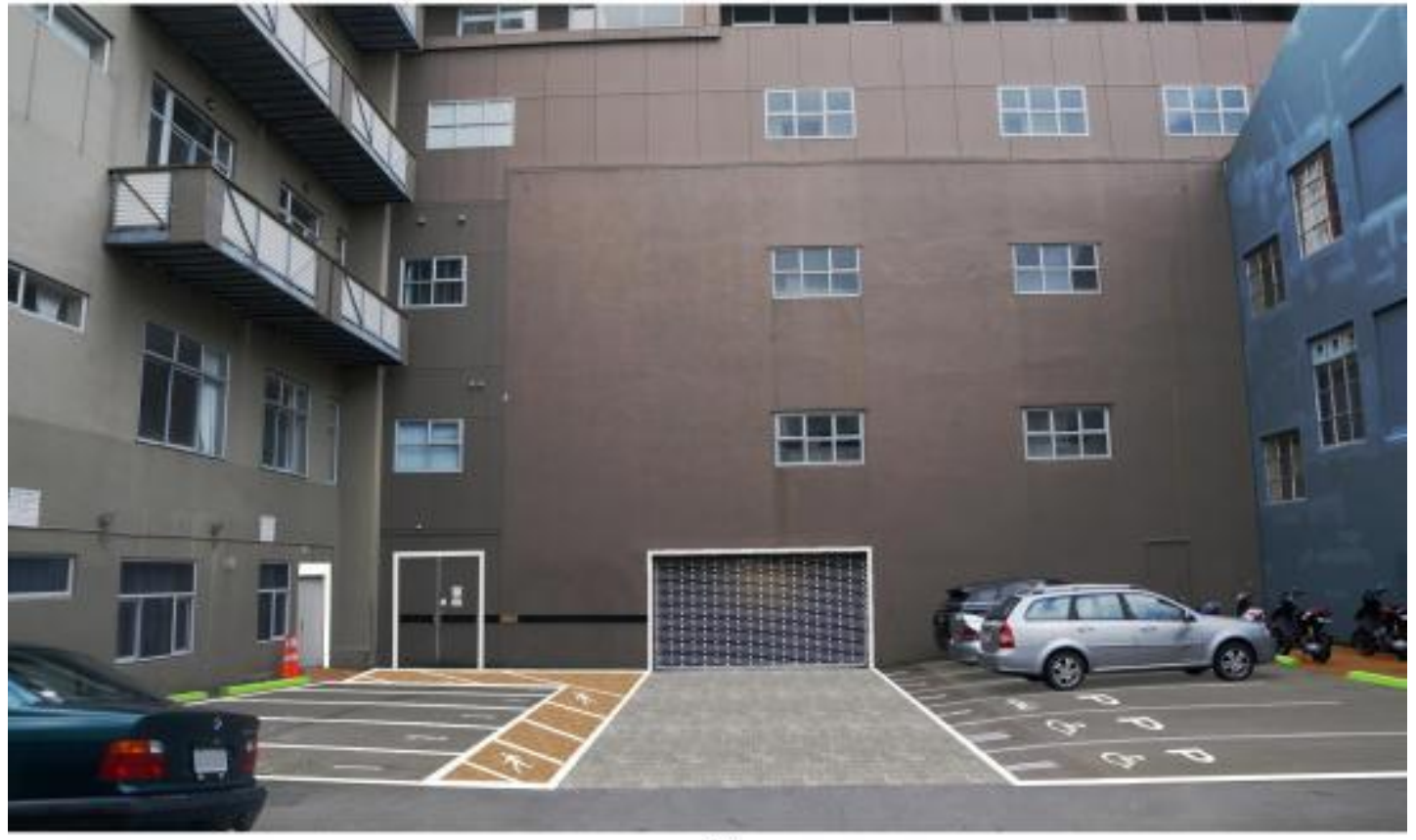

B 
Q11.1 Please score IMAGE B from scale -2 (Dislike) to 2 (Like)

\begin{tabular}{|c|c|c|c|c|c|}
\hline & $\begin{array}{l}\text { Dislike } \\
(-2)\end{array}$ & $\begin{array}{l}\text { Somewhat } \\
\text { Dislike } \\
(-1)\end{array}$ & $\begin{array}{l}\text { Neither Like nor } \\
\text { Dislike } \\
(0)\end{array}$ & $\begin{array}{l}\text { Somewhat Like } \\
\text { (1) }\end{array}$ & $\begin{array}{r}\text { Like } \\
(2)\end{array}$ \\
\hline Image B (2) & & & & & \\
\hline
\end{tabular}

Q11.2 How would you describe 'Image B' on the following scale from 1 to 5 (1 being the lowest and 5 being the highest)

\begin{tabular}{|c|c|c|c|c|c|c|}
\hline & 1 & 2 & 3 & 4 & 5 & \\
\hline & $1(1)$ & $2(2)$ & (3) & (4) & (5) & \\
\hline Ugly & & & & & & Beautiful \\
\hline Annoying & & & & & & Pleasing \\
\hline Impossible & & & & & & Realizable \\
\hline Boring & & & & & & Interesting \\
\hline Constrained & & & & & & Energetic \\
\hline
\end{tabular}

Q11.3 Do you have any suggestion for improving the visual quality of image B (you can type in the blank box)?

Yes (1)

No (2)

Page Break 
Q12 Rate how visually appealing you find image $C$ for a space surrounded with building on 3 sides

\section{IMAGE BEFORE}

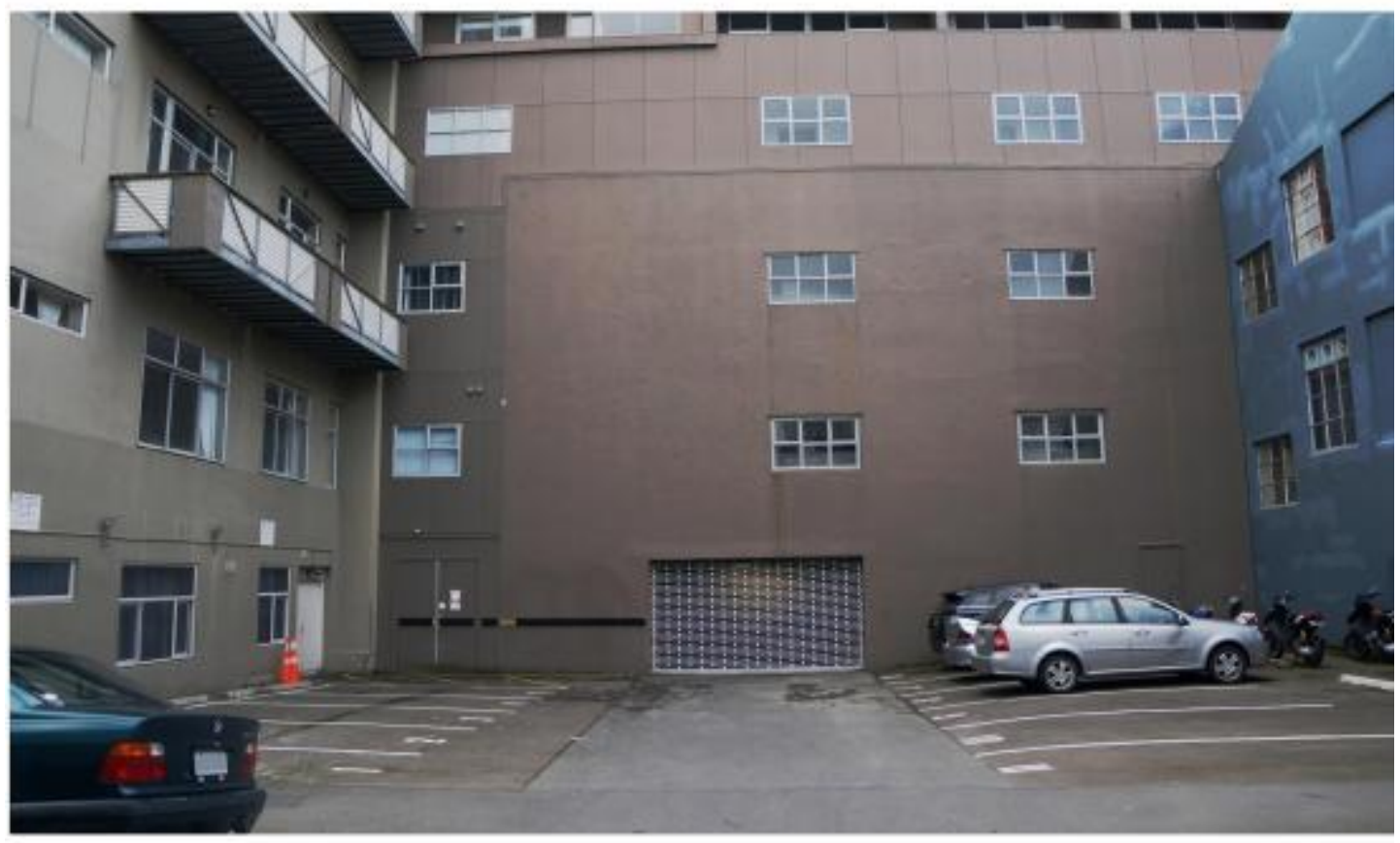

IMAGE AFTER

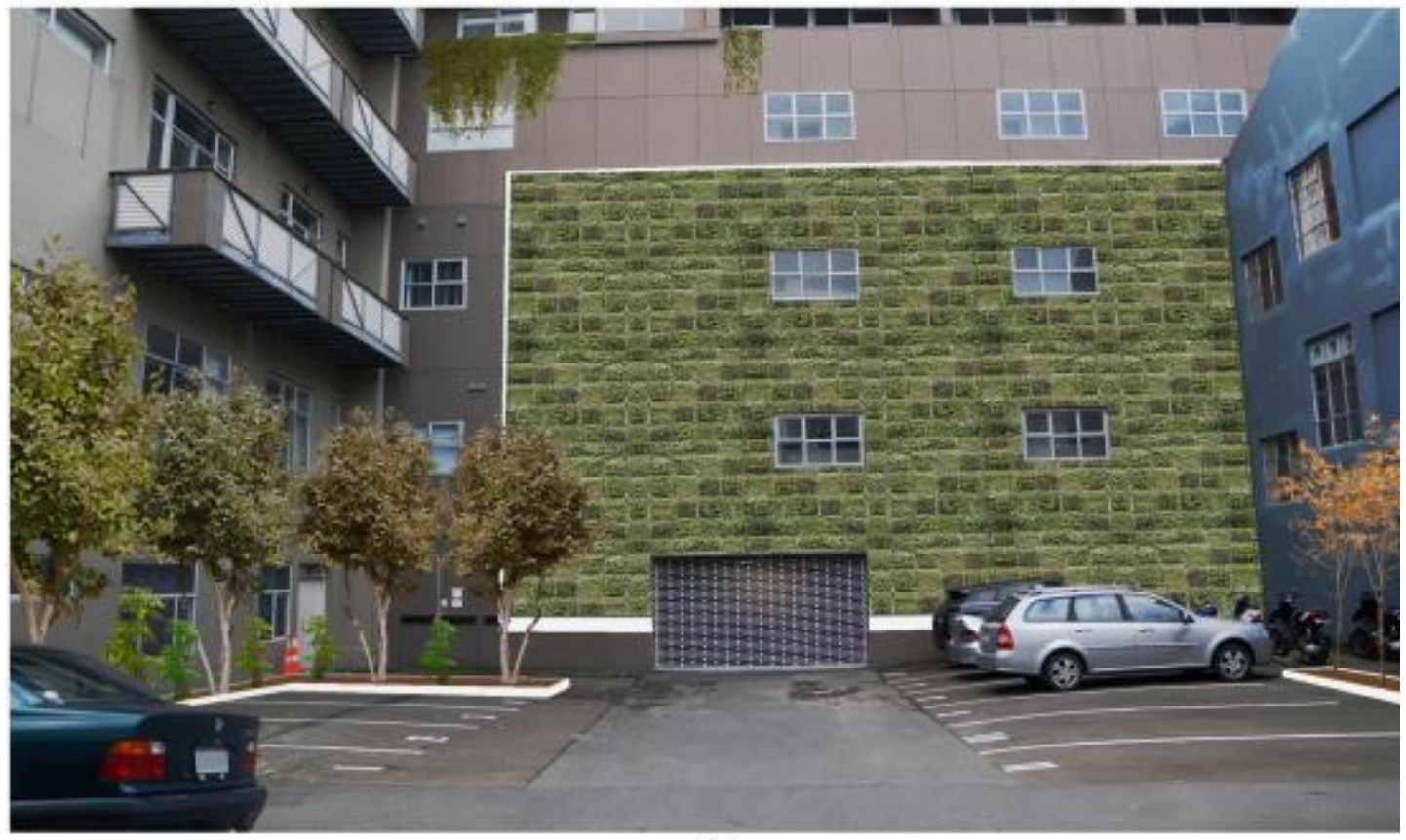

C 
Q12.1 Please score IMAGE C from scale -2 (Dislike) to 2 (Like)

\begin{tabular}{|c|c|c|c|c|c|}
\hline & $\begin{array}{l}\text { Dislike } \\
(-2)\end{array}$ & $\begin{array}{l}\text { Somewhat } \\
\text { Dislike } \\
(-1)\end{array}$ & $\begin{array}{l}\text { Neither Like nor } \\
\text { Dislike } \\
(0)\end{array}$ & $\begin{array}{c}\text { Somewhat Like } \\
\text { (1) }\end{array}$ & $\begin{array}{r}\text { Like } \\
(2)\end{array}$ \\
\hline lage $C(2)$ & & & & & \\
\hline
\end{tabular}

Q12.2 How would you describe 'Image C' on the following scale from 1 to 5 (1 being the lowest and 5 being the highest)

\begin{tabular}{|c|c|c|c|c|c|c|}
\hline & 1 & 2 & 3 & 4 & 5 & \\
\hline & $1(1)$ & $2(2)$ & (3) & (4) & (5) & \\
\hline Ugly & & & & & & Beautiful \\
\hline Annoying & & & & & & Pleasing \\
\hline Impossible & & & & & & Realizable \\
\hline Boring & & & & & & Interesting \\
\hline Constrained & & & & & & Energetic \\
\hline
\end{tabular}

Q12.3 Do you have any suggestion for improving the visual quality of image $C$ (you can type in the blank box)?

Yes (1)

No (2)

Page Break 
Q13 Rate how visually appealing you find image $A$ for a space surrounded with building on 2 sides

\section{IMAGE BEFORE}

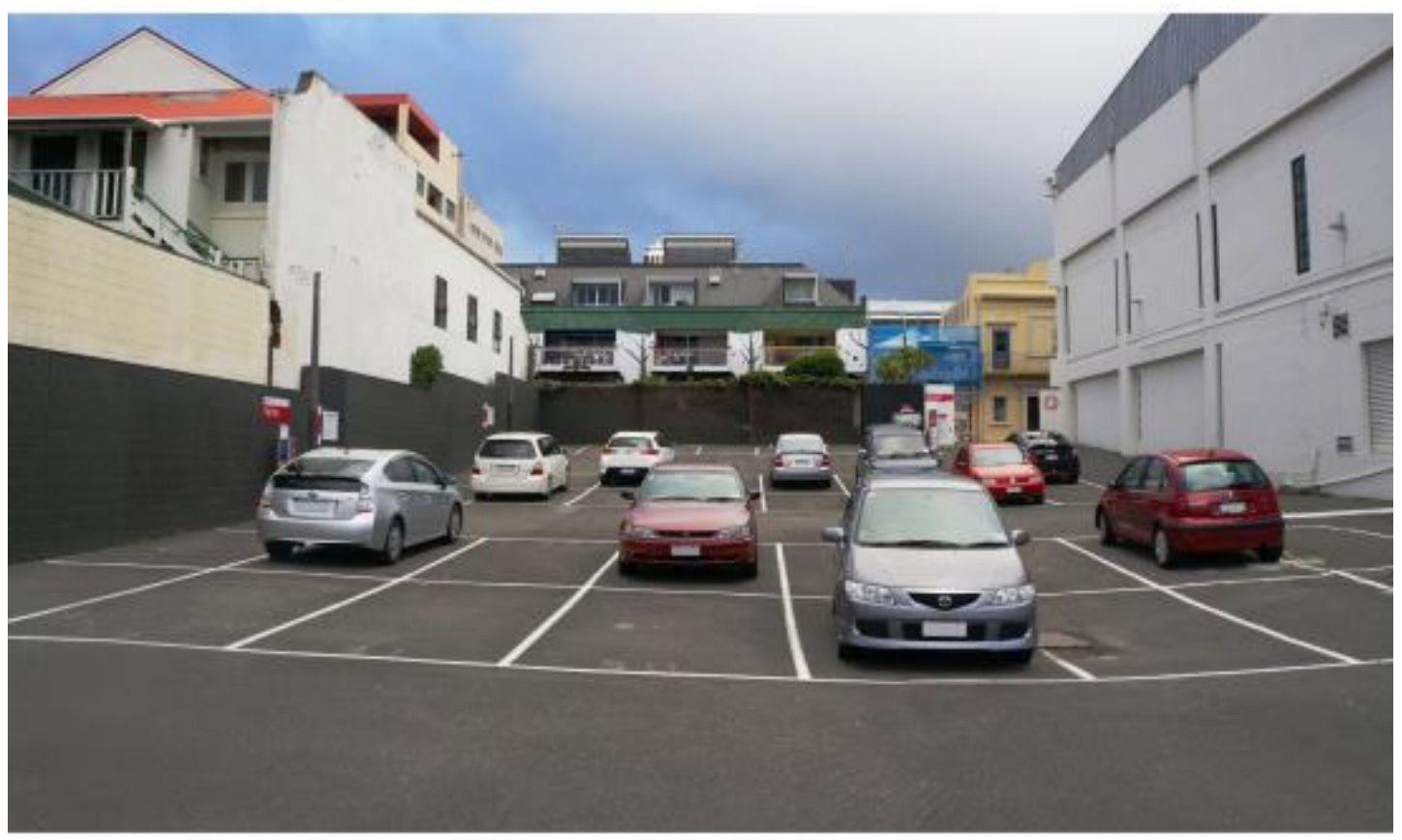

\section{IMAGE AFTER}

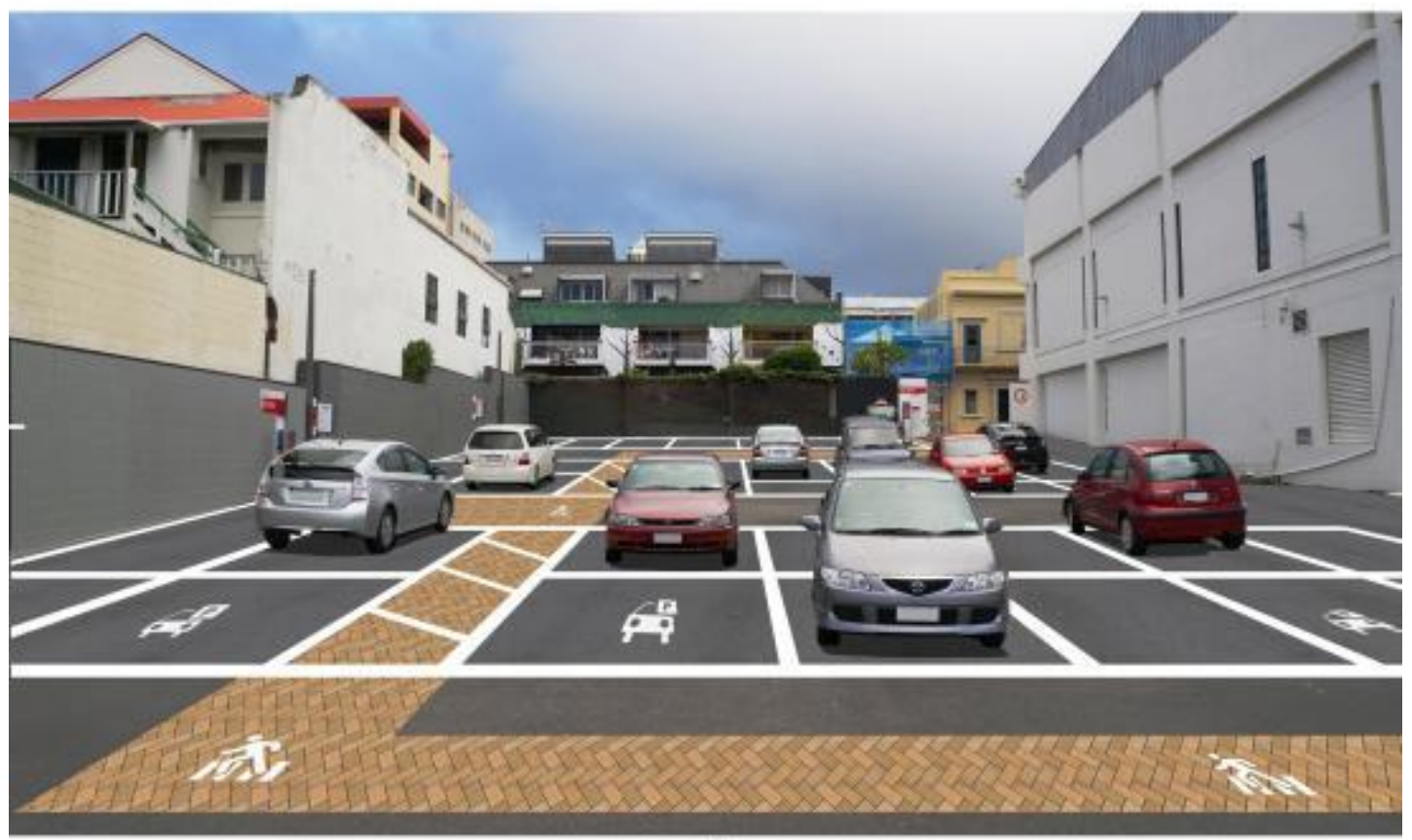

A 
Q13.1 Please score IMAGE A from scale -2 (Dislike) to 2 (Like)

\begin{tabular}{|c|c|c|c|c|c|}
\hline & $\begin{array}{l}\text { Dislike } \\
(-2)\end{array}$ & $\begin{array}{l}\text { Somewhat } \\
\text { Dislike } \\
(-1)\end{array}$ & $\begin{array}{l}\text { Neither Like nor } \\
\text { Dislike } \\
(0)\end{array}$ & $\begin{array}{l}\text { Somewhat Like } \\
\text { (1) }\end{array}$ & $\begin{array}{r}\text { Like } \\
(2)\end{array}$ \\
\hline Image A (2) & & & & & \\
\hline
\end{tabular}

Q13.2 How would you describe 'Image A' on the following scale from 1 to 5 (1 being the lowest and 5 being the highest)

\begin{tabular}{|c|c|c|c|c|c|c|}
\hline & 1 & 2 & 3 & 4 & 5 & \\
\hline & $1(1)$ & $2(2)$ & (3) & (4) & (5) & \\
\hline Ugly & & & & & & Beautiful \\
\hline Annoying & & & & & & Pleasing \\
\hline Impossible & & & & & & Realizable \\
\hline Boring & & & & & & Interesting \\
\hline Constrained & & & & & & Energetic \\
\hline
\end{tabular}

Q13.3 Do you have any suggestion for improving the visual quality of image A (you can type in the blank box)?

Yes (1)

No (2)

Page Break 
Q14 Rate how visually appealing you find image B for a space surrounded with building on 2 sides

IMAGE BEFORE

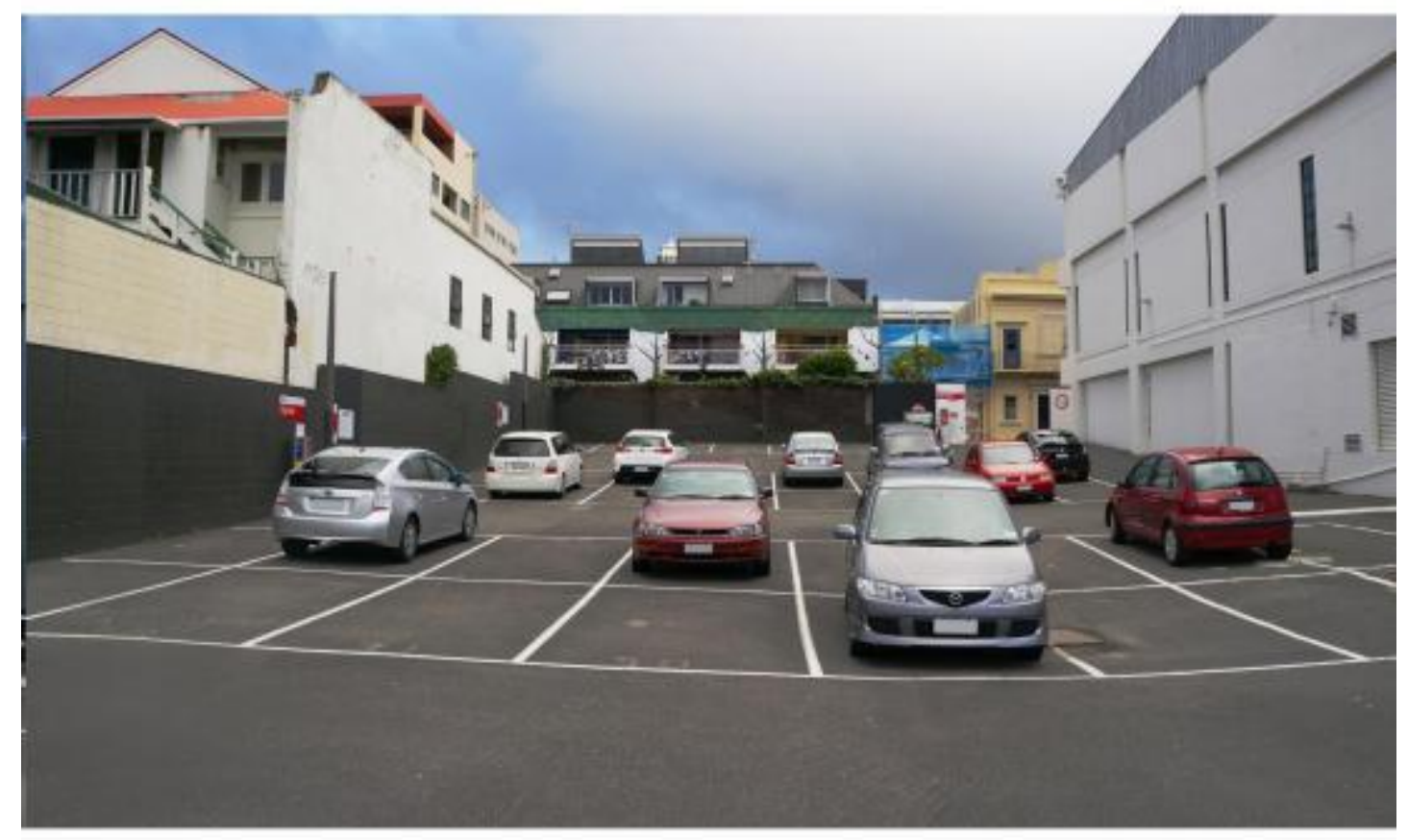

IMAGE AFTER

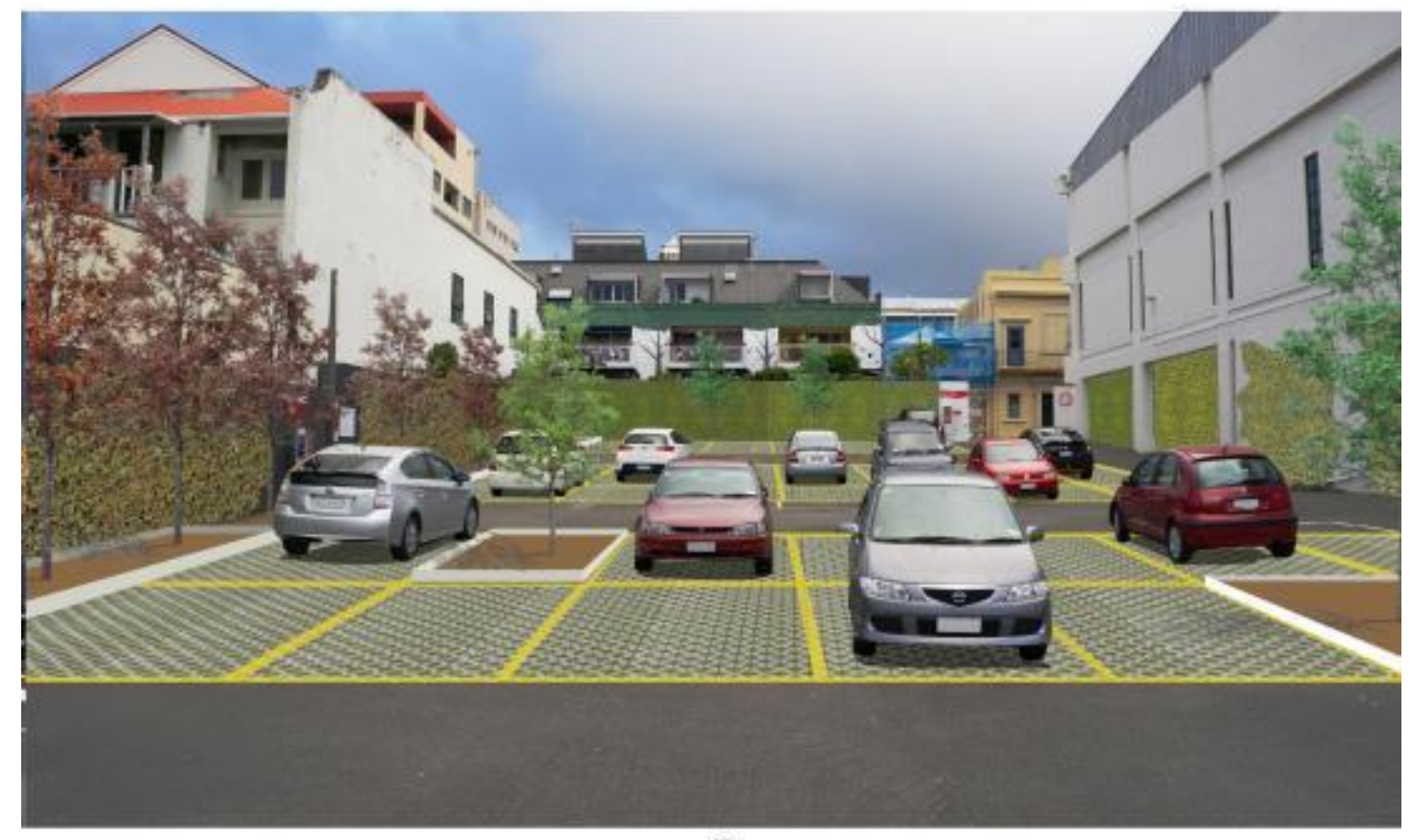

B 
Q14.1 Please score IMAGE B from scale -2 (Dislike) to 2 (Like)

\begin{tabular}{|c|c|c|c|c|c|}
\hline & $\begin{array}{c}\text { Dislike } \\
(-2)\end{array}$ & $\begin{array}{l}\text { Somewhat } \\
\text { Dislike } \\
(-1)\end{array}$ & $\begin{array}{l}\text { Neither Like nor } \\
\text { Dislike } \\
(0)\end{array}$ & $\begin{array}{c}\text { Somewhat Like } \\
\text { (1) }\end{array}$ & $\begin{array}{r}\text { Like } \\
(2)\end{array}$ \\
\hline lage $B(2)$ & & & & & \\
\hline
\end{tabular}

Q14.2 How would you describe 'Image B' on the following scale from 1 to 5 (1 being the lowest and 5 being the highest)

\begin{tabular}{|c|c|c|c|c|c|c|}
\hline & 1 & 2 & 3 & 4 & 5 & \\
\hline & $1(1)$ & $2(2)$ & (3) & (4) & (5) & \\
\hline Ugly & & & & & & Beautiful \\
\hline Annoying & & & & & & Pleasing \\
\hline Impossible & & & & & & Realizable \\
\hline Boring & & & & & & Interesting \\
\hline Constrained & & & & & & Energetic \\
\hline
\end{tabular}

Q14.3 Do you have any suggestion for improving the visual quality of image B (you can type in the blank box)?

Yes (1)

No (2)

Page Break 
Q15 Rate how visually appealing you find image $C$ for a space surrounded with building on 2 sides

\section{IMAGE BEFORE}

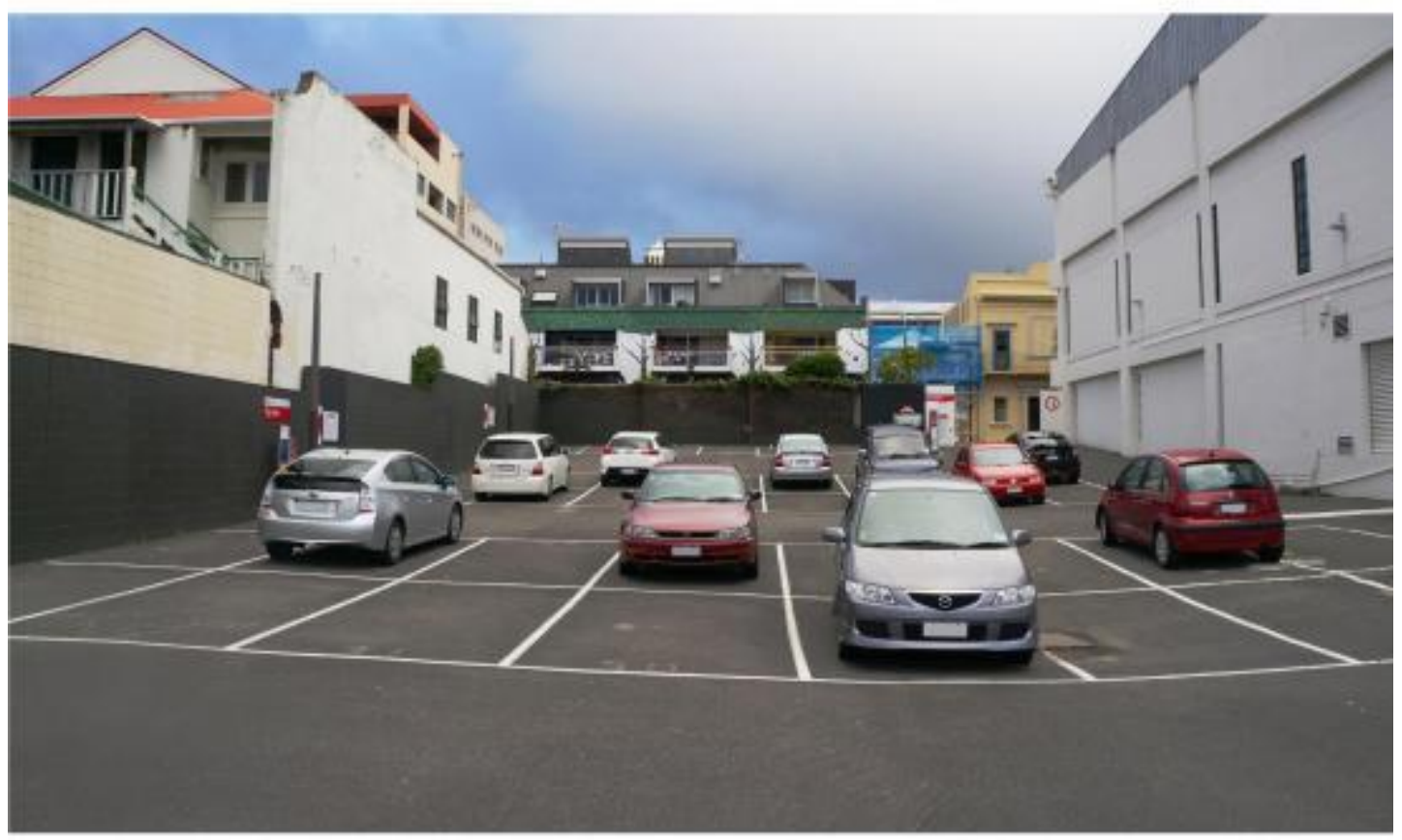

\section{IMAGE AFTER}

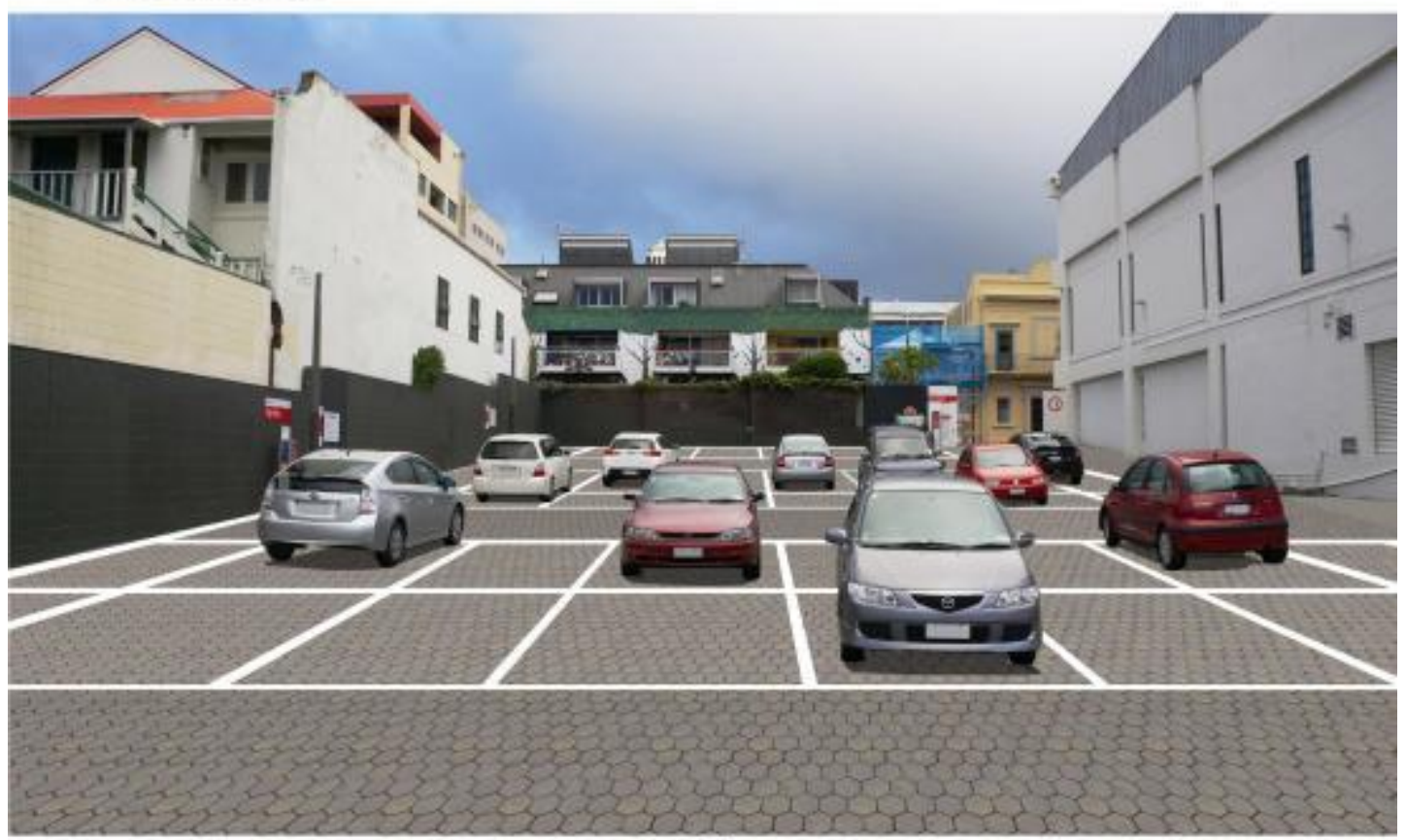

C 
Q15.1 Please score IMAGE C from scale -2 (Dislike) to 2 (Like)

\begin{tabular}{|c|c|c|c|c|c|}
\hline & $\begin{array}{l}\text { Dislike } \\
(-2)\end{array}$ & $\begin{array}{l}\text { Somewhat } \\
\text { Dislike } \\
(-1)\end{array}$ & $\begin{array}{l}\text { Neither Like nor } \\
\text { Dislike } \\
(0)\end{array}$ & $\begin{array}{l}\text { Somewhat Like } \\
\text { (1) }\end{array}$ & $\begin{array}{r}\text { Like } \\
(2)\end{array}$ \\
\hline lage $C(2)$ & & & & & \\
\hline
\end{tabular}

Q15.2 How would you describe 'Image C' on the following scale from 1 to 5 (1 being the lowest and 5 being the highest)

\begin{tabular}{|c|c|c|c|c|c|c|}
\hline & 1 & 2 & 3 & 4 & 5 & \\
\hline & $1(1)$ & $2(2)$ & (3) & (4) & (5) & \\
\hline Ugly & & & & & & Beautiful \\
\hline Annoying & & & & & & Pleasing \\
\hline Impossible & & & & & & Realizable \\
\hline Boring & & & & & & Interesting \\
\hline Constrained & & & & & & Energetic \\
\hline
\end{tabular}

Q15.3 Do you have any suggestion for improving the visual quality of image $C$ (you can type in the blank box)?

Yes (1)

No (2)

Page Break 
Q16 Rate how visually appealing you find image $A$ for a space on the rooftop

IMAGE BEFORE

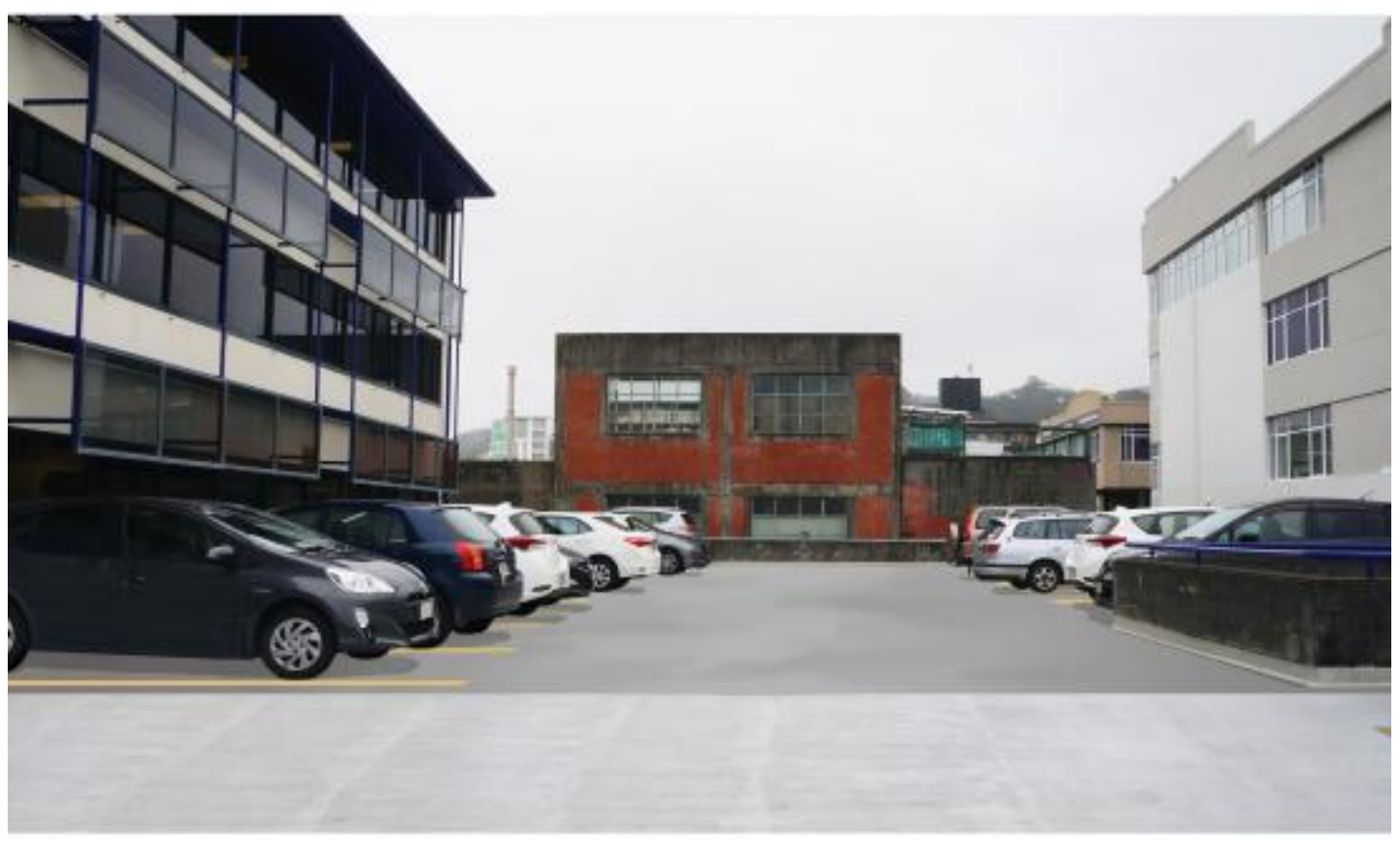

IMAGE AFTER

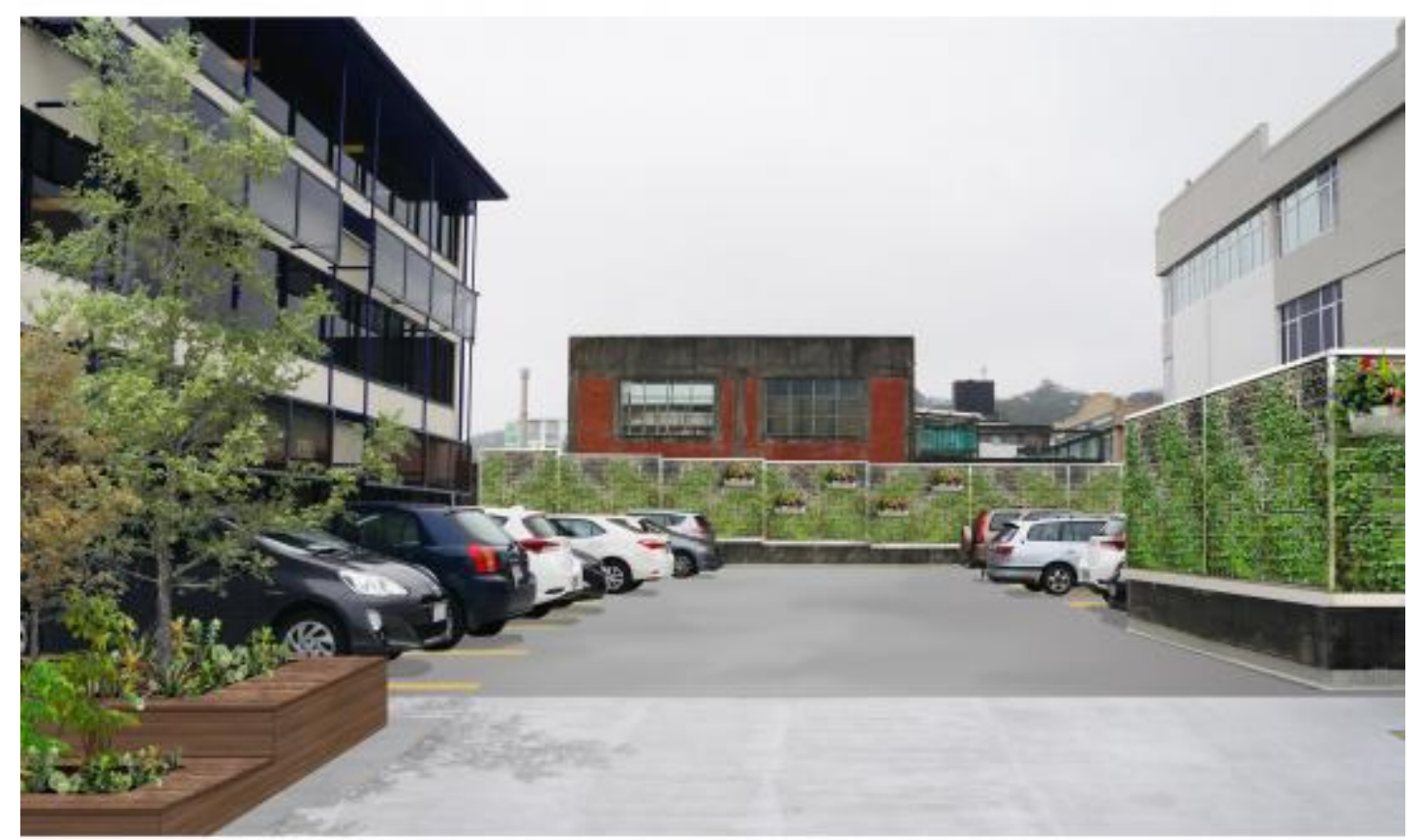

A 
Q16.1 Please score IMAGE A from scale -2 (Dislike) to 2 (Like)

\begin{tabular}{|c|c|c|c|c|c|}
\hline & $\begin{array}{c}\text { Dislike } \\
(-2)\end{array}$ & $\begin{array}{l}\text { Somewhat } \\
\text { Dislike } \\
(-1)\end{array}$ & $\begin{array}{l}\text { Neither Like nor } \\
\text { Dislike } \\
(0)\end{array}$ & $\begin{array}{c}\text { Somewhat Like } \\
\text { (1) }\end{array}$ & $\begin{array}{r}\text { Like } \\
(2)\end{array}$ \\
\hline age A (2) & & & & & \\
\hline
\end{tabular}

Q16.2 How would you describe 'Image A' on the following scale from 1 to 5 (1 being the lowest and 5 being the highest)

\begin{tabular}{|c|c|c|c|c|c|c|}
\hline & 1 & 2 & 3 & 4 & 5 & \\
\hline & $1(1)$ & $2(2)$ & (3) & (4) & (5) & \\
\hline Ugly & & & & & & Beautiful \\
\hline Annoying & & & & & & Pleasing \\
\hline Impossible & & & & & & Realizable \\
\hline Boring & & & & & & Interesting \\
\hline Constrained & & & & & & Energetic \\
\hline
\end{tabular}

Q16.3 Do you have any suggestion for improving the visual quality of image A (you can type in the blank box)?

Yes (1)

No (2)

Page Break 
Q17 Rate how visually appealing you find image $B$ for a space on the rooftop
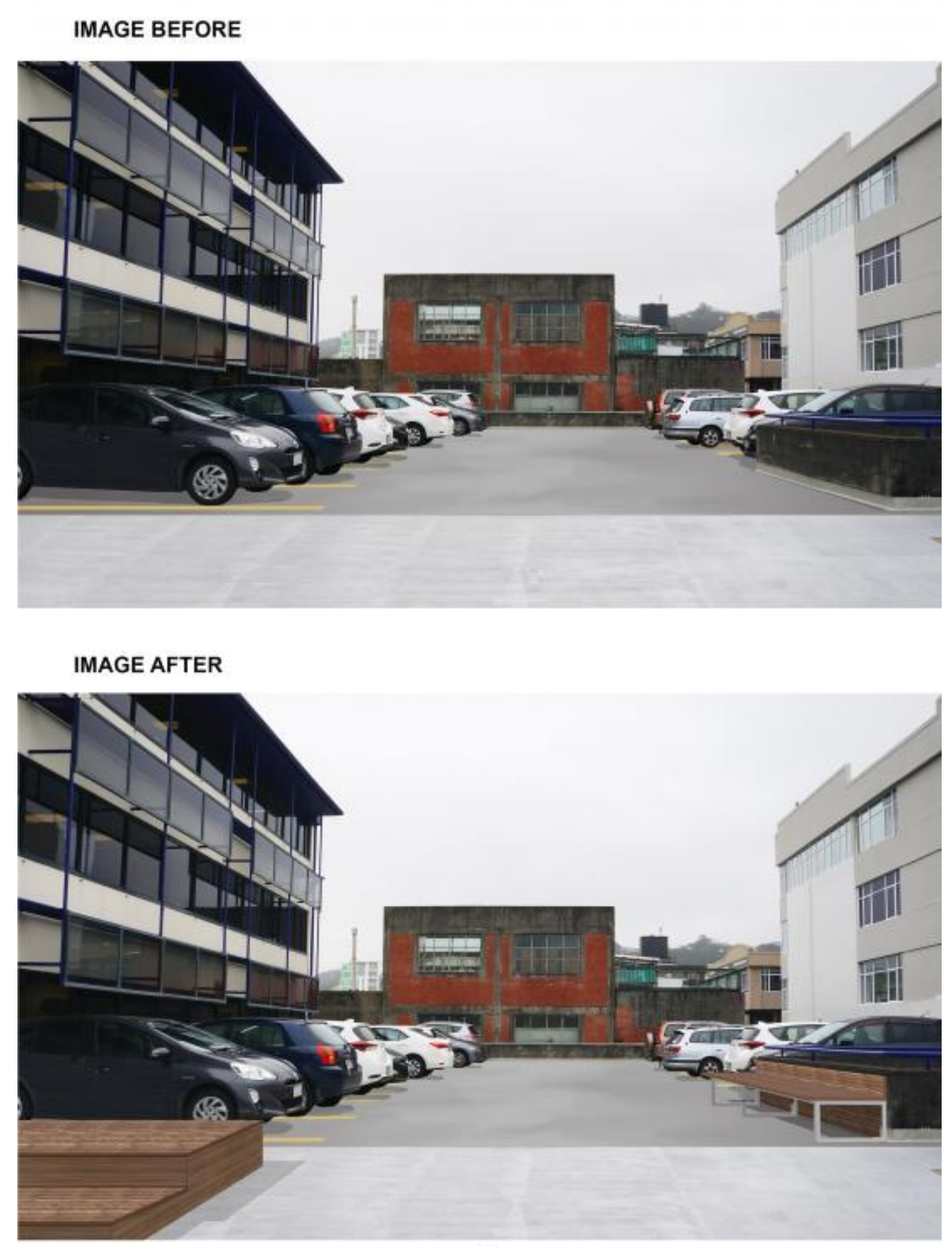

B 
Q17.1 Please score IMAGE B from scale -2 (Dislike) to 2 (Like)

\begin{tabular}{|c|c|c|c|c|c|}
\hline & $\begin{array}{c}\text { Dislike } \\
(-2)\end{array}$ & $\begin{array}{l}\text { Somewhat } \\
\text { Dislike } \\
(-1)\end{array}$ & $\begin{array}{l}\text { Neither Like nor } \\
\text { Dislike } \\
(0)\end{array}$ & $\begin{array}{c}\text { Somewhat Like } \\
\text { (1) }\end{array}$ & $\begin{array}{r}\text { Like } \\
(2)\end{array}$ \\
\hline lage $B(2)$ & & & & & \\
\hline
\end{tabular}

Q17.2 How would you describe 'Image B' on the following scale from 1 to 5 (1 being the lowest and 5 being the highest)

\begin{tabular}{|c|c|c|c|c|c|c|}
\hline & 1 & 2 & 3 & 4 & 5 & \\
\hline & $1(1)$ & $2(2)$ & (3) & (4) & (5) & \\
\hline Ugly & & & & & & Beautiful \\
\hline Annoying & & & & & & Pleasing \\
\hline Impossible & & & & & & Realizable \\
\hline Boring & & & & & & Interesting \\
\hline Constrained & & & & & & Energetic \\
\hline
\end{tabular}

Q17.3 Do you have any suggestion for improving the visual quality of image B (you can type in the blank box)?

Yes (1)

No (2)

Page Break 
IMAGE BEFORE

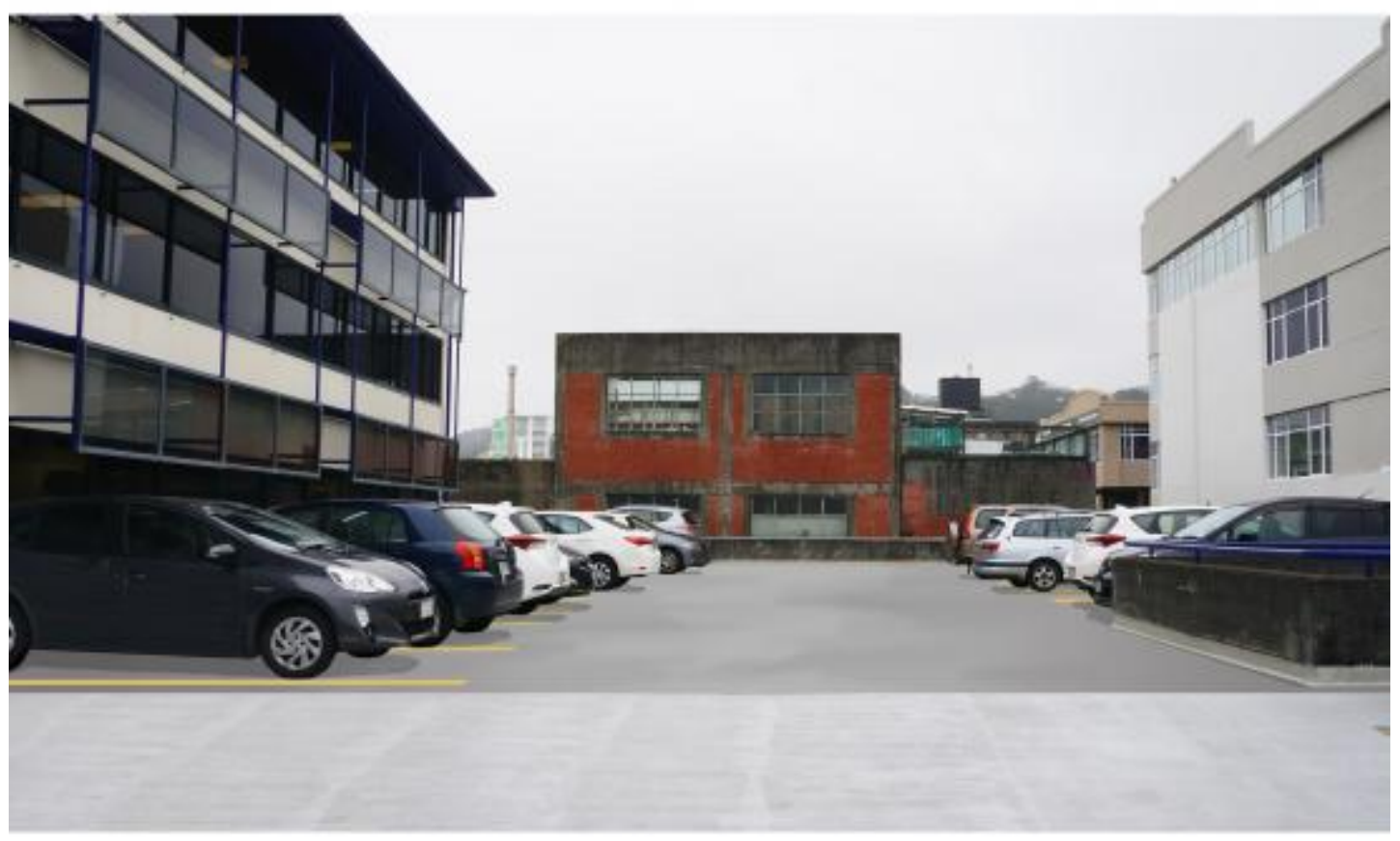

IMAGE AFTER

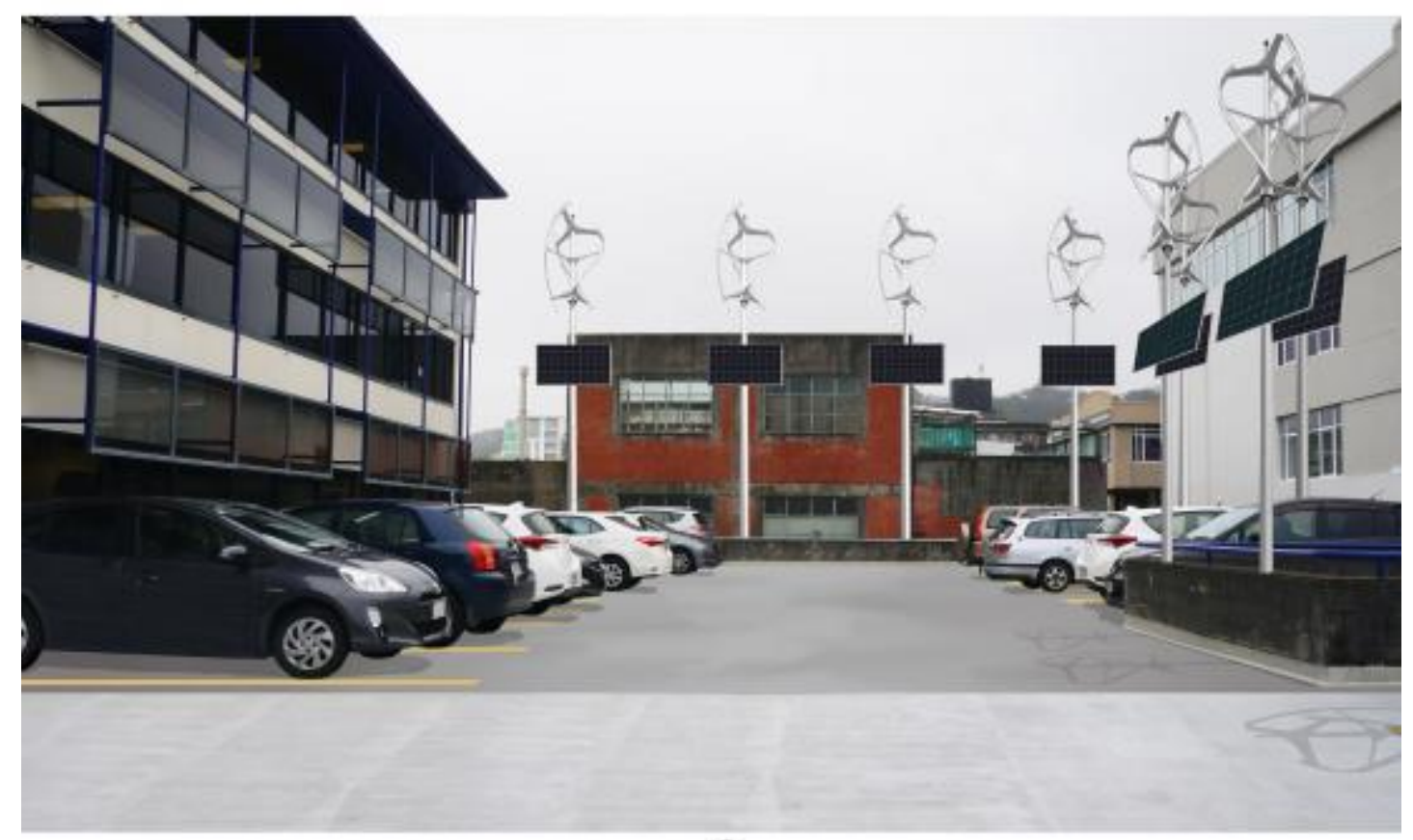

C 
Q18.1 Please score IMAGE C from scale -2 (Dislike) to 2 (Like)

\begin{tabular}{|c|c|c|c|c|c|}
\hline & $\begin{array}{c}\text { Dislike } \\
(-2)\end{array}$ & $\begin{array}{l}\text { Somewhat } \\
\text { Dislike } \\
(-1)\end{array}$ & $\begin{array}{l}\text { Neither Like nor } \\
\text { Dislike } \\
(0)\end{array}$ & $\begin{array}{l}\text { Somewhat Like } \\
\text { (1) ( }\end{array}$ & $\begin{array}{r}\text { Like } \\
(2)\end{array}$ \\
\hline lage $C(2)$ & & & & & \\
\hline
\end{tabular}

Q18.2 How would you describe 'Image C' on the following scale from 1 to 5 (1 being the lowest and 5 being the highest)

\begin{tabular}{|c|c|c|c|c|c|c|}
\hline & 1 & 2 & 3 & 4 & 5 & \\
\hline & $1(1)$ & $2(2)$ & (3) & (4) & (5) & \\
\hline Ugly & & & & & & Beautiful \\
\hline Annoying & & & & & & Pleasing \\
\hline Impossible & & & & & & Realizable \\
\hline Boring & & & & & & Interesting \\
\hline Constrained & & & & & & Energetic \\
\hline
\end{tabular}

Q18.3 Do you have any suggestion for improving the visual quality of image $C$ (you can type in the blank box)?

Yes (1)

No (2)

Page Break 
Q19 In PART 2 of this survey, you are asked to think about a future scenario.

Please suggest one function for space below (between buildings on 2 sides) on the assumption that the parking is removed.

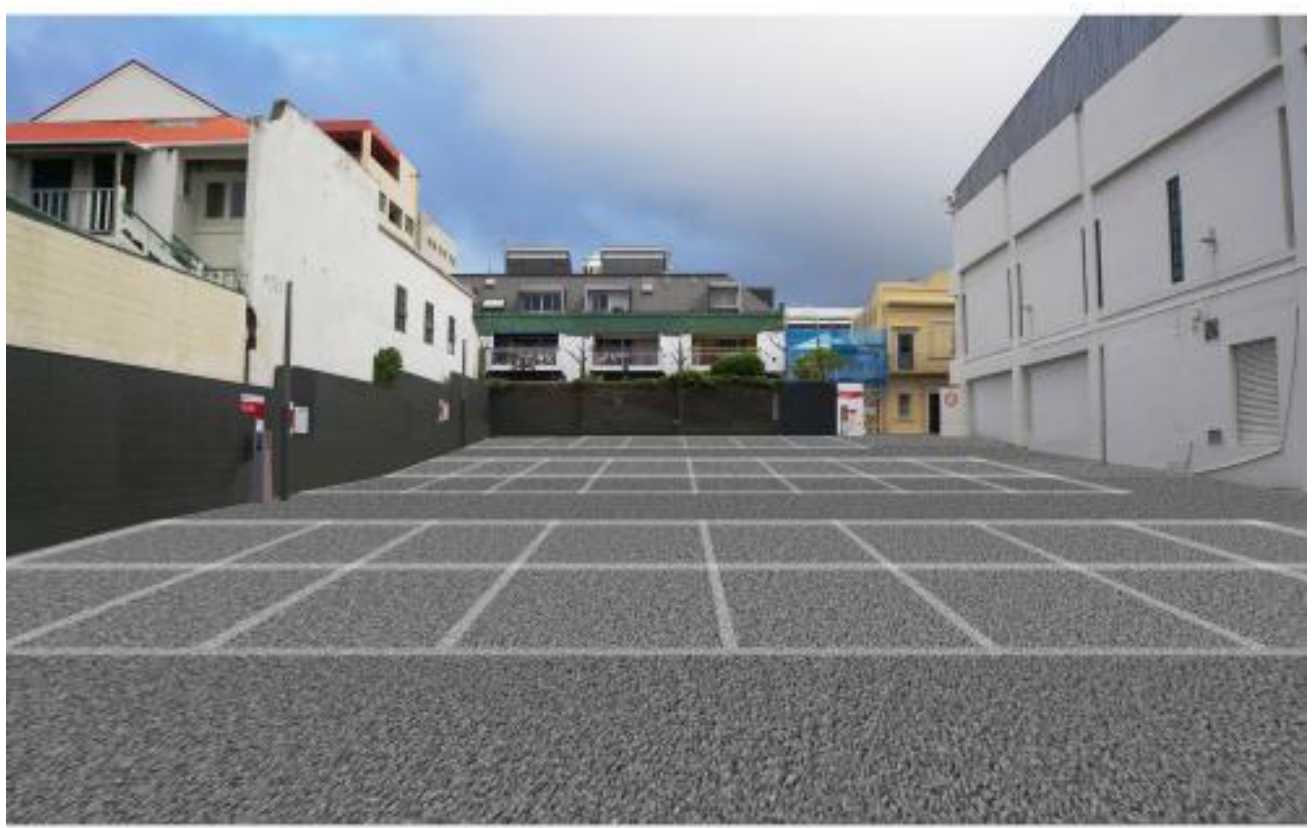


Q19.1 Please select one or (max) two functions

Provide a community garden

Design a sports area

Install Wind Turbines

Install Solar Panels

Create graffiti on wall

Provide food market

Create an exhibition space

Design a space for green park

Q20 Please suggest one function for space below (between 3 sides) on the assumption that the parking is removed.

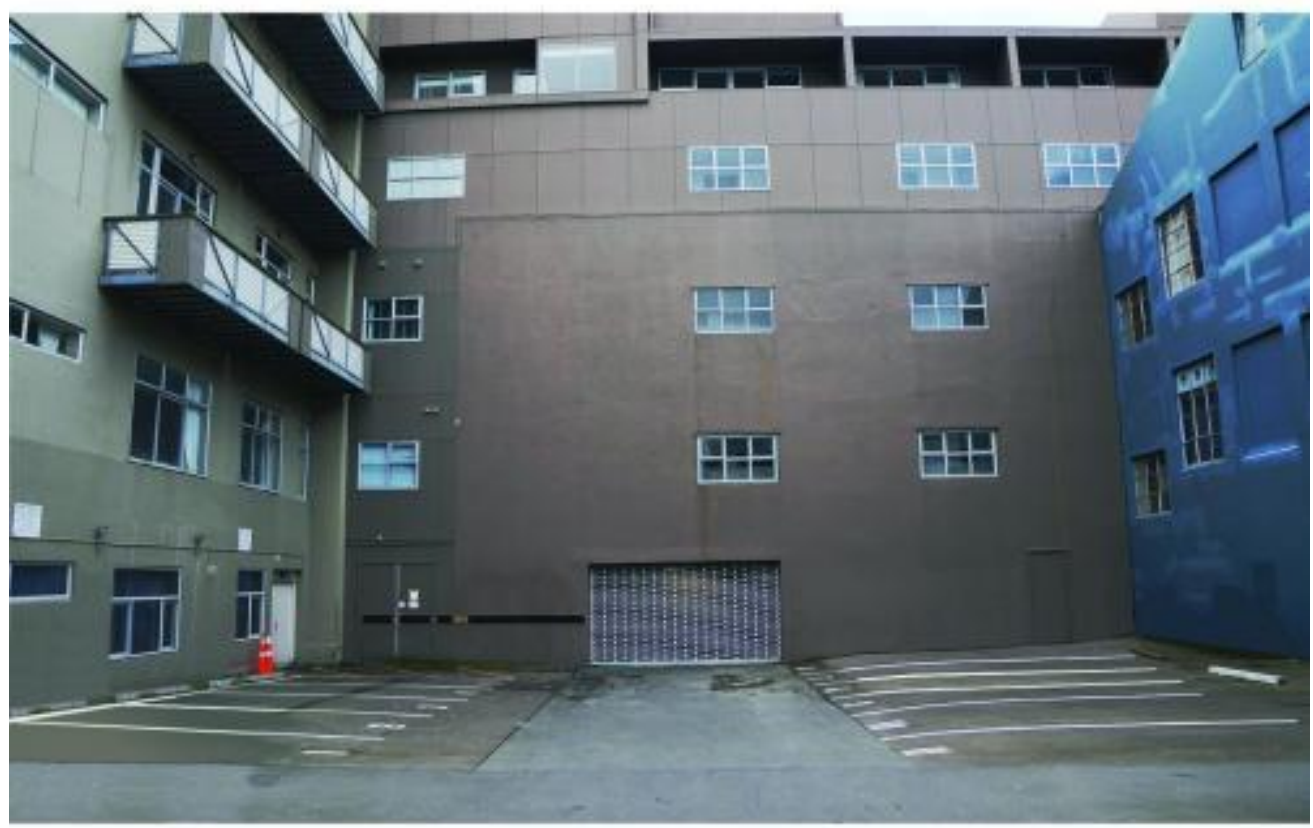


Q20.1 Please select one or ( $\max$ ) two functions

Provide a community garden

Design a sports area

Install Wind Turbines

Install Solar Panels

Create graffiti on wall

Provide food market

Create an exhibition space

Design a space for green park

Page Break 
Q21 Please suggest one function for space below (backyard) on the assumption that the parking is removed.

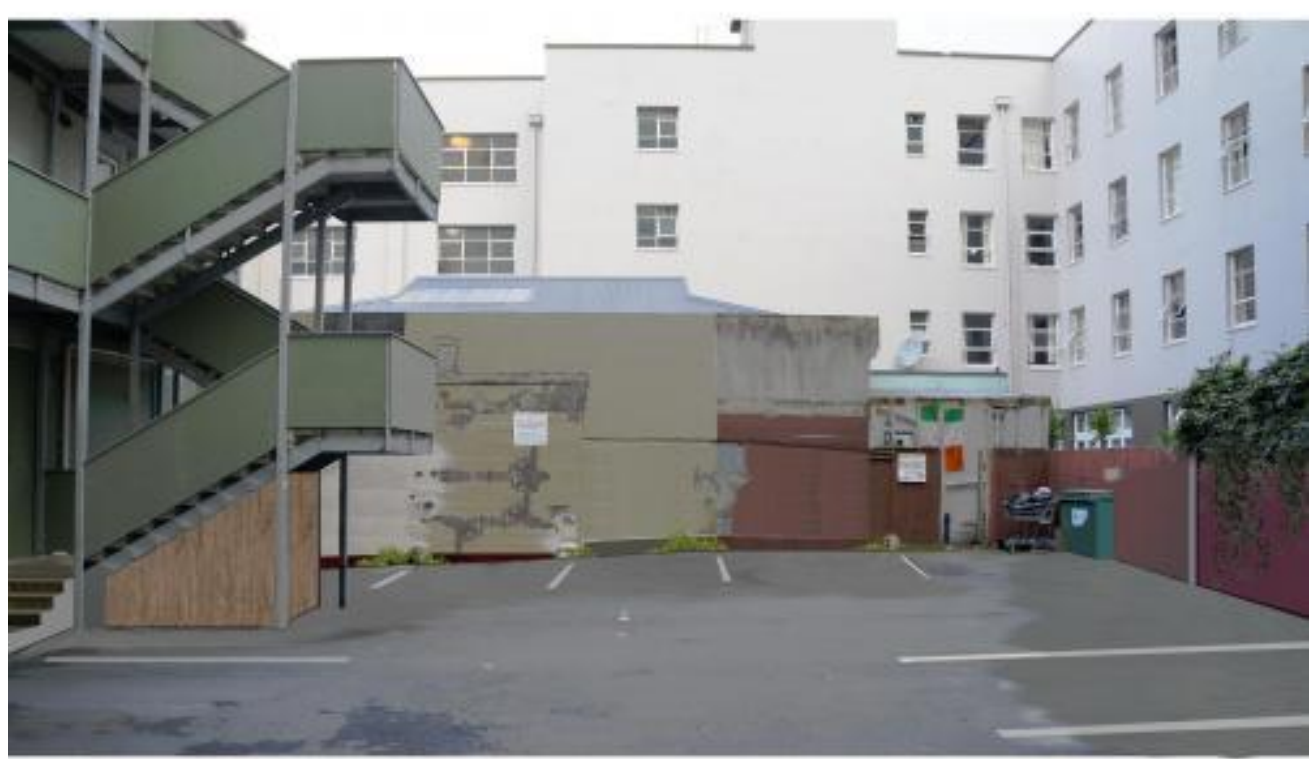

Q21.1 Please select one or ( $\max )$ two functions

Provide a community garden

Design a sports area

Install Wind Turbines

Install Solar Panels

Create graffiti on wall

Provide food market

Create an exhibition space

Design a space for green park 
Q22 Please suggest one function for space below (rooftop) on the assumption that the parking is removed.

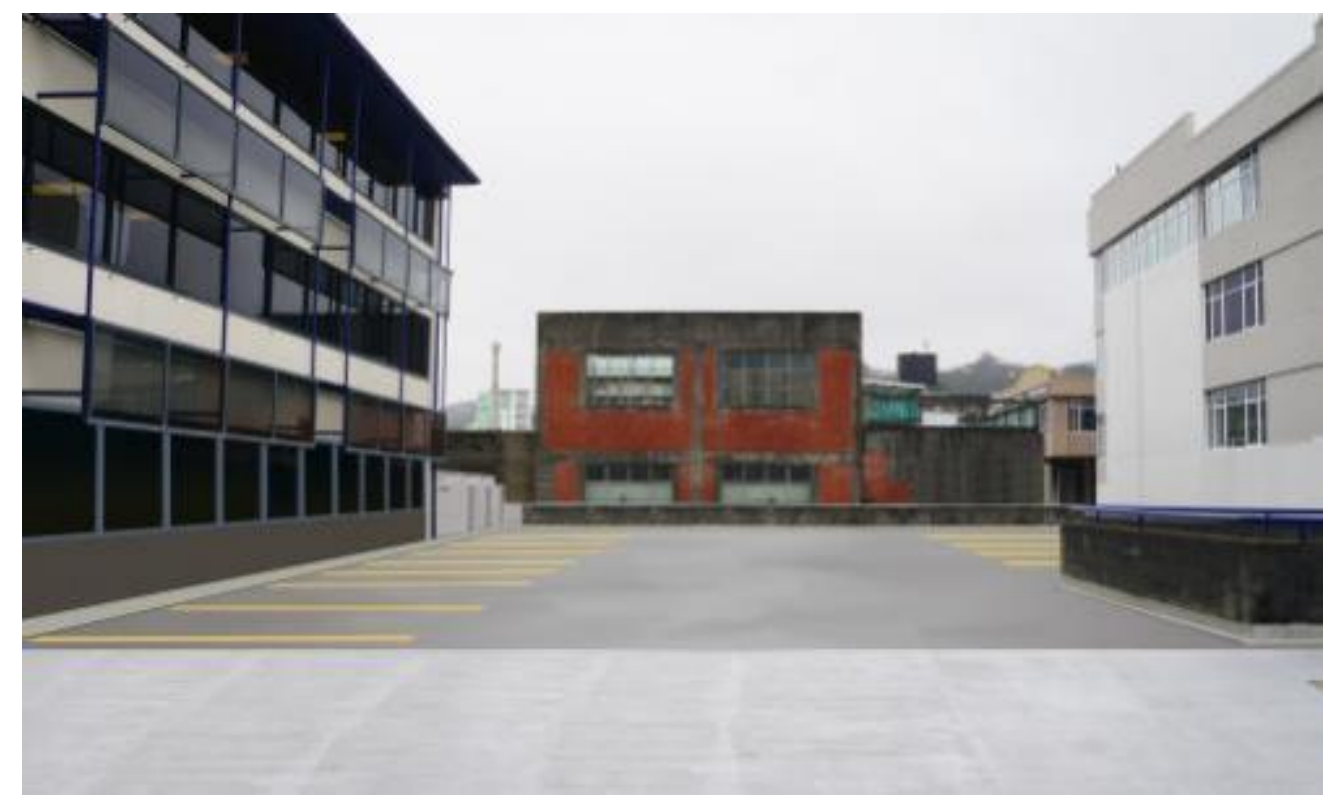

Q22.1 Please select one or (max) two functions

Provide a community garden

Design a sports area

Install Wind Turbines

Install Solar Panels

Create graffiti on wall

Provide a food market

Create an exhibition space

Design a space for green park

\section{End of Block: SURVEY STARTS}


Q23 Would you like to participate in the lucky draw?

Yes ( you will redirected to new page for signing up) (1)

No (2)

Q24 Do you want to be a part of focus group discussion probing the reasons behind visual preferences?

Yes (1)

No (3)

\section{Chapter 8: Focus Group Discussion}




\section{HOW CAN WELLINGTON CITY BE IMPROVED?}

participate in this study about Urban Leftover Spaces \& EARN \$10 CASH AS A REWARD

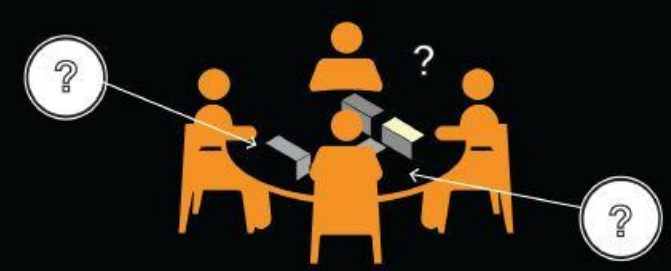

Who can participate?

Anyone from a Non-Built environment background / expertise

( 18 years old or above)

What's involved?

This FOCUS GROUP will discuss your opinions on the designs (that you imagine) for Leftover Spaces in Wellington City.

The discussion should take about 60-75 minutes (at max) to complete and is approved

from VUW ethics committee.

Discussion will be held in Victoria University, School of Architecture on 27th August at $12: 30 \mathrm{pm}-1: 45 \mathrm{pm}$

if you want to participate please send an email, apply before 24th August, 2018 (Friday)

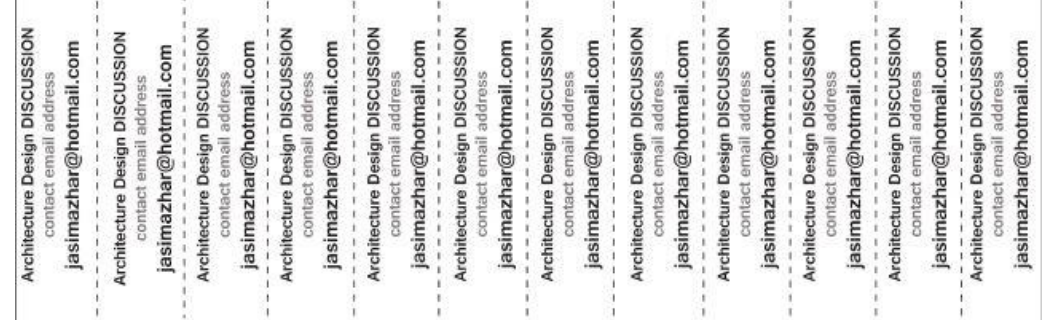

Appendix Figure 8.1 Invitation poster for Study 3 


\begin{tabular}{|c|c|c|c|c|c|}
\hline Male & \multicolumn{5}{|c|}{$\begin{array}{l}\text { AVERAGE (frequencies) OF MOST RECURRING } \\
\text { LIKEABLE DESIGN ATTRIBUTES }\end{array}$} \\
\hline \multirow{11}{*}{$\begin{array}{c}\text { Female } \\
\text { particip } \\
\text { ants } \\
\text { group }\end{array}$} & & $\mathbf{M}$ & $\mathbf{F}$ & $\mathbf{M}$ & $\mathbf{F}$ \\
\hline & Provide more vegetation & $39 \%$ & $40 \%$ & $84 \%$ & $86 \%$ \\
\hline & $\begin{array}{l}\text { Change of surface } \\
\text { materials / colours }\end{array}$ & $25 \%$ & $17 \%$ & $43 \%$ & $41 \%$ \\
\hline & $\begin{array}{l}\text { Improve maintenance } \\
\text { and cleanliness }\end{array}$ & $19 \%$ & $15 \%$ & $16 \%$ & $13 \%$ \\
\hline & Create a clear pathway & $24 \%$ & $29 \%$ & $12 \%$ & $13 \%$ \\
\hline & Remove boundary walls & $31 \%$ & $44 \%$ & $76 \%$ & $83 \%$ \\
\hline & \multicolumn{5}{|c|}{$\begin{array}{c}\text { AVERAGE (frequencies) OF LESS RECURRING } \\
\text { DESIGN ATTRIBUTES }\end{array}$} \\
\hline & Create seating space & $22 \%$ & $14 \%$ & $42 \%$ & $49 \%$ \\
\hline & $\begin{array}{l}\text { Install wind turbines and } \\
\text { solar panels }\end{array}$ & $13 \%$ & $11 \%$ & $50 \%$ & $42 \%$ \\
\hline & Remove car park & $17 \%$ & $8 \%$ & $\mathrm{~N} / \mathrm{A}$ & $\mathrm{N} / \mathrm{A}$ \\
\hline & Allow graffiti on the wall & $15 \%$ & $20 \%$ & $\mathrm{~N} / \mathrm{A}$ & $\mathrm{N} / \mathrm{A}$ \\
\hline & & $18-35$ & $35+$ & 18-35 & $35+$ \\
\hline $18-25$ & Provide more vegetation & $22 \%$ & $18 \%$ & $28 \%$ & $34 \%$ \\
\hline $\begin{array}{l}\text { and } 26+ \\
\text { years }\end{array}$ & $\begin{array}{l}\text { Change of surface } \\
\text { materials / colours }\end{array}$ & $13 \%$ & $09 \%$ & $15 \%$ & $13 \%$ \\
\hline $\begin{array}{c}\text { old } \\
\text { particip }\end{array}$ & $\begin{array}{l}\text { Improve maintenance } \\
\text { and cleanliness }\end{array}$ & $13 \%$ & $14 \%$ & $14 \%$ & $16 \%$ \\
\hline ants & Create seating space & $14 \%$ & $15 \%$ & $16 \%$ & $18 \%$ \\
\hline group & Remove boundary walls & $34 \%$ & $31 \%$ & $26 \%$ & $24 \%$ \\
\hline
\end{tabular}




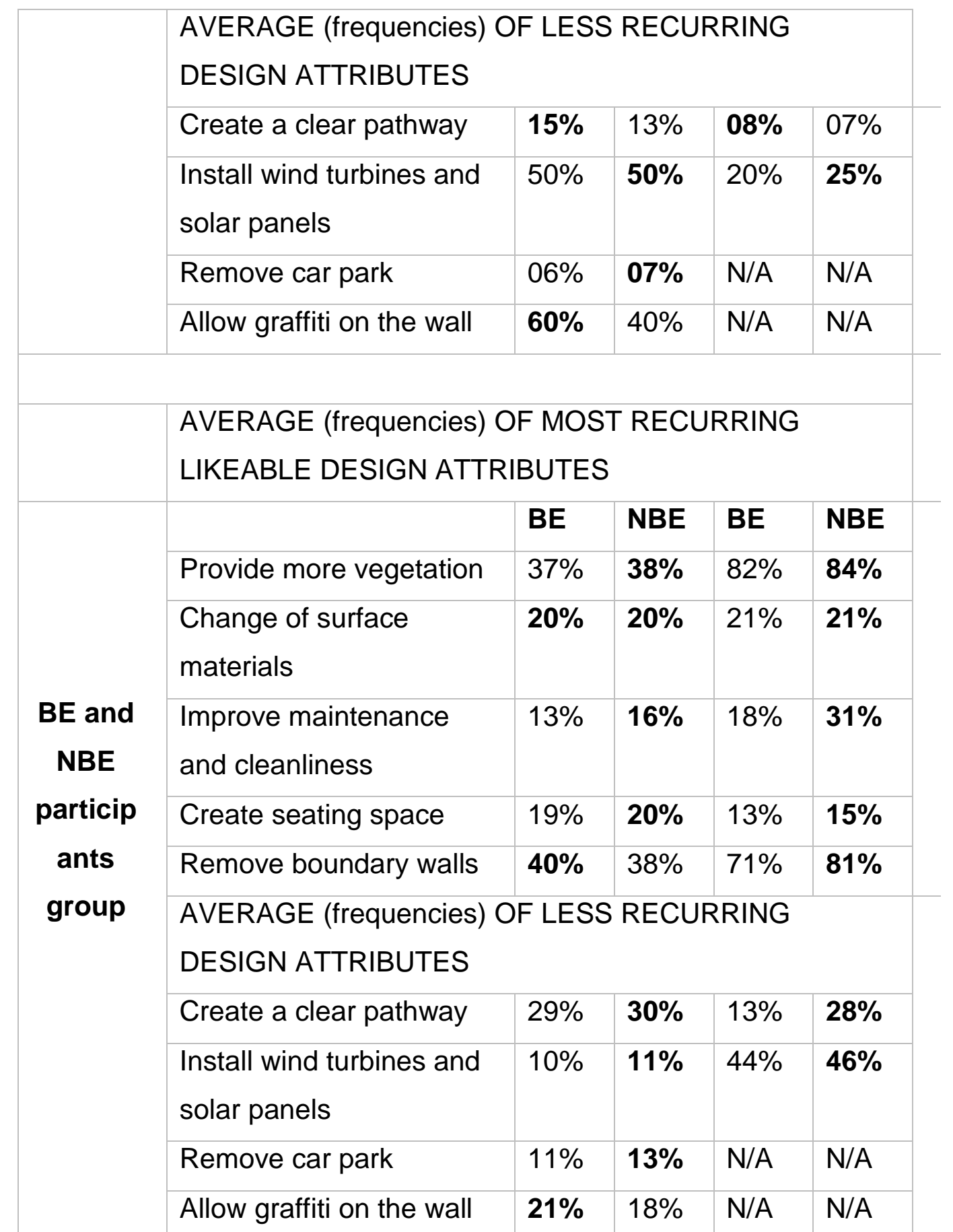

Table 9.1 Preference scores of two studies for all six types of leftover space 
